\title{
ELECTRON MICROSCOPY OF BUBBLES AND DISLOCATIONS IN HELIUM IMPLANTED METALS
}

by

Kevin John Stevens 
ELECTRON MICROSCOPY OF BUBBLES AND DISLOCATIONS IN HELIUM IMPLANTED METALS

by

Kevin John Stevens

Submitted for the degree of Doctor of Philosophy in Physics at Victoria University of Wellington

December 1993 


\section{Abstract}

The theoretical contrast in transmission electron microscope images of a superlattice of helium gas bubbles in copper is computed using the two-beam and many-beam dynamical theories of electron diffraction with the aim of measuring the density and size of dislocation loops associated with the bubble array. A wide range of parameters (foil thickness, diffracting vector, excitation error, defocus, and depth, radius, and strain-field of the bubble) is considered to construct a library of theoretical images and intensity profiles for a single, isolated bubble. Various criteria are applied to obtain a measurement of the bubble radius from the simulations but the results are inaccurate because of the sensitive dependence of the intensity profile on the imaging parameters. A better measurement is obtained by comparing simulations to an experimental through-focal series. Intensity profiles from a single stack of bubbles are modelled and electron diffraction from superlattices is simulated. The results obtained suggest that the bubble ordering is of limited extent.

A library is made of the theoretical contrast when imaging a system of dislocation loops punched out along the $<110>$ directions by the growth of gas bubbles ordered on a superlattice aligned with the host fcc matrix. These image simulations use the displacement fields surrounding loops and bubbles predicted by isotropic elasticity theory. For a variety of structures involving loops and bubbles, the following imaging parameters were investigated: beam direction, foil normal, diffracting vector, excitation error, number of beams, and defocus. These simulations indicate that it should be possible to image the small dislocations at high density thought to be present in the bubble lattice, provided wellfocussed micrographs taken under strong two-beam conditions can be obtained. In practice it proved difficult to tilt specimens containing superlattices to strong two-beam conditions because of the deterioration in crystallinity resulting from the implantation. However, the method was successfully tested by imaging loops associated with bubbles produced in lower concentrations by low dose implantations. 


\section{Preface}

A brief review is given in chapter 1 of past work done on the theories of formation of superlattices of gas bubbles and of the punching of dislocation loops from overpressured bubbles. How the displacement field solution of a dislocation with a mixed screw and edge nature is obtained from elasticity theory is outlined. This gives the background needed for a discussion later in the chapter of the mechanism by which dislocation loops are punched from bubbles.

The theory of image formation in a transmission electron microscope is reviewed in chapter 2. The differential equations of Howie and Whelan provide the starting point for the discussion. The more complicated many-beam and weak-beam theories are then discussed. The latter theory is particularly useful because of the potentially higher resolution it offers. When imaging gas bubble superlattices, or related defects such as voids, defocus is an important parameter and so the background to this contrast mechanism is summarised. High resolution image formation based on the multislice theory is discussed briefly. However, this theory is not used extensively in this thesis because of the limitations of the technique for studying dislocations. Instead, images of dislocations are more commonly interpreted with the rapid image simulation technique of Head et al. This technique is based on a generalised cross-section construction that uses the form of the anisotropic elasticity solution for the displacements around a long straight dislocation.

The displacement field solutions for bubbles and loops are obtained in chapter 3 . These solutions are used extensively in chapters 5 and 6 to simulate the diffraction from combinations of gas bubbles and the punched dislocation loops that may exist in implanted metals containing gas-bubble superlattices.

Before attempting to match experimental images of complex structures in irradiated metals with simulated images it was decided to learn the techniques that would be needed by examining first the simpler structures occurring in lightly deformed or quenched copper specimens. The micrographs of simpler structures which the simulations are used to interpret, together with the equipment and techniques used, are presented in chapter 4.

In chapter 5 the measurement of the radius of a bubble using a through-focal series is described. The method used is based on investigating how the positions of the maximum 
and minimum in an intensity profile through the Fresnel fringes about a bubble vary with the degree of defocus, in comparison to the theory. An accurate measurement of the bubble volume fraction, allows an estimate to be made of the number of host metal atoms displaced from bubble cavities. Those that do not escape to the surface must be accommodated in the host matrix, perhaps as dislocations.

In chapter 7 experimental images of implanted specimens containing gas bubbles are compared to the simulations of chapter 6 . The results indicate that, for prethinned specimens implanted to low dose the density of dislocation loops is lower than would be expected if bubble growth were to occur solely by dislocation punching.

The main chapters of the thesis are chapters 4 through to 7 . Much of the work described is of a complicated nature so as an aid to the reader abbreviated summaries of these chapters are included below.

\section{Abbreviated Summaries of the Four Main Chapters}

\section{Chapter 4 Experimental Techniques and Equipment}

TEM imaging, specimen preparation by quenching and by implantation, image simulation and processing with experimental results for comparison. Particular emphasis is given to the new line of work for Victoria University, namely - the production by quenching, and examination under TEM, of bubbles and dislocations at low density (to avoid the image overlap that occurs at high defect densities).

- Description of the equipment used. The quenching rig is discussed in greater detail because this is new to Victoria University.

- Comparison of experimental and simulated images of dislocation loops punched out from isolated bubbles and of long straight dislocations.

- Comparison of loop spacings on a glide cylinder with Bullough and Newman theory.

- Evaluation of the potential and the limitations of High Resolution EM for imaging bubbles. 


\section{Chapter 5. Simulations, Bubble Images and Diffraction}

Images are simulated for an isolated bubble and intensity profiles were obtained for bubbles in a stack. The image appearance is investigated for a range of values for the important parameters associated with microscope settings and bubble structures.

- A library of simulated images is presented to show the effects of systematic changes in the imaging parameters

- A comparison is made between actual experimental bubble images and those obtained in the simulations.

- The main results are (i) the apparent bubble radius varies significantly with the degree of defocus (ii) bubble images are not always dark in overfocus and white in underfocus (iii) stacks of bubbles have imaging characteristics which are different from those for isolated bubbles.

- The comparison between simulation and experiment for the superlattice spots produced in electron diffraction from the bubble array suggests that the bubble ordering in the gas bubble superlattice is of limited extent.

\section{Chapter 6. Simulations: Combinations of Bubbles and Dislocation Loops}

A program used to simulate the diffraction of electrons from structures containing loops near bubbles is described. The geometry of the structures and the diffraction conditions are specified through starting parameters selected as inputs to the program. Parameters are chosen to match those typical of the gas bubble superlattice. The appearance of the images for a range of imaging conditions is investigated.

- It is shown that the imaging behaviour of a structure containing a set of loops and bubbles is complicated.

- It is suggested that in well focussed micrographs taken under strong twobeam conditions it should be possible to identify the presence of dislocations 
Experimental images taken from implanted specimens containing gas bubbles are compared with images calculated in the simulations described in the previous chapter.

- In specimens containing superlattices it is found that the Kikuchi lines are very diffuse (this is attributed to a deterioration in the crystallinity) and it is difficult to tilt such specimens to obtain the required strong two-beam conditions. Consequently, no firm conclusions have been reached on the dislocation densities in this case.

- For lower dose implantations into prethinned discs the population of bubbles and dislocations are at much lower densities. In this case there is sufficient crystallinity retained to allow the specimens to be tilted to obtain strong twobeam conditions.

- The density of dislocations found around bubbles in these low dose implantations is much lower than expected under the assumption that bubble growth is by dislocation punching. 


\section{Contents}

page

Title page

(i)

Abstract

(ii)

Preface

Contents

List of figures

List of tables

Acknowledgements

\section{Chapter 1. Introduction}

1.1 Aim of thesis

1.2 Isotropic elasticity theory

1.2.1 The Burgers Vector

1.2.2 Stress and Strain

1.2.3 Displacement Field Solutions for Screw and Edge Dislocations

1.3 Dislocation loop punching theory

1.3.1 Greenwood, Foreman and Rimmer theory

1.3.2 The Theory of Bullough and Newman for the Spacings of Loops

1.3.3 Dubinko theory for loop spacing and ordering of bubbles

1.3.4 Wolfer theory 
1.3.5 Helical dislocations

1.4 Competing mechanisms of bubble superlattice formation

\section{Chapter 2. The Theory of Image Formation in the TEM}

2.1 The differential equations of Howie and Whelan

2.1.1 Introduction

2.1.2 The Dispersion Surface

2.1.3 Description of the Howie-Whelan differential equations

2.1.4 Derivation of the Howie-Whelan differential equations

2.1.4.1 Scattering factors and the scattering geometry

2.1.4.2 Construction of the crystal potential

2.1.4.3 The column approximation and solving the Schrodinger wave equation

2.1.4.4 Extinction distances

2.1.4.5 Different forms of the Howie-Whelan differential equations

2.1.5 Analytic solutions to the Howie-Whelan differential equations

2.2 Many beam matrix formulation of diffraction

2.2.1 Bloch waves

2.2.2 Darwin amplitudes

2.2.3 Imperfect crystal

2.3 Weak beam theory

2.3.1 Analytical solution for the Bloch wave coefficients

2.3.2 Bloch wave amplitudes in a deformed crystal

2.3.3 The relative size of the Bloch wave coefficients

2.3.4 Strength of the Scattering between Bloch Waves 
2.4 Multislice theory

2.4.1 Introduction

2.4.2 Calculation of the wave function at the foil exit surface 2-38

2.4.3 The lens transfer function

2.5 Defocussing the wave function

2.6 The generalised cross-section construction

2.7 Summary

\section{Chapter 3. Defect Displacement Fields}

3.1 Burgers equation

3.2 Angular dislocation

3.3 Hexagonal dislocation loop

3.4 Bubble displacement field

3.5 Straight Dislocation - Anisotropic Elasticity Solution

3.6 Summary

\section{Chapter 4. Experimental Techniques and Equipment}

4.1 Description of the TEM

4.2 Specimen preparation

4.2.1 Preparation of target

4.2.2 Description of the accelerator and the target irradiation

4.2.3 Quenching

\subsubsection{Introduction}

4.2.3.2 The Design of the Quenching Cell

\subsubsection{3}

Quenching Results

4.2.4 Punching, jet electropolishing and ion beam thinning

4.3 Miscellaneous TEM techniques

4.4 The image simulation and processing system

4.5 Large resolvable loops - Maher and Eyre rules 
4.6 Experimental micrographs and analysis of: 4-34

4.6.1 Inclined dislocations 4-34

4.6.2 Isolated loops $\quad 4-47$

4.6.3 Spacing of punched loops 4-55

4.6.4 Helices $4-57$

4.7 HREM, lattice fringe pictures and multislice simulations 4-58

$\begin{array}{lll}4.8 & \text { Summary } & 4-65\end{array}$

\section{Chapter 5. Simulations, Bubble Images and Diffraction}

5.1 Introduction 5-1

5.1.1 Introduction $5-1$

5.1.2 In-Focus contrast at the centre of an equilibrium bubble 5-6

5.1.3 The dependence of the contrast at the centre of an equilibrium bubble on excitation error and depth

5.2 Isolated bubbles - dependence of the contrast on defocus, strain and diffracting vector

5.2.1 Defocus and Bubble Radius

5.2.1.1 Measurement of the Bubble Radius using the Position of the First Minimum or Maximum in the Intensity Profile

5.2.1.2 Measurement of the Bubble Radius using the Position of the First Maximum in the Modulus of the Slope in the Intensity Profile

5.2.1.3 Measurement of the Bubble Radius using the Position of the First Maximum in the Visibility of the Intensity Profile

5.2.1.4 Conclusions 
5.3 Contrast from bubble stacks

5.4 Helium in a range of metals and other gas-metal combinations

5.5 Comparison to an experimental through-focal series

5.6 Facetted Cavities

5.7 Superlattice spots

Chapter 6. Simulations: Combinations of Bubbles and Dislocation Loops

6.1 The simulation program

6.1.1 Introduction

6.1.2 Calculation of the Scattering Matrices

6.1.3 The Numerical Integration

6.1.4 The Displacement Field

6.1.5 The Program Geometry

6.2 Simulations

6.2.1 Introduction

6.2.2 Simulations of Individual Defects

6.2.3 Simulations of Punched-out Dislocation Loops

6.2.3.1 The Layer Structure

6.2.3.2 Simulations as the Bubble Pressure changes

6.2.3.3 The appearance of a Superlattice

6.2.3.4 Simulations as the excitation error changes

6.2.3.5 Out-of-focus Simulations

6.3 Summary: Best Conditions for Imaging Loops and

Prospects for Finding Loops 


\section{Chapter 7. Experimental Evidence for Dislocation Loops}

\section{Near Bubbles}

7.1 Variation in the structure with changes in the dose, the depth and the irradiation conditions

7.1.1 The Density of Dislocation Loops

7.1.1.1 Introduction

7.1.1.2 Experimental Problems in imaging a High

Density of Defects

7.1.1.3 Experimental Results from Low Dose Irradiations

7.1.1.4 The Theoretical Density of Dislocation

Loops in the Low Dose Irradiations

7.1.2 Experimental Evidence for the Punching of

Dislocation Loops by Small Bubbles

$7-14$

7.1.2.1 Quenched Metals

7-14

7.1.2.2 Tritium Charging

$7-15$

7.1.2.3 Low Energy Implantations

7.1.2.4 Low Dose Irradiations of a Prethinned

Copper Disc

7.1.2.5 Electron Irradiations

7.1.3 Implications for Implantations to a Higher Dose

Chapter 8. Conclusions. 


\section{List of Figures}

Figure

page

1.1 FS / RH definition of the Burgers vector

1.2 Stress cube

$\begin{array}{llr}1.3 \text { Screw dislocation } & 1-10\end{array}$

1.4 Edge dislocation $\quad 1-12$

1.5 Bubble and dislocation loop parameters 1-13

1.6 Bubble and surrounding atom planes before dislocation punched out 1-18

1.7 Bubble and surrounding atom planes after dislocation punched out 1-18

$\begin{array}{lll}1.8 & \text { Shear stress vs distance from loop } & 1-21\end{array}$

1.9 Two-dimensional nature of the interaction between loops 1-25

1.10 Bubble pressure required for loop punching vs bubble radius $\quad 1-29$

1.11 Prismatic punching sequence 1-31

2.1 The Ewald sphere construction $\quad 2-3$

2.2 The dispersion surface construction $2-5$

2.3 Geometry used to prove some approximations 2-22

2.4 Wave propagating through a perfect and composite sandwich crystal 2-29

2.5 Extinction distance vs number of beams included in finding the eigenvalues 2-31

2.6 Dispersion surface geometry at weak beam conditions 2-35

$\begin{array}{lll}2.7 & \text { Bubble embedded in crystal matrix } & 2-42\end{array}$

$\begin{array}{lll}2.8 & \text { Generalised cross-section geometry } & 2-44\end{array}$

3.1 Point force acting on a dislocation loop 3-3

3.2 Angular dislocation 3-5

3.3 A hexagonal dislocation loop constructed from angular dislocations 3-8

3.4 Discontinuity in the displacement field for a loop when the discontinuity lies outside the loop

3.5 Discontinuity in the displacement field for a loop when the discontinuity lies inside the loop

3.6 Images of the displacement field for a loop 
3.7 Sections through the displacement field of a loop

3.8 Sections through the displacement field of an isolated bubble and two bubbles separated by $50 \AA$

4.1 The annealing apparatus

4.2 The particle accelerator

4.3 The quenching equipment

4.4 Vacuum, pressure, electrical and water connections for the quenching equipment

4.5 Cutaway schematic of quenching cell

4.6 Jet electropolishing system

4.7 Ion beam thinning apparatus

4.8 Rocking curves

4.9 On zone Bloch wave simulation of intensity and phase vs thickness

4.10 Maher and Eyre rules

4.11 Experimental micrographs and simulations of long straight threading dislocations

4.12 Experimental micrographs and simulations of dislocation loops in copper formed by quenching from an argon atmosphere

4.13 Application of Maher and Eyre rules 4-56

4.14 Spacing of quenched loops and comparison to Bullough-Newman theory 4-57

4.15 Helical dislocations in quenched copper 4-57

4.16 Facetted particles whose atom positions are used to generate facetted voids 4-60

4.17 Supercells of a facetted cavity and a multislice simulation 4-63

4.18 Lattice fringe picture in a helium irradiated copper specimen 4-64

5.1 Transmitted intensity at centre of bubble vs thickness of foil

5.2 Depth dependence of the transmitted intensity at the centre of a bubble for non-zero excitation error

5.3 Rocking curve of the transmitted intensity at the centre of a bubble 
5.4(a) Contrast profiles from different radii bubbles as defocus varies

5.4(b) Effective radius using Fresnel fringe peak position criterion of different radii bubbles vs defocus

5.5 Intensity profiles of a $10 \AA$ radius bubble for a defocus of $-9000 \AA$ to $9000 \AA 5-22$

5.6 Fresnel fringe peak positions of a $10 \AA$ radius bubble vs defocus

5.7 Effective radius using maximum magnitude of the slope peak position criterion of different radii bubbles at $4000 \AA$ defocus

5.8 Effective radius using maximum magnitude of the visibility peak position criterion of different radii bubbles at $4000 \AA$ defocus

5.9 In focus simulation of a $10 \AA$ radius bubble with non-zero displacement field

5.10 Through-focal series of a $10 \AA$ a radius bubble with non-zero displacement field

5.11 Contrast profiles and Fresnel fringe peak positions vs defocus for $\mathbf{g}=[220]$

5.12 Intensity at the centre of bubbles stacks vs thickness

5.13 Rocking curves of the intensity at the centre of bubble stacks

5.14 Transmitted intensity at the centre of bubble stacks vs defocus for $\mathrm{w}=0.0$ as the number of bubbles in the stack varies

5.15 Transmitted intensity at the centre of bubble stacks vs defocus for $\mathrm{w}=15.0$ as the number of bubbles in the stack varies

5.16 Intensity profiles of bubble stacks at various defocus levels and $w=0.0$ as the number of bubbles in the stack varies

5.17 Intensity profiles of bubble stacks at various defocus levels and $\mathrm{w}=15.0$ as the number of bubbles in the stack varies

5.18 Intensity profiles of bubble stacks at various defocus levels and $w=0.0$ as the number of bubbles in the stack varies for a constant foil thickness

5.19 Contrast profiles of a helium bubble in a range of metals

5.20 Experimental and theoretical positions of the maximum and minimum in intensity profiles through a helium gas bubble in copper

5.21 Through focal series of facetted cavities in aluminium

5.22 Projections of an octahedral cavity for the beam directions of figure 5.24 5-60

5.23 Simulated images of a cavity with $\{111\}$ and $\{100\}$ facets 
5.24 Simulated and experimental image profiles through facetted cavities

5.25 Comparison of experimental peaks in intensity profiles to theory

5.26 Power spectrum of a $10 \AA$ radius bubble as the crystal is tilted

5.27 Simulated SAD intensity profiles through superlattice spots for different domain structure factors

5.28 Simulated SAD photos of superlattice spots for different domain structure factors

6.1 Flowchart of the image simulation program

6.2 Check of the geometry of the structure

6.3 Stereographic projections for $\mathbf{B}=[001], \mathbf{B}=[011]$ and $\mathbf{B}=[111]$.

6.4 Simulations of individual loops next to a bubble

6.5 Simulations for various B's, g's and F's, BF and DF

6.6 Layer structure for interstitial dislocation loops and bubbles

6.7 Simulations as the depth of the defects varies

6.8 Simulations as the defect depth varies, through the transition layer region.

$$
\mathbf{B}=[001]=\mathbf{F}, \mathbf{g}=[-1,1,-1] \text { and the thickness of the foil }=2 \varepsilon_{\mathrm{g}}
$$

6.9 Simulations as the bubble pressure varies

6.10 Simulations of two bubbles with twelve dislocation loops around each.

The bubble superlattice constant is varied

6.11 Simulations of a bubble with twelve dislocation loops as the distance between the loops and the bubble is increased

6.12 Simulations with $\mathbf{B} \neq \mathbf{F}$, i.e. the foil surface is inclined to the beam direction

6.13 TEM images as the excitation error $\mathrm{w}$ varies

6.14 Weak beam simulations of loop/bubble structures

6.15 Simulations for various defocus values of one loop next to a bubble

6.16 Typical ball model representation of bubbles on an fcc lattice 
7.1 Comparison of CBED patterns in unirradiated and irradiated copper specimens

7.2 Weak and two-beam micrographs of a helium gas bubble superlattice in copper

7.3 Two-beam micrographs of low dose helium irradiated copper 


\section{List of Tables}

Table

1.1 Loop spacings for a ten loop row

4.1 Quenching results 4-18

4.2 Jet Electropolishing conditions $\quad$ 4-24

5.1 The differences between defects similar to gas bubbles $\quad 5-2$

5.2 Extinction distances used in the simulations for various $\mathbf{g}$

5.3 Table of parameters required to simulate different combinations of a gas bubble and the surrounding metal matrix

6.1 g.b values of [110] type Burgers vectors 6-15

6.2 Angles between $\mathbf{n}$ and $\mathbf{B}$ for the six [110] type edge on dislocation loops 6-15

6.3 Image classifications for edge dislocations in an fcc crystal 6-15 


\section{Acknowledgements}

The supervision and assistance received from Drs Peter Johnson ${ }^{1,2}$ and Colin Cook $^{1}$ whilst writing this thesis is gratefully acknowledged. Assistance with specimen preparation by Cliff Snell ${ }^{3}$, Tong Young ${ }^{1}$ and David Housden ${ }^{1}$ was appreciated. As was the photographic work of Karen Reader ${ }^{2}$, Laurel Quirke ${ }^{2}$, Margaret Alison ${ }^{2}$ and Teeba Lundy ${ }^{2}$, and the help with the computing received from Tony Martindale 6 and Grant Carter ${ }^{1}$. The work was also helped by discussions and experimental work with Drs David Cockayne ${ }^{5}$, Rob Glaisher ${ }^{5}$ and Zou Jin ${ }^{5}$, as well as Robert Thomson ${ }^{2}$, and Drs David Mazey and Peter Gilberd ${ }^{1,4}$ (who also provided considerable help during the writing up stages). The stereographic projections and ball model figures were produced by the EMS programs of Pierre Stadelman 7 , the installation of which was helped by Blair Hall ${ }^{11}$. The quenching equipment was built from a modified design of Dr W. Wampler ${ }^{8}$ by Cliff Snell ${ }^{3}$ and John Hawkins ${ }^{9}$. Nestor Zaluzec ${ }^{10}$ provided some programs, parts of which were useful. It is a pleasure to acknowledge the support of my family during the long and sometimes trying process of working on this thesis.

1. Physics Department, Victoria University of Wellington.

2. Electron Microscope Facility, Victoria University of Wellington.

3. Mineral Sciences Laboratory, Victoria University of Wellington.

4. Industrial Research Limited, Gracefield, Wellington.

5. Electron Microscope Unit, Sydney University.

6. Computer Services Centre, Victoria University of Wellington.

7. I2M-EPLF, Swiss Federal Institute of Technology, CH-1015 Laussanne, Switzerland.

8. Sandia National Laboratories, Albuquerque, New Mexico, U.S.A.

9. Mechanical Workshop, Victoria University of Wellington.

10. Argonne National Laboratories, Illinois, U.S.A.

11. Physics Department, Massey University 


\section{CHAPTER 1: INTRODUCTION}

\subsection{Aim of Thesis}

The physical properties of crystalline materials can be strongly influenced by the presence of dislocations and other defects in the atomic lattice. The ion-implantation of foreign atoms into a metal at low temperature may result in dislocation and defect densities that are amongst the highest that can be produced in a material. The aim of the present work is to develop and evaluate techniques, based on transmission electron microscopy (TEM), for measuring dislocation densities in the particular case of metals implanted with helium.

Inert gases such as helium are insoluble in metals and precipitate out to form gas bubbles on a microscopic scale (1 to $2 \mathrm{~nm}$ in diameter). At low temperatures $\sim 0.2 \mathrm{~T} \mathrm{~m}$ (where $\mathrm{T}_{\mathrm{m}}$ is the melting temperature of the metal), the mobility of vacancies in the metal is low and the ordering of bubbles to form a gas-bubble superlattice is a common feature. The superlattice has the same symmetry as the host metal (i.e., fcc superlattice in an fcc metal, etc.) and has rational alignment with the underlying metal lattice. The lattice constant, al, of $6-8 \mathrm{~nm}$, is a factor $\sim 20$ times that of the metal. At these low temperatures, bubble growth is thought to be by an athermal pressure-driven process where metal atoms are forcibly ejected from bubbles by the high pressure of the gas they contain. If the gas pressure exceeds the equilibrium pressure the bubble is said to be overpressured. The punching of interstitial dislocation loops from overpressured bubbles is the process most commonly proposed [1]. These prismatic loops are expected to have diameters comparable with the bubble size. Such loops have been observed around large isolated bubbles of approximately $30 \mathrm{~nm}$ diameter [2-5] and around precipitates having a coefficient of thermal expansion which is different from that of the host matrix [6]. Some theories of bubble ordering are based on the presence of such loops on the common glide cylinders linking nearestneighbour bubbles in the array [7].

The assumptions underlying these theories of bubble growth and bubble ordering have led to the suggestion by Johnson [8] that the density of dislocations associated with the gas bubble superlattice could be close to, or even exceed, the upper density limit proposed 
by Cotterill [9]. Cotterill has considered the limiting strain energy that a metal can withstand and yet retain a crystalline character. For copper the limiting dislocation density has been estimated to be $10^{16}$ to $10^{17} \mathrm{~m}^{-2}$. Kittel [10] has estimated that electron microscopy can measure dislocation densities of about $10^{15}$ to $10^{16} \mathrm{~m}^{-2}$. Johnson [8] has pointed out that if all the metal atoms displaced from bubbles were to be accommodated in dislocation loops lying on the common glide cylinders between nearest-neighbour bubbles in the ordered bubble array, the Cotterill limit would be exceeded by several factors of ten.

Stress relief processes will tend to lower the strain energy through a coarsening of the dislocation structure. At low temperatures, however, thermally driven processes of stress relief will be somewhat restricted and it could be expected that the dislocation structure might remain at high density. High levels of damage accompany the ionimplantation process. Each atom in the metal will be displaced from its site in the lattice some 10 to 20 times over the course of the implantation as a result of collisions between incoming helium ions and metal atoms. This collision damage results in two effects which could be expected to influence the dislocation structure. Firstly, it provides an athermal mechanism for stress relief by inducing atom movement and secondly, it is a source of interstitial-vacancy pairs that can contribute to the defect structure. These two effects could act in opposing senses so it is not clear what the overall effect of collision damage on dislocation density will be. However, if all the metal atoms displaced from bubble cavities are held locally in dislocations having sizes comparable with the spacing of the bubbles, then dislocation densities will approach or exceed the Cotterill limit [9].

The development of microstructure in metals during light ion-bombardment of metals is similar to other radiation damage processes. The radiation damage in nuclear fission reactors and potential fusion reactors provide a motivation for studying ionimplantation. In fission reactors $\alpha$-decay from nuclear fuels and $(n, \alpha)$ reactions cause helium to build up in the fuel and reactor components. Fusion reactors will use magnetic containment to confine the reacting hydrogen isotopes in the plasma. Helium produced in nuclear reactions will escape from the magnetic fields and damage the reactor walls. This bombardment will result in blistering, flaking and swelling of the walls. The radiation damage resulting from bombardment of reactor materials by ions having a range of energies has been simulated using monoenergetic ion beams from charged particle accelerators. 
Ion implantation is also a technique for preparing metals with desired surface properties $[11,12]$. For example nitrogen implantation of metals can lead to longer lasting hip replacements. The dislocation structure induced in the metal is important for properties such as wear resistance and corrosion resistance.

A bubble in which the gas pressure is zero or has a low value is termed a void. The ordering of voids to form a superlattice was discovered before the ordering of bubbles. Ordered void lattices have now been observed for the following: the fcc metals $\mathrm{Ni}$ [13] and $\mathrm{Al}[14,15]$, in $\mathrm{Ni}-\mathrm{Al}$ solid solution alloys [16], in stainless steel [17] as well as for a variety of bcc metals such as Mo [18] (see references [19-21] for reviews). Ordered anion voids have been observed in the alkaline earth halides: $\mathrm{SrF}_{2}, \mathrm{CaF}_{2}$ and $\mathrm{BaF}_{2}$ [22]. Theories and experimental work on void ordering influenced the later work on bubble ordering.

Barnes and Mazey [23] first found ordered helium bubbles in metals following helium ion-implantation. Subsequently Sass and Eyre [24] discovered ordered bubbles in Mo and Johnson and Mazey [25-28] found ordered He bubble arrays in the fcc metals $\mathrm{Cu}$, $\mathrm{Ni}$, and stainless steel and in the hcp Ti [29]. Ordered hydrogen bubble arrays were also found in $\mathrm{Cu}$ following proton irradiation $[30,31]$. Implantation of other inert gases such as $\mathrm{Ar}, \mathrm{Xe}$ and $\mathrm{Kr}$ also result in gas bubbles [32-37] which in some cases are ordered. Other metals subjected to helium implantation have been Au and V [38, 39]. A recent discovery is that a large lattice (a macrolattice) with ordering on a scale 20 times bigger than that of the superlattices can occur in $\mathrm{Au}$ [38]. All of the ordering has the same symmetry as the host matrix. A feature of superlattices in fcc metals is structural variants which are domains of ordered bubbles that have the same symmetry as the host metal but the basis vectors of the domain are rotated away from the basis vectors of the host metal [40-45]. Other work has concentrated on deducing how far the implanted ions penetrate into the metal. Depth profiling techniques determine how many of the implanted ions stay in the target and how these ions are distributed $[28,46-56]$. In-situ electron irradiations and heating the helium irradiated material in the hot-stage of a TEM has also given information on the concentrations and mobilities of interstitials and vacancies in the microstructure [28].

The Burgers vectors and loop normals of isolated dislocations can be measured by various methods. For example, for large, resolvable loops (i.e., both sides of the loop can be resolved) the Maher and Eyre rules [57] can be used. The images of small point defect 1- 3 
clusters can be interpreted by comparison with simulated images as discussed by Eyre et al. [58-61], Saldin et al. [62-64], Holmes [60, 65] and Wilkens et al. [66]. Hirsch, Howie and Whelan et al.[67-78] investigated the case of dislocations lying parallel to the foil surface. Head et al.[79-82] interpreted the images for isolated, long straight dislocations that thread through the foil.

The difficulty in applying the techniques developed for isolated dislocations to ionimplanted materials is the much higher density and smaller size of the defects. Most of the above techniques rely on taking electron micrographs of the same defect for a range of strong two-beam conditions. The two-beam conditions are near simple, low index, beam directions. With a high density of defects it is difficult to find the same defect at the required beam directions because the appearance of the complicated structure varies markedly as the specimen is tilted. Also because of the large population of bubbles it can be difficult to distinguish between which defects are loops and which are bubbles. This is an even greater problem when the bubble ordering is poor. At strong two-beam conditions, the strain fields of any defects will give broad black/white lobe images in bright field (chapter six). If the density of defects is very high, these strain field images overlap considerably. A broad black image may be obtained instead of distinct black/white lobes.

Computer simulations of the electron diffraction from isolated bubbles and loops have been done previously [58-66, 83-88]. To distinguish between bubbles and loops in this thesis simulations are made of the diffraction from loops inserted between bubbles ordered on a bubble lattice. The aim is to find conditions where the loop contrast is strong and the bubble contrast is as weak as possible. This will enable the loops in ion-implanted materials to be counted and their sizes determined.

The dislocation structure associated with ordered bubble arrays has not been the subject of detailed investigation in previous work. However, the indications are that the dislocation images are on a fine scale, comparable with bubble sizes, and are at very high density. Weak beam images [89-96] have a higher resolution than images taken under strong two-beam conditions. This improved resolution may be useful in studying these high defect density materials. A technique based on simulating images for comparison with weak beam micrographs is used to overcome the increased problems in interpreting the experimental micrographs. 


\subsection{Isotropic Elasticity Theory}

The displacement fields of screw and edge dislocations used by Hirsch et al. [67-78] and Cockayne et al. [89-96] for image simulation, are solutions in isotropic elasticity theory. These solutions will be obtained in section 1.2 after the necessary elasticity theory has been reviewed. The theoretical background to isotropic elasticity in section 1.2 follows the treatment of Hirth and Lothe [97].

\subsubsection{The Burgers Vector}

In the so-called FS/RH (Finish $\underline{\operatorname{Start}}$ / ight $\underline{H}$ and) convention of Bilby et al. [98], the Burgers vector of a dislocation is conventionally defined by a line integral taken around the dislocation line, as shown in figure 1.1 (on the next page). The Burgers vector (b) is the vector required to complete the circuit in figure 1.1(b). The same atoms in the closed circuit (FS) in figure 1.1(a) when a dislocation is present are connected up in the perfect crystal of figure 1.1(b). The circuit is traversed in the direction that the fingers of the right hand $(\mathrm{RH})$ would curl if the thumb pointed out of the page. The thumb points along the positive dislocation line direction. The mathematical expression for this is

$$
\mathbf{b}=\int_{\mathrm{c}} \frac{\partial \mathbf{R}}{\partial \mathrm{l}} \mathrm{dl} .
$$

In equation $1.1 \mathbf{R}$ is the displacement field representing the distortion of the crystal due to the dislocation.

Parts (c) and (d) of figure 1.1 show how to find the Burgers vector of an edge dislocation loop. Conventionally the loop normal $\mathbf{n}$ is defined as a vector, which is perpendicular to the inserted plane of atoms, and points upwards in the foil. The dislocation line (u) defines the boundary of an area over which slip displacement has occurred. For a dislocation loop the dislocation line bends around in a circle. The positive sense of the 

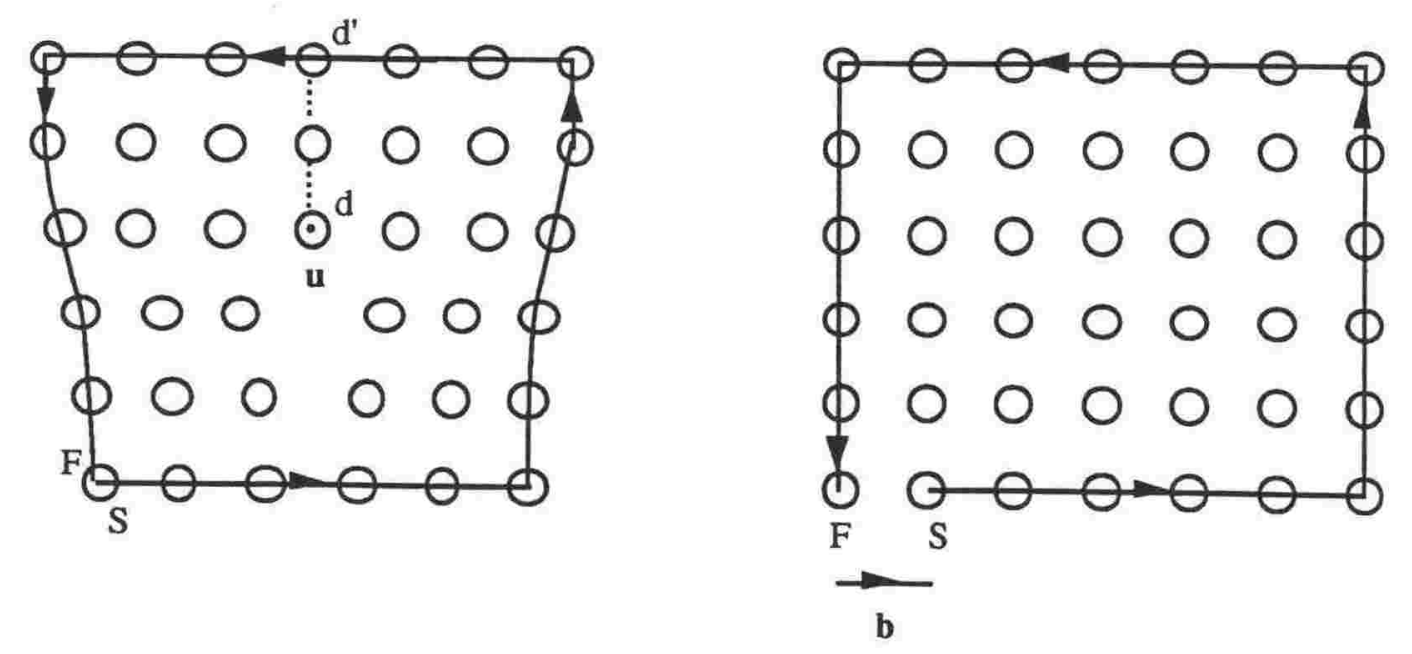

(a)

(b)

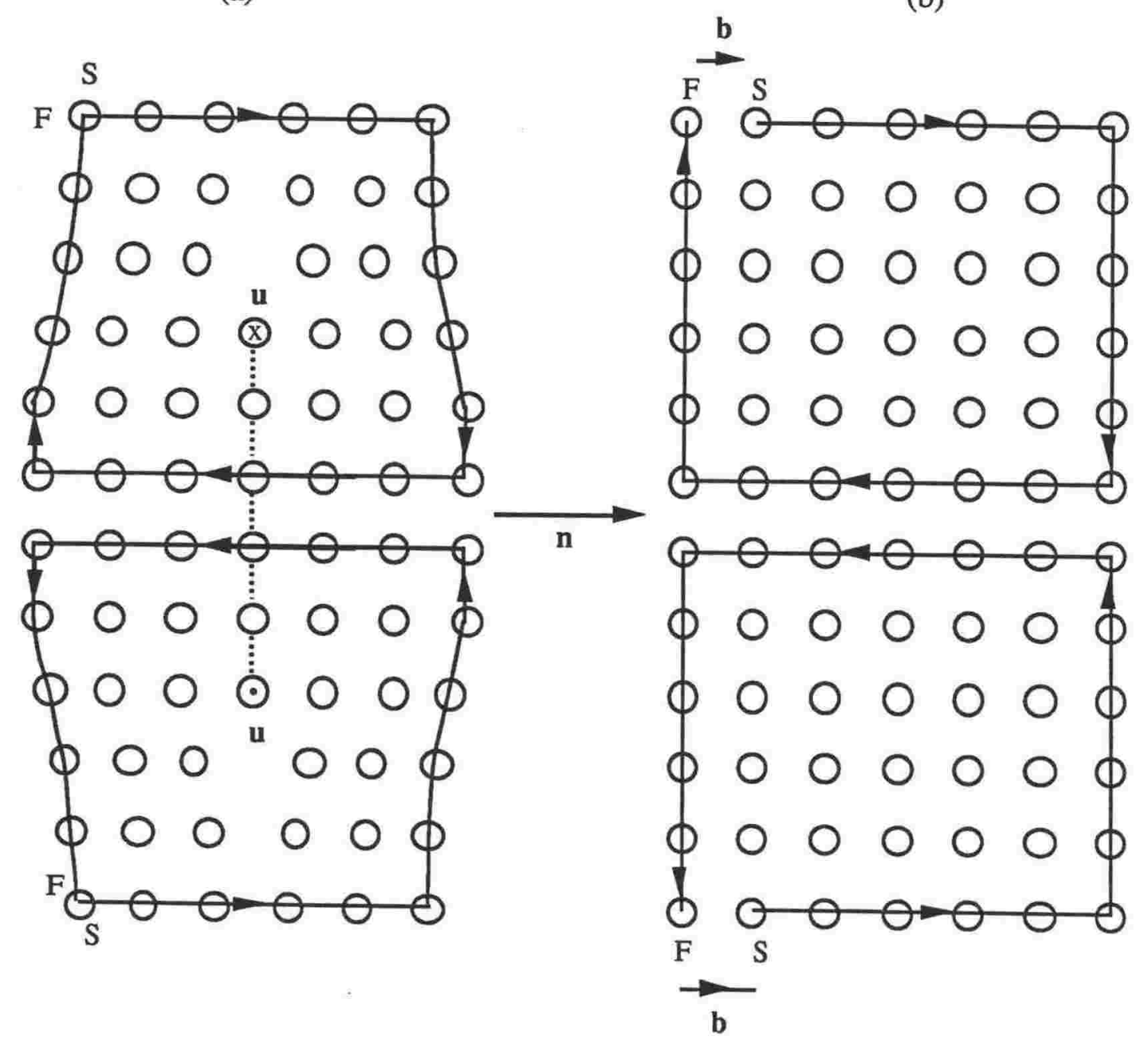

(c)

(d)

Figure 1.1: Circuits taken for the line integral of equation 1.1 in a simple cubic crystal:

(a) around an edge dislocation. The dislocation line is out of the page. The dashed line dd' marks the intersection of the plane of interstitial atoms perpendicular to the plane of the paper with the paper.

(b) around the perfect crystal.

(c) around an interstitial edge dislocation loop. The dashed line indicates a row of interstitial atoms in the plane of the diagram.

(d) around the crystal 
dislocation line is the clockwise direction when looking from above the foil down upon the loop. When the thumb of the left hand ( $\mathrm{LH})$ points in the direction of $\mathbf{n}$ the fingers curl along the positive direction of the dislocation line. Therefore the circuits around the top and bottom of the loop are in different directions by the RH rule. Thus $\mathbf{b}$ is found by a RH rule, but $\mathbf{n}$ by a $\mathrm{LH}$ rule.

\subsubsection{Stress and Strain}

The stress in a material is represented by a stress tensor. By considering the stress tensor $\sigma_{\mathrm{ij}}$ on an infinitesimal cube of material and requiring that the torques on this volume are zero for equilibrium, it can be shown that

$$
\sigma_{\mathrm{ij}}=\sigma_{\mathrm{ji}}
$$

Equation 1.2 uses a three-dimensional coordinate system, so that $\mathrm{i}$ and $\mathrm{j}$ can take the values 1,2 or 3 . The stress at a point in space is a three-dimensional vector. $\sigma_{\mathrm{ij}}$ is the ith component of this stress on the jth face of the cube as shown in figure 1.2. The jth cube face is orientated so that it has an outward drawn normal parallel to the $x_{j}$ axis.

The strain tensor $\left(\varepsilon_{i j}\right)$ is related to the displacements $u_{j}$ of the material from the positions that the material occupied in the unstressed continuum in a coordinate system $x_{j}$ by

$$
\varepsilon_{i j}=\frac{1}{2}\left(\frac{\partial u_{i}}{\partial x_{j}}+\frac{\partial u_{j}}{\partial x_{i}}\right)
$$

By definition of the strain

$$
\varepsilon_{\mathrm{kl}}=\varepsilon_{\mathrm{lk}}
$$

The indices $\mathrm{k}$ and $\mathrm{l}$ can take the values 1,2 or 3 . The condition for equilibrium of the system in the $\mathrm{x}_{\mathrm{i}}$ direction, when there are no applied external forces is

$$
\frac{\partial \sigma_{i j}}{\partial x_{j}}=0 .
$$

Equation 1.5 is derived by considering the stress on one face of the cube at $x_{i}$ and the stress on the opposite face of the cube at $x_{i}+d x_{i}$. The repeated index means make a summation 
over this index. That is $\mathrm{j}$ is cycled over its allowed values of 1,2 and 3 , independent of the value of $i$.

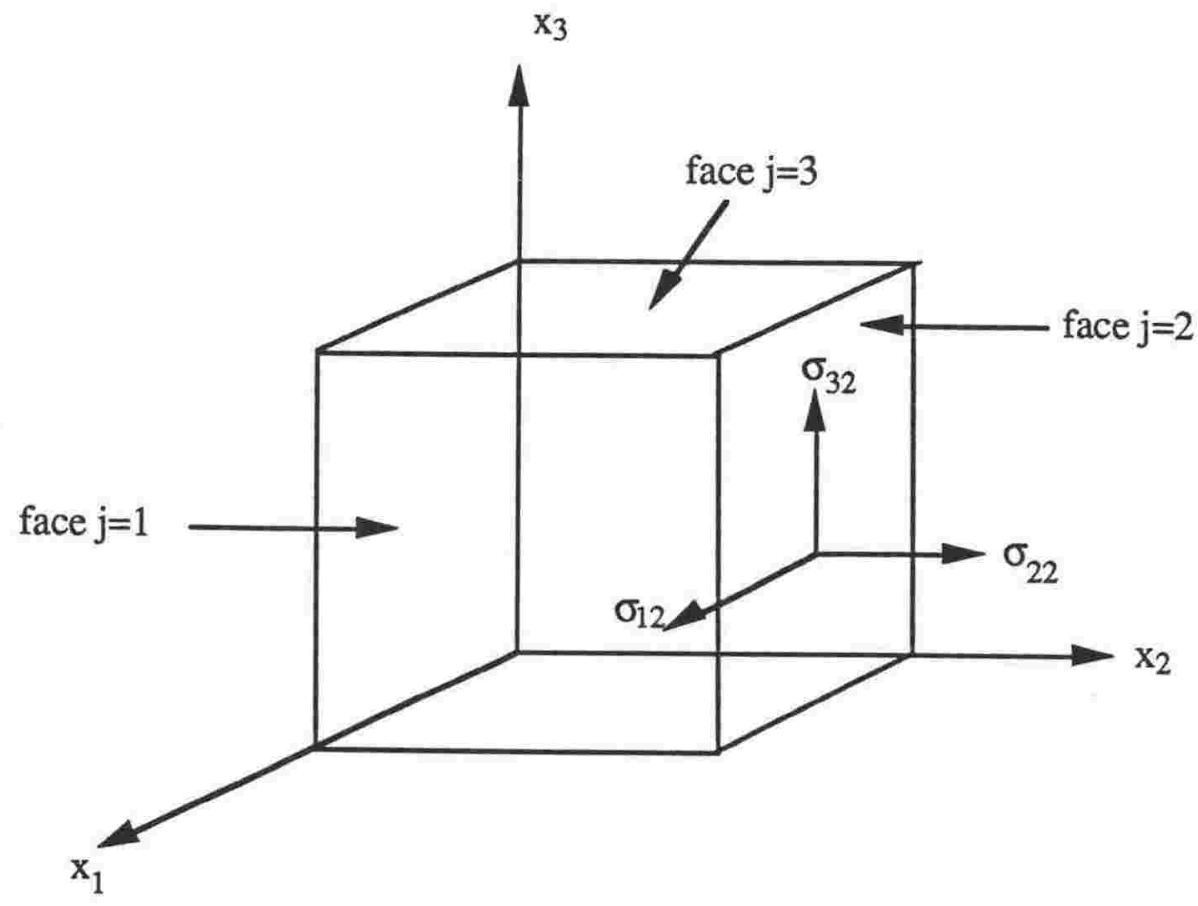

Figure 1.2: The cube construction and coordinate system used to label the stress tensor. The components of the stress tensor on the $j=2$ face of the cube are shown.

The elastic constants, $\mathrm{c}_{\mathrm{ijk}}$, of the material are defined by the relation between the stress and strain

$$
\sigma_{\mathrm{ij}}=\mathrm{c}_{\mathrm{ijk} l} \varepsilon_{\mathrm{kl}} \text {. }
$$

Then the stress can be more directly related to the displacements by

$$
\sigma_{i j}=c_{i j k l} \frac{\partial u_{k}}{\partial x_{1}}
$$

Equation 1.7 can be written out in matrix form, representing the elastic constants by a 9 by 9 matrix. In the matrix form the stress and strain are represented by 9 element column vectors. The symmetry relations of equations 1.2 and 1.4 between the elements of the stress and strain tensors and the order of differentiation when the strain energy is considered can be used to derive symmetry relations between these elastic constants:

$$
c_{i j k l}=c_{j i k l}=c_{i j l k}=c_{j i l k}=c_{k l i j} .
$$


These relations reduce the number of independent elastic constants from 81 to 21 . It is therefore possible to use a contracted notation on the subscripts of the elastic constant tensor. The contracted notation reduces the number of these subscripts from 4 to 2 . Mapping of the subscripts is done in pairs, i.e. $c_{\mathrm{ijkl}}$ is relabelled as $\mathrm{c}_{\mathrm{mn}}$ according to

$$
11 \rightarrow 1,22 \rightarrow 2,33 \rightarrow 3,23 \text { or } 32 \rightarrow 4,31 \text { or } 13 \rightarrow 5 \text { and } 12 \text { or } 21 \rightarrow 6 \text {, }
$$

where $\mathrm{ij}$ or $\mathrm{kl}$ are the pair of integers on the left hand side of the arrows. They are relabelled as $\mathrm{m}$ or $\mathrm{n}$ according to the integers on the right of the arrows above. For example the relabelling maps $c_{1123}$ to $c_{14}$. For cubic crystals the elastic constants must be invariant with respect to $90^{\circ}$ rotations. This requirement further reduces the number of independent elastic constants to 3 , namely $c_{11}, c_{12}$ and $c_{44}$. Several alternative elastic constants can be defined such as Poisson's ratio (v)

$$
v=\frac{c_{12}}{c_{11}+c_{12}}
$$

\subsubsection{Displacement Field Solutions for Screw and Edge Dislocations}

To find the displacement field solution to the equilibrium equation 1.5 for a screw dislocation with Burgers vector $\mathbf{b}_{\mathrm{s}}$ parallel to the $\mathrm{x}_{3}$ direction, the following boundary conditions are used:

$$
\mathrm{u}_{1}=0, \mathrm{u}_{2}=0 \text { and } \mathrm{u}_{3}=\mathrm{u}_{3}\left(\mathrm{x}_{1}, \mathrm{x}_{2}\right) \text {. }
$$

These boundary conditions require that the only distortion due to the dislocation is along the axis of the dislocation line but is independent of the coordinate in this direction. The solution is (as shown in figure 1.3):

$$
\mathrm{u}_{3}=\frac{\left|\mathbf{b}_{\mathrm{s}}\right|}{2 \pi} \tan ^{-1}\left(\frac{\mathrm{x}_{2}}{\mathrm{x}_{1}}\right)=\frac{\left|\mathbf{b}_{\mathrm{s}}\right|}{2 \pi} \theta \text {. }
$$




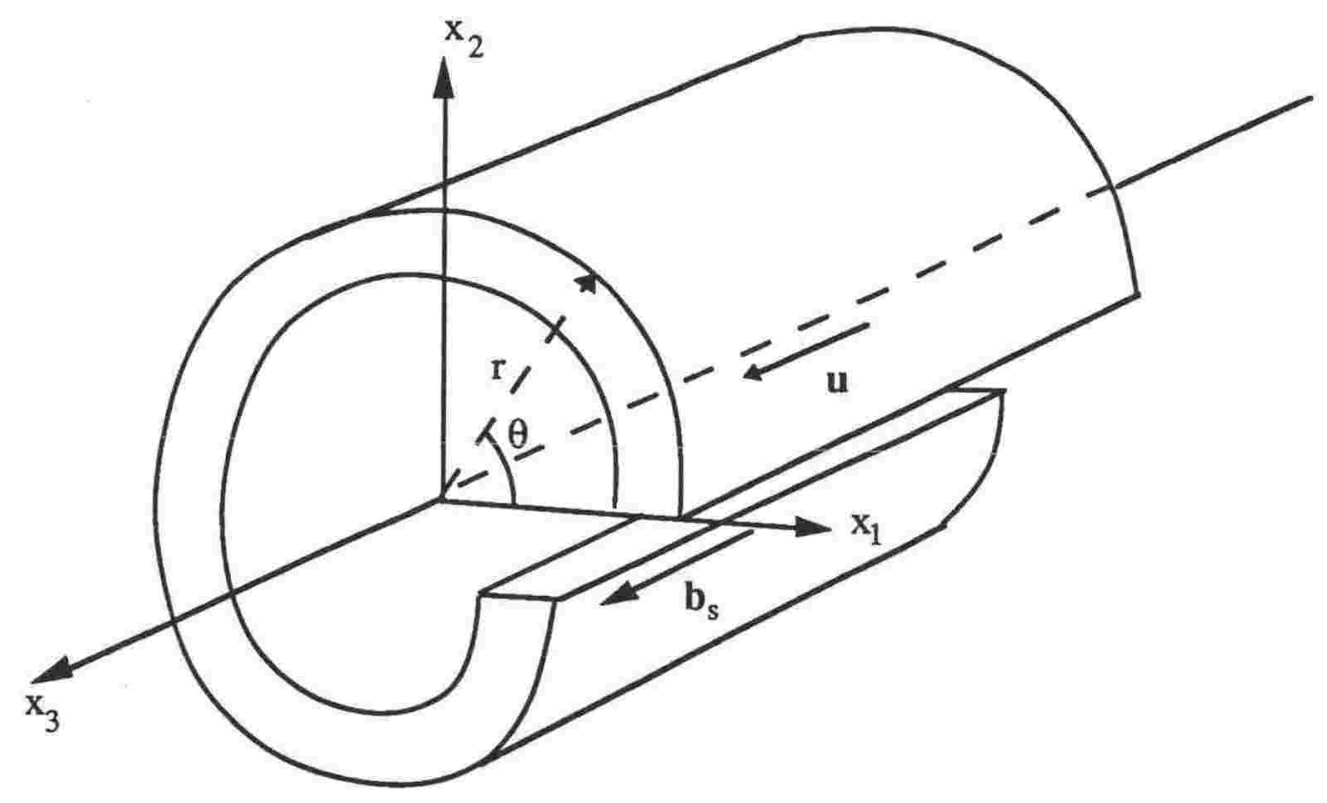

Figure 1.3: A right-handed screw dislocation that has a dislocation line along the axis of the cylinder. In the perfect crystal the cylinder is undeformed. A screw dislocation is formed by cutting the cylinder along one side in the plane formed by the $x_{1}$ and $x_{3}$ axes and then deforming the material below the cut relative to the material above the cut in a direction parallel to the $x_{3}$ axis.

Finding the solution to the equilibrium equations for an edge dislocation requires different boundary conditions. The boundary conditions for an edge dislocation with $\mathbf{b}_{\mathrm{e}}$ parallel to the $x_{1}$ axis and $u$ parallel to the $x_{3}$ axis are those of plane strain:

$$
\begin{gathered}
u_{1}=u_{1}\left(x_{1}, x_{2}\right), u_{2}=u_{2}\left(x_{1}, x_{2}\right), u_{3}=0, \text { and } \\
\frac{\partial u_{i}}{\partial x_{3}}=0 \quad, \text { for } i=1,2 \text { or } 3 .
\end{gathered}
$$

Consider the equilibrium equations and express the stresses in terms of the Airy stress function $\psi$ by

$$
\begin{gathered}
\sigma_{11}=\frac{\partial^{2} \psi}{\partial x_{2}^{2}} \\
\sigma_{22}=\frac{\partial^{2} \psi}{\partial x_{1}^{2}} \\
\text { and } \sigma_{12}=-\frac{\partial^{2} \psi}{\partial x_{1} \partial x_{2}} .
\end{gathered}
$$


Using equation 1.5 for the equilibrium between the stresses and equation 1.7 between stress and strain we obtain

$$
\nabla^{4} \psi=0
$$

By making the substitution

$$
\phi=\nabla^{2} \psi
$$

equation 1.15 becomes the familiar Laplace's equation that can be solved for $\phi$ starting with Legendre polynomials then using the boundary conditions to truncate the series and so on. The Airy stress function can be then found. This enables the stress to be obtained from equation 1.14. The strain is obtained from the stress by inverting equation 1.6. The displacement field around the edge dislocation follows by integrating the stress using equation 1.7:

$$
\mathbf{R}=\frac{1}{2 \pi}\left\{\mathbf{b}_{\mathrm{e}}\left(\frac{\sin 2 \theta}{4(1-v)}\right)+\mathbf{b}_{\mathrm{e}}{ }^{\wedge} \mathbf{u}\left[\frac{(1-2 v)}{2(1-v)} \log (\mathrm{r})+\frac{\cos 2 \theta}{4(1-v)}\right]\right\},
$$

where the displacement field vector $\mathbf{R}=\left(\mathrm{u}_{1}, \mathrm{u}_{2}, \mathrm{u}_{3}\right)$ and $\mathrm{r}$ has its usual meaning in cylindrical coordinate systems. In equation 1.17 the " $\wedge$ " symbol has been used to denote a crossproduct of two vectors. Figure 1.4 shows the form of the solution for $\mathbf{R}$ in equation 1.17 .

A general dislocation has both a screw and edge like character. The Burgers vector (b) can be divided into its edge ( $\left.\mathbf{b}_{\mathrm{e}}\right)$ and screw $\left(\mathbf{b}_{\mathrm{s}}\right)$ components

$$
\mathbf{b}=\mathbf{b}_{\mathrm{e}}+\mathbf{b}_{\mathrm{s}} \text {. }
$$

Then the solution for the displacement field is a combination of the solutions for the displacement fields of the edge and screw dislocations

$$
\mathbf{R}=\frac{1}{2 \pi}\left\{\mathbf{b}_{\mathrm{s}} \theta+\mathbf{b}_{\mathrm{e}}\left(\frac{\sin 2 \theta}{4(1-v)}\right)+\mathbf{b}_{\mathrm{e}} \wedge\left[\left(\frac{(1-2 v)}{2(1-v)} \log (\mathrm{r})+\frac{\cos 2 \theta}{4(1-v)}\right]\right\} .\right.
$$




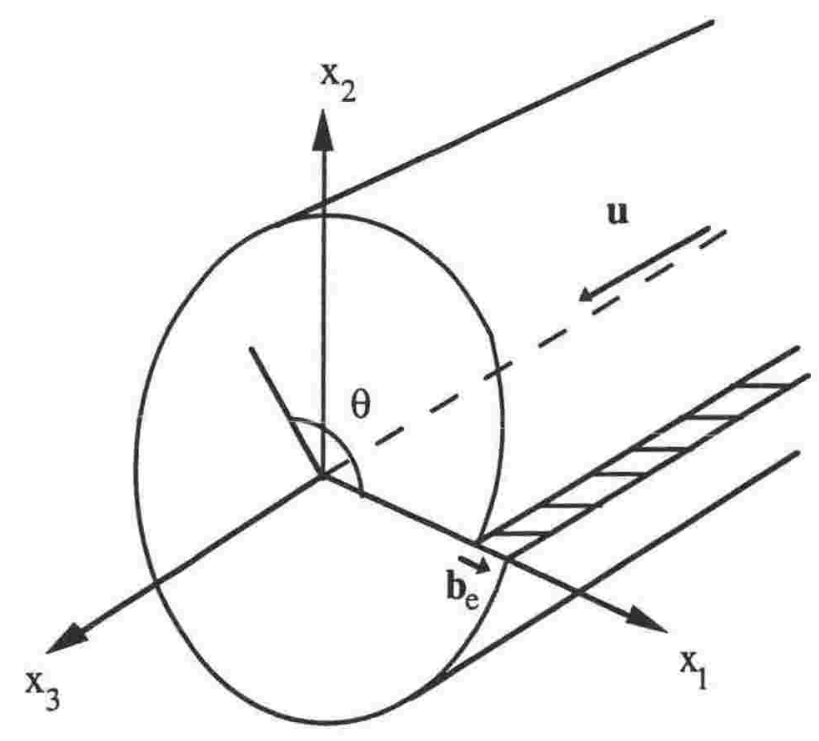

Figure 1.4: An edge dislocation that has a dislocation line along the axis of the cylinder. In the perfect crystal the cylinder is undeformed. An edge dislocation is formed by cutting the cylinder along one side in the plane formed by the $\mathrm{x}_{1}$ and $\mathrm{x}_{3}$ axes and then deforming the material above the cut relative to the material below the cut. 


\subsection{Dislocation Loop Punching Theory}

\subsubsection{Greenwood, Foreman and Rimmer theory}

Greenwood, Foreman and Rimmer (GFR) were the first to estimate the pressure required for a bubble to create a dislocation loop at the bubble surface [1]. The estimate assumes the validity of isotropic elasticity theory. The neglect of the elastic anisotropy in the GFR model may be significant for some metals. It may prevent any obvious relationship (for example, between the shear modulus and the superlattice spacing) being demonstrated by the theory.

Let the radius of the dislocation loop be $\mathrm{r}_{l}$ and the bubble radius be $\mathrm{r}$ as shown in figure 1.5. Also let the surface tension of the host metal be $\gamma$ and the gas pressure in the bubble be $\mathrm{P}$.

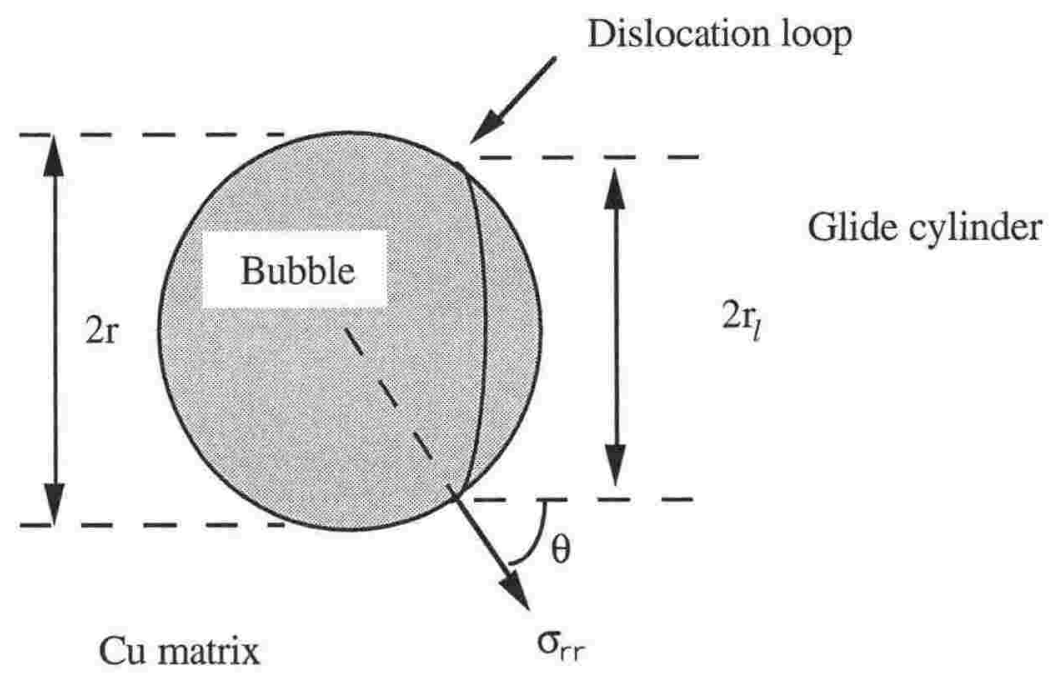

Figure 1.5: Bubble and dislocation loop parameters.

After being punched out by the bubble the edge dislocation loops can move (i.e., glide) along the glide cylinder. The GFR model for dislocation punching compares the energy of the system before and after a loop is punched out. The assumption is made that after punching, the loop has glided far enough away from the bubble that loop-bubble interactions can be neglected. In this way a derivation can be made of a necessary but not sufficient condition for loop punching. This derivation is outlined below. 
The energy to form the dislocation loop is approximately equal to the length of the dislocation line times the strain energy per unit length of the dislocation:

$$
\mathrm{E}=\frac{2 \pi \mathrm{r}_{l}}{(1-v)}\left(\frac{\mu \mathrm{b}^{2}}{4 \pi}\right) \ln \left(\frac{\mathrm{r}_{l}}{\mathrm{r}_{\mathrm{d}}}\right),
$$

where $\mu, \mathrm{r}_{\mathrm{d}}(\approx \mathrm{b})$ and $\mathrm{b}$ are the shear modulus, dislocation core cut-off parameter and magnitude of the Burgers vector respectively. The idea of a core cut-off parameter is necessitated by the solution for the displacement field of a long straight dislocation found in section 1.2.3. The strain energy per unit length of a dislocation is found by integrating over $0.5 \mathrm{x}$ stress $\mathrm{x}$ strain per unit volume over an annulus about the dislocation. The integrand is inversely proportional to the distance from the dislocation line, so the log term that results from the integration has a singularity in it if one of the limits of the integration is placed at the centre of the dislocation. Physically the need to use a cut-off parameter is a consequence of treating a crystal lattice as a continuum. That is, atoms are displaced by finite amounts near the core of a dislocation and do not have an infinite strain energy at the centre of the dislocation. To avoid this problem the solutions for the displacement fields are assumed to be valid from a radius equal to the core cut-off parameter or bigger.

Equation 1.20 is valid for an edge dislocation only. A screw dislocation will have an energy lower than that of the edge dislocation by a factor of $1-v$. Equation 1.20 is derived by integrating over the stress and strain about a long straight edge or screw dislocation. Strictly the resultant strain energy is only the strain energy contained in the elastic continuum between the core cut-off parameter and the outer radius of the annulus. If the outer radius is infinite the strain energy diverges, so in practice it is necesary to impose a limit on the integrand beyond which the solutions found for the stress and strain fields are considered to not be good models of what actually occurs in a crystal about a dislocation. Smallman [99] quotes a typical value for the outer radius of the annulus of $2.5 \mu \mathrm{m}$. As opposite sides of the dislocation loop will have dislocation line directions that lie in opposite directions the resultant displacement field outside the loop will be less than that due to the closest side of the dislocation alone. The way that Greenwood et al. [1] used the solution for the strain energy assumes that the outer limit of the annulus is equal to the radius of the 
The energy to form the dislocation loop is approximately equal to the length of the dislocation line times the strain energy per unit length of the dislocation:

$$
E=\frac{2 \pi r_{l}}{(1-v)}\left(\frac{\mu b^{2}}{4 \pi}\right) \ln \left(\frac{r_{l}}{r_{d}}\right),
$$

where $\mu, r_{d}(\approx b)$ and $b$ are the shear modulus, dislocation core cut-off parameter and magnitude of the Burgers vector respectively. The idea of a core cut-off parameter is necessitated by the solution for the displacement field of a long straight dislocation found in section 1.2.3. The strain energy per unit length of a dislocation is found by integrating over $0.5 \mathrm{x}$ stress $\mathrm{x}$ strain per unit volume over an annulus about the dislocation. The integrand is inversely proportional to the distance from the dislocation line, so that the log term that results from the integration has a singularity in it if one of the limits of the integration is placed at the centre of the dislocation. Physically the need to use a cut-off parameter is a consequence of treating a crystal lattice as a continuum. That is, atoms are displaced by finite amounts near the core of a dislocation and do not have an infinite strain energy at the centre of the dislocation. To avoid this problem the solutions for the displacement fields are assumed to be valid from a radius equal to the core cut-off parameter or bigger.

Equation 1.20 is valid for an edge dislocation only. A screw dislocation will have an energy that is lower than that of the edge dislocation by a factor of $1-v$. Equation 1.20 is derived by integrating over the stress and strain about a long straight edge or screw dislocation. Strictly the resultant strain energy is only the strain energy contained in the elastic continuum between the core cut-off parameter and the outer radius of the annulus. If the outer radius is infinite the strain energy diverges, so in practice it is necesary to impose a limit on the integrand beyond which the solutions found for the stress and strain fields are considered to not be good models of what actually occurs in a crystal about a dislocation. Smallman [99] quotes a typical value for the outer radius of the annulus of $2.5 \mu \mathrm{m}$. As opposite sides of the dislocation loop will have dislocation line directions that lie in opposite directions the resultant displacement field outside the loop will be less than that due to the closest side of the dislocation alone. The way that Greenwood et al. [1] used the solution for the strain energy assumes that the outer limit of the annulus is equal to the radius of the 
dislocation loop $(\approx 10 \AA)$. This choice of the outer radius of the annulus neglects a large amount of the strain energy associated with a dislocation loop and means that the GFR inequality (equation 1.27) significantly underestimates the pressure required for a bubble to punch out a loop. Kroupa [100] found a solution for the strain energy of a prismatic dislocation loop in an infinite and isotropic solid. This solution was used by Wolfer [101103] (section 1.3.4) to obtain a more accurate estimate of the pressure required to punch out a dislocation loop. However, the GFR inequality is still widely used in the literature.

When $P=2 \gamma / r$ the pressure within the bubble balances the surface stress and the bubble is said to be at equilibrium pressure. (The surface stress tries to reduce the surface area of the bubble cavity to lower the surface free energy). In terms of this simple model the effects of the metal surrounding the cavity are neglected and the system is treated as if it were a gas bubble in air. This is valid only if the bubble can grow to equilibrium without straining the surrounding metal. For this to be the case, for example, the metal atoms displaced by the bubble would have to diffuse away to leave the bubble without a surrounding displacement or strain field.

The decrease in the free energy of the bubble caused by creating the loop is approximately

$$
\begin{aligned}
\mathrm{dF} & =-\mathrm{P}_{\text {resultant }} \mathrm{dV} \\
& =-\left(\mathrm{P}-\frac{2 \gamma}{\mathrm{r}}\right) \pi \mathrm{r}_{l}^{2} \mathrm{~b} .
\end{aligned}
$$

$\mathrm{P}_{\text {resultant }}$ is the overpressure inside the bubble or the amount by which the pressure inside the bubble exceeds the equilibrium pressure. For the mechanism of punching out a loop to be energetically allowed using equations 1.20 and 1.21 the following condition must be satisfied:

$$
\left(\mathrm{P}-\frac{2 \gamma}{\mathrm{r}}\right) \pi \mathrm{r}_{l}^{2} \mathrm{~b}>\frac{2 \pi \mathrm{r}_{l}}{(1-\mathrm{v})}\left(\frac{\mu \mathrm{b}^{2}}{4 \pi}\right) \ln \left(\frac{\mathrm{r}_{l}}{\mathrm{r}_{\mathrm{d}}}\right) .
$$

That is the decrease in free energy of the bubble caused by creating a loop must exceed the energy required to form the loop. 
The radially symmetric displacement field about the bubble, ignoring foil surface effects, is:

$$
\mathrm{u}_{\mathrm{r}} \sim \frac{1}{\mathrm{r}^{2}}
$$

This can readily be seen by the analogy between the Green's function theories of elasticity and of electrostatics. That is, the mathematical techniques required to find the solution (e.g. those used to solve equation 1.16) are similar in elasticity and electrostatics. The displacement field in elasticity theory about a bubble is similar mathematically to the potential in electrostatics about a solid sphere of uniformly distributed charge. The radial stress is

$$
\begin{aligned}
\sigma_{\mathrm{Ir}} & \sim \frac{\mathrm{du} \mathrm{r}_{\mathrm{r}}}{\mathrm{dr}} \\
& \sim \frac{1}{\mathrm{r}^{3}}
\end{aligned}
$$

The component of the total force acting on the loop along the glide cylinder per unit length at the bubble surface is

$$
\text { Force/unit length }=2 \pi \mathrm{r}_{l} \cos (\theta) \sigma_{\mathrm{rr}} \text {. }
$$

By maximising the force per unit length the most likely radius of the loop is found to be

$$
\mathrm{r}_{l}=\frac{\mathrm{r}}{\sqrt{2}}
$$

This result was not explicitly stated in the original GFR paper [1], but is readily obtained from it. Then the pressure required for punching of a dislocation loop of this radius is

$$
\left(P-\frac{2 \gamma}{r}\right)>\frac{\mu b}{(1-v) \pi r \sqrt{2}} \ln \left(\frac{r}{r_{d} \sqrt{2}}\right)
$$

Willis and Bullough [104] derived the displacement field about two interacting bubbles that could be underpressured or overpressured. Their displacement field is proportional to the amount by which the pressure exceeds the equilibrium pressure. The GFR model assumes that a bubble is overpressured. Yet the GFR model assumes that this 
overpressure does not create a significant backstress upon the bubble due to the displacement of the surrounding metal atoms. Jones [54] estimated that the effect of the volume backstress is not significant when the elastic constants are unaltered by the irradiation but, nonetheless also points out that Eernisse and Picraux [105] have measured the maximum integrated yield stress in $30 \mathrm{keV}$ helium ion irradiated copper and found it to exceed the bulk yield stress for the unirradiated material. In this particular case the volume backstress would seem significant and it means the GFR inequality underestimates the pressure required to punch out a loop. However, without a knowledge of how the elastic constants are changed on a microscopic scale by the irradiation it is impossible to obtain a better estimate of the pressure required by the bubble to punch out dislocation loops.

Provided the inequality of equation 1.27 is satisfied the bubble will grow by displacing the surrounding atom planes. This is shown schematically in figures 1.6 and 1.7. Typically gas bubbles have internal pressures of $\sim \mathrm{GPa}$ and radii that are close to satisfying this inequality [106]. There is some dispute about the applicability of the methods by which bubble pressures should be measured. In specimens containing superlattices the gas and damage profiles are approximately gaussian. X-ray diffraction measurements of the pressure [107] in bulk specimens irradiated at a number of different energies, so as to obtain a macroscopically uniform gas and damage profile, may be misleading if applied to the superlattice case. X-ray diffraction techniques require that spots are formed by scattering from the implanted ions in solid bubbles and are therefore not applicable to bubbles containing gas. Electron energy loss spectroscopy (EELS) based methods rely upon measuring the broadening and shift in the energy of a transition between energy levels when it is excited by the electron beam in a TEM [108]. Methods based on EELS [109] use the shift in the ${ }^{1} \mathrm{~S}_{0}$ to $2^{1} \mathrm{P}_{1}$ transition of an electron in a helium atom that results from the gas being at high pressure. A brief attempt was made in the present work to detect helium in a specimen containing a superlattice using an EELS setup at Sydney University. However, this failed because of the difficulties in exciting the inert helium. The pressure measured by other authors using X-ray diffraction or EELS are the average pressure in the bubbles. The real pressure in a bubble could be much higher. Evans and Mazey [36] used the spacing of the diffraction spots from solid $\mathrm{Kr}$ contained in bubbles in 
Ti to obtain the lattice spacing of the krypton. The volume of the bubble gave the number of $\mathrm{Kr}$ atoms in the bubble. An equation of state was then used to estimate the pressure within

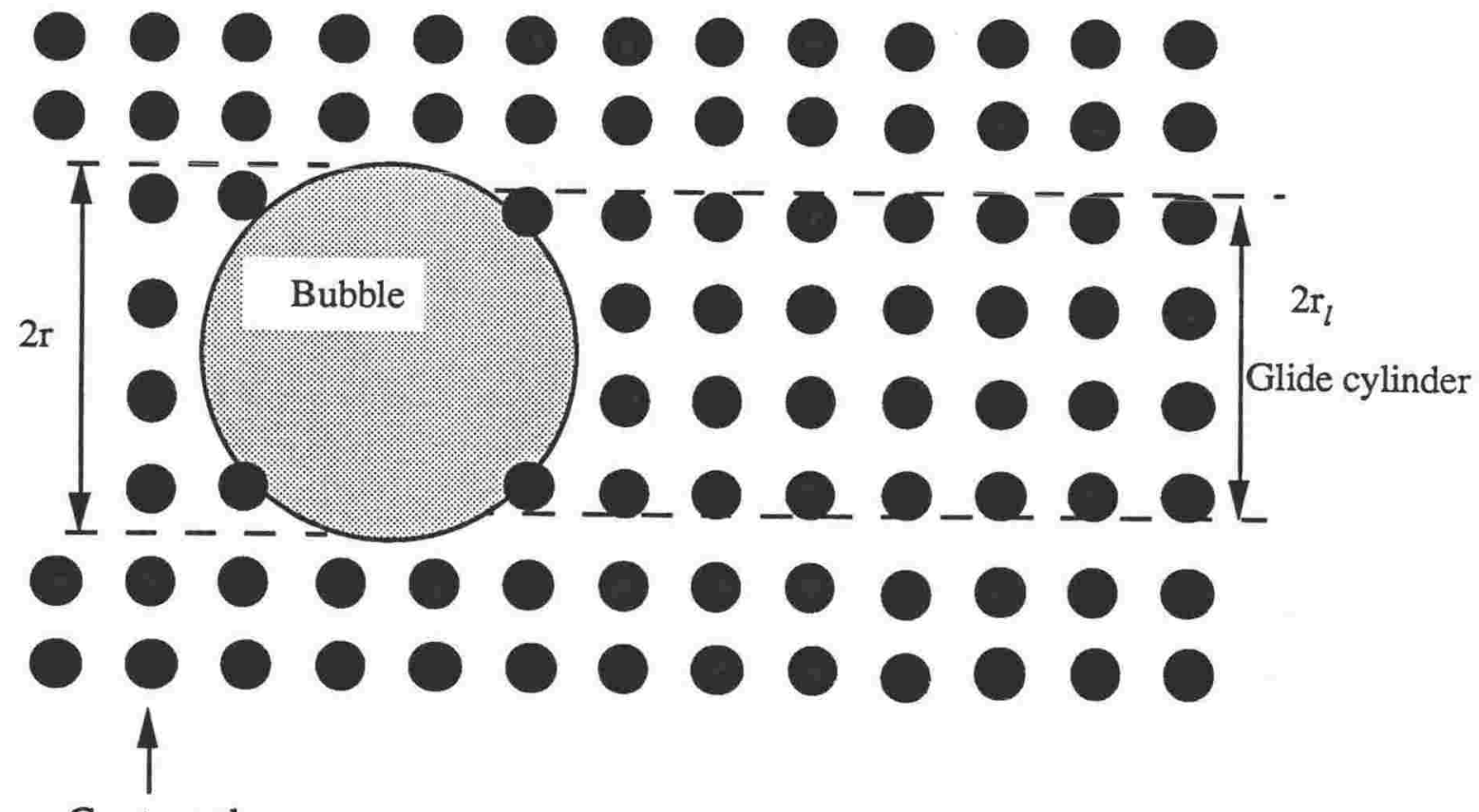

$\mathrm{Cu}$ atom planes

Figure 1.6: A bubble and surrounding atom planes before the dislocation is punched out.

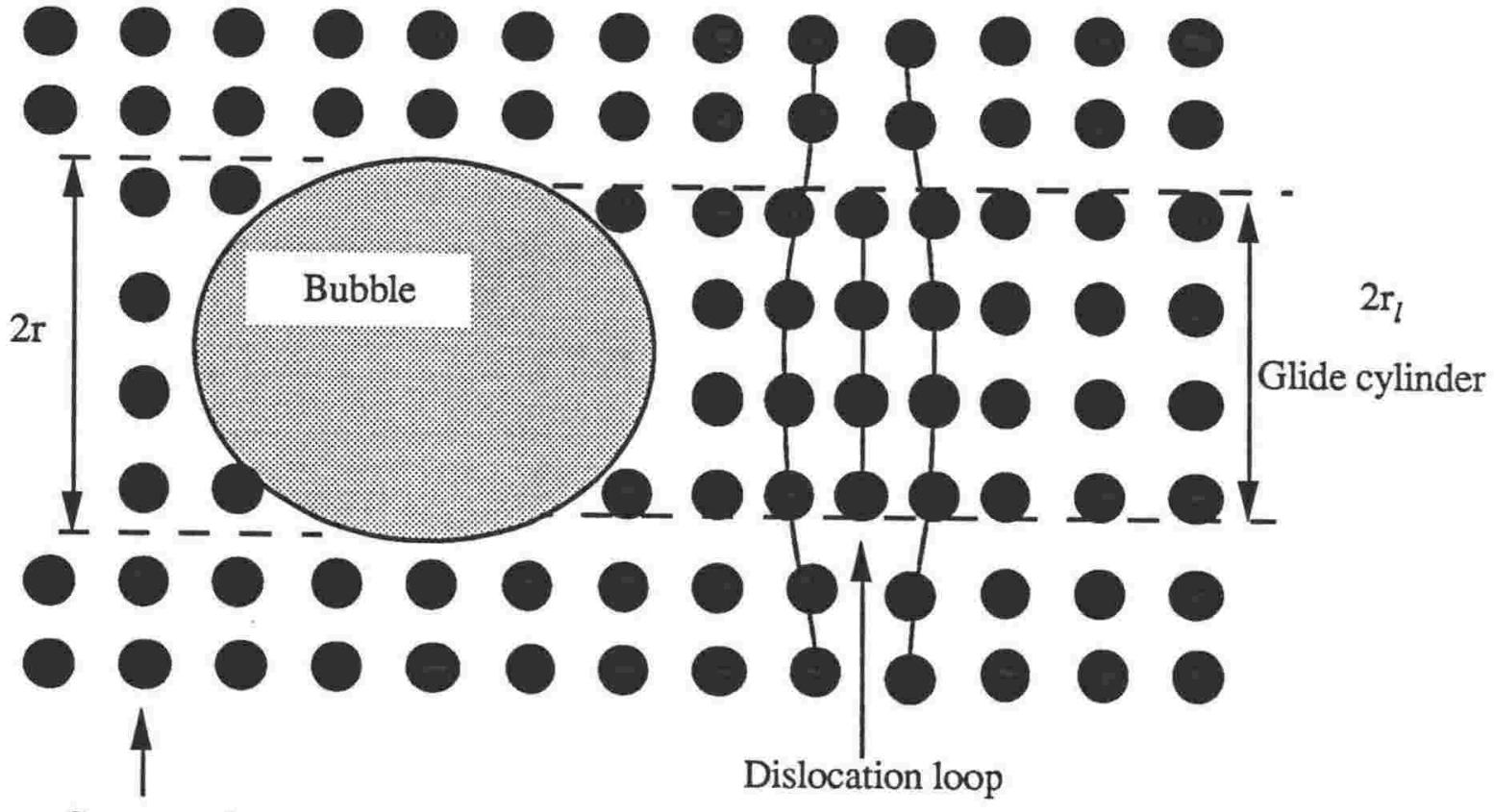

$\mathrm{Cu}$ atom planes

Figure 1.7: A bubble and surrounding atom planes after the dislocation is punched out.

the bubble. Their subsequent comparison of the pressure with the GFR inequality has been criticised because it failed to include the equilibrium gas pressure (Donnelly, Mitchell and $1-18$ 
van Veen [110]). (That is the bubble pressure instead of the overpressure was compared to the right hand side of equation 1.27). Wolfer's model (section 1.3.4) gives a pressure for loop punching that is higher than that required by the GFR model.

In terms of the GFR model, once the bubble has created the loop it relaxes back into a spherical shape. The whole process of punching a loop will be repeated if the increase in bubble radius is insufficient to lower the pressure enough to invalidate the inequality. The centre of mass of the bubble moves in the direction in which the bubble punches out the loop. Matrix atoms from the side of the bubble on which the loop was punched can diffuse around the surface. The surface diffusion will enhance the movement of the centre of mass of the bubble along the glide cylinder. The bubble then moves in the direction that the loop was punched. Thus by emitting a loop the bubble grows and can move. This property may be important in explaining the alignment of gas bubbles into superlattices.

The dislocation loops may have a habit plane of (110) or (111), the habit plane of a loop being the plane of the interstitial atoms making up the loop. The lowest energy configuration occurs when the Burgers vector has the smallest magnitude $\mid \mathbf{b l}$ corresponding to the $<110>$ directions in a fcc crystal. After a loop is formed it can glide (i.e., move) along these directions with very little extra energy by being thermally excited over the barrier of the atomic potential (the so called Peierls barrier). Alternatively, the loop can be moved along the glide cylinder by stress.

The GFR model uses continuum elasticity theory to model the loop punching process. More recently [111], "molecular dynamic" calculations applied to atoms in a lattice surrounding a high pressure bubble show that the loops should form a few atom layers from the bubble (before gliding away from the bubble). This is in contrast to the loop forming right at the bubble surface as envisaged here. 


\subsubsection{The Theory of Bullough and Newman for the Spacings of Loops}

Bullough and Newman [112] have predicted the equilibrium spacing for loops punched by precipitates or similar defects such as gas bubbles. In chapter 4 this theory will be compared to experimental data obtained from loops punched by argon bubbles quenched into copper. Bullough and Newman start their development by obtaining the solution for the shear stress on the cylindrical surface $r=a$, where $a$ is the dislocation loop radius. They arrive at the solution by making a cylindrical cut in an elastic medium over the surface $r=a$. Then tractions are applied to the two sides of the cut to create the loop. The cut is then rewelded. The solution for the stress about the loop is found by requiring continuity of the stress and displacements across the cut. The resulting shear stress is:

$$
\operatorname{prz}_{\mathrm{rz}}(\mathrm{a}, \mathrm{z})=\pi \alpha \mu \mathrm{z} \int_{0}^{\infty} \eta^{2} \mathrm{~J}_{1}^{2}(\mathrm{a} \eta) \exp (-\eta \mid \mathrm{z}) \mathrm{d} \eta
$$

where the $\mathrm{z}$-axis is perpendicular to the plane of the loop and

$$
\alpha=\frac{\mathrm{ba}}{2 \pi(1-v)} .
$$

They state that the integral can be evaluated in terms of tabulated elliptic integrals (Watson [113]). Watson (p390) does not explicitly evaluate this integral but transforms it into

$$
\frac{\Gamma(2.5) \mathrm{b}^{2}}{\pi^{1.5}} \int_{0}^{\frac{\pi}{2}} \frac{\cos ^{2} \phi}{\left(\alpha^{2}+\mathrm{b}^{2} \cos ^{2} \phi\right)^{2.5}} \mathrm{~d} \phi .
$$

This can expressed in terms of elliptic integrals of the first and second type by using equations 3.158 .11 and 3.162 .15 on pages $252 / 3$ and $257 / 8$ of Gradshteyn and Ryzhik [114]. Bullough and Newman define the dimensionless variable

$$
\mathrm{v}=\frac{4 \mathrm{a} \pi(1-\mathrm{v})}{\mathrm{b} \mu} \mathrm{p}_{\mathrm{rZ}}^{\mathrm{crit}}
$$


where prit is the critical resolved shear stress (i.e., the shear stress resolved parallel to the axis of the glide cylinder that must be exerted on a loop for the loop to continue gliding away from the gas bubble that created it). Using Gradshteyn and Ryzhik [114], equation 1.28 becomes

$$
\frac{v_{\mathrm{rZ}}}{\mathrm{p}_{\mathrm{IZ}}^{\mathrm{crit}}}=\frac{\rho}{\left(1+\rho^{2}\right)^{1.5}}\left[\left(\frac{1-\rho^{2}}{\rho^{2}}\right) E(k)+F(k)\right]
$$

where $\mathrm{E}$ and $\mathrm{F}$ are complete elliptic integrals of the second and first kind respectively, and

$$
\rho=\frac{z}{2 a}
$$

The elliptic integrals were evaluated by the "NAG" numerical applications package on a Silicon Graphics 4D/340S computer. The resulting values of the elliptic integrals were tested against the tabulated values in the mathematical companion to the CRC handbook of physical constants [115]. The values gave good agreement, to 4 figures after the decimal place, over the important range of the values tabulated, corresponding to $\rho$ values that are physically plausible. The resulting plot of the shear stress on a logarithmic scale is shown in figure 1.8. This is similar to figure 1 of Bullough and Newman.

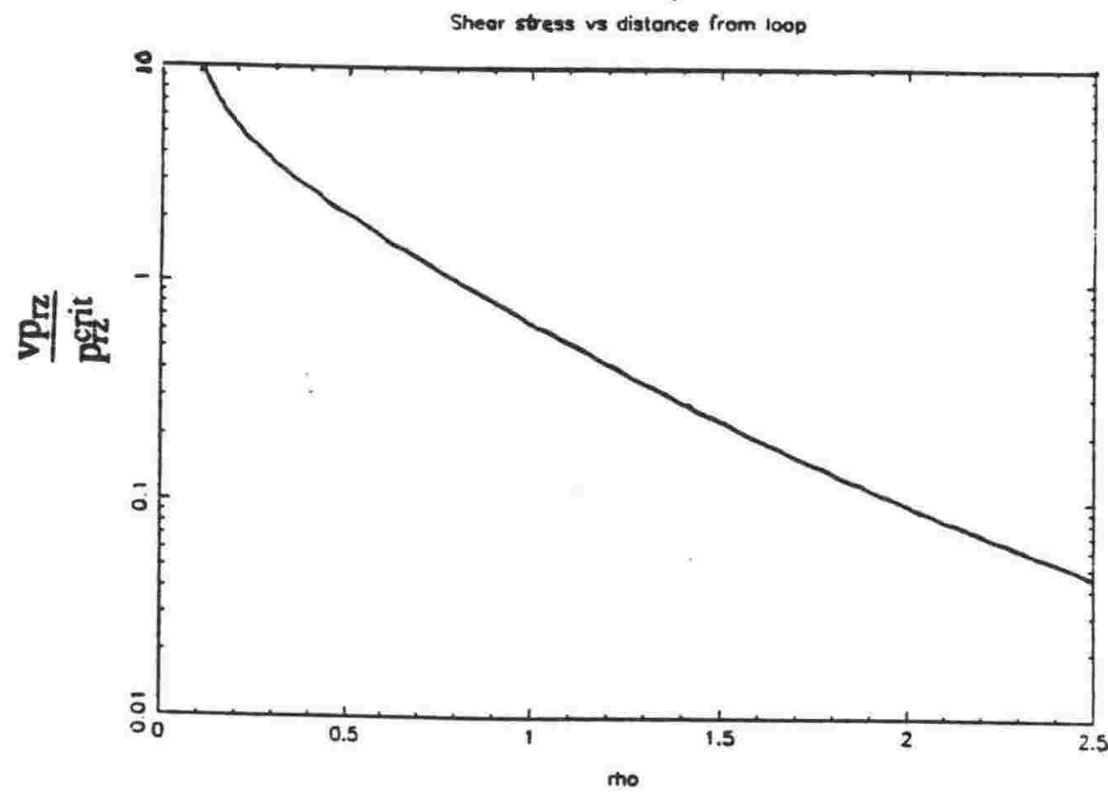

Figure 1.8: Plot of shear stress vs. distance from a loop. Note the logarithmic scale on the vertical axis. Rho $(\rho)$ was defined in equation 1.33. 
The equilibrium loop spacings of a row of loops punched out along the same glide cylinder by a gas bubble can be calculated by first assuming that two loops are created close together. One loop, the last to be formed, is pinned in position by the backstress of the first loop and the stress from the bubble, the backstress being the stress that points back towards the bubble caused by loops that were punched out earlier. The shear stress on the loop that was formed first (and thus lies furthest from the bubble), due to the second loop, is calculated. The first loop is then moved through a distance proportional to the amount by which the shear stress exceeds the critical shear stress of the material. The constant of proportionality is chosen so that the calculations are completed in reasonable time and the final positions of the loops are insensitive to the value of the constant. The stress the bubble exerts on the loops is ignored, i.e. the bubble is assumed only to create and pin the loops. The shear stress on the first loop is then recalculated and this loop is again moved. The process is repeated until the first loop is far enough away from the loop nearest the bubble that the shear stress on it falls below the critical resolved shear stress. At this point the first loop stops moving. The second loop is assumed to be pinned in position near the bubble until the third loop is punched out. This way of moving the loops seems reasonable because in equal time intervals, the distance through which a loop is moved is proportional to the shear stress upon it. The computer can be considered to be sampling the position of the loops in equal successive intervals of time.

At this stage a third loop is created nearest the bubble. The loop that was previously closest to the bubble is moved away from this new loop far enough to avoid the singularity in the shear stress at $\rho=0.0$ in equation 1.32. (Values for this initial loop spacing of 0.1 to 0.5 , in units of the loop diameter, have been chosen depending on the value used for v). The resultant shear stress on the first and second loops are then calculated and both are moved further away from the bubble. The movements continue until they both reach an equilibrium position at which another loop can be created. This process is repeated until the desired number of loops have been punched by the bubble. The loops punched later in the sequence will not glide as far as the earlier loops because of the higher backstress on the later loops. For this reason the equilibrium loop spacings increase with the distance of the loop from the bubble. The accuracy of the results depends on several factors: the 
frequency with which the loop positions are sampled in time and the initial loop spacing that is used to avoid the singularity. The major assumptions in the theory of Bullough and Newman are that the stress of the bubble on the loops can be ignored and that the loops are of equal diameter.

Theoretical results for the loop spacings for $i=0$ to 8 are given in table $1.1\left(\rho_{i}\right.$ is proportional (equation 1.33) to the spacing between the $(i+1)$ th the loop and the $(i+2)$ th loop punched out). The equilibrium values for different critical shear stresses, were calculated using equation 1.32. They are compared to the original Bullough-Newman results. The small disagreement may result from Bullough and Newman evaluating the elliptic integrals by interpolation from tabulated values whereas the present results calculate the elliptic integrals at all of the required positions.

Table 1.1 Loop spacings for a ten loop row.

(a) Bullough-Newman results:

\begin{tabular}{|l|c|c|c|c|c|c|c|c|c|}
\hline & \multicolumn{10}{|c|}{$\rho_{\mathrm{i}}$} \\
\hline $\mathrm{v}$ & 0 & 1 & 2 & 3 & 4 & 5 & 6 & 7 & 8 \\
\hline 0.5 & 1.23 & 0.951 & 0.791 & 0.684 & 0.606 & 0.546 & 0.491 & 0.478 & 0.317 \\
\hline 0.25 & 1.55 & 1.25 & 1.07 & 0.952 & 0.862 & 0.792 & 0.731 & 0.703 & 0.549 \\
\hline 0.05 & 2.47 & 2.05 & 1.83 & 1.68 & 1.57 & 1.48 & 1.41 & 1.35 & 1.25 \\
\hline
\end{tabular}

(b) Present results (using equation 1.32):

\begin{tabular}{|c|c|c|c|c|c|c|c|c|c|}
\hline & \multicolumn{10}{|c|}{$\rho_{\mathrm{i}}$} \\
\hline $\mathrm{v}$ & 0 & 1 & 2 & 3 & 4 & 5 & 6 & 7 & 8 \\
\hline 0.5 & 1.25 & 0.984 & 0.842 & 0.747 & 0.683 & 0.635 & 0.596 & 0.540 & 0.350 \\
\hline 0.25 & 1.57 & 1.27 & 1.10 & 0.992 & 0.909 & 0.843 & 0.792 & 0.735 & 0.571 \\
\hline 0.05 & 2.54 & 2.11 & 1.88 & 1.72 & 1.61 & 1.52 & 1.44 & 1.36 & 1.24 \\
\hline
\end{tabular}

Barnes and Mazey [2] measured the spacing of loops which had been punched from gas bubbles quenched into copper. The results (for loops with diameters all of about the same size) were compared to the theoretical Bullough and Newman results. Others [5] have obtained results similar to those of Barnes and Mazey. This comparison was used to find a value for the critical shear stress. Barnes and Mazey compared the value for the critical 
shear stress they obtained with the macroscopic measurements of Barrett [116]. Barnes and Mazey state that the value obtained was "higher by an order of magnitude, which is greater than the experimental error. This difference is not explained but is possibly due to the lattice hardening which could be attributable to dislocation locking caused by quenching." When the integral of equation 1.28, evaluated as above, is compared with the data of Barnes and Mazey the disagreement remains about the same. The failure of the theory of Bullough and Newman is probably a result of the loops not all being of the same size. As the bubble grows the size of the loops punched out also grow but the theory does not take this into account.

\subsubsection{Dubinko Theory for loop spacing and ordering of bubbles}

Johnson [117] invoked the alignment of dislocation loops punched out by bubbles to explain how bubble ordering is energetically favourable as shown in figure 1.9. Later Dubinko [7] combined the dislocation loop punching mechanism of Greenwood, Foreman and Rimmer [1], and the loop spacing theory of Bullough and Newman [112] into a theory for bubble ordering. Dubinko applied this approach to the bubbles and loops thought to exist in the superlattice case. In chapters 6 and 7 it will be seen that the experimental evidence suggests that there is not a high density of dislocation loops punched by the small gas bubbles present in specimens containing superlattices. Therefore this section on Dubinko's theory is somewhat critical. The theory of Dubinko is explained and criticised in the rest of this section.

Dubinko considered the forces in one dimension along a glide cylinder and ignored the interactions between loops from different bubbles that may tend to align the loops (figure 1.9). Initially only two bubbles are included in the model. The second bubble is positioned along one of the $\mathrm{n}$ glide cylinders of the first bubble (for an fcc crystal there are $\mathrm{n}$ $=12<110>$ glide cylinders per bubble). This second bubble acts as a stopper for the loops punched by the first bubble along this glide cylinder. The other $\mathrm{n}-1$ glide cylinders are unobstructed. At first sight this positioning of the bubbles along glide cylinders of other bubbles is a major assumption. It implies that the initial bubble positions are not 
random. Dubinko points out that a bubble that is not positioned on another bubble's glide cylinder cannot be repulsed from that bubble by a repulsion between dislocation loops, because the loops will lie only on a glide cylinder of a bubble. Instead the bubbles will be attracted to each other by the attractive force between two bubbles postulated by Willis and Bullough [104]. It is thought that such bubbles would coalesce to minimise surface energy. In fact Dubinko assumes that any bubbles not positioned on a glide cylinder of a nearest neighbour bubble will join that bubble to form a new bubble. This process would continue until all the bubbles are positioned along glide cylinders of their nearest neighbours. The theory then assumes the initial inter-bubble spacings along these glide cylinders are random. Dubinko then explains how the bubbles could move along these glide cylinders by punching out dislocation loops to obtain a regular inter-bubble spacing.

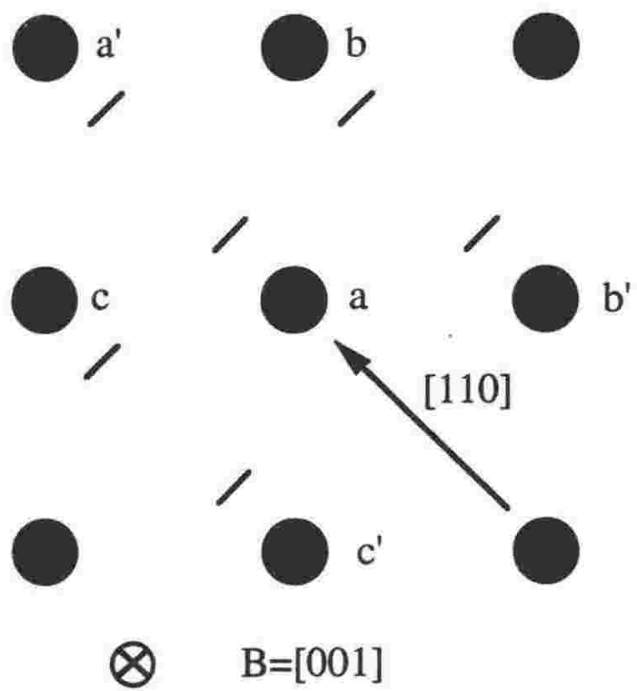

Figure 1.9: In a real crystal the interaction between overpressured bubbles could be expected to depend strongly on crystallographic direction. For example, dislocation loop punching from bubble a, in the [110] glide direction towards a', is likely to be helped by the presence of dislocation loops punched antiparallel to this direction by bubbles at $\mathrm{b}$ and $\mathrm{c}$ and inhibited by the loop from the bubble at a'. The dislocations are drawn on the figure with $(1,-1,0)$ habit planes.

In the initial two-bubble approximation two different types of one-dimensional equilibrium equation need to be solved. The first type is for a line through the bubble along a pair of unobstructed glide cylinders. The second type is for a line through the bubble that includes one unobstructed glide cylinder and a second glide cylinder obstructed by a second 
bubble. Included in the one-dimensional equilibrium equations is the force between loops, between a bubble and a loop and between a loop and the matrix (the so called Peierls force). Dubinko neglects the bubble-bubble attractions in the equilibrium equations because they are much smaller than the interactions of bubbles with loops, or between loops. The Peierls force is similar to the critical shear stress used in the Bullough-Newman theory. It causes the loops furthest from the bubble to come to rest when the force on these loops drops below the Peierls force. It also means that the bubbles cannot move apart indefinitely under the repulsion between the loops on a common glide cylinder between the bubbles. Instead the first bubble is held at a finite distance from the second bubble by the back-stress from the dislocation loops on the unobstructed glide cylinder. These loops are pinned in position by the Peierls force. The only interactions between loops that are included in the equilibrium equations are for loops on the same glide cylinder that are not separated by a bubble. Approximate expressions for the equilibrium loop and bubble spacings were obtained by taking asymptotic expansions of the exact solutions to the two equilibrium equations.

Dubinko then goes on to treat the superlattice case using the same assumptions as above. He takes the solution for the glide cylinder between bubbles, applies it to all $\mathrm{n}$ glide cylinders and then sums the result. The parts of the earlier solutions due to the unobstructed glide cylinders are totally ignored. To obtain the movement of the bubble along a glide cylinder in one dimension the number of loops punched out in one direction is subtracted from the number of loops punched out on the opposite glide cylinder and multiplied by a factor proportional to the Burgers vector of the loops. This gives the resultant volume punched out in one direction by the bubble which can be related back to the change in the position of the centre of mass of the bubble in the direction in which the loops have been punched. Effects due to the change in loop size with bubble growth are neglected.

Predictions can be made about the equilibrium inter-bubble spacing and the radius at which the bubbles stop growing. These predictions are in broad agreement with experiment for a range of materials. The discrepancies can be explained by the failure of some of the assumptions above, especially the failure of the bubbles to lie along the glide cylinders of other bubbles. The bubbles can readily move along the $\langle 110\rangle$ directions only. So the short range order eventually evolves into a gas bubble superlattice having the same 
symmetry as the host matrix. However, Evans [118] has criticised Dubinko's model for bubble ordering because "it would seem that a loop from one bubble punched out toward an imperfectly aligned bubble will be partially absorbed by that bubble and move it away from linear alignment."

The shear modulus $(\mu)$ is smallest in aluminium and largest in rhenium amongst the materials for which gas bubble formation has been commonly found. The gas bubbles have radii varying from about 8 to $20 \AA$ depending on the implantation conditions. But this bubble size or the superlattice constant is not related in an obvious way to $\mu$. Therefore it is impossible to pick a material which would be the most likely to form dislocation loops if the punching mechanism is valid. Also the effects of elastic anisotropy are not taken into account in the dislocation loop punching mechanism. So the effects of using anisotropic materials on the likelihood of obtaining loops is unknown.

\subsubsection{Wolfer theory}

The GFR theory does not consider how the Helmholtz free energy of the system varies with the distance between the bubble and the loop and also assumes that after the loop has been punched there is no more interaction between the bubble and the loop, i.e., effectively the loop glides off a large distance from the bubble. In the GFR model the bubble takes on its final volume the moment the loop has been punched. Wolfer [102,103] extended the GFR theory for loop punching by including the variation in the Helmholtz free energy that occurs during the process and used a more accurate form for the strain field surrounding a dislocation loop and bubble.

The neglect of the interaction between a loop and a bubble is unrealistic and Wolfer and Drugan [101] derived the strain and displacement field about a loop that has been punched by a bubble as a function of the distance between the loop and bubble. A solution for the displacement field due to the loop was sought in a form that can be expressed as the gradient and sum of two harmonic functions ( $\phi$ and $\psi$ ). One of the harmonic functions which is differentiated to calculate the displacement field is shown in equation 1.34. The solution takes the form: 


$$
\begin{gathered}
\mathbf{u}=\nabla(\phi+z \psi)-4(1-v) \widehat{\mathbf{z}} \psi \\
\phi^{1}=\sum_{n=0}^{\infty} F_{n} R^{n} P_{n}(\cos \theta)
\end{gathered}
$$

where $\mathrm{R}$ and $\theta$ define the position of a segment of the loop from the centre of the bubble. $P_{n}$ is a Legendre polynomial of degree $n$, and the $F_{n}$ are the coefficients that are required for the solution. The stress can be derived by differentiating this displacement field solution in cylindrical or spherical coordinates. The boundary condition, that the stress is zero on the surface of the bubble, enables the coefficients to be found. The stress field due to the bubble can similarly be found.

Integrating the stress due to the bubble, over the area of the loop that does not intersect the bubble surface, gives the work done by the bubble in forming the loop. The integration also gives the dependence on separation of the subsequent interaction energy between the loop and the bubble. Wolfer and Drugan [101] obtain asymptotic expansions of this infinite sum in three limiting cases, in the first "the loop is far removed from the cavity." The second solution is for "the loop is close to the cavity surface, and the loop and cavity radii are similar in magnitude." The third solution is for "the loop radius is small in comparison with the cavity radius but its distance to the surface is larger than its diameter." They construct an approximate interpolation formula, for all bubble and loop separations, using expressions derived for the above three cases.

In a subsequent paper Wolfer [102] derives the pressure required for the punching of a dislocation loop from a single isolated bubble by considering the change in Helmholtz free energy of the system as the loop emerges from the bubble and moves away. Included among the energy terms is the difference in the Helmholtz free energy of the bubble for the initial bubble volume and for the volume when the loop is at a certain distance from the bubble. Also included is the change in strain energy due to the pressure decrease in the bubble as its volume increases. The final terms included are the loop energy, the energy of interaction of the loop with its image beyond the surface of the bubble and the energy of interaction of the loop with the stress field of the bubble. 


$$
\begin{gathered}
u=\nabla(\phi+z \psi)-4(1-v) \widehat{\mathbf{z}} \psi \\
\phi^{1}=\sum_{n=0}^{\infty} F_{n} R^{n} P_{n}(\cos \theta)
\end{gathered}
$$

where $\mathrm{R}$ and $\theta$ define the position of a segment of the loop from the centre of the bubble. $P_{n}$ is a Legendre polynomial of degree $n$, and the $F_{n}$ are the coefficients that are required for the solution. The stress can be derived by differentiating this displacement field solution in cylindrical or spherical coordinates. The boundary condition, that the stress is zero on the surface of the bubble, enables the coefficients to be found. The stress field due to the bubble can similarly be found.

Integrating the stress due to the bubble, over the area of the loop that does not intersect the bubble surface, gives the work done by the bubble in forming the loop. The integration also gives the dependence on separation of the subsequent interaction energy between the loop and the bubble. Wolfer and Drugan [101] obtain asymptotic expansions of this infinite sum in three limiting cases, in the first "the loop is far removed from the cavity." The second solution is for where "the loop is close to the cavity surface, and the loop and cavity radii are similar in magnitude." The third solution is for where "the loop radius is small in comparison with the cavity radius but its distance to the surface is larger than its diameter." They construct an approximate interpolation formula, for all bubble and loop separations, using expressions derived for the above three cases.

In a subsequent paper Wolfer [102] derives the pressure required for the punching of a dislocation loop from a single isolated bubble by considering the change in Helmholtz free energy of the system as the loop emerges from the bubble and moves away. Included among the energy terms is the difference in the Helmholtz free energy of the bubble for the initial bubble volume and for the volume when the loop is at a certain distance from the bubble. Also included is the change in strain energy due to the pressure decrease in the bubble as its volume increases. The final terms included are the loop energy, the energy of interaction of the loop with its image beyond the surface of the bubble and the energy of interaction of the loop with the stress field of the bubble. 
The solution for the radial displacement field at the cavity surface can be integrated to find the volume change of the bubble as the separation of the loop and bubble changes. The radius of the bubble calculated from the volume is then used to calculate the area of the bubble surface and the change in bubble surface energy. The equation of state for helium as a function of temperature then gives the bubble pressure as a function of volume or defect separation. The pressure thus found for dislocation loop punching is significantly higher than that derived by GFR. Also the pressure plateaus out as the bubble radius increases instead of being approximately inversely proportional to bubble radius as the GFR mechanism predicts as shown in figure 1.10.

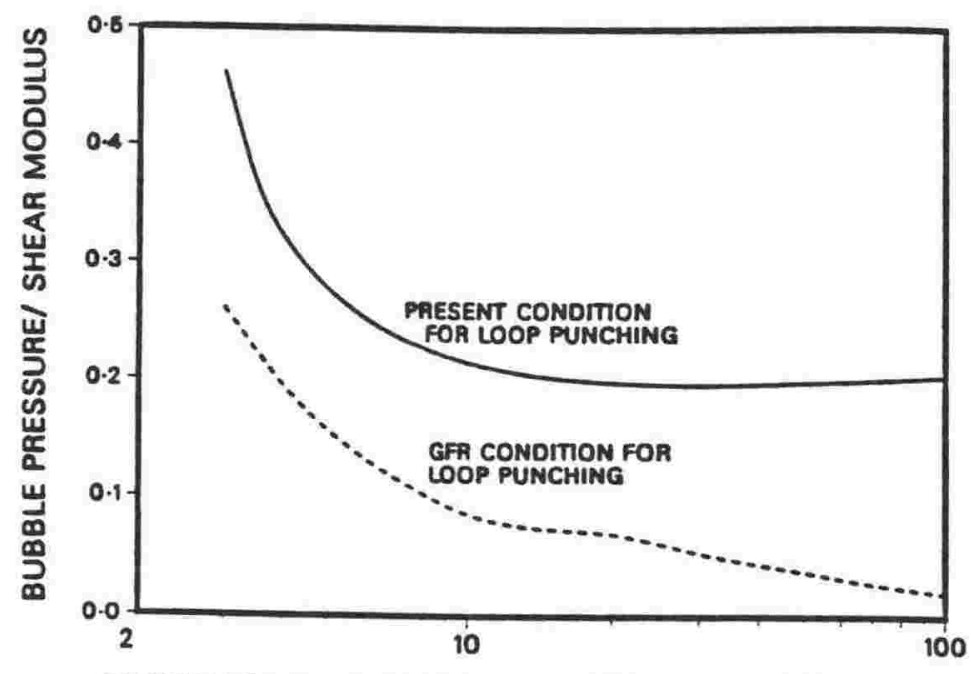

BUBBLE RADIUS IN UNITS OF BURGERS VECTOR

Figure 1.10: The pressure for loop punching as a function of bubble radius

The higher density of helium predicted lead Wolfer and Drugan to conclude that the helium stays "in the solid state at room temperature for all bubbles regardless of their size. Hence, they should perhaps be called helium precipitates".

The critical pressure for loop punching is about a fifth of the shear modulus. This implies that "in order for a bubble to form a prismatic dislocation loop, the pressure has to produce on a suitable glide cylinder a local shear stress equal to the theoretical shear strength of the solid."

Wolfer [103] extends the theory to the punching of dislocation loops in bubble arrays; predictions are made about bubble swelling and blistering. The formation of a 
bubble superlattice arises naturally out of this treatment and that of Dubinko [7] and of Johnson, Malcolm and Mazey [43]. As a bubble punches a loop, the centre of gravity of the bubble is shifted along the glide cylinder of the loop. Thus the punching mechanism allows the bubbles to move around on the glide cylinders. The bubble punches out loops most easily along cylinders which do not have another bubble or loop lying upon them already. The bubbles then tend to become evenly spaced and to stop growing when it becomes energetically unfavourable to punch out any more loops.

\subsubsection{Helical Dislocations}

Helical dislocations are often observed in quenched metals (Jones and Mitchell [4], Thomas and Whelan [3], Smallman and Eikum [6], and Partridge [5]). Some theories of the mechanism by which dislocation loops are punched by bubbles or by precipitates (Seitz [119], Weertman [120], Jones and Mitchell [4]) claim that the helices are a precursor of the dislocation loops. (Seitz was the first to use the term "prismatic dislocation loop" for a dislocation loop that is not circular but is constructed from straight dislocation segments). To form a helix a dislocation with two screw segments separated by a small edge segment is emitted by a bubble along a [110] direction in a fcc metal. This allows the bubble to grow as shown in figure 1.11 .

The screw segments can climb along the [110] direction to relieve the stress due to the bubble. But the stress will also rotate the dislocation line direction. The resultant mixed character dislocation can glide only on the [110] glide cylinder defined by the Burgers vector and dislocation line direction. The dislocation line can wrap itself around the glide cylinder to form a helix.

The helix can have a left or right handed character and when viewed perpendicular to the axis of the glide cylinder it looks like a sine wave. When rotated away from this position the helix develops cusps then loops at what were previously peaks in the sine wave. The rotation of the pairs of screw segments to the dislocation line can continue further until edge type loops are formed when the two screw segments meet up. The loops are connected by a long straight screw dislocation. This long screw dislocation can glide in 
a plane perpendicular to $\mathbf{b}$ and so is not restricted to the glide cylinder. If the screw dislocation glides away from the glide cylinder a row of edge dislocation loops punched out along a [110] glide cylinder remain behind. The number of loops formed depends upon the number of turns in the helix. The failure of the Bullough-Newman theory to take into account the role of helices in the formation of rows of dislocation loops may partially explain the discrepancy between loop spacing predictions made by their theory and experiment.
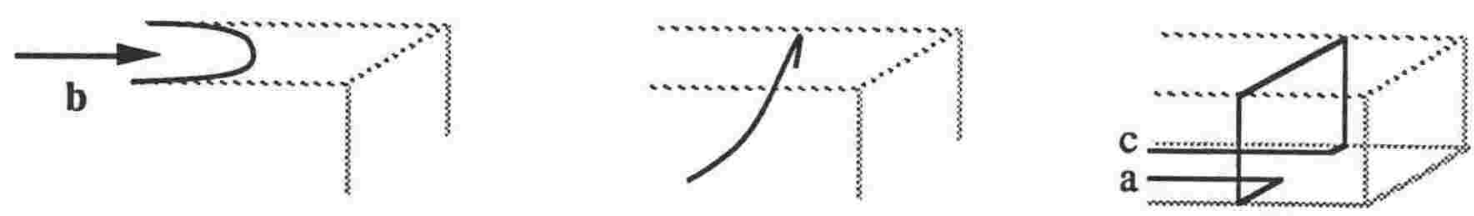

Figure 1.11: The prismatic punching sequence. The solid black line indicates the dislocation line. The parts of the dislocation which have $\mathbf{b}$ parallel to this line are pure screw segments and will have a displacement field about them like that shown in figure 1.3. The parts of the dislocation which have $b$ perpendicular to this line are edge segments. The rest of the line has a mixed edge and screw character. The edge components are restricted to moving along the glide cylinder but the screw components are not confined to this cylinder. As the dislocation is emitted by the bubble the screw components denoted by a and c twist around the glide cylinder to form a helical dislocation. The edge component that forms on the left hand side of the figure grows bigger during this process but it cannot twist around the cylinder.

\subsection{Competing Mechanisms of Bubble Superlattice Formation}

Besides the dislocation punching mechanism there are two other factors that could be important in bubble ordering. They are elastic interactions between the displacement fields of the bubbles and the diffusion of vacancies and gas atoms. Bubble superlattices are similar to void superlattices for which ordering theories based on elastic interactions have been proposed (Evans et al.[121], Stoneham [122] and Tewary and Bullough [123]). These theories use Eshebly's solution [124] for the displacement field about a void or spherical shell to calculate the energy of interaction between two voids. These voids are thought to nucleate randomly then order by elastic interactions. The papers then discuss 
various ways to sum this interaction energy over the entire void lattice using Green's functions or Fourier transform techniques. The interaction energy can be minimised to find that the equilibrium void spacing agrees approximately with experiment. Recently more accurate computer simulations that predict void spacings and sizes have cast doubt on these models.

Bubble lattices are different from void lattices in that they are formed by irradiations at temperatures $\sim 0.2 \mathrm{~T}_{\mathrm{m}}$ where the thermal mobility of vacancies is low. Voids on the other hand rely upon the increased mobility of vacancies, that occurs at higher temperatures, for their formation. An obscuring factor is that the irradiating gas atoms can cause vacancies to be knocked around by collisions. So a significant vacancy movement by athermal processes could occur even at room temperature. The growth of the bubbles is linked to theories of Ostwald ripening that will not be treated in detail here (Evans [125] and Dubinko et al. [126] for an example).

It has been suggested by Evans and others [118] that the diffusion of interstitial metal atoms could play a role in bubble ordering. The model proposed is based on the anisotropic diffusion of self-interstitial atoms (SIA) which have been produced as the result of collision damage. In metals there is some evidence that SIA diffusion may be confined to two dimensions - i.e., planar diffusion. Evans has shown that the ordering of bubbles in three dimensions can be explained if SIA diffusion is confined to one set of planes only. Bubble alignment is attributed to the effect of one bubble shadowing a neighbouring bubble from the diffusing flux of SIA's. A bubble misaligned with respect to neighbouring bubbles will collect more diffusing metal atoms on the side projecting out of the shadow zone and so will be moved towards the shielded region. In this way the bubbles will become ordered. 


\subsection{Summary}

In this chapter the aim of the thesis is mentioned and some of the background necessary to achieve this aim is introduced. The introduction briefly reviews past work done on the ordering of bubbles and voids to form superlattices. There are several alternative theories for bubble ordering. Perhaps the most important theory is based on the punching of dislocation loops from overpressurized bubbles. The aim of the thesis is to develop and evaluate methods based on TEM for measuring the density and size of the loops in ion-implanted metals.

To achieve this aim it is necessary to understand how a dislocation loop can be punched out by a bubble. The mechanisms by which loops are punched are described by isotropic elasticity theory. It was therefore necessary to revise elasticity theory in this chapter before discussing the mechanisms by which loops are punched out.

The nature of several different types of dislocations is described to provide the reader with a good physical model for the structures investigated in this thesis. This is important especially in the later chapters where experimental micrographs of dislocations and bubbles are presented. The image formation theory used in transmission electron microscopy (TEM) is introduced in chapter 2 and elasticity theory is developed further in chapter 3. It will become apparent in later chapters that the appearance of dislocations in micrographs is not connected in an obvious way to the dislocation structures themselves. Some understanding is needed of the process by which images are formed when electrons are scattered off different types of dislocations so that the structure is deduced correctly from experimental micrographs. 


\section{Chapter 2: THE THEORY OF IMAGE FORMATION IN THE TEM}

\subsection{The Differential Equations of Howie and Whelan}

\subsubsection{Introduction}

The early theory of the diffraction of electrons was influenced by the more mature theory of X-ray diffraction. In this section the ideas and nomenclature of X-ray diffraction relevant to electron diffraction are summarised. From this start, in the initial sections of this chapter the theory of electron diffraction is developed and used to derive the differential equations of Howie and Whelan. The following sections deal in turn with the many-beam theory, which is necessary to understand the weak beam technique, and the multislice formalism used to interpret high resolution electron micrographs. The final sections of this chapter deal with how the wave function at the exit surface of the foil can be defocussed to simulate through-focal series, and how the generalised cross-section construction of Head et al. [79-82] enables the rapid simulation of images of dislocations. A more detailed coverage can be found in Hirsch et al. [67] and Cowley [127]. An outline only is given in this chapter.

The analysis of the diffraction of X-rays can be expressed in terms of either Bragg reflection from atomic planes or Laue equations based on the diffraction from individual atoms. In the Bragg case, effectively the planes of atoms are treated as partially reflecting mirrors.

In the Laue case the constructive interference from a row of atoms lying in a plane parallel to the surface forms cones of allowed direction with axes coinciding with the atom row. Where these cones intersect a distant screen, hyperbolae are formed upon which the intensity is large. In between these hyperbolae the path differences of the waves scattered by the atoms give destructive interference. The scattering from a two-dimensional grating of atoms gives an array of intense spots at the intersection points of two sets of hyperbolae. The observation of strong spots in the scattering from a three-dimensional stack of atoms requires the satisfaction of the three Laue equations. The Laue equations require the path differences to be an integral multiple of the wavelength. The reciprocal lattice can be 
constructed by applying the Laue equations. In this thesis the term diffraction vector will be used to describe the vector pointing from the origin in reciprocal space to an intense spot at which constructive interference occurs.

The idea of a reciprocal lattice can be carried over from the theory of X-ray diffraction to describe electron diffraction. A $100 \mathrm{keV}$ electron has a wavelength of 0.037 $\AA$. A typical X-ray will have a wavelength of about 1-2 $\AA$. The curvature of the hyperbolae is inversely proportional to the wavelength. The hyperbolae occurring in X-ray diffraction patterns will have, therefore, much greater curvature than those found in electron diffraction patterns.

Darwin [128] obtained solutions for the intensity of the diffracted spots in the Bragg case for X-rays. The intensity of these spots cannot be predicted by the Laue equations. The Darwin approach was to find reflection and transmission coefficients for each plane of atoms. The amplitudes of the diffracted beams were then found by summing over the amplitudes diffracted from each plane. The X-ray diffraction process is modelled using differential equations and a two-beam approximation. The approach is limited to modelling the diffraction where the crystal is tilted so that the central beam, which is undeviated, and only one other have large amplitudes. In this case the crystal is said to be tilted to strong two-beam conditions.

Howie and Whelan later used a similar approach to model electron diffraction; the analysis is again based on differential equations and a two-beam approximation. The equations were developed from the theory for electron diffraction of Bethe. Bethe [129] used a set of differential equations and an eigenvalue equation to model the electron diffraction; the formalism uses the Bloch wavefunction solutions to the Schrodinger wave equation (SWE) to describe the propagation of the electron wave through a perfect crystal. Kato [130-132] extended the Bethe theory in the Laue case to wedge shaped and polyhedral crystals and then approximated the results to describe Fraunhofer and Fresnel diffraction. These papers contain a good description of the dispersion surface and boundary conditions used at the crystal surface to solve the differential equations. The Bethe formalism is described in sections 2.1.2, 2.1.4 and 2.2 in sufficient detail for a reader to understand the derivation of the Howie-Whelan differential equations in section 2.1.4. 


\subsubsection{The Dispersion Surface}

For the electron waves scattered from a plane of atoms to interfere constructively the Bragg condition must be close to being satisfied. The Bragg condition requires the waves reflected from successive planes of atoms have a path difference equal to an integral multiple of the electron wavelength. Suppose for a given set of planes in reciprocal space the start of the reflected wavevector is made coincident with the start of the incident wavevector. The diffraction vector $\mathrm{g}$ is then defined to be the vector that starts at the end of the incident wavevector $\mathbf{K}$ and terminates at the end of the scattered wavevector, $\mathbf{K}^{\prime}$ as shown in figure 2.1. The planes giving rise to the diffraction are perpendicular to $\mathrm{g}$. The Bragg condition requires the magnitude of the vector $\mathbf{g}$ to be inversely proportional to the spacing between the diffracting planes. The Brillouin zone boundary for this particular $\mathbf{g}$ is the plane that is perpendicular to $\mathbf{g}$ and passes through $0.5 \mathrm{~g}$ as shown in figure 2.1 . All of the closest $\mathbf{g}$ 's in the crystal will form the Brillouin zone surface [10].

Figure 2.1: The Ewald sphere construction (not to scale). The circle shows the portion of a two-dimensional slice through the reciprocal lattice that intersects with the Ewald sphere. The incident wave with wavevector $(\mathbf{K})$ will be strongly scattered into the spot in the reciprocal lattice with a vector $\mathbf{g}$ because this spot lies on the surface of the Ewald sphere. The scattered wave will have a wavevector of $\mathbf{K}$ '. 
An electron wave with a specified wavelength and direction $\mathbf{K}$ incident on a crystal will be most strongly scattered in the direction closest to satisfying the Bragg condition. To see most easily which plane of atoms (i.e. g) the electrons will be most strongly scattering from, it is useful to introduce the idea of a Ewald sphere in reciprocal space as shown in figure 2.1. The Ewald sphere has a centre at the start of the electron wavevector and a radius equal to the magnitude of the electron wavevector. An elastically scattered wavevector must terminate on the Ewald sphere. The Ewald sphere construction is useful because constructive interference occurs when the end of a $\mathbf{g}$ vector terminates on the Ewald sphere. The construction is used to show which g's will be strongly excited in the diffraction pattern.

The dispersion surface is introduced in the Bethe theory. The shape of this surface corresponds with the allowed wavevectors for a given total energy (kinetic energy plus potential energy). The term "allowed wavevector" is used to describe a wavevector that satisfies the boundary conditions at the top surface of the foil. The dispersion surface is constructed approximately from a set of Ewald spheres centred on the diffraction vectors (instead of the start of the electron wavevector). The dispersion surface follows the Ewald spheres except near the Brillouin zone boundaries where the dispersion surface is distorted due to the Fourier coefficients of the potential. Bloch waves (eigenfunctions of the SWE) from different branches of the dispersion surface will spend different amounts of time in the vicinity of the lower potential surrounding the atoms of the crystal. Different Bloch waves will then experience a different potential and because they have the same total energy their kinetic energies will not be the same.

The allowed wave vectors of the dispersion surface can be found by solving for the Bloch wave solutions to the SWE using the Bethe formalism. In practice the only part of the dispersion surface calculated is the part excited by a given incident electron wave. The rest of the dispersion surface could be found by varying the direction of the incident electron. The dispersion surface construction readily allows the excitation of the various Bloch waves by the incident electron to be represented schematically as in figure 2.2. Section 2.3 , on the weak beam method, deals with a more sophisticated theory of scattering between the Bloch waves in a deformed crystal. 


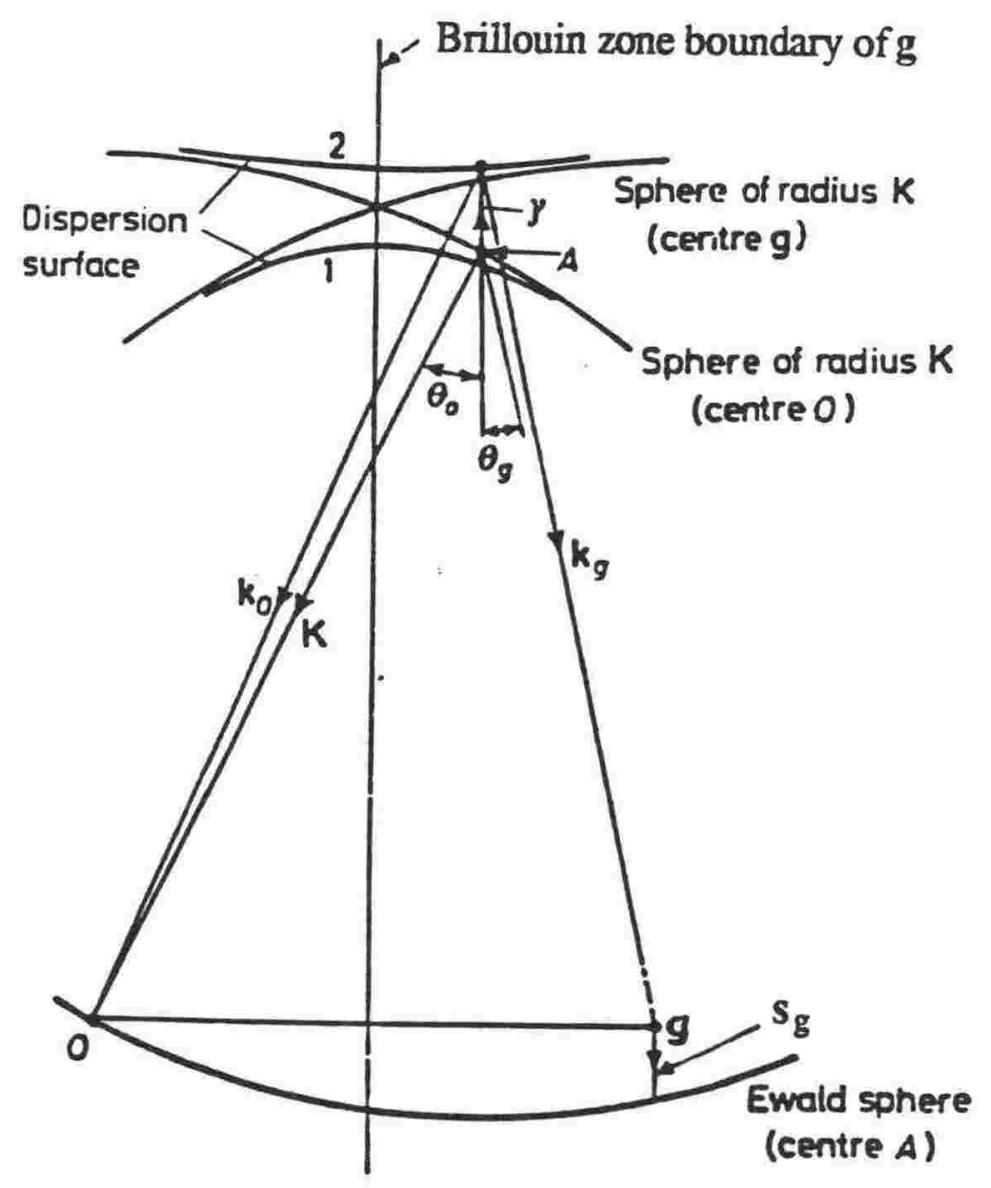

Figure 2.2: The figure shows the excitation error and other vectors used in reciprocal space to construct the dispersion surface and the Ewald spheres. The excitation error for the gth diffracted beam is the vertical distance between the gth diffracted spot in reciprocal lattice space and the Ewald sphere. The Bloch wave vectors $\mathbf{k}_{0}$ and $\mathbf{k}_{\mathrm{g}}$ for dispersion surface 2 only are shown. The wave vectors associated with dispersion surface 1 and the other dispersion surfaces have been omitted. Dispersion surfaces 1 and 2 are shown on the figure only because these are the two closest to the energy of the incident electron beam. The Bloch waves associated with these two dispersion surfaces will be the most strongly excited at the top surface of the crystal. The Bloch waves on the second dispersion surface will have a higher kinetic energy than the Bloch waves on the first dispersion surface. $\mathbf{K}$ is the electron wavevector in the crystal and it points in approximately the direction of the electron wave, $\chi$, incident in vacuum on the crystal surface. $\mathbf{K}$ has been refracted slightly towards the surface normal by the mean potential in the crystal. The vector $\gamma$ is shown for only the second dispersion surface. Later in this chapter superscripts will be used to identify the dispersion surface to which the Bloch waves and $\gamma$ 's belong. 
Those wave vectors which satisfy the boundary conditions of continuity of the wave function and its derivative can be readily identified using the following procedure. At the top surface of the crystal the component of the incident wave vector parallel to the surface must be the same as that of the excited Bloch waves. The Bloch waves satisfying this boundary condition are found by drawing the line parallel to the foil normal through the point $\mathrm{A}$ on figure 2.2. The allowed Bloch wave vectors are given by connecting the points where this line intersects the dispersion surface to the end of the diffracting vectors of the crystal. The wave vector components parallel to the foil normal do not have to be conserved. Hirsch et al. [67] prove that as a consequence of the boundary conditions nearly all of the electron intensity incident upon the top surface of a foil will propagate into the specimen. Very little of the electron intensity is reflected from the top surface of the foil.

Because the crystal has a finite thickness the Ewald sphere does not have to pass exactly through $\mathbf{g}$ to excite a Bloch wave with wave vector $\mathbf{k}_{\mathrm{g}}$. The sharp spot predicted by the Laue equations is smeared out parallel to the Brillouin zone boundary. The smearing of the amplitudes has a $\sin (\mathrm{x}) / \mathrm{x}$ variation. Therefore when the crystal is tilted so that $\mathbf{s}_{\mathrm{g}}$ is finite, the Ewald sphere will still cut through the region about $\mathbf{g}$ in which constructive interference occurs.

\subsubsection{Description of the Howie-Whelan Differential Equations}

The Howie-Whelan differential equations are a pair of complex, linear, coupled equations describing how the beam amplitudes vary through a crystal in which the atoms may have been displaced from their Bravais lattice positions. Hence they are useful for interpreting the electron microscope images of defects. They are obtained from the Bethe formalism in the Laue case by using a two-beam approximation.

The simplest displacement of the atoms in a crystal from their Bravais lattice positions occurs when a stacking fault is introduced. A stacking fault can be created by cutting a perfect crystal into two pieces and displacing one piece relative to the other. This displacement introduces a change of phase in the electron waves passing through the fault. Whelan and Hirsch $[68,69]$ obtained analytical solutions using the dynamical theory with 
no absorption (i.e., no inelastic scattering events) for the beam amplitudes diffracted from a stacking fault. They plotted the solutions as intensity profiles across the exit surface of the crystal. They verified the theory by comparing these profiles against experimental micrographs. Hashimoto, Howie and Whelan $[70,71]$ extended the theory of diffraction from deformed crystals to phenomenologically include absorption. Their computed intensity profiles for bent crystals and stacking faults again agree with experiment.

Howie and Whelan $[72,73]$ derived differential equations to model the diffraction from crystals continuously deformed by dislocations. Analytical solutions cannot be obtained for the diffracted intensities due to the complexity of the displacement fields about dislocations.

\subsubsection{Derivation of the Howie-Whelan differential equations}

\subsubsection{Scattering Factors and the Scattering Geometry}

The strength of the elastic scattering of electrons from atoms can be found using the Born approximation. Quantitatively the strength of the scattering is given by the atomic electron scattering factor. Kinematical theories include the effects of single scattering only and are of use in situations where the diffracted beams have a weak amplitude. (In the kinematical theory, the beam amplitudes oscillate with the foil thickness in a perfect crystal, giving rise to thickness fringes, even though no scattering from the diffracted beam to the central beam occurs). Dynamical theories include multiple scattering effects and are useful when several of the beam amplitudes are strongly excited. Kinematic theories [67] were not used in this thesis as it was thought that the assumption of kinematical scattering may be invalid when several defects overlap. However, this was not tested by computing kinematic images. Considerably more computational time must be expended to produce an image using the more accurate dynamical scattering theory with a nonzero displacement field so that sometimes the less accurate kinematic theory is preferred.

The Howie-Whelan differential equations are based on dynamical theory. However, kinematic theory (i.e., the idea of scattering factors) is still used to construct the periodic 
crystal potential. The first Born approximation is used to treat the change in beam amplitudes, resulting from an infinitesimally thin slice of crystal. Multiple scattering effects are included by reapplying the first Born approximation to the new beam amplitudes as they pass through successive slices of the crystal. This approach is similar to that of Darwin.

The Mott formula connects the electron $\left(f_{e}\right)$ and $X$-ray $\left(f_{X}\right)$ scattering factors [133]. The atomic electron scattering factor at a particular angle $\theta$ is calculated from the corresponding X-ray scattering factor calculated by Doyle and Turner [134] by means of the following expression

$$
\mathrm{f}_{\mathrm{e}}\left(\mathrm{S}_{\mathrm{g}}\right)=\frac{\mathrm{m}_{\mathrm{e}} \mathrm{e}^{2}}{2 \mathrm{~h}^{2}}\left[\frac{\mathrm{Z}-\mathrm{f}_{\mathrm{X}}\left(\mathrm{S}_{\mathrm{g}}\right)}{\mathrm{S}_{\mathrm{g}}^{2}}\right]
$$

Symbols used in equation 2.1 and in the following discussion are defined in a list of symbols in the appendices. The variables $\mathrm{e}, \mathrm{m}_{\mathrm{e}}$ and $\mathrm{h}$ have their usual meanings. The units have been omitted in the list of symbols, but in electron microscopy length for example is conventionally measured in Angstrom units. With the exception of Angstroms the mks system is used in this thesis although some authors, e.g. Cowley [127], use cgs units. The equations quoted by these authors need to be converted from the cgs to mks systems to be compared with the equations quoted here.

A better understanding of the Mott formula results from a consideration of the different mechanisms by which $\mathrm{X}$-rays and electrons are scattered from atoms. X-rays propagate as an electromagnetic field. An X-ray interacts with an atomic electron by accelerating the electron with an oscillating electric field. The accelerating electron in turn radiates an electric field, so that effectively the $\mathrm{X}$-ray has been scattered by the electron. The X-ray will not interact as strongly with the atomic nucleus because the nucleus has a larger mass than an electron. Alternatively, the interaction between an electron and X-ray photon can be thought of as being due to the "exchange" of an electron between the two particles. The electron "exchanged" between the photons is allowed to exist for a short time by the Heisenberg uncertainty principle.

In contrast to X-rays, the electrons scatter off both the atomic electrons and the nucleus through the Coulomb force, (or equivalently by the exchange of photons between 
the particles). The $\mathrm{Z}$ term in equation 2.1 arises because the electrons interact with the nuclear charge, whereas the X-rays do not. The Coulomb force has a range greater than the distance that the exchange electron can travel in an X-ray scattering process. For this reason electrons will be more strongly scattered than X-rays if both pass through the same thickness of material. The electron scattering factor is a factor of $10^{4}-10^{5}$ larger than the $\mathrm{X}$ ray scattering factor.

In equation 2.1, angles are measured in terms of $S_{g}$ where $S_{g}$ varies with angle according to equation 2.2. $\mathrm{S}_{\mathrm{g}}$ is a distance in reciprocal space, i.e., it corresponds to a direct lattice frequency in real space. $S_{g}$ can be calculated from the angle between the incident and scattered beam. This is equivalent to applying the Bragg equation to the planes responsible for the diffraction:

$$
\mathrm{S}_{\mathrm{g}}=\frac{\sin \theta_{\mathrm{g}}}{\lambda}=\frac{1}{2 \mathrm{~d}_{\mathrm{g}}}
$$

The electron wavelength needed to calculate $S_{\mathrm{g}}$ using equation 2.2 is calculated from the energy of the electrons by the relativistic formula

$$
\lambda=\left[\frac{2 m_{e} e E}{h^{2}}\left(1+\frac{e E}{2 m_{e} c^{2}}\right)\right]^{-\frac{1}{2}} .
$$

At $S_{g}=0$ equation 2.1 cannot be used, and the tabulated values for $f_{e}$ of Doyle and Turner [134] must be used.

Doyle and Turner [134] used relativistic Hartee-Fock wave functions to obtain theoretical values for the scattering factors for $\mathrm{X}$-rays and electrons. To calculate the $\mathrm{X}$-ray scattering factor an integral was taken, over all space, of the total charge density of the atom. The electron scattering factor can be obtained from an integral over the atomic potential.

Fujiwara $[135,136]$ gave a more sophisticated treatment of relativistic effects in electron diffraction by applying the Bethe formalism to the Dirac wave equation rather than the SWE. He found that relativistic effects are adequately included in the theory of Bethe by using a relativistic wavelength and relativistic mass in the SWE. The spin of the electron 
has negligible effects on the diffraction from non-magnetic materials. Equation 2.3 and the relativistic electron mass are all that are required to make the theory in this chapter relativistic. A $100 \mathrm{keV}$ electron will have a relativistic correction factor $\gamma=1 / \sqrt{ }\left(1-\mathrm{v}^{2} / \mathrm{c}^{2}\right)$ equal to about 1.19. Relativistic effects are significant in electron microscopy and must be included in any modelling of the electron diffraction.

\subsubsection{Construction of the Crystal Potential}

The Howie-Whelan differential equations are derived by solving the SWE so it is necessary to construct the crystal potential for inclusion in this equation. The X-ray scattering factors in section 2.1.4.1 can be predicted by theory (Doyle and Turner [134]). The electron scattering factors are then calculated from equation 2.1. The first Born approximation relates the electron scattering factors to a Fourier transform over the crystal potential. This allows the Fourier coefficients of the crystal potential to be calculated. This section shows how to construct the crystal potential from tables of X-ray scattering factors derived from theory.

In the first Born approximation, the structure factors describe the degree of interference between the waves scattered from different atoms in the unit cell of the crystal. The structure factors are calculated from the electron scattering factors by a sum over the atoms in the unit cell

$$
F_{g}=\sum_{j} f_{e, j}\left(S_{g}\right) \exp \left(-B_{j} S_{g}\right) \exp \left(2 \pi i g \cdot r_{j}\right)
$$

A subscript has been added to the electron scattering factor in equation 2.4 to indicate that the unit cell can contain different types of atoms. The second factor, $\exp \left(-\mathrm{B}_{\mathrm{j}} \mathrm{S}_{\mathrm{g}}\right)$, called the Debye-Waller factor takes into account the thermal vibration of the atoms, i.e., the diffuse electron-phonon scattering.

The potential in a perfect crystal containing many unit cells is constructed by a Fourier series over the Fourier coefficients of the potential 


$$
\mathrm{V}(\mathbf{r})=\frac{h^{2}}{2 m_{\mathrm{e}} \mathrm{e}} \sum_{\mathrm{g}} \mathrm{U}_{\mathrm{g}} \exp (2 \pi \mathrm{ig} \cdot \mathbf{r})=\sum_{\mathrm{g}} \mathrm{V}_{\mathrm{g}} \exp (2 \pi \mathrm{ig} \cdot \mathbf{r})
$$

The new coefficients $\mathrm{U}_{\mathrm{g}}$ introduced in equation 2.5 are proportional to $\mathrm{V}_{\mathrm{g}}$. The use of these new coefficients will result in a simpler equation being obtained when the potential is substituted into the SWE because some common factors can be cancelled out. The Fourier coefficients of the potential are calculated from the structure factors using

$$
V_{g}=\frac{h^{2} F_{g}}{2 \pi m_{e} e V_{c}}
$$

Consider the situation when the crystal is deformed by a displacement field $\mathbf{R}(\mathbf{r})$. The potential at position $\mathbf{r}$ will be displaced by $\mathbf{R}(\mathbf{r})$. The new potential at $\mathbf{r}$ is the potential which was at $\mathbf{r}-\mathbf{R}(\mathbf{r})$. The potential $V(\mathbf{r})$ in a deformed crystal, that follows after transforming $\mathbf{r}$ to $\mathbf{r}-\mathbf{R}(\mathbf{r})$, is

$$
\mathrm{V}(\mathbf{r})=\frac{h^{2}}{2 m_{\mathrm{e}} \mathrm{e}} \sum_{\mathrm{g}} \mathrm{U}_{\mathrm{g}} \exp (-2 \pi \mathrm{ig} \cdot \mathbf{R}(\mathbf{r})) \exp (2 \pi \mathrm{ig} \cdot \mathbf{r})
$$

The Fourier coefficients are thus phase shifted by an amount proportional to the deformation. The structure of the reciprocal lattice is not significantly affected by the deformation around one dislocation and therefore it is still valid to calculate the potential using the reciprocal lattice positions.

\subsubsection{The Column Approximation and Solving the SWE}

A solution for the SWE of the following form can be postulated:

$$
\psi(\mathbf{r})=\sum_{\mathrm{g}} \phi_{\mathrm{g}}(\mathbf{r}) \exp \left(2 \pi \mathrm{i}\left(\chi+\mathrm{g}+\mathbf{s}_{\mathrm{g}}\right) \cdot \mathbf{r}\right)
$$

The wave function is a sum of terms involving plane waves, or beams, propagating through the crystal. In this section equations are derived which describe how the beam amplitudes 
$\left(\phi_{\mathrm{g}}(\mathbf{r})\right)$ vary as the electrons pass through the periodic crystal potential constructed in the previous section.

Energy conservation applied to the Ewald sphere construction requires that the wave vector $\chi$ in equation 2.8 satisfies

$$
\chi^{2}=\left(\chi+g+s_{g}\right)^{2}=\frac{2 m_{e} e E}{h^{2}} .
$$

The SWE is

$$
\nabla^{2} \psi(\mathbf{r})+\frac{8 \pi^{2} m_{e} e^{2}}{h^{2}}[E+V(\mathbf{r})] \psi(\mathbf{r})=0
$$

where the unit of $\mathrm{E}$ and $\mathrm{V}(\mathbf{r})$ is volts. Substituting the solution in equation 2.8 into the SWE we obtain

$$
\begin{gathered}
\sum_{\mathbf{g}}\left\{\frac{1}{4 \pi^{2}} \nabla^{2} \phi_{\mathrm{g}}(\mathbf{r})+\frac{\mathrm{i}}{\pi}\left(\chi+\mathbf{g}+\mathbf{s}_{\mathrm{g}}\right) \cdot \nabla \phi_{\mathrm{g}}(\mathbf{r})+\sum_{\mathrm{h}} \mathrm{U}_{\mathrm{g}-\mathrm{h}} \phi_{\mathrm{h}}(\mathbf{r}) \exp \left[2 \pi \mathrm{i}(\mathbf{h}-\mathbf{g}) \cdot \mathbf{R}+2 \pi \mathrm{i}\left(\mathbf{s}_{\mathrm{h}}-\mathbf{s}_{\mathrm{g}}\right) \cdot \mathbf{r}\right]\right\} \\
\mathrm{x} \exp \left(2 \pi \mathrm{i}\left(\chi+\mathbf{g}+\mathbf{s}_{\mathrm{g}}\right) \cdot \mathbf{r}\right)=0 .
\end{gathered}
$$

The assumption is made that each coefficient of the exponential terms outside the curly brackets in equation 2.11 is individually zero. Provided that the beam amplitudes do not vary rapidly on the scale of the electron wavelength, the gradient squared term can be neglected to give

$$
\left(\chi+\mathrm{g}+\mathrm{s}_{\mathrm{g}}\right) \cdot \nabla \phi_{\mathrm{g}}(\mathbf{r})=\pi \mathrm{i} \sum_{\mathrm{h}} \mathrm{U}_{\mathrm{g}-\mathrm{h}} \phi_{\mathrm{h}}(\mathbf{r}) \exp \left[2 \pi \mathrm{i}(\mathbf{h}-\mathbf{g}) \cdot \mathbf{R}+2 \pi \mathrm{i}\left(\mathbf{s}_{\mathrm{h}}-\mathbf{s}_{\mathrm{g}}\right) \cdot \mathbf{r}\right] .
$$

Because the electron wave vector has a large $\mathrm{z}$ component and relatively small $\mathrm{x}$ and $\mathrm{y}$ components, the partial differential equations in 2.12 can be reduced to the ordinary differential equations

$$
\frac{\mathrm{d} \phi_{\mathrm{g}}(\mathbf{r})}{\mathrm{dz}}=\pi \mathrm{i} \frac{1}{\left(\chi+\mathbf{g}+\mathbf{s}_{\mathrm{g}}\right)_{\mathrm{z}}} \sum_{\mathrm{h}} \mathrm{U}_{\mathrm{g}-\mathrm{h}} \phi_{\mathrm{h}}(\mathbf{r}) \exp \left[2 \pi \mathrm{i}(\mathbf{h}-\mathbf{g}) \cdot \mathbf{R}+2 \pi \mathrm{i}\left(\mathbf{s}_{\mathrm{h}}-\mathbf{s}_{\mathrm{g}}\right) \cdot \mathbf{r}\right]
$$




$$
\cong \sum_{\mathrm{h}} \frac{\pi \mathrm{i}}{\xi_{\mathrm{g}-\mathrm{h}}} \phi_{\mathrm{h}}(\mathbf{r}) \exp \left[2 \pi \mathrm{i}(\mathbf{h}-\mathbf{g}) \cdot \mathbf{R}+2 \pi \mathrm{i}\left(\mathbf{s}_{\mathrm{h}}-\mathbf{S}_{\mathrm{g}}\right) \cdot \mathbf{r}\right] .
$$

The $\xi_{\mathrm{g}-\mathrm{h}}$ term in equation 2.13 is an extinction distance that will be defined in section 2.1.4.4. The approximations made in obtaining equation 2.13 from equation 2.11 are collectively known as the "column approximation". The validity of this approximation in the strong two-beam case was confirmed by Howie and Basinski [75]. Howie and Sworn [77] validated the column approximation in the weak-beam case.

\subsubsection{Extinction Distances}

The substitution has been made in equation 2.13 that

$$
\frac{\mathrm{U}_{\mathrm{g}-\mathrm{h}}}{\left(\chi+\mathrm{g}+\mathrm{s}_{\mathrm{g}}\right)_{\mathrm{z}}} \cong \frac{\mathrm{U}_{\mathrm{g}-\mathrm{h}}}{\mathrm{K}} \cong \frac{1}{\xi_{\mathrm{g}-\mathrm{h}}} .
$$

Where the extinction distance is defined by

$$
\xi_{\mathrm{g}}=\frac{\mathrm{K} \cos \theta_{\mathrm{g}}}{\mathrm{U}_{\mathrm{g}}} .
$$

For $\xi_{0}, \theta_{0}$ should replace $\theta_{\mathrm{g}}$ in equation 2.15. In electron diffraction the $\theta_{0}$ and $\theta_{\mathrm{g}}$ values are typically $1^{\circ}$ to $2^{\circ}$ so $\cos \theta$ can be taken as unity.

The analytical solutions to the Howie-Whelan differential equations (found later in section 2.1.5) have the form of damped sinusoidal functions. The extinction distances appear in the arguments of the sinusoids. The beam amplitudes oscillate with a period set by the extinction distances. Thus the intensity of a beam will fall to zero (i.e., become extinct) and then build up back to near its original value (in the absence of absorption) when the electron wave passes through a thickness of crystal equal to the extinction distance.

Experimentally electron micrographs are taken at temperatures above absolute zero, so the effects of a non-zero temperature must be included in any modelling. $\mathrm{U}_{\mathrm{g}}$ is the only temperature dependent term on the right hand side of equation 2.15 and $\mathrm{U}_{\mathrm{g}}$ is proportional to the structure factor (refer to equations 2.5 and 2.6). The structure factor is calculated 
using equation 2.4. Since the Debye-Waller coefficient in equation 2.4 is temperature dependent the extinction distance depends on temperature. The dependence has been checked by Howie and Valdre [76] by measuring the variation in the spacing of thickness fringes as various materials were heated.

The zeroth extinction distance corresponds to the mean potential of the crystal refracting the electrons. The wave vector is refracted according to

$$
\mathrm{K}_{\mathrm{z}}=\chi_{\mathrm{z}}+\frac{1}{2 \xi_{0}}
$$

$\mathrm{K}$ and $\chi$ differ only due to the mean potential in the crystal. Because the mean potential is about $10 \mathrm{eV}$, whereas the electron energy is typically $120 \mathrm{keV}, \mathrm{K}$ can be substituted for $\chi$ to a good approximation. The wavevectors have a magnitude of approximately $27 \AA^{-1}$ whereas the extinction distances are equal to about $300 \AA$, so $\mathrm{K}_{\mathrm{z}} \approx \chi_{\mathrm{z}}$.

Molière [137] introduced a complex potential to explain the intensity of transmitted X-rays at the angle of Bragg reflection. Yoshioka [138] applied Molière's approach to the case of electron diffraction and extended it to develop a phenomenological model for the effects of inelastic scattering. The complex potential he uses is equivalent to the complex extinction distances of Hashimoto et al. $[70,71]$ in equation 2.17. Yoshioka uses the same formalism for the scattering as that used by Kainuma [139] to explain the dependence of the position of the Kikuchi lines on crystal tilt .

Absorption of the electron beam amplitudes can be included in the differential equations, phenomenologically, by replacing the real extinction distances by extinction distances that have both a real and imaginary part

$$
\frac{1}{\xi_{0}} \rightarrow \frac{1}{\xi_{0}}+\frac{\mathrm{i}}{\xi_{0}^{\prime}} \text { and } \frac{1}{\xi_{\mathrm{g}}} \rightarrow \frac{1}{\xi_{\mathrm{g}}}+\frac{\mathrm{i}}{\xi_{\mathrm{g}}^{\prime}} \text {. }
$$

The Howie-Whelan differential equations cannot be used to model Kikuchi lines when the beam amplitudes are damped by the complex potential, even though physically the intensity lost from the beams may be transferred into the Kikuchi lines. The electron diffraction 
theory used in this thesis neglects the contributions of any inelastically scattered electrons to the images or diffraction patterns.

\subsubsection{Different Forms of the Howie-Whelan Differential Equations}

In the two-beam case equation 2.13 above reduces to:

$$
\begin{aligned}
& \frac{\mathrm{d} \phi_{0}}{\mathrm{dz}}=\frac{\pi \mathrm{i}}{\xi_{0}} \phi_{0}+\frac{\pi \mathrm{i}}{\xi_{\mathrm{g}}} \phi_{\mathrm{g}} \exp \left[2 \pi \mathrm{i}\left(\mathrm{s}_{\mathrm{g}} \mathrm{z}+\mathrm{g} \cdot \mathbf{R}\right)\right] \\
& \text { and } \frac{\mathrm{d} \phi_{\mathrm{g}}}{\mathrm{dz}}=\frac{\pi \mathrm{i}}{\xi_{0}} \phi_{\mathrm{g}}+\frac{\pi \mathrm{i}}{\xi_{\mathrm{g}}} \phi_{0} \exp \left[-2 \pi \mathrm{i}\left(\mathrm{s}_{\mathrm{g}} \mathrm{z}+\mathrm{g} \cdot \mathbf{R}\right)\right] .
\end{aligned}
$$

This is the displacement field form of the Howie-Whelan differential equations. These equations are similar to those obtained by Darwin in the X-ray case and so the beam amplitudes are often called the Darwin amplitudes. The variables can be transformed by using

$$
\begin{aligned}
\phi_{0}^{\prime \prime} & =\phi_{0} \exp \left(\frac{-\pi \mathrm{iz}}{\xi_{0}}\right) \\
\text { and } \phi_{\mathrm{g}}^{\prime \prime} & =\phi_{\mathrm{g}} \exp \left(2 \pi \mathrm{is}_{\mathrm{g}} \mathrm{z}-\frac{\pi \mathrm{iz}}{\xi_{0}}+2 \pi \mathrm{ig} \cdot \mathbf{R}\right) .
\end{aligned}
$$

Dropping the primes and explicitly including the complex part of the extinction distances equations 2.18 become

$$
\begin{gathered}
\frac{\mathrm{d} \phi_{0}}{\mathrm{dz}}=-\frac{\pi}{\xi_{0}^{\prime}} \phi_{0}+\pi\left(\frac{\mathrm{i}}{\xi_{\mathrm{g}}}-\frac{1}{\xi_{\mathrm{g}}^{\prime}}\right) \phi_{\mathrm{g}} \\
\text { and } \frac{\mathrm{d} \phi_{\mathrm{g}}}{\mathrm{dz}}=\pi\left(\frac{i}{\xi_{\mathrm{g}}}-\frac{1}{\xi_{\mathrm{g}}^{\prime}}\right) \phi_{\mathrm{o}}+\left(-\frac{\pi}{\xi_{0}^{\prime}}+2 \pi \mathrm{i}\left(\mathrm{s}_{\mathrm{g}}+\beta_{\mathrm{g}}^{\prime}\right)\right) \phi_{\mathrm{g}} .
\end{gathered}
$$


These equations are referred to as the derivative of the displacement form of the HowieWhelan differential equations. The crystal deformation is included by the term

$$
\beta_{\mathrm{g}}^{\prime}=\frac{1}{2 \pi} \mathbf{g} \cdot \frac{\mathrm{d} \mathbf{R}}{\mathrm{dz}}
$$

Whether equations 2.18 or 2.20 are used to model the electron diffraction depends on the structure of the scatterer. For some structures, although the displacement field may be found from elasticity theory, the derivatives of the field are complicated. This is the case for prismatic dislocation loops and so equation 2.18 in the form that includes absorption is used. In contrast, long straight dislocations are modelled using equations 2.20 and 2.21 .

\subsubsection{Analytic solutions to the Howie-Whelan differential equations}

The solution to the zero displacement field form of the Howie-Whelan differential equations can be found as follows. Let $\mathrm{T}$ and $\mathrm{S}$ represent the transmitted and diffracted beam amplitudes respectively. For simplicity of the solution, the variable $\mathrm{z}$ is transformed to $\mathrm{Z}=\mathrm{z} \pi / \xi_{\mathrm{g}}$. The solution at a depth $\mathrm{Z}$ in the crystal, when the initial conditions for $\mathrm{T}$ and $\mathrm{S}$ are known at a depth $\mathrm{Z}_{0}$, is

$$
\begin{aligned}
& T(z)=C_{1} \exp \left(\alpha_{1}\left(Z-Z_{0}\right)\right)+C_{2} \exp \left(\alpha_{2}\left(Z-Z_{0}\right)\right) \\
& S(z)=C_{3} \exp \left(\alpha_{1}\left(Z-Z_{0}\right)\right)+C_{4} \exp \left(\alpha_{2}\left(Z-Z_{0}\right)\right) .
\end{aligned}
$$$$
\text { and }
$$

Head et al. [79-82] usually just assume that $\mathrm{T}=1$ and $\mathrm{S}=0$ at $\mathrm{Z}=0$. More flexible initial conditions are necessary when including the change in potential occurring between a bubble and the matrix. For example in section 2.5 the case is considered when the bubble is not positioned at $\mathrm{Z}=0$.

The $\mathrm{C}$ and $\alpha$ coefficients in equation 2.22 can be derived by substituting these trial solutions into equation 2.20 and assuming that $\mathrm{T}\left(\mathrm{Z}_{0}\right)$ and $\mathrm{S}\left(\mathrm{Z}_{0}\right)$ are known. The two initial 
These equations are referred to as the derivative of the displacement form of the HowieWhelan differential equations. The crystal deformation is included by the term

$$
\beta_{\mathrm{g}}^{\prime}=\frac{1}{2 \pi} \mathrm{g} \cdot \frac{\mathrm{d} \mathbf{R}}{\mathrm{dz}}
$$

Whether equations 2.18 or 2.21 are used to model the electron diffraction depends on the structure the electrons are to be scattered from. For some structures, although the displacement field may be found from elasticity theory, the derivatives of the field are complicated. This is the case for prismatic dislocation loops and so equation 2.18 in the form that includes absorption is used. In contrast, long straight dislocations are modelled using equations 2.20 and 2.21 .

\subsubsection{Analytic solutions to the Howie-Whelan differential equations}

The solution to the zero displacement field form of the Howie-Whelan differential equations can be found as follows. Let $\mathrm{T}$ and $\mathrm{S}$ represent the transmitted and diffracted beam amplitudes respectively. For simplicity of the solution, the variable $\mathrm{z}$ is transformed to $\mathrm{Z}=\mathrm{z} \pi / \xi_{\mathrm{g}}$. The solution at a depth $\mathrm{Z}$ in the crystal, when the initial conditions for $\mathrm{T}$ and $\mathrm{S}$ are known at a depth $\mathrm{Z}_{0}$, is

$$
\begin{aligned}
& \qquad \mathrm{T}(\mathrm{z})=\mathrm{C}_{1} \exp \left(\alpha_{1}\left(\mathrm{Z}-\mathrm{Z}_{0}\right)\right)+\mathrm{C}_{2} \exp \left(\alpha_{2}\left(\mathrm{Z}-\mathrm{Z}_{0}\right)\right) \\
& \text { and } \quad \mathrm{S}(\mathrm{z})=\mathrm{C}_{3} \exp \left(\alpha_{1}\left(\mathrm{Z}-\mathrm{Z}_{0}\right)\right)+\mathrm{C}_{4} \exp \left(\alpha_{2}\left(\mathrm{Z}-\mathrm{Z}_{0}\right)\right) \text {. }
\end{aligned}
$$

Head et al. [79-82] usually just assume that $\mathrm{T}=1$ and $\mathrm{S}=0$ at $\mathrm{Z}=0$. More flexible initial conditions are necessary when including the change in potential occurring between a bubble and the matrix. For example in section 2.5 the case is considered when the bubble is not positioned at $\mathrm{Z}=0$.

The $\mathrm{C}$ and $\alpha$ coefficients in equation 2.22 can be derived by substituting these trial solutions into equation 2.20 and assuming that $\mathrm{T}\left(\mathrm{Z}_{0}\right)$ and $\mathrm{S}\left(\mathrm{Z}_{0}\right)$ are known. The two initial 
conditions and the two differential equations of equation 2.22 are enough to derive the six unknown coefficients. The solutions for the $\alpha$ 's are

$$
\alpha_{1,2}=-\eta+i w \pm \sqrt{\left((i-\eta)^{2}-w^{2}\right)},
$$

where $\mathrm{w}=\mathrm{s}_{\mathrm{g}} \xi_{\mathrm{g}}$ is the excitation error. The absorption coefficients are defined by

$$
\eta=\frac{\xi_{\mathrm{g}}}{\xi_{0}^{\prime}} \text { and } \mathrm{A}=\frac{\xi_{\mathrm{g}}}{\xi_{\mathrm{g}}^{\prime}}
$$

$\eta$ is called the normal absorption coefficient and A is called the anomalous absorption coefficient. A is conventionally set equal to $\eta$ and so A does not normally appear in the solutions for the coefficients. Effectively, the imaginary parts of the potential for the gth beam and the 0 th beam are assumed to be the same. The coefficients of the exponential terms are:

$$
\begin{gathered}
C_{1}=\frac{\left[(i-\eta) S\left(Z_{0}\right)-\left(\alpha_{2}+\eta\right) T\left(Z_{0}\right)\right]}{\alpha_{1}-\alpha_{2}} \text { and } C_{2}=T\left(Z_{0}\right)-C_{1} \\
C_{3}=\frac{\left(\alpha_{1}+\eta\right) C_{1}}{i-\eta} \text { and } C_{4}=\frac{\left(\alpha_{2}+\eta\right) C_{2}}{i-\eta}
\end{gathered}
$$

There are several ways of setting the absorption coefficients commonly found in the literature. Head et al. found that the absorption coefficients that gave the best match between images calculated from theory and images obtained from the the microscope were somewhat different from the theoretical values derived by earlier authors. The absorption coefficients of Head et al. [82], obtained from this matching procedure, are used in this thesis. It is necessary to know the alternative values used in the literature when comparing or checking program output against published results.

Hashimoto et al. $[70,71]$ set the absorption coefficients at 0.1 to get agreement of their theoretical plots with experiment. A more sophisticated theory of inelastic scattering effects due to single electron excitations and plasmon scattering was developed by Howie [74] from the work of Yoshioka [138]. Humphreys and Hirsch [140] gave theoretical 
values for the absorption coefficients as a function of diffracting vector. The imaginary part of the potential was calculated using Howie's theory (where applicable). They also used Hall and Hirsch's [78] theory for the scattering from phonons. Also the influence of the objective aperture in removing some of the inelastically scattered electrons from the final image was considered. The theory of inelastic scattering that results when all the terms mentioned above are included is perhaps too complicated. For this reason, the theory of inelastic scattering is used here only to broadly justify the phenomenological approach taken in this thesis of having a complex extinction distance. 


\subsection{Many Beam Matrix Formulation.}

\subsubsection{Bloch Waves}

When the crystal is tilted so that more than two beams are strongly excited, or all but the central beam are weakly excited, more than two beams may need to be included in modelling the electron diffraction. The object of this section is to derive equations linking these beam amplitudes in a deformed crystal. The scattering matrix formalism outlined in this section was developed from the Bethe theory (first mentioned in section 2.1) by Sturkey [141], Niehrs and Wagner [142], and Fujimoto [143].

The linearly independent set of Bloch wave solutions to the SWE have the form:

$$
\mathbf{b}^{\mathrm{i}}\left(\mathbf{k}_{0}^{\mathrm{i}}, \mathbf{r}\right)=\sum_{\mathbf{g}} \mathrm{C}_{\mathrm{g}}^{\mathrm{i}} \exp \left(2 \pi \mathrm{i}\left(\mathbf{k}_{0}^{\mathrm{i}}+\mathbf{g}\right) \cdot \mathbf{r}\right)
$$

The index $i$ indicates the branch of the dispersion surface that the Bloch wave lies on and the sum is over all the reciprocal lattice vectors $g$. A set of plane waves associated with each branch of the dispersion surface is used to construct a Bloch wave of that branch as shown in figure 2.2. The ith column of the $\mathrm{C}$ matrix provides the coefficients of the plane waves included in the summation for the ith Bloch wave. Any solution to the SWE can be expressed as a linear combination of these Bloch waves,

$$
\begin{aligned}
\psi & =\sum_{\mathrm{i}} \psi^{\mathrm{i}} \mathrm{b}^{\mathrm{i}}\left(\mathbf{k}_{0}^{\mathrm{i}}, \mathbf{r}\right) \\
& =\sum_{\mathbf{g}} \phi_{\mathrm{g}} \exp \left(2 \pi \mathrm{i}\left(\chi+\mathbf{g}+\mathbf{s}_{\mathbf{g}}\right) \cdot \mathbf{r}\right) \\
& =\sum_{\mathbf{g}} \phi_{\mathrm{g}}^{\prime \prime} \exp (2 \pi \mathrm{i}(\mathbf{K}+\mathbf{g}) \cdot \mathbf{r}) .
\end{aligned}
$$

Whereas the plane waves in equation $2.27 \mathrm{~b}$ in general have a non-zero excitation error $\left(\mathbf{s}_{\mathrm{g}}\right)$ in the phase of the exponential, the plane waves included in the summation for a Bloch wave in equation (2.26) do not. At strong two-beam conditions in a perfect crystal with no 
absorption, $\phi_{\mathrm{g}}$ and $\phi_{0}$ vary sinusoidally. In this case the $\psi^{\mathrm{i}}$ are constant, and of the two important Bloch waves, one will be a cosine wave and the other a sine wave. Equation $2.27 \mathrm{c}$ is obtained from equation $2.27 \mathrm{~b}$ by transforming the Darwin amplitudes by the zero displacement field form of equation 2.19. The wave vector in vacuum $(\chi)$ has first been replaced by the wave vector in the crystal $(\mathbf{K})$ using equation 2.16 . In the rest of this section the transformed Darwin amplitudes are used but the double primes have been dropped.

The wave function $\psi$ in equation $2.27 \mathrm{a}$ is substituted into the SWE in the form for an absorbing potential (equation 2.7). Consider the coefficient of the gth plane wave contributing to the ith Bloch wave in the resulting equation. All the plane waves are dependent on the spatial coordinates but the sum over the plane waves when they have individually been multiplied by their coefficients must equal zero independent of the spatial coordinates. The only way to satisfy this requirement is to set all the coefficients to zero:

$$
\begin{gathered}
\left(\mathrm{K}^{2}-\left(\mathbf{k}_{0}^{\mathrm{i}}+\mathrm{g}\right)^{2}\right) \mathrm{C}_{\mathrm{g}}^{\mathrm{i}}+\sum_{\mathrm{h}}^{\prime} \mathrm{U}_{\mathrm{h}} \mathrm{C}_{\mathrm{g}-\mathrm{h}}^{\mathrm{i}}+\mathrm{i} \sum_{\mathrm{h}} \mathrm{U}_{\mathrm{h}}^{\prime} \mathrm{C}_{\mathrm{g}-\mathrm{h}}^{\mathrm{i}}=0 . \\
\text { where } \mathrm{U}_{\mathrm{h}} \rightarrow \mathrm{U}_{\mathrm{h}}+\mathrm{i} \mathrm{U}_{\mathrm{h}}^{\prime}
\end{gathered}
$$

The transformation of the potential resulting in a non-zero imaginary component (so as to include absorption) is given in equation $2.28 \mathrm{~b}$. The Fourier transform from which the periodic potential is constructed has been relabelled from a sum over the set of $\mathbf{g}$ diffracting vectors, to a sum over $\mathbf{h}$. The index $\mathbf{h}$ must be introduced to distinguish it from that used in the sum over the Bloch waves in equation 2.27a. The $\mathrm{C}_{\mathrm{g}-\mathrm{h}}$ cross terms arise from the two sums over the $\mathbf{g}$ and $\mathbf{h}$ diffracting vectors. The amplitude of the wave vector in the crystal $(\mathrm{K})$ is different from that in the vacuum $(\chi)$ because the wave vector is refracted by the mean potential of the crystal $\left(\mathrm{U}_{0}\right) . \mathrm{K}$ is related to the parameters $\chi$ and $\mathrm{U}_{0}$ as follows:

$$
\mathrm{K}^{2}=\frac{8 \pi^{2} \mathrm{~m}_{\mathrm{e}} \mathrm{eE}}{\mathrm{h}^{2}}+\mathrm{U}_{0}=\chi^{2}+\mathrm{U}_{0}
$$


The dash above the first sum over $\mathbf{h}$ in equation $2.28 \mathrm{a}$ indicates that the $\mathbf{h}=\mathbf{0}$ term is excluded. Instead the Oth Fourier coefficient of the potential is included in equation $2.28 \mathrm{a}$ in the $\mathrm{K}^{2}$ term.

With reference to figure 2.3, the following approximations are made:

$$
\mathrm{K}^{2}-\left(\mathbf{k}_{0}^{\mathrm{i}}+\mathbf{g}\right)^{2} \cong \mathrm{K}^{2}-\left(|\mathbf{K}|+\left|\gamma^{\mathrm{i}}-\mathrm{s}_{\mathrm{g}}\right| \cos \theta_{\mathrm{g}}\right)^{2} \cong 2 \mathrm{~K}\left(\mathrm{~s}_{\mathrm{g}}-\gamma^{\mathrm{i}}\right) \cos \theta_{\mathrm{g}}
$$

$\left|\gamma^{i}\right|$ is the distance parallel to the Brillouin zone boundary between the ith dispersion surface and the Ewald sphere for the 0th reflection. Figure 2.2 shows $|\gamma|$ f for the case when $i=2$. The $\gamma^{j}$ are the eigenvalue solutions to the following eigenvalue equation obtained by converting equation $2.28 \mathrm{a}$ into matrix form:

$$
\mathbf{A} \mathbf{C}^{\mathrm{i}}-\boldsymbol{\gamma}^{\mathrm{i}} \mathbf{C}^{\mathrm{i}}=0
$$

In a crystal with a complex potential the $\gamma^{j}$ will have, in general, non-zero imaginary parts. The wave vectors of the $\mathrm{n}$ Bloch waves will have non-zero imaginary parts also and so the Bloch waves will be attenuated as they pass through the crystal. The $\mathrm{n}$ by 1 matrix $\mathrm{C}^{\mathrm{i}}$ is the ith column of the $\mathrm{n}$ by $\mathrm{n} \mathbf{C}$ matrix. The matrix elements of the $\mathrm{n}$ by $\mathrm{n}$ matrix $\mathbf{A}$ used in equation 2.31 are:

$$
\begin{aligned}
& A_{00}=\frac{i}{2 \xi_{0}^{\prime}} \text { and } A_{g g}=s_{g}+\frac{i}{2 \xi_{0}^{\prime}} \\
& A_{g h}=\frac{1}{2 \xi_{g-h}}+\frac{i}{2 \xi_{g-h}^{\prime}} .
\end{aligned}
$$

where the imaginary extinction distances are defined in a similar way to the real extinction distances defined earlier and are related to the imaginary (i.e., absorbing) parts of the potential by

$$
\xi_{0}^{\prime}=\frac{\mathrm{K}}{\mathrm{U}_{0}^{\prime}} \cos \theta_{0} \text { and } \xi_{\mathrm{g}}^{\prime}=\frac{\mathrm{K}}{\mathrm{U}_{\mathrm{g}}^{\prime}} \cos \theta_{\mathrm{g}} \text {. }
$$



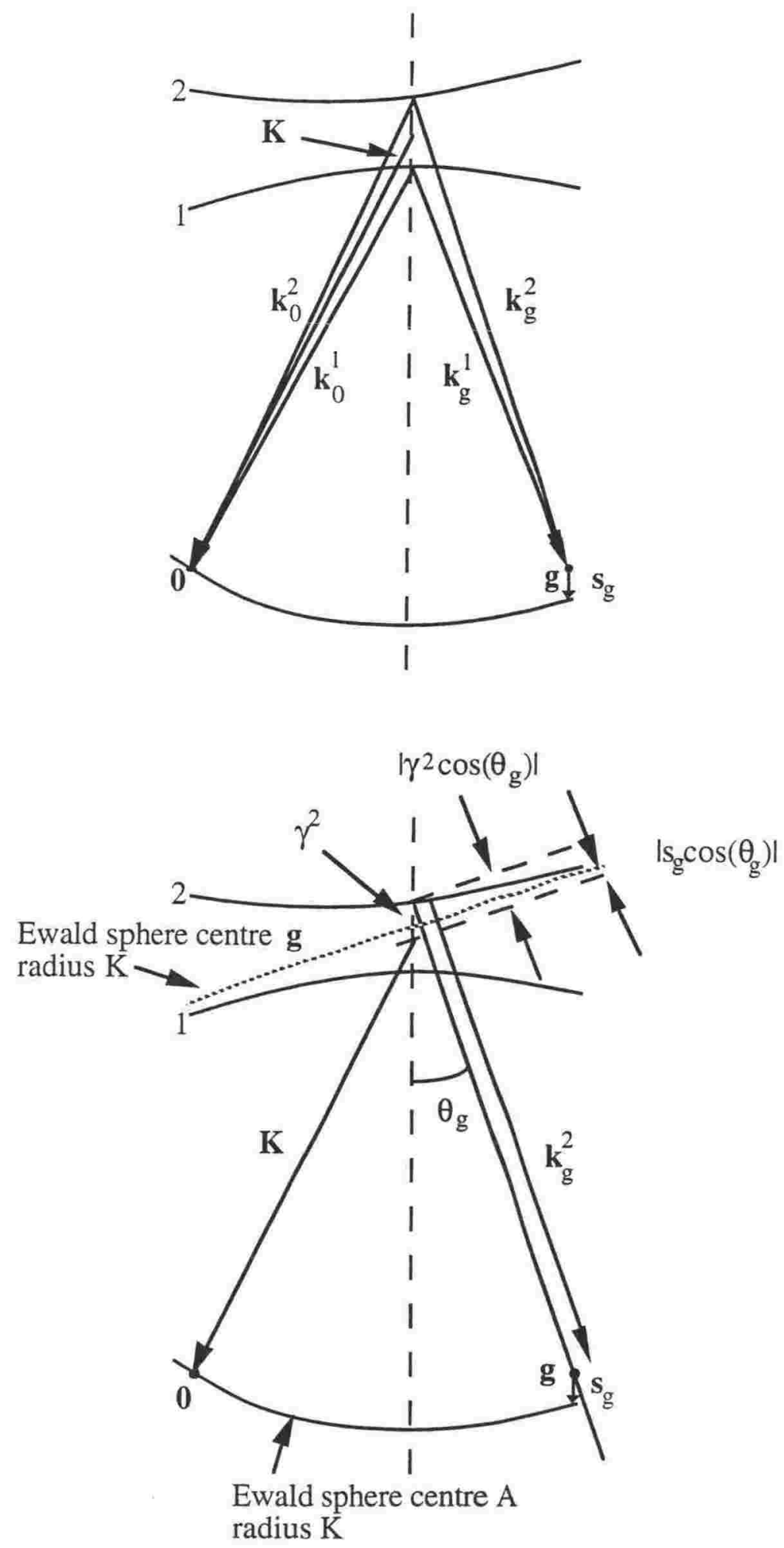

Figure 2.3: The figure shows the geometry used to justify the approximations in equation 2.30. For simplicity on the top part of the figure the vectors are shown without the detail shown in the bottom part of the figure. Only two branches of the dispersion surface are shown in the figure but the approximations are valid for all the branches. 


\subsubsection{Darwin Amplitudes}

The boundary conditions at the top surface of the foil require that the beam amplitudes $\phi_{\mathrm{g}}$ outside and inside the crystal and the Bloch wave amplitudes $\psi^{\mathrm{i}}$ are continuous, i.e.,

$$
\sum_{i} \psi^{i} C_{g}^{i}=\phi_{g}
$$

Equation 2.34 is true only at the top surface of the foil where $z=0$. There is no restriction on the $\mathrm{x}$ and $\mathrm{y}$ coordinates used at the top surface because the wave vector of the incident wave and the Bloch wave vectors have the same $\mathrm{x}$ and $\mathrm{y}$ components. Away from the top surface the exponential terms in equations 2.26 and 2.27 are not, in general, equal.

The eigenvectors can be solved from the eigenvalue equation. There are no cross terms between the ith and $j$ th Bloch waves $(i \neq j)$ in equations 2.31 to 2.34 . If there is no inelastic scattering in a crystal, and if there is no deformation introduced by the presence of dislocations or other defects, there will be no scattering between different Bloch waves. The incident electron wave is used to find the Bloch wave amplitudes ( $\psi^{\mathrm{i}}$ ) and to determine which Bloch waves are excited at the top surface which will then propagate through to the bottom surface without any scattering between waves.

Substitute the expression for the Bloch wave as a sum over plane waves from equation 2.26 into equation 2.27 a. Equation $2.35 \mathrm{c}$ can be derived by comparing the result of the substitution to equation $2.27 \mathrm{c}$ and using equation $2.35 \mathrm{~b}$. The coefficients of the Bloch waves and the Darwin amplitudes are linked in equations $2.35 \mathrm{a}$ and $2.35 \mathrm{c}$ by the matrix form of equations 2.34 and 2.27 ,

$$
\begin{gathered}
\psi=\mathrm{C}^{-1} \phi \quad \text { at } \mathrm{z}=0, \\
\mathbf{k}_{0}^{\mathrm{i}}-\mathbf{K}=\gamma^{\mathrm{i}} \\
\phi=\mathrm{C}\left\{\exp \left(2 \pi \mathrm{i} \gamma^{\mathrm{i}} \mathrm{z}\right)\right\}_{\mathrm{D} \psi} \quad \text { at } \mathrm{z} \neq 0 .
\end{gathered}
$$

The beam amplitudes leaving a slab of crystal of thickness $\delta z$ are then 


$$
\begin{gathered}
\phi(\mathrm{z}+\delta \mathrm{z})=\phi^{\prime}=\mathrm{C}\left\{\exp \left(2 \pi \mathrm{i} \gamma^{i}(\mathrm{z}+\delta \mathrm{z})\right)\right\}_{\mathrm{D}} \psi \\
=\mathrm{C}\left\{\exp \left(2 \pi \mathrm{i} \gamma^{i} \delta \mathrm{z}\right)\right\}_{\mathrm{D}} \mathrm{C}^{-1} \phi(\mathrm{z})
\end{gathered}
$$

where the $\mathrm{D}$ subscript signifies a diagonal matrix. The derivation of equation 2.36 uses the fact that the Bloch wave amplitude does not vary in a perfect crystal in which there is no inelastic scattering.

Expanding the diagonal matrix to the first order in $\delta \mathrm{z}$ gives for the change in beam amplitudes due to the slab of crystal

$$
\begin{aligned}
\delta \phi & =\phi^{\prime}-\phi \\
& =2 \pi \mathrm{i} C\left\{\gamma^{1}\right\} \mathrm{D}^{-1} \phi \delta z .
\end{aligned}
$$

Using the eigenvalue equation (equation 2.31), enables this to be reduced to the differential equation:

$$
\frac{\mathrm{d} \phi}{\mathrm{dz}}=2 \pi \mathrm{iA}(\mathrm{z}) \phi
$$

The Darwin beam amplitudes will vary even in a deformation free crystal in which no inelastic scattering occurs.

\subsubsection{Darwin Amplitudes in an Imperfect Crystal.}

For an imperfect crystal define

$$
\mathbf{Q}=\left\{\exp \left(2 \pi i \beta_{\mathrm{g}}\right)\right\}_{\mathrm{D}}
$$

where

$$
\beta_{g}=\mathbf{g} \cdot \mathbf{R}(\mathrm{z}) .
$$


In a slab of crystal at depth $\mathrm{z}$ which is displaced by $\mathbf{R}(\mathrm{z})$, the Fourier coefficients of the potential in the off-diagonal elements of $\mathbf{A}$, are phase shifted. For example

$$
\mathrm{A}_{\mathrm{g} 0} \rightarrow \frac{\mathrm{Ug}_{\mathrm{g}}}{2 \mathrm{~K}} \exp \left(-2 \pi \mathrm{i} \beta_{\mathrm{g}}\right)
$$

Then the depth-dependent matrix describing the system is

$$
\mathbf{A}(\mathrm{z})=\mathbf{Q}^{-1}(\mathrm{z}) \mathbf{A Q}(\mathrm{z})
$$

where $\mathrm{A}$ is the perfect crystal matrix. Equation 2.42 can be checked by multiplying out the right hand side after substituting for $\mathbf{Q}$ from equation 2.39 , and substituting for $\mathbf{A}$ from equation 2.32 .

In the two-beam case equations 2.42 and 2.38 can be used to derive the HowieWhelan differential equations in the form of equations 2.18. However, the Darwin beam amplitudes must be transformed correctly to obtain equation 2.18 . 


\subsection{Weak Beam Theory}

Weak beam conditions occur when the crystal is tilted so that all the diffracted beams are weakly excited but the central beam is still strong. Weak beam images of dislocations turn out to have a higher resolution than images taken under strong two-beam conditions, i.e., they are more sensitive to the position of the dislocation core and to the structure of the dislocation. For example weak beam techniques can allow the separation of images of partial dislocations which are close together and would appear as a single broad image under strong two-beam conditions.

A two-beam theory is used in this section to explain how the increased resolution of a weak beam image arises. The treatment of weak beam theory given here will follow that in Cockayne [89] and Hirsch et al. [67]. The electron wave that is incident upon a dislocation can be approximated by the two Bloch waves corresponding to those branches of the dispersion surface closest to the tail of the wavevector of the incident electron. The scattering of the electron wave by the dislocation into the weakly excited reflection with which an image is to be formed requires the inclusion of other Bloch waves in the model. The amplitudes of these additional Bloch waves will be small, so there will be negligible interaction between them. Instead, the important interactions are between the additional Bloch waves and one or other of the two Bloch waves with which the incident electron wave is constructed. This approximation (that only the interaction between pairs of Bloch waves are significant) will be used to model the amplitudes of all of the Bloch waves. In this thesis the two-beam theory will not be used to simulate any weak beam images as its use can give rise to inaccuracies but it does show how the increased resolution arises. The weak beam simulations presented in chapter 6 are calculated using a many beam theory in which all the interactions between the Bloch waves will be included.

\subsubsection{Analytical Solution For The Bloch Wave Coefficients}

The first step in modelling the diffraction process in a weak beam situation is to obtain analytical solutions for the Bloch wave coefficients. Elimination of $\phi_{\mathrm{g}}$ and $\frac{\mathrm{d} \phi_{\mathrm{g}}}{\mathrm{dz}}$ from 
the Howie-Whelan differential equations in the form appropriate for zero displacement and no absorption (equation 2.20) gives a second order differential equation in $\phi_{0}$

$$
\frac{\mathrm{d}^{2} \phi_{0}}{\mathrm{dz}}-2 \pi \mathrm{is} \frac{\mathrm{d} \phi_{0}}{\mathrm{dz}}+\left(\frac{\pi}{\xi_{\mathrm{g}}}\right)^{2} \phi_{0}=0
$$

Trying solutions of the form $\exp (2 \pi i \gamma z)$ gives a characteristic equation

$$
\gamma^{2}-\gamma s-\frac{1}{4 \xi_{g}^{2}}=0
$$

This has solutions

$$
\gamma^{2,1}=\frac{1}{2}\left(s \pm \sqrt{s^{2}+\frac{1}{\xi_{g}^{2}}}\right)
$$

Then the two independent solutions (i.e., one for each $\mathrm{C}$ and $\gamma$ pair) for $\phi_{0}$ and $\phi_{\mathrm{g}}$ are

$$
\begin{gathered}
\phi_{0}=C_{0}^{2,1} \exp \left(\pi i z\left(s \pm \sqrt{s^{2}+\frac{1}{\xi_{g}^{2}}}\right)\right) \\
\text { and } \phi_{g}=C_{g}^{2,1} \exp \left(\pi i z\left(s \pm \sqrt{s^{2}+\frac{1}{\xi_{g}^{2}}}\right)\right)
\end{gathered}
$$

Substituting these solutions back into equation 2.18 with $\mathbf{R}=0$ gives

$$
\frac{C_{g}^{1}}{C_{0}^{1}}=w-\sqrt{1+w^{2}} \text { and } \frac{C_{g}^{2}}{C_{0}^{2}}=w+\sqrt{1+w^{2}}
$$

Defining

$$
\mathrm{w}=\cot \beta
$$

the Bloch wave coefficients of equation 2.47 can be expressed as 


$$
C_{0}^{1}=C_{g}^{2}=\cos \frac{\beta}{2} \text { and } C_{0}^{2}=-C_{g}^{1}=\sin \frac{\beta}{2}
$$

The matrix elements of the $\mathrm{C}$ matrix in equation 2.49 can be substituted back into equation 2.47 to check the solution using simple trigonometric relations. Using the boundary conditions $\phi_{0}=1$ and $\phi_{\mathrm{g}}=0$ for the beam amplitudes at the foil surface, the coefficients of the Bloch waves in the two-beam approximation using equation $2.35 \mathrm{a}$ can be found:

$$
\psi^{1}=\cos \frac{\beta}{2} \text { and } \psi^{2}=\sin \frac{\beta}{2}
$$

\subsubsection{Bloch Wave Amplitudes in a Deformed Crystal}

In section 2.3.1 the Bloch wave amplitudes in an undeformed crystal were obtained. In this section the effects of a deformation of the crystal on the Bloch wave amplitudes are investigated. For a crystal slab deformed by $\mathbf{R}$ the Bloch waves, using the column approximation and replacing $\mathbf{r}$ in equation 2.26 by $\mathbf{r}-\mathbf{R}$, become

$$
\sum_{\mathbf{g}} C_{\mathbf{g}}^{\mathrm{i}}\left(\mathbf{k}_{0}^{\mathrm{i}}\right) \exp \left(2 \pi \mathrm{i}\left(\mathbf{k}_{0}^{\mathrm{i}}+\mathbf{g}\right) \cdot(\mathbf{r}-\mathbf{R})\right)=\exp \left(-2 \pi \mathrm{i} \mathbf{k}_{0}^{\mathrm{i}} \cdot \mathbf{R}\right) \sum_{\mathbf{g}} \mathrm{C}_{\mathbf{g}}^{\mathrm{i}}\left(\mathbf{k}_{0}^{\mathrm{i}}\right) \exp (-2 \pi \mathrm{i} \mathbf{g} \cdot \mathbf{R}) \exp \left(2 \pi \mathrm{i}\left(\mathbf{k}_{0}^{\mathrm{i}}+\mathbf{g}\right) \cdot \mathbf{r}\right)
$$

The matrix $\mathbf{C}$ for the slab becomes, after dropping the constant phase factor outside the summation,

$$
\left(\begin{array}{cc}
C_{0}^{1} & C_{0}^{2} \\
C_{g}^{1} \exp (-i \alpha) & C_{g}^{2} \exp (-i \alpha)
\end{array}\right)
$$

where $\alpha=2 \pi$ g.R. Using equations $2.36,2.52,2.49$ and figure 2.4 , the beam amplitudes after the beams have passed through a thickness $t_{1}$ of perfect crystal then another slab of thickness $t_{2}$ of perfect crystal which is displaced relative to the top layer by $\mathbf{R}$, are: 


$$
\begin{aligned}
& \left(\begin{array}{l}
\phi_{0}(t) \\
\phi_{g}(t)
\end{array}\right)=\left(\begin{array}{cc}
\cos \frac{\beta}{2} & \sin \frac{\beta}{2} \\
-\sin \frac{\beta}{2} \exp (-i \alpha) \cos \frac{\beta}{2} \exp (-i \alpha)
\end{array}\right)\left(\begin{array}{cc}
\exp \left(2 \pi i \gamma^{1} t_{2}\right) & 0 \\
0 & \exp \left(2 \pi i \gamma^{2} t_{2}\right)
\end{array}\right) \\
& x\left(\begin{array}{cc}
\cos \frac{\beta}{2} & -\sin \frac{\beta}{2} \exp (i \alpha) \\
\sin \frac{\beta}{2} & \cos \frac{\beta}{2} \exp (i \alpha)
\end{array}\right)\left(\begin{array}{rr}
\cos \frac{\beta}{2} & \sin \frac{\beta}{2} \\
-\sin \frac{\beta}{2} & \cos \frac{\beta}{2}
\end{array}\right) \\
& x\left(\begin{array}{cc}
\exp \left(2 \pi i \gamma^{1} t_{1}\right) & 0 \\
0 & \exp \left(2 \pi i \gamma^{2} t_{1}\right)
\end{array}\right)\left(\begin{array}{cc}
\cos \frac{\beta}{2} & -\sin \frac{\beta}{2} \\
\sin \frac{\beta}{2} & \cos \frac{\beta}{2}
\end{array}\right)\left(\begin{array}{l}
\phi_{0}(0) \\
\phi_{g}(0)
\end{array}\right)
\end{aligned}
$$

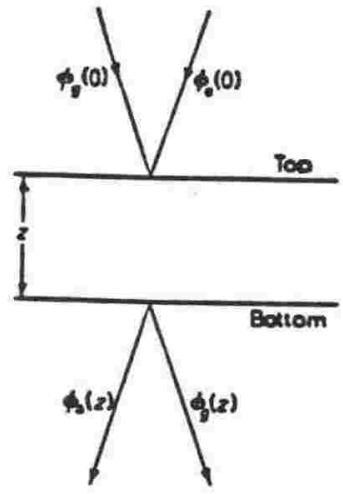

(a)

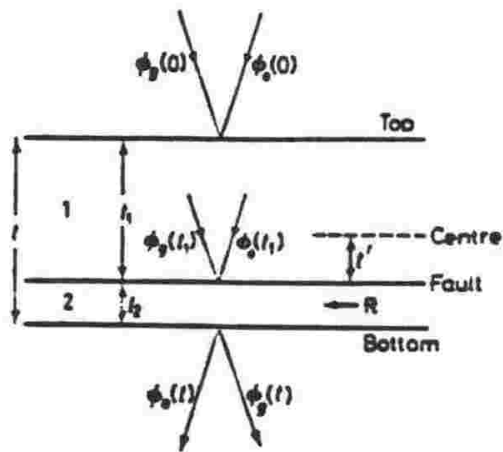

(b)

Figure 2.4: Waves propagating through a crystal (a) and a composite crystal (b). In (b) the bottom layer of crystal (section 2 of thickness $t_{2}$ ) has been displaced relative to the top layer (section 1 of thickness $t_{1}$ ) by $\mathbf{R}$.

The Darwin amplitudes $\left(\phi_{0}(\mathrm{t}), \phi_{\mathrm{g}}(\mathrm{t}), \phi_{0}(0)\right.$ and $\left.\phi_{\mathrm{g}}(0)\right)$ are replaced in equation 2.53 at $\mathrm{z}=\mathrm{t}$ $=t_{1}+t_{2}$ and $z=0$ by

$$
\left(\begin{array}{l}
\phi_{0}(z) \\
\phi_{g}(z)
\end{array}\right)=\left(\begin{array}{c}
C_{0}^{1} C_{0}^{2} \\
C_{g}^{1} C_{g}^{2}
\end{array}\right)\left(\begin{array}{cc}
\exp \left(2 \pi i \gamma^{1} z\right) & 0 \\
0 & \exp \left(2 \pi i \gamma^{2} z\right)
\end{array}\right)\left(\begin{array}{l}
\psi^{1} \\
\psi^{2}
\end{array}\right)
$$

By multiplying the matrices together a relation between the Bloch wave amplitudes at the top $(\psi)$ and bottom $\left(\psi^{\prime}\right)$ of the deformed slab is obtained 


$$
\begin{aligned}
\psi^{\prime 1} & =\left\{\cos ^{2} \frac{\beta}{2}+\sin ^{2} \frac{\beta}{2} \exp (\mathrm{i} \alpha)\right\} \psi^{1}+\sin \frac{\beta}{2} \cos \frac{\beta}{2}(1-\exp (\mathrm{i} \alpha)) \exp \left(2 \pi \mathrm{i} \Delta \mathrm{kt}_{1}\right) \psi^{2} \\
\psi^{\prime 2} & =\left\{\sin ^{2} \frac{\beta}{2}+\cos ^{2} \frac{\beta}{2} \exp (\mathrm{i} \alpha)\right\} \psi^{2}+\sin \frac{\beta}{2} \cos \frac{\beta}{2}(1-\exp (\mathrm{i} \alpha)) \exp \left(-2 \pi \mathrm{i} \Delta \mathrm{kt}_{1}\right) \psi^{1} .
\end{aligned}
$$

In equation $2.55 t_{1}$ appears but $t_{2}$ and $\gamma$ do not because of the non-commutativity of some of the matrices. That is the terms involving $\mathrm{t}_{2}$ and $\gamma$ can be cancelled out but those involving $\mathrm{t}_{1}$ cannot. The phase factor arises from a change in wave vector due to scattering from dispersion surface 2 to dispersion surface 1, i.e,

$$
\Delta \mathrm{k}=\gamma^{2}-\gamma^{1}
$$

The physical effects of the change in wavevector in equation 2.56 can be understood by returning to the idea of extinction distances. Rearranging equation 2.45 for the extinction distance as a function of the eigenvalues, we obtain

$$
\xi_{g}=\frac{1}{\sqrt{\left(\gamma^{2}-\gamma^{1}\right)^{2}-s^{2}}}
$$

The extinction distance is roughly inversely proportional to the change in wave vector in equation 2.56. As the number of beams used in finding the eigenvalues is increased, the eigenvalues and consequently the extinction distances are found more accurately. The Bloch waves are constructed from a sum over a set of plane waves. As the set of plane waves grows bigger the parameters associated with the Bloch wave can be calculated more accurately. Figure 2.5 shows a calculation by the author of how a typical extinction distance in copper varies with the number of beams.

After dropping the dashed superscripts, and expanding the exponential in equation 2.55 to first order in $\delta \alpha$ using a Taylor series expansion we obtain equations for the change in the Bloch wave amplitudes with depth:

$$
\delta \psi^{1}=i \delta \alpha\left\{\sin ^{2} \frac{\beta}{2} \psi^{1}-\sin \frac{\beta}{2} \cos \frac{\beta}{2} \exp \left(2 \pi \mathrm{i} \Delta \mathrm{kt}_{1}\right) \psi^{2}\right\}
$$


and $\left.\delta \psi^{2}=i \delta \alpha \mid \cos ^{2} \frac{\beta}{2} \psi^{2}-\sin \frac{\beta}{2} \cos \frac{\beta}{2} \exp \left(-2 \pi \mathrm{i} \Delta \mathrm{kt} \mathrm{t}_{1}\right) \psi^{1}\right\}$.

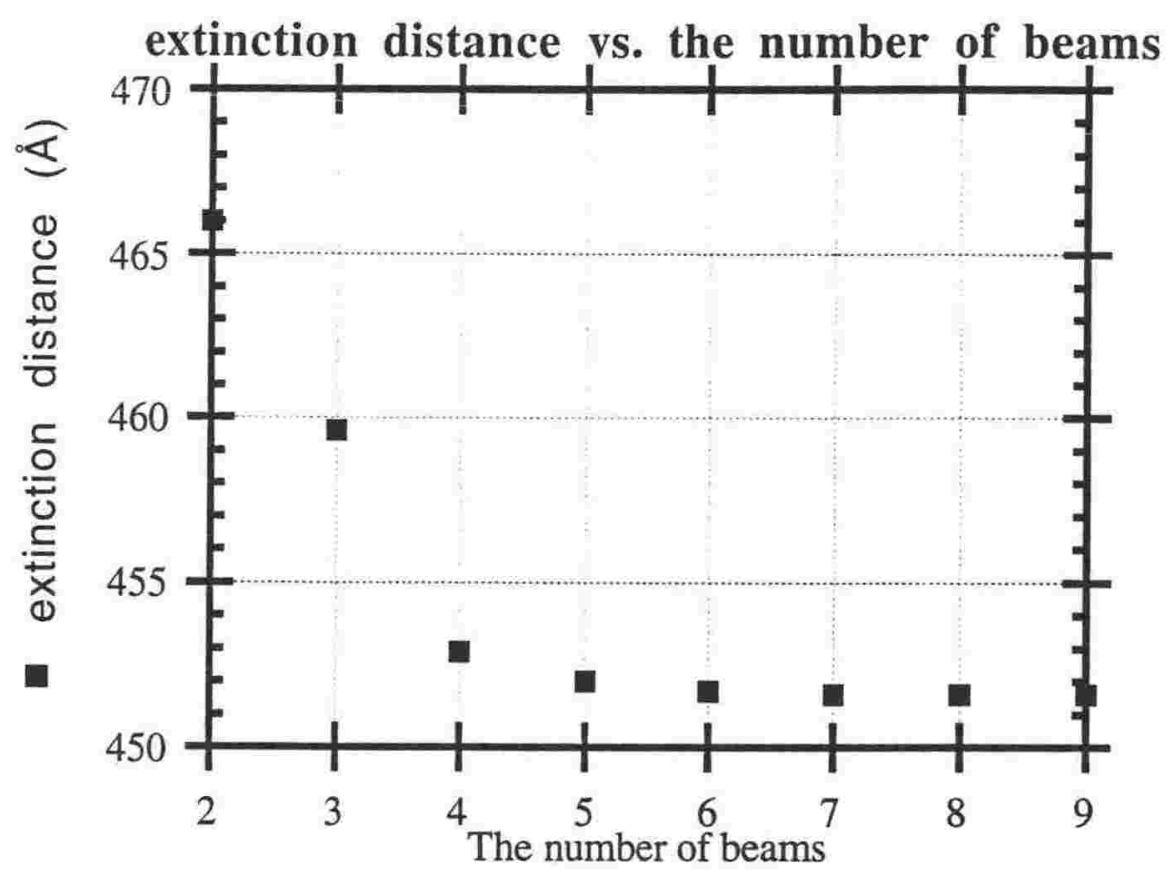

Figure 2.5: The variation in the (220) extinction distance with the number of beams included in the calculation.

Equation 2.58 becomes

$$
\begin{gathered}
\left.\frac{\mathrm{d} \psi^{1}}{\mathrm{dz}}=2 \pi \mathrm{i} \frac{\mathrm{d}(\mathrm{g} \cdot \mathbf{R}(\mathrm{z}))}{\mathrm{dz}} \mid \sin ^{2} \frac{\beta}{2} \psi^{1}-\sin \frac{\beta}{2} \cos \frac{\beta}{2} \exp \left(2 \pi \mathrm{i} \Delta \mathrm{kt} \mathrm{t}_{1}\right) \psi^{2}\right\} \\
\text { and } \left.\frac{\mathrm{d} \psi^{2}}{\mathrm{dz}}=2 \pi \mathrm{i} \frac{\mathrm{d}(\mathbf{g} \cdot \mathbf{R}(\mathrm{z}))}{\mathrm{dz}} \mid \cos ^{2} \frac{\beta}{2} \psi^{2}-\sin \frac{\beta}{2} \cos \frac{\beta}{2} \exp \left(-2 \pi \mathrm{i} \Delta \mathrm{kt} \mathrm{t}_{1}\right) \psi^{1}\right\} \text {, }
\end{gathered}
$$

using

$$
\delta \alpha=2 \pi \frac{\mathrm{d} \mathbf{g} \cdot \mathbf{R}}{\mathrm{dz}} \delta \mathrm{z} .
$$

Equations 2.59 give the change in Bloch wave amplitudes as a function of the distortion in the crystal. Even though the slab of thickness $t_{2}$ is a perfect crystal it can be made to have an infinitesmal thickness of $\delta z$. The Bloch wave amplitudes for a deformed crystal have 
and eigenvalues are approximately $0.02 \AA^{-1}$ in weak beam conditions. When the crystal is tilted away from strong two-beam conditions the parts of the dispersion surfaces that satisfy the boundary conditions at the surface become closer to the Ewald spheres. Because the energy of the Bloch waves approach that which occurs when a wavevector lies on the Ewald sphere, equation 2.30 shows that the excitation error becomes approximately equal to the eigenvalues as stated in equation 2.64. If the conditions on the right-hand side of equation 2.64 are not true then by dividing one of equations 2.63 into the other we obtain

$$
\left|C_{n}^{n}\right| \approx\left|C_{n}^{m}\right| »\left|C_{n}^{p}\right| \quad \text {, if } s_{m} \approx s_{n}, p \neq m \text { or } n
$$

Equation 2.65 follows from using the fact that $\mathbf{C}$ is Hermitian when the coordinate origin is chosen so that the potential has a centre of symmetry. The last part of equation 2.65 is a restatement of the inequality in equation 2.64 .

In weak beam conditions, all the beam amplitudes are weak except the central beam, i.e., $\phi_{0}=1$ but $\phi_{\mathrm{g}} \approx 0$ for the other g's. Then the Bloch wave amplitudes are (from equation 2.35a)

$$
\psi^{\mathrm{j}}=\mathrm{C}_{0}^{\mathrm{j}}
$$

This results from the first column of $\mathbf{C}^{-1}$ being the same as the first row of $\mathbf{C}$, when $\mathbf{C}$ is Hermitian. When $s$ is large in equation $2.48, \beta=\varepsilon \approx 0$. Using equations 2.49 and 2.66 when $\beta \approx 0$, we obtain for the relative size of the Bloch wave amplitudes

$$
\psi^{1}(0) \approx 1 \text { and } \psi^{2}(0) \approx \frac{\varepsilon}{2}
$$

The first Bloch wave is associated with the first dispersion surface and the others are labelled with an index increasing as $\left|s_{n}\right|$ or $|\gamma n|$ increases as shown in figure 2.6. Physically the Bloch wave amplitudes are bigger if the part of the dispersion surface associated with the jth wave is close to one of the Ewald spheres upon which kinetic energy and crystal momentum are conserved. 
If the crystal is tilted to near two-beam conditions for the -gth reflection and a weak beam image is formed from an objective aperture around the gth beam then the amplitude of the gth beam at the exit surface of the foil is

$$
\begin{aligned}
\phi_{g}(t) & =\sum_{j} \psi^{j}(t) C_{g}^{j} \exp \left(2 \pi i \gamma^{j} t\right) \\
& \approx \psi^{3}(t) C_{g}^{3} \exp \left(2 \pi i \gamma^{3} t\right)+\psi^{4}(t) C_{g}^{4} \exp \left(2 \pi i \gamma^{4} t\right) .
\end{aligned}
$$

The first part of equation 2.68 is a restatement of the 2 nd part of equation 2.35. The rest of equation 2.68 follows because the amplitudes of the 2 nd, 3rd and 4th Bloch waves are small and of comparable size (from the two-beam approximation of equation 2.67). From equations 2.64 and 2.65 the relative size of the relevant coefficients of the $\mathrm{C}$ matrix are

$$
\left|C_{\mathrm{g}}^{3}\right|=\left|C_{g}^{4}\right| \gg\left|C_{g}^{1}\right|,\left|C_{g}^{2}\right| \text { as } s_{3} \approx s_{4} \gg s_{2} \text {. }
$$

The excitation errors of the third and fourth beams are approximately equal and are much larger than that of the second beam as shown in figure 2.6 when the crystal is tilted to near two-beam conditions for the second beam. Equation 2.69 justifies the neglect of all but the Bloch wave coefficients included in the second part of equation 2.68.

\subsubsection{Strength of the Scattering between Bloch Waves}

At the top surface of the foil the first and second Bloch waves are significantly excited only. The important scattering contribution to $\phi_{\mathrm{g}}$ through equation 2.68 is from Bloch waves 1 and 2 into waves 3 and 4 only (this is shown in the dispersion surface of figure 2.6). Therefore a two-beam approximation can be made between one member of each set of waves at a time. Following the treatment in D. Cockayne [89], the variables are transformed according to

$$
\psi^{j}=\psi^{j} \exp \left(-2 \pi i g \cdot \mathbf{R} \sin \frac{2}{2}\right), \quad \psi^{i}=\psi^{i} \exp \left(-2 \pi i \operatorname{g} \cdot \mathbf{R} \cos 2 \frac{\beta}{2}\right)
$$




$$
z k_{z}^{j}=z k_{z}^{j}+g \cdot R \sin 2 \frac{\beta}{2}, \quad z k_{z}^{i j}=z k_{z}^{i}+g \cdot R \cos 2 \frac{\beta}{2}
$$

and $\Delta \mathrm{k}=\mathrm{k}_{\mathrm{z}}^{\mathrm{i}}-\mathrm{k}_{\mathrm{z}}^{\mathrm{j}}=\gamma^{\mathrm{i}}-\gamma^{\mathrm{j}}$.

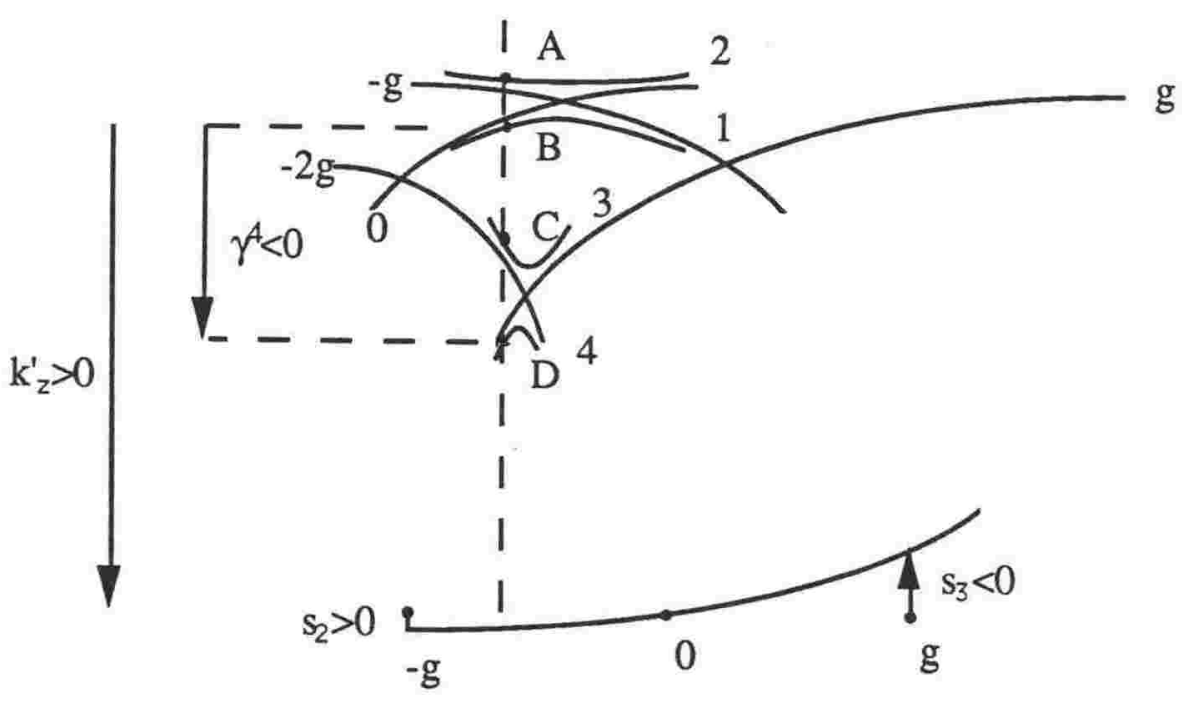

Figure 2.6: Dispersion surface showing the scattering between 4 beams when the crystal is tilted to near strong two-beam conditions for -g. (Not to scale). The Ewald spheres around the main diffracting vectors and the branches of the dispersion surface are shown.

Then 2nd part of equation 2.59 describing the scattering between Bloch waves becomes (where $\mathrm{j}=3,4$ and $\mathrm{i}=1,2$ )

$$
\begin{aligned}
& \qquad \frac{d \psi^{\prime} \mathrm{j}}{\mathrm{d} z}=-2 \pi \mathrm{i} \frac{\mathrm{d}(\mathrm{g} \cdot \mathbf{R})}{\mathrm{dz}} \sin \frac{\beta}{2} \cos \frac{\beta}{2} \exp (-2 \pi \mathrm{i} \Delta \mathrm{kz}) \psi^{\mathrm{i}} \\
& \text { integrating gives } \psi^{\prime} \mathrm{j}=\psi^{\prime} \mathrm{j}(0)-\pi \mathrm{i} \sin \beta \psi^{\prime} \mathrm{i}\left(\mathrm{z}_{1}\right) \int_{\mathrm{z}_{1}}^{\Delta \mathrm{z}+\mathrm{z}_{1}} \frac{\mathrm{d}(\mathbf{g} \cdot \mathbf{R})}{\mathrm{dz}} \exp (-2 \pi \mathrm{i} \Delta \mathrm{kz}) \mathrm{dz} \text {. }
\end{aligned}
$$

The range of integration is transformed by the variables

$$
\Delta \mathrm{k}=\mathrm{k}_{\mathrm{z}}^{\mathrm{i}}-\mathrm{k}_{\mathrm{z}}^{\mathrm{j}}+\frac{\mathrm{d}(\mathrm{g} \cdot \mathbf{R})}{\mathrm{dz}}\left(\cos ^{2} \frac{\beta}{2}-\sin ^{2} \frac{\beta}{2}\right) \text { and } \mathrm{z}=\mathrm{z}^{\prime}+\mathrm{z}_{1}+\frac{\Delta \mathrm{z}}{2}
$$


Assuming that the $s_{1}$ term (defined in equation 2.73) is constant over the range of integration so that it can be moved outside the integral, equation 2.71 then becomes:

$$
\begin{gathered}
\psi^{\mathrm{j}}=\psi^{\mathrm{j}}(0)-\pi \mathrm{i} \sin \beta \psi^{\prime \mathrm{i}}\left(\mathrm{z}_{1}\right) \exp \left(-2 \pi \mathrm{i}\left(\mathrm{z}_{1}+\frac{\Delta \mathrm{z}}{2}\right) \Delta \mathrm{k}\right) \mathrm{s}_{1} \int_{\frac{-\Delta z}{2}}^{\frac{\Delta z}{2}} \exp (-2 \pi \mathrm{i} \Delta \mathrm{kz}) \mathrm{d} \mathrm{z}^{\prime} \\
\text { where } \frac{\mathrm{d}(\mathbf{g} \cdot \mathbf{R})}{\mathrm{dz}}=\mathrm{s}_{1} .
\end{gathered}
$$

Evaluation of the integral gives

$$
\int_{\frac{-\Delta z}{2}}^{\frac{\Delta z}{2}} \exp \left(-2 \pi i \Delta k z^{\prime}\right) d z^{\prime}=\Delta z \frac{\sin (\pi \Delta k \Delta z)}{\pi \Delta k \Delta z} .
$$

For $\mathrm{w} \approx 1$ to 11.5 , i.e., for moderately large $\mathrm{s}, \beta \approx \pi / 4$ to 0.087 radians and so the $\sin (\beta)$ factor outside the integral is non-zero. Then the scattering and the integral are maximised if $\Delta \mathrm{k}=0$, i.e.,

$$
\mathrm{k}_{\mathrm{z}}^{\mathrm{i}}-\mathrm{k}_{\mathrm{z}}^{\mathrm{j}}=\frac{\mathrm{d}(\mathbf{g} \cdot \mathbf{R})}{\mathrm{dz}}=-\mathrm{s}_{\mathrm{g}}
$$

Therefore the maximum scattering between dispersion surfaces occurs between B and D, followed by $\mathrm{B}$ and $\mathrm{C}$ in figure 2.6 , near the dislocation core, as this is the only place where the curvature of the diffracting planes is large enough to equal the excitation error. Equation 2.75 is a necessary condition for the amplitude of the Bloch waves to change and for the intensity in the dark field image to be significantly different from the background intensity. Equation 2.75 is only close to being satisfied near the core of the dislocation. This is the reason that weak beam images have a higher resolution than images taken at strong twobeam conditions. 


\subsection{Multislice Theory}

\subsubsection{Introduction}

Multislice theory is a physical optics approach that is mainly applicable to images of perfect crystals obtained by high resolution electron microscopy. Cowley and Moodie [144-152] in a series of papers in the late 1950's and early 1960's gradually built up the multislice theory for diffraction. They initially considered the images formed in the backfocal plane of a lens by either light waves or electron waves. Initially the physical optics approach was applied to the in-focus Fourier image of a point source and then the image off the back-focal plane of a lens was modelled, i.e., defocussed images were modelled. Further developments considered the Fourier image of finite sources, such as the Gaussian source by which a TEM filament is approximated and the extension of the theory to periodic images or phase gratings which could be considered to be very thin weakly scattering crystals. The problem of a further extension of the theory to describe electron diffraction from thicker crystals centred on the question of how to create phase gratings from the crystal potential and how to incorporate the effects of dynamical scattering.

The approach that Cowley and Moodie use is to divide the three-dimensional crystal into a sequence of two-dimensional slices perpendicular to the electron beam direction. The dynamical diffraction through the crystal is considered as being due to multiple transmission through the slices, with propagation by Huygens principle between these slices. The effects of a finite objective aperture are included by multiplying the Fourier image by a circular step function which passes the intensity inside the aperture but blocks the intensity outside it.

Fujiwara [135] used a Green's function method to extend the first order Born approximation to higher order elastic scattering by iterated integrations, demonstrating the equivalence of the Born approximation (which is the basis of the Bethe theory using Bloch waves) to the multislice method. However, multislice computation of the diffraction is quicker than using the Bloch wave formalism for a large number of beams (Cowley [127]). Practical calculations using the theory took longer to develop (Goodman and Moodie [153]). The theory was first applied to experimental micrographs by the Melbourne group 
in the early 1970's (O'Keefe et al. [154-159]) and it has since become the standard method for interpreting high resolution images.

A brief, non-rigorous, outline of the multislice theory is presented in sections 2.4 .2 and 2.4.3. Although defects such as bubbles and dislocations can be included by the method of periodic continuation (Fields and Cowley [160]), the resultant images can be difficult to interpret except in situations such as looking at a screw dislocation end-on. In high resolution microscopy, bubble images are not as difficult to interpret as those of dislocations but still represent a non-standard application of the multislice theory. The multislice theory is used in chapter 4 to simulate high resolution images of bubbles.

\subsubsection{Calculation of the Wave Function at the Exit Surface of the Foil}

The refractive index (n) of a crystal is related to the potential $(\phi(\mathbf{r}))$ of the scattering atoms and the relativistic energy of the electrons by (Cowley [119])

$$
\mathrm{n}=\left\{1+\frac{\phi(\mathbf{r})}{\mathrm{E}_{0}}\left(1+\frac{\mathrm{eE_{0 }}}{2 \mathrm{~m}_{0} \mathrm{c}^{2}}\right)\right\}^{\frac{1}{2}} \approx 1+\frac{\phi(\mathbf{r})}{2 \mathrm{E}} .
$$

The phase change of the electron wave due to a slice of thickness $\Delta \mathrm{z}$ is

$$
\left[\left(1+\frac{\phi(\mathbf{r})}{2 \mathrm{E}}\right)-1\right] \Delta \mathrm{z} \frac{2 \pi}{\lambda}=\frac{\pi}{\lambda \mathrm{E}} \Delta \mathrm{z} \phi\left(\mathrm{x}, \mathrm{y}, \mathrm{z}_{\mathrm{n}}\right)=-\sigma \phi \Delta \mathrm{z}
$$

The first term in equation 2.77 arises because the wavelength is shortened in the crystal by a factor equal to the refractive index. The potential on the right hand side is the mean potential in the nth slice. In equation 2.77 the quantity $\sigma$ is called the interaction constant. The transmission function for the nth slice is

$$
\mathrm{q}_{\mathrm{n}}\left(\mathrm{x}, \mathrm{y}, \mathrm{z}_{\mathrm{n}}\right)=\exp \left\{-i \sigma \phi\left(\mathrm{x}, \mathrm{y}, \mathrm{z}_{\mathrm{n}}\right) \Delta \mathrm{z}-\mu\left(\mathrm{x}, \mathrm{y}, \mathrm{z}_{\mathrm{n}}\right) \Delta \mathrm{z}\right\}
$$

This function is multiplied with the incident electron wave function to calculate a phase change. The second term in the exponential phenomenologically includes the absorption of 
the wave, i.e., it includes the effects of the imaginary part of the potential. In the absence of absorption the right hand side of equation 2.78 gives the same phase change as equation 2.77 in the limit of small slice thickness. If the phase change of the electron wave due to the entire thickness of the crystal can be calculated by one multiplication of the wave function by equation 2.78 then the weak phase object approximation (WPOA) is valid.

After passing through a slice, the wave is propagated to the next slice by convoluting the real space wave function with

$$
\mathrm{p}(\mathrm{x}, \mathrm{y})=\frac{\mathrm{i}}{\mathrm{R} \lambda} \exp \left\{\frac{-\mathrm{ik}\left(\mathrm{x}^{2}+\mathrm{y}^{2}\right)}{2 \Delta \mathrm{z}}\right\} \text {. }
$$

This propagation function arises from the Kirchhoff integral scattering formula and is just a mathematical statement of Fresnel diffraction effects and Huygens principle. In reciprocal space the wave function can be multiplied by the following factor instead of being convoluted

$$
\mathrm{P}(\mathrm{u}, \mathrm{v})=\frac{\mathrm{i}}{\mathrm{R} \lambda} \exp \left\{\pi \mathrm{i} \lambda \Delta \mathrm{z}\left(\mathrm{u}^{2}+\mathrm{v}^{2}\right)\right\}
$$

This term is the Fourier transform of the previous real space representation of the propagation function in equation 2.79 .

The wave function at the exit surface of the crystal can be calculated by a series of multiplications by the transmission function and convolutions with the propagation function

$$
\psi(x)=q_{N}(x)\left[\ldots\left[q_{2}(x)\left[q_{1}(x)\left[q_{0}(x) * p_{0}(x)\right]^{*} p_{1}(x)\right]^{*} p_{2}(x)\right]^{*} \ldots\right]^{*} \mathrm{p}_{N}(x)
$$

In equation 2.81 the subscripts indicate the slice to which the phase grating and propagation function belong. Alternatively the reciprocal space form of this equation can be used. This latter form is usually preferred since it requires less computational time. 
After the wave function at the exit surface of the foil has been calculated by the methods of section 2.4.2, the effects of lens aberrations can be included by means of the lens transfer function. Cowley [127] and Saxton [161] show that the micrograph contrast, $c(k)$, of a weak-phase object is

$$
c(\mathbf{k})=\psi(\mathbf{k}) w(\mathbf{k})+\psi^{*}(-\mathbf{k}) w^{*}(-\mathbf{k}) .
$$

The effect of the objective lens upon the wave can be included by multiplying the wave function, $\psi(\mathbf{k})$, in the WPOA, by the contrast transfer function of the lens, $w(\mathbf{k})$,

$$
\mathrm{w}(\mathbf{k})=\exp \left\{-\mathrm{i}\left[\gamma\left(\mathbf{k}_{0}+\mathbf{k}\right)-\gamma\left(\mathbf{k}_{0}\right)\right]\right\} \mathrm{E}\left(\mathbf{k}_{0}+\mathbf{k}, \mathbf{k}_{0}\right)
$$

where the phase factor is $\quad \gamma(\mathbf{k})=0.5 \pi \mathrm{C}_{\mathrm{s}} \lambda^{3} \mathrm{k}^{4}+\pi \lambda \mathrm{Dk}^{2}$, and the envelope function is

$$
E\left(k_{1}, k_{2}\right)=\exp \left\{-0.5 \pi^{2} \lambda^{2}\left(k_{1}^{2}-k_{2}^{2}\right)^{2}-2 \pi^{2} \beta^{2} I\left(C_{s} \lambda^{2} k_{1}^{2}-d\right) k_{1}-\left.\left(C_{s} \lambda^{2} k_{2}^{2}-d\right) k_{2}\right|^{2}\right\}
$$

and the defocus term is $\quad \mathrm{D}=\Delta+0.5 \mathrm{~A} \cos ^{2}\left(\varphi-\varphi_{0}\right)$.

$\mathbf{k}$ is the spatial frequency, $\varphi$ its azimuth and $\mathrm{C}_{\mathrm{s}}$ the spherical aberration coefficient. $\Delta$ is the defocus (overfocus positive), A the axial astigmatism and $\varphi_{0}$ the azimuth at which the defocus is maximum. $\mathbf{k}_{0}$ is the tilt of the illumination relative to the optical axis, $\beta$ is the rms width in section of the angular brightness distribution of the illumination and $\mathrm{d}$ the rms effective focus spread.

For thicker specimens the effect of beam divergence is modelled by adding together the intensities from a distribution of point sources instead of multiplying the wave function by the envelope function (E) in equation $2.83[162,163]$. The effects of energy spread in the electrons emitted by the filament are included by adding together images at different defocus levels. The intensities in the diffraction pattern are not affected by that part of the 
contrast transfer function that changes the phase of the wave scattered by the specimen, but the intensities are affected by the beam divergence and chromatic aberrations.

\subsection{Defocussing the Wave Function}

After the wave function has been calculated by one of the methods outlined in sections 2.1 to 2.3, it can be defocussed (Rühle and Wilkens [83]). To defocus the wave function it is necessary to find the phases of the beams at the exit surface of the crystal as the effect of defocussing is to further phase shift these beams. The method of defocussing the wave function outlined below is valid for thick specimens and is equivalent to including only the defocus term in the WPOA approximation.

As shown in the dispersion surfaces in figure 2.2 the effect of the mean potential is to refract or change the modulus of the electron wave vector. In figure 2.2 the refraction occurs as an electron is transmitted into the crystal at the top surface of the foil. Similar effects occur in figure 2.7 as an electron passes from the matrix to a bubble as the mean potential that an electron is travelling through will change. The component of the real part of the wave vector $\left(\kappa_{0}\right)$ inside the crystal which is determined by the mean potential of the crystal (where $V_{0}<0$ in the sign convention used by Rühle and Wilkens [83]) is, (a comparison of equation 2.84 to equation 2.77 shows that they are essentially the same equation),

$$
\kappa_{0}=-\frac{\mathrm{eV}_{0} \mathrm{k}_{0}}{2 \mathrm{E}}>0
$$

where $\mathrm{k}_{0}$ is the modulus of the wavevector of the electrons outside the crystal and $\mathrm{E}$ is the energy of the electrons. The difference in the wave vectors between the bubble and the crystal regions is

$$
\kappa_{0, \mathrm{~b}}-\kappa_{0}=\frac{\mathrm{e}\left(\mathrm{V}_{0}-\mathrm{V}_{0, \mathrm{~b}}\right) \mathrm{k}_{0}}{2 \mathrm{E}}
$$


where the b subscript denotes variables related to the bubble instead of the crystal. Both the undeviated and diffracted beams that pass through the bubble should be phase shifted relative to the the electrons that do not pass through the bubble by a factor of

$$
\exp \left[2 \pi i t\left(\kappa_{0, b}-\kappa_{0}\right)\right]
$$

This change in phase will not affect the in-focus images, but will change the out-of-focus simulations.

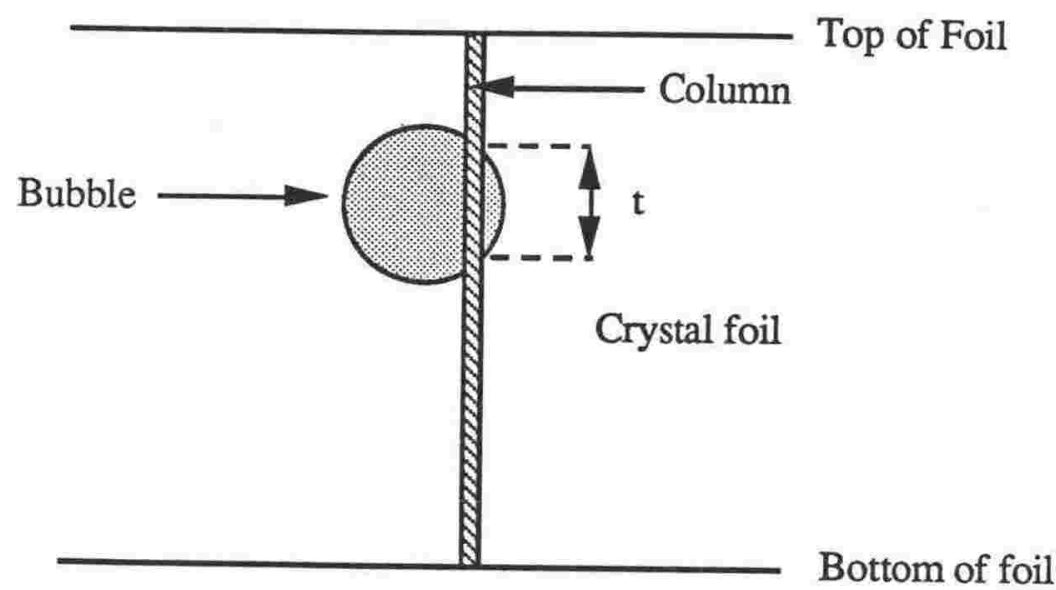

Figure 2.7: Column passing through a bubble embedded in a foil.

The wave function of the nth beam at the exit surface of the foil, $\psi_{n}\left(T, r^{\prime}\right)$, calculated from the theories in sections 2.1 to 2.3 can be then defocussed using [83]

$$
\psi_{\mathrm{n}}(\mathrm{T}+\xi, \mathbf{r})=\frac{-\mathrm{i}}{\lambda \xi} \int_{0}^{\mathrm{r}_{0}} \psi_{\mathrm{n}}\left(\mathrm{T}, \mathbf{r}^{\prime}\right) \exp \left[\frac{\mathrm{i} \pi \mathrm{k}_{0}\left(\mathrm{r}-\mathrm{r}^{\prime}\right)^{2}}{\xi}\right] \mathrm{d} \mathbf{r}^{\prime},
$$

where $\xi$ is the defocus, and $\mathrm{T}$ is the foil thickness in the beam direction.

If the structure has rotational symmetry, with no displacement field, then the expression for the defocussed wave function can be reduced to an integral over the reduced radius, $\rho^{\prime}$, where

$$
\rho^{\prime}=\frac{\left|\mathbf{r}^{\prime}\right|}{r_{0}}
$$


and $\mathrm{r}_{0}$ is the radius of the bubble. Equation 2.87 becomes

$$
\psi_{n}\left(\mathrm{~T}+\xi_{,}, \mathrm{r}\right)=\psi_{\mathrm{n}, \mathrm{p}}\left[1-\frac{2 \mathrm{i}}{\beta} \exp \left(\frac{\mathrm{i} \rho^{2}}{\beta}\right) \int_{0}^{1} \Delta_{\mathrm{n}}\left(\rho^{\prime}\right) \mathrm{J}_{0}\left(\frac{2 \rho \rho^{\prime}}{\beta}\right) \exp \left(\mathrm{i} \frac{\rho^{\prime 2}}{\beta}\right) \rho^{\prime} \mathrm{d} \rho^{\prime}\right]
$$

where $\mathrm{J}_{0}$ is a zero order Bessel function. $\beta$ and $\Delta_{\mathrm{n}}$ are defined by

$$
\beta=\frac{\xi}{\pi \mathrm{k}_{0} \mathrm{r}_{0}^{2}} \quad \text { and } \quad \Delta_{\mathrm{n}}=\frac{\psi_{\mathrm{n}}}{\psi_{\mathrm{n}, \mathrm{p}}}-1
$$

$\Delta_{\mathrm{n}}$ is the difference between the wave function that has passed through the bubble and the perfect crystal wave function, normalised by the perfect crystal wave function.

Several equations in the original paper of Rühle and Wilkens [83], from which equations 2.84 to 2.90 are derived, are wrong. The right hand side of their equation 3.6 is missing a factor of $-\mathrm{i} /(\lambda \xi)$ and $\psi_{\mathrm{n}}$ should be replaced by $\Delta_{\mathrm{n}}$. The $\sqrt{ }(\pi / 2)$ factor in equation A.1.4 should be multiplying the exponential term only. Using the definition of Fresnel integrals in Abramowitz and Stegun [164], the Fresnel integrals should be functions of $\sqrt{ }[2 /(\pi \beta)]$.

\subsection{The Generalised Cross-section Construction}

Head et al. [79-82] speeded up the simulation of images of long straight dislocations inclined to the foil surface by making use of the fact that equal displacements occur at points lying along a line parallel to a dislocation. The Howie-Whelan differential equations are coupled linear differential equations so that only two linearly independent solutions exist. All the solutions can be found by taking linear combinations of these two linearly independent solutions. Geometrically the displacement field or $\beta$ parameter that enters the Howie-Whelan differential equations will be the same for all columns that intersect a line parallel to the dislocation as shown in figure 2.8. Linear combinations of the results of two 
numerical integrations down one column that passes through the line ds are taken. The numerical integrations are made on columns of approximately twice the foil thickness The combinations are used to generate the linearly independent solutions required for the intensity along a line in the image. If for example an image containing 128 by 128 columns is required then the generalised cross-section construction of Head et al. will save on the time needed to produce this picture by a factor of approximately 64 , a difference between getting an image in about 10 seconds compared to the approximately 10 minutes required if an integration had to be made down every column in the image.

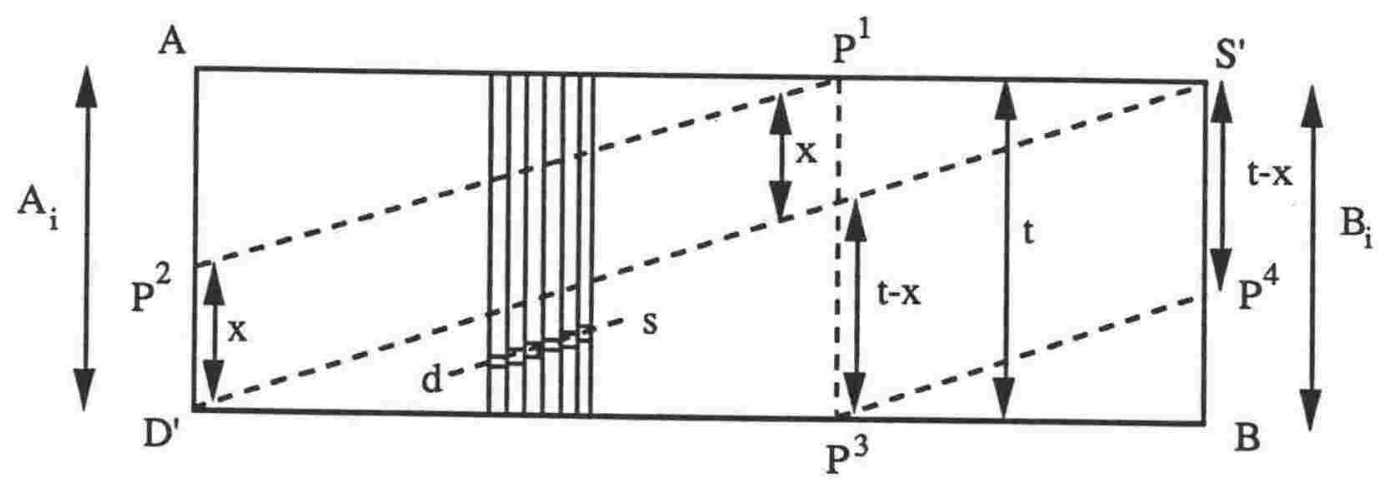

Figure 2.8: Side elevation of an untilted foil. The displacements at points in neighbouring columns are the same if they lie on a line such as ds which is parallel to the defect line (projection D'S'). The foil normal is parallel to the beam direction in this figure. The foil thickness is t. The other parameters in the figure are described in the text.

The $(\mathrm{T}, \mathrm{S})$ formalism used in section 2.1 is used to represent the beam amplitudes, where $\mathrm{T}$ is the transmitted beam and $\mathrm{S}$ is the diffracted beam. An incident beam will be used that has $\mathrm{T}=1$ and $\mathrm{S}=0$. The length of picture to be simulated is divided into $\mathrm{N}$ equal length segments. An artificially thick cross-section through the foil is created. Numerical integrations are made through the columns in the plane perpendicular to the page of the paper which projects onto AD'. When these integrations reach $\mathrm{D}^{\prime}$ they are continued from $S^{\prime}$ until B is reached. The columns are subdivided into segments identified by the index $\mathrm{i}$. The segments on the part of the integration from $A$ to $D^{\prime}$ are denoted by $A_{i}$. The segments on the part of the integration from $\mathrm{S}^{\prime}$ to $\mathrm{B}$ are denoted by $\mathrm{B}_{\mathrm{i}}$.

As the numerical integration through $A D^{\prime}$ proceeds the $a_{i}$ and $b_{i}$ coefficients in equation 2.91 are calculated at the top surface of the foil by solving 


$$
\mathrm{T}=\mathrm{a}_{\mathrm{i}} \mathrm{T}_{\mathrm{A}_{\mathrm{i}}}^{(1)}+\mathrm{b}_{\mathrm{i}} \mathrm{T}_{\mathrm{A}_{\mathrm{i}}}^{(2)}=1 \text { and } \mathrm{S}=\mathrm{a}_{\mathrm{i}} \mathrm{S}_{\mathrm{A}_{\mathrm{i}}}^{(1)}+\mathrm{b}_{\mathrm{i}} \mathrm{S}_{\mathrm{A}_{\mathrm{i}}}^{(2)}=0
$$

The 1 and 2 superscripts above the $\mathrm{T}$ and $\mathrm{S}$ denote the first and second linearly independent solutions to the Howie-Whelan differential equations which the program is integrating. As the first half of the numerical integration proceeds down the column the $a_{i}$ and $b_{i}$ coefficients are stored in a matrix. The numerical integration is continued through to B with the $\mathrm{T}$ and $\mathrm{S}$ values on each $\mathrm{B}_{\mathrm{i}}$ being stored in another matrix.

A two-dimensional picture can then be generated from the parameters stored during the integrations through the artificially thick cross section. Consider a point $\mathrm{P}^{\mathrm{I}}$ required for the picture on the top surface of the foil. This point has a particular value of $\beta_{\mathrm{g}}$ due to the dislocation. The point is projected parallel to the line D'S' onto the line AD'. The projected point has the same value of $\beta_{\mathrm{g}}{ }^{\prime}$ due to the dislocation. The original point has a wave with $\mathrm{T}$ $=1$ and $\mathrm{S}=0$ incident upon it. If this $\mathrm{T}$ and $\mathrm{S}$ was integrated from $\mathrm{P}^{1}$ to $\mathrm{P}^{3}$ the same $\beta_{\mathrm{g}}{ }^{\prime}$ values would occur in this column as occurred in the earlier numerical integration between $\mathrm{P}^{2}$ and $\mathrm{P}^{4}$. Because the linearly independent solutions for $\mathrm{T}$ and $\mathrm{S}$ have already been integrated through the desired $\beta_{\mathrm{g}}{ }^{\prime}$ values, there is no need to repeat the integration. Instead the $a_{i}$ and $b_{i}$ values at $\mathrm{P}^{2}$ can be retrieved from the matrix they were stored in and used to construct the final solution. The final solutions are calculated by

$$
\mathrm{T}_{\mathrm{i}}^{\mathrm{F}}=\mathrm{a}_{\mathrm{i}} \mathrm{T}_{\mathrm{B}_{\mathrm{i}}}^{(1)}+\mathrm{b}_{\mathrm{i}} \mathrm{T}_{\mathrm{B}_{\mathrm{i}}}^{(2)} \text { and } \mathrm{S}_{\mathrm{i}}^{\mathrm{F}}=\mathrm{a}_{\mathrm{i}} \mathrm{S}_{\mathrm{B}_{\mathrm{i}}}^{(1)}+\mathrm{b}_{\mathrm{i}} \mathrm{S}_{\mathrm{B}_{\mathrm{i}}}^{(2)}
$$

The final solution (denoted by the F superscripts) at the exit surface of the foil is generated and stored in the matrix representing the image. If the foil is tilted, i.e., the foil normal is not coincident with the beam direction, then the integration needs to extend further than over just twice the foil distance (Head et al. [79-82]).

If the Burgers vector $\mathbf{b}$ is reversed in direction to $\mathbf{- b}$ then the image obtained for $\mathbf{- b}$ will be the same as that for $\mathbf{b}$ rotated through $180^{\circ}$. This is a consequence of the symmetry of the $\beta_{\mathrm{g}}$ term appearing in the Howie-Whelan differential equations for the two different Burgers vectors. This is proved in an appendix of Head et al., and further reduces the 
amount of computation required to generate a series of theoretical images, for a range of $\mathbf{b}$, to compare against experimental micrographs.

Humble [81] incorporated image dislocations at the foil surfaces to obtain boundary conditions that are more physically reasonable for the strain field of the dislocation at the foil surfaces. In the simulations of long straight dislocations, in chapter 4 , image dislocations are ignored because the updated computer code was unavailable. Instead the original Head et al. programs that assume that the dislocations are embedded in an infinite medium are used.

\subsection{Summary}

In this chapter an outline has been given of the theory required in this thesis to interpret images obtained with a TEM. The theory describes the interaction of electrons with crystalline materials. The theories outlined in this chapter are briefly summarised below in order of increasing resolution.

The most commonly used set of equations for modelling the diffraction of electrons in a crystal containing dislocations are the Howie-Whelan differential equations. These equations are derived in section 2.1 by considering the plane wave solutions to the SWE and the dispersion surface construction familiar to solid state physicists. Images of superlattices of bubbles are typically taken at out-of-focus conditions to obtain good contrast. The wavefunction calculated by integrating the Howie-Whelan differential equations can be defocussed using the equations in section 2.5. Simulations of images can be obtained most quickly in some situations by use of the generalised cross-section construction of Head et al. and the Howie-Whelan differential equations. The method of Head et al. is discussed in section 2.6.

Weak beam images show the position of a dislocation core more accurately than the strong two-beam images which are interpreted using the Howie-Whelan differential equations. The weak beam technique is then potentially useful for studying the materials containing the high density of defects considered in this thesis. An understanding of why the increased resolution occurs is obtained by considering the scattering effects between the 
Bloch wave solutions to the SWE. The weak beam theory in section 2.3 is developed from the many beam theory of section 2.2. In an undeformed crystal there will be no scattering between dispersion surfaces. The theory of weak beam diffraction calculates the imaging conditions that give the maximum scattering between dispersion surfaces in a deformed crystal. The maximum scattering is associated with the strongly curved lattice planes near the dislocation core.

Structure images of planes of atoms require the use of the multislice theory of electron diffraction for interpretation. Modelling in the multislice formalism usually includes a larger number of beams than the calculations performed with the other theories in this chapter. In this formalism the crystal is subdivided into a sequence of two-dimensional phase gratings that lie parallel to the foil surface (section 2.4). The diffraction of an electron wave is calculated by multiplying the electron wave function by the transmission function of a phase grating before propagating the function onto the next slice. This procedure is continued until the electron wave has passed through all the slices spanning the crystal thickness. Whereas the multislice technique can be readily applied to the imaging of small bubbles, the images calculated for scattering from dislocations are difficult to interpret. 


\section{CHAPTER 3: DEFECT DISPLACEMENT FIELDS}

\subsection{Burgers Equation}

In this chapter an outline is given of the derivation of the displacement fields about a range of defects. The displacement fields are required in later chapters to simulate the images of these defects. This section derives Burgers equation which is an expression for the displacement field of a dislocation of arbitrary shape in an isotropic material. The derivation follows that in Hirth and Lothe [97]. Burgers equation contains a line integral over the dislocation line so that in practice the displacement field can be only evaluated for dislocations of simple geometry. Section 3.2 outlines how the displacement field of an angular dislocation is derived from Burgers equation. This solution will be used in section 3.3 to construct a hexagonal dislocation loop.

In an isotropic material the number of independent elastic constants is further reduced from the three constants used in an anisotropic material with cubic symmetry to two elastic constants only. The two independent elastic constants in an isotropic material can be relabelled as

$$
\mu=c_{44}=\frac{1}{2}\left(c_{11}-c_{12}\right) \text { and } \lambda=c_{12}
$$

where $\mu$ is the shear modulus and $\lambda$ the Lamé constant. By examination, the elastic constants in equation 3.1 satisfy

$$
c_{\mathrm{ijkl}}=\mu\left(\delta_{\mathrm{ik}} \delta_{\mathrm{jl}}+\delta_{\mathrm{il}} \delta_{\mathrm{jk}}\right)+\lambda \delta_{\mathrm{ij}} \delta_{\mathrm{kl}}
$$

where $\delta_{\mathrm{ij}}$ is the Kronecka delta function. Then the vector form of the equilibrium condition, equation 1.5 , becomes

$$
(\lambda+\mu) \nabla(\nabla . \mathbf{u})+\mu \nabla^{2} \mathbf{u}+\mathbf{F}=0
$$


where $\mathbf{F}$ is a point force vector. By substitution into equation 3.3 the solution to the equilibrium equation for a continuous distribution of point forces has the form

$$
u_{i}(\mathbf{r})=\int u_{i j}\left(\mathbf{r}-\mathbf{r}^{\prime}\right) F_{j}\left(\mathbf{r}^{\prime}\right) d V^{\prime}
$$

where $\mathrm{u}_{\mathrm{ij}}$ is called the Green's function for the elastic displacements and $d V^{\prime}$ ' is an element of volume. The Greens function is

$$
\mathrm{u}_{\mathrm{ij}}(\mathbf{r})=\frac{1}{8 \pi \mu}\left[\delta_{\mathrm{ij}} \nabla^{2} \mathrm{r}-\left(\frac{\lambda+\mu}{\lambda+2 \mu}\right) \frac{\partial^{2} \mathrm{r}}{\partial \mathrm{x}_{\mathrm{i}} \partial \mathrm{x}_{\mathrm{j}}}\right]
$$

Effectively the Greens function is the ith component of the displacement that results when a unit point force $F_{j} \delta\left(\mathbf{r}^{\prime}\right)$ is applied in the jth direction.

The work (W) done by the mth component of a point force $\left(\mathrm{F}_{\mathrm{m}}\right)$ at the position $\mathbf{r}$ to create a displacement $u_{m}$ is

$$
\mathrm{W}=\mathrm{F}_{\mathrm{m}} \mathrm{u}_{\mathrm{m}}(\mathbf{r}) \text {. }
$$

The force $F_{m}$ is assumed to be independent of $u_{m}$. The stress $\sigma_{i j}$ at $\mathbf{r}^{\prime}$ is caused by the components $\mathrm{F}_{\mathrm{m}}$ of a point force $\mathbf{F}$ at $\mathbf{r}$. The total stress due to $\mathbf{F}$ is then found by a sum over its $\mathrm{m}$ components. That is the repeated index in the equations denotes that a summation is to be made. The work done to create a closed dislocation loop is, as shown using figure 3.1 ,

$$
W=-\int_{A} d A_{j} b_{i} \sigma_{i j}\left(\mathbf{r}^{\prime}-\mathbf{r}\right) .
$$

To evaluate the integral in equation 3.7 the displacement must be found because the stress is a function of the displacement (equation 1.7). The displacement is 


$$
\mathrm{u}_{\mathrm{k}}\left(\mathbf{r}^{\prime}\right)=\mathrm{u}_{\mathrm{mk}}\left(\mathbf{r}^{\prime}-\mathbf{r}\right) \mathrm{F}_{\mathrm{m}} \text { using } \mathrm{F}_{\mathrm{m}} \int \delta(\mathbf{r}) \mathrm{dV}=\mathrm{F}_{\mathrm{m}} \text { in equation 3.4. }
$$

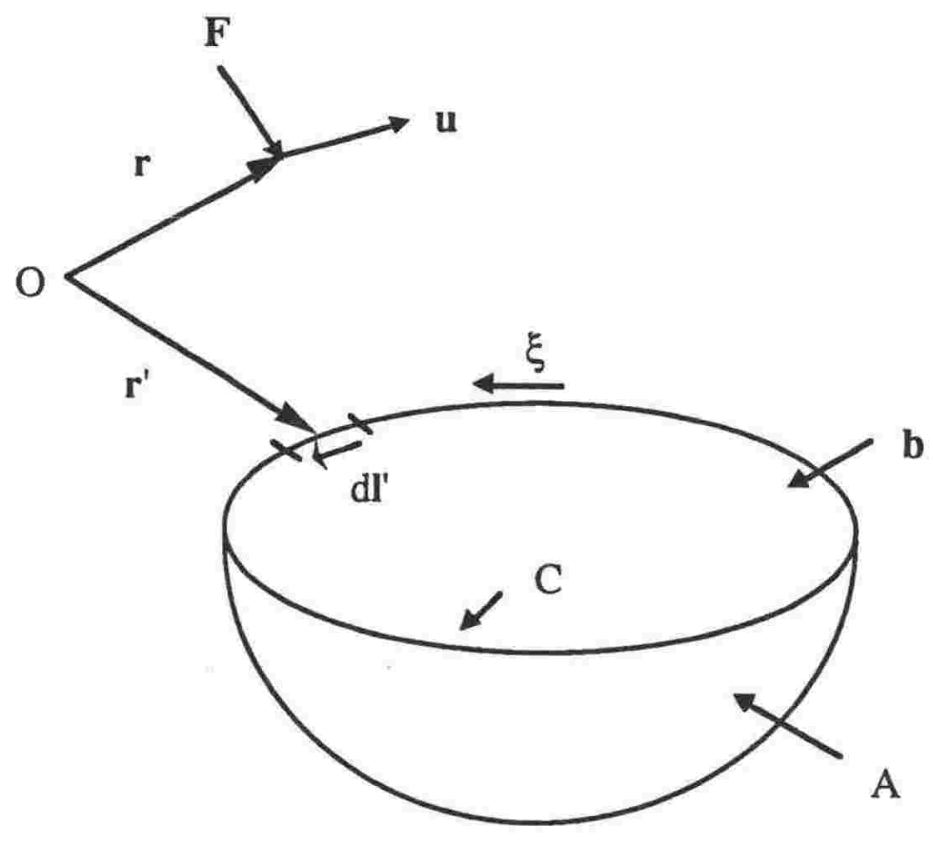

Figure 3.1: A point force $\mathbf{F}$ acting within an elastic continuum containing a closed dislocation loop. The figure shows the relationship between the force, several position vectors and the area used in the integral in equation 3.7.

Substituting for the stress in terms of the displacement using equation 1.7 gives

$$
W=-\int_{A} d A_{j} b_{i} c_{i j k l} \frac{\partial F_{m} u_{m k}\left(r^{\prime}-r\right)}{\partial x_{1}^{\prime}}
$$

Equating equations 3.6 and 3.9, cancelling out the common $\mathrm{F}_{\mathrm{m}}$ term and substituting for the elastic constants from equation 3.2 gives

$$
\mathrm{u}_{\mathrm{m}}(\mathbf{r})=-\lambda \int_{\mathrm{A}} \mathrm{d} \mathrm{A}_{\mathrm{j}} \mathrm{b}_{\mathrm{j}} \frac{\partial \mathrm{u}_{\mathrm{mk}}}{\partial \mathrm{x}_{\mathrm{k}}^{\prime}}-\mu \int_{\mathrm{A}} \mathrm{d} \mathrm{A}_{\mathrm{j}} \mathrm{b}_{\mathrm{i}} \frac{\partial \mathrm{u}_{\mathrm{mi}}}{\partial \mathrm{x}_{\mathrm{j}}^{\prime}}-\mu \int_{\mathrm{A}} \mathrm{d} \mathrm{A}_{\mathrm{j}} \mathrm{b}_{\mathrm{i}} \frac{\partial \mathrm{u}_{\mathrm{mj}}}{\partial \mathrm{x}_{\mathrm{i}}^{\prime}}
$$

Substituting the solution for the Green's function for the elastic displacements in equation 3.5 into equation 3.10 gives 


$$
\begin{aligned}
\mathrm{u}_{\mathrm{m}}(\mathbf{r})= & -\frac{1}{8 \pi} \int_{\mathrm{A}} \mathrm{b}_{\mathrm{m}} \frac{\partial \nabla^{2} \mathrm{R}}{\partial \mathrm{x}_{\mathrm{j}}^{\prime}} d \mathrm{~A}_{\mathrm{j}}-\frac{1}{8 \pi} \int_{\mathrm{A}}\left(\mathrm{b}_{\mathrm{i}} \frac{\partial \nabla^{2} \mathrm{R}}{\partial \mathrm{x}_{\mathrm{i}}^{\prime}} d \mathrm{~A}_{\mathrm{m}}-\mathrm{b}_{\mathrm{i}} \frac{\partial \nabla^{\prime 2} \mathrm{R}}{\partial \mathrm{x}_{\mathrm{m}}^{\prime}} d \mathrm{~A}_{\mathrm{i}}\right) \\
& +\frac{1}{4 \pi}\left(\frac{\lambda+\mu}{\lambda+2 \mu}\right) \int_{\mathrm{A}}\left(\mathrm{b}_{\mathrm{i}} \frac{\partial}{\partial \mathrm{x}_{\mathrm{i}}^{\prime}} \frac{\partial^{2} \mathrm{R}}{\partial \mathrm{x}_{\mathrm{m}}^{\prime} \partial \mathrm{x}_{\mathrm{j}}^{\prime}} d \mathrm{~A}_{\mathrm{j}}-\mathrm{b}_{\mathrm{i}} \frac{\partial}{\partial \mathrm{x}_{\mathrm{j}}^{\prime}} \frac{\partial^{2} \mathrm{R}}{\partial \mathrm{x}_{\mathrm{m}}^{\prime} \partial \mathrm{x}_{\mathrm{j}}^{\prime}} \mathrm{dA} \mathrm{A}_{\mathrm{i}}\right)
\end{aligned}
$$

Equation 3.11 can be simplified using equations 3.12 to 3.15. Poisson's ratio can be expressed in terms of the elastic constants by

$$
\frac{\lambda+\mu}{\lambda+2 \mu}=\frac{1}{2(1-v)} .
$$

The solid angle $(\Omega)$ subtended by the dislocation is

$$
\Omega=\int_{\mathrm{A}} \frac{\mathbf{R} \cdot \mathrm{dA}}{\mathrm{R}^{3}} .
$$

(Integration of equation 3.13 gives $4 \pi$ steradians as expected.) Stokes theorem is

$$
\int_{A}\left(\frac{\partial \phi}{\partial x_{j}} d A_{i}-\frac{\partial \phi}{\partial x_{i}} d A_{j}\right)=\varepsilon_{i j k} \int_{C} \phi d_{k}
$$

where the Einstein permutation operator is

$$
\varepsilon_{\mathrm{ijk}}=\mathbf{e}_{\mathrm{i}} \cdot\left(\mathbf{e}_{\mathrm{j}} \times \mathbf{e}_{\mathrm{k}}\right) .
$$

Then in a result first derived by Burgers in 1939 the displacement in equation 3.11 simplifies to

$$
\mathbf{u}(\mathbf{r})=-\frac{\mathbf{b} \Omega}{4 \pi}-\frac{1}{4 \pi} \int_{C} \frac{\mathbf{b} \times \mathrm{d} \mathbf{l}^{\prime}}{\mathrm{R}}+\frac{1}{8 \pi(1-v)} \nabla \int_{\mathrm{C}} \frac{(\mathbf{b} \times \mathbf{R}) \cdot \mathrm{dl}}{\mathrm{R}}
$$


This is the desired expression for the displacement field of a dislocation of arbitrary shape in an isotropic material.

\subsection{Angular Dislocation}

Yoffe [165] evaluated the line integrals in equation 3.16 for the case of an angular dislocation that lies along the $\xi$ and $\mathrm{z}$ axes as shown in figure 3.2.

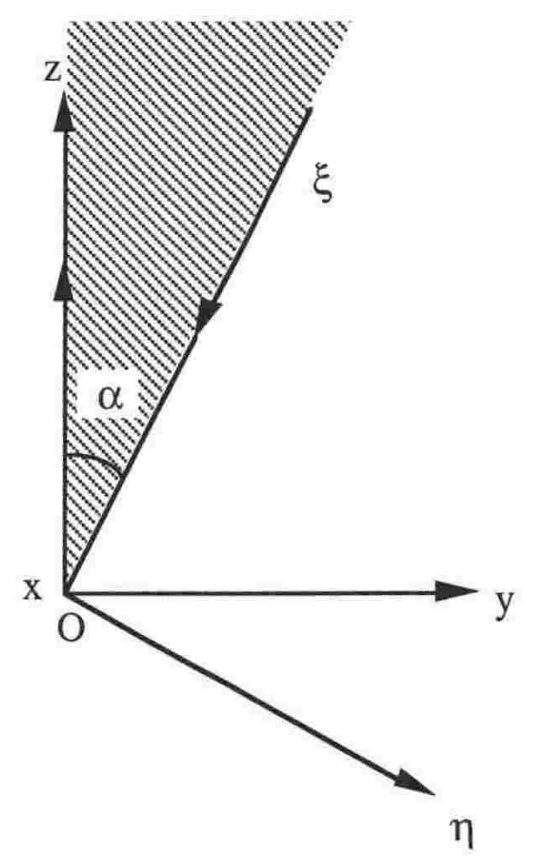

Figure 3.2: An angular dislocation lying along the $\mathrm{z}$ and $\xi$ axes. The other axes are required to evaluate the integrals in Burgers equation. The $\eta$ axis is at right angles to the $\xi$ and $\mathrm{x}$ axes.

The displacements due to the $\mathrm{x}$-component of the Burgers vector $\mathrm{b}_{\mathrm{x}}$, at the point $\mathrm{P}(\mathrm{x}, \mathrm{y}, \mathrm{z})$ or $(\mathrm{x}, \eta, \xi)$ are

$$
\begin{gathered}
\mathrm{u}_{\mathrm{x}}=\mathrm{b}_{\mathrm{x}} \frac{\Omega}{4 \pi}+\frac{\mathrm{xb}_{\mathrm{x}}}{8 \pi \mathrm{r}(1-\mathrm{v})}\left(\frac{\mathrm{y}}{(\mathrm{r}-\mathrm{z})}-\frac{\eta}{(\mathrm{r}-\xi)}\right), \\
\mathrm{v}_{\mathrm{x}}=\frac{\mathrm{b}_{\mathrm{x}}}{8 \pi(1-\mathrm{v})}\left(\frac{\eta \sin (\alpha)}{\mathrm{r}-\xi}-\frac{\mathrm{y} \eta}{\mathrm{r}(\mathrm{r}-\xi)}+\frac{\mathrm{y}^{2}}{\mathrm{r}(\mathrm{r}-\mathrm{z})}+(1-2 \mathrm{v})(\cos (\alpha) \ln (\mathrm{r}-\xi)-\ln (\mathrm{r}-\mathrm{z}))\right), \\
3-5
\end{gathered}
$$




$$
\text { and } \quad \mathrm{w}_{\mathrm{x}}=\frac{\mathrm{b}_{\mathrm{x}}}{8 \pi(1-\mathrm{v})}\left(\frac{\eta \cos (\alpha)}{\mathrm{r}-\xi}-\frac{\mathrm{y}}{\mathrm{r}}-\frac{\eta \mathrm{z}}{(\mathrm{r}(\mathrm{r}-\mathrm{x}))}-(1-2 \mathrm{v}) \sin (\alpha) \ln (\mathrm{r}-\xi)\right) \text {, }
$$

where $\mathrm{u}_{\mathrm{x}}, \mathrm{v}_{\mathrm{x}}$ and $\mathrm{w}_{\mathrm{x}}$ are the components of the displacement field in the $(\mathrm{x}, \mathrm{y}, \mathrm{z})$ coordinate system and $\Omega$ is the solid angle subtended at $P$ by the shaded area

$$
\Omega=\tan ^{-1}\left(\frac{y}{x}\right)-\tan ^{-1}\left(\frac{\eta}{x}\right)+\tan ^{-1}\left(\frac{x r \sin (\alpha)}{\left(x^{2} \cos (\alpha)+\eta y\right)}\right) .
$$

Similar expressions occur for the displacement field components due to the $\mathrm{y}$ and $\mathrm{z}$ components of the Burgers vector:

$$
\begin{gathered}
\mathrm{u}_{\mathrm{y}}=\frac{\mathrm{b}_{\mathrm{y}}}{8 \pi(1-v)}\left(\frac{\mathrm{x}^{2} \cos (\alpha)}{\mathrm{r}(\mathrm{r}-\xi)}-\frac{\mathrm{x}^{2}}{\mathrm{r}(\mathrm{r}-\mathrm{z})}-(1-2 v)(\cos (\alpha) \log (\mathrm{r}-\xi)-\log (\mathrm{r}-\mathrm{z}))\right) \\
\mathrm{v}_{\mathrm{y}}=\mathrm{b}_{\mathrm{y}} \frac{\Omega}{4 \pi}+\frac{\mathrm{b}_{\mathrm{y}} \mathrm{x}}{8 \pi(1-v)}\left(\frac{\mathrm{y} \cos (\alpha)}{\mathrm{r}(\mathrm{r}-\xi)}-\frac{\sin (\alpha) \cos (\alpha)}{\mathrm{r}-\xi}-\frac{\mathrm{y}}{\mathrm{r}(\mathrm{r}-\mathrm{z})}\right) \\
\mathrm{w}_{\mathrm{y}}=\frac{\mathrm{b}_{\mathrm{y}} \mathrm{x}}{8 \pi(1-v)}\left(\frac{\mathrm{z} \cos (\alpha)}{\mathrm{r}(\mathrm{r}-\xi)}-\frac{\cos ^{2}(\alpha)}{\mathrm{r}-\xi}+\frac{1}{\mathrm{r}}\right) \\
\mathrm{u}_{\mathrm{z}}=\frac{\mathrm{b}_{\mathrm{z}} \sin (\alpha)}{8 \pi(1-v)}\left((1-2 v) \log (\mathrm{r}-\xi)-\frac{\mathrm{x}^{2}}{\mathrm{r}(\mathrm{r}-\xi)}\right) \\
\mathrm{v}_{\mathrm{z}}=\frac{\mathrm{b}_{\mathrm{z}} \mathrm{x} \sin (\alpha)}{8 \pi(1-v)}\left(\frac{\sin (\alpha)}{\mathrm{r}-\xi}-\frac{\mathrm{y}}{\mathrm{r}(\mathrm{r}-\xi)}\right) \\
\text { and } \quad \mathrm{w}_{\mathrm{z}}=\mathrm{b}_{\mathrm{z}} \frac{\Omega}{4 \pi}+\frac{\mathrm{b}_{\mathrm{z}} \mathrm{x} \sin (\alpha)}{8 \pi(1-v)}\left(\frac{\cos (\alpha)}{\mathrm{r}-\xi}-\frac{\mathrm{z}}{\mathrm{r}(\mathrm{r}-\xi)}\right)
\end{gathered}
$$

Equations 3.17, 3.18 and 3.19 can be used to find the displacement field due to an angular dislocation with any Burgers vector. 


\subsection{Hexagonal Dislocation Loop}

The displacement field of an hexagonal dislocation loop can be constructed from six angular dislocations as shown in figure 3.3. The angular dislocations have the same Burgers vector. Outside the hexagon the dislocation lines of overlapping angular dislocation segments are in opposite directions. Therefore the dislocation line only extends around the hexagon. Alternatively the construction of a hexagonal dislocation can be viewed as evaluating the line integrals of equation 3.16 over six angular dislocations. The contributions to the displacement field of those parts of the circuits traversed in opposite directions cancel each other out. The resultant displacement field due to a hexagonal dislocation loop is calculated by adding the individual displacement fields of the angular dislocations together. However, the displacements due to the individual angular dislocations expressed in the six different coordinate systems of figure 3.3 must first be rotated into a common coordinate system before the resultant displacement is calculated.

If $\mathbf{b}$ is set parallel to the $\mathrm{x}$ axis in Saldin's [62-64] model for a loop, which uses the FS/RH rule of Bilby et al. to define $\mathbf{b}$ (chapter one), then a vacancy loop will be simulated. If $\mathbf{b}$ is set antiparallel to the $\mathrm{x}$ axis an interstitial loop is formed. The $\mathrm{x}$ axis is antiparallel to the loop normal defined in section 1.2. The Burgers vectors in the $\mathrm{x}, \mathrm{y}, \mathrm{z}$ system and with respect to the coordinate system of each angular dislocation are

$$
\left(b_{x}, b_{y}, b_{z}\right) \text { and }\left(b_{x_{j}}, b_{\eta_{j}}, b_{\xi j}\right) \text { respectively. }
$$

The Burgers vector can be rotated from one coordinate system into another by a rotation matrix:

$$
\begin{gathered}
\mathrm{b}_{\mathrm{x}_{\mathrm{j}}}=\mathrm{b}_{\mathrm{x}} \quad \forall \mathrm{j}, \mathrm{j}=1 \text { to } 6,\left(\begin{array}{c}
\mathrm{b}_{\eta_{\mathrm{j}}} \\
\mathrm{b}_{\xi_{\mathrm{j}}}
\end{array}\right)=\mathbf{A}_{\mathrm{j}}\left(\begin{array}{l}
\mathrm{b}_{\mathrm{y}} \\
\mathrm{b}_{\mathrm{z}}
\end{array}\right), \\
\mathbf{A}_{\mathrm{j}}=\left(\begin{array}{cc}
\cos \theta_{\mathrm{j}} & \sin \theta_{\mathrm{j}} \\
-\sin \theta_{\mathrm{j}} & \cos \theta_{\mathrm{j}}
\end{array}\right),
\end{gathered}
$$




$$
\alpha=\frac{2 \pi}{N}, N=6 \text { and } \theta_{j}=(j-1) \alpha .
$$

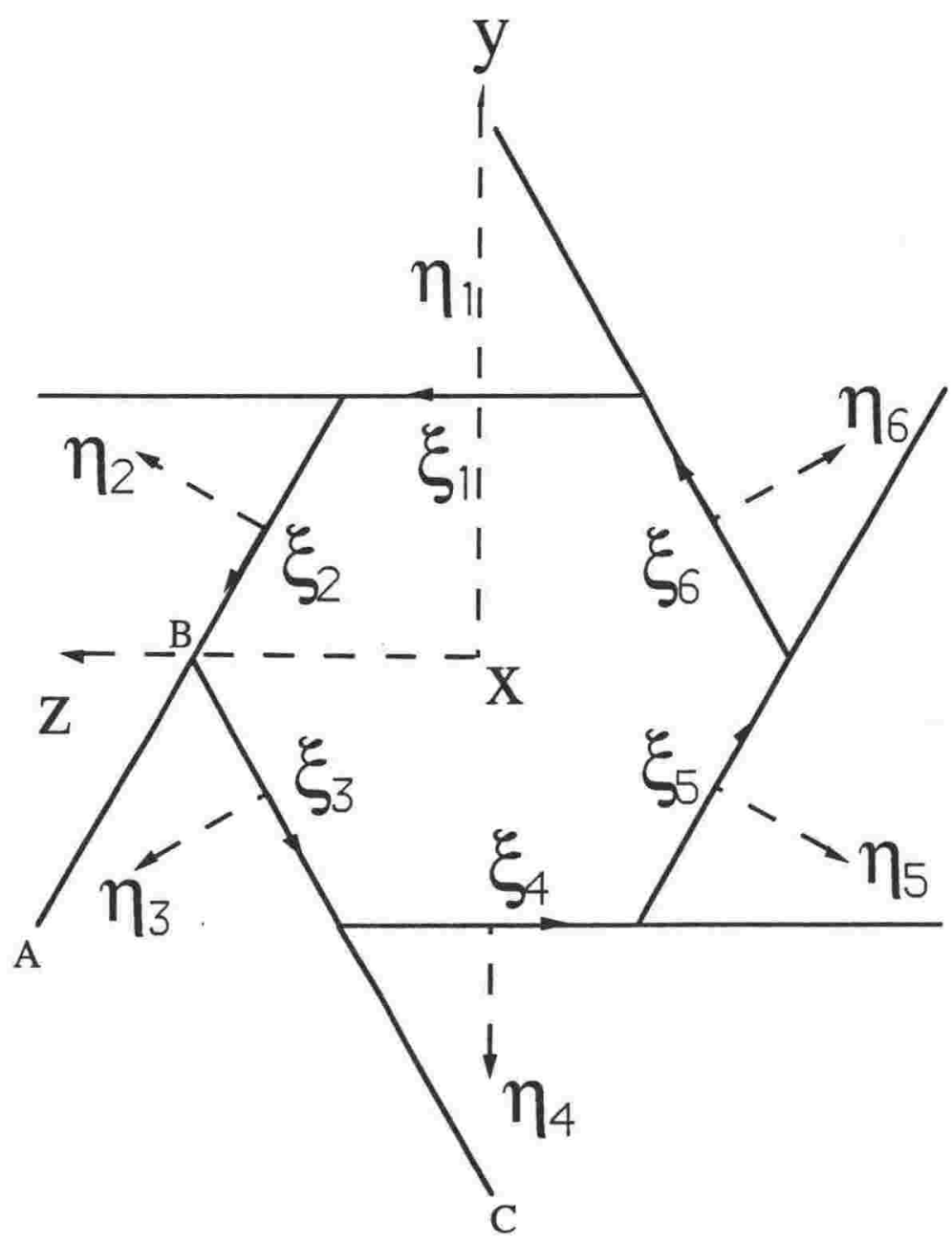

Figure 3.3: The coordinate systems used to construct an hexagonal dislocation loop from six angular dislocations such as $\mathrm{ABC}$.

To calculate the displacement field about each angular dislocation the coordinates must be similarly transformed according to

$$
\mathrm{x}_{\mathrm{j}}=\mathrm{x} \quad \forall \mathrm{j} \quad \text { and }\left(\begin{array}{c}
\eta_{\mathrm{j}}+\mathrm{h} \cos \frac{\alpha}{2} \\
\xi_{\mathrm{j}}
\end{array}\right)=\mathbf{A}_{\mathrm{j}}\left(\begin{array}{l}
\mathrm{y} \\
\mathrm{z}
\end{array}\right) \text {. }
$$

Yoffe's equations for the displacement field of an angular dislocation (equations 3.17 to 3.19) use a slightly different notation, and her variables are substituted for the ones below:

$$
y \rightarrow \eta_{j}, z \rightarrow \xi_{j}+h \sin \frac{\alpha}{2}, \eta \rightarrow \eta_{j-1} \text {, and } \xi \rightarrow \xi_{j-1}-h \sin \frac{\alpha}{2}
$$


The resultant displacement due to the hexagonal dislocation loop is the sum of the individual displacements due to the angular dislocation loops. Before adding the displacements together they must be rotated into the common $\mathrm{x}, \mathrm{y}, \mathrm{z}$ coordinate system by

$$
U=\sum_{j=1}^{N} u_{j} \text { and }\left(\begin{array}{c}
V \\
W
\end{array}\right)=\sum_{j=1}^{N} \mathbf{A}_{j}^{-1}\left(\begin{array}{c}
v_{j} \\
w_{j}
\end{array}\right) \text {. }
$$

The equations of Yoffe give a discontinuity in the displacement field outside the loop. This discontinuity can be transferred to the inside of the loop by subtracting or adding constants to the displacement field as shown in Saldin [64] and figures 3.4 and 3.5.

Figure 3.6 shows the resultant displacement field calculated using the equations for the displacement field about an angular dislocation and the rotation matrices above for an edge type dislocation loop with an [011] Burgers vector. The representation of the components of the displacement field about a dislocation by grey levels has not been seen by this author elsewhere in the literature. This technique is invaluable for visualising the dislocation structure and checking the program geometry. Figure 3.7 shows sections through the components of the displacement field that lie in this plane. 


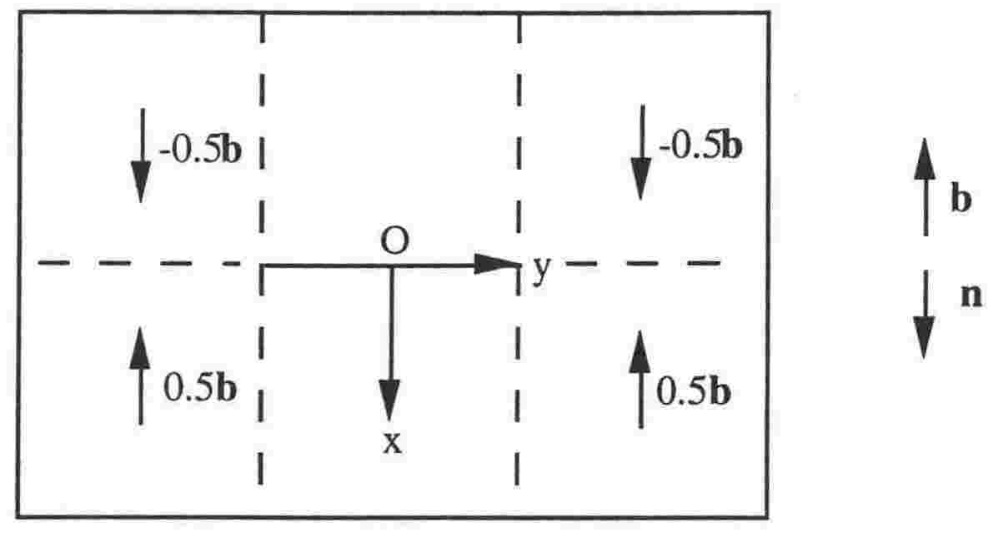

Figure 3.4: The figure shows the maximum magnitude and direction of the displacement field that occurs near the plane of the loop. There is a discontinuity in the displacement field due to the construction of a hexagonal dislocation loop from six angular dislocations. The discontinuity lies outside the loop.
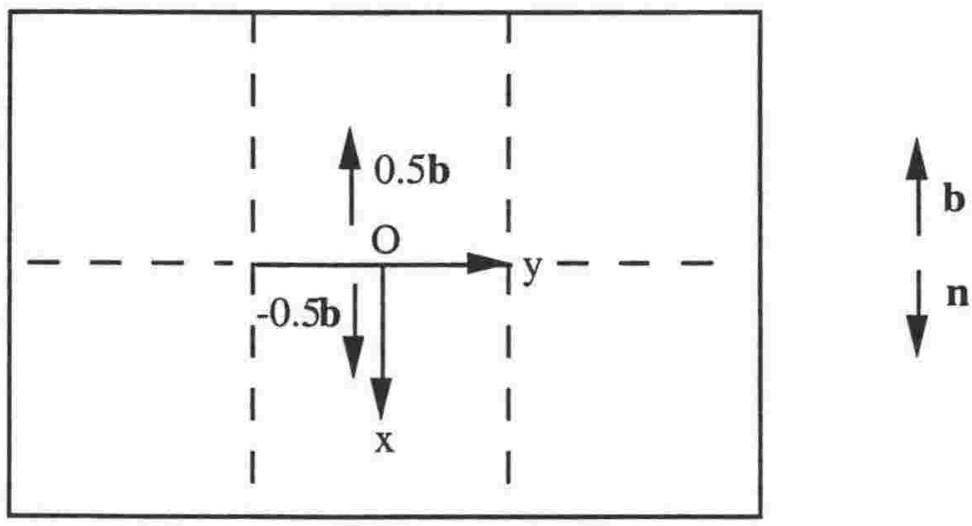

Figure 3.5: The discontinuity in the displacement field after it has been transferred to the inside of the loop. It is permissible to add or subtract different constants from the displacement field on either side of the loop. This is because the image contrast is a function of the derivative of the displacement field and not its magnitude. Across the plane of the loop the derivative has a singularity but for $\mathrm{x} \neq 0$ the derivative is finite. Image simulations integrate to near the plane of the loop then step across to the other side of the loop before continuing the integration. In this way the singularity is avoided. 


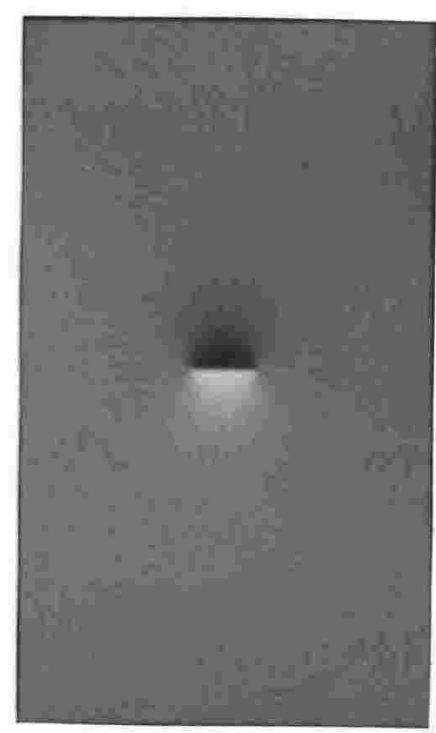

$\downarrow^{\mathrm{R}_{\mathrm{ox}_{2}}}$

(a)

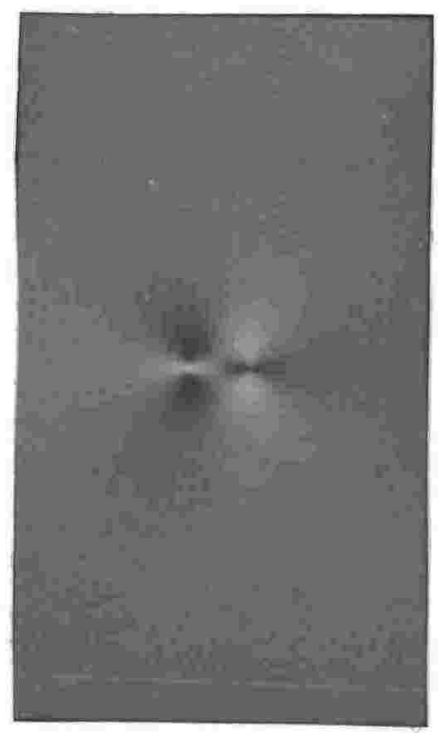

$\mathrm{R}_{\mathrm{Ox}}$

(b)

3- 11 


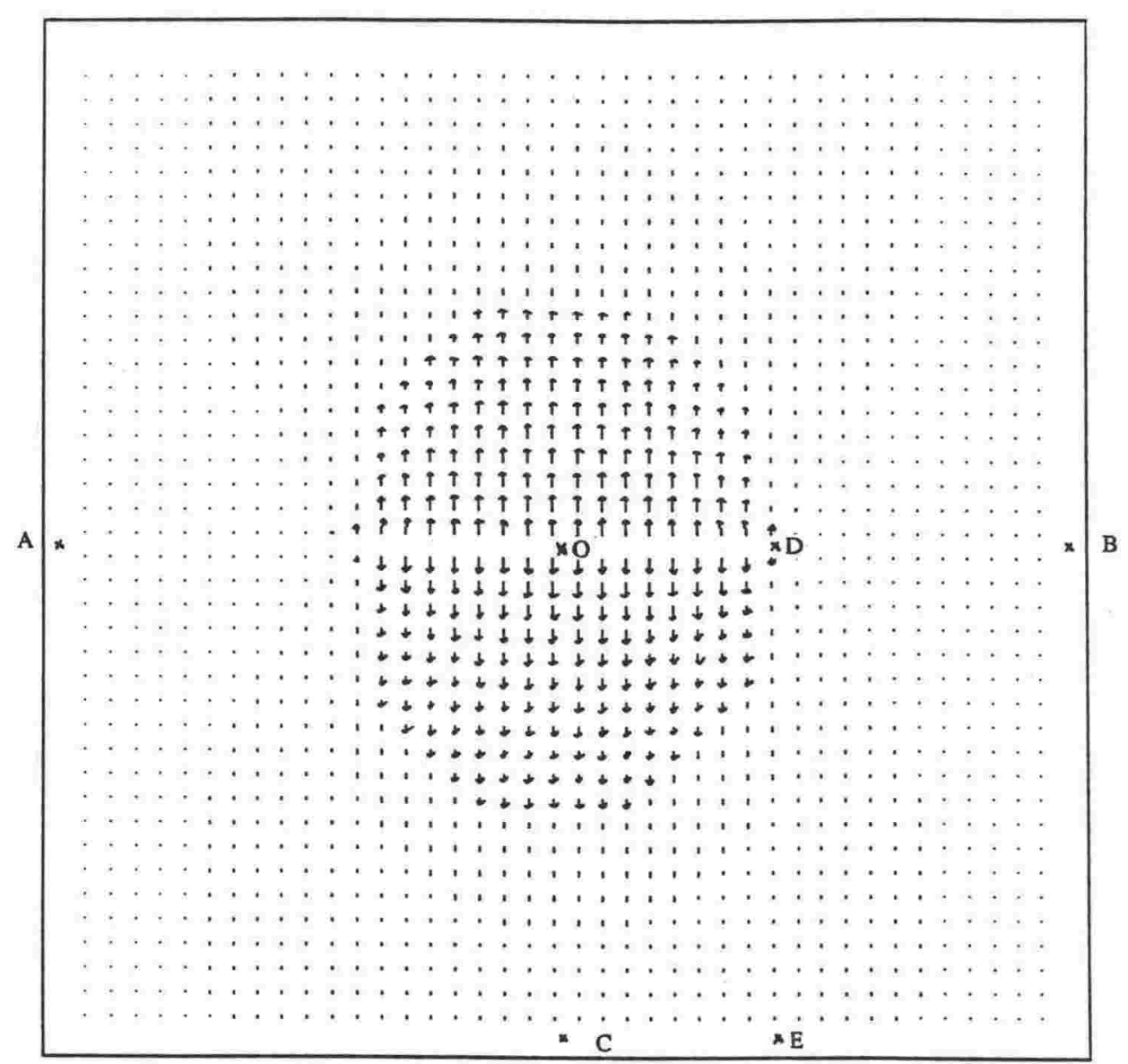

(c)

Figure 3.6: Displacement field for an edge type dislocation loop with an [011] Burgers vector. The plane of the loop is perpendicular to the plane of the figure. The components of the displacement field in two directions in the plane of the paper are shown as grey scale images in (a) and (b), and as a vector is constructed from these in (c). The images show the magnitude of the components of the displacement field in a region of size $100 \AA$ by $200 \AA$ near the loop. Part (a) shows the magnitude of the component parallel to the $\mathrm{OX}_{2}$ direction, this direction being indicated by the vector alongside the picture. The white grey level represents a displacement parallel to the $\mathrm{OX}_{2}$ direction. A black grey level represents a displacement antiparallel to the $\mathrm{OX}_{2}$ direction. The discontinuity across the plane of the loop is clearly visible. This discontinuity has been transferred into the centre of the loop by the process outlined in figures 3.5 and 3.6. Part (b) shows the magnitude of the component of the displacement vector parallel to the $\mathrm{OX}_{3}$ direction. This component is smaller than that in (a) indicating that the displacement vector is predominantly parallel to the loop normal, and is responsible for the residual contrast of a dislocation loop, i.e., the contrast when $\mathbf{g} . \mathbf{b}$ $=0$. 
Displacement vs distance

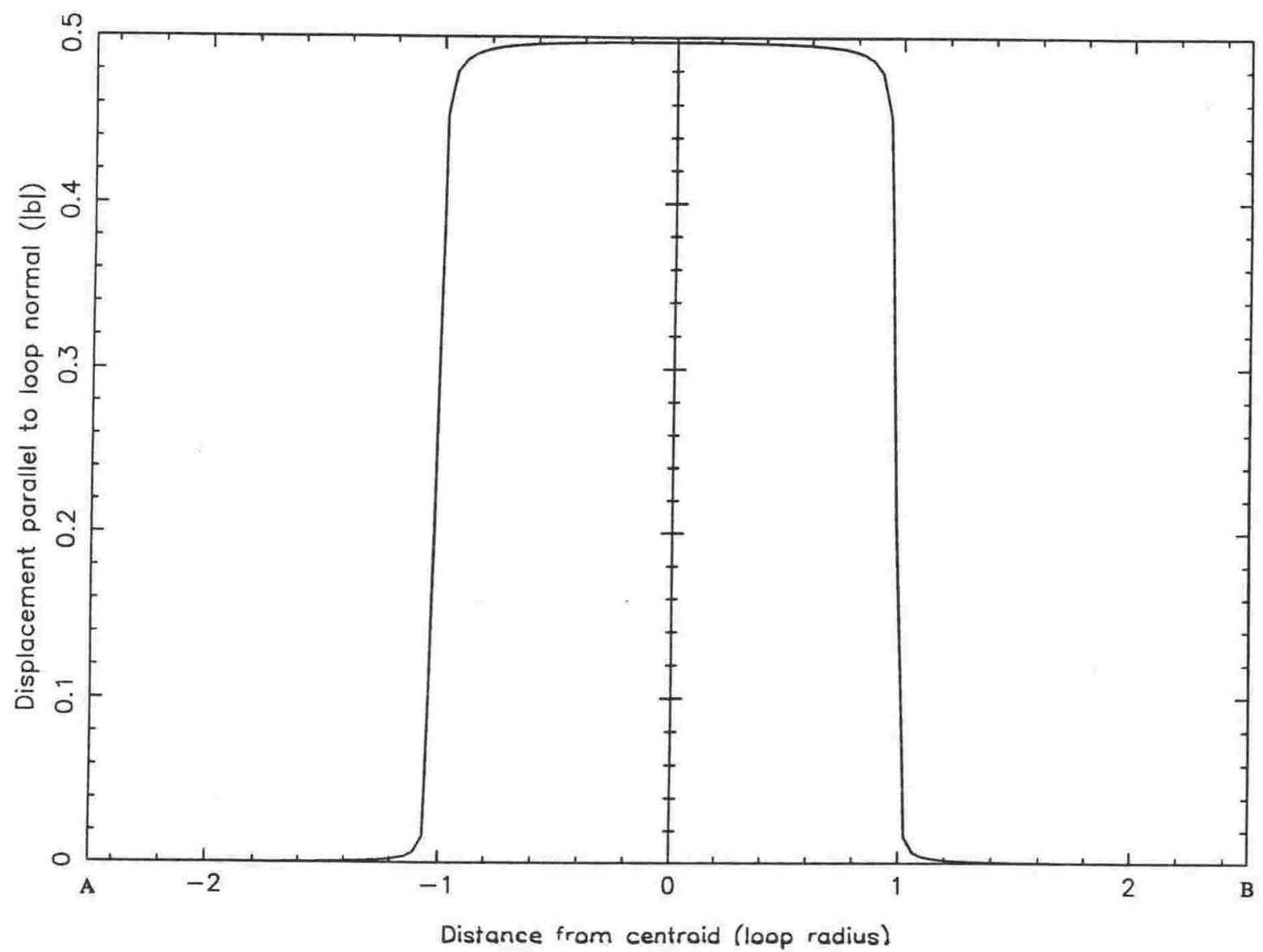

Displacement vs distance

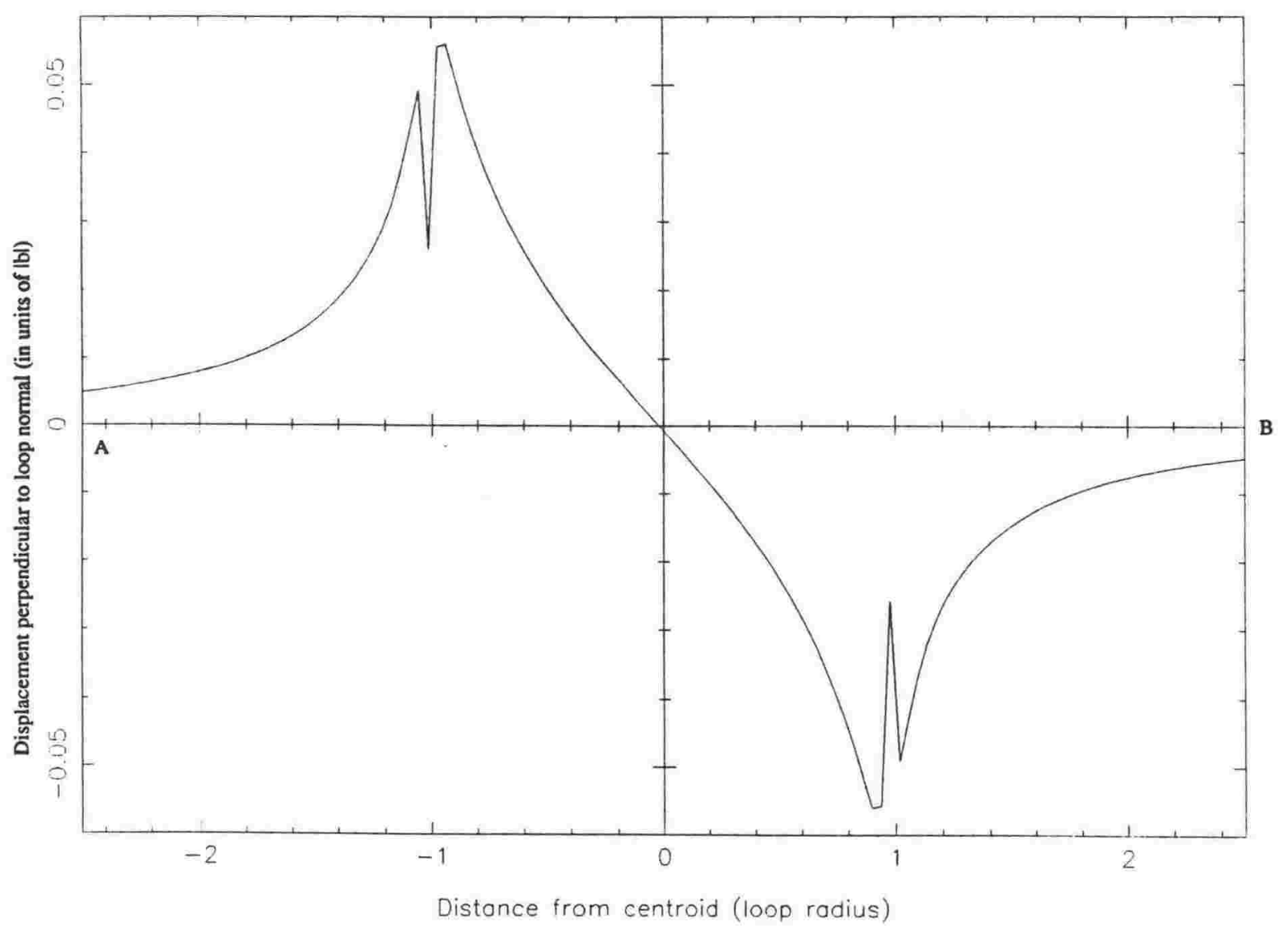

Figure $3.73-13$ 

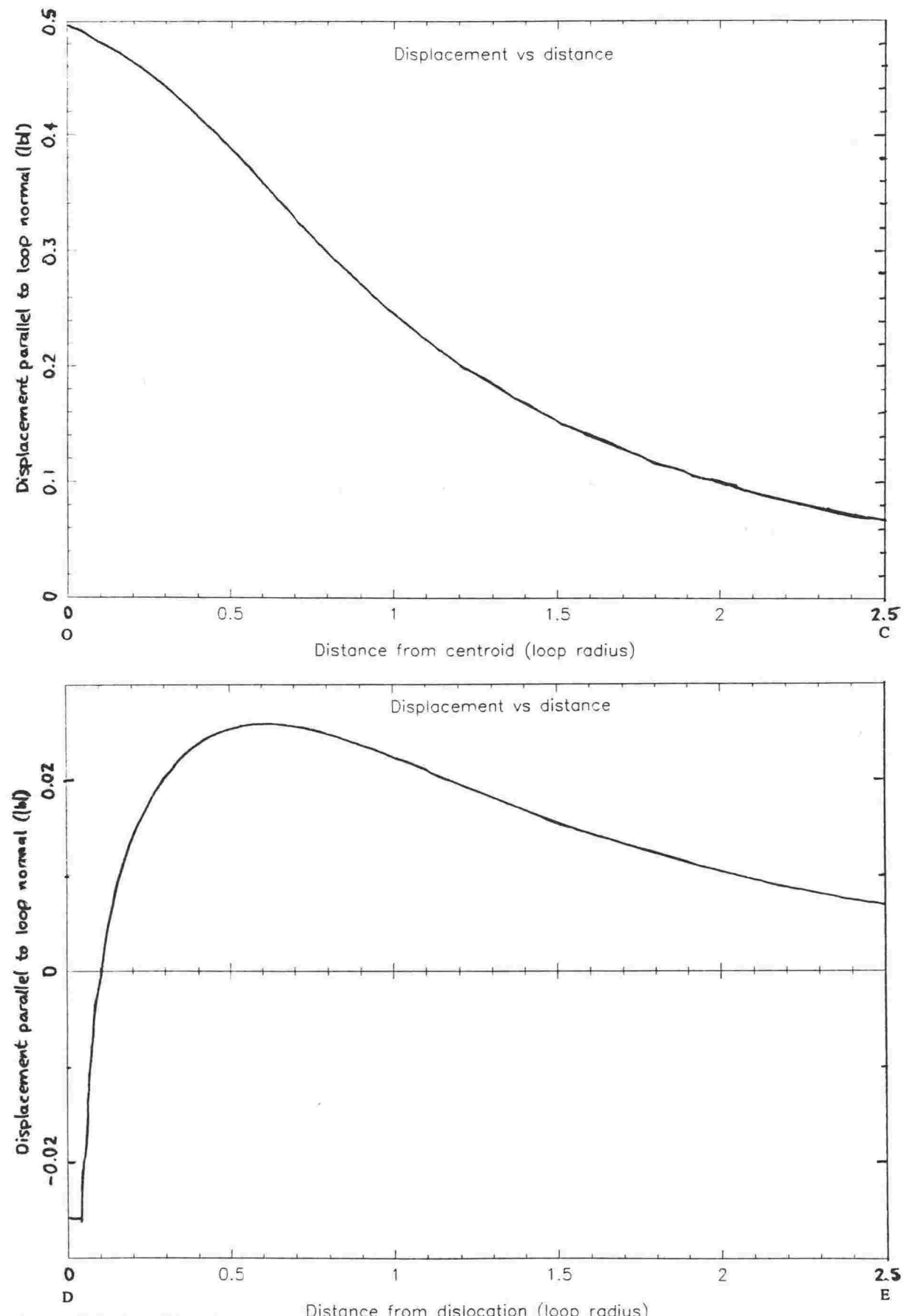

Figure 3.7: Profiles through the components of the displacement field shown as grey scale images in parts (a) and (b) of figure 3.6. In any profile the horizontal axis is labelled with a pair of points that also appear on figure 3.6 to indicate the directions along which the profile has been taken. 


\subsection{Bubble Displacement Field}

In this section several ways of calculating the displacement field about a bubble are outlined. The displacement field for a centre of dilatation or spherical inclusion, ignoring the effects of the foil surface is (Hirth and Lothe [97] or Timoshenko and Goodier [165])

$$
\mathbf{u}(\mathbf{r})=\frac{\delta \hat{\mathbf{r}}}{\mathrm{r}^{2}}
$$

where $\delta$ is a measure of the strength of dilatation or overpressure in the bubble:

$$
\delta=\left(\mathrm{P}-\frac{2 \gamma}{\mathrm{a}}\right) \frac{\mathrm{a}^{3}}{2 \mu}
$$

For a void $\delta<0$ and for a bubble $\delta>0$, that is the surrounding material is pushed out by the pressure in the bubble. $\mu$ is the shear modulus of rigidity of the material, $\gamma$ the surface tension, "a" the bubble radius and $\mathrm{P}$ is the bubble pressure first introduced in chapter one.

Mindlin and Cheng [167] quoted a solution for the displacement field around a centre of dilatation in a semi-infinite solid using the techniques in Love [168] for finding displacements around nuclei of strain. Their solution is

$$
\mathbf{u}(\mathbf{r})=2(1-v) \widehat{\mathbf{k}} \nabla^{2} \mathrm{Q}-\nabla \frac{\partial \mathrm{Q}}{\partial \mathrm{z}}
$$

where the unit vector $\mathbf{k}$ defines the $\mathrm{z}$ axis which is antiparallel to the foil normal. The other variables introduced in equation 3.27 are:

$$
\begin{aligned}
& Q(\mathbf{r})=\delta\left[\log \left(R_{1}+z-d\right)+(1-4 v) \log \left(R_{2}+z-d\right)+\frac{2 z}{R_{2}}\right] \\
& \text { where } R_{1}^{2}=x^{2}+y^{2}+(z-d)^{2}, R_{2}^{2}=x^{2}+y^{2}+(z+d)^{2},
\end{aligned}
$$

and $\mathrm{d}$ is the distance from the centre of the bubble to the foil surface. (Ingram [169] misquotes the $\mathrm{z}$ component. Equation 3.27 is the original solution of Mindlin and Cheng 
and not that quoted by Ingram.) The point on the foil surface directly above the centre of the bubble is taken as the origin $(\mathrm{z}=0)$.

The analytical expression of equation 3.27 for the displacement field has zero strain at one of the surfaces of the foil and treats the rest of the foil as a semi-infinite medium. To obtain an approximate displacement field in a foil it is necessary to include the effects due to both surfaces of the foil. This is done by taking each surface in turn and then taking the mean of the displacement fields for the two cases. This is more practical than trying to merge the two solutions for the displacement field in any other way that would, necessarily, introduce a stacking fault or other discontinuity in the displacement field.

The component of the displacement field in the $\mathrm{z}$ direction does not need to be calculated if the beam direction is parallel to the foil normal. In this situation any diffracting vector will be perpendicular to the $\mathrm{z}$ direction and the $\mathrm{z}$-component of the displacement field will not contribute to the differential equations describing the electron diffraction.

The program described in chapter six, which is used for simulating combinations of bubbles and loops, can treat the case when the foil normal is tilted acutely to the direction of the electron beam. To do this a coordinate system was created with one axis parallel to the foil normal. The other two axes are the projections of the edges of the picture onto a plane parallel to the foil surfaces. The picture coordinates and the diffracting vector are rotated into this coordinate system. The new coordinates can be used to calculate the $\mathrm{x}$ and $\mathrm{y}$ components of the displacement field in the same way as when the foil normal was parallel to the beam direction. The diffracting vectors may have non-zero $\mathrm{z}$ components in the coordinate system based on the foil normal and the $\mathrm{z}$ component of the displacement must then be calculated.

Ingram and Mindlin and Cheng do not explicitly quote the result for the displacement field. Instead they leave it to the reader to evaluate the partial derivatives. More explicitly the displacement components are:

$$
\begin{gathered}
u_{x}=-\frac{\partial^{2} Q}{\partial x \partial z} \quad, \quad u_{y}=-\frac{\partial^{2} Q}{\partial y \partial z} \\
\text { and } u_{z}=2(1-v)\left\{\frac{\partial^{2} Q}{\partial x^{2}}+\frac{\partial^{2} Q}{\partial y^{2}}\right\}+(1-2 v) \frac{\partial^{2} Q}{\partial z^{2}} .
\end{gathered}
$$




$$
\begin{gathered}
\frac{1}{P} \frac{\partial^{2} Q}{\partial x \partial z}=\frac{-1}{\left(R_{1}+z-d\right)^{2}} \frac{\partial R_{1}}{\partial x}\left(\frac{\partial R_{1}}{\partial z}+1\right)+\frac{1}{\left(R_{1}+z-d\right)} \frac{\partial^{2} R_{1}}{\partial x \partial z}-\frac{a}{\left(R_{2}+z+d\right)^{2}} \frac{\partial R_{2}}{\partial x}\left(\frac{\partial R_{2}}{\partial z}+1\right) \\
+\frac{a}{\left(R_{2}+z+d\right)} \frac{\partial^{2} R_{2}}{\partial x \partial z}-\frac{2}{R_{2}^{2}} \frac{\partial R_{2}}{\partial x}+\frac{4 z}{R_{2}^{3}} \frac{\partial R_{2}}{\partial x} \frac{\partial R_{2}}{\partial z}-\frac{2 z}{R_{2}^{2}} \frac{\partial^{2} R_{2}}{\partial x \partial z}, \\
\frac{1}{P} \frac{\partial^{2} Q}{\partial z^{2}}=\frac{-1}{\left(R_{1}+z-d\right)^{2}}\left(\frac{\partial R_{1}}{\partial z}+1\right)+\frac{1}{\left(R_{1}+z-d\right)} \frac{\partial^{2} R_{1}}{\partial z^{2}}-\frac{a}{\left(R_{2}+z+d\right)^{2}}\left(\frac{\partial R_{2}}{\partial z}+1\right)^{2}+\frac{a}{\left(R_{2}+z+d\right)} \frac{\partial^{2} R_{2}}{\partial z^{2}} \\
-\frac{4}{R_{2}^{2}} \frac{\partial R_{2}}{\partial z}+\frac{4 z}{R_{2}^{3}}\left(\frac{\partial R_{2}}{\partial z}\right)^{2}-\frac{2 z}{R_{2}^{2}} \frac{\partial^{2} R_{2}}{\partial z^{2}} \\
\text { and } \frac{1}{P} \frac{\partial^{2} Q}{\partial x^{2}}=\frac{-1}{\left(R_{1}+z-d\right)^{2}}\left(\frac{\partial R_{1}}{\partial x}\right)^{2}+\frac{1}{\left(R_{1}+z-d\right)} \frac{\partial^{2} R_{1}}{\partial x^{2}}-\frac{a}{\left(R_{2}+z+d\right)^{2}}\left(\frac{\partial R_{2}}{\partial x}\right)^{2}+\frac{a}{\left(R_{2}+z+d\right)} \frac{\partial^{2} R_{2}}{\partial x^{2}} \\
+\frac{4 z}{R_{2}^{3}}\left(\frac{\partial R_{2}}{\partial x}\right)^{2}-\frac{2 z}{R_{2}^{2}} \frac{\partial^{2} R_{2}}{\partial x^{2}} \cdot
\end{gathered}
$$

The partial derivatives of $\mathrm{Q}$ in terms of $\mathrm{y}$ are obtained by replacing $\mathrm{x}$ with $\mathrm{y}$ in the derivatives of $Q$ in terms of $x$. Singularities occur in several terms at $x=y=z=0$. This is not a problem when calculating the $\mathrm{x}$ and $\mathrm{y}$ displacements because a zero occurs in the partial derivatives by which the terms with singularities are multiplied. However, the singularities in the $\mathrm{z}$ component are not "cancelled" out like this. Computation of this $\mathrm{z}$ component shows that these singularities give an unrealistically large displacement over a large enough region of the foil to make image simulation impractical and inaccurate. Instead when the foil normal was tilted away from the beam direction the simple inverse square form for the displacement field in equation 3.25 was used to calculate the $\mathrm{z}$ component of the displacement. However, this simpler form for the $\mathrm{z}$ component of the displacement ignores the effects of the foil surface. The $\mathrm{x}$ and $\mathrm{y}$ components were always calculated using Mindlin and Cheng's solution for the displacements.

Mindlin and Cheng use the method of images when considering the displacement field of a bubble. Two image particles are used and, to account for the two surfaces of the 
foil, two coordinate systems centred at the top and bottom surfaces are required. (The equations for the $\mathrm{x}$ and $\mathrm{y}$ components of the displacement field are odd functions of $\mathrm{x}$ and $\mathrm{y}$ so it is only necessary to change the $\mathrm{z}$ coordinate between calculating the effects of either image particle.)

Figure 3.8 shows the sections through the displacement field due to an isolated bubble and due to two bubbles separated by $50 \AA$. The bubbles in these profiles have overpressures of $10 \mathrm{GPa}$. The displacement field has a smaller maximum and falls off more quickly than the sections through the displacement field of an edge dislocation loop in figure 3.7. The simulations in chapter six show that loops and bubbles have similar contrast; this occurs because the displacement fields of the defects have a similar strength.

Bubbles are expected to have contrast characteristics similar to spherical inclusions outside the bubble, but similar to voids inside the bubble. Internally, voids and bubbles have no displacement field whereas precipitates have a displacement field for which the magnitude is proportional to the distance from the centre. The boiling point of helium is approximately $4 \mathrm{~K}$ so the helium is in gaseous form, unless the bubbles have a very high pressure, so there cannot be a non-zero displacement field inside a bubble. Crystalline precipitates can also have a different orientation from that of the host matrix. The coherency of the precipitate can cause other diffraction behaviour that is different from that due to a bubble.

Isotropic elasticity theory is used in this thesis to calculate the displacement field around a bubble, even though copper is anisotropic. That is, only Poisson's ratio (v) and the shear modulus $(\mu)$ are used in Mindlin and Cheng's theory to construct the displacement field. This is equivalent to including only two of the three independent elastic constants for copper in the calculations. Ingram [169] examined the scattering from aluminium, a material for which the assumption of isotropy is reasonable. The extension of the simulations to anisotropic copper using isotropic elasticity theory is of unknown validity. However, the experience that others have gained, in comparing simulations of loops done with anisotropic elasticity theory to those done with isotropic elasticity theory, is that the effects of ignoring the anisotropy are not too significant. This is discussed further in chapter 6. 

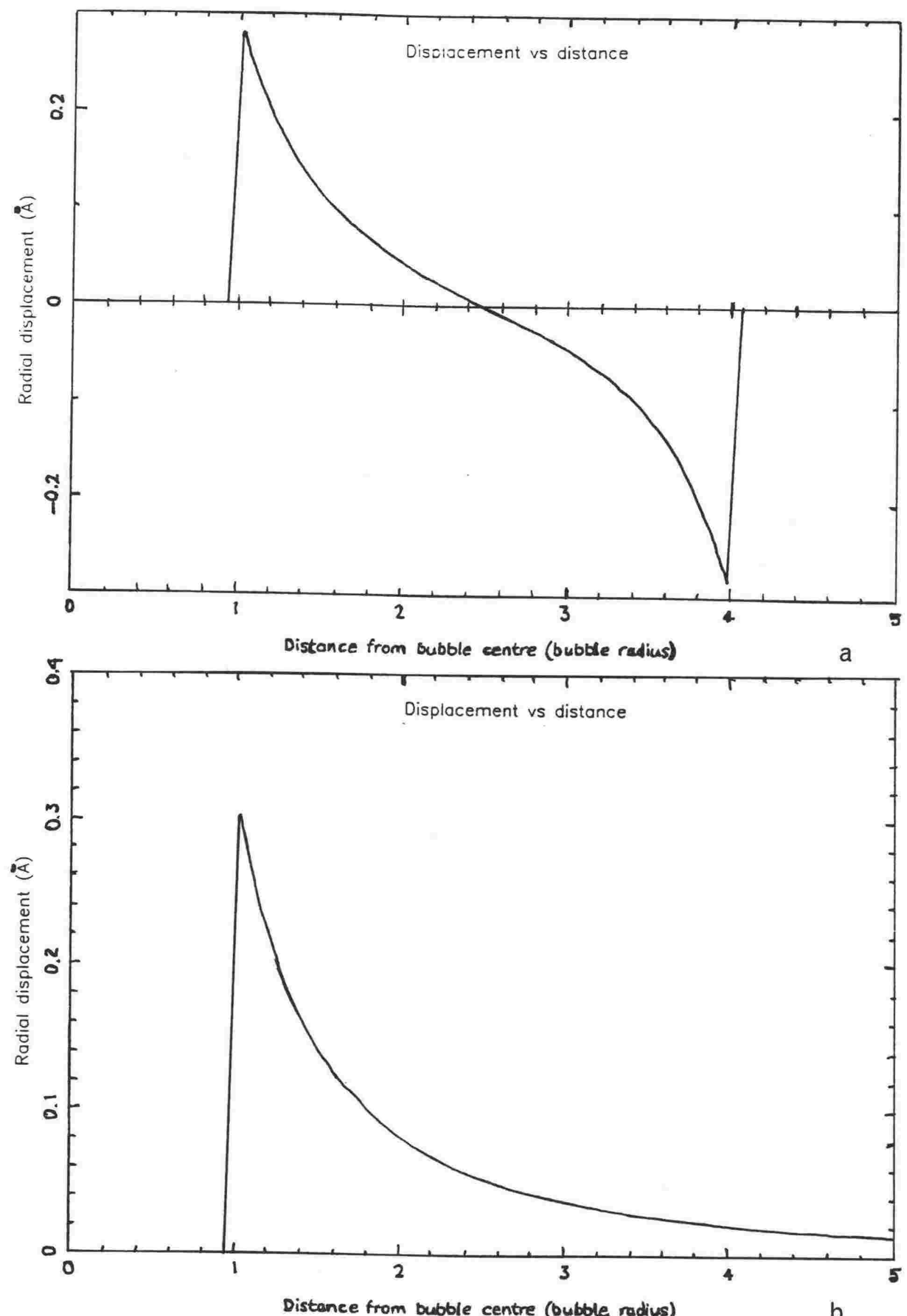

Figure 3.8: Sections through the displacement field (a) due to two bubbles separated by 50 $\AA$ and (b) due to an isolated bubble. The displacement fields of the two bubbles pushes the material between the bubbles in opposite directions. 
The two-dimensional simulations presented in chapters five and six are much more representative of the diffraction from bubbles than Ingram's work. Ingram [169] produced intensity profiles parallel to the diffracting vector only, with the foil normal parallel to the beam direction. The only restriction on the foil normal in the present work is that the angle must be acute.

\subsection{Straight Dislocation - Anisotropic Elasticity Solution}

The form of the displacement field used by Head et al. [82] is outlined in this section. Head et al. identify the Burgers vectors of long straight dislocations that are inclined to the foil by matching the entire contrast of an image. This approach can be used instead of just using the $\mathbf{g} \cdot \mathbf{b}=0$ and residual contrast techniques of Hirsch et al. [67] to identify $\mathbf{b}$.

For a line dislocation parallel to the $\mathrm{x}_{3}$ direction in a solid of infinite extent, the displacement field surrounding the dislocation will be independent of $\mathrm{x}_{3}$ (using the $\mathrm{x}_{1}, \mathrm{x}_{2}$, $\mathrm{x}_{3}$ coordinate system for which the elastic constants are defined in chapter 1 ). Therefore a solution to the equilibrium equation 1.5 is sought, of the form

$$
\mathrm{u}_{\mathrm{k}}=\mathrm{A}_{\mathrm{k}} \mathrm{f}\left(\mathrm{x}_{1}+\mathrm{px}_{2}\right)
$$

where $\mathrm{p}$ is a constant, $\mathrm{A}_{\mathrm{k}}$ is a vector and $\mathrm{f}$ is an arbitrary function (to be determined below). After substituting the trial solution for $\mathrm{u}_{\mathrm{k}}$ into equation 1.5 and using equation 1.7 , we obtain

$$
\left(c_{i 1 k 1}+c_{i 1 k 2} p+c_{i 2 k 1} p+c_{i 2 k 2} p^{2}\right) A_{k}=0 .
$$

To have a non-trivial solution, we require that the determinant of equation 3.32 is zero, i.e.,

$$
\operatorname{det}\left(c_{i 1 k 1}+c_{i 1 k 2} p+c_{i 2 k 1} p+c_{i 2 k 2} p^{2}\right)=0 \text {. }
$$


This sextic equation has six roots for $\mathrm{p}$. No real solutions exist and so the solutions occur in complex conjugate pairs. The complex conjugate of $\mathrm{p}$ is denoted by $\bar{p}$ and the subscript $\alpha$ is used to distinguish the different pairs of solutions for $\mathrm{p}$. The solution shows $\mathrm{f}$ to be a $\log$ function [82]

$$
\mathrm{u}_{\mathrm{k}}=\frac{1}{2 \pi \mathrm{i}} \sum_{\alpha} \mathrm{A}_{\mathrm{k} \alpha} \mathrm{D}_{\alpha} \log \left(\mathrm{x}_{1}+\mathrm{p}_{\alpha} \mathrm{x}_{2}\right)-\frac{1}{2 \pi \mathrm{i}} \sum_{\alpha} \overline{\mathrm{A}}_{\mathrm{k} \alpha} \overline{\mathrm{D}}_{\alpha} \log \left(\mathrm{x}_{1}+\overline{\mathrm{p}}_{\alpha} \mathrm{x}_{2}\right)
$$

where $\alpha$ takes the value 1,2 or 3 . The $\alpha$ subscript has been added to each $A_{k}$ value to distinguish the different solutions for $A_{k}$ obtained when the different values for $p_{\alpha}$ are substituted into equation 3.32 . $\mathrm{D}_{\alpha}$ is a scaling constant.

The simulation of images, using the Howie-Whelan differential equations in the $\beta_{\mathrm{g}}{ }^{\prime}$ form of equation 2.20 , requires the evaluation of the derivative

$$
\beta_{\mathrm{g}}^{\prime}=\frac{\mathrm{d}(\mathbf{g} \cdot \mathbf{R})}{\mathrm{dZ}} \propto \frac{\partial\left(\mathrm{g}_{\mathrm{k}} \mathrm{u}_{\mathrm{k}}\right)}{\partial \mathrm{x}_{2}}=\sum_{\alpha} \frac{\mathrm{P}_{\alpha} \mathrm{x}_{1}+\mathrm{Q}_{\alpha} \mathrm{x}_{2}}{\left(\mathrm{x}_{1}+\mathrm{R}_{\alpha} \mathrm{x}_{2}\right)^{2}+\left(\mathrm{S}_{\alpha} \mathrm{x}_{2}\right)^{2}} .
$$

$R_{\alpha}$ is the real part of $p_{\alpha}$ and $S_{\alpha}$ is the imaginary part of $p_{\alpha} . P_{\alpha}$ and $Q_{\alpha}$ are functions of $p_{\alpha}$, $\mathrm{A}_{\mathrm{k} \alpha}, \mathbf{g}, \mathbf{b}$ and the elastic constants. The elastic constants and the other parameters are transformed into $\mathrm{P}_{\alpha}, \mathrm{Q}_{\alpha}, \mathrm{R}_{\alpha}$ and $\mathrm{S}_{\alpha}$ so that equation 3.35 may be used in the numerical integrations required to find the image contrast. The ultimate speed of the program is set by the subroutine to evaluate the displacement field as it will be called many times during the computation of an image. Therefore equation 3.35 is expressed in as simplified form as possible.

In general the dislocation line will not lie along an axis of the coordinate system used to define the elastic constants. In this situation various transformations of the variables must be made to use equation 3.35. The details involved in performing these are explained in Head et al. [82]. 


\subsection{Summary}

To simulate the image of a defect, either the displacement field or the derivative of the displacement field about the defect is required. The following defects are considered in this chapter: an angular dislocation, an hexagonal dislocation loop, an overpressured bubble and a long straight dislocation.

In section 3.2 the analytical solution for the displacement field of an angular dislocation is determined by solving Burgers equation, an equation which is derived in section 3.1 and which gives the displacement field in terms of line integrals.

An hexagonal dislocation loop can be constructed from six angular dislocations positioned so that the dislocation line directions of touching angular dislocations are antiparallel outside the loop. Thus, the dislocation line only extends around the hexagon and the displacement field is the sum of the individual displacements of the constituent angular dislocations. An hexagonal loop will be used in later chapters to represent the dislocation loop punched out by an overpressured gas bubble.

Later work also requires that the displacement field around an overpressured bubble be known. A bubble at equilibrium will not distort the material surrounding it. (An equilibrium bubble contains gas at a pressure that just balances the surface tension of the metal). When a bubble is overpressured the displacement field in the surrounding matrix is proportional to the degree of overpressure (i.e., the difference between the gas pressure inside the bubble and the gas pressure required for equilibrium). In section 3.4 the displacement field is determined to be roughly inversely proportional to the square of the distance from the centre of the bubble. The distortion of the displacement field due to the foil surfaces can be modelled by including image particles beyond the foil surfaces. These effects due to the foil surface have not been included when considering the fields about the other types of defects discussed in this chapter. 


\section{CHAPTER 4: EXPERIMENTAL TECHNIQUES AND EQUIPMENT}

\subsection{Description of the TEM}

The transmission electron microscope (TEM) at Victoria University is a Philips 420ST that has been steadily upgraded from the standard model. An image intensifier has been attached to the column below the plate camera to aid high resolution work and to make it easier to focus on superlattices of bubbles. The image intensifier can provide a further twenty fold magnification and improve the contrast of the final image displayed on a TV monitor.

A wide-angle camera is positioned above the viewing screen. This camera collects its image over a wider angle than the image intensifier so it is especially useful in dark field and weak beam work in which low intensities occur. In these situations it is difficult to focus accurately using the faint image on the viewing screen or the image produced by the image intensifier. Images are fainter with the tungsten filaments used than with $\mathrm{LaB}_{6}$ filaments. The camera is normally out of the path of the electrons travelling down the column but it can be brought in by a mechanism that is driven by compressed air. The image magnification is greater than that for the normal viewing screen by a factor of eight. On-line images from both cameras can be fed via co-axial cable into either a computer system (described in section 4.4) or a video tape recorder.

When tilting a specimen with the super twin lenses in the TEM it was usually possible to reach one major pole and several minor ones only. To obtain accurate foil normals, glide cylinder axes and dislocation line directions etc. analysis covering a large range of beam directions is essential. Therefore the super twin lenses were replaced by a set of twin pole-pieces. This gave an increase in the available tilt from approximately $\pm 12^{\circ}$ to $\pm 50^{\circ}$. This change enabled several major poles to be reached that are widely separated in the standard stereographic triangle. The super twin lens has a $\mathrm{C}_{\mathrm{s}}$ of $1.2 \mathrm{~mm}$ that gave a nominal point to point resolution of $3 \AA$, (using as the resolution criterion the position of the first zero in the contrast transfer function at Scherzer focus). The twin pole pieces have a $\mathrm{C}_{\mathrm{s}}$ 
of $1.4 \mathrm{~mm}$, which gives a point to point resolution of $3.4 \AA$. The loss in resolution is not important because high-resolution techniques were not really appropriate to the structures studied in this thesis (section 4.3).

A low dose unit is also available. It can move the incident electron beam away from the region being examined to another area or away from the specimen altogether and is useful in keeping the irradiation dose to a minimum. This is useful when a large series of photos of a particular region must be taken at different beam directions with various diffracting vectors. Most of the tilting and focussing can be done on a region near the area containing the structure of interest if the foil is flat enough to allow both regions to be simultaneously at strong two-beam conditions.

\subsection{Specimen Preparation}

\subsubsection{Preparation of the Target}

Targets for ion-implantation are prepared from foil of $99.99 \%$ purity by cutting to a rectangular shape $35 \mathrm{~mm}$ by $10 \mathrm{~mm}$. To remove surface roughness the targets are mechanically polished using diamond paste. As the polishing progresses, the size of the diamond paste used is decreased down through the grades ( $6 \mu \mathrm{m}, 3 \mu \mathrm{m}$ and finally $1 \mu \mathrm{m})$ to remove surface roughness on an increasingly fine scale.

A smooth crystalline target with little surface contamination is required if good bubble superlattices are to be formed during the irradiation. Polycrystalline specimens are grown using a furnace shown in figure 4.1 (T. Corfiatis [170]). Annealing is done by holding the foils at a temperature of approximately $900^{\circ} \mathrm{C}$ for a few hours. The specimens are then cooled overnight with a steady temperature gradient back to room temperature. This causes grains to grow to a size 0.1 to $1 \mathrm{~mm}$ across. Bath electropolishing of the foils in orthophosphoric acid is then used to produce a clean, highly polished surface finish. 


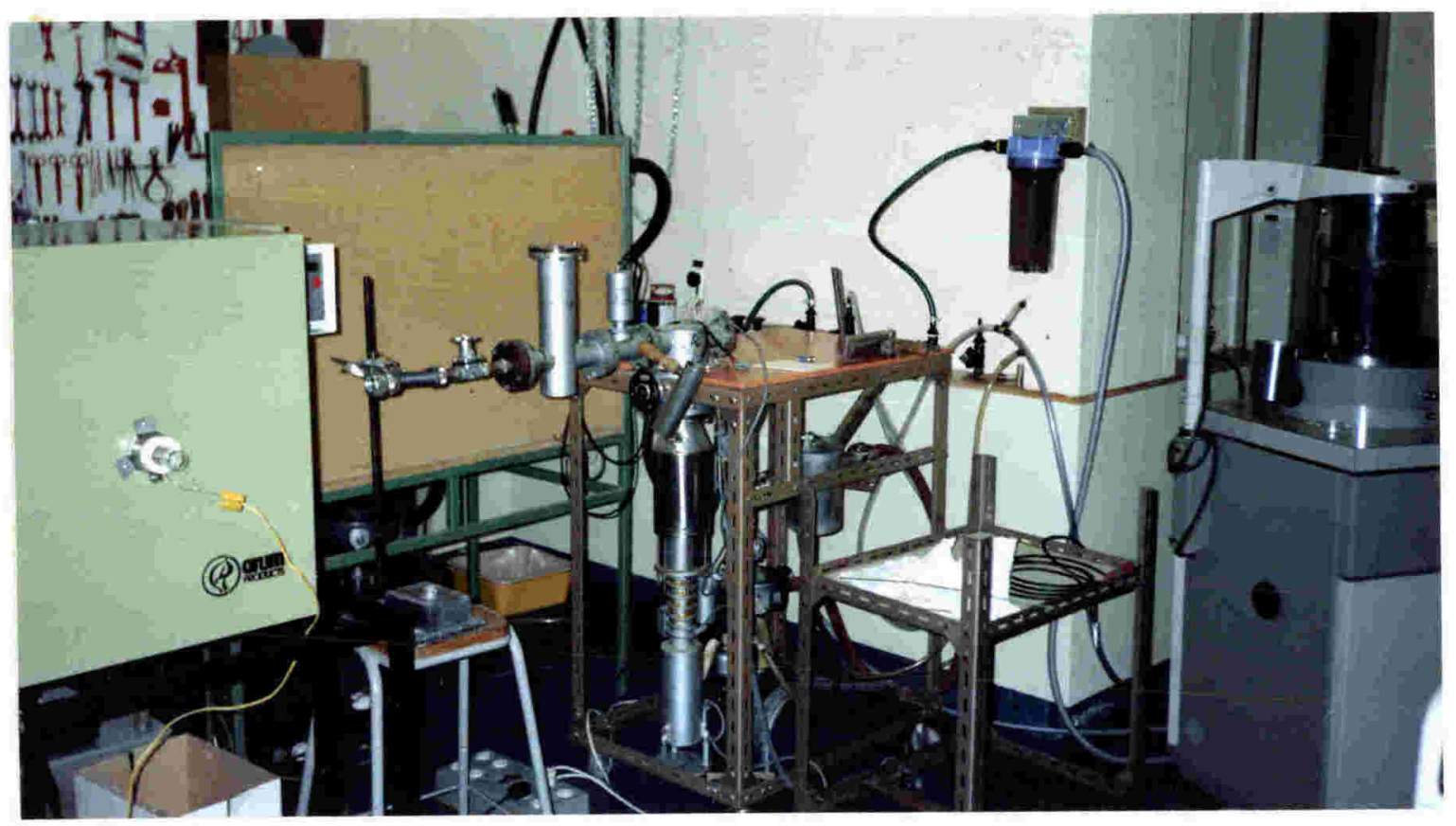

Figure 4.1: The furnace and vacuum apparatus used for annealing metal foils. The foils are laid on a strip that is chemically inert and has a high melting point. The metal support is then inserted into a quartz tube that is clamped to the vacuum equipment and protrudes into the furnace. The tube is first roughed out with a rotary vacuum pump. A diffusion pump is then used to improve the vacuum further.

\subsubsection{Description of the Accelerator and the Target Irradiation}

Once the foils have been mechanically polished, annealed and bath electropolished they are inserted into the accelerator to be implanted with ions. The accelerator is a PN-400 Van de Graaff accelerator modified so it can provide $1 \mathrm{MeV}$ ions, but which is normally used to provide approximately $160 \mathrm{keV}$ helium or deuterium ions. The current hitting the target is not stable when the accelerator is run below about $100 \mathrm{kV}$. Photographs of the accelerator are shown in Figure 4.2. The accelerator and beam transport system were designed for light ion irradiations. Heavy ion irradiations cannot be performed because of limitations in the accelerator and beam transport components. Light ion irradiations are done by ionising helium, hydrogen or deuterium gas in the ion source.

The charge sprayed onto a moving belt at the low voltage end of the accelerator accumulates at the high voltage terminal. To avoid dielectric breakdown occuring the high voltage terminal of the tank is insulated from its surroundings. To achieve this the tank contains a mixture of insulating gases at a pressure of 100 psi consisting of sulphur 


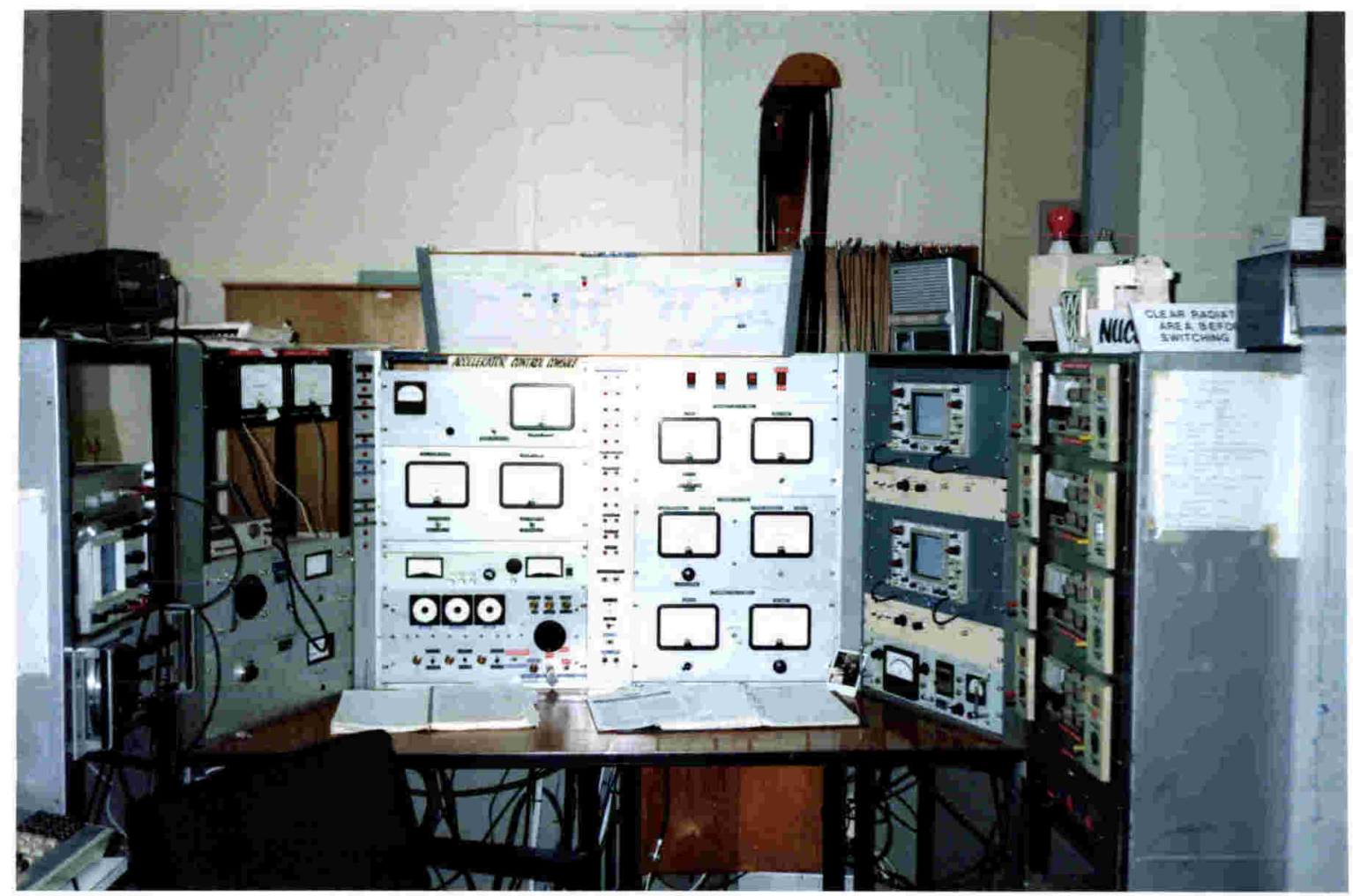

(a)

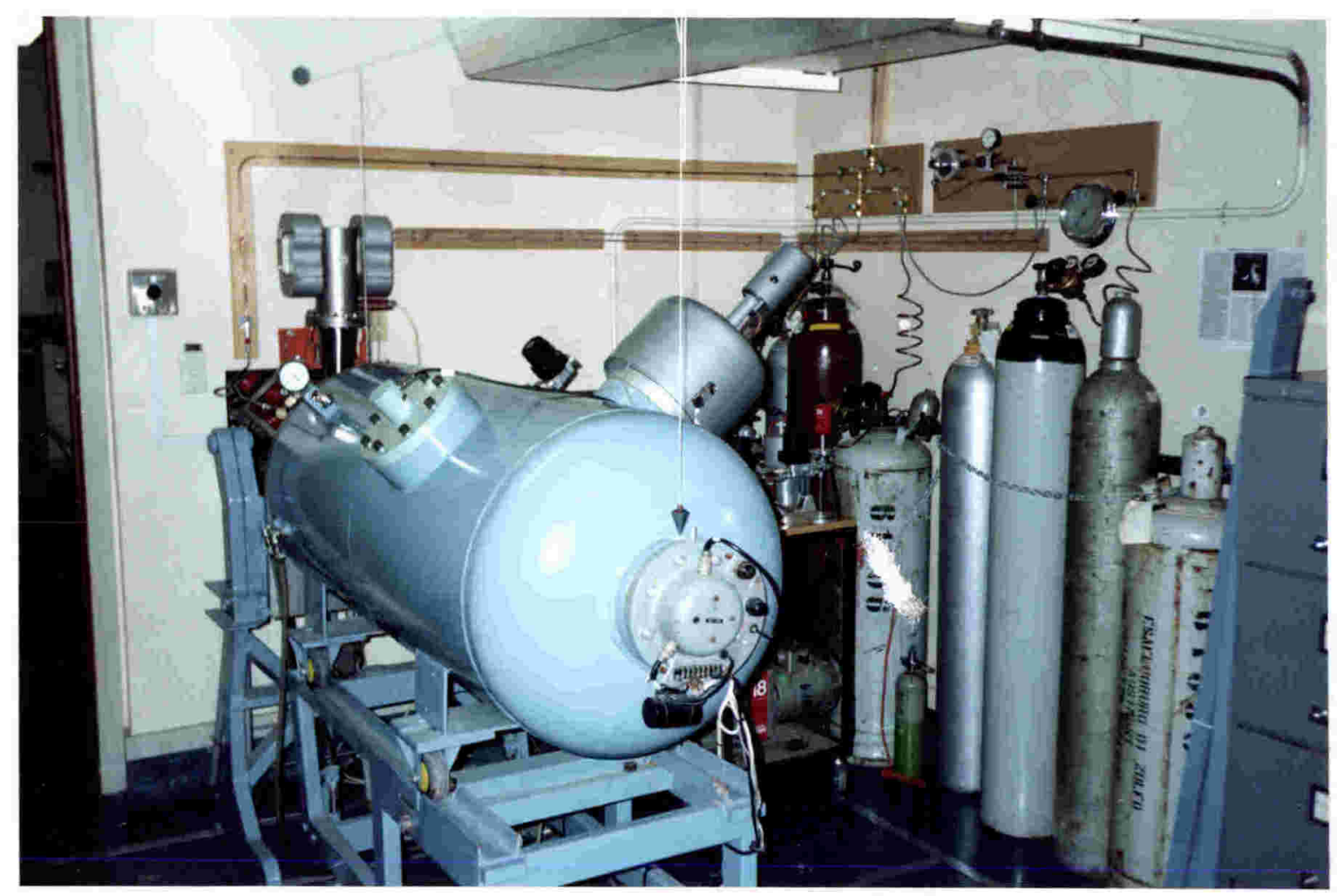

Figure 4.2 (b) 


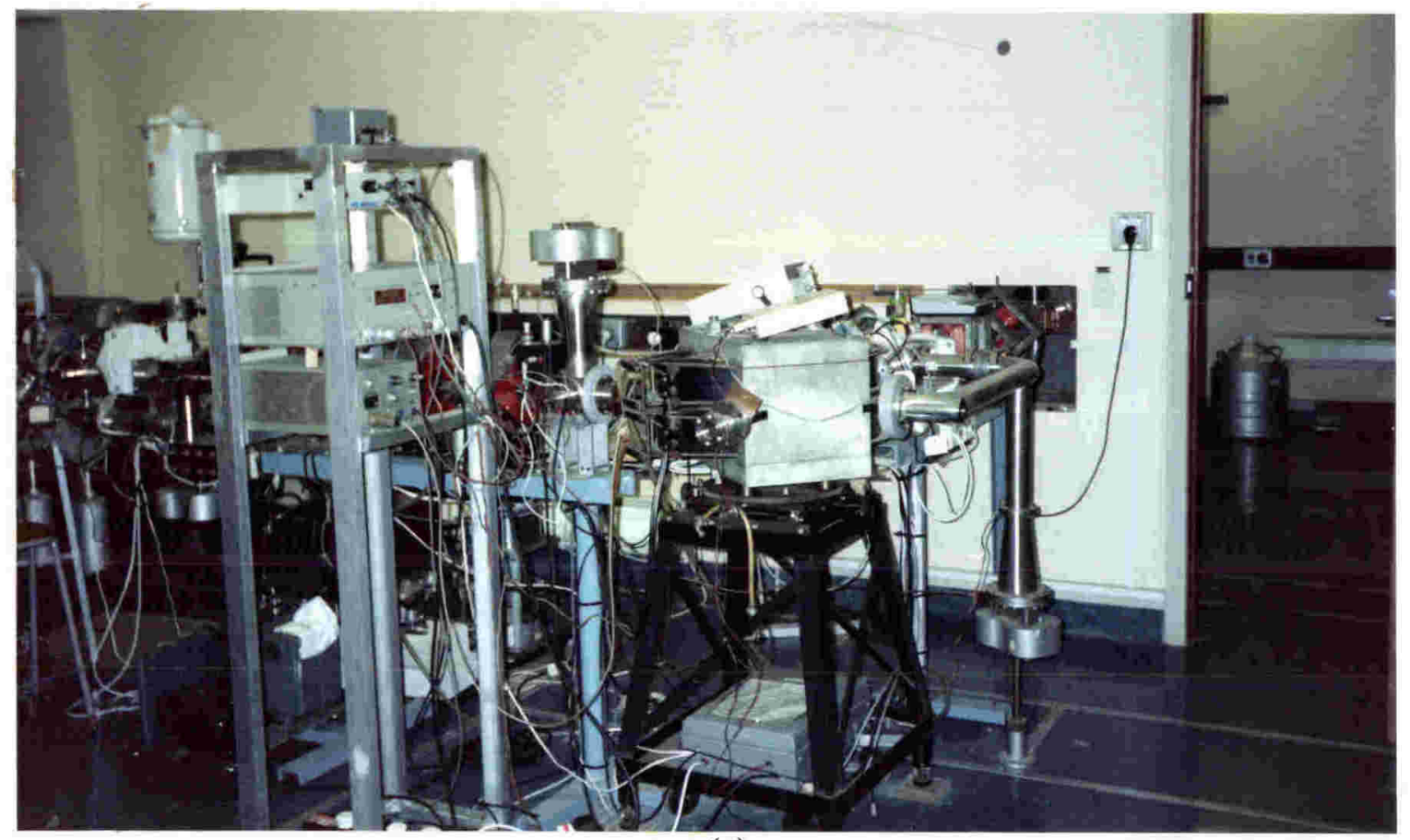

(c)

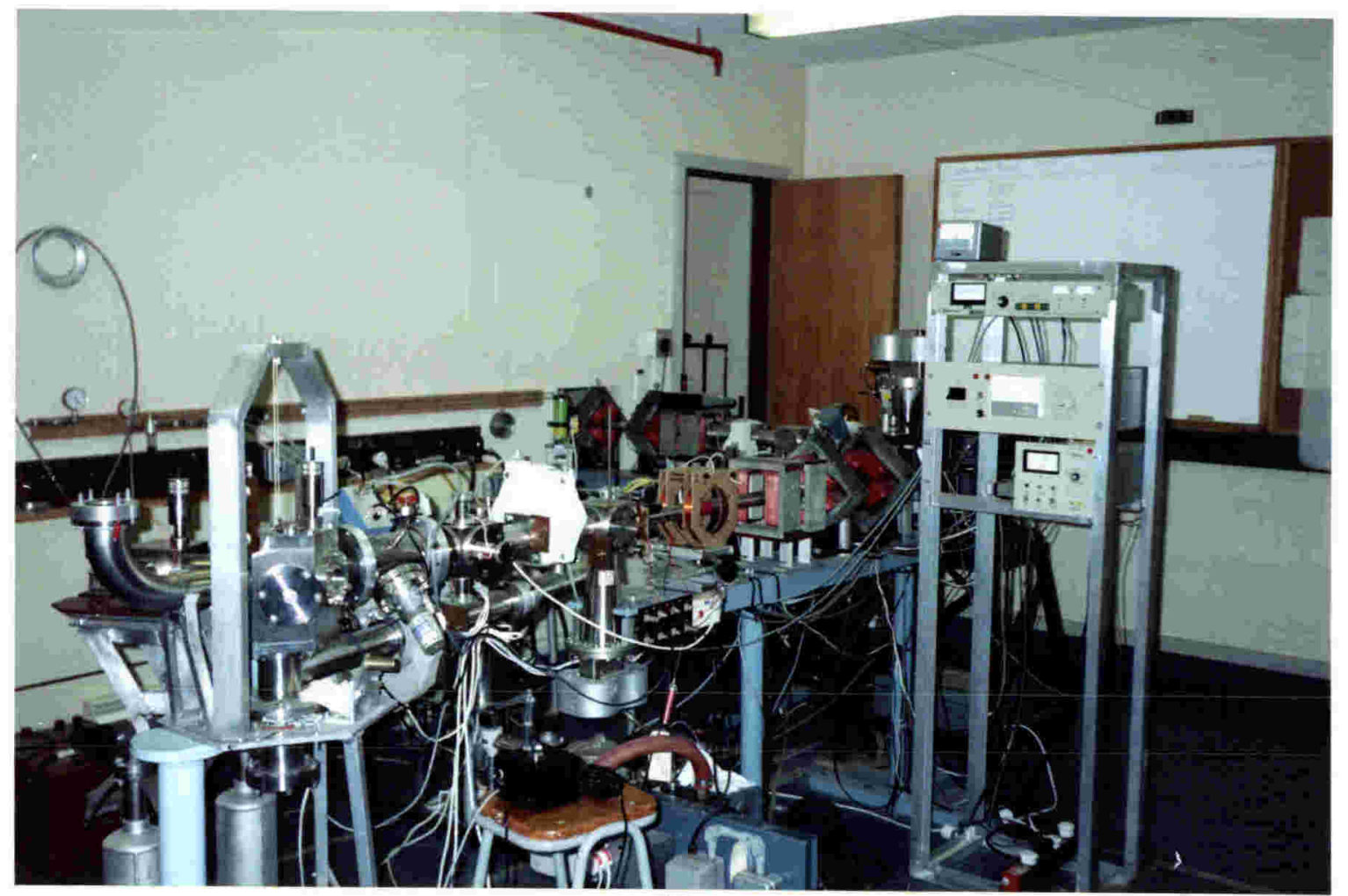

(d)

Figure 4.2: (a) Accelerator control console. The currents to the magnets on the beam line are controlled from this console. The oscilloscopes show the horizontal and vertical beam profiles before and after the energy analysing magnet. The ammeters display the currents: along the belt, down the beam line, drawn off by the corona, and hitting the sides of the slit boxes and the target. (b) The tank room. The motor driven corona assembly emerges from the top right of the pressure vessel. (c) analysing magnet and beam line, (d) quadrupole focussing magnets, steering magnets and target chamber. 
hexaflouride, nitrogen and carbon dioxide. The exact proportions of the gas mixture used depends on whether or not high voltage work is to be done. The energy of the accelerated ions is primarily set by the amount of belt charge transferred to the high voltage terminal. The energy of the accelerated ions is partially determined by the adjustable position of the corona points that remove charge from the high voltage terminal of the accelerator. For example moving the corona points in towards the high voltage terminal of the tank decreases the amount of charge allowed to build up there. The potential will then drop and the ions will be accelerated to a lower final energy. The terminal voltage, or equivalently the energy of the ions leaving the tank, can be estimated by multiplying the effective resistance of the column resistors by the column current.

It is desirable for the operator to know how much current is going down the beam line. Besides the column current, the corona current and the belt-charge current are also monitored. The current going down the beam line is then equal to the belt-charge current minus the corona current minus the column current.

Vacuum valves can be used to isolate the accelerator from the rest of the system. This causes the initial gas pressure in the ion source to be raised and the ion source to strike, i.e., to begin to ionise the gas. A vacuum is maintained and monitored in the system with a set of ion pumps and a rotary backed diffusion pump. Three pneumatically driven vacuum valves allow the tank, the beam line and the target chamber to be isolated from one another.

There are several controls that adjust the current coming out of the source. The amount of gas being ionised depends on how much gas is allowed to build up before being pumped away or accelerated down the beam line. The "gas " control adjusts how much gas is bled from the small gas bottles inside the accelerator into the radio frequency ion source. The pressure inside the gas bottles will also affect the current. The "beam " control adjusts a small initial potential applied to direct the ions down the beam. The "focus " control adjusts the focus of an electrostatic lens in the tank.

Isolating the target chamber by using the appropriate vacuum valves enables specimens to be inserted and removed without losing the vacuum in the rest of the system. Once the target is inserted, and the target chamber evacuated, the target can be heated to outgas it before the irradiation is done. The target heating is done by passing a current 
through a heating coil in thermal contact with the specimen. The temperature is monitored by a thermocouple attached to the target block.

The ions that are accelerated down the beam line are passed through a series of quadrupole magnets (for beam focussing), steering magnets and an analysing magnet (for selecting the correct ion species) before striking the metal foil target. The currents in these electromagnets determine the strengths of the respective magnetic fields. As the ions travel down the beam line they also pass through a sequence of beam profilers, slit boxes, vacuum valves and Helmholtz coils (for scanning the beam).

The energy and type (i.e., the charge to mass ratio) of the ions that strike the target is selected by the analysing magnet that steers the beam through $90^{\circ}$. The strength of the magnetic field in the analysing magnet is measured using a Hall probe. Plots of ion-energy vs. magnetic field can be used to calibrate the magnetic field in the analysing magnet against energy.

The ion beam intensity at the target needs to be sufficiently high to complete the ion irradiations within a reasonable time (i.e., two to five hours). This requires an efficient beam transport system between the ion source and the target (which is some five metres away). Magnetic lenses are distributed along the beam line. Even with these magnets to do the steering and focussing further mechanical alignment of the magnets and the accelerator is sometimes required to achieve the maximum beam intensity. The focussing is done before and after the analysing magnet by two pairs of magnetic quadrupole lenses (Callaghan [171]). One magnet in each pair squeezes the beam vertically and the other horizontally. It is also necessary to steer the ions down the beam line between these magnets and another two sets of dipole magnets, one before and one after the analysing magnet are for this purpose. The north poles of each magnet in a pair are rotated by $90^{\circ}$ relative to each other so that one magnet steers the beam vertically, the other horizontally. The focussing and steering is done by adjusting the current through these lenses.

The position and state of focus of the ion beam is monitored by means of two NEC beam profiling units, one located before the analysing magnet and one after. These units sample the beam profile by continually passing a wire through the beam. The axis of rotation of the wire is perpendicular to the direction the ions are moving in and is at $45^{\circ}$ to the vertical and horizontal directions. The wire has a $45^{\circ}$ bend in it, with the end of the wire 
the vertical and horizontal directions. The wire has a $45^{\circ}$ bend in it, with the end of the wire only passing through the beam. This means that once every rotation the wire sweeps out the beam profile vertically and then horizontally. The ions strike the wire and cause secondary electrons to be emitted. The secondary electrons are collected by the electronics of the beam profiling unit. The variation of voltage as a function of time, displayed on an oscilloscope, provides an indicator of the beam profile (ion beam current as a function of position).

Slits (acting as collimators) are positioned in the beam line immediately after the analysing magnet (these slits are called the image slits) and before the target chamber (the target slits). The current hitting the slits is monitored to allow adjustment of the magnet currents, the tank voltage, and the beam position. The image slits provide an error signal (difference between the current hitting the left and right slits) which is related to the energy of the ions passing through the analysing magnet. This error signal is fed back to the accelerator to stabilise the accelerating voltage [54 and 172].

The accumulated ion dose to which the target is subjected will determine the structure obtained. The current hitting the target is fed into an integrator unit to monitor the total number of ions striking the target during an irradiation. How large a region of the specimen is irradiated is determined by the separation of the slits and by a pair of Helmholtz coils. Triangular wave trains can be applied to the Helmholtz coils to scan the beam vertically and horizontally over the target. The scan system provides an approximately uniform dose across the specimen surface. The beam is scanned so that the target slit edges intercept some of the current which would otherwise hit the target. There are four edges to the target slits, two that are horizontal and two that are vertical. If some current is hitting all four edges of the target slits the area of the specimen that is being irradiated is known. In practice the uncertainties associated with the beam profile, the scanning process and the method used to measure the current lead to uncertainties in determining the ion dose received by the target. Also the area of the target that has been irradiated is uncertain. The different types of structures that can be obtained are discussed in chapter seven. 


\subsubsection{Quenching}

\subsubsection{Introduction}

Quenching is a technique in which specimens are cooled very quickly from an atmosphere of gas at elevated temperatures to room temperature by immersion in a coolant such as distilled water. The advantage of specimens formed from quenching is that the density of bubbles and dislocations is lower than that characteristic of ion-implanted metals. Quenched specimens are much easier to examine in the TEM to gain experience in applying the image matching techniques to dislocation loops before extending the work to ionimplanted metals. Quenching produces a concentration of gas atoms in the metal at room temperature that is much higher than the equilibrium concentration. This occurs because the equilibrium concentration of gas atoms at elevated temperatures is much higher than the equilibrium concentration at room temperature. During the rapid cooling of the specimen the gas atoms try to escape from the metal but their mobility is significantly reduced before they can reach the surface and escape from the foil. Instead the atoms are trapped in the metal and it is energetically favourable for the trapped atoms to clump together to form gas bubbles. The gas bubbles as they grow displace the surrounding metal atoms into interstitial dislocation loops. Vacancy dislocation loops can form by the collapse of discs or platelets of vacancies.

The heating of the metal in particular causes the amount of hydrogen in the matrix to be increased dramatically over the normal equilibrium concentrations at room temperature and pressure. The concentrations of hydrogen in copper obey the following equation

$$
\frac{\mathrm{c}_{\mathrm{H}}}{\mathrm{c}_{0}}=\left(\frac{\mathrm{p}}{\mathrm{p}_{0}}\right)^{\frac{1}{2}} \exp \left(\frac{-\mathrm{E}_{\mathrm{s}}}{\mathrm{kT}}\right)
$$

where $c_{H}$ is the equilibrium concentration of hydrogen at a temperature $T, E_{S}$ is the free enthaply of solution, $\mathrm{k}$ is Boltzmann's constant, $\mathrm{p}$ the gas pressure and $\mathrm{c}_{0}$ is the equilibrium concentration of hydrogen at the melting point of copper at one atmosphere of pressure $\left(\mathrm{p}_{0}\right)$. 


\subsubsection{The Design of the Quenching Cell}

In this section the design and operation of the quenching cell is discussed. The initial attempts to quench hydrogen gas into copper, involved admitting gas into a cell turned from a solid piece of numonic steel, then heating the cell to about $900-950^{\circ} \mathrm{C}$. The cooling was done by raising a bucket of water that pushed the furnace up leaving the stationary cell to be immersed in the water. This achieved only limited success probably because the cooling rate of the copper specimens was too slow. The rate was low because the entire cell had to be cooled down.

A large amount of cuprous oxide was formed on some copper specimens during the heating process so a rotary vacuum pump was added to pump the air out of the cell before the hydrogen gas was admitted. This solved the oxidation problem but not the problem with the slow rate of cooling.

Another problem was that the extraction of the thin specimens from the cell after quenching often resulted in the specimens being distorted. Attempts to use thicker specimens to solve this problem failed because the specimens fractured as they cooled nonuniformly. When trying to electropolish fractured specimens the acid flows down through the cracks, widening them until a hole through the specimen is produced. The hole, which causes the premature shut-off of the electropolishing unit (section 4.2.4), has edges which are too thick to allow satisfactory examination using TEM.

The limited success of this initial approach, and variations on it, made it necessary to redesign the system. Figures 4.3 to 4.5 show the new design based on a design of $\mathrm{W}$. Wampler [173-175]. Most steels are not resistant to hydrogen embrittlement. In fact the numonic cell used in the initial approach failed several times. Holes could form through the side of the cell; the top of the cell could shear off at the thread where a cap is screwed down to seal the cell. Fused quartz is resistant to hydrogen embrittlement and so the high pressure fittings and vacuum fittings used with the numonic cell were reconnected via stainless steel components to a quartz tube of $10 \mathrm{~mm}$ inside diameter and $16 \mathrm{~mm}$ outside diameter. The stainless steel components are outside the furnace and so are not embrittled to the same extent as the numonic cell. The stainless steel fittings were araldited to the quartz. 

of up to about 16 atmospheres.

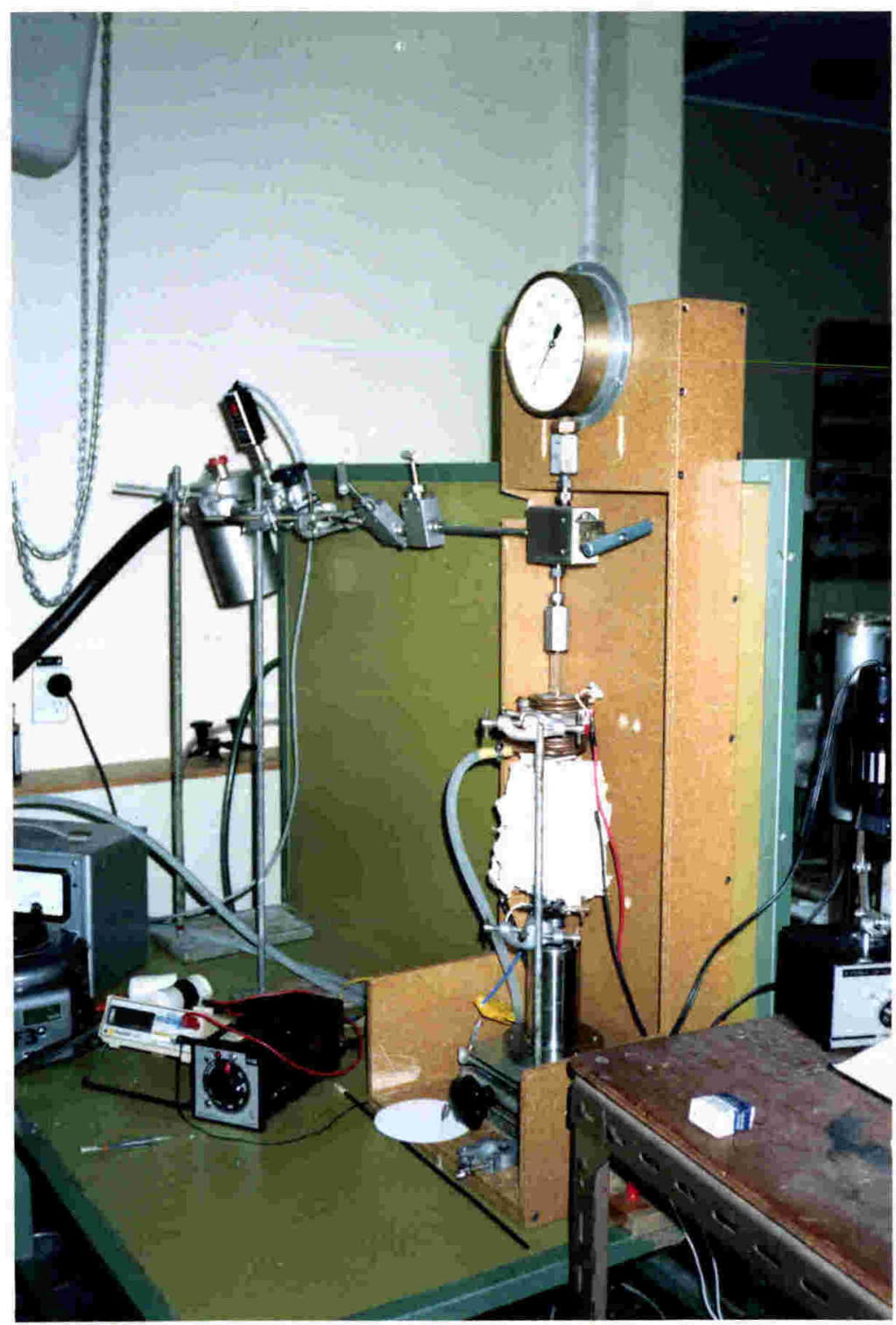

Figure 4.3: The quenching apparatus. The wood section supports the pressure gauge. The quartz tube is suspended from this gauge and supported underneath by the container of distilled water. The specimen holder is resting against the bottom of the assembly on a circle of filter paper. On the left hand side of the photo is the vacuum apparatus, the variac for the furnace and the temperature controller. During operation the central high pressure valve is connected to a gas cylinder via steel tubing that can tolerate high pressures. A steel safety cage is also screwed onto the wood box. The apparatus is described in detail in the text.

The system can be pressurised with gases other than hydrogen such as nitrogen and argon. Because of the inflammability of hydrogen and its proximity to a furnace the quartz tube and furnace section of the system was placed in a steel mesh cage. If an explosion 


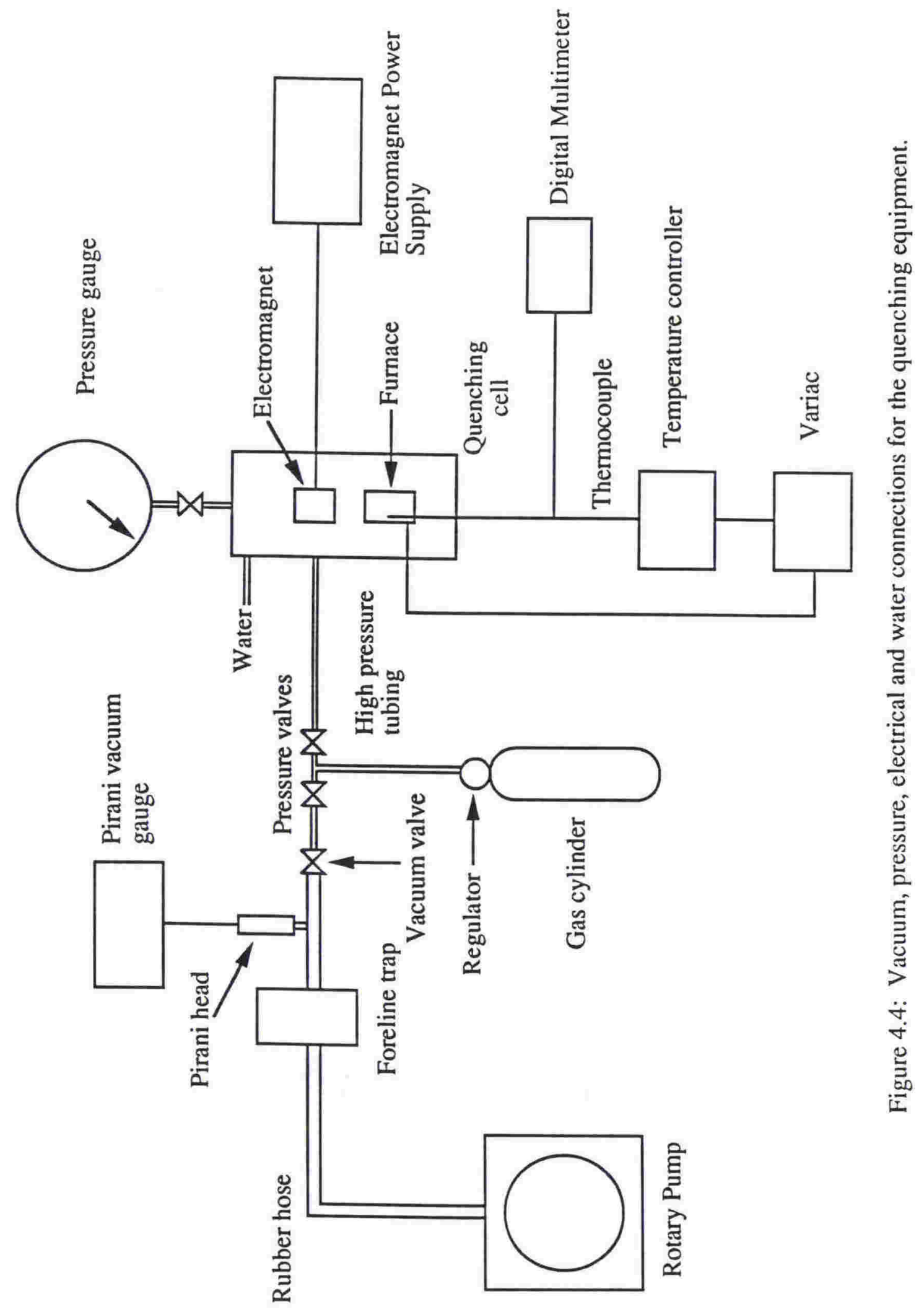




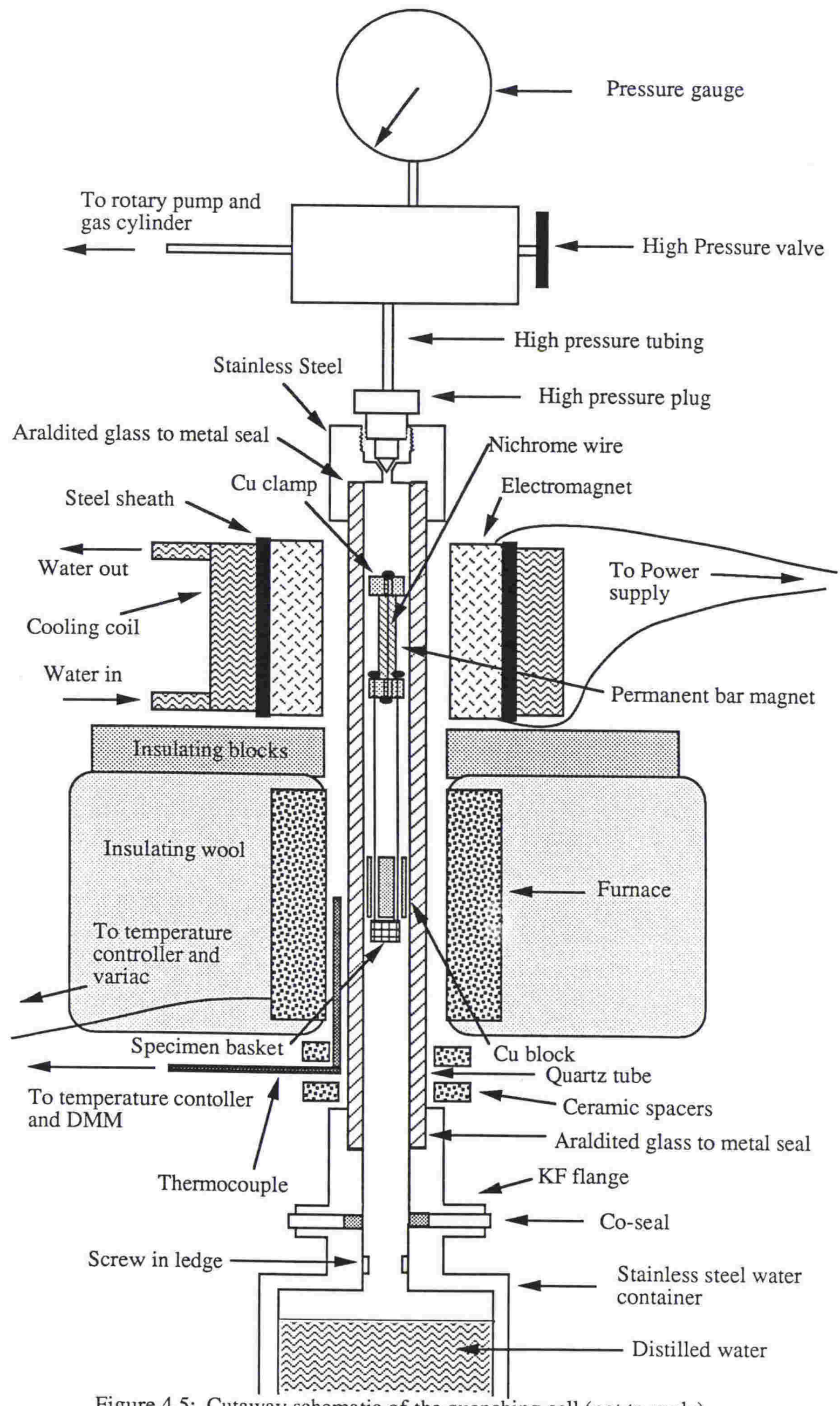

Figure 4.5: Cutaway schematic of the quenching cell (not to scale). 
occurred the cage is designed to deform and so absorb some of the energy of the explosion. The cage would also prevent shards of glass etc. from flying across the room.

The specimens of $3 \mathrm{~mm}$ diameter are loaded into a nichrome basket at the bottom of the specimen holder that is attached via more nichrome wire to a magnet. The specimens are separated with pieces of nichrome wire to stop them from sticking together when heated. The wire spacers also allow both sides of the specimens to be exposed to the gas.

The specimens are loaded into the system by turning on the electromagnet and sliding the specimen holder assembly up into the magnetic field with a rod. The rod is padded out with tissue to fit snugly the inside of the quartz tube. A hole was drilled through the top of the table on which the quenching apparatus sat to allow the long rod to be inserted into the tube. When the magnet on top of the specimen assembly is held by the magnetic field of the electromagnet the steel rod is withdrawn. The length of the specimen holder is such that when its magnet is held inside the electromagnet the bottom of the specimen holder will lie in the centre of the furnace.

The electromagnet has approximately 800 turns of copper wire wound onto a perspex former. The magnetic field inside this coil was enhanced by placing it in snug fitting steel cylinder. The iron in the steel has a higher magnetic permeability than air. This causes the density of field lines to increase through the same region near the magnet compared to the field strength when the steel sheath was not there by deceasing the magnetic reluctance of the flux return path.

The specimens must remain held even if the quartz tube is jostled when valves are turned and specimens loaded. During a run the position of the specimen holder can be viewed in the quartz tube to provide a check that a premature quench has not occurred. (This would not be possible if a steel cylinder was to replace the quartz tube.) To hold the specimen holder assembly securely in place, a current of approximately 1.8 to $2.0 \mathrm{~A}$ (DC) is passed through the coils of the electromagnet. This causes the magnet assembly to heat up. Also as it is heated the resistance of the copper wire increases. For this reason the voltage applied to the electromagnet usually had to be slightly increased during a run to maintain the current needed to hold the specimens in place. The perspex former partially melted when the magnet was first used, even though the nearby furnace was turned off. This problem 
was solved by cooling the coil and magnet by passing water through a coil of copper tubing soldered to the surrounding steel sheath. Although the electromagnet still heats up to about $80^{\circ} \mathrm{C}$ during a 30 minute run this is not enough to melt the perspex.

The furnace is a coil of wire wrapped around a former that can be slid over the quartz tube. Insulating glass fibre is tied around the furnace to decrease the thermal load on the furnace. The tying is done with glass fibre "string" that has a wire core. This "string" was also used to tie a thermocouple in position inside the furnace but outside the quartz tube. The temperature is monitored by measuring the Emf across the chromel-alumel thermocouple with a digital multimeter. A table in the Handbook of Chemistry and Physics was used to convert this voltage into a temperature. A temperature controller can be used to control the power supplied to the furnace windings.

After the specimen holder has been inserted then the stainless steel cylinder holding the water (into which the specimens are to be quenched) is raised using a retort stand. The height of the retort stand is adjusted so that the cylinder meets the stainless steel joint at the bottom of the quartz tube. This joint is sealed with a co-seal and kon-flat clamp.

When the system is closed with the co-seal, the quartz tube can become stressed and may break. To avoid breakages the quartz tube had to be clamped at the top and bottom steel joints to which it is araldited. The breakages were especially a problem in an earlier design in which considerable torque had to be exerted on a cajon valve to tighten it. The initial attempts to seal the system involved a copper gasket and cajon valve. However, the gas pressure could not be held reliably with this arrangement. With the co-seal design the wing nut on the clamp is tightened by hand so the quartz tube is not unduly stressed. If a crack occurs in the part of the quartz tube that is araldited to the stainless steel, it is not visible. The presence of a crack is suspected if a sufficiently low pressure cannot be attained. The quartz tube can be replaced by heating the araldite with a Bunsen burner.

After the specimen holder has been inserted and the system sealed by the co-seal the air is evacuated from the quartz tube via a rotary pump and foreline trap. The distilled water remains in the container at the bottom of the system even when pumped on by the rotary pump although water vapour does limit the lowest pressure that can be obtained. A typical vacuum is one of about $150 \pm 20 \mu \mathrm{m}$ of $\mathrm{Hg}$. The vacuum is measured with a pirani gauge. 
The furnace is switched on when a sufficiently low pressure is reached and the specimens are heated to $1000^{\circ} \mathrm{C}$ in about 25 to 30 minutes with a variac set to 60 volts. The voltage is reduced to about 55 volts to maintain this temperature.

A system of valves allows the vacuum system components that cannot withstand high pressures to be isolated. Other valves bring in a pressure gauge which, if not measuring the pressure the specimens actually experience, at least indicates whether there is a leak of gas. The system is then pressurised.

The suspended specimens are held under pressure inside the furnace at $1000^{\circ} \mathrm{C}$ for approximately 5 minutes. Small pressure leaks can be tolerated if gas is admitted occasionally to maintain the system pressure. If the windows are open during the 5 minute holding time at $1000{ }^{\circ} \mathrm{C}$ when hydrogen is being used and no sparks occur in the laboratory, the gas diffuses quickly enough to avoid explosions (although an occasional popping sound may be heard). The specimens are dropped by switching off the electromagnet current: they slide through the quartz tube into the distilled water.

The pressure is released from the system by undoing some of the high pressure tubing (steel tubing that has a narrow bore and thick walls) connected to the vacuum system. This leaves a high pressure valve with one side at atmospheric pressure and the other side at up to approximately 16 atmospheres. A nearby window is opened to prevent the gas that is to be released building up in the room. By opening the valve the system pressure is released and the gas escapes into the laboratory.

The viton co-seal allows the system to be broken between the top of the stainless steel container of distilled water and the bottom fitting connected to the quartz tube. This allows the extraction of the specimens from the distilled water.

Initially the specimens were suspended in a nichrome basket. They can remain in the nichrome basket when it hits the water and can be readily lifted out. However, they may come to rest on the bottom of the steel cylinder. They must then be flushed out with the distilled water. Light specimens are hardest to flush out because they may float on the water. Flushing out the specimens is a time consuming process. To avoid having to do this the nichrome basket was replaced with a basket constructed from stainless steel mesh of 
approximately $2 \mathrm{~mm}$ spacing. A lid is latched to the top of the basket to prevent the specimens being knocked out when the basket hits the water.

\subsubsection{Quenching Results}

It is possible that hydrogen bubbles in the specimen could result from dissociation of the water caused by a hot specimen coming into contact with it. However, attempts to obtain bubbles that have punched out loops by rapid quenches into water from air failed. The quench from air also gave very badly oxidised specimens that were difficult to electropolish. Similarly quenches from vacuum with no gas admitted into the system failed. This seems to cast doubt on the idea that the dissociation of the water is a significant factor in obtaining bubbles.

To find several rows of loops emanating from the same bubble several axes of glide cylinders need to lie in the foil. If the axes intersect the foil surface the dislocation loops punched out by the bubble can easily escape. If the distance along a glide cylinder that intersects the foil surface, from the bubble to the foil surface, is large then the loops come to rest inside the foil. Experimentally, bubbles punching out a row of loops in just one direction are found in thinner regions of the metal. Bubbles punching out several rows of bubbles however are more commonly found in thicker regions. In these regions, because longer exposure times (about 10 - 30 seconds) are needed photography is more demanding. It is not unusual in taking a series of photos for a range of beam directions with these long exposure times to find several of the photos are blurred. The blurring makes image matching impossible. The use of foils with [111] surface normals would be required to obtain several rows that have [110] directions that are perpendicular to [111] in thin regions. In practice it is necessary to produce many polycrystalline specimens with different foil normals to obtain a grain with a [111] foil normal. An alternative that was not investigated might have been to use the image processing system to grab images directly off the cameras or to use $\mathrm{LaB}_{6}$ filament to obtain shorter exposure times.

Table 4.1 summarises the results of quenching six different metals from an atmosphere of argon or hydrogen. Four or more specimens of each metal were quenched in 
each gas at a pressure from 60 to 135 psi. These metals were selected because it was possible to obtain reasonably large thin regions using the electropolishing system described in section 4.2.4. It was hoped to study the variation in loop spacing, the size and density of the bubbles and loops as the shear modulus varied between the metals. This proved to be impractical and good results were obtained in quenched copper only and so more than 100 copper specimens were quenched.

\begin{tabular}{|c|c|c|}
\hline Metal & $\mathrm{Ar}$ & $\mathrm{H}_{2}$ \\
\hline $\mathrm{Al}$ & some dislocation loops & $\begin{array}{l}\text { lots of dislocation loops but } \\
\text { no rows }\end{array}$ \\
\hline $\mathrm{Cu}$ & crossed rows of loops & $\begin{array}{l}\text { some rows, lots of isolated } \\
\text { loops, material fractures and } \\
\text { the usual electropolishing } \\
\text { conditions fail }\end{array}$ \\
\hline Mo & loops but no rows & loops but no rows \\
\hline $\mathrm{Ni}$ & loops but no rows & loops but no rows \\
\hline $\mathrm{Ti}$ & $\begin{array}{l}\text { very dirty oxidised } \\
\text { specimens, hard to see any } \\
\text { dislocations against this } \\
\text { background }\end{array}$ & $\begin{array}{l}\text { can no longer put a hole in } \\
\text { the material, a white oxide } \\
\text { forms on one side of the } \\
\text { specimen during polishing }\end{array}$ \\
\hline V & $\begin{array}{l}\text { blackened and badly } \\
\text { oxidised, } 3 \mathrm{~mm} \text { discs } \\
\text { disintegrate during the } \\
\text { quench }\end{array}$ & $\begin{array}{l}\text { blackened and badly } \\
\text { oxidised, } 3 \mathrm{~mm} \text { discs } \\
\text { disintegrate during the } \\
\text { quench }\end{array}$ \\
\hline
\end{tabular}

Table 4.1 Quenching results from atmospheres of argon and hydrogen.

\subsubsection{Punching, Jet Electropolishing and Ion Beam Thinning}

Once the foils have been irradiated and removed from the accelerator they are punched out into $3 \mathrm{~mm}$ discs. Specimens are also punched into $3 \mathrm{~mm}$ discs before being inserted into the quenching system. During this project a punch that had been constructed 
in the mechanical workshop was replaced with a commercial Gatan punch that gave flatter specimens. After punching, the discs must be thinned to electron transparency by jet electropolishing or ion beam thinning before insertion into the TEM.

Two systems were used to perforate specimens using jet electropolishing. The old system sprayed the acid onto a specimen through a glass spout using compressed air (A.L. Malcolm [45]). In this old system the specimen was held at the desired voltage by one end of an electrode that is attached to a pair of tweezers holding the specimen in the acid jet, the other end of the electrode is connected to a power supply. A problem with the old system was the difficulty of judging when the perforation had occurred. A strong light is shone from behind the specimen. Electropolishing was stopped when a hole was detected. The need to realign the light and binocular each time the specimen is replaced slowed down the thinning process.

When looking at irradiated surfaces where the damage structure occurs in a thin layer below the irradiated surface it is necessary to thin the specimen from the back surface only. A problem with the old system was the need to use vinament and the use of compressed air. The vinament was painted onto the specimen edges so that the acid did not erode both sides of the specimen. The vinament was removed by immersing the specimen in acetone. Acetone can degrade the TEM images obscuring the dislocations and bubbles. If the acetone did not remove all the vinament it is necessary to peel off the protective vinament layer. The peeling can bend the specimen. The compressed air valve had a sensitive adjustment. By undoing it too much, or turning it the wrong way when trying to shut the jet of acid down, it could result in a fine spray of acid on the users face or lab coat.

Specimen thinning to perforation was done mainly using a new Tenupol system that thins the discs electrochemically (figure 4.6). The new unit has a LED light source on one side of the specimen and a photocell on the other side to detect when perforation has occurred. When the light emitted by the LED in the infrared is detected by the photocell the unit is automatically switched off. The amount of light that must be detected for this to occur is specified by a "sensitivity " knob. For copper $50 \%$ by volume orthophosphoric acid is used. The acid transmits IR light when the solution is new, i.e., it is transparent in the visible. However, when the solution has been well used it becomes a light blue colour 
and it does not transmit IR light as efficiently as a new solution. The acid is sprayed onto the specimen by a pumping system connected to two jets, one for either side of the specimen. The pumping speed, or "flowrate", can be easily varied by adjusting the current to the pump motor.

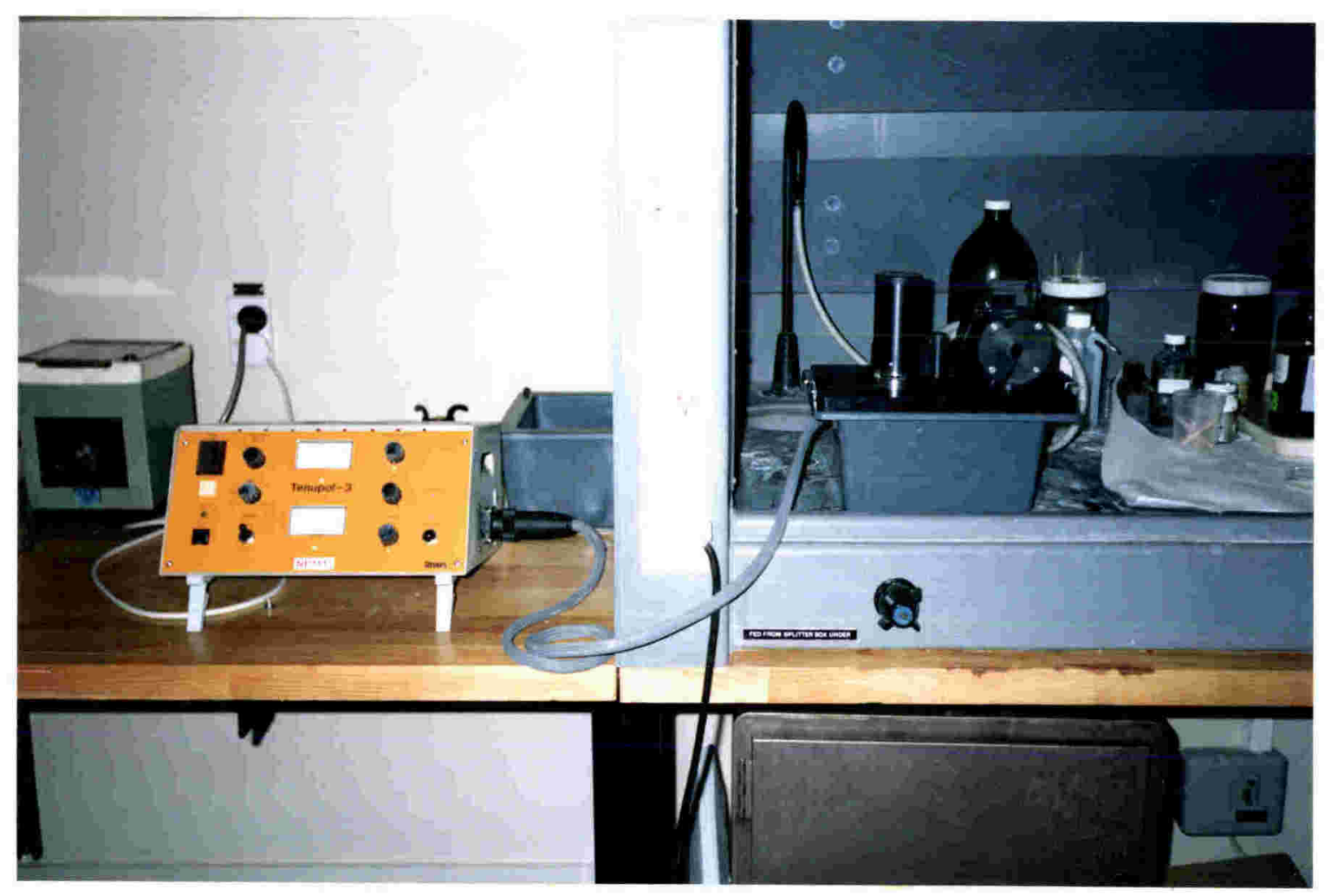

Figure 4.6: The Tenupol apparatus for electrochemical thinning. The acid is placed in the bottom of the container in the fume cupboard. The part of the apparatus in the fume cabinet has a pump on its left hand side that sprays the acid onto the specimen that is inserted at the top right of the cell. The electronics for the pump, the electrodes and the LED are outside the fume cabinet.

In the Tenupol system thinning from one side only can be done by covering one side of the specimen with a protective section of overhead projector transparency (D. Housden [176]). There is no need to peel or float any protective vinament layer off the specimen so both the mechanical distortion of the disc and the contamination of the specimen surface is reduced. This still allows the light to be transmitted through the specimen when a hole has been formed. The mechanism which automatically shuts off the unit then works as usual.

The temperature of the electropolishing solution is controlled by cooling coils passing through the main bath of electrolyte. This is important when using potentially 
explosive combinations of chemicals. By passing water through the coils the temperature of the electrolyte can be kept slightly below room temperature (approximately 16 to $17^{\circ} \mathrm{C}$ ).

After perforation, to stop the electropolishing process and clean the thin regions, the specimens are quickly removed from between the jets and sequentially immersed in two baths of doubly distilled water at approximately $40{ }^{\circ} \mathrm{C}$. The water removes the orthophosphoric acid. Methanol is then sprayed onto the specimen from a squeeze bottle to wash away the water and dry the specimen (T. Corfiatis [170]). Attempts to dry and clean off the acid in one step using methanol alone resulted in heavily contaminated specimens. The contamination was visible with both SEM and TEM. This idea was supported by the observation that contamination did not occur to the same extent when distilled water was used prior to drying with methanol. Some contamination can still occur when using the distilled water. It is important to immerse the specimen holder numerous times in the baths of distilled water and to wash plenty of methanol across the specimens.

The acid tended to age and after about a dozen good specimens being prepared the amount of thin material around the holes starts to become too small to be of much use. This is probably because of the increasing number of copper ions in the solution as the solution is used. The number of specimens that could be obtained before the solution needed replacing depends on the thickness of the specimens being thinned. It was also necessary to start the acid solution off by introducing some copper ions. This was done by adding about $200 \mathrm{ml}$ of an older solution to the new solution. Failure to do this gives specimens with thick edges for the first four or five specimens before large thin regions are obtained. This is of course a waste of specimens and high purity copper.

The current - voltage characteristic of the electropolishing apparatus did not display the predicted plateau region [177]. The optimum electropolishing conditions are meant to occur at this plateau region. This appears to be valid for bath electropolishing only and not for the jet electropolishing used in the Tenupol.

Satisfactory results, i.e., large thin regions, were obtained by using a "sensitivity " setting of 5.0, a "voltage " setting of 15 volts and "flowrate" of 1.0. These conditions gave a current of about $0.8 \mathrm{~A}$ when thinning from both sides of the specimen using an acid solution prepared as above. Assuming laminar flow occurs in the jets the lowest flowrate 
available on the system should give the largest thin regions. This is because at these conditions the velocity profiles across the jets are the most uniform. Various other combinations of these parameters were tested and found to give poorer results, but these settings are not necessarily the optimum settings. Finding better settings would require using an even larger amount of blank (unirradiated) copper specimens than the approximately eighty specimens used to deduce the above settings. When polishing from one side only it is better to use about 10 volts. This gives a current of about 0.22 to 0.30 amps. However, the current depends on the age of the solution. The electropolishing conditions used for copper and a range of other metals are shown in table 4.2. Typically these are different from those listed in standard electropolishing references [177 and 178]. Although the solution may be the same as used by other authors the voltages are almost always different because of the different experimental equipment used.

Although large thin regions are easily obtained in nickel this material is magnetic and so the TEM imaging is a bit unusual. For example, this metal has the same TEM characteristics as copper when untilted, but if the specimen is tilted by more than about $12^{\circ}$ different regions are observed. The eucentric position can be still found. The same object in the specimen remains approximately fixed in place as tilting occurs but it may no longer be illuminated by the beam. This makes necessary the constant readjustment of the beam deflection during tilting.

If upon examination in the TEM, specimens do not show large thin regions an attempt can be made to thin the specimen further using the ion beam thinning apparatus shown in figure 4.7. The results of ion beam thinning are variable. Thin regions may be obtained that are often coated with a hydrocarbon layer that is visible in the TEM. Also the material loses its crystallinity if high current levels are used and ring patterns characteristic of amorphous material are obtained instead of crystalline diffraction patterns. If the current is kept below about $30 \mu \mathrm{A}$ then thin crystalline regions that are large and clean are attainable. 


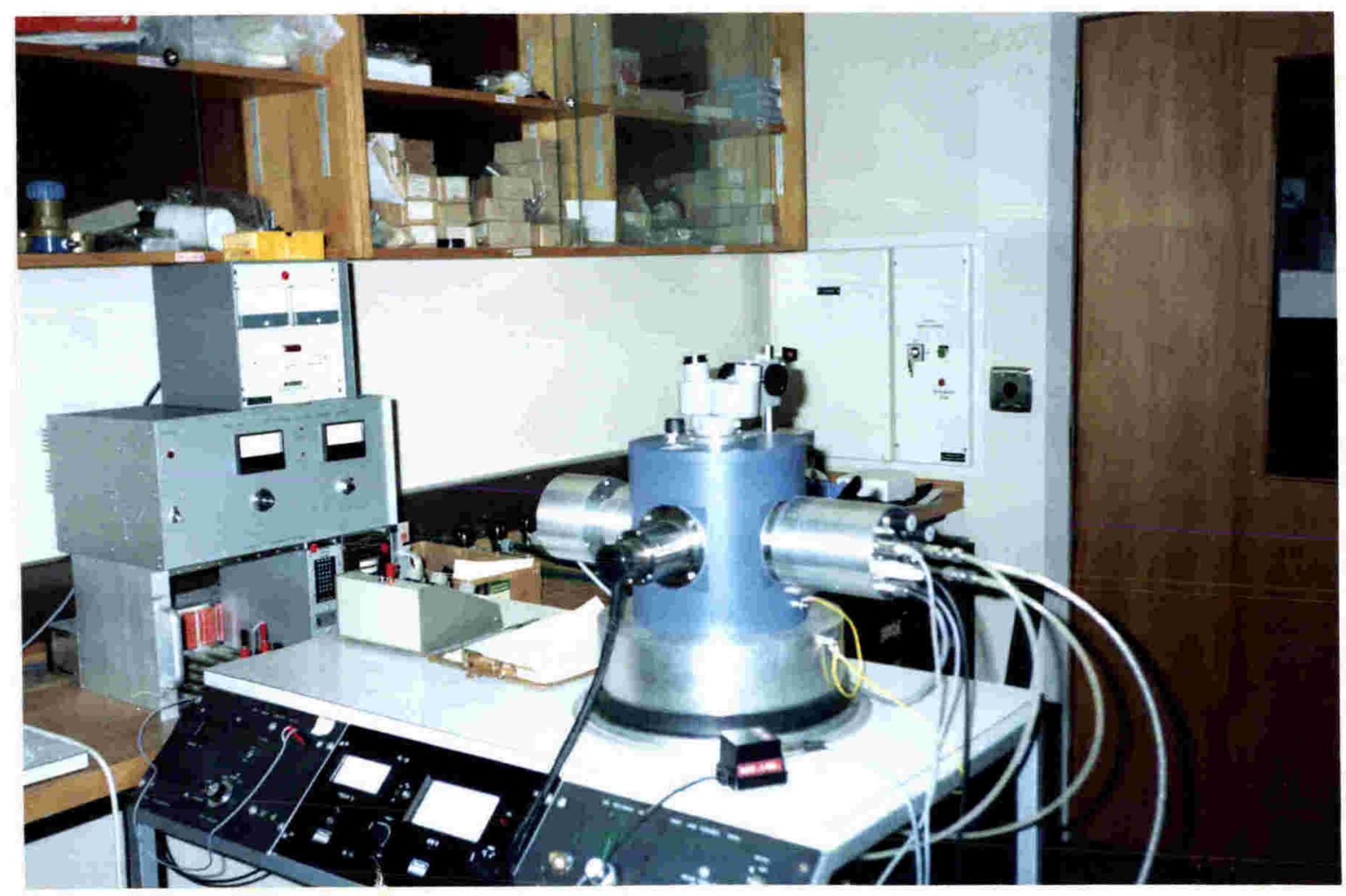

Figure 4.7: Ion beam thinning apparatus. The $3 \mathrm{~mm}$ disc is clamped into a holder inserted into the left hand side of the blue cylinder. Gas is bled into the cylinder through the valves on the right. The gas is ionised by the power supply in the background. The current hitting the specimen is integrated by the unit under the power supply. The pressure is monitored with meters located above the power supply and on the front of the table. A light can be shone onto the specimen from above and below the specimen. The binoculars and the light below the specimen can be used to detect when the foil is perforated and whether the holes in the specimen are being widened.

\subsection{Miscellaneous TEM Techniques}

Kikuchi lines, which are named after their discoverer [179], are important in electron microscopy because they provide an accurate determination of the direction of the electron beam relative to the crystal. He explained the lines in terms of how a beam of electrons passing through a crystal can produce a divergent secondary wave. This secondary wave originates from the inelastic electron scattering later postulated by Yoshioki [138]. Once produced, the secondary wave undergoes elastic scattering. Laue [180] attempted to explain the intensity of the Kikuchi lines using a dynamical theory. However he did not explain 


\begin{tabular}{|c|c|c|c|}
\hline Metal & $\begin{array}{l}\text { Jet electropolishing } \\
\text { solution }\end{array}$ & Voltage (Volts) & Current (Amps) \\
\hline $\mathrm{Al}$ & $\begin{array}{l}8 \% \text { Perchloric acid } \\
+ \text { glycerol +methanol } \\
\text { +distilled water }\end{array}$ & 15.0 & $0.15-0.18$ \\
\hline $\mathrm{Cu}$ & $\begin{array}{l}50 \% \\
\text { Orthophosphoric } \\
\text { acid } \\
+50 \% \text { Distilled water } \\
\end{array}$ & $\begin{array}{l}15.0 \text { from both sides } \\
10.0 \text { from back }\end{array}$ & $\begin{array}{l}0.75-0.8 \\
0.22-0.3\end{array}$ \\
\hline Mo & $\begin{array}{l}14 \% \text { Sulphuric acid } \\
+86 \% \text { Methanol } \\
\end{array}$ & 35.0 & 0.7 \\
\hline $\mathrm{Ni}$ & $\begin{array}{l}50 \% \\
\text { Orthophosphoric } \\
\text { acid } \\
+50 \% \text { Distilled water } \\
\end{array}$ & 15.0 & $0.2-0.25$ \\
\hline $\mathrm{Ti}$ & $\begin{array}{l}\begin{array}{l}14 \% \text { Sulphuric acid } \\
+86 \% \text { Methanol }\end{array} \\
\end{array}$ & 25.0 & 0.45 \\
\hline V & $\begin{array}{l}14 \% \text { Sulphuric acid } \\
+86 \% \text { Methanol }\end{array}$ & 20.0 & 0.35 \\
\hline
\end{tabular}

Table 4.2 The jet electropolishing conditions that were used for a range of metals.

the variation in the intensity as the crystal is tilted. Kainuma [139] later corrected this defect in the theory of Laue.

The waves interfere constructively at the Kikuchi lines according to the usual Bragg equation with the lines occurring in pairs. (J Gjonnes [181] presents a more rigourous theory based on perturbation theory). Every diffracting vector $\mathbf{g}$ has a Kikuchi line associated with it. If $\mathbf{g}$ is near the electron beam direction, then $\mathbf{- g}$ is also, so the lines occur in pairs. These pairs are slightly different, one is above the background intensity, the other 
below it. The bright line corresponds to the $\mathrm{g}$ that is more strongly excited or nearer to strong two-beam conditions.

When the electron beam is exactly down a crystal zone axis the Kikuchi line for any g will occur midway between the undeviated beam and g. As the crystal is tilted the intensity of the spots in the diffraction pattern in the direction in which the Ewald sphere is tilted become stronger. Suppose the incident electron beam is exactly down a zone axis and then the crystal is tilted through the Bragg angle, $\theta_{\mathrm{B}}$, corresponding to a particular reflection g. The gth reflection will now be strongly excited. The associated bright (or excess) line will pass exactly through the g spot in the diffraction pattern and the dark (or deficient) line for $-\mathbf{g}$ will pass through the origin. This position of the Kikuchi line for a reflection is a more accurate guide to the position of the electron beam than merely looking at the relative spot intensities. The sign of the excitation error (defined in figure 2.1) can be obtained from the position of the Kikuchi line for g. Tilting from one pole to another may be done by referring to standard Kikuchi maps of the position of the lines about the major axes in the crystal (Loretto [182]).

Accurate dislocation line directions and foil normals (Head et al. [82]) are required for the image simulations. This requires the electron beam direction to be known more accurately than to just the nearest major axis. The TEM camera length on the LED display is typically out by up to $30 \%$. Instead the ratio of the spacings between lines must be used to index the g's and B's. The technique in Head et al. is used in this thesis to obtain the beam directions. To measure foil normals etc. from micrographs the rotation between the image and the diffraction pattern needs to be known. This was done using long lathes of a $\mathrm{MoO}_{3}$ crystal as described in Head et al. [82]. Attempts were made to use the EMS programs of Stadelman [183] to index the diffraction patterns and to find the B's but the beam directions that are obtained seem to be wrong.

Often the Kikuchi lines appear more prominently viewed in convergent beam diffraction (CBED) conditions. CBED is achieved if the "spotsize" (strength of the excitation of the first condenser lens) is increased and the beam converged down by using the control for the current in the second condenser lens. This is preferable to ordinary selected area diffraction with the beam spread and a small spotsize setting. CBED is also 
useful for measuring the thickness of the crystal foil. Better Kikuchi lines are obtained if the foil is of moderate thickness. If the foil is too thin then not enough inelastic scattering occurs and if too thick the intensity is too low to obtain good Kikuchi lines.

Electron micrographs taken with an aperture selecting just the central beam that is undeviated to form the image are called bright field (BF) pictures. Micrographs taken with an aperture selecting a particular diffracted vector are called dark field (DF) pictures. Pictures of superlattices of gas bubbles are usually taken in overfocussed bright field conditions to get good contrast. The central undeviated beam interferes with all of the diffracting vectors and so the resultant BF image contains contributions from the central beam to these g's due to the scattering off the structure. The deviated beams have a lesser intensity than the central beam. Therefore an image taken using DF will be influenced mainly by the interference between the particular $\mathrm{g}$ selected and the central beam. This characteristic of DF is important when attempting to match experimental micrographs against theoretical simulations. This is because a two-beam theory is needed when the crystal is tilted to strong two-beam conditions for the particular $\mathbf{g}$ selected by the aperture. Accurate matching of $\mathrm{BF}$ pictures may require the use of the many beam dynamical theory which is computationally more demanding and time consuming.

The effects of lens aberrations such as astigmatism and spherical aberration can be minimised by deflecting the beams to bring the gth reflection into the centre of the pattern. The beam deflection coils in dark field are used for this purpose. These controls are situated on the right-hand side of the microscope. Photos taken by this process are called centred dark field (CDF) photos. The success of this technique in minimising the effects of the aberrations depends, of course, on how well the central beam is aligned down the optic axis of the microscope in the first place using the standard alignment procedure described in the operating manual of the TEM.

Another advantage of using CDF instead of BF is that the rocking curve is symmetrical in CDF (a rocking curve is a plot of transmitted intensity versus the excitation error $\mathrm{w})$. The corresponding BF curve is asymmetric and rapidly converges to zero for $\mathrm{w}<$ 0 . This means that in a slightly bent region the CDF micrograph will give a larger region of well illuminated material in regions where $w<0$ that are not too dark to interpret. The 
disadvantage of using CDF pictures is the extra time required to align the diffracted beam with the optic axis of the TEM beyond that required to take a BF picture. This time constraint can become important when a large series of photos is required in the same region of the specimen because the specimen is accumulating radiation damage throughout the alignment procedure.

When a crystal is tilted so that the electron beam is down a pole, the excitation error for all the reflections is large and negative. Rocking curves produced by the Howie-Whelan differential equations, which are being used in a many beam situation, show that both the $\mathrm{BF}$ and DF intensities are low as demonstrated in figure 4.8. Bloch wave simulations produced by the EMS programs are shown in figure 4.9 , for the intensity of the central beam and the [200] and [220] beams when at a [001] axis vs. thickness of the crystal. These situations can be compared with the situation when the crystal is at strong two-beam conditions. The $[200]$ reflections have a w of about -6 in the simulations done using Bloch waves. Physically many diffracting vectors become strongly excited when on-axis and the central beam loses its intensity and BF images are dark. Because there are many reflections gaining electrons from the central beam, no DF picture will have much intensity either. This shows that the blacking out of an image when on zone in itself is not evidence for the existence of dislocations. In the case of metals containing a bubble lattice when excited close to zone the broad dark image has a granular appearance on a very fine scale. This granularity has been attributed by Johnson and Mazey to the presence of dislocation loops, or some other small centres of strain, at high concentration. On tilting the specimen to be precisely on zone axis, the granularity seems to merge to form a totally black continuous image. Since this black continuous image occurs not only in crystals that have been ionimplanted but also in perfect crystals (Head et al. [82]), it does not itself indicate high dislocation densities.

Weak beam (WB) techniques rely upon weakly exciting all the g's and using CDF for one of these $g$ 's. By imaging with a larger $w$ the resolution is increased but difficulties in interpreting the result can be increased also. A two-beam theory with large w can be used but there are many evenly excited g's in the image. The neglect of these other g's may 
image intensity

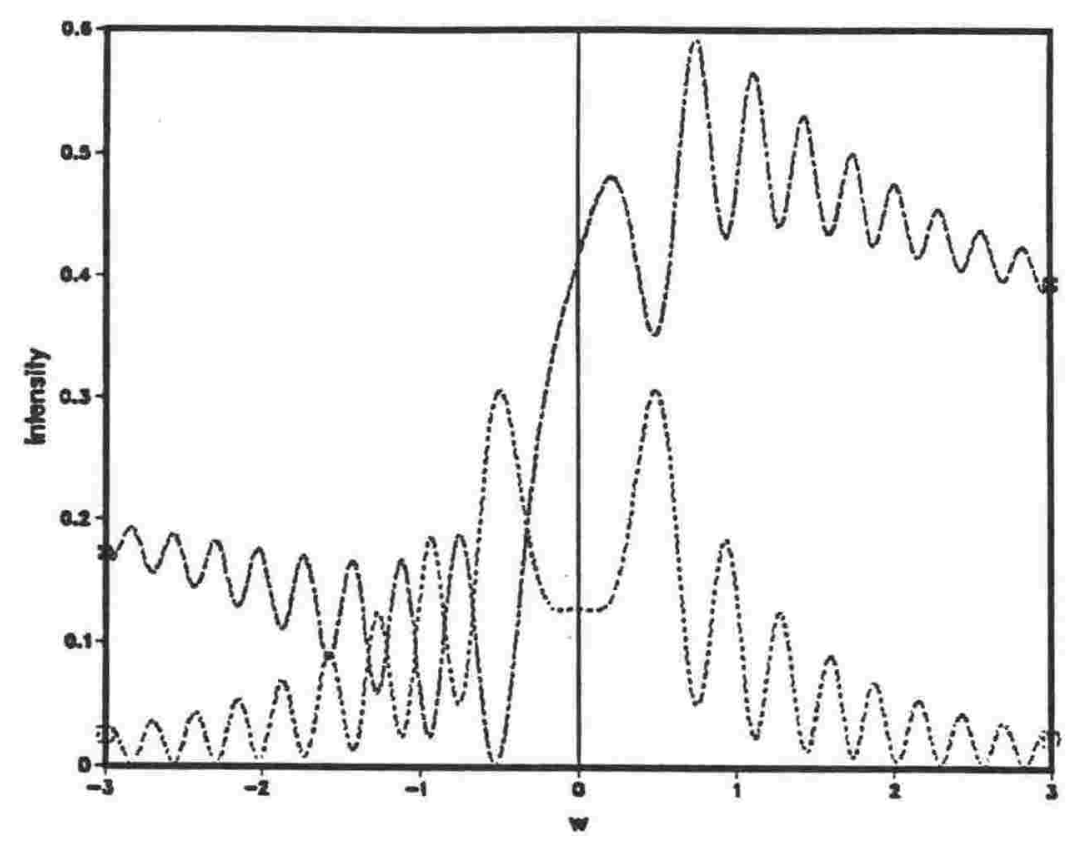

Legend

a $\mathrm{Br}$

?.I PF.........

(a)

image intensity

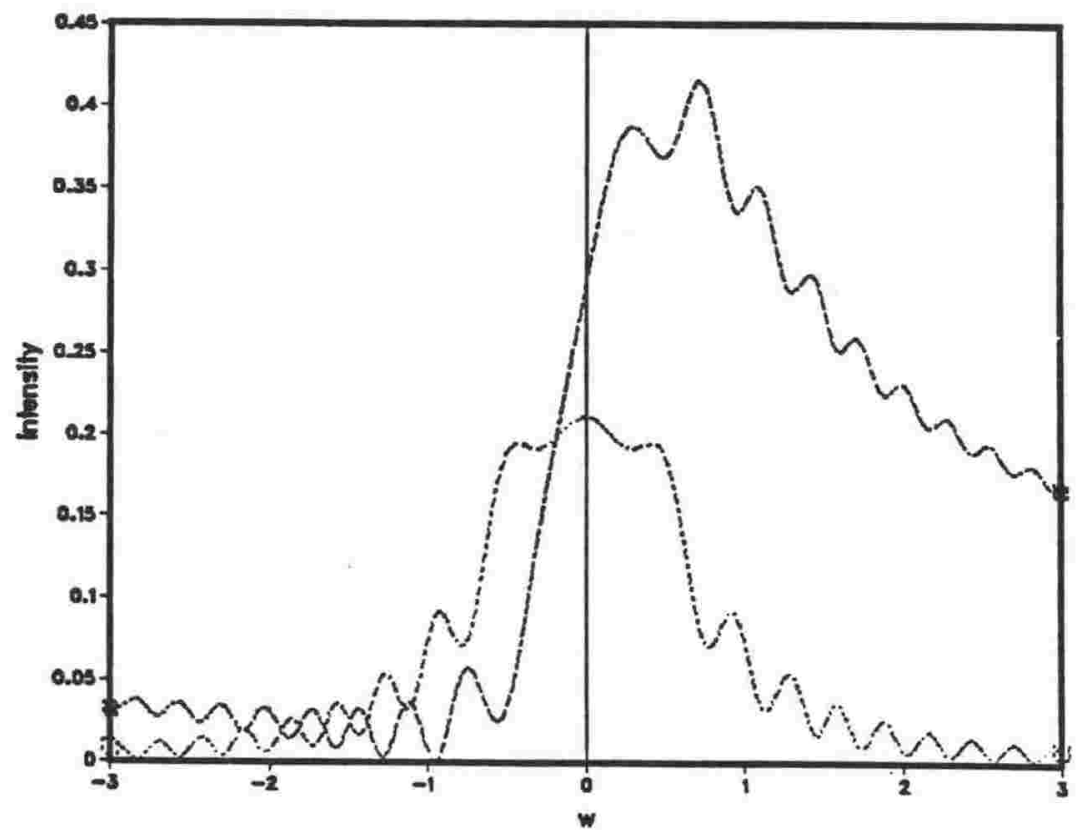

Legend

a B B

a pr.........

(b)

Figure 4.8: $\mathrm{BF}$ and DF rocking curves for two values of the anomalous absorption coefficient. The foil thickness is $4.0 \xi_{\mathrm{g}}$, and the anomalous absorption coefficients are: (a) 0.05 and (b) 0.10 . 


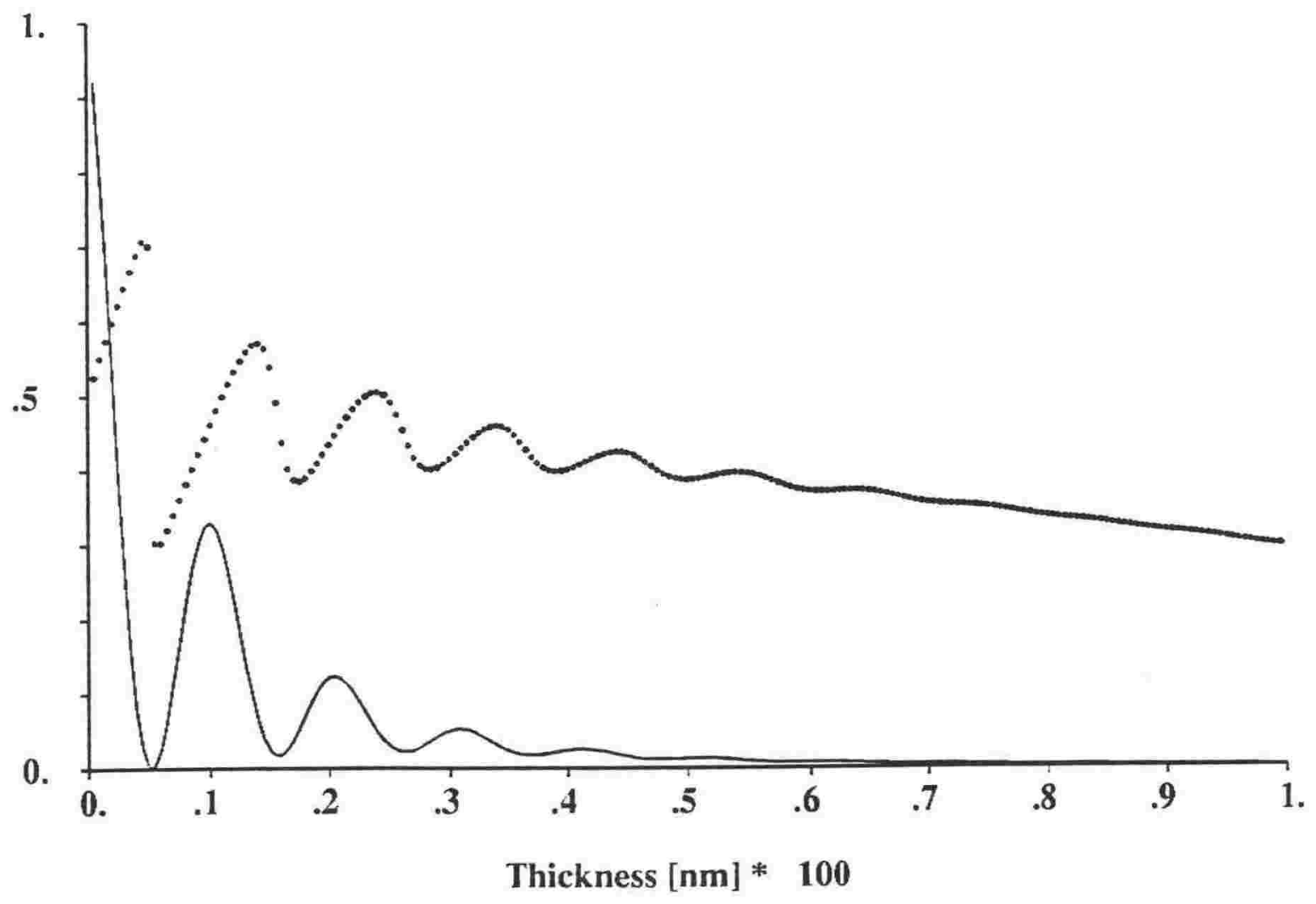

(a)

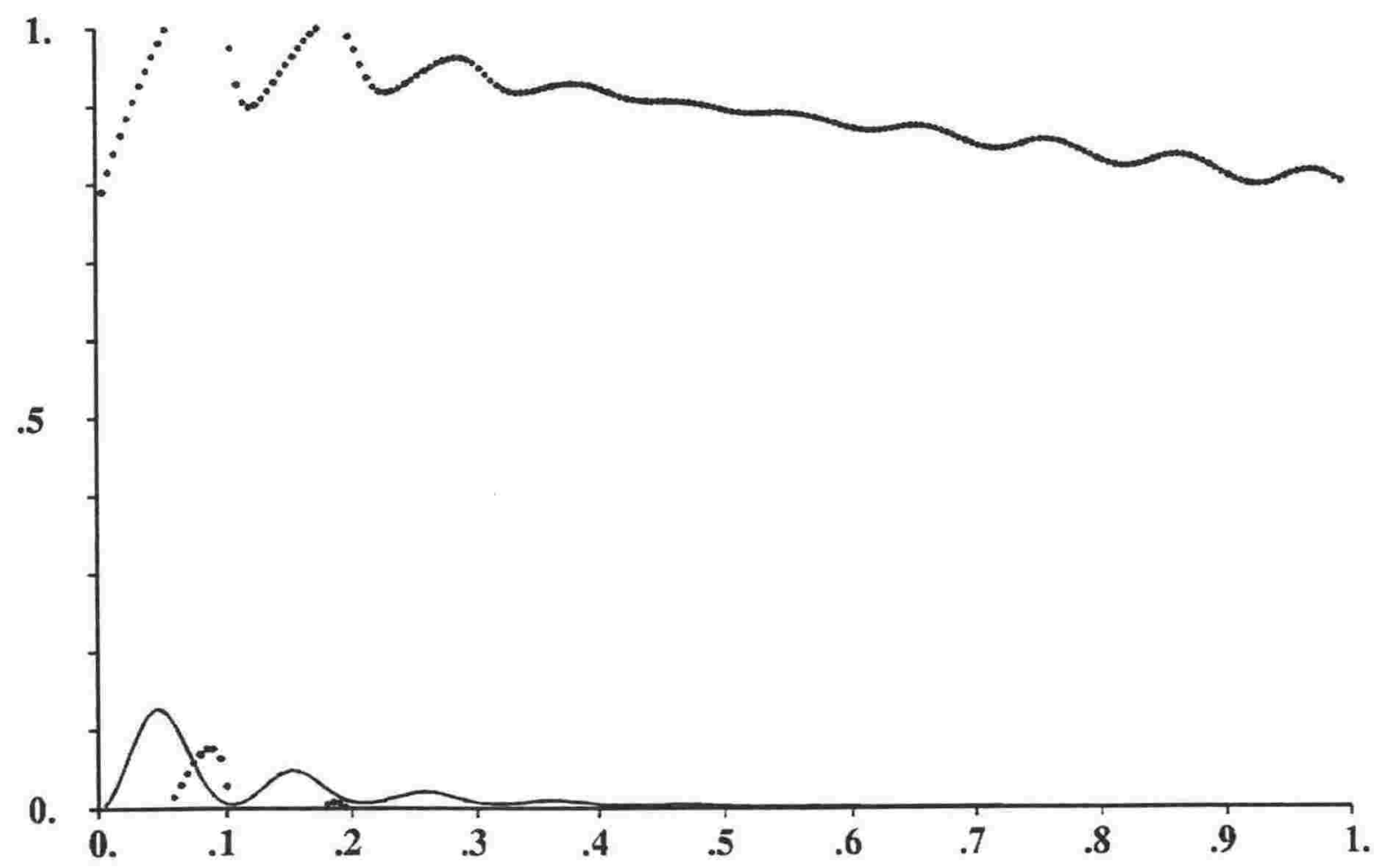

Thickness $[\mathrm{nm}] * 100$

(b) 


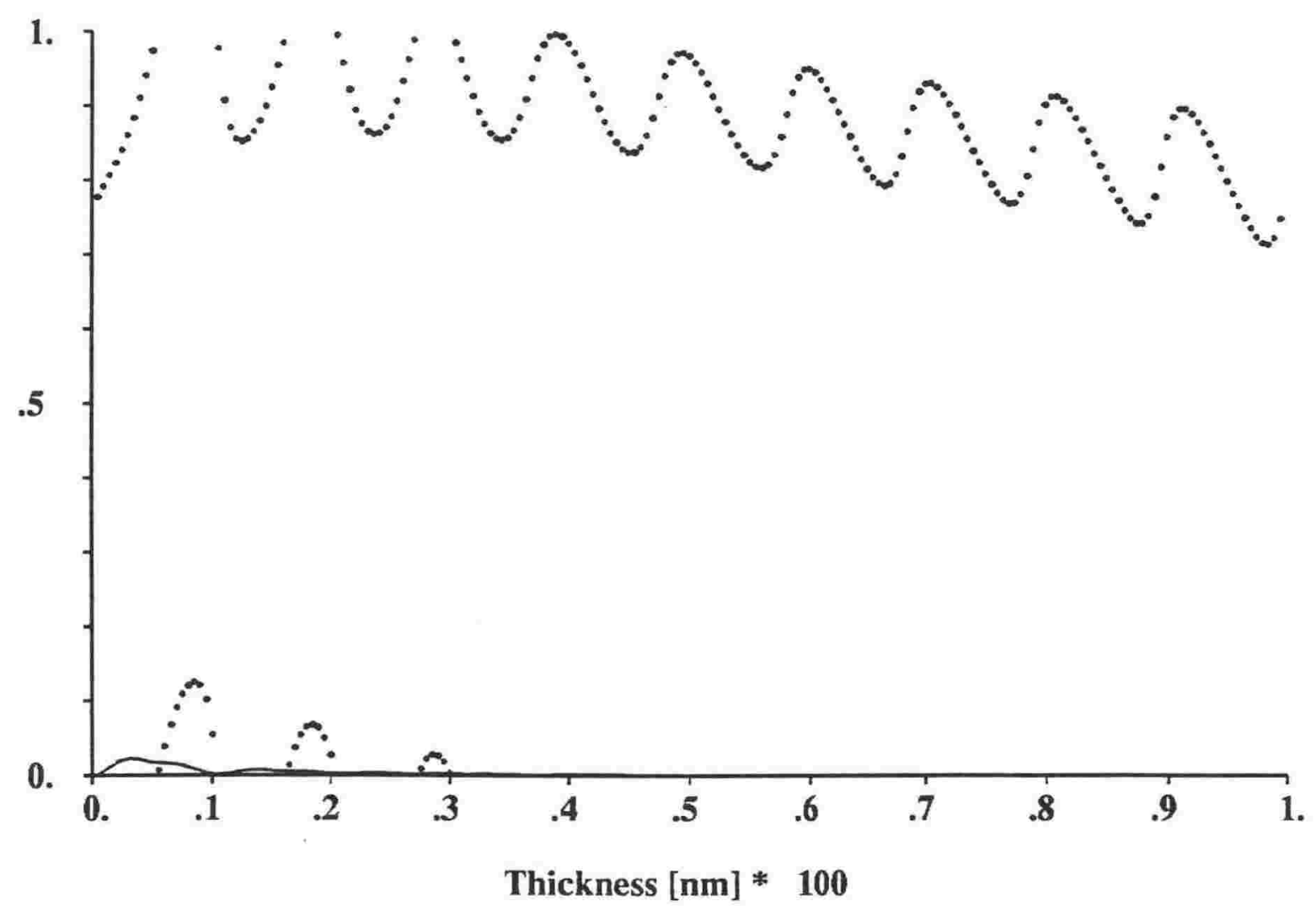

(c)

Figure 4.9: Nine beam Bloch wave simulation of the on-axis intensity and phase vs. the crystal thickness. The solid line is the intensity and the dotted line is the phase of the beams. (a) [000] beam, (b) [200] beam and (c) [220] beam. The vertical scale is labelled for the intensity values. The phase ranges from $+\pi$ radians (corresponding to 1 on the vertical axis) to $-\pi$ radians ( 0 on the vertical axis). 
result in inaccuracies and many-beam theory may have to be used. The crystal tilting technique to obtain WB conditions is described in Eddington [178].

The highest resolution technique for imaging perfect crystals is to put a large aperture around the central, undeviated beam and the inner g's. The crystal must be accurately lined up on an axis and the TEM well stigmated and focussed. A crossed lattice fringe image of the atom planes may be attainable. However, this technique is limited by the resolution of the microscope. It is difficult to image metals because of their small lattice constant. Interpretation of the images is usually done by the multislice formulation of electron diffraction.

It is difficult to interpret HREM images of dislocations unless the dislocations are viewed end-on. The number of missing lattice fringes cannot be counted to obtain the Burgers vector or the number of dislocations in any simple way. However knowledge of the positions of the atoms in the dislocation core are not required in this work. Even finding missing lattice fringes, indicative of dislocations, against a strong background of perfect crystal lattice is difficult by comparison with BF or DF images of dislocations taken at strong two-beam conditions. At strong two-beam conditions the background perfect crystal gives a fairly uniform intensity and the dislocation images stand out strongly for dislocations from the size of a point defect or larger.

If the undeviated beam is tilted off the optic axis by using the same deflection coils mentioned above then a higher resolution image may be obtained. The multislice programs cannot interpret this situation because a simple geometry is required. Also the images are more strongly affected by aberrations so that the images are even more difficult to interpret than the orthodox HREM images.

Several ways of visualising directions and structures are useful. Stereographic projections are introduced in several books (Eddington [178] and Moon [184]). Another useful construction is the Thompson tetrahedron mentioned in, for example, Hirth and Lothe [97]. The tetrahedron displays the [110] and [111] directions that Burgers vectors and glide cylinder axes lie on in an fcc material. The EMS software also can be used to generate three-dimensional "ball model" diagrams of atoms, bubble arrays and loop structures. 
To identify Burgers vectors using the invisibility criterion for a dislocation, i.e. g.b $=0$, it is best to use the diffracting vectors [111], [200] and [220] in turn. (The g.b = 0 criterion comes out of the Howie-Whelan differential equations using the approximation that the elasticity is isotropic.) This is because $\mathbf{g}=[111]$ gives the greatest number (three) of occurrences of $\mathbf{g} \cdot \mathbf{b}=0$ for the case where $\mathbf{b}=0.5[110]$ (the preferred form for $\mathbf{b}$ in an fcc crystal). The [200] and [220] reflections give at least two extinctions and one extinction respectively. In exciting the desired reflections the electron beam should be directed down a simple pole (i.e., one having low indices). [111] reflections occur for both [110] and [112] poles; [220] reflections for [100], [111] and [112] poles; and [200] reflections for either [100] or [110] poles. The poles used in any image matching exercise should be selected from these options.

\subsection{The Image Simulation and Processing System}

The computer simulation and image processing systems have been described in detail elsewhere [185] and so will be only briefly summarised here. Micrographs could be digitised by a CCD camera or images captured directly off the TEM from the Gatan image intensifier or wide-angle camera. The cameras were connected to a Matrox board mounted in a Dasher 286 Data General computer. The image could be transferred to a Data General MV 4000 for processing by Semper 5 software.

Since the article mentioned above was published the system has been upgraded: the Semper 5 software has been replaced with Semper 6.4 and the Data General MV 4000 has been superseded by a Sun sparcstation 1 . A videopix board mounted in the sparcstation allows images to be both digitised and processed on the same computer which speeds up the process. Before purchase of the videopix board the images were still digitised by the Matrox boards and transferred to the sparcstation by network NFS software.

In addition to obtaining a photographic output from a polaroid freeze frame recorder it is possible to convert images into postscript files. These files can then be sent to one of several laser printers. This printed output is not as realistic as the photographic output but the results are more reproducible. The output from a Mitsubishi thermal printer is more 
realistic looking than that from a laser printer although the cost is greater. Both are much cheaper than photographic output. Images can be sent to the thermal printer via a video connection from a camera or from the Sun computer via a 386 computer. The 386 computer has a VGA / EGA board that can display the image on the computer screen (using a software package written by Foster-Findlay) after the image is routed through the RGB connection of the printer. That is a thermal print can be made of whatever is on the screen. The thermal printer has adjustable black and white levels, and contrast controls. This gives greater ease in matching the simulations to experimental micrographs because the image does not have to be recalculated or the grey levels rescaled (using a new look up table (lut)) every time the contrast and brightness are adjusted.

The outputs from the EMS software are in the form of postscript files that can be sent to the laser printer. Alternatively, a postscript previewer that is available in the openwindows operating system can be used to display the images on the computer screen.

\subsection{Large Resolvable Loops - Maher and Eyre Rules}

The Maher and Eyre rules allow the interstitial or vacancy character of large resolvable loops to be determined. The theoretical basis for the method comes from the variation of the position of the dislocation image in a kinematical theory with the curvature of the local lattice planes (equation 2.75). The method uses the FS/RH convention of Bilby et al. to define the Burgers vector, so that the lattice curvature will depend on the interstitial or vacancy character of the loop as shown in figure 4.10. A diffracting vector $\mathbf{g}$ and its opposite diffracting vector $-\mathbf{g}$ at the same sign of excitation error are used. Alternatively images with the same $\mathrm{g}$ but different sign of the excitation error can be used. In both approaches the idea is to vary the sign of $(\mathbf{g} \cdot \mathbf{b}) \mathrm{s}_{\mathrm{g}}$.

It is observed how the image of the loop changes in size in a pair of images taken with the same geometry but using either a $\pm \mathrm{g}$ pair (with $\mathrm{s}_{\mathrm{g}}$ of the same sign) or a given $\mathbf{g}$ with two values of $\mathrm{sg}$ having opposite sign (figure 4.10). The variation in the image can be then compared with figure 4.10 to find whether the loop has a vacancy or interstitial character. Maher et al. [57-59] used image simulations based on dynamical theory to 
identify "safe " orientations of the loop normal and Burgers vector where this method of analysis works. The orientations where the analysis does not work because of dynamical effects are referred to as "unsafe". Edge dislocation loops are always in "safe" orientations. This is because the loop normal has to be rotated away from $\mathbf{b}$ to be at an "unsafe " orientation. The interstitial or vacancy character of some loops is determined in section 4.6.2.

\subsection{Experimental Micrographs and Simulations.}

\subsubsection{Experimental Micrographs and Simulations of Long Straight Dislocations}

In this section the image matching techniques are applied to images of long straight dislocations. The experimental techniques used to obtain micrographs are the same for loops and long straight dislocations. They are detailed in Head et al. [82] and are briefly summarised here. Many micrographs are needed of the same region. They should be taken under strong two-beam conditions for at least three different beam directions (relative to the specimen) and with various diffracting vectors. The diffraction patterns (and hence the beam directions) are indexed using the techniques in section 4.3. The beam directions should be as widely apart as possible to obtain good determinations of the foil normal and dislocation line direction using stereographic projections. To obtain the foil normal it is necessary to mark the directions connecting three recognisable features (these vectors need to be determined), that are non-colinear, lying in the midplane of the foil. The angles are measured between the projections of a vector and the g's used to obtain the three images. By simple stereographic techniques the vector can be then determined. The foil normal is measured by taking by eye the average of a set of three vectors formed by the cross product of different combinations of the three vectors connecting the three recognisable features.

The foil thickness can be estimated from the beam directions and the foil normal using the techniques outlined in the previous paragraph. The thickness can be adjusted a bit to obtain a good match because of the uncertainty in the extinction distances. The excitation error also can be varied slightly from exact two-beam conditions because the foils are usually a bit bent. However, the excitation error may not 


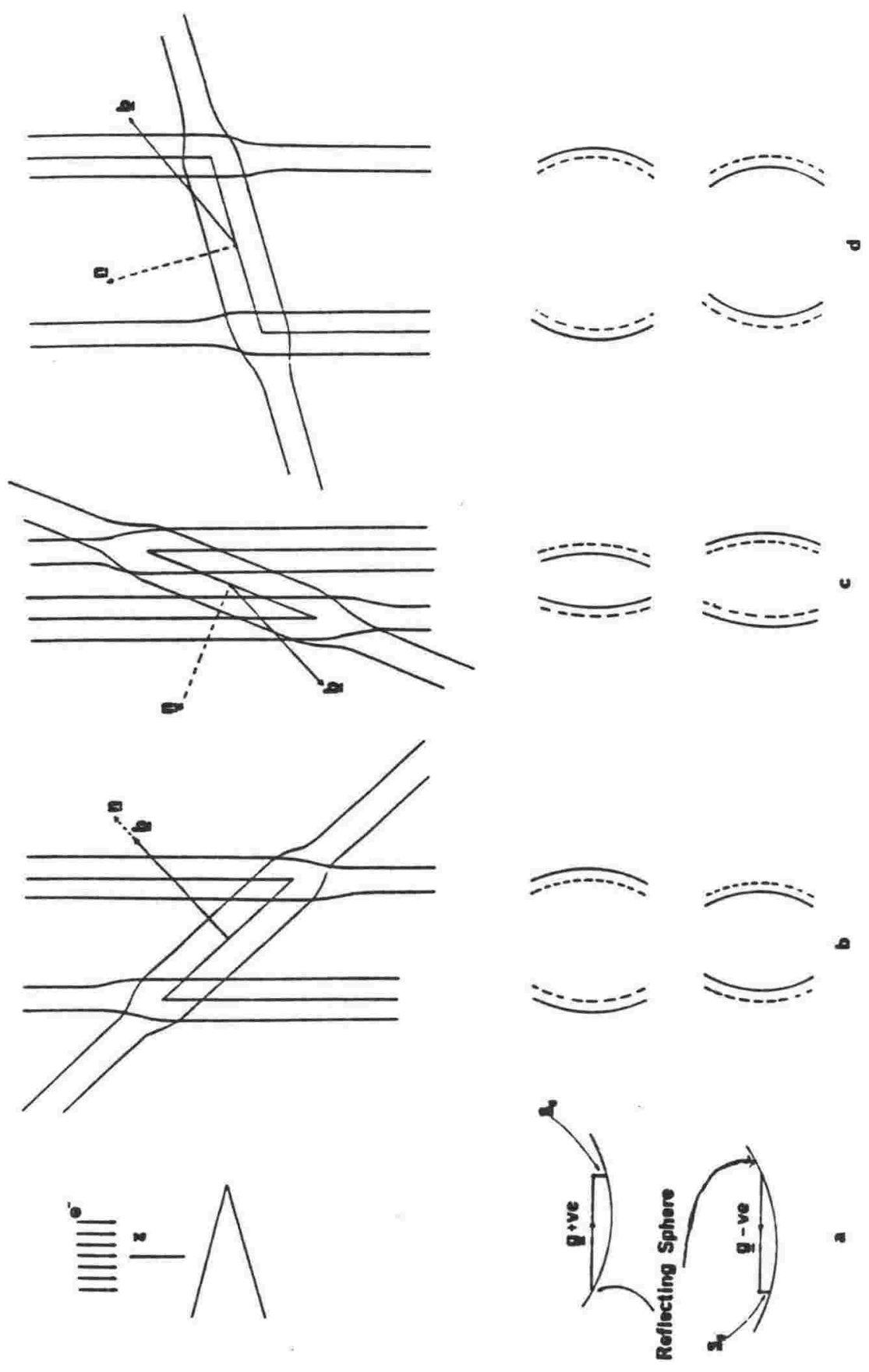

Figure 4.10: Schematic diagram of an interstitial loop and the corresponding position of the intensity minimum (continuous arc) with respect to the dislocation core (broken arc) for $\mathbf{g}$ +ve and $\mathbf{g}$-ve keeping $\mathbf{s}_{\mathrm{g}}>0$ : (a) crystal and diffracting geometries; (b) edge loop; (c) rotation of $\mathbf{n}$ through the $\mathbf{n} . \mathbf{z}=0$ configuration; and (d) rotation of $\mathbf{n}$ through the $\mathbf{n} \cdot \mathbf{b}=0$ configuration. 
correspond exactly to the region from which the CBED was taken. The CBED pattern is used to determine the approximate excitation error only. In performing simulations the different images are taken for different $g$ 's and therefore $\xi_{g}$ 's. Thus although the foil thickness for the different images is a constant number of angstroms thick, it is a different number of extinction distances thick.

Because copper is anisotropic there is some residual contrast when $\mathbf{g} \cdot \mathbf{b}=0$. However, the intensity of defects with $\mathbf{g} \cdot \mathbf{b}=0$ is usually much fainter than other defects in the same photo and this criterion can be still used to narrow down the possible choice of $\mathbf{b}$. For the images of figure 4.11 this procedure was used to narrow the possible Burgers vectors down to the following possibilities: $0.5[110], 0.5[-1,-1,0], 0.5[0,1,-1], 0.5[0$, $1,1]$. Opposite b's give the same image rotated by $180^{\circ}$ so it is necessary to make a choice between two b's only. The pair of Burgers vectors are then simulated using the Head et al. onedis program with the results shown in figure 4.11. The experimental images are shown on pages $4.38,4.44$ and 4.45 ; calculated images for comparison appear on 4.37 through to 4.46. The validity of the $\mathbf{g} \cdot \mathbf{b}=0$ analysis of course can be checked by simulation also. The effects of varying the foil thickness are shown in figure 4.11(a). The best match for the foil thickness of $4.6 \xi_{\mathrm{g}}$, suitably scaled by the different extinction distances required as $\mathbf{g}$ changes, was used as the foil thickness in parts (b) and (c). The simulations show that the Burgers vector is $0.5[0,-1,1]$. In figure 4.11 a range of values for $w$ is used; the middle value gives the best match with experiment. In figure 4.11(a) a larger range of w was used than in the other parts of this figure. This was done because the number of extinction distances that the foil thickness is equal to will vary as w changes (because a change in w will alter the effective extinction distance).

Better matches probably would be attainable if a larger set of experimental micrographs were taken. In practice it would have been easier to take more experimental images at different $\mathbf{g}$ to identify $\mathbf{b}$ using the $\mathbf{g} \cdot \mathbf{b}=0$ invisibility criterion. This was not done as the motivation for examining straight dislocations was to gain experience of image matching before applying the same techniques to dislocation loops. 


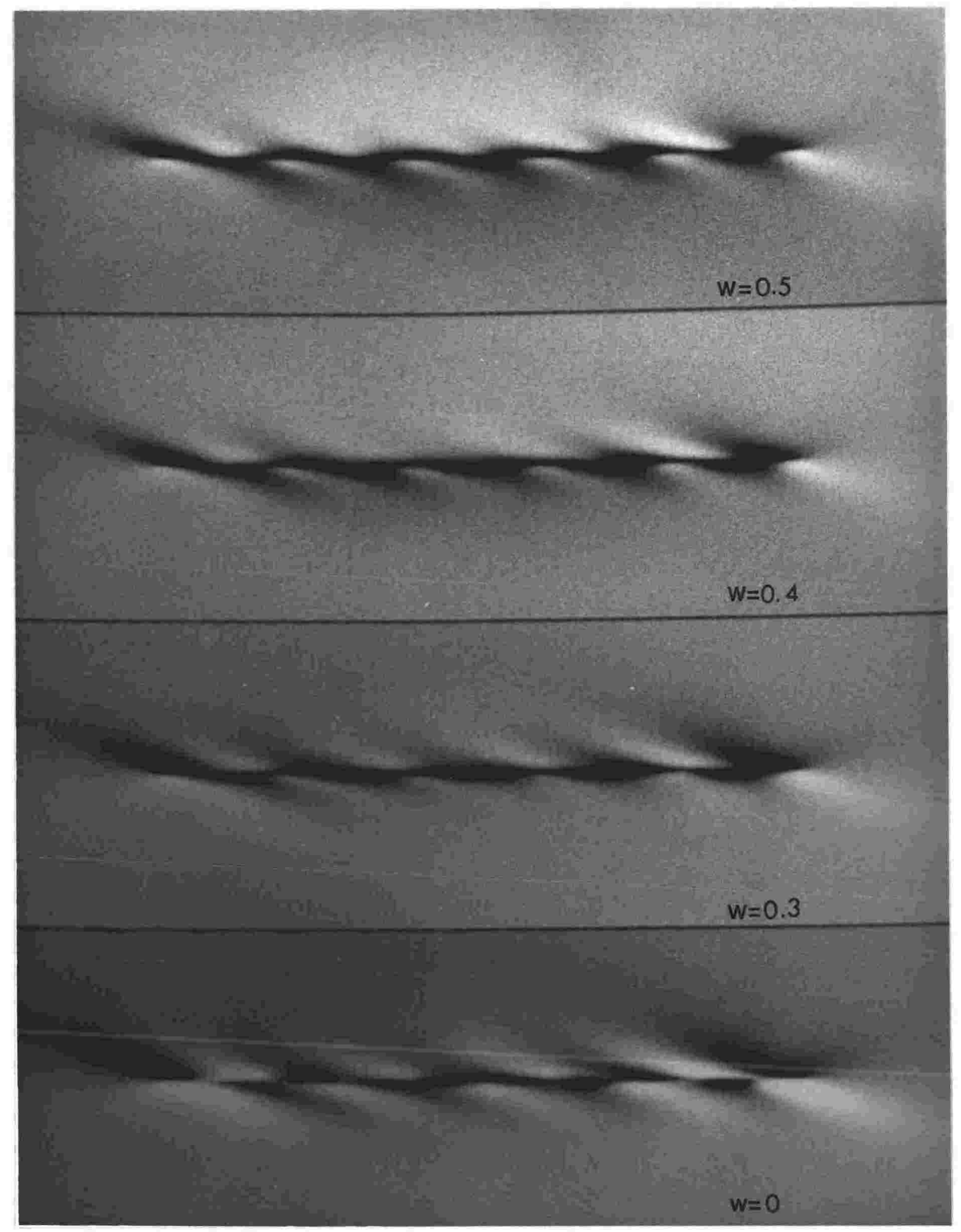

Figure 4.11 (a), (i) $t=4.45 \xi_{g}$ 

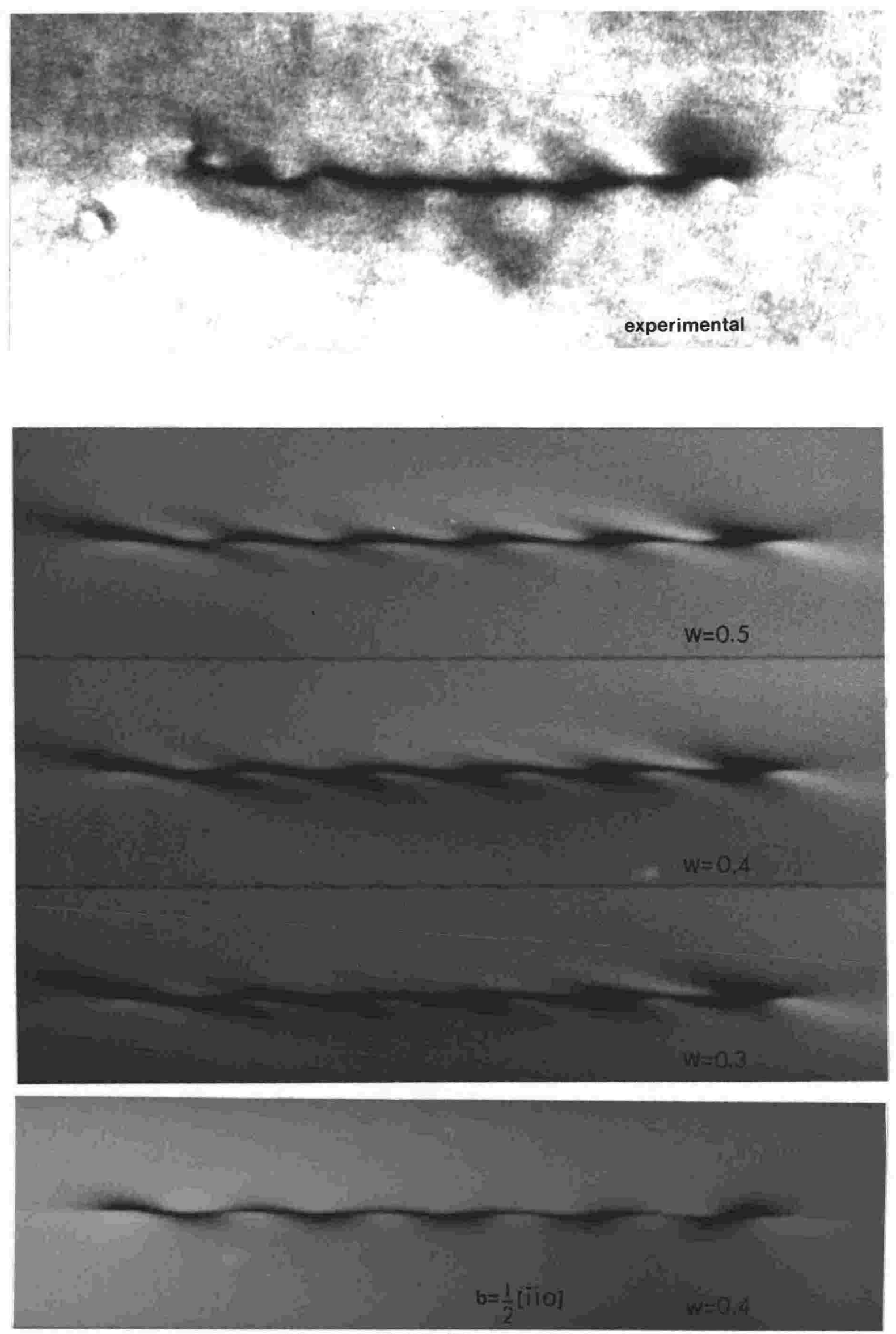

Figure 4.11 (a), (ii) $\mathrm{t}=4.6 \xi_{\mathrm{g}}$ 


$$
W=0.5
$$

$w=0.4$

Figure 4.11 (a), (iii) $\mathrm{t}=4.75 \xi_{\mathrm{g}}$ 


$$
w=0.7
$$

$$
w=0.6
$$

$w=0.5$

$w=0.4$

Figure 4.11 (a), (iv) $t=5.05 \xi_{\mathrm{g}}$

$4-40$ 

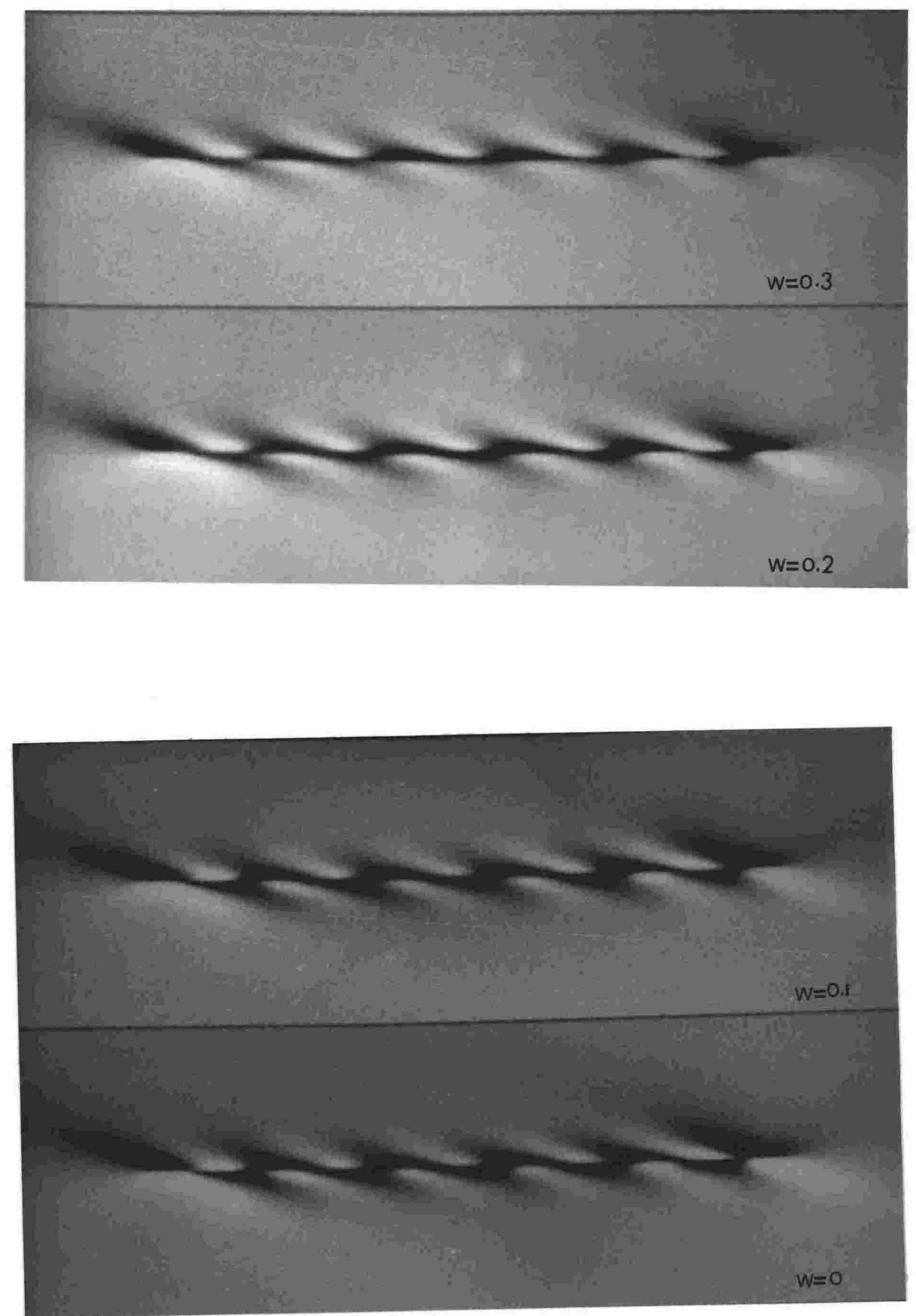

Figure 4.11 (a), (iv) $t=5.05 \xi_{g}$ 


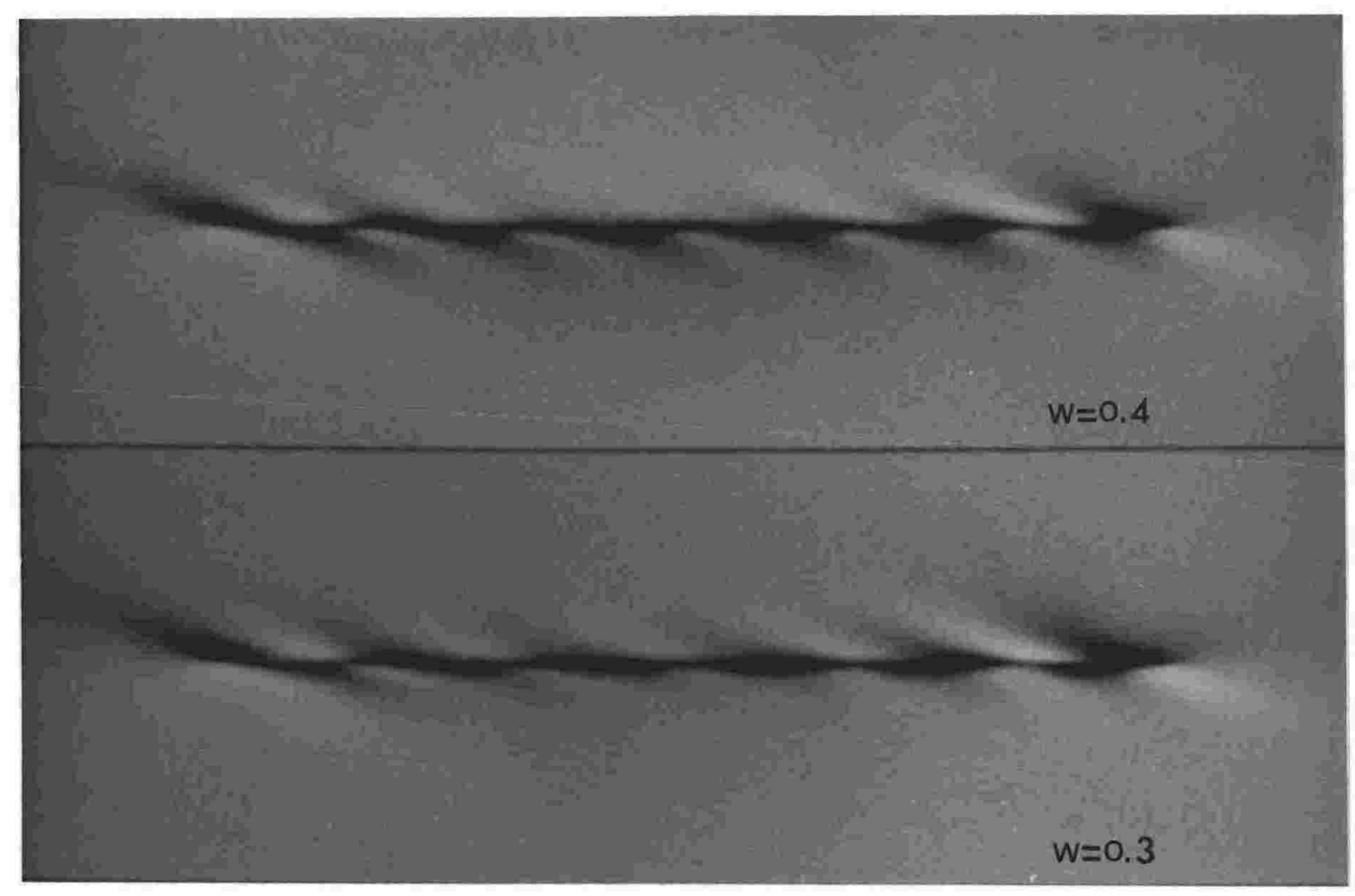

Figure $4.11(\mathrm{a}),(\mathrm{v}) \mathrm{t}=5.35 \xi_{\mathrm{g}}$ 

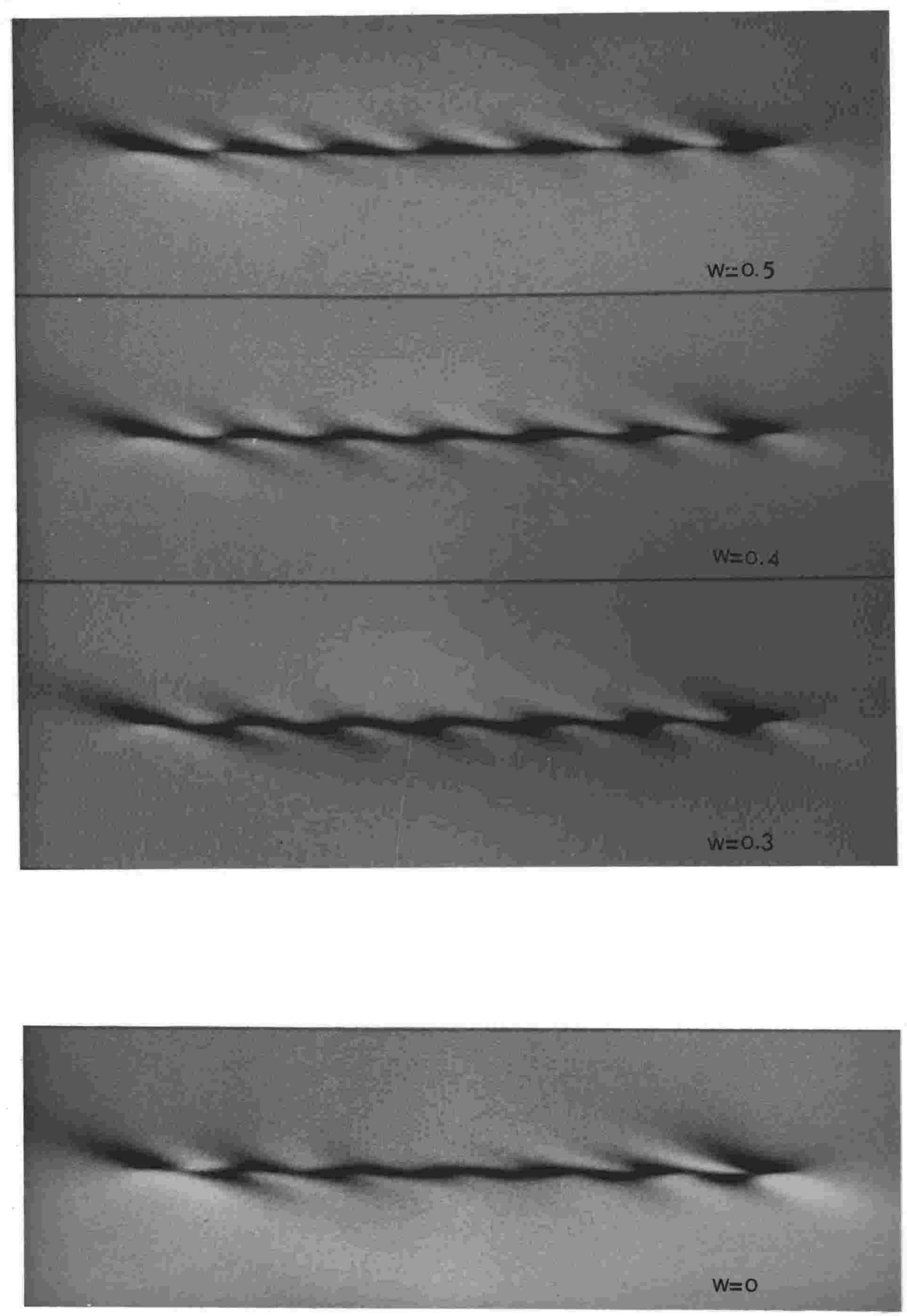

Figure $4.11(\mathrm{a}),(\mathrm{vi}) \mathrm{t}=5.65 \xi_{\mathrm{g}}$ 


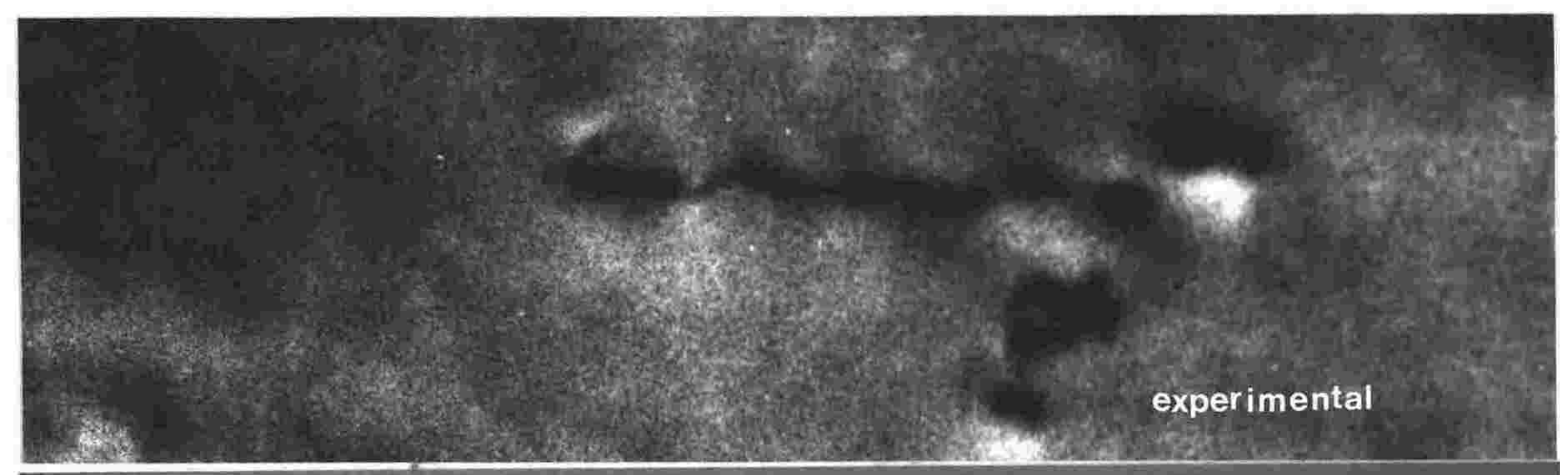

$W=0.6$

$w=0.5$

$w=0.4$

Figure 4.11 (b) 

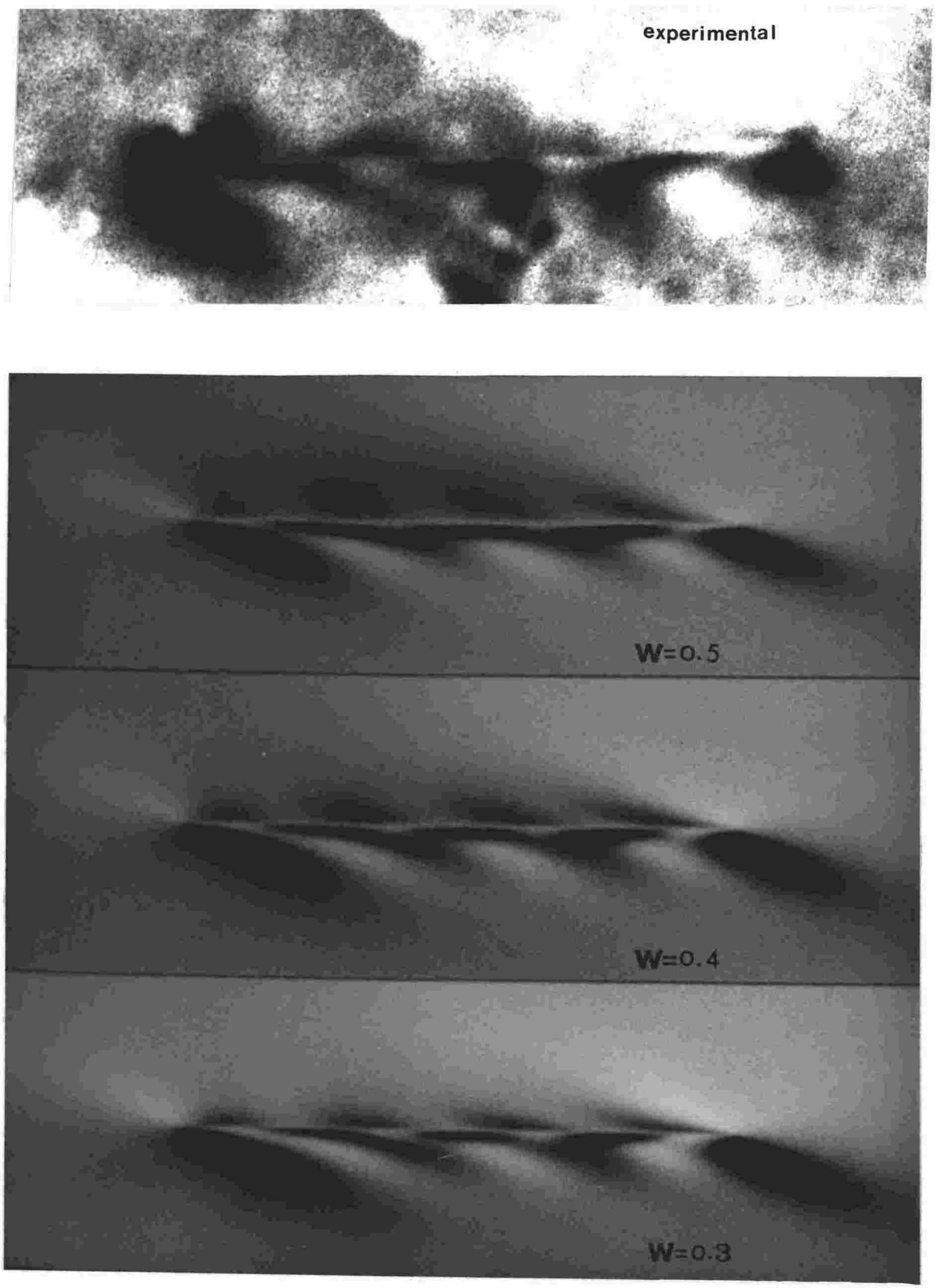

Figure 4.11 (c) 


\section{$\mathrm{b}=\frac{1}{2}[\mathrm{i} i \mathrm{i}] \quad \mathrm{W}=0.4$}

(c)

Figure 4.11: Comparison of experimental micrographs and simulations for $\mathbf{b}=0.5[-1,-1$, $0]$ and $0.5[0,-1,1]$ in copper (a simulation is for $\mathbf{b}=0.5[0,-1,1]$ unless otherwise marked on the figure). The common simulation parameters are a foil normal $(\mathbf{F})=[3,1,7]$, a dislocation line direction $(\mathbf{u})=[-4,-6,5]$, and the elastic constants are: $\mathbf{c}_{11}=1.684 \times 10^{-10}$

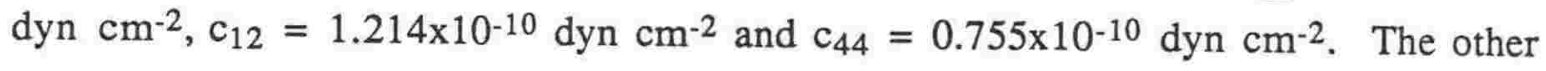
parameters are:

(a) $\mathbf{B}=[6,7,13], \mathbf{g}=[-1,-1,1]$ and the anomalous absorption coefficient is 0.07 .

Combinations are made of a foil thickness (t) of (i) $4.45 \xi_{\mathrm{g}}$, (ii) $4.6 \xi_{\mathrm{g}}$, (iii) $4.75 \xi_{\mathrm{g}}$, (iv) $5.05 \xi_{\mathrm{g}}$, (v) $5.35 \xi_{\mathrm{g}}$, (vi) $5.65 \xi_{\mathrm{g}}$, and $\mathrm{w}=0$ to 0.7 . The simulations for 4.6 $\xi_{\mathrm{g}}$ are the best match to the experimental micrographs and so this foil thickness (scaled with $\mathbf{g}$ ) is used in parts (b) and (c).

(b) $\mathbf{B}=[1,0,16], \mathbf{g}=[0,-2,0]$, the foil thickness is $4.1 \xi_{\mathrm{g}}, \mathbf{w}=0.3,0.4$ and 0.5 , and the anomalous absorption coefficient is 0.08 .

(c) $\mathbf{B}=[14,15,15], \mathbf{g}=[0,2,-2]$, the foil thickness is $2.94 \xi_{\mathrm{g}}$, w $=0.3,0.4$ and 0.5 , and the anomalous absorption coefficient is 0.1 . 


\subsubsection{Isolated Loops}

In this section experimental micrographs of isolated loops are compared to theoretical images. The experimental micrographs are used to find the parameters for the simulations. The same experimental techniques that are used to find the parameters required to simulate long straight dislocations in section 4.6.1 are used to match dislocation loops. The experimental techniques were summarised in sections 4.3 and 4.6.1. The term "isolated loops" is used to indicate that the contrast of a loop can be considered as due to that loop alone. It is assumed that nearby loops do not significantly influence the contrast of this loop. Therefore the experimental images of the loops in figure 4.12 are matched against simulations of isolated loops.

The foil thickness is estimated using a straight dislocation that threads through the foil. The foil normal is found using directions connecting the midpoints of straight dislocations that thread through the foil. The midpoints of loops cannot be used as they do not necessarily lie in the centre of the foil.

It is not possible to find the habit planes of isolated loops directly from experimental micrographs because the direction through the black / white lobes is approximately parallel to $g$. The direction of the line of no contrast between the lobes is not necessarily parallel to the habit plane.

Hirth and Lothe [97] state that: "the glide plane of a straight dislocation is that plane which contains both the dislocation line and the Burgers vector ". Thus the glide plane of a straight dislocation is set by the edge component of the Burgers vector. A straight dislocation that is of pure screw type has no restrictions on where it can glide. The restrictions on dislocations with an edge character arise because if the dislocation attempted to move perpendicular to its glide plane then matter would have to be moved. This would require work to be done by an external force. In the absence of external forces dislocations are restricted to moving on their glide planes.

The glide cylinder of a loop is set by the edge component of the Burgers vector and

the loop normal. Equivalently the dislocation line of a loop curves around in a circle so that a loop with a non-zero edge component to the Burgers vector is restricted to moving in one direction instead of a plane. Consider the situation when the Burgers vector is parallel (or 

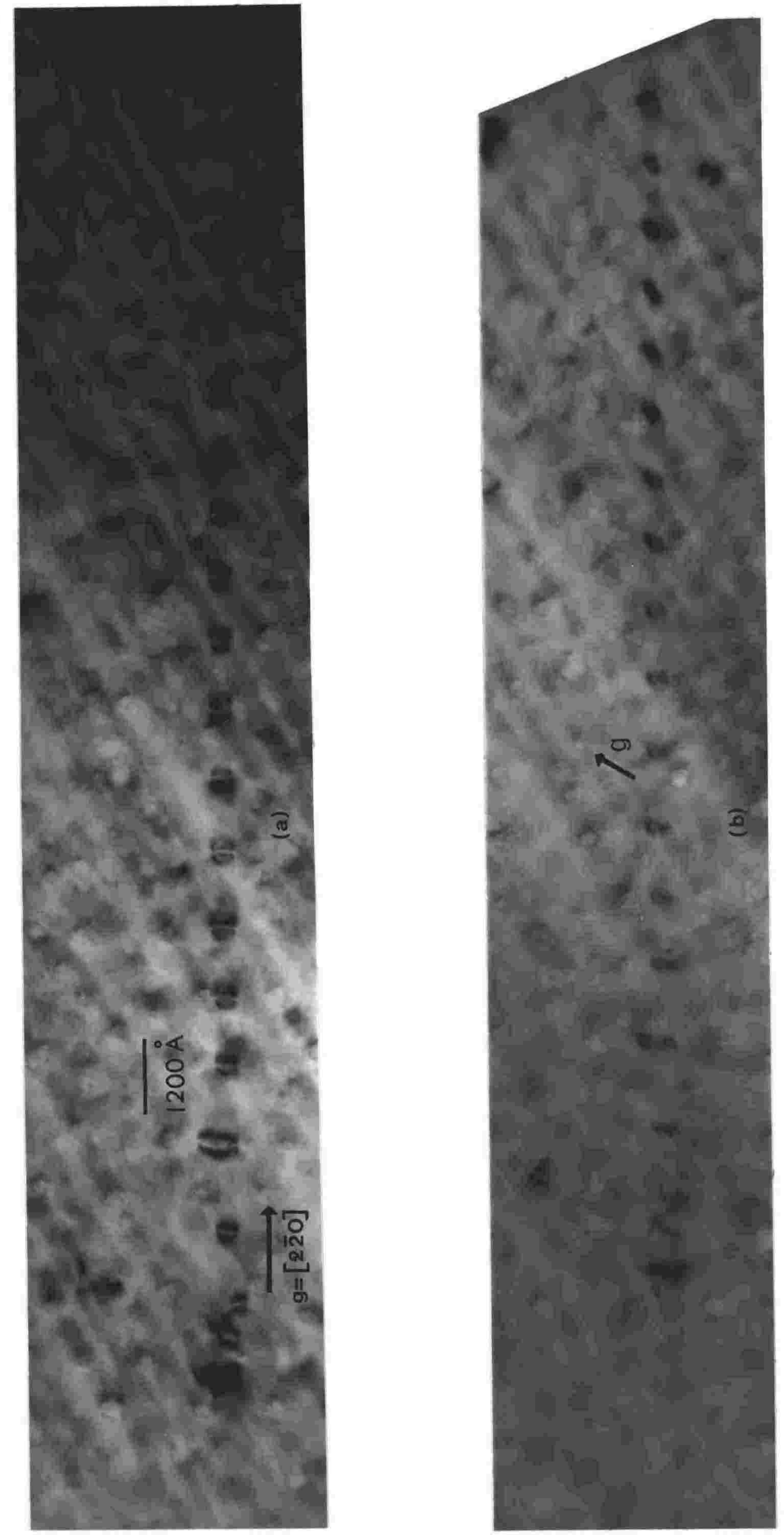

Figure 4.12, experimental images.

$4-48$ 


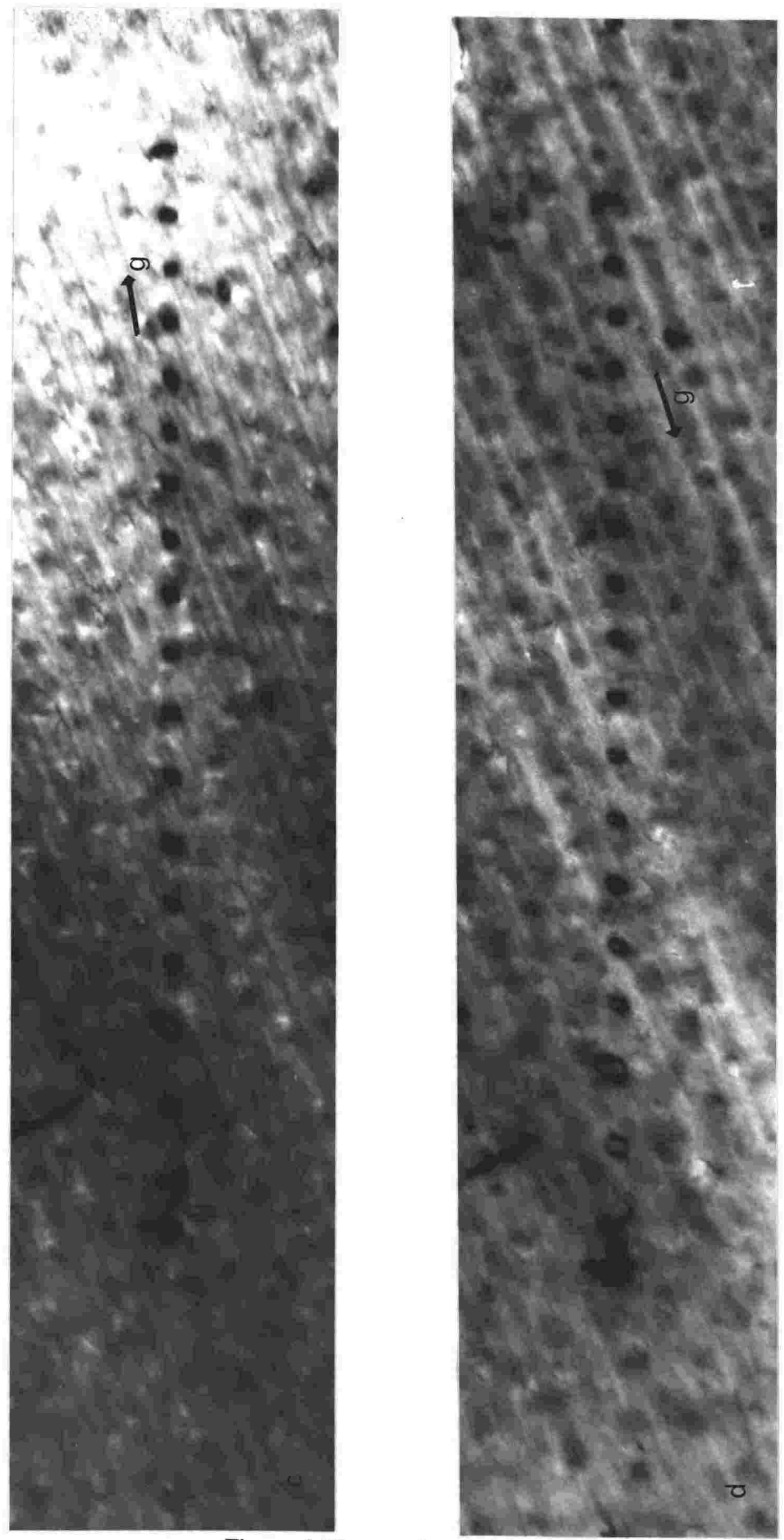

Figure 4.12, experimental images. 
$\mathrm{d}=380 \AA$

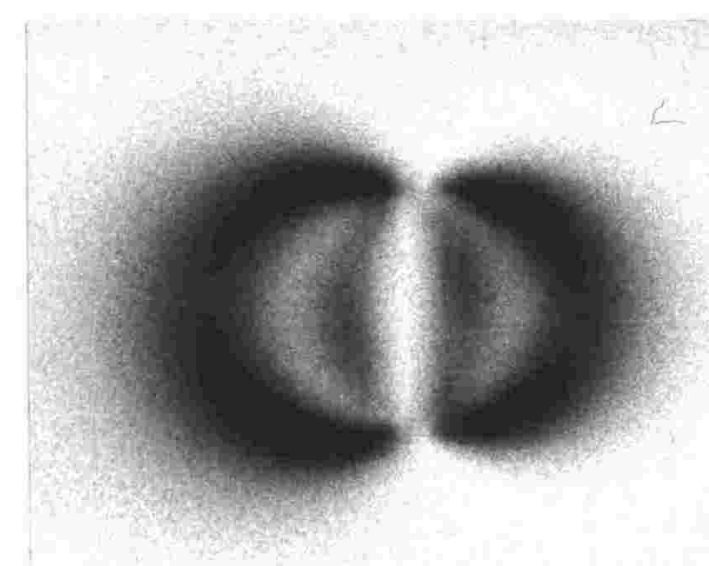

$\mathrm{d}=480 \AA$

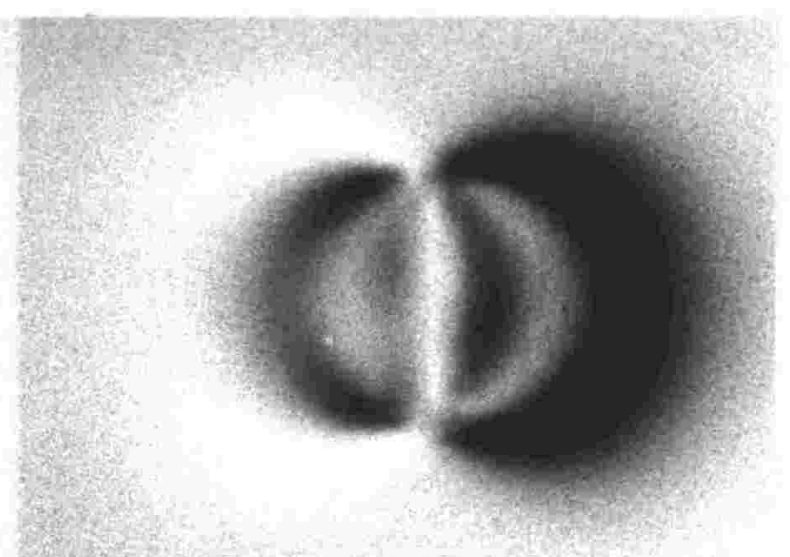

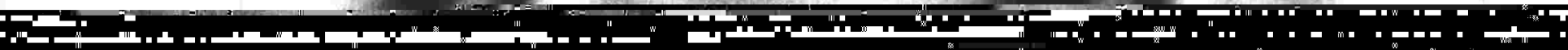

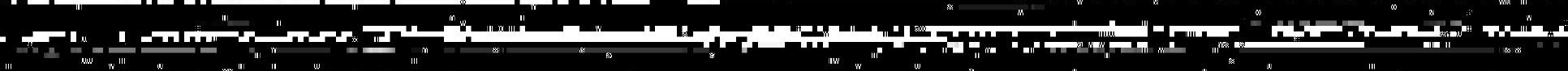

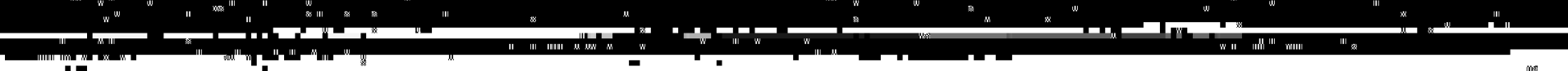
.

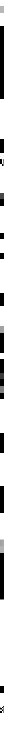




$$
\mathrm{d}=980 \AA
$$

$$
d=1080 \AA
$$
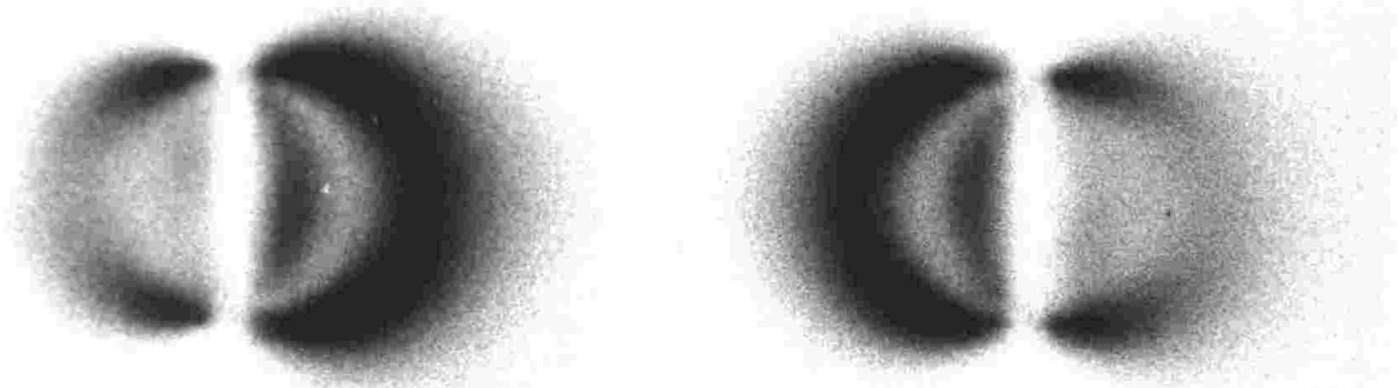
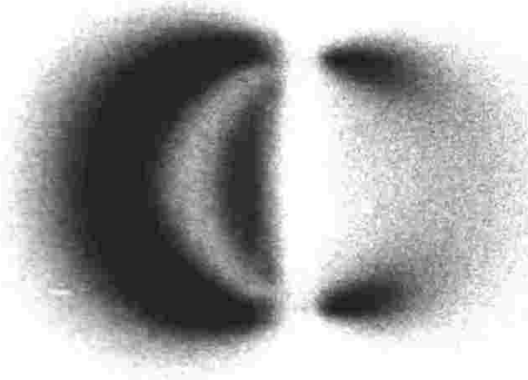

$d=|| 80 \AA$

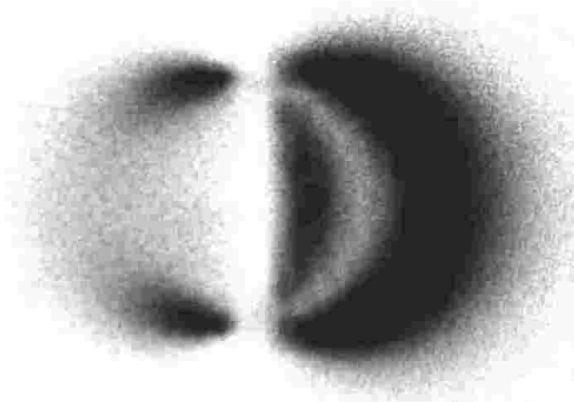

$\mathrm{d}=1380 \AA$

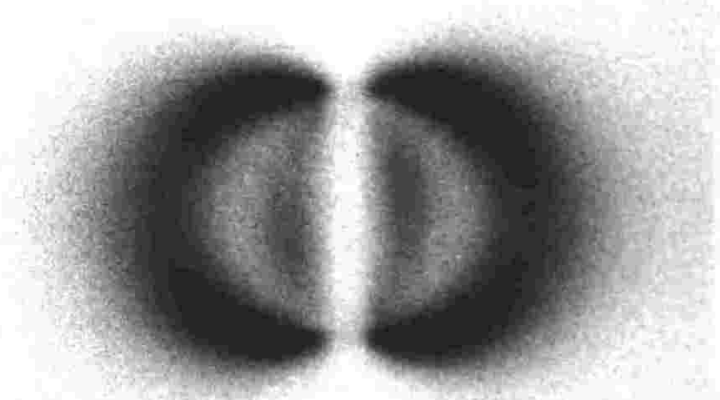

$d=1280 \AA$

Figure 4.12 (a) 


$$
\mathrm{d}=1580 \AA
$$

$$
\mathrm{d}=1680 \AA
$$
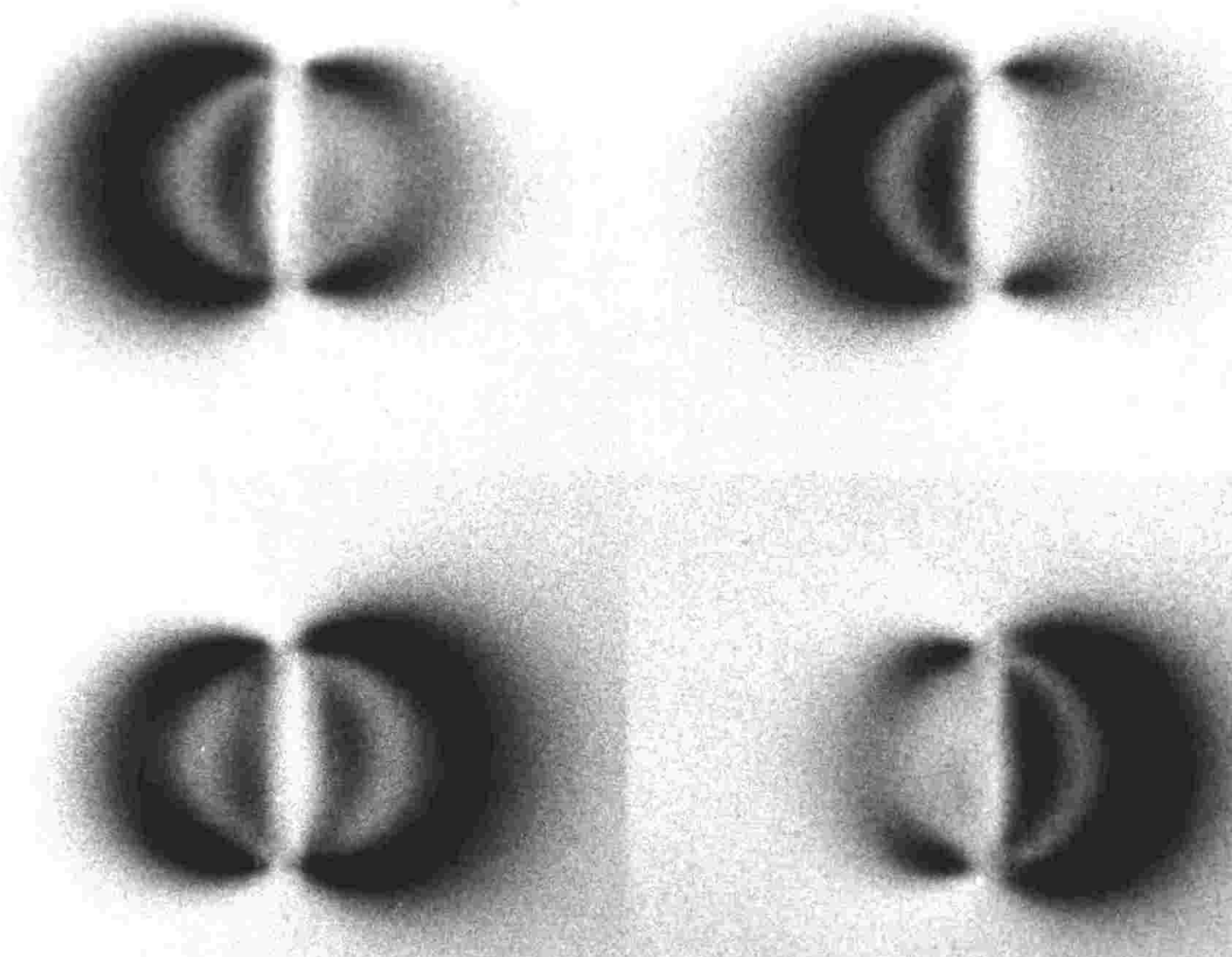

$$
d=1780 \AA
$$

$$
d=1880 \AA
$$

$$
d=1980 \AA
$$

$$
\mathrm{d}=2080 \AA
$$

Figure 4.12 (a) 


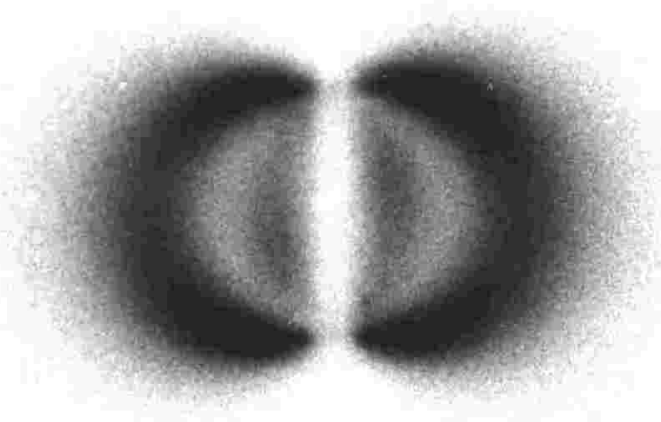

a

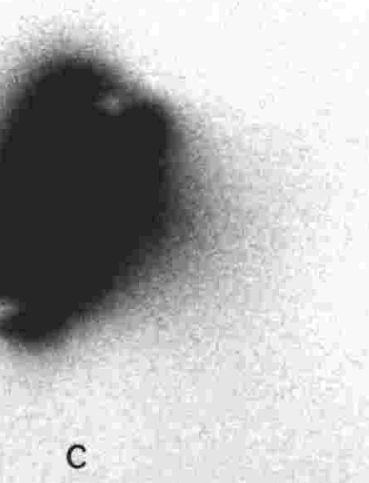

C

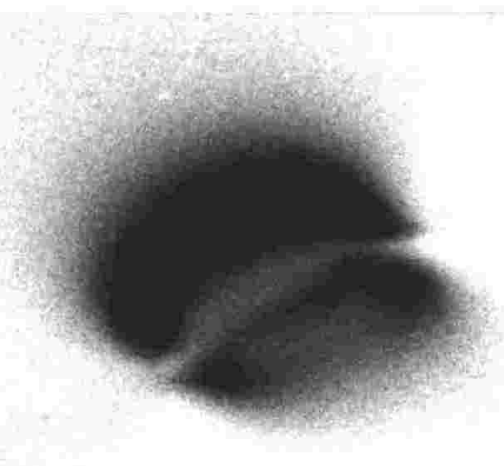

b

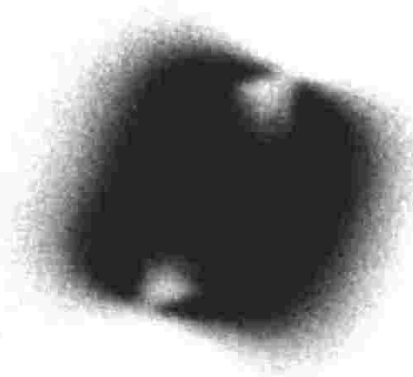

d

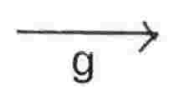

$400 \AA$

Figure 4.12: Comparison of experimental micrographs and simulations for an edge dislocation loop with $\mathbf{b}=0.5[1,-1,0]$ in copper. The experimental image labelled as (a) should be matched against the simulations labelled as (a) and similarly for parts (b), (c) and (d). The foil normal $(\mathbf{F})=[6,7,11]$ for all the simulations. The other parameters are:

(a) $\mathbf{B}=[2,2,3], \mathbf{g}=[2,-2,0]$, a foil thickness of $5.5 \xi_{\mathrm{g}}$, w $=0.0$ and an anomalous absorption ratio of 0.1 . The depth of the loop " $\mathrm{d}$ " is varied in steps of $100 \AA$.

(b) $\mathbf{B}=[4,5,4], \mathbf{g}=[2,0,-2]$, a foil thickness of $5.5 \xi_{\mathrm{g}}, \mathrm{w}=0.0$ and anomalous absorption ratio of 0.1 .

(c) $\mathbf{B}=[11,2,9], \mathbf{g}=[1,-1,-1]$, a foil thickness of $8.6 \xi_{\mathrm{g}}$, w $>0.0$ and an anomalous absorption ratio of 0.07 .

(d) $\mathbf{B}=[15,2,13], \mathbf{g}=[-1,1,1]$, a foil thickness of $8.6 \xi_{\mathrm{g}}$, $\mathbf{w}>0.0$ and an anomalous absorption ratio of 0.07 .

The foil thickness in terms of $\xi_{\mathrm{g}}$ has been scaled with $\mathbf{g}$ as was done in section 4.6.1. 
antiparallel) to the loop normal. The axis of the glide cylinder will be parallel to the Burgers vector. If the loop normal $\mathbf{n}$ is rotated away from $\mathbf{b}$ the axis of the glide cylinder will follow n. Locally this rotation of $\mathbf{n}$ can be thought of as movement of the small segments of the prismatic dislocation loop along the surface of the glide cylinder. Some of the segments move in different senses along the glide cylinder. Other segments rotate around and grow longer.

The axis of the glide cylinder can be measured by using stereographic projections. In figure 4.12 the glide cylinder axis found experimentally is $[1,-1,0]$. Therefore the loops must have had loop normals and Burgers vectors of $[1,-1,0]$ or $[-1,1,0]$ when they were still mobile as none of these loops have moved out of the row. There are some loops near the row which could have been squeezed out of the row after their $\mathbf{n}$ was rotated before the entire row reached equilibrium.

In figure 4.12 images of an edge type loop is simulated with a $\mathbf{b}$ and $\mathbf{n}$ equal to [1, $-1,0]$. The g's used are shown in the figure caption of figure 4.12 . The simulations of figure 4.12 (a) and (b) confirm that $\mathbf{b}=0.5[1,-1,0]$. Varying w gives a more convincing fit for figure $4.12(\mathrm{a})$.

The loops in figure 4.12 are about $330 \AA$ in diameter which means that the loop extends over several layers. The layer structure construction, referred to in chapter six as a means of minimising the amount of computation required to simulate small dislocation loops, is therefore not valid. The simulations of large loops shown in figure 4.12(a) shows there is some periodicity in the images as the depth of the loop is varied. The simulations represented in the other parts of figure 4.12 assume the loop is positioned approximately in the middle of the foil. This can be compared to the experimental micrographs, where the glide plane is inclined acutely to the foil surface, so the loops are at different depths. The depth of the loops increases as the distance from the bubble is increased. The simulations for increasing depth, $\mathrm{d}$, can be matched against the loops that are increasingly further away from the bubble.

To differentiate between whether a loop is of vacancy or interstitial type requires micrographs taken with opposite $\mathbf{g}$ or (g.b) $\mathrm{s}_{\mathrm{g}}$ as shown in Maher and Eyre [57]. Images (c) and (d) are $a \pm g$ pair with $w>0$. Simulations of interstitial and vacancy loops for the imaging parameters of figure 4.12 (c) and (d) are presented in figure 4.13 to verify the 
interstitial nature of the punched out loops. The Maher and Eyre rules require that the loop normal is at an acute angle to the beam directions of figure 4.12 (c) and (d). (The beam direction is taken as antiparallel to the direction of motion of the incident electrons.) The Burgers vector is parallel to the loop normal for an interstitial loop. Therefore $\mathbf{b}=0.5[1$, $-1,0]$ was used in the simulations and not $\mathbf{b}=0.5[-1,1,0]$. The $w=0$ simulations of figure 4.13 (a) and (b) will be identical for $\pm \mathbf{b}$. Simulation of a vacancy loop in $-\mathbf{g}$ and $w=$ 0.5 conditions gave approximately the same image as obtained from an interstitial loop in $+\mathrm{g}$ and $w=0.5$ conditions as expected from the Maher and Eyre rules.

\subsubsection{Spacing of Punched Loops}

Measurement of the spacings between loops and the loop diameters in figure 4.12 shows that the $\rho$ parameter of the Bullough and Newman theory [112] actually decreases for the loops furthest from the bubble as shown in figure 4.14. The Bullough-Newman theory predicts that $\rho$ should increase because of the backstress of the loops that were punched out earlier. Thus the assumptions of the Bullough-Newman theory do not apply in the present case. It should be noted that the theory does not allow for loops of different diameters and does not take into account the role of helices in forming rows of loops. The approximately regular spacing of the eight loops between loops 4 and 12 is interesting and may indicate some blocking of dislocation movement on the glide cylinder owing to the presence of defects in the matrix. 


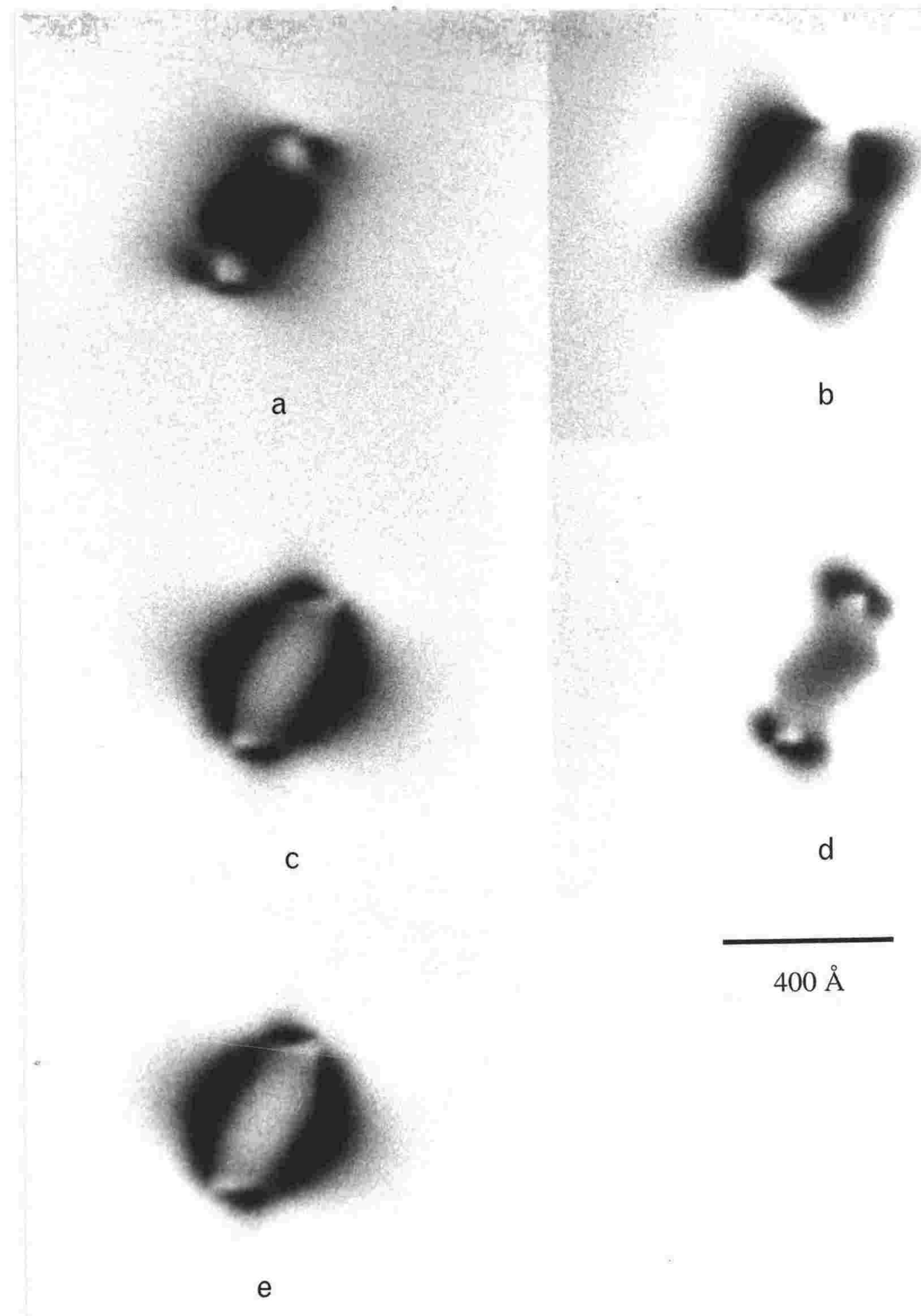

Figure 4.13: Simulations with imaging parameters as for figure 4.12 (c) and (d) with variable (g.b) $\mathrm{s}_{\mathrm{g}}$ for both vacancy $(\mathbf{b}=0.5[-1,1,0])$ and interstitial loops $(\mathbf{b}=0.5[1,-1$, 0]).
(a) $\mathbf{g}=[-1,1,1], \mathbf{b}=0.5[1,-1,0], \mathrm{BF}$ and $\mathrm{w}=0.5$ ("inside contrast").
(b) $\mathbf{g}=[-1,1,1], \mathbf{b}=0.5[1,-1,0], \mathrm{BF}$ and $\mathrm{w}=-0.5$ ("outside contrast").
(c) $\mathbf{g}=[1,-1,-1], \mathbf{b}=0.5[1,-1,0], \mathrm{DF}$ and $\mathrm{w}=0.5$ ("outside contrast").
(d) $\mathbf{g}=[1,-1,-1], \mathbf{b}=0.5[1,-1,0], \mathrm{BF}$ and $\mathrm{w}=-0.5$ ("inside contrast").
(e) $\mathbf{g}=[-1,1,1], \mathbf{b}=0.5[-1,1,0], \mathrm{BF}$ and $\mathrm{w}=0.5$ ("outside contrast"). 
× $\operatorname{spacing}(\AA)$

ه $\operatorname{diameter}(\AA)$

a rho

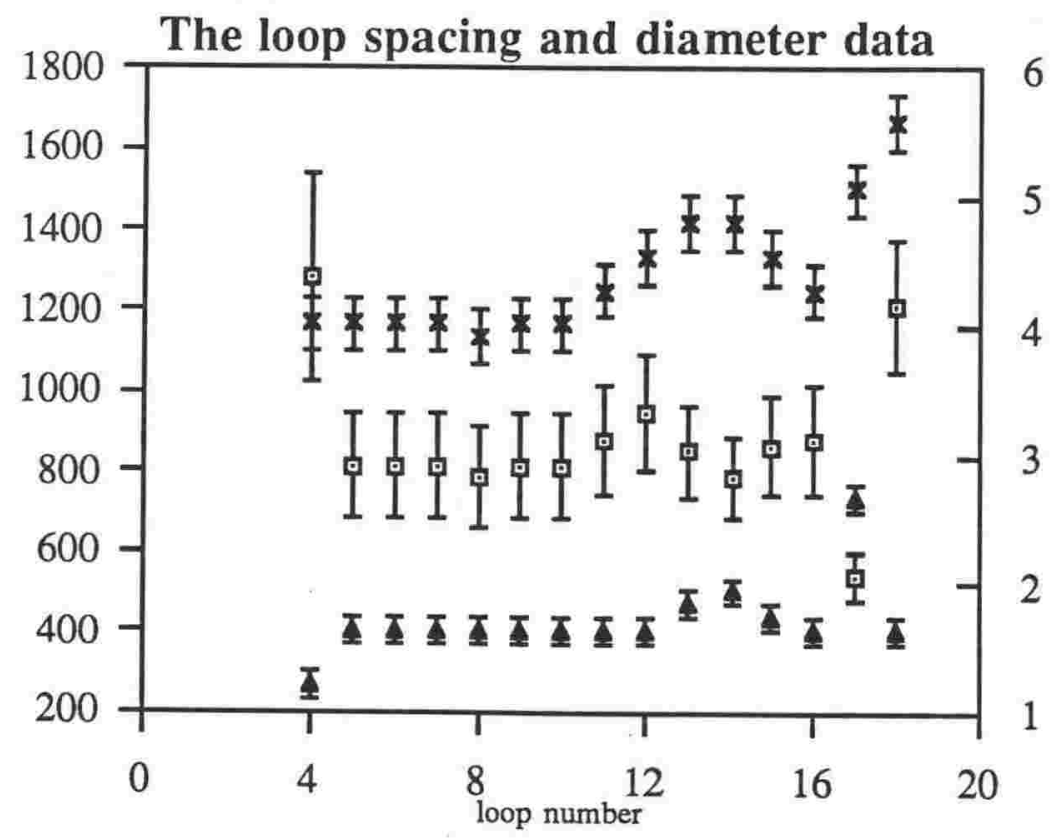

Figure 4.14: The loop spacing and diameter data taken from figure 4.12 (a). The left hand vertical axis is for the spacing and diameter data in $\AA$ and the right hand vertical axis is for $\rho$ (=loop spacing/loop diameter).

\subsubsection{Helices}

Figure 4.15 shows a helix, probably emitted from a bubble, that has not collapsed to form a row of loops via the prismatic punching sequence of Seitz [119]. No simulations of this image were done as the displacement field is unknown.

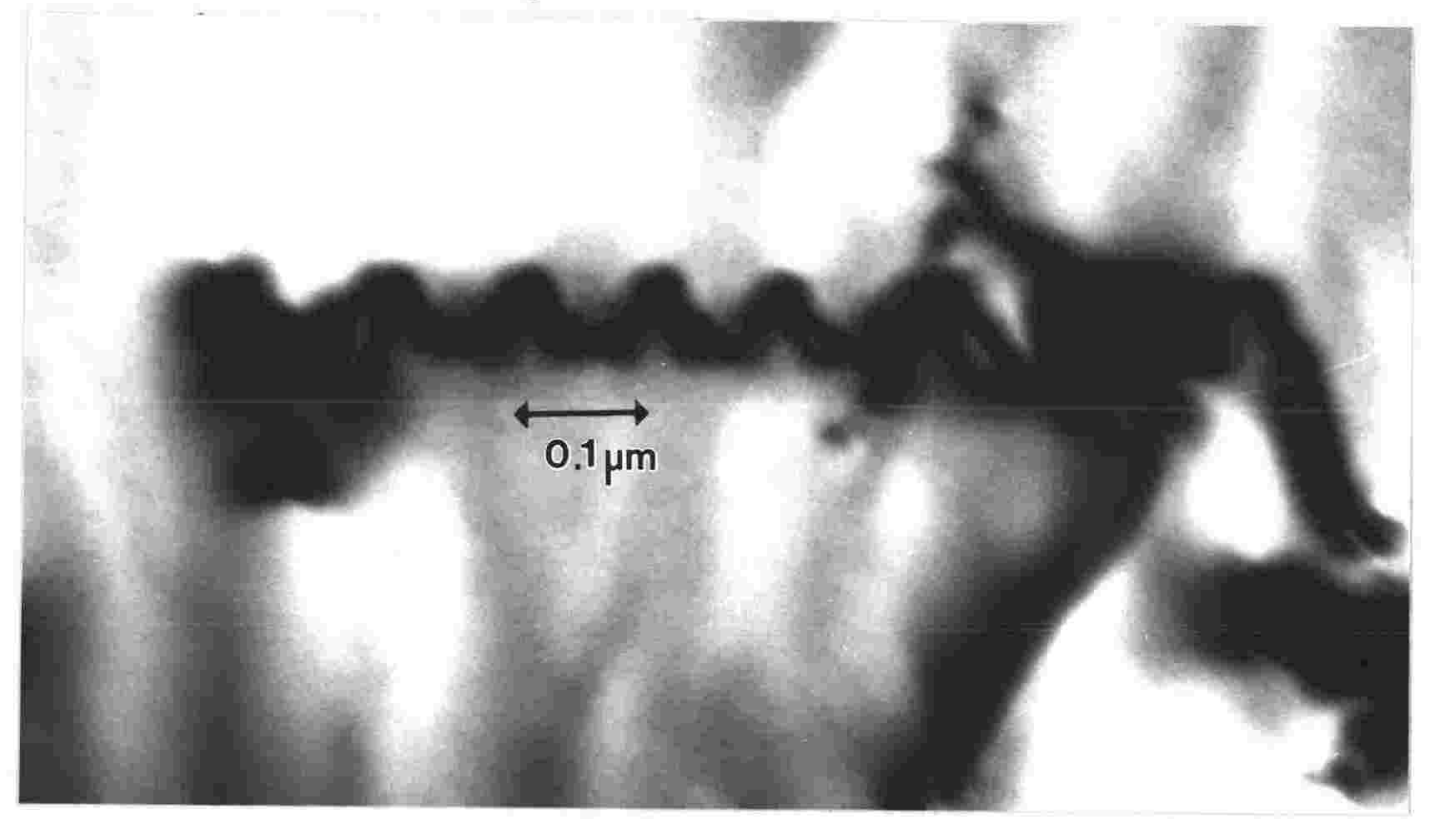

Figure 4.15: Helix punched by a bubble in quenched copper. 


\subsection{HREM, Lattice Fringe Pictures and Multislice Simulations}

The EMS programs of Stadelman can be used to generate high resolution images of bubbles. Supercells are created by a separate Fortran program that EMS then uses to generate the phase grating of the slices the electron wave is to pass through, and to do the multislice propagation. The number of iterations with any one supercell phase grating can be specified. Therefore the diffraction from complex structures can be simulated by varying the supercell. M. Flueli [186] has used EMS to simulate HREM images of particles such as gold islands on a thin film of amorphous carbon. Simulation of cavities or precipitates is similar from Babinet's principle as the Fourier transforms of the phase gratings will be similar.

M. Flueli [186] partially gives the code for generating the atom positions required in various facetted particles. Figure 4.16 shows perspective drawings of the different particles generated by these programs. Similarly the atom positions in a supercell used to simulate a facetted cavity can be found by looping over the perfect crystal atom positions in the supercell and removing those positions which match up with the atom positions in the facetted particle. A HREM simulation of a cavity at Scherzer focus is shown in figure 4.17 (a) that was generated using the atom positions shown in the supercells in figure 4.17 (b). For comparison with the HREM simulation of figure 4.17 , figure 4.18 shows some lattice fringes in copper that are typical of those obtained with the TEM at Victoria University. 


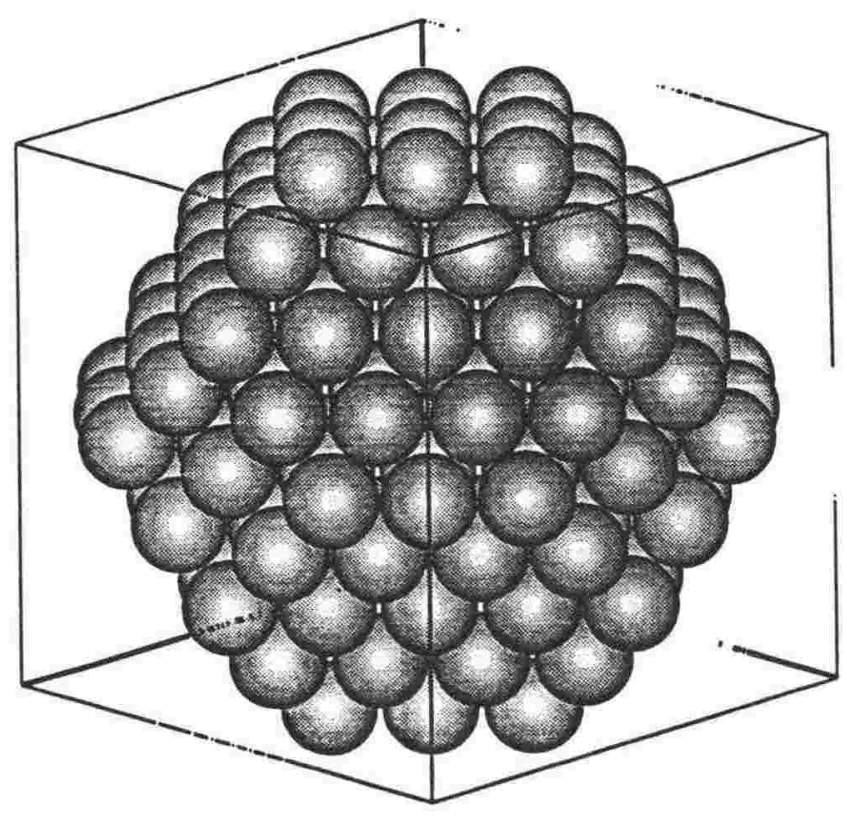

Super-cell : superx / [ 9, 9, 4]

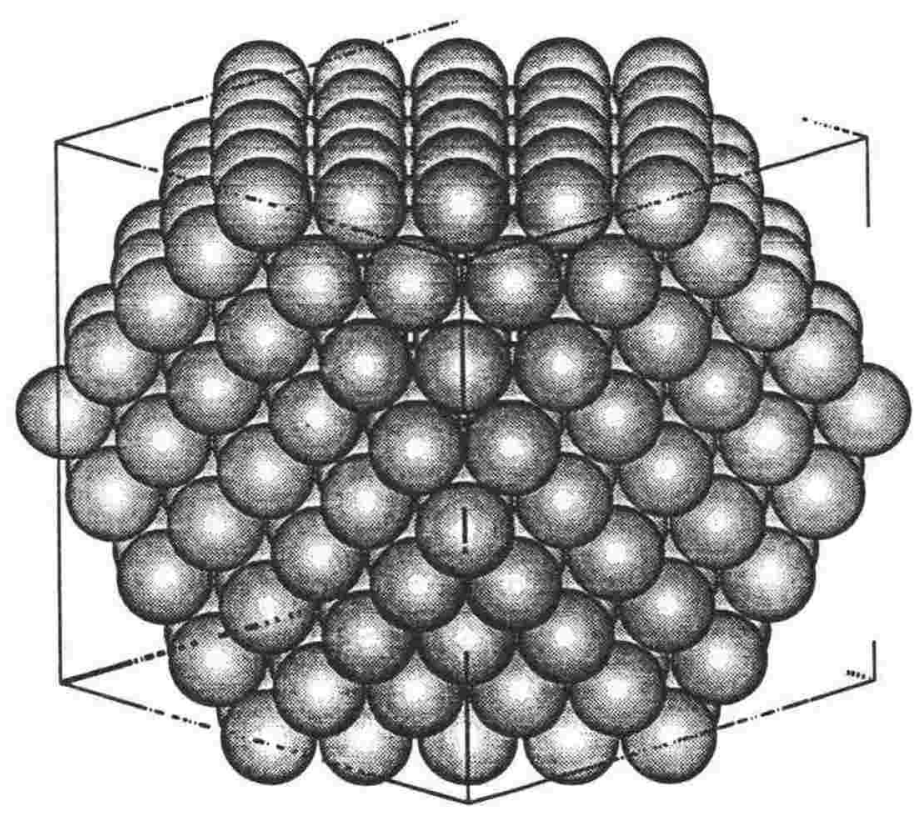

Figure 4.16

Super-cell : superx / [ 9, 9, 4] 


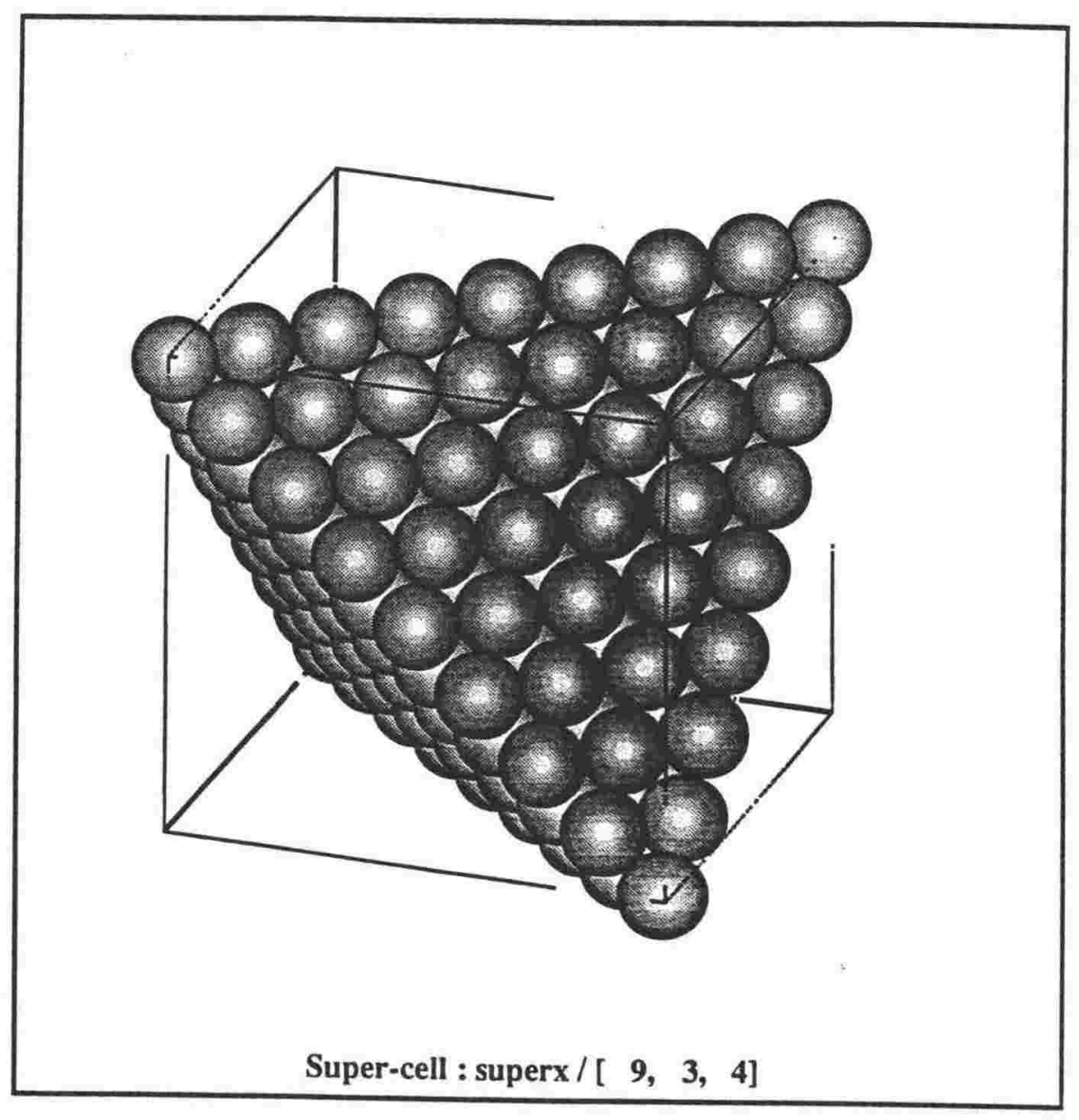

Figure 4.16: Facetted particles whose atom positions were used to generate facetted voids. $4-60$ 


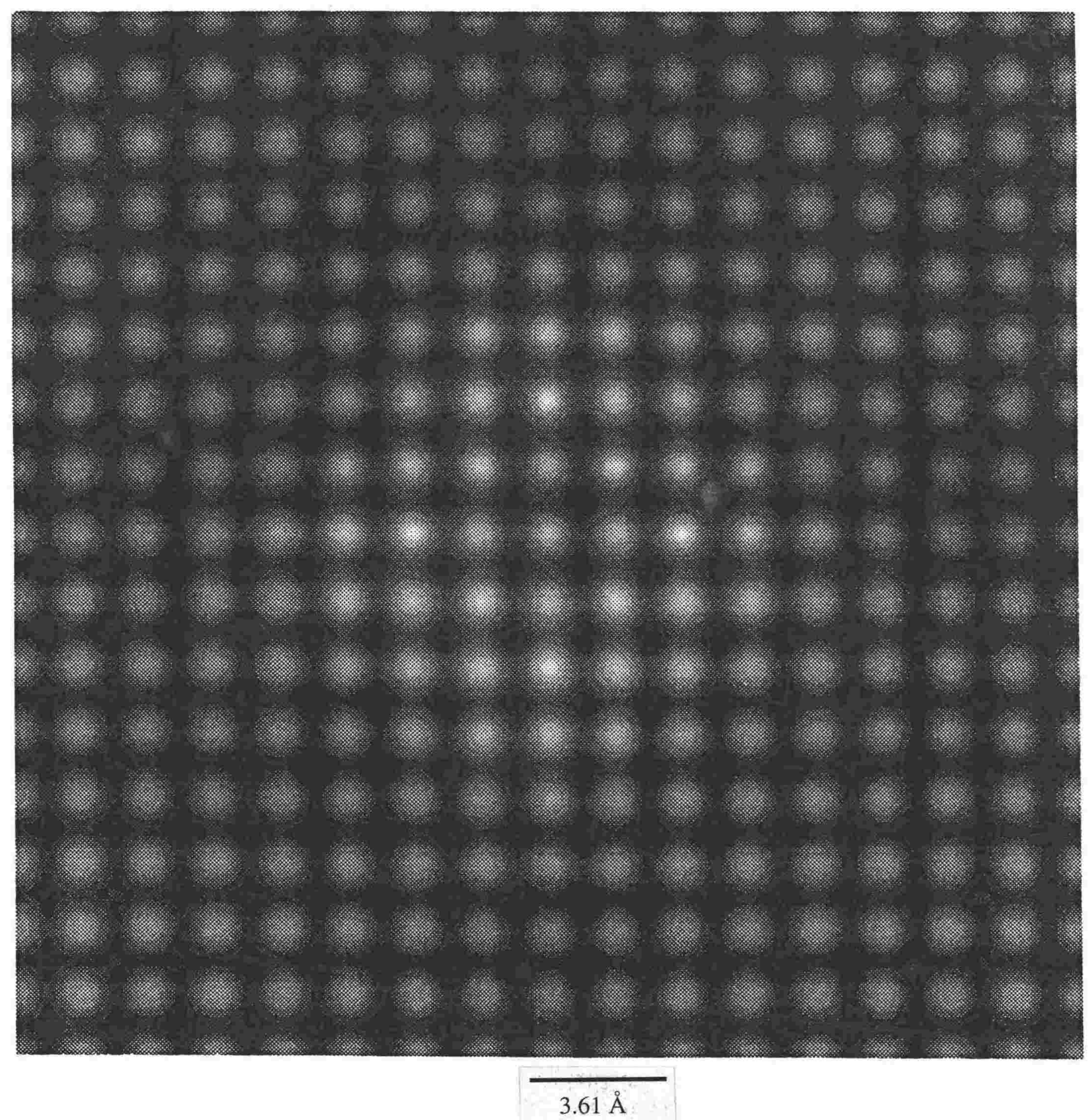

Figure 4.17

(a)

4- 61 

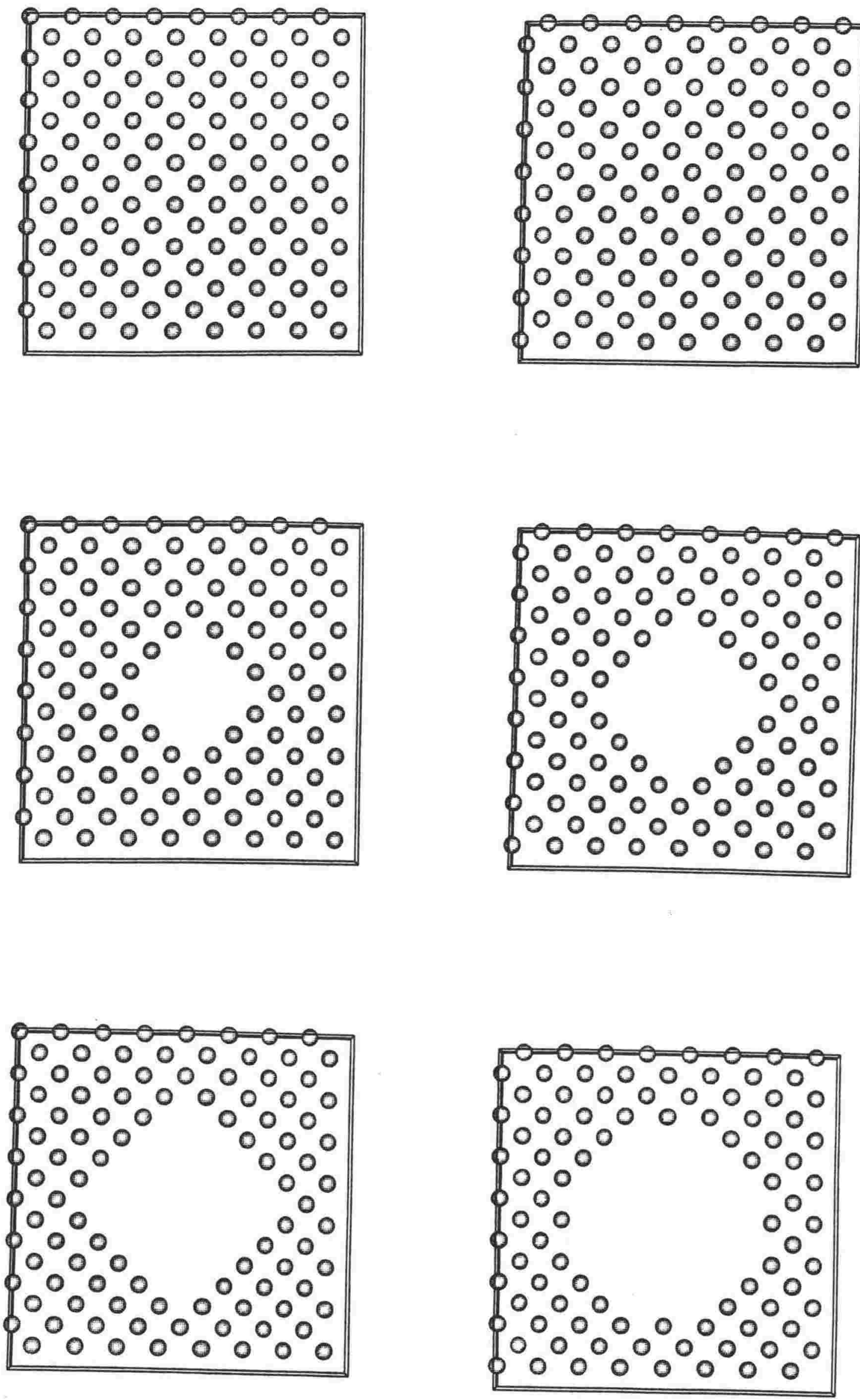

Figure 4.17 (b) 


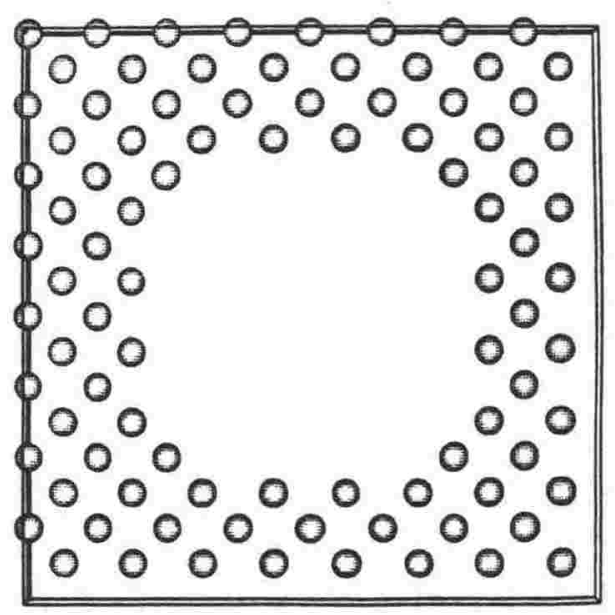

(b)

Figure 4.17: HREM simulation at Scherzer focus of an octahedral shaped cavity, with hexagonally facetted [111] type planes, in copper. The supercells used are shown in part (b) and the simulation is shown in part (a). The imaging parameters are: $\mathbf{B}=[001], \mathrm{C}_{\mathrm{s}}=$ $1.1 \mathrm{~mm}$, the electron energy is $300 \mathrm{keV}$, beam divergence $=0.6 \mathrm{mrad}$, defocus spread $=6$ $\mathrm{nm}$ and an aperture radius of $12 \mathrm{~nm}^{-1}$. The Scherzer focus for these parameters is $57 \mathrm{~nm}$. The dominant periodicities in the image is due to the [200] diffracted beam. The cavity has been embedded in a foil a few slices of perfect crystal larger than the cavity. 


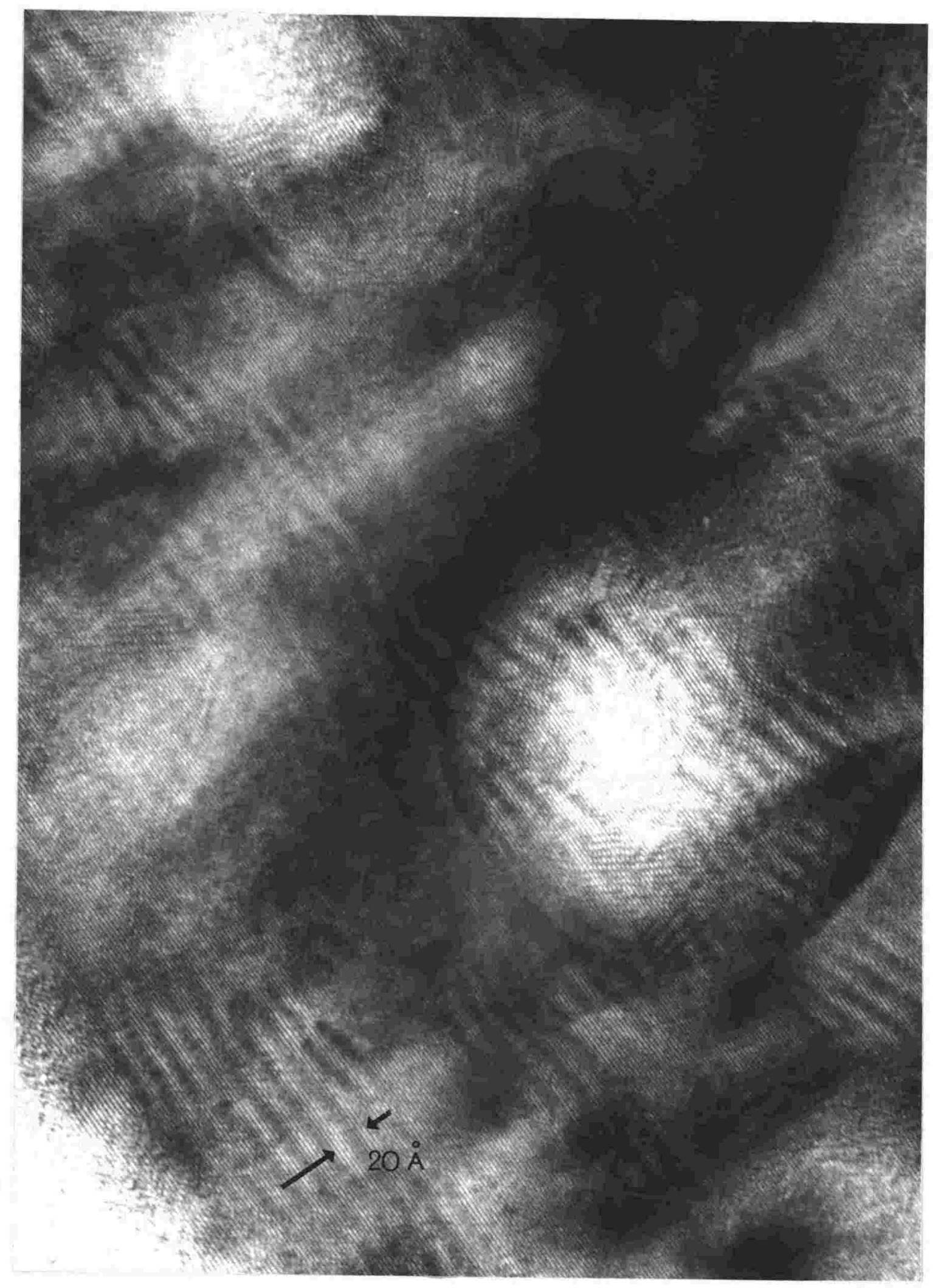

Figure 4.18: Crossed lattice fringes in a helium irradiated copper specimen. Lower magnification $\mathrm{BF}$ images of the same region showed up the bubbles more clearly than the lattice fringe picture. 


\subsection{Summary}

This chapter reviews the experimental techniques and equipment used to obtain the results on the TEM imaging of bubbles and dislocations in metals presented in this thesis. Two alternative methods were used to produce the defects in the specimens. One method was to expose the metal to one of several gases at high temperature and pressure and then quench to a low temperature to trap the gas in the metal. Quenching is a technique to create in a specimen a low density of bubbles and dislocations. The quenched specimens are much easier to examine in the TEM to gain experience in applying the image matching techniques to dislocation loops without the problems resulting from the overlapping of images as shown in section 4.6.2. A comparison of the spacings of the loops in quenched specimens with the Bullough and Newman theory showed the assumptions on which the theory is based did not apply in the case studied. The second method used was the ionimplantation of helium into the metal using a charged particle accelerator. In this case gas bubbles and dislocations are formed at high concentrations and the gas bubbles may order to form a gas-bubble superlattice.

The specimens are prepared for examination in the TEM by a variety of methods. Foils are mechanically polished, heat treated in vacuo and then bath electropolished to a high surface finish. The heat treatment consists of annealing in a furnace at temperatures sufficient to give both vacancies and self interstitials a significant mobility. This treatment reduces the density of dislocations and grows the grains in the polycrystalline specimens to about 0.1 to $1 \mathrm{~mm}$ across. The prepared foils are then loaded with gas either by quenching or irradiation.

Following gas loading the foils are punched to produce specimens that are discs with a diameter of $3 \mathrm{~mm}$. The discs are backthinned, usually by jet electropolishing in a Tenupol, to produce electron transparent specimens. Ion beam thinning with argon ions can be used to clean up the specimens or extend the thinned areas around a hole formed by the electropolishing. Some long straight dislocations occurring in lightly deformed blank specimens have been examined in section 4.6.1 to learn the experimental techniques for imaging dislocations in the TEM. Micrographs of specimens that have been subjected to ion-implantation will be discussed in chapter seven. 


\section{CHAPTER 5: SIMULATIONS; BUBBLE IMAGES AND DIFFRACTION}

\subsection{Introduction}

\subsubsection{Introduction}

In this chapter the diffraction from bubble structures in metals is considered in order to understand better experimental diffraction patterns and images. The simulated appearance of a bubble or stack of bubbles is used to identify the effects of changes in a range of parameters. The sample parameters considered include the thickness of the foil, the depth of the defects below the foil surface and the bubble pressure. The imaging parameters considered include the defocus, the diffracting vector and the excitation error. Experimental micrographs are compared to the simulations to obtain the real radius of both a bubble and a facetted cavity. In addition to simulating images of bubbles, the superlattice spots obtained in selected area diffraction patterns are simulated in an attempt to deduce information about the ordering of bubbles into domains.

Whilst most of the work presented in this chapter is similar to that of other authors in that it uses the same theoretical techniques, it is original work because the specific bubble structures considered have not been treated in this way elsewhere. The section on electron diffraction from bubble arrays leading to superlattice spots is unique both in this sense and in using a different theoretical formalism from that of other authors. Where possible, the published simulations of other authors were first reproduced to check the accuracy of the programs developed here. A problem in doing this is that sometimes not all of the parameters necessary to perform the simulations are explicitly stated in their publications. However, where comparisons could be made, good agreement was obtained between the results of programs written for this thesis and published results.

The more accurate the measurement of the bubble radius the better the experimental data for testing the theories of bubble growth and ordering. Measurements of the bubble radii, for example, allow an estimate to be made of how much of the total implanted helium is contained in the visible bubbles. The helium not contained in bubbles is thought to reside 
in the copper matrix or in small gas bubbles lying below the resolution limit of the microscope. (A variety of experiments based on nuclear reaction depth profiling have shown that essentially all the implanted helium is retained in the implanted layer). The pressure is an important parameter in some proposed mechanisms of superlattice formation such as those based on dislocation punching from bubbles [7]. It can be deduced from an accurate measurement of the bubble radius under the assumption that the bubble is at equilibrium pressure. Most of the theories of superlattice formation allow predictions to be made of the ratio of bubble spacing to bubble radius. The average spacing of bubbles can be found experimentally from measurements of the separation of the superlattice spots in a selected-area electron diffraction pattern (SADP) [45]. In addition to its simplicity, another advantage of using diffraction patterns rather than direct images is that it possible to remove the effects of defocus.

Table 5.1 shows the differences between the types of defects discussed in this chapter. The columns indicate whether or not the defects have a surrounding displacement field and contain gas. If a defect contains gas the mean potential inside the defect is different from that in vacuum. Some authors use the term "cavity" interchangeably with the term "void". However, these two types of defects are distinguished in table 5.1 in the way they are used in this thesis.

\begin{tabular}{|c|c|c|}
\hline Defect Type & Contains gas? & Displacement Field? \\
\hline Cavity & No & No* \\
\hline Void & No & Yes (radially inward) \\
\hline Equilibrium bubble & Yes & No \\
\hline Overpressured bubble & Yes & Yes (radially outward) \\
\hline
\end{tabular}

Table 5.1: The distinguishing characteristics of the different types of defects considered in this chapter. $(*$ In some simulations the displacement field is neglected to simplify the analysis).

Investigations of how the bubble structure varies with depth, dose and irradiation conditions is facilitated if the TEM imaging parameters are deconvoluted from the experimental micrographs. Defocus is the important imaging parameter when looking at 
images of bubbles. The defocus dependence of the appearance of isolated cavities that are strain-free was studied by Rühle and Wilkens [83]. Broadly speaking, isolated cavities appear as black (i.e., below the background intensity) when over-focussed and white (i.e., above the background intensity) when under-focussed. Rühle and Wilkens [83] deduced the cavity radii from the positions of the Fresnel fringes in direct TEM micrographs. They suggest that when the cavities are less than or about $10 \AA$ in radius, measurements of cavity radii could be more than $10 \%$ in error.

Previous work on the TEM contrast from defects similar to gas bubbles has been reported in the literature $[83,84,88,169,187,188]$. The defects examined are voids [169], cavities [84 and 88], spherical inclusions and coherent spherical precipitates [187 and 188]. This previous work on the electron scattering from such defects took a different approach from the present work. Rühle and Wilkens [83] used an analytical approach at strong two-beam conditions to obtain their defocussed image profiles of isolated cavities. Foreman et al. [88] assumed kinematic scattering conditions to study the diffraction of electrons from isolated, strain-free, facetted cavities. In simulations they obtained defocussed images as well as one-dimensional intensity profiles across the cavities. Both groups found that the diffraction was independent of the depth of the cavity because the effects of a non-zero excitation error were ignored. Ashby and Brown [187 and 188] considered the in-focus contrast and contour plots of the intensity of the image formed by the electrons scattered from spherical inclusions. Their inclusions were large, isolated from one another and had a surrounding strain field. They also considered the dependence of the results on the depth of the defect, the excitation error and the strength of the strain field (but the displacement field was always radially outward). E Johnson et al. [84] were the first to make a stack of cavities the subject of simulations of defocussed images and profiles. They took the cavities to be strain-free and used numerical integration to calculate the beam amplitudes.

In this chapter many of the parameters considered by the above authors in isolation are combined together to characterise the appearance of stacks of small gas-bubbles. The bubbles can have a strain-field associated with them and the results will in some situations depend on the depth of the defect. Images of these defects exhibit contrast resulting from strain, thickness and structure factor effects. The images are strongly dependent on the 
amount of defocus. Calculations based on the theory of electron scattering at two-beam conditions are used to find the dependence of the apparent bubble radius on the above imaging conditions. The approach used here is more appropriate to helium bubble superlattices than those used by previous authors who concentrated on defects of larger radius with smaller changes in mean potential between the cavity and the matrix.

Two different formulations of the theory of electron diffraction are used in this chapter. In the first the effects of strain are neglected. This allows the wave function at the exit surface of the foil to be generated analytically from the Howie-Whelan differential equations of section 2.1. The wavefunction is then defocussed using the theory of Rühle and Wilkens [83]. In the second approach the effects of a non-zero displacement field (R) are included. This requires the differential equations to be numerically integrated. The first method was used initially because it is simpler, faster, and perfectly adequate for an equilibrium bubble.

The extinction distances are important as they set the relative scale of the bubble radius in the Howie-Whelan differential equations. If experimental micrographs are to be interpreted with these equations then the bubble radius must be converted into a fraction of an extinction distance. This is because the form of the factor in the scattering matrix of the bubble that phase shifts the diffracted $\mathrm{S}$ beam requires the radius to be specified in terms of $\xi_{\mathrm{g}}$ if $\mathrm{w}$ is non-zero (references 83 and 84 , equations 2.32 and 2.38 , and the comment at the end of section 2.2.3). Authors such as E Johnson et al. [84] do not specify particular extinction distances in their theoretical intensity profiles. They assume that all distances have already been converted into fractions of the extinction distance. However the phase changes caused by the change in mean potential from the matrix to the equilibrium bubble is incorporated by multiplying the wavefunctions passing through the bubble by a phase factor of the form exp[-i $x$ (the change in the wavevector) $\mathrm{x}$ radius]. The change in the wavevector is a function of the mean potentials and the radius must be specified in $\AA$ for it to be substituted into this equation. If the radius is specified as a fraction of an extinction distance but the extinction distance is not given, then this phase factor cannot be calculated. Therefore the work of E Johnson et al. [84] is of limited use for making detailed comparisons between experiment and theory for particular metals, bubble radii and gas pressures. 
The extinction distances used throughout this chapter are shown in table 5.2. (The temperature dependence of the extinction distances results from the Debye-Waller factor in equation 2.4).

\begin{tabular}{|c|l|l|l|}
\hline $\mathbf{g}$ & $\xi_{\mathrm{g}}($ at $0 \mathrm{~K})$ & $\xi_{\mathrm{g}}($ at $293 \mathrm{~K})$ & $\xi_{\mathrm{g} /} \xi_{\mathrm{g}}^{\prime}$ \\
\hline$[111]$ & 291.0 & 297.6 & 0.07 \\
\hline$[200]$ & 324.5 & 334.3 & 0.08 \\
\hline$[220]$ & 439.3 & 466.4 & 0.1 \\
\hline
\end{tabular}

Table 5.2: Extinction distances, in $\AA$, and anomalous absorption ratios for $\mathrm{Cu}$ and $100 \mathrm{keV}$ electrons. The anomalous absorption ratios are taken from the literature, as discussed in chapter 2.

An image of a bubble is strongly affected by many parameters. In this chapter the in-focus contrast at the centre of an equilibrium bubble embedded in a copper matrix is first characterised. Starting with section 5.1, the parameters varied, in the order in which they are presented, are foil thickness, excitation error and depth of the bubble below the surface of the foil. In section 5.2 contrast profiles through the centre of a bubble are investigated. The parameters varied are, in order, defocus, bubble radius, strain (i.e. bubble pressure) and diffracting vector. In section 5.3 contrast profiles through the centre of a stack of equilibrium bubbles are investigated. The parameters varied are foil thickness, number of bubbles in the stack, excitation error and defocus. In section 5.4 the theoretical results in earlier sections, for a helium bubble, and the conclusions drawn from them are tested in a wider range of metals. Experimental through-focal series are compared to theoretical profiles in section 5.5 to determine the radius of a bubble. Facetted cavities are treated in section 5.6 with experimental images and profiles being compared to simulations in order to determine the geometry and size of a cavity. In section 5.7 superlattice spots are simulated to probe the number of bubbles in a domain (a patch of bubbles which are locally ordered). The appearance of a stack of bubbles is even more complicated but has crudely been used to probe the three-dimensional nature of the ordering between bubbles. 


\subsubsection{In-focus Contrast at the Centre of an Equilibrium Bubble}

It is easiest to first consider how the contrast at the centre of the image of an equilibrium bubble depends on the thickness of the foil. This section calculates this behaviour and the later sections will consider how the contrast of the entire image of the bubble varies with this and other parameters.

The in-focus intensity at the centre of the bubble image is not affected by the displacement field so the intensity in the centre will be the same for images of bubbles and voids of the same size. Ingram's calculations [169] for this case show the behaviour typical of this intensity. Voids of large radius $(\approx 80 \AA)$ are considered in that paper whereas the interest in this thesis is centred on small bubbles of $\approx 10 \AA$ radius.

The in-focus contrast, at the centre of an equilibrium bubble image, for an excitation error (w) that is zero (i.e. the crystal is tilted to exactly satisfy the Bragg condition), is determined by the thickness contrast alone. The variation in the intensity of the undiffracted transmitted beam as a function of depth, $\mathrm{z}$, below the foil surface is shown in the upper curve of figure 5.1. (Alternatively the curve gives the thickness dependence of the intensity of the [000] transmitted beam on the exit side of a foil). An electron passing through the centre of a bubble of diameter, $d$, will have a path length in the metal of $z-d$, where $z$ is the foil thickness. Away from the bubble image an electron will have a path $\mathrm{z}$. This allows the difference between the intensity at the centre of the bubble image $\left(\mathrm{I}_{\mathrm{c}}\right)$ and the background intensity at points outside the bubble image $\left(\mathrm{I}_{\mathrm{b}}\right)$ to be found for foils of different thickness. The lower curve in figure 5.1 shows this difference in intensity $\left(I_{c}-I_{b}\right)$ as a function of foil thickness $\mathrm{z}$ for the particular case of a $20 \AA$ diameter bubble with $\xi_{\mathrm{g}}=334.3 \AA$ (corresponding to strong two-beam conditions with $\mathbf{g}=[200]$ ). (Figure 5.1 would be at least approximately true for $\mathbf{g}=[220]$ and a bubble diameter scaled, by the ratio of the extinction distances, to $27.9 \AA$. The only differences will be due to the different anomalous absorption ratios for the two $\mathrm{g}$ values as shown in table 5.2). 


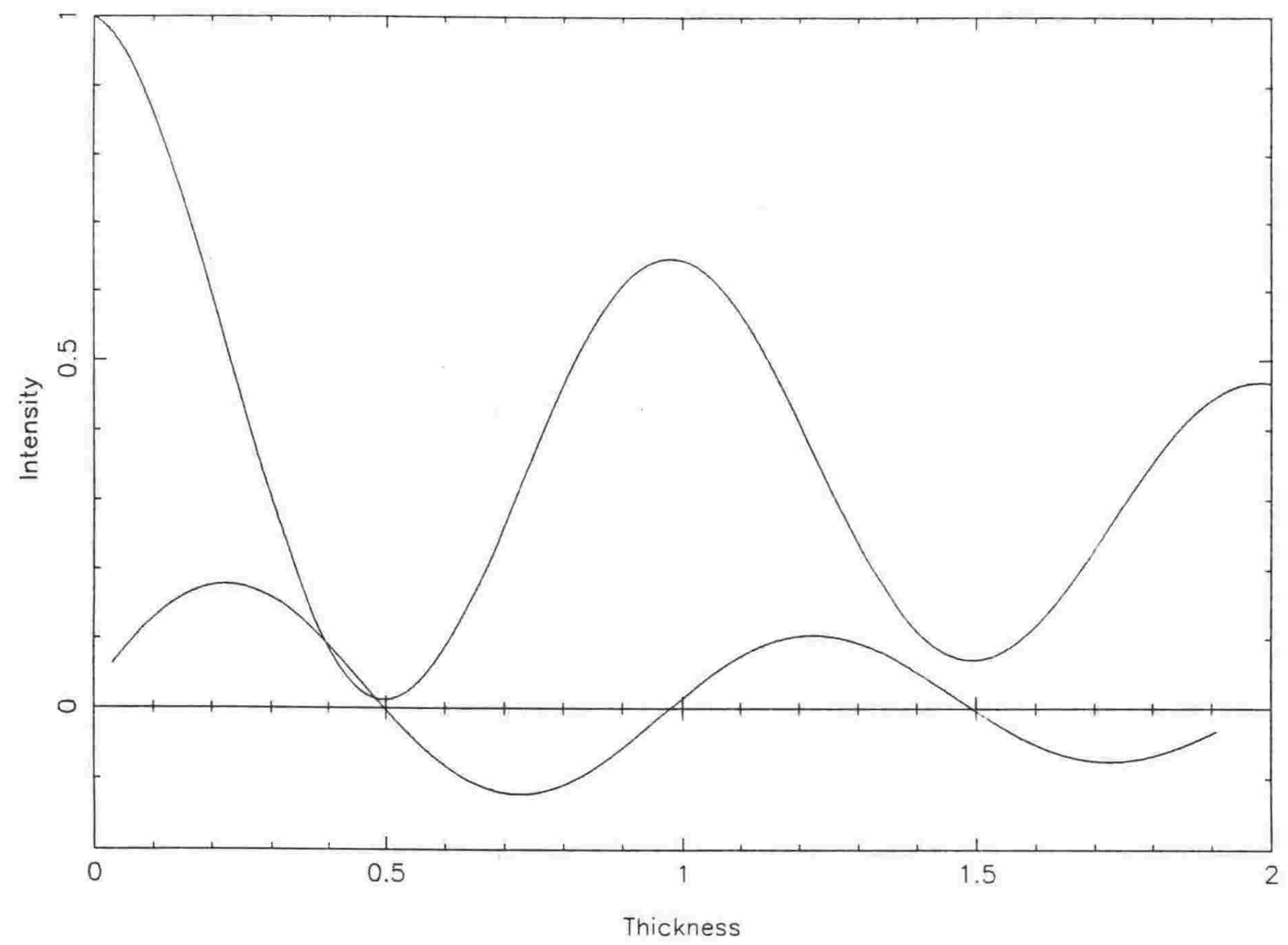

Figure 5.1: The top curve shows the in-focus, $w=0.0$, bright-field, intensity of the undiffracted [000] transmitted beam as a function of foil thickness. The thickness is in units of the extinction distance and the intensity is assumed to have unit magnitude at the top surface of the foil. Note that the intensity is oscillatory with minima at thicknesses corresponding to odd-integer multiples of half the extinction distance. The lower curve is the in-focus, bright field, contrast at the centre of the image of a $20 \AA$ diameter equilibrium bubble versus the thickness of the foil. In this case the intensity is expressed as the difference $I_{c}-I_{b}$ where $I_{c}$ is the intensity at the centre of the bubble image and $I_{b}$ is the intensity outside the (isolated) bubble image. This curve shows that the intensity at the centre of a bubble is strongly dependent on the thickness of the foil. 
Ashby and Brown [187 and 188] described qualitatively the contrast at the centre of the image of a cavity. However, for equilibrium bubbles containing helium gas and having sizes typical of those found in the present case the results of figure 5.1 are more useful both because they are quantitative and because they are based on parameters that are more appropriate to this case. The lower curve in figure 5.1 peaks in places where the original intensity versus $\mathrm{z}$ curve is varying the most rapidly. It shows that the contrast in an image of a bubble can oscillate from white (central image intensity above background) to black (central image intensity below background) as the thickness of the foil increases. Note that for $w=0.0$ the intensity does not depend on the depth of the bubble below the foil surface. The upper curve in figure 5.1 would have a periodicity of $\xi_{\mathrm{g}}$ if the beams were not attenuated by inelastic scattering. Because of attenuation the upper curve peaks at $331.5 \AA$, slightly before one extinction distance is reached.

\subsubsection{The Dependence of the Contrast at the Centre of an Equilibrium Bubble on the Excitation Error and the Depth}

If the specimen is tilted so that $\mathrm{w} \neq 0$ the diffraction becomes dependent on the depth of the bubble because the scattering matrix for the bubble is no longer the identity matrix. Consequently, the diffracted intensity depends on the wave function at the top surface of the bubble. The dependence on the depth of the bubble is strong for large bubbles as shown in figure 10.19 of Hirsch et al. [67]. The results for a $20 \AA$ diameter equilibrium bubble are shown in figure 5.2 for $\mathbf{g}=$ [200], three states of focus (in-focus, $2500 \AA$ overfocus and $5000 \AA$ overfocus) and six values of w. As can be seen in the figure the intensity is strongly dependent on the depth of the bubble below the foil surface. In some combinations of defocus and tilt (i.e. w) the intensity is altered enough by the depth to cause the image of a bubble to oscillate from black to white. As $w$ is increased the period of the oscillations in the transmitted intensity decreases while the amplitude initially increases before decreasing until, at $w=15.0$, the curves have very little dependence on depth.

Figure 5.3 shows the rocking curve of the diffraction from an isolated equilibrium bubble (a rocking curve is a plot of the transmitted intensity of any beam versus angle (measured by $\mathrm{w}=\mathrm{s}_{\mathrm{g}} \xi_{\mathrm{g}}$ ) as the crystal is tilted away from strong two-beam conditions). To 


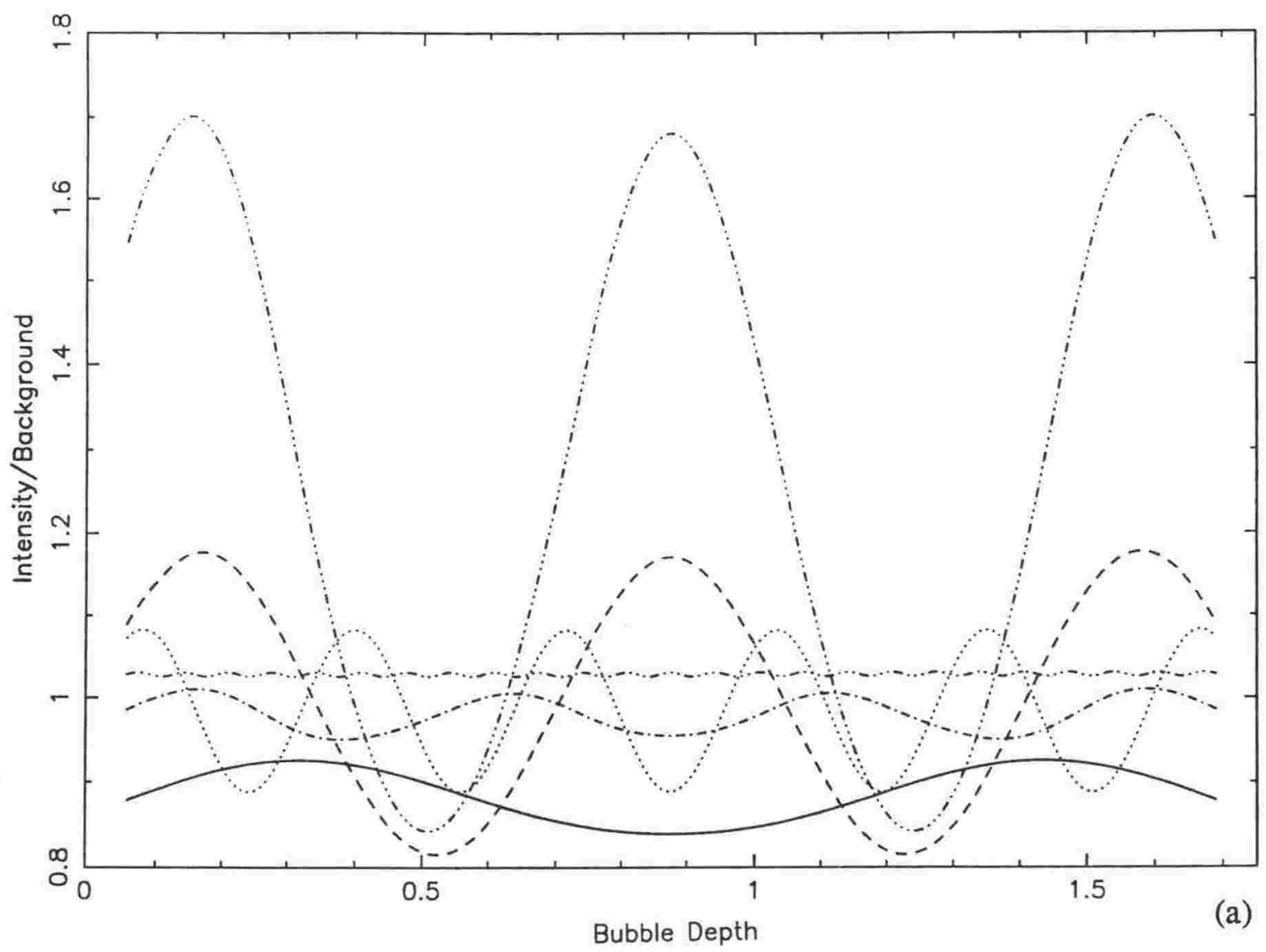

Transmitted Intensity/Background vs Bubble Depth

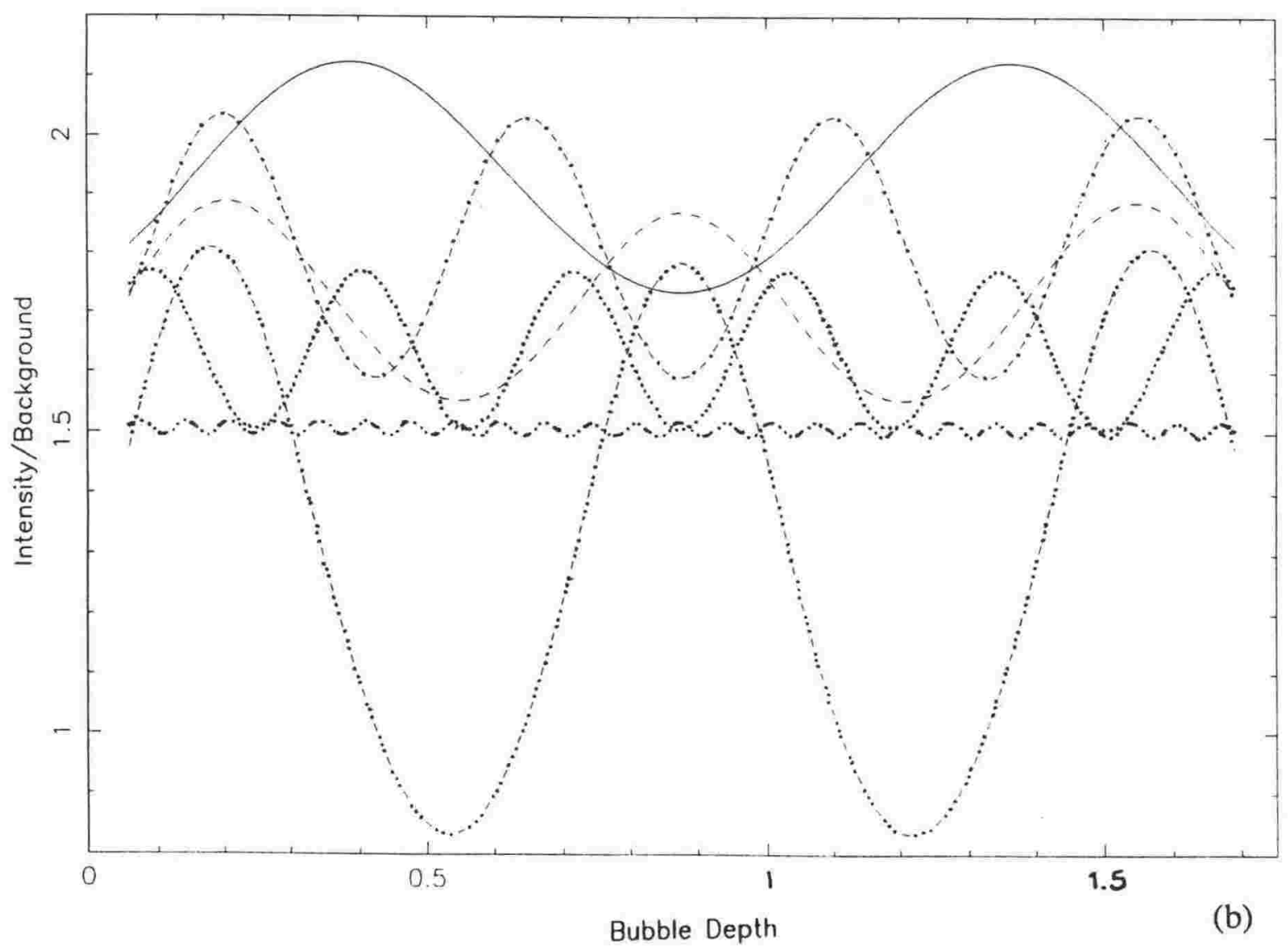

5 - 9 Figure 5.2 (caption on following page) 

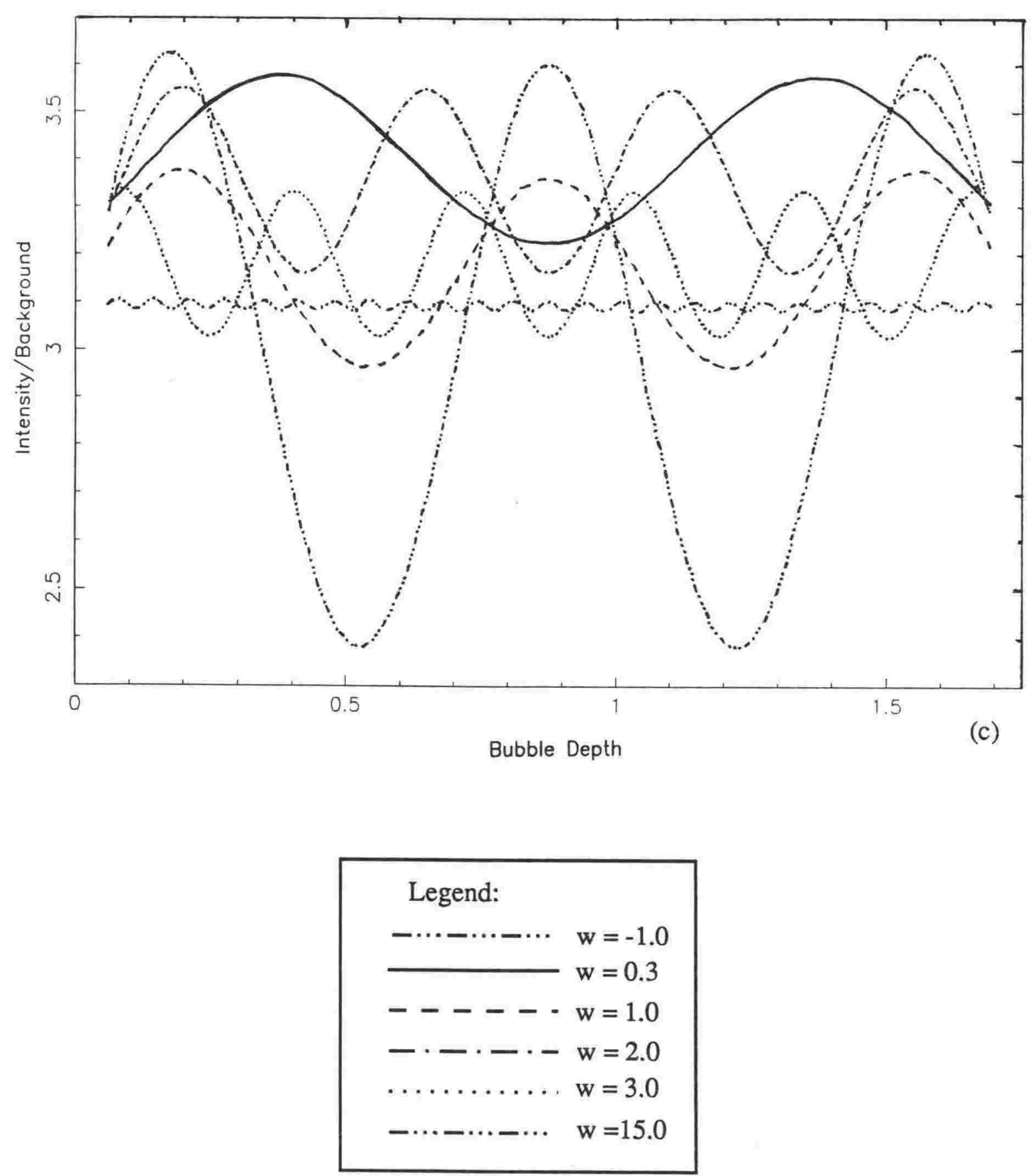

Figure 5.2: The intensity at the centre of the image of a $20 \AA$ diameter equilibrium bubble as a function of the depth of the bubble below the foil surface for $w=-1.0,0.3,1.0,2.0,3.0$ and 15.0 and $\mathbf{g}=$ [200]. The electron energy is $100 \mathrm{keV}$ and the foil thickness is equal to $1.75 \xi_{\mathrm{g}}$. The three figures are for: (a) in focus, (b) $2500 \AA$ overfocus and (c) $5000 \AA$ overfocus. Note the suppressed zero on the vertical scale. The curves show that under some conditions a bubble can change from black to white and back again as the depth of the bubble changes. The same symbol has been used in the curves for $\mathrm{w}=-1.0$ and $\mathrm{w}=15.0$ but the curves are readily distinguished because the curve for $\mathrm{w}=15.0$ is more rapidly oscillating. 
Transmitted Intensity vs w

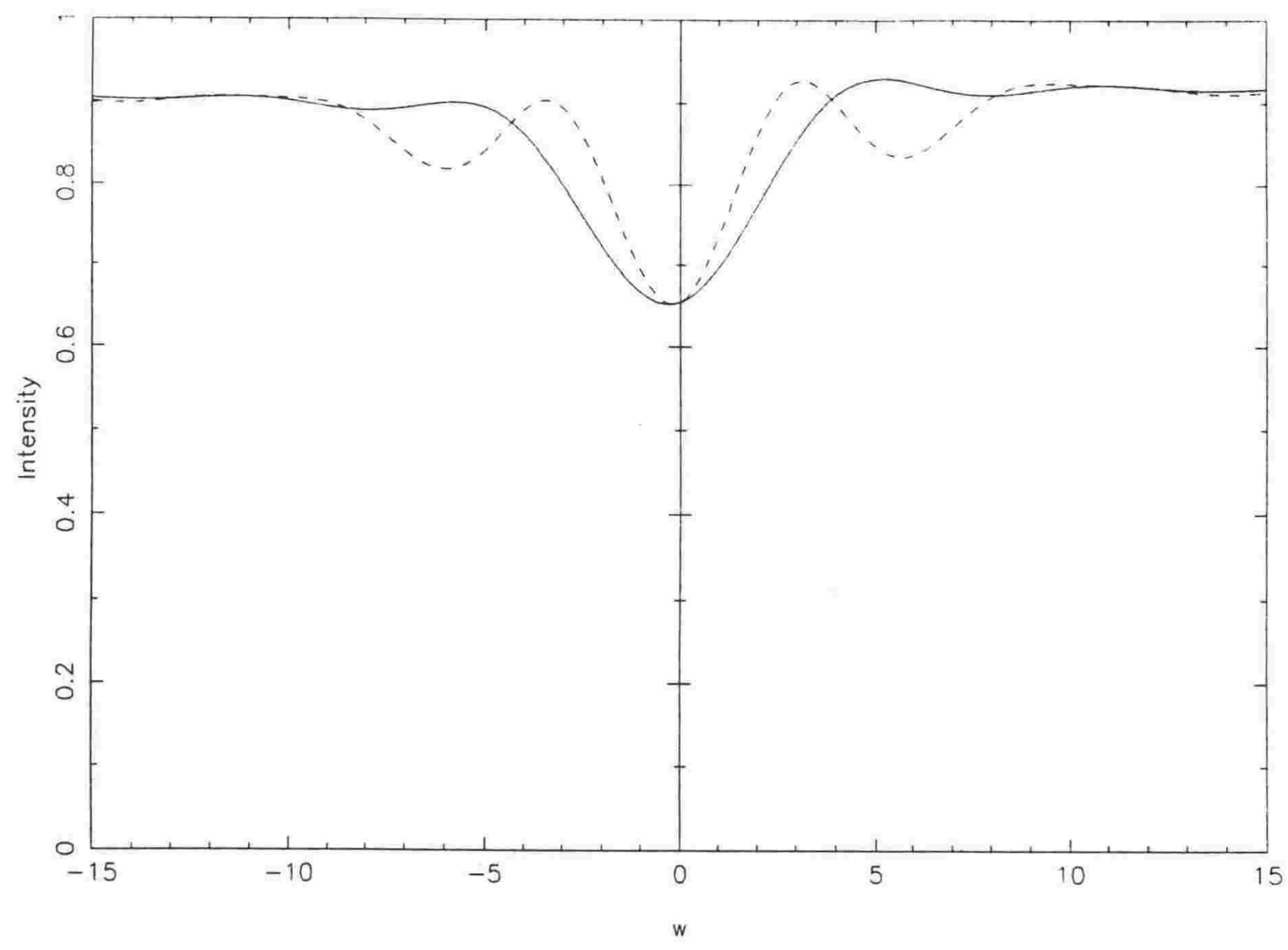

Figure 5.3: The solid line is the rocking curve for a column passing through pure crystal 60 $\AA$ thick, for $\mathbf{g}=[200]$ and an electron energy of $100 \mathrm{keV}$. The dashed line is the corresponding rocking curve of the [000] transmitted intensity at the centre of the image of a $20 \AA$ diameter equilibrium bubble which is in the middle of a foil $80 \AA$ thick. Oscillations occur in the rocking curve when an equilibrium bubble is present. 
find the effect of a bubble on the intensity, two electron paths are compared. The first path, in sequence, is down through a $30 \AA$ column of crystal, a $20 \AA$ equilibrium bubble and a further $30 \AA$ of crystal. The second path is down through a column of crystal of height 60 $\AA$. As expected, at $\mathrm{w}=0$, the intensity at the bottom of the bubble column is the same as that at the bottom of the perfect crystal column. Away from $w=0$ the dashed line, representing the bubble column, exhibits strong dips in the intensity. These dips are a manifestation of an effect that is more pronounced when the electron beam passes through more than one bubble. The discussion of the origin of these dips is deferred therefore until section 5.3 where the case of several bubbles in a stack is considered. Note that the dashed curve is roughly symmetric about the origin and converges to the usual background intensity of the solid curve at large values of $w$.

When $w=0$ the scattering matrix of the bubble (equation 2.32 ) is just the identity matrix as can be seen by inspecting the Howie-Whelan differential equations derived in chapter two. The intensities are then the same for the two different columns. However, if $\mathrm{w}$ is non-zero the curves are different because of the phase change of the diffracted beam relative to the transmitted beam in the column that passes through the bubble.

\subsection{Isolated Bubbles - Dependence of the Contrast on Defocus, Strain and Diffracting Vector.}

\subsubsection{Defocus and Bubble Radius}

The purpose of this section is to investigate the accuracy of measuring bubble radii from images using different methods, including the following: (i) the radial distance to the first minimum or maximum in the Fresnel pattern (the circular fringe pattern which is superimposed on the bubble image in defocussed intensity profiles); (ii) the radial distance to where the contrast varies most rapidly; (iii) the radial distance to where the contrast is rapidly varying and is significantly different from background. How the measured radius varies when using these different criteria is investigated for a single bubble containing helium gas at equilibrium pressure, using the faster method of simulation. Note that the 
different criteria for measuring the radius are used on the same intensity profiles, i.e., the method of simulating the intensity profiles is not in any way affected by the choice of criteria for measuring the radius of the bubble. The contrast depends upon several different variables - defocus, strain and diffracting vector - and is calculated for strong two-beam conditions. Since it is the defocus that affects the intensity profiles most significantly, the emphasis in this section is on calculating defocussed intensity profiles.

In order to calculate defocussed intensity profiles it is necessary to know the phase of the wavefunction at the exit surface of the crystal. The $\mathrm{T}$ and $\mathrm{S}$ beams of the analytical solution of chapter 2 can be found as a function of the reduced radius $\rho$. ( $T$ is the amplitude of the undeviated beam and $\mathrm{S}$ is that of the diffracted beam with vector $\mathbf{g}$ ). Head et al. [82] transform the $\mathrm{T}$ and the $\mathrm{S}$ variables in the Howie-Whelan differential equations into a simpler form by multiplying them by different phase factors. Consider the phases of the $\mathrm{T}$ and $\mathrm{S}$ beams that pass through a column that intersects the bubble and the $\mathrm{T}$ and $\mathrm{S}$ beams that pass through a column of pure crystal only. It can be shown that the effect of this transformation on these beams is to reproduce the phase factor of Rühle and Wilkens (equations 2.84 to 2.86 ). The phase of the wavefunction is correct as the effects of the transformations used to derive the Howie-Whelan differential equations have been included.

To calculate the defocussed profiles the wavefunction is substituted into the integral of equation 2.89. Note that the derivation of this equation requires that the wavefunction diffracted from a bubble is cylindrically symmetric which means that this formalism can only be used when the displacement field is zero. The zero-order Bessel function that is a factor in the integrand of equation 2.89 is calculated using a NAG library routine on a Silicon Graphics computer. The integral is evaluated numerically by a Runge-Kutta process and the speed of the programs is such that interpolation of the results is not needed even where large images or profiles are involved. Rühle and Wilkens [83] assumed a particular form for the wavefunction to derive analytical results for the defocussed profiles and, as a result, they did not have to resort to using numerical integration. Gruschel [85-87], however, adopted the more general numerical integration approach used here.

The phase change in the wavefunction that occurs as a result of the change in refractive index between matrix and bubble (equivalent to a change in mean potential as shown in equation 2.76) must be known in order to calculate a defocussed intensity profile. 
The mean potential for copper was calculated as 22.64 volts using the $0 \mathrm{keV}$ electron scattering factors of Doyle and Turner [134]. Since the mean potential for helium is calculated to be 1.76 volts, the difference in mean potentials is 20.88 volts. This value is more than twice the value that E Johnson [84] used in his simulations and so a greater phase change will occur if the electrons pass through bubbles of the same radius. In practice significantly smaller bubbles than E Johnson used are considered in this thesis so the phase change is less. Note that the convention in the present work is that mean potentials are taken with a positive sign whereas in the work of Rühle and Wilkens the mean potentials are taken as negative.

Part of the motivation for calculating intensity profiles is to enable accurate bubble radii to be measured from through-focal series. The criteria mentioned above for measuring the bubble radius are used in sequence in the next three sections with some comparison of the relative merits of the different methods being made as the discussion progresses, but with the main conclusions being drawn in the fourth section. In copper, gas bubbles containing helium introduced by ion-implantation typically have a radius of $10 \AA$. Defocussed intensity profiles for bubbles with radii in the range $5 \AA$ to $15 \AA$ have been simulated and the results shown in figure 5.4(a) as a function of reduced radius. In this figure the contrast is weak for small bubbles of radius less than about $10 \AA$. Hence for these bubbles it is more difficult to measure the bubble radius experimentally than it is for larger bubbles using any of the criteria mentioned above. However, images that have a weak contrast can have their contrast increased by image processing on the computer or by the choice of exposure times and grade of photographic paper in the darkroom. Increasing the contrast in this way makes it easier for the eye to see the structures but the process will not affect the position of the first minimum or maximum in the intensity profile. At the overfocus levels chosen the image of a bubble may be white or black at the centre, depending on the degree of defocus. 

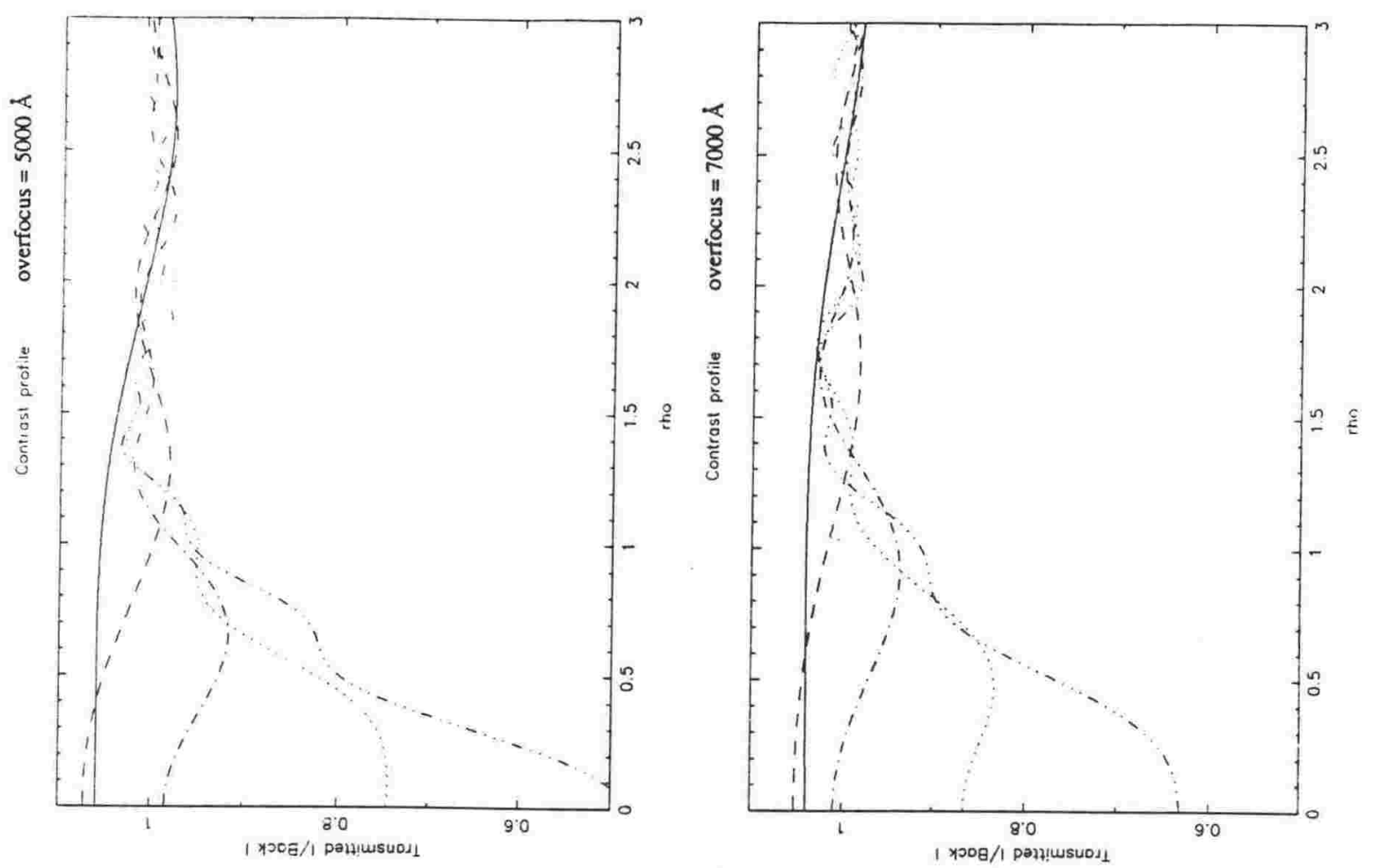

Figure 5.4 (a) (caption on following page)
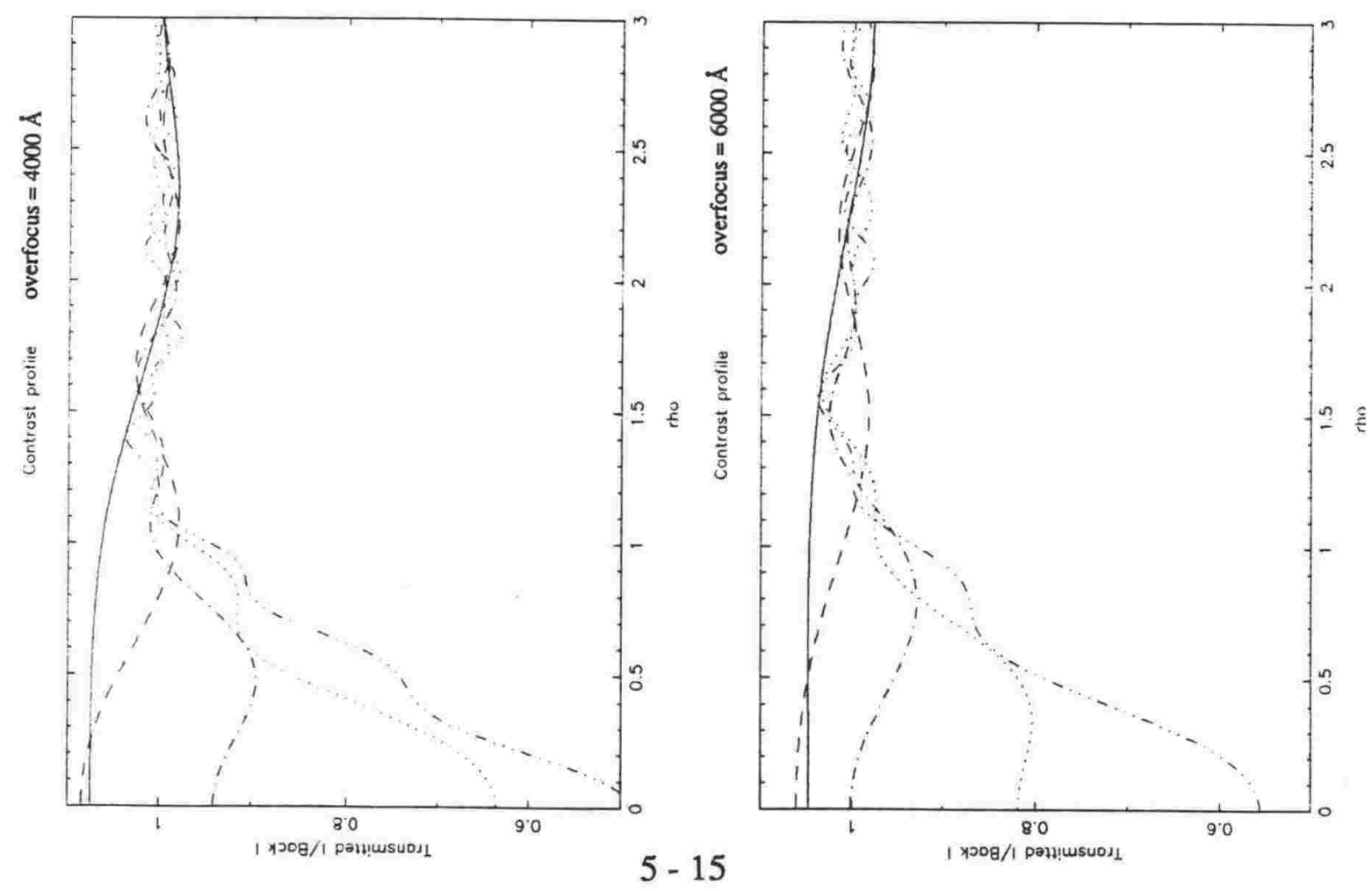

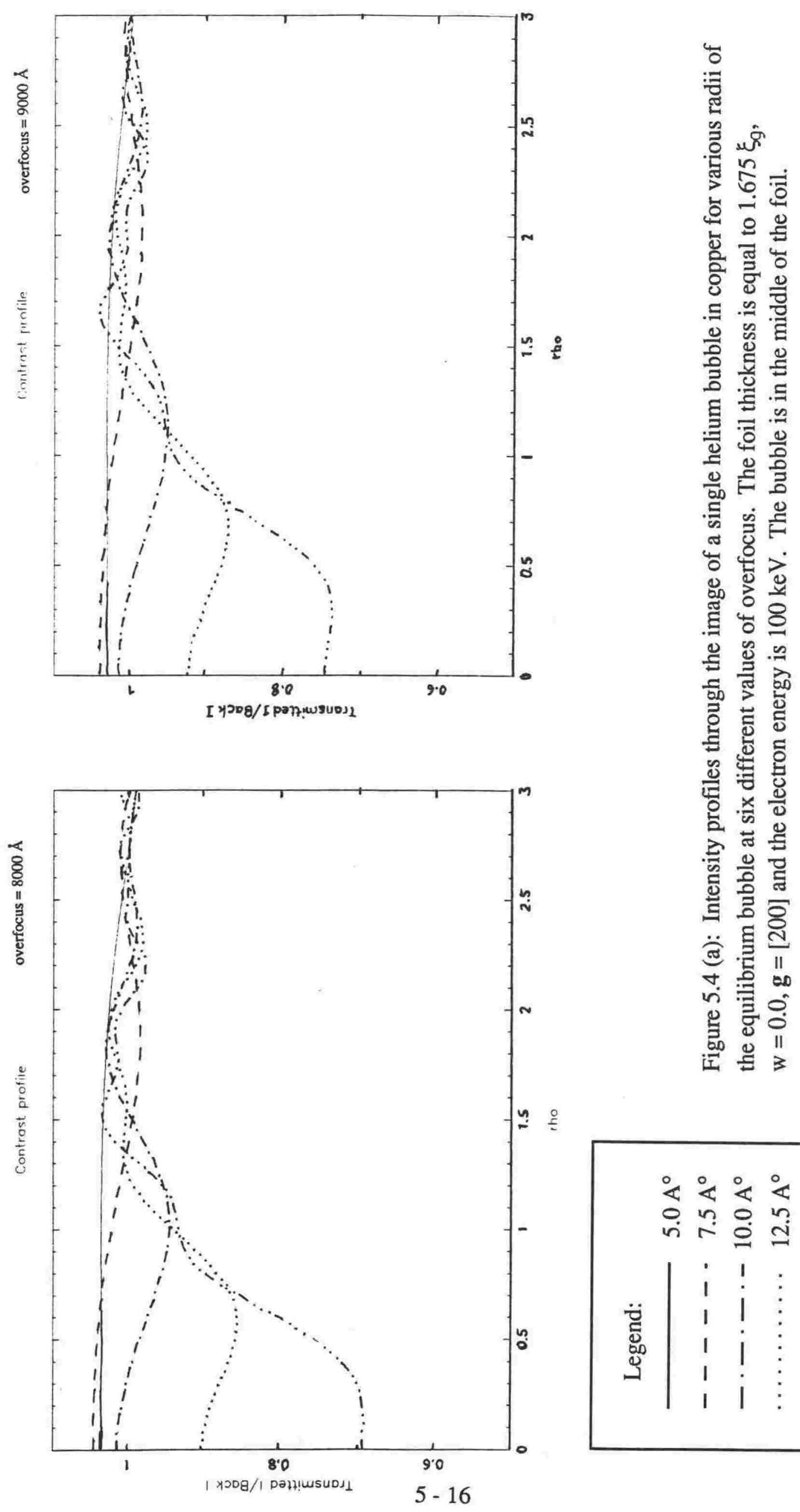

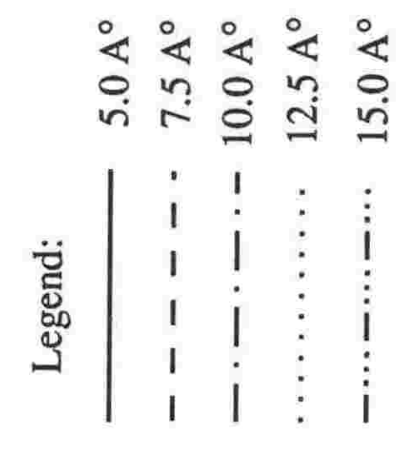



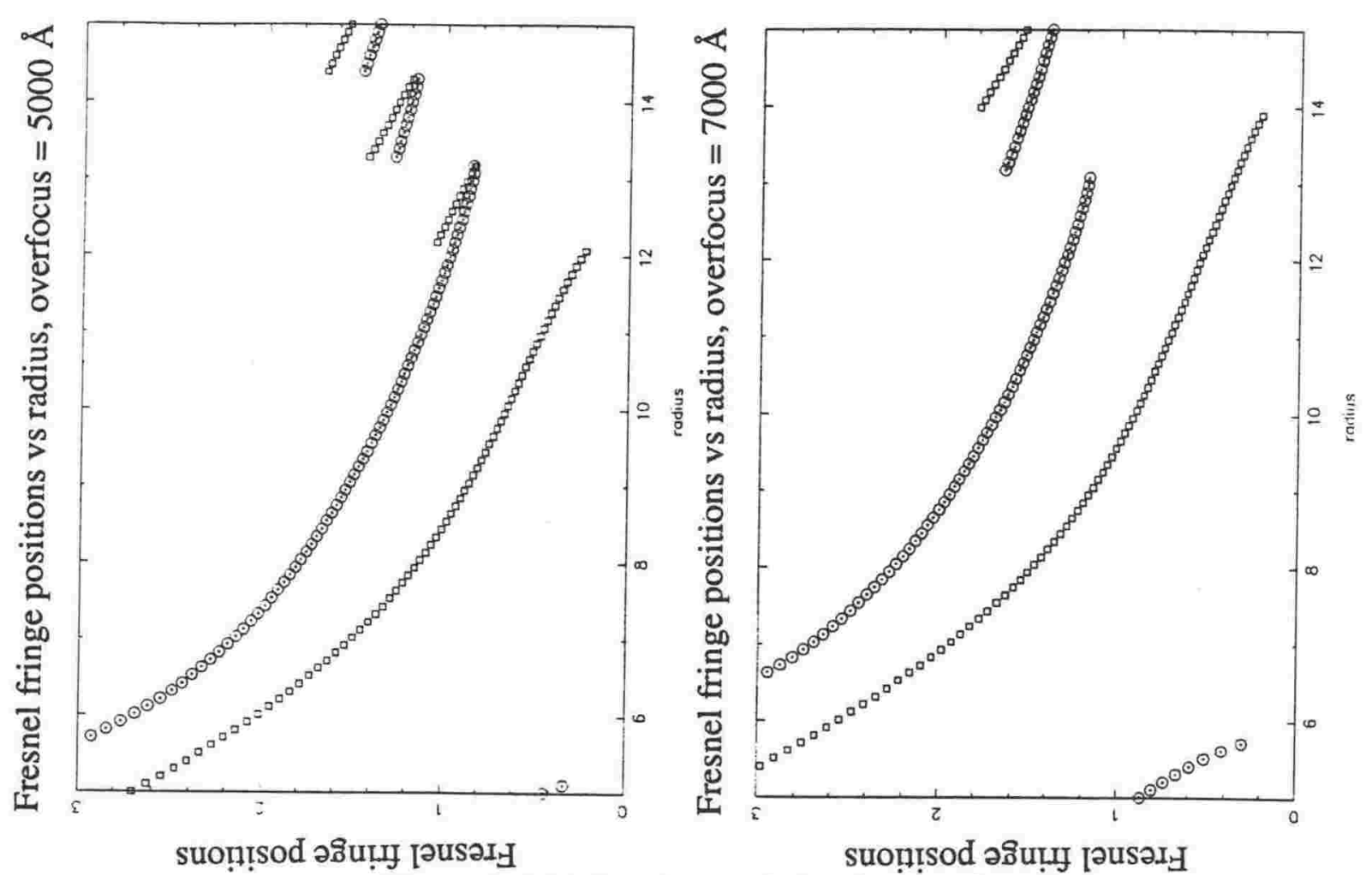

Figure 5.4 (b) (caption on following page)
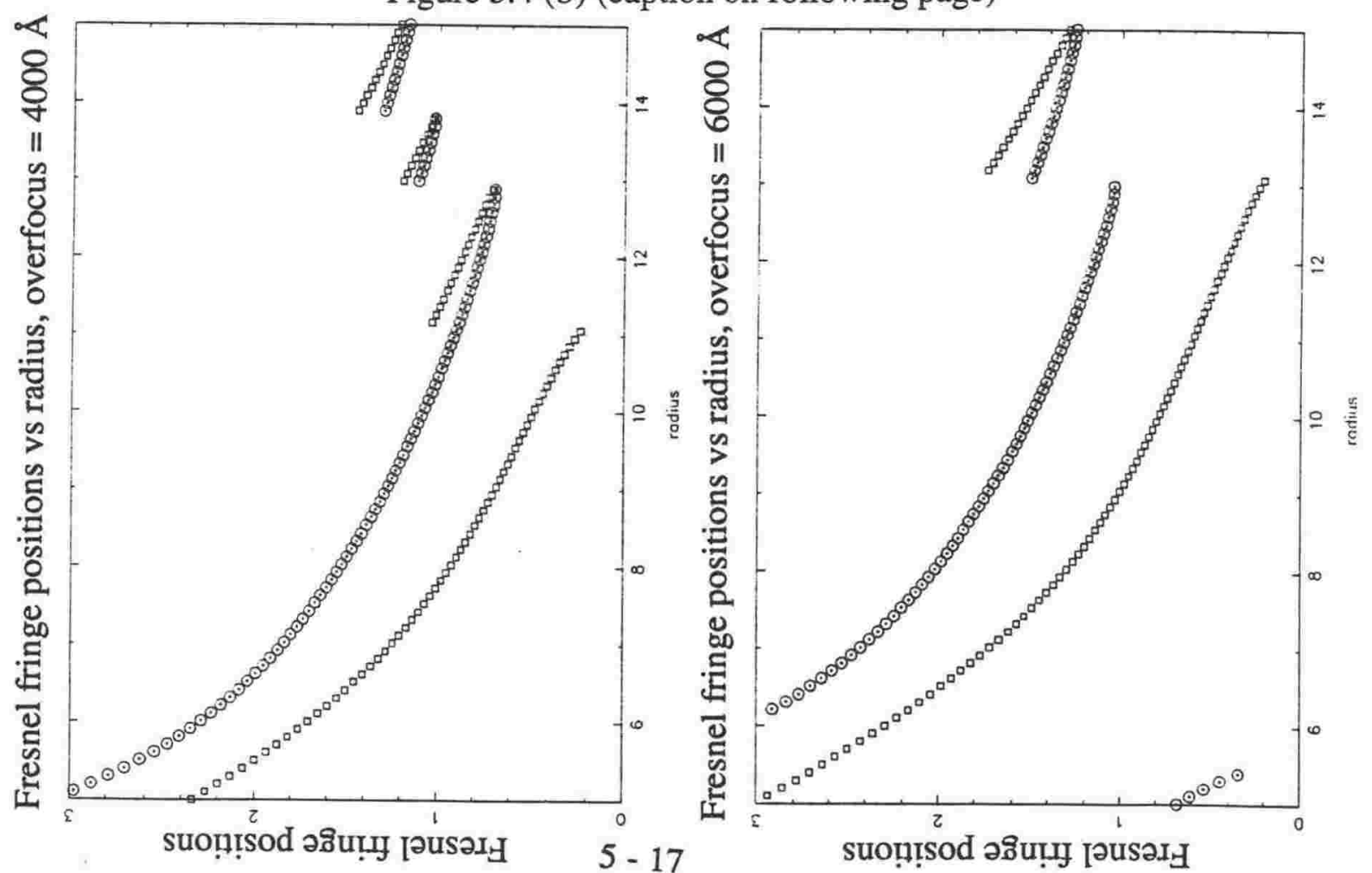


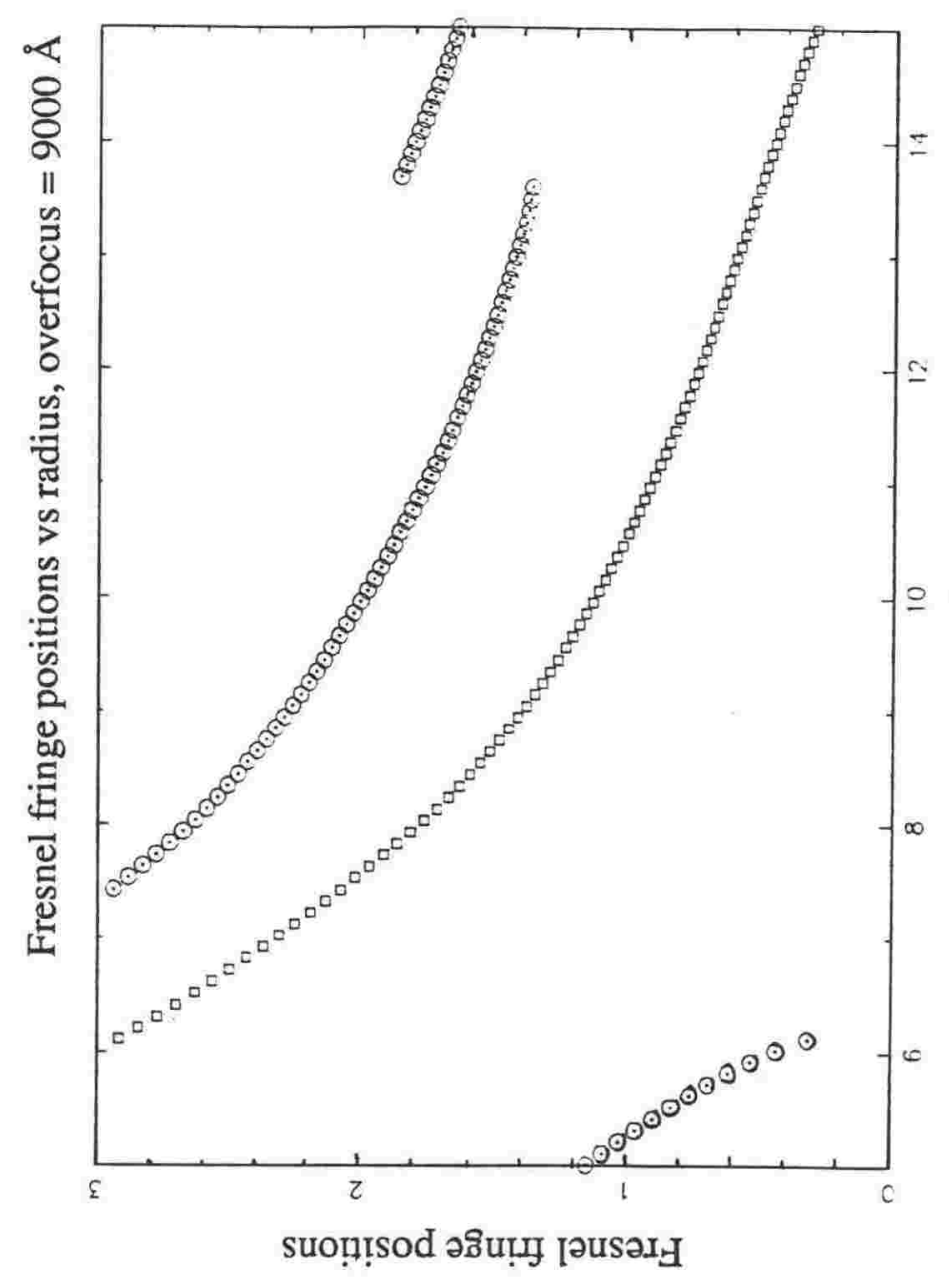

E气 Е

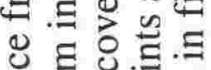

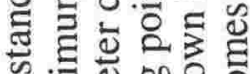
. 蛋.

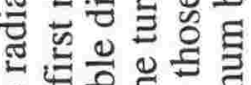

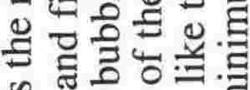
. 틀 结记

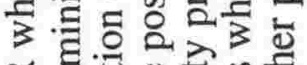

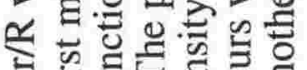

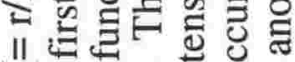

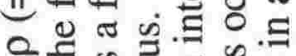
은

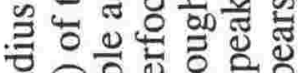

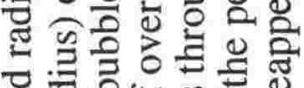

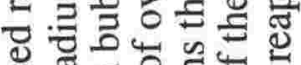

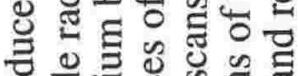

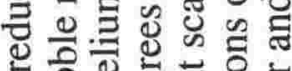

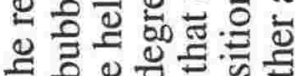
造记 o을

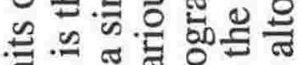

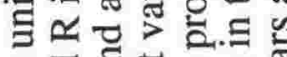

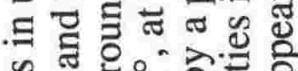

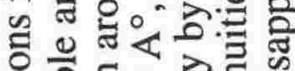
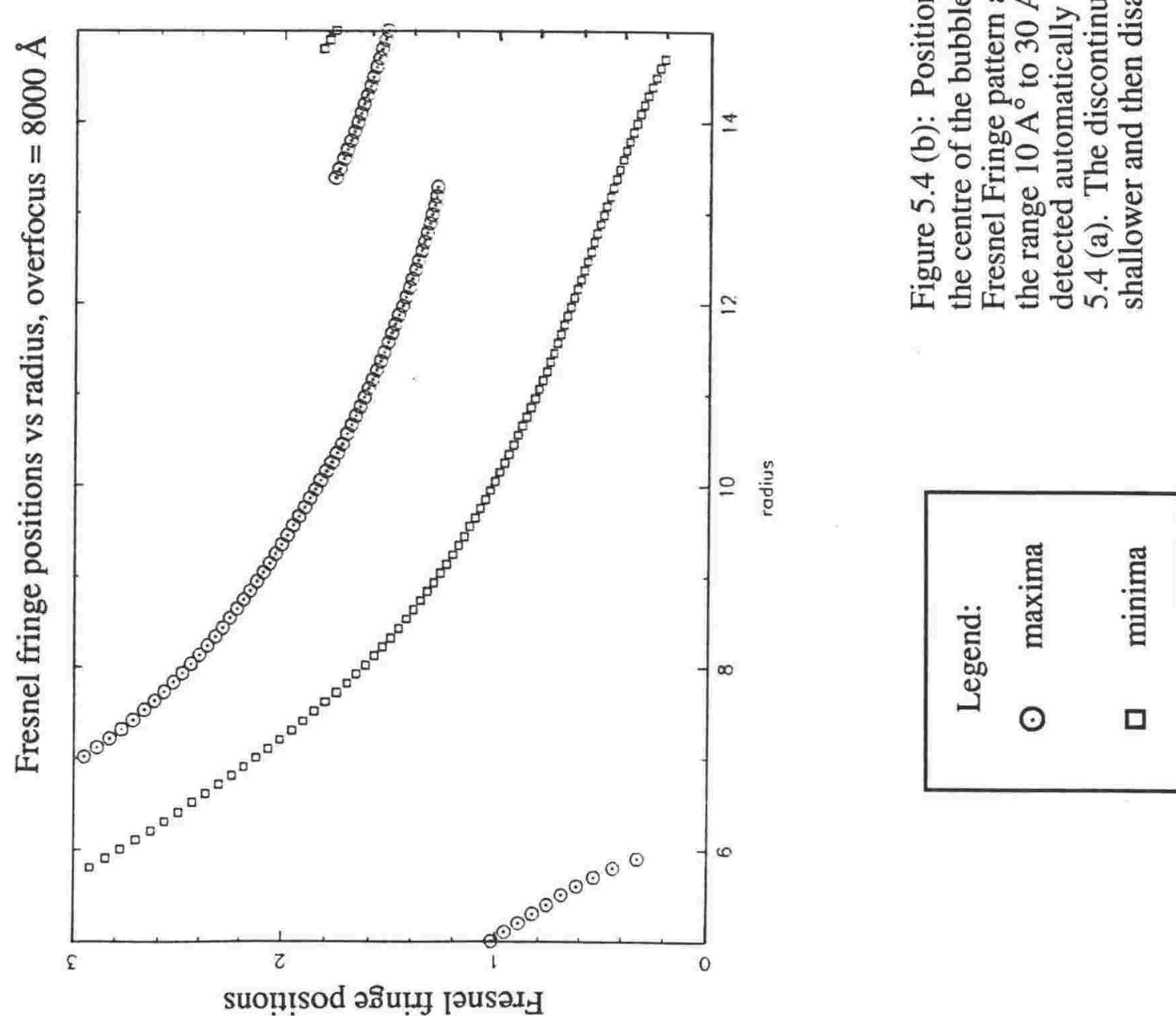
Figure 5.4(b) shows the bubble radii that would be measured using the radial distance to either the first maximum or minimum in the Fresnel fringes as the criterion for the radius of the bubble. Note that two criteria for measuring the bubble radius are compared in the same figure. Rühle and Wilkens [83] used the same criteria to measure the bubble radius of larger equilibrium bubbles with similar results being obtained. The experimental results using this method would be accurate if the positions of the first minimum or maximum in the intensity profiles occurred at $\rho=1$. For a defocus of $4000 \AA$ and a bubble radius of $10 \AA$ the radial distance to the first minimum is about $\rho=0.48$ and to the first maximum about $\rho=1.1$. For the same defocus but a bubble of radius $15 \AA$ the first global maximum occurs at $\rho=1.4$ but the program that detects the maxima will detect the first maximum at $\rho=1.2$. The maximum at $\rho=1.2$ is a local maximum and not a global maximum. The earlier bumps in the curve for a $15 \AA$ bubble are only inflexions and not local maxima or minima.

Several conclusions can be drawn from figure 5.4. In the plot for an overfocus of $4000 \AA$, the first minimum occurs at a position that is less than the actual bubble radius for bubbles with radius larger than about $8 \AA$. For example, a bubble of radius $10 \AA$ has the first minimum at about $5 \AA$ whereas a bubble of radius $6 \AA$ has the first minimum at about $10 \AA$. Images of small bubbles have minima that give values for the radii that can be in error by $200 \%$. Using the position of the maximum at an overfocus of $4000 \AA$ gives even more inaccurate results for small bubbles but better values for larger bubbles. If a large overfocus is used the first minimum lies closer to the centre of the bubble than the first maximum over most of the range of bubble sizes. The profiles suggest that the conventional wisdom that bubbles are dark in overfocus only applies for bubbles with a radius larger than about $12.5 \AA$.

The intensity profiles assume that a coherent plane wave is incident on a bubble. In practice, beam divergence, finite filament size, and ripples of the current and voltage in the high voltage supply (causing a spread in the energies of the electrons) will cause a loss in coherence. This will damp out the Fresnel fringes (i.e., the size of the oscillations will be 
reduced and they will not extend as far from the bubble). Fresnel peaks further out than the first maximum and minimum in the intensity profiles have not been studied here as these peaks are not observed experimentally.

To find out whether the inaccuracy in determining the bubble radius is large just for the particular degrees of defocus included in figure 5.4 or whether it is a general characteristic, further plots were obtained for a bubble with a radius of $10 \AA$ over a wider range of defocus values. Figure 5.5 shows a selection of these plots. For underfocus values from $-9000 \AA$ to $-2000 \AA$, and overfocus values from $2500 \AA$ to $9000 \AA$, the image of the bubble appears white at the centre. For smaller values of defocus the intensity at the centre of the image of the bubble oscillates from black to white.

The variation in the positions $(\rho)$ of the first minimum and maximum in the intensity profiles as the defocus changes is shown in figure 5.6. The central region from $-1000 \AA$ to $1000 \AA$ has been omitted because in this region the contrast rapidly oscillates. The oscillations occur for a defocus (i.e., $\beta$ ) near zero because of the rapidly oscillating nature of the Bessel function in the integrand of equation 2.89 in this region. The discontinuities, such as that in the minimum at a defocus of $-1800 \AA$, occur when peaks in the intensity profiles disappear or reappear. The peaks are usually about $30 \%$ to $100 \%$ different from the ideal value of $\rho=1$. For example, at a defocus of $-8000 \AA$ the maximum occurs at a $\rho$ of 0.7 (i.e., the position of the peak is $30 \%$ out). The most accurate results from a single micrograph would be obtained by using the position of the first maximum in the intensity profiles for large underfocus values.

The plots of the positions of the first maximum and minimum versus bubble radius show that the values deduced from BF micrographs using the above criteria can be significantly different from the actual value for bubbles of radius $5 \AA$ to $15 \AA$. Equation 2.89 for the intensity profiles depends on $\beta$ (a parameter proportional to defocus and inversely proportional to the square of the bubble radius) and on the wavefunction in equation 2.90. To attain a given value of $\beta$, a larger value of defocus is required for a larger bubble than for a smaller bubble. This explains why the curves tend to move to the right (i.e., towards larger radius) as defocus increases in figure 5.4. The detail of the curves does change because the wavefunction which appears in the integral in equation 2.89 is not 


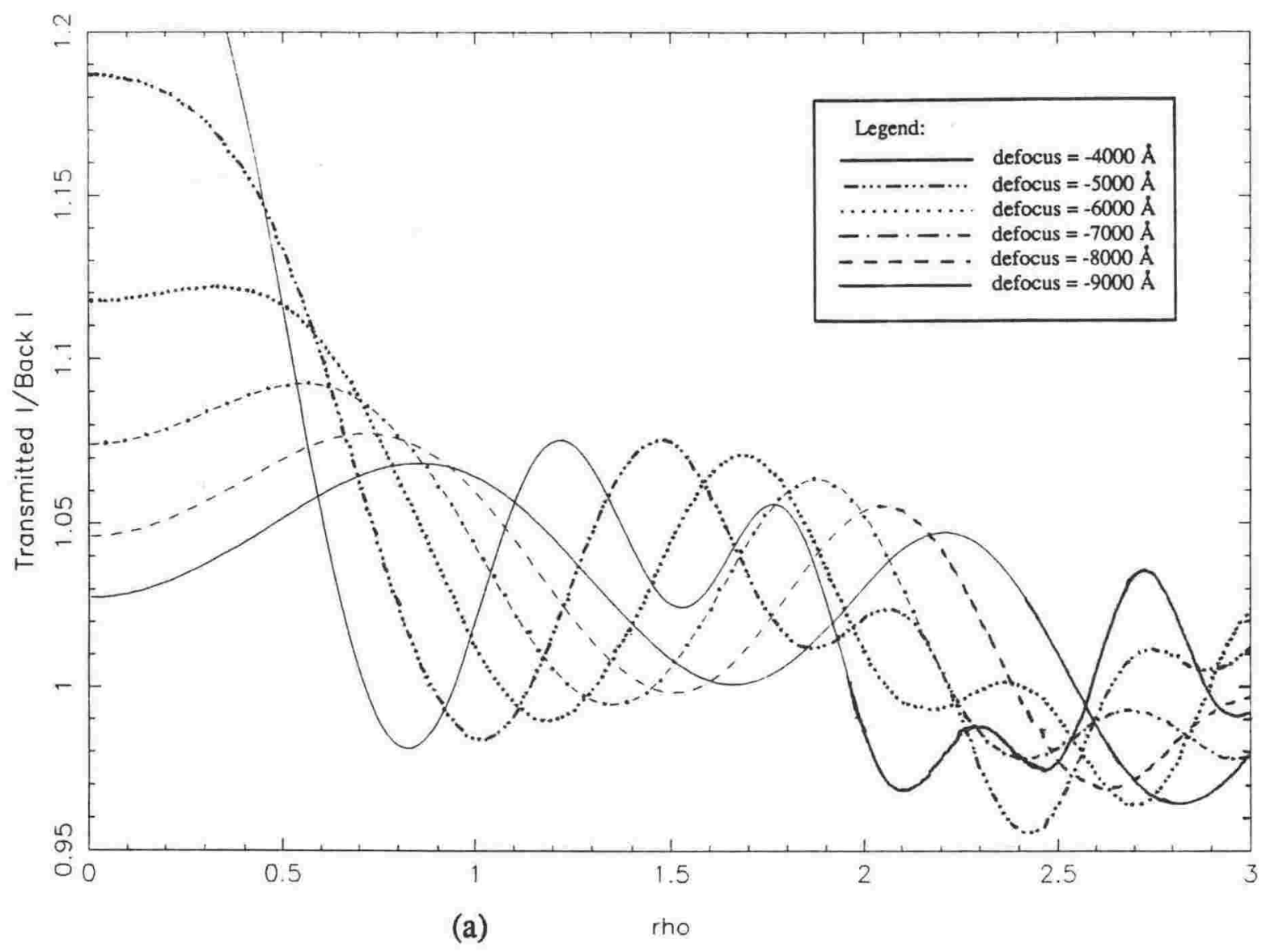

Figure 5.5 (caption on following page)

Contrast profile

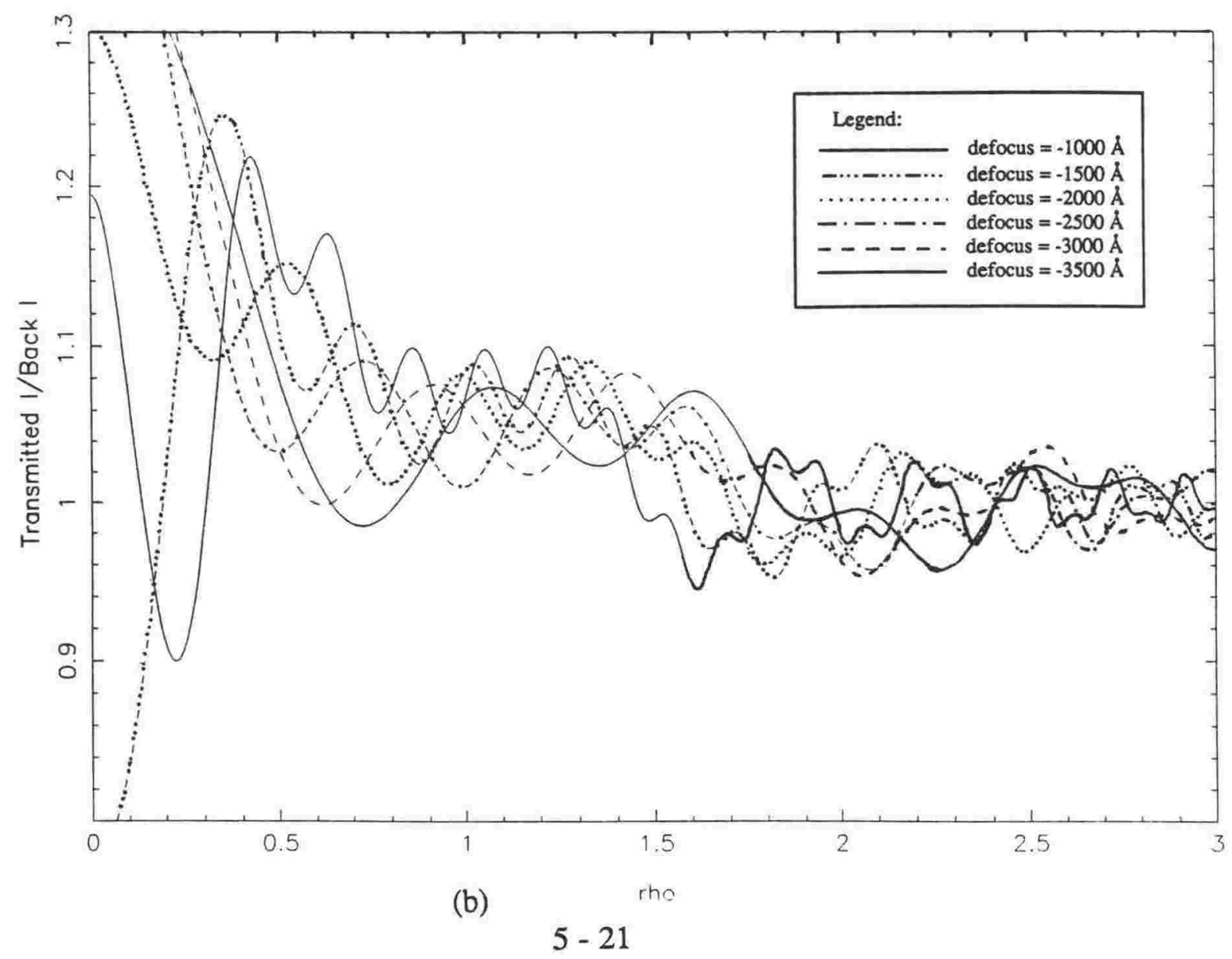




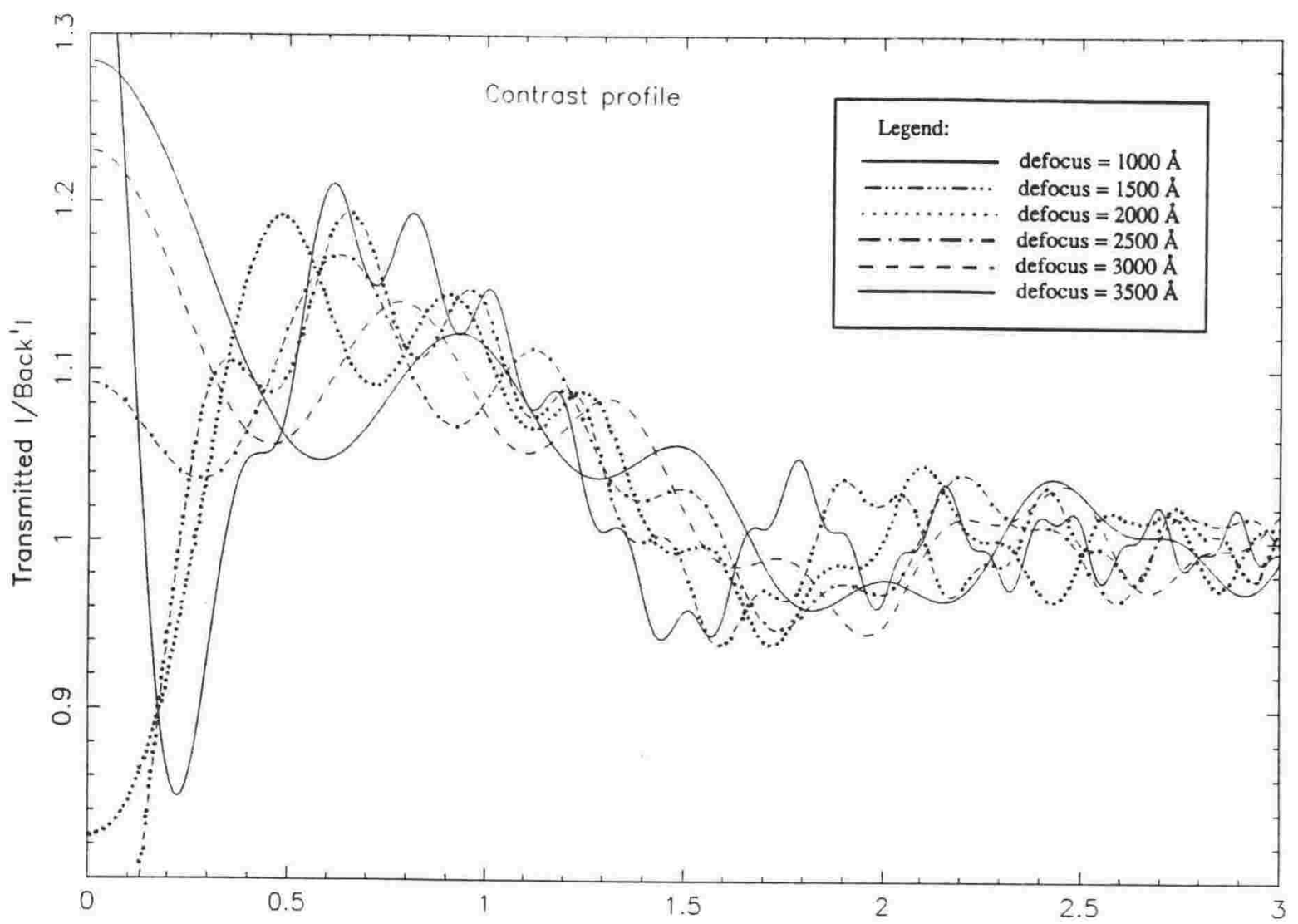

(c)

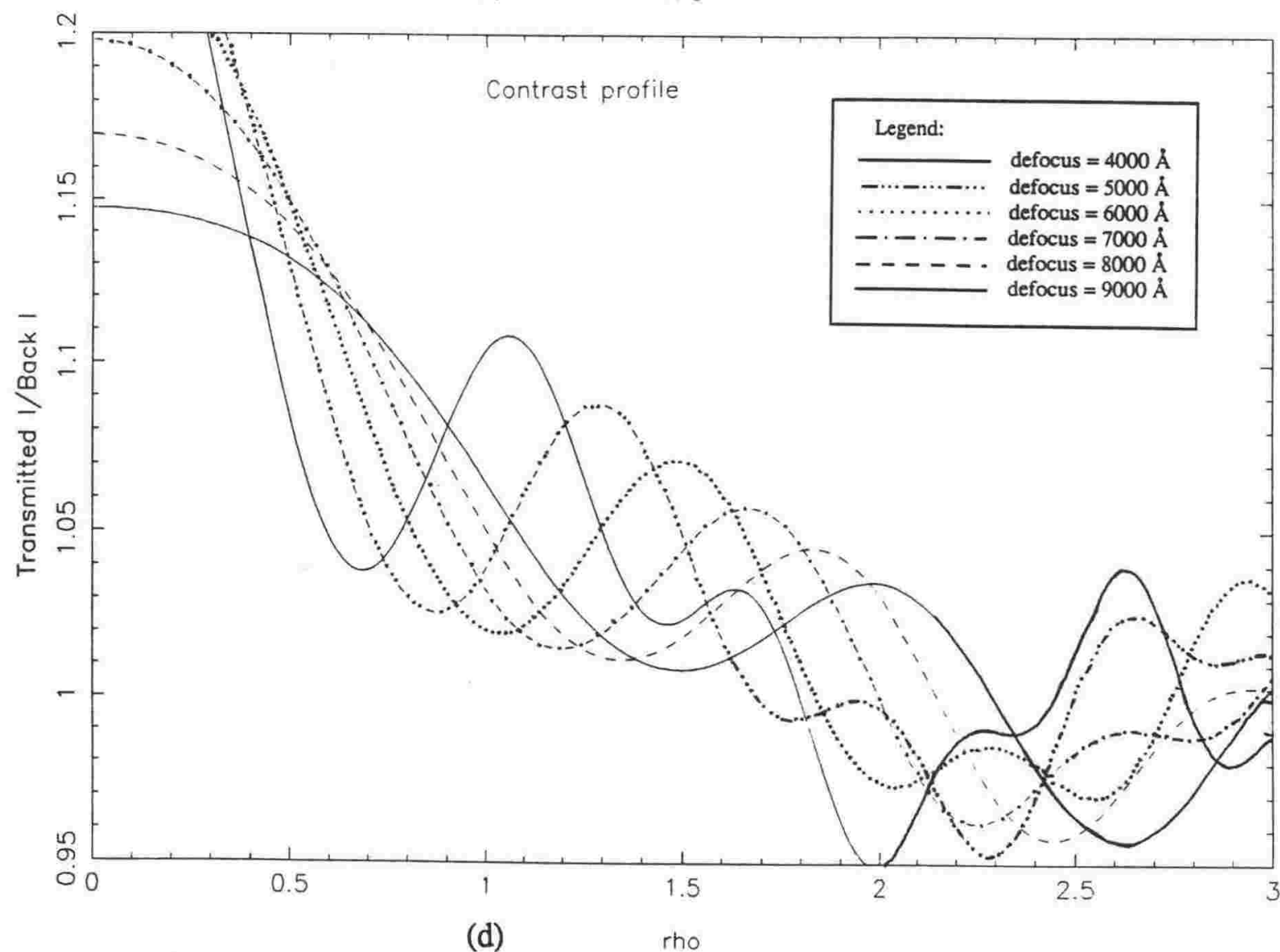

Figure 5.5: Intensity profiles for a helium gas bubble at equilibrium with a radius of $10 \AA$ in copper for a defocus range of $-9000 \AA$ to $9000 \AA$ : (a) $-9000 \AA$ to $-4000 \AA$, (b) $-3500 \AA$ to $-1000 \AA$, (c) $1000 \AA$ to $3500 \AA$ and (d) $4000 \AA$ to $9000 \AA$. Positive values of the defocus correspond to overfocus, and negative values of the defocus to underfocus. The other variables are $\mathbf{g}=$ [200], a foil thickness of $1.675 \xi_{\mathrm{g}}, \mathrm{w}=0.0$, a bubble depth equal to half the foil thickness and an electron energy of $100 \mathrm{keV}$. 


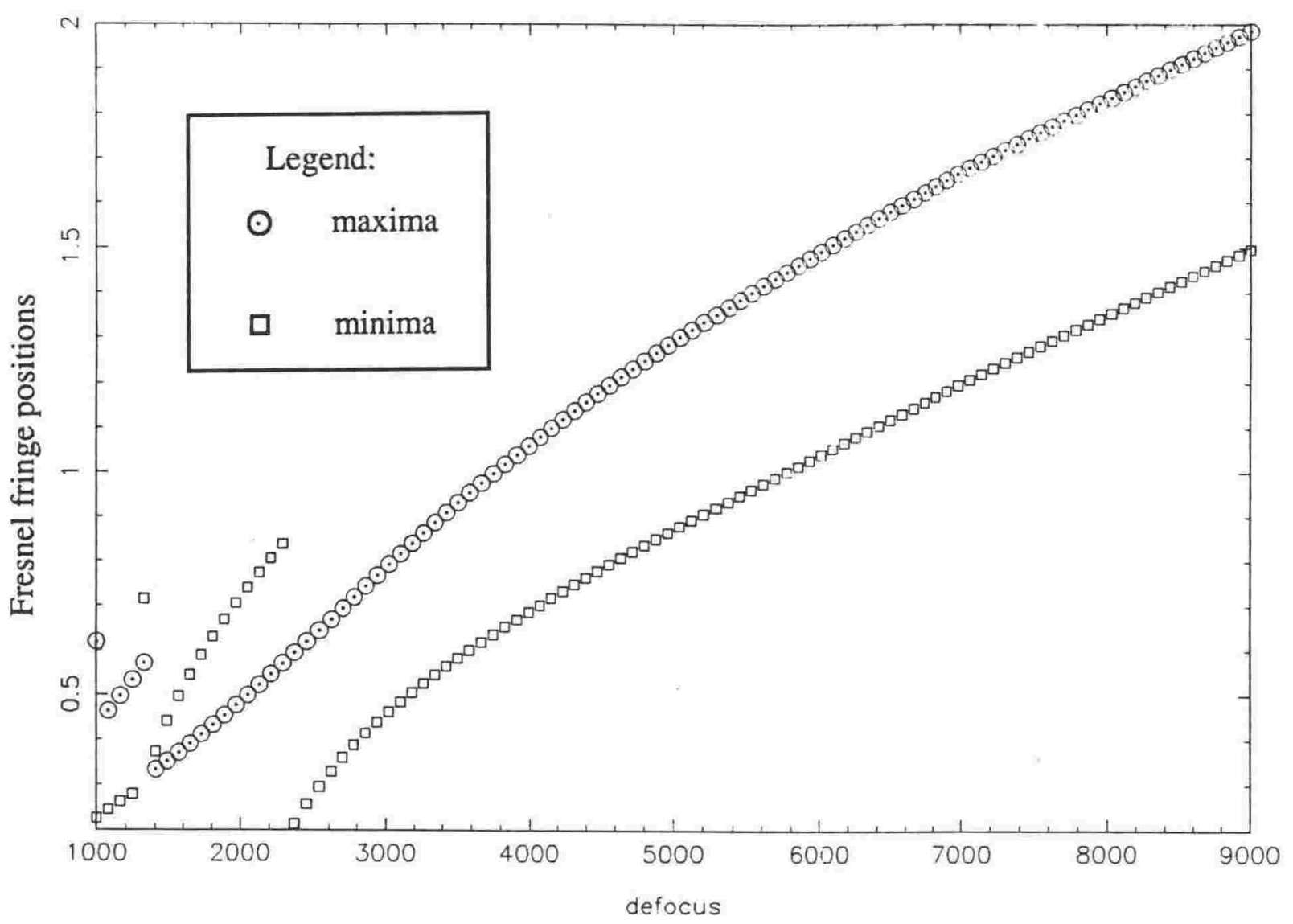

Fresnel fringe positions vs defocus

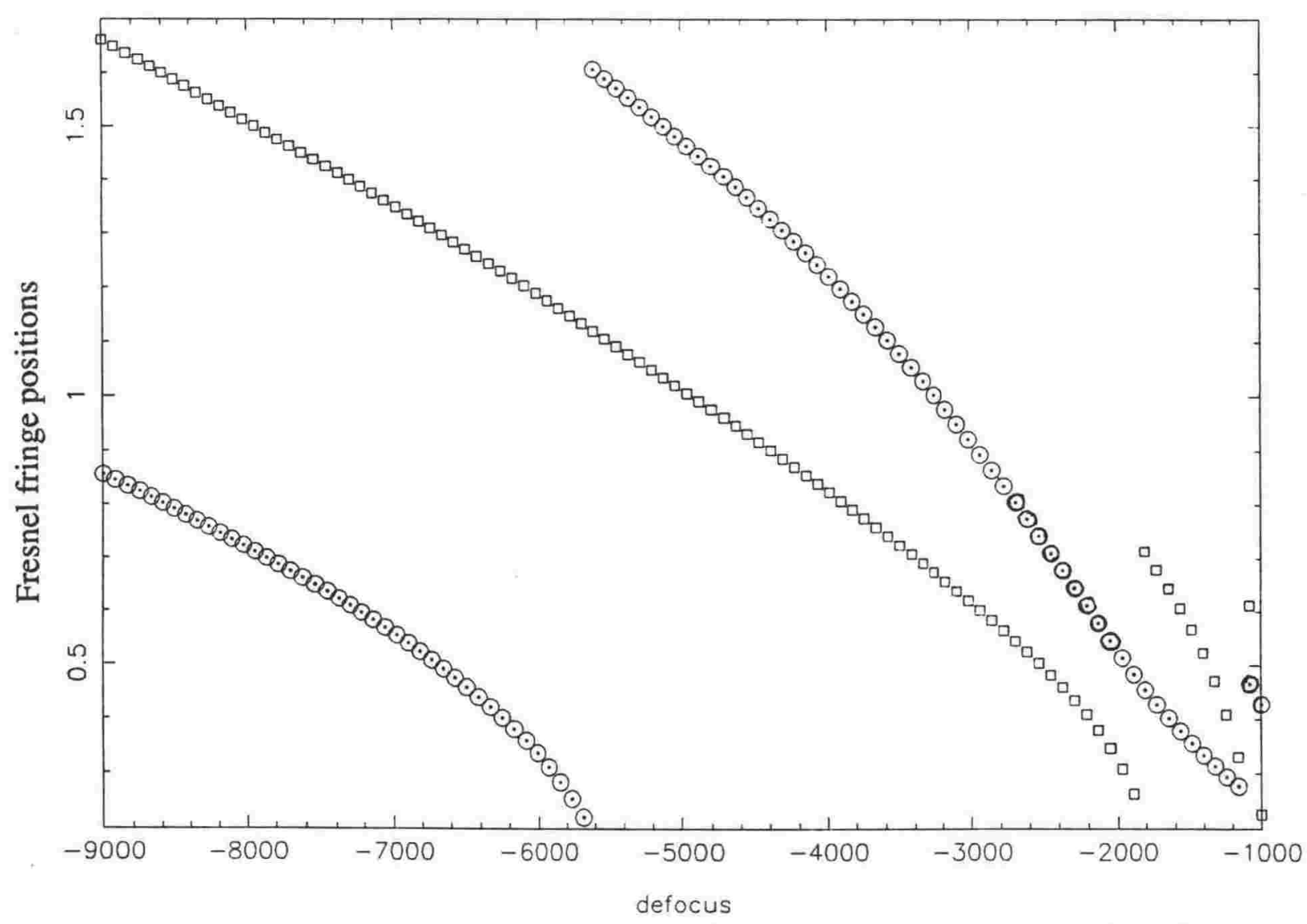

Figure 5.6: Positions in units of the reduced radius of the first minimum and maximum in the intensity profile for a $10 \AA$ radius equilibrium bubble for defocus values from $-9000 \AA$ to $9000 \AA$. The other variables are the same as those used in figure 5.5. The positions of the minimum and maximum in the intensity profiles can be significantly away from the ideal value of $\rho=1$. 
a function of $\beta$. Hence the inaccuracies of bubble radii measurement found for a $10 \AA$ radius bubble across a large range of defocus will occur for all the bubble radii considered.

In assessing the significance and generality of these results it should be pointed out that the defocussed intensity profiles are altered if the depth of the bubble is changed because a defocussed profile depends on the phase of the wavefunction at the exit surface of the crystal. This is not so when simulating in-focus profiles as in section 5.1.

Measurement of the Bubble Radius using the Position of the First Maximum in the Modulus of the Slope in the Intensity Profile

The third criterion investigated for determining bubble radius is based on the ability of the eye to detect rapid changes in intensity with position in the image. The radius is taken as the radial distance from the centre of the image to the position where the modulus of the slope of the intensity, $\mid \mathrm{d} \mathrm{I} / \mathrm{drl}$, has the maximum value. This criterion, suggested by Johnson [189], is similar to that used by Rühle and Wilkens [83] but differs from it in ignoring the contrast. The results for a defocus of $4000 \AA$ are shown in figure 5.7. A comparison of these results with those of figure 5.4 (b) shows the values of the radius found with this criterion are generally less than those found using either of the two criteria based on the Fresnel fringe pattern. Notice also that there is only the one discontinuity in figure 5.7 (at $\mathrm{r}$ $=10.6 \AA$ ) compared with three in figure 5.4 (b).

\subsubsection{Measurement of the Bubble Radius using the Position of the First Maximum in the Visibility of the Intensity Profile}

In considering the measurement of the radius of a spherical inclusion, Ashby and Brown took as the radius the distance from the centre of the image to a point, in a direction parallel to the operative diffraction vector, where the intensity differed from that of the background by a certain amount (not necessarily the maximum or minimum amount). Rühle and Wilkens [83] extended this approach by including also the slope of the intensity profile. They took the visibility $\mathrm{V}$ to have the particular form: 


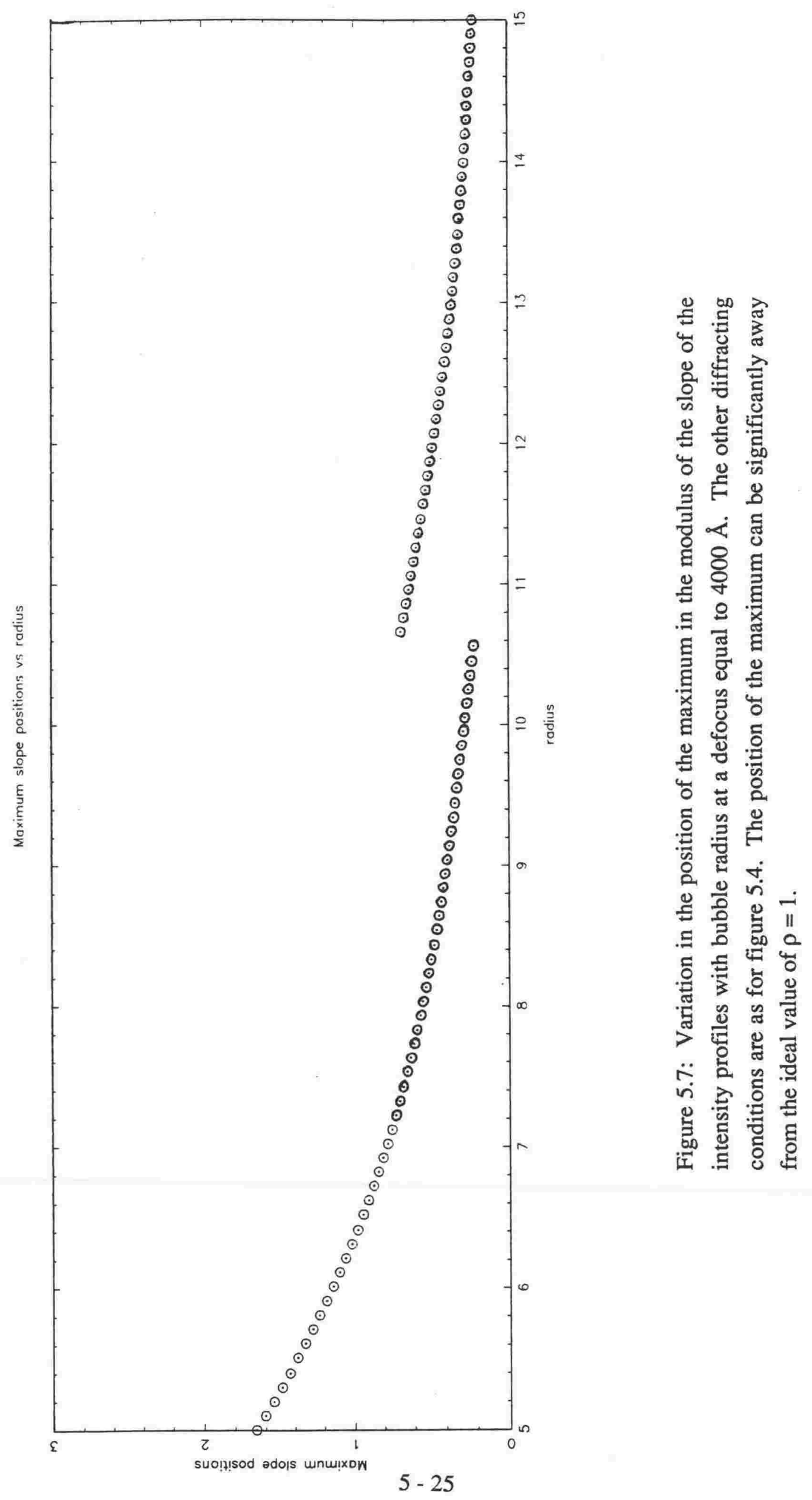




$$
\mathrm{V} \alpha\left|\left(\mathrm{I}(\mathrm{r})-\mathrm{I}_{0}\right) \frac{\mathrm{dI}}{\mathrm{dr}}\right|
$$

where $\mathrm{I}$ is the intensity, $\mathrm{I}_{0}$ the background intensity and $\mathrm{r}$ the distance from the centre of the defect. The radius is taken as the distance from the centre of the image to the point in the profile where V has the largest value. The criterion of Rühle and Wilkens in using the maximum in $\mathrm{V}$ as a measure of the bubble radius was investigated here and the results are presented in figure 5.8. The results show that the positions of the maxima are not significantly closer to $\rho=1.0$ and that three discontinuities occur.

\subsubsection{Conclusions}

The results obtained in this thesis show that using the position of either the first minimum (or maximum) of the Fresnel fringe pattern gives rise to a bubble radius that is often out by more than $100 \%$. The position, and hence radius, varies discontinuously with defocus. Taking the radius as the radial distance to the point in the profile, closest to the centre of the bubble, where the maximum in the magnitude of the slope of the intensity curve occurs also gives discontinuities. Although the peaks in $\mathrm{V}$ give a value for $\rho$ which is closer to the ideal value of 1 for a bubble that has a radius of $5 \AA$ or $15 \AA$, a bubble of intermediate radius has a $\rho$ that is still significantly different from 1 . The slope criterion in figure 5.7 gave the smoothest variation of the effective bubble radius with defocus. The criteria used in figures 5.7 and 5.8 give a value for $\rho$ closer to 1 than the Fresnel fringe position criterion does over more of the defocus values tested. Cavity volume fractions calculated from bubble radii based on any of the criteria could be in error by factors of eight.

The criteria used above to measure the bubble radius rely on measuring the position of the first maximum or minimum in some function of the intensity profile. Any criteria other than those investigated explicitly here will also have to be functions of the intensity and the derivatives of the intensity. Because intensity profiles through experimental images are noisy, it is difficult to use methods that rely on higher order derivatives of the intensity profiles. Hence the methods investigated above are thought to be among the best available. 


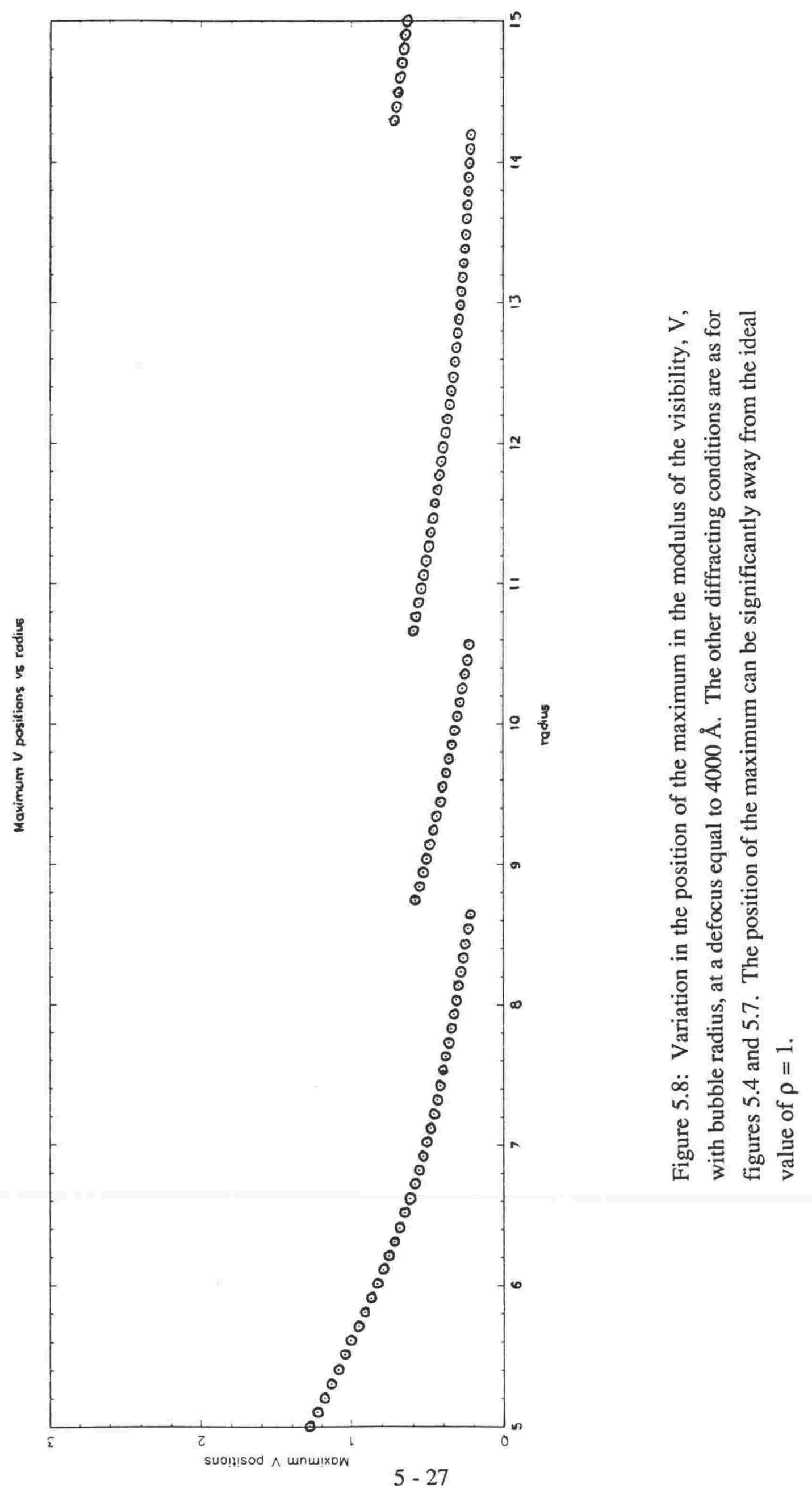




\subsubsection{Non-zero Displacement Field}

So far the discussion has centred on cavities or bubbles that are strain-free $(\mathbf{R}=\mathbf{0})$. It is interesting to investigate at this point the image of a bubble having a strain field which is non-zero $(\mathbf{R} \neq \mathbf{0})$. The images formed by overpressured bubbles surrounded by a nonzero displacement field will not have the cylindrical symmetry of the simulations considered in the previous sections of this chapter. The out-of-focus intensity at the centre of an image of a bubble is determined by a convolution over all real space (section 2.5). The non-zero displacement field for bubbles that are not at equilibrium will affect this calculation and so the results obtained in this thesis will be different from those of previous authors.

The image of a single bubble having a non-zero displacement field and no nearby dislocation loops can be simulated numerically using the program described in chapter six. (The program is designed for simulating images from combinations of bubbles and loops with non-zero displacement fields and can be run with the number of loops set to zero). The gas overpressure in the bubble has been set to $10 \mathrm{GPa}$, which is approximately that required for the punching of dislocation loops. The simulated in-focus image for a diffracting vector $\mathbf{g}=[200]$ is shown in figure 5.9. The non-zero displacement field around the bubble (resulting from the overpressure) gives rise to asymmetrical black and white lobes in the simulated image. Whereas the thickness contrast dominates the intensity inside the bubble image, the non-zero displacement field dominates the intensity outside.

Simulated images for varying degrees of defocus have also been calculated for the case of a non-zero displacement field. The defocussing of the wave function at the exit surface of the foil can be approximated using a Fourier series as explained in the section in chapter two on the multislice method. This approach is used here in preference to the analytical expressions of Rühle and Wilkens [83] or the numerical integration of Gruschel [86]. The Fourier series approach allows images to be simulated for structures with strain fields that are non-zero whereas the other two techniques are restricted to the calculations of the contrast of cavities that are strain-free and cylindrically symmetric. However, the disadvantage of using Fourier analysis is that instead of using interpolation to magnify the picture, larger arrays must be calculated if errors are to be avoided. Fourier series have been used previously [88], but only for structures with a zero displacement field. 


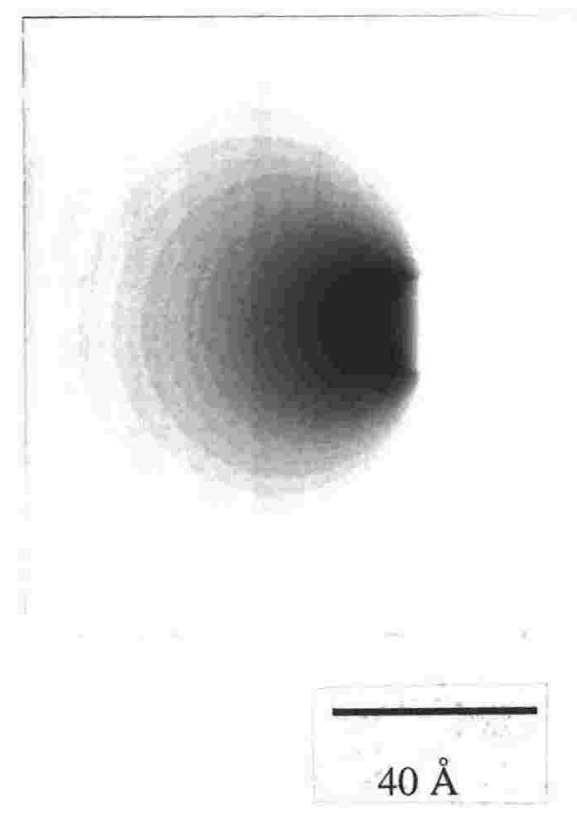

Figure 5.9: In-focus image of bubble with non-zero displacement field, with diffracting conditions as for figure 5.4. The bubble overpressure, the amount by which the pressure inside the bubble exceeds equilibrium pressure, is $10 \mathrm{GPa}$. The overpressure creates a nonzero displacement field around the bubble which is imaged up by the TEM as a pair of black and white lobes. The thickness contrast dominates the intensity inside the bubble image, whereas the non-zero displacement field dominates the intensity outside. The visibility can be measured along a line at any angle to $\mathrm{g}$ in the image and is largest along a line parallel to $\mathbf{g}$ as $\mathbf{g . R}$ is largest for this direction. 
Figure 5.10 shows some defocussed images of bubbles. In a through-focal series taken over a larger range of defocus the size of the lobes will increase with defocus. For non-zero values of $w$ the black/white lobes will be reduced to a single black or white image.

\subsubsection{Diffracting Vector}

The above profiles were all for the same $\mathbf{g}=[200]$ diffracting vector. To find out whether using a different $\mathbf{g}$ (and therefore $\xi_{\mathrm{g}}$ ) would affect the conclusions, intensity profiles were calculated also for $\mathbf{g}=[0,2,-2]$ and are shown in figure 5.11 . The plots obtained are not significantly different from those of figures 5.5 and 5.6 so the conclusions reached above for $\mathbf{g}=[200]$ should also hold for other diffracting vectors. Since the simple black / white behaviour of the contrast expected for a change from overfocus to underfocus does not stand up to scrutiny for $\mathbf{B}=[011]$ (which is orthogonal to both of the diffracting vectors considered), it is unlikely to do so for other beam directions.

\subsection{Contrast From Bubble Stacks.}

For the image of a single strain-free bubble, the difference in the intensity at the centre of the image from the background intensity outside was calculated in section 5.1 and figure 5.1. For ordered bubble structures the bubble lattice has a rational orientation with respect to the host matrix. If the electron beam in the microscope is directed down a zone axis in the metal foil it will also be directed down columns of bubbles. If the alignment of the bubbles were perfect, a given stack of bubbles would project to form a single image. For example, in the gas bubble superlattice in copper, a typical bubble radius is $\approx 10 \AA$ and the spacing between nearest-neighbour bubbles is $\approx 50 \AA$ (along <011>). In this case, for perfect bubble ordering, a single stack of bubbles along any zone axis would project into a single image of radius $10 \AA$.

In this section the in-focus intensity contrast at the centre of the projected bubble image for a stack of strain-free bubbles is calculated using the same approach as was adopted in section 5.1 for a single bubble. There the intensities of the electrons passing 


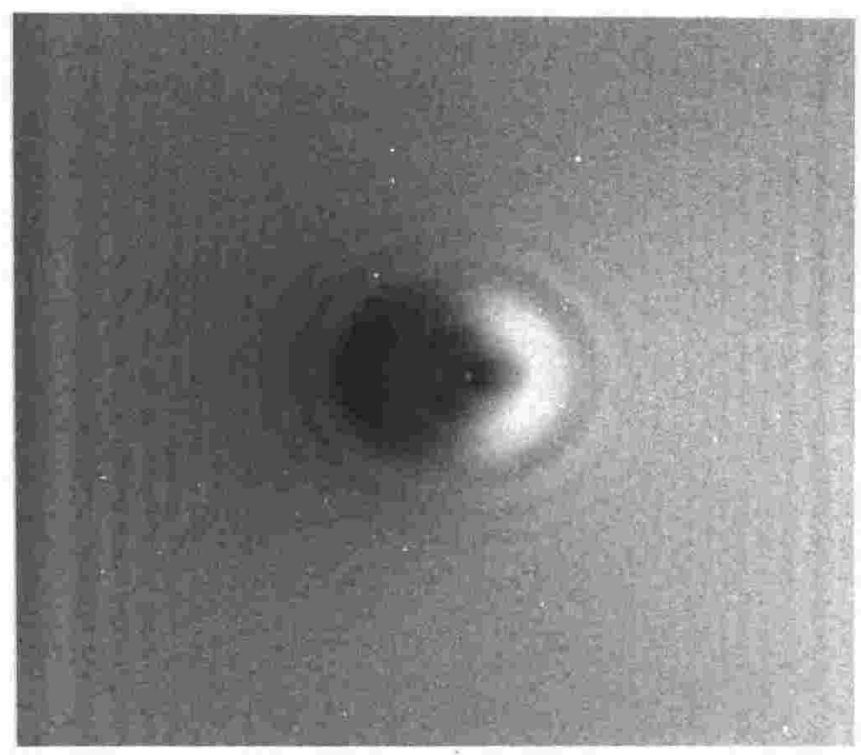

(a)

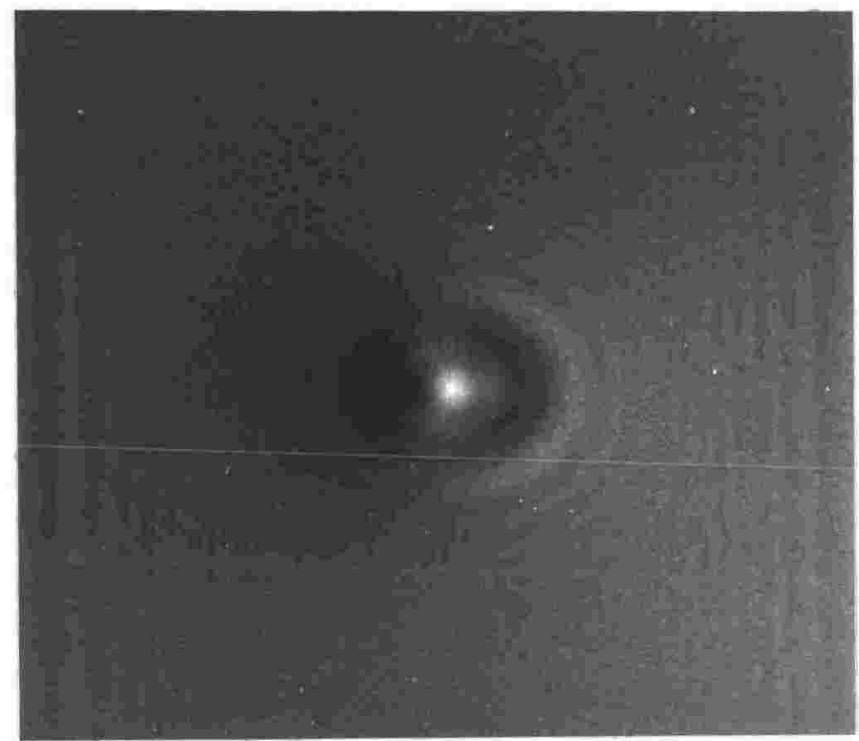

$40 \AA$

(b)

Figure 5.10: Defocussed images for the bubble of figure 5.9. The size of the lobes and the structure of the Fresnel fringes vary with defocus: (a) defocus $=339.4 \mathrm{~nm}$ and (b) defocus $=-339.4 \mathrm{~nm}$ (underfocus). 


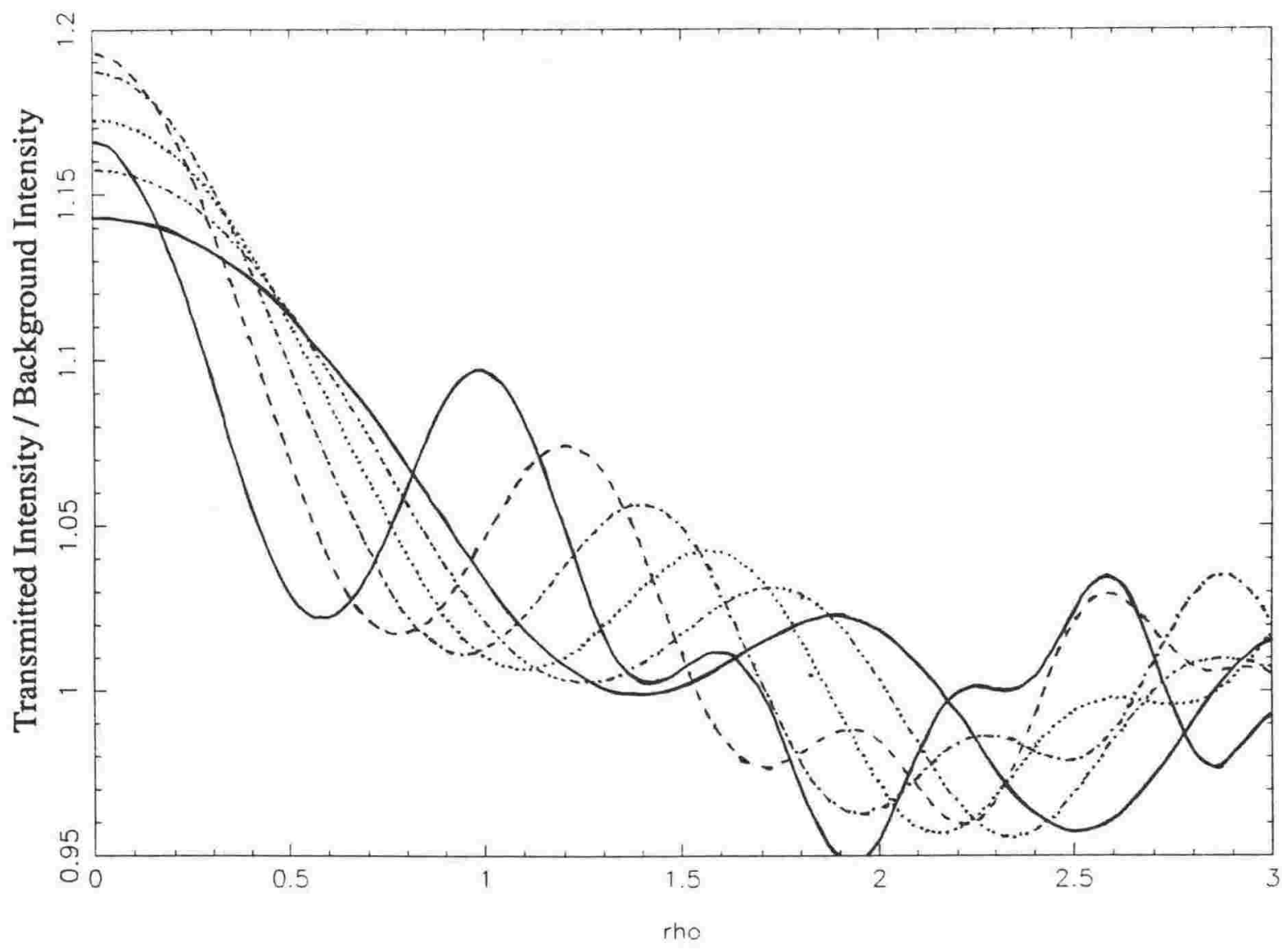

Fresnel fringe positions vs defocus

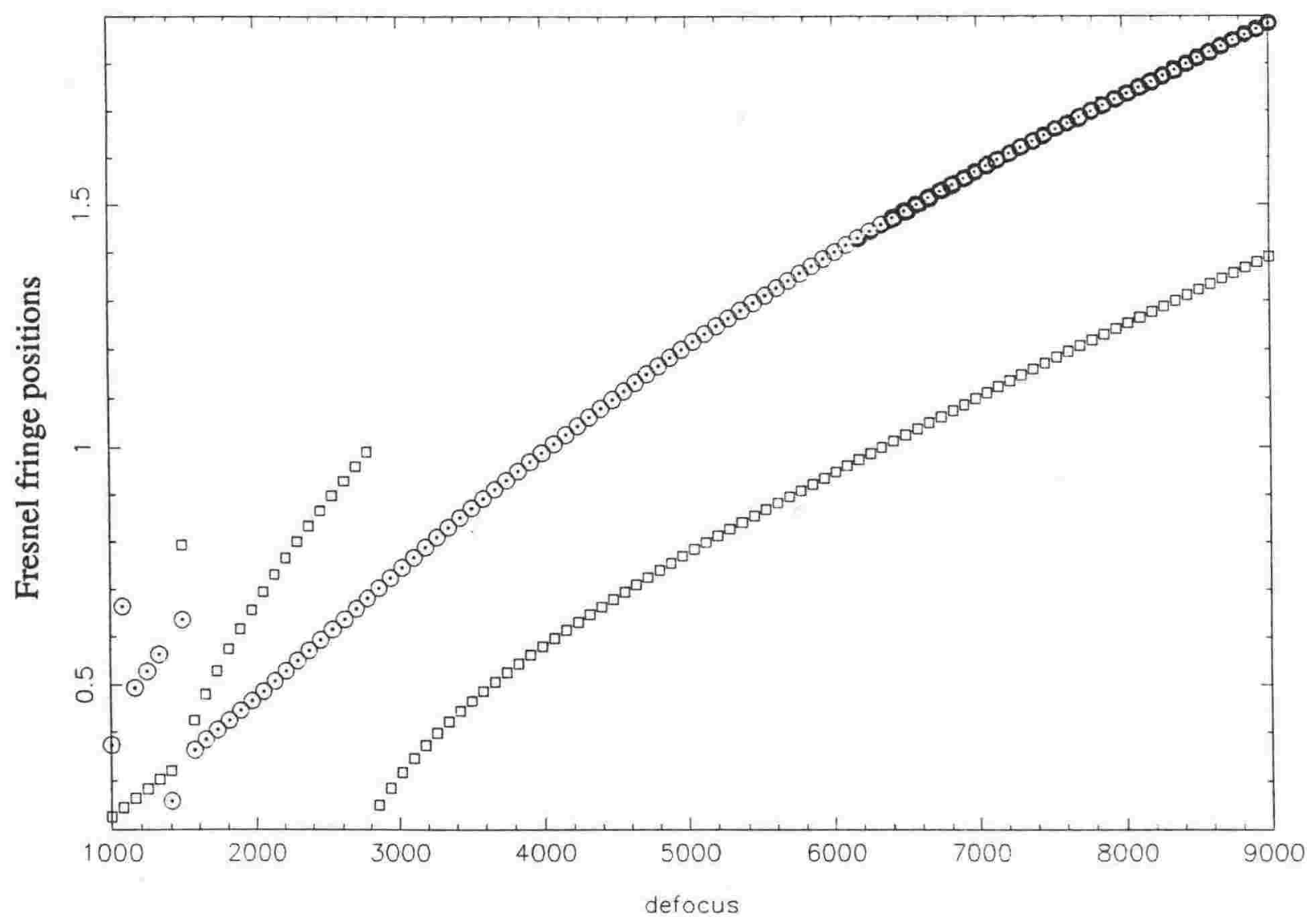

Figure 5.11: Contrast profiles and positions of the maximum and minimum in the intensity profiles for a $20 \AA$ diameter, strain-free, helium gas bubble in copper using $g=[0,2,-2]$. The other parameters are as for figures 5.5 and 5.6. 


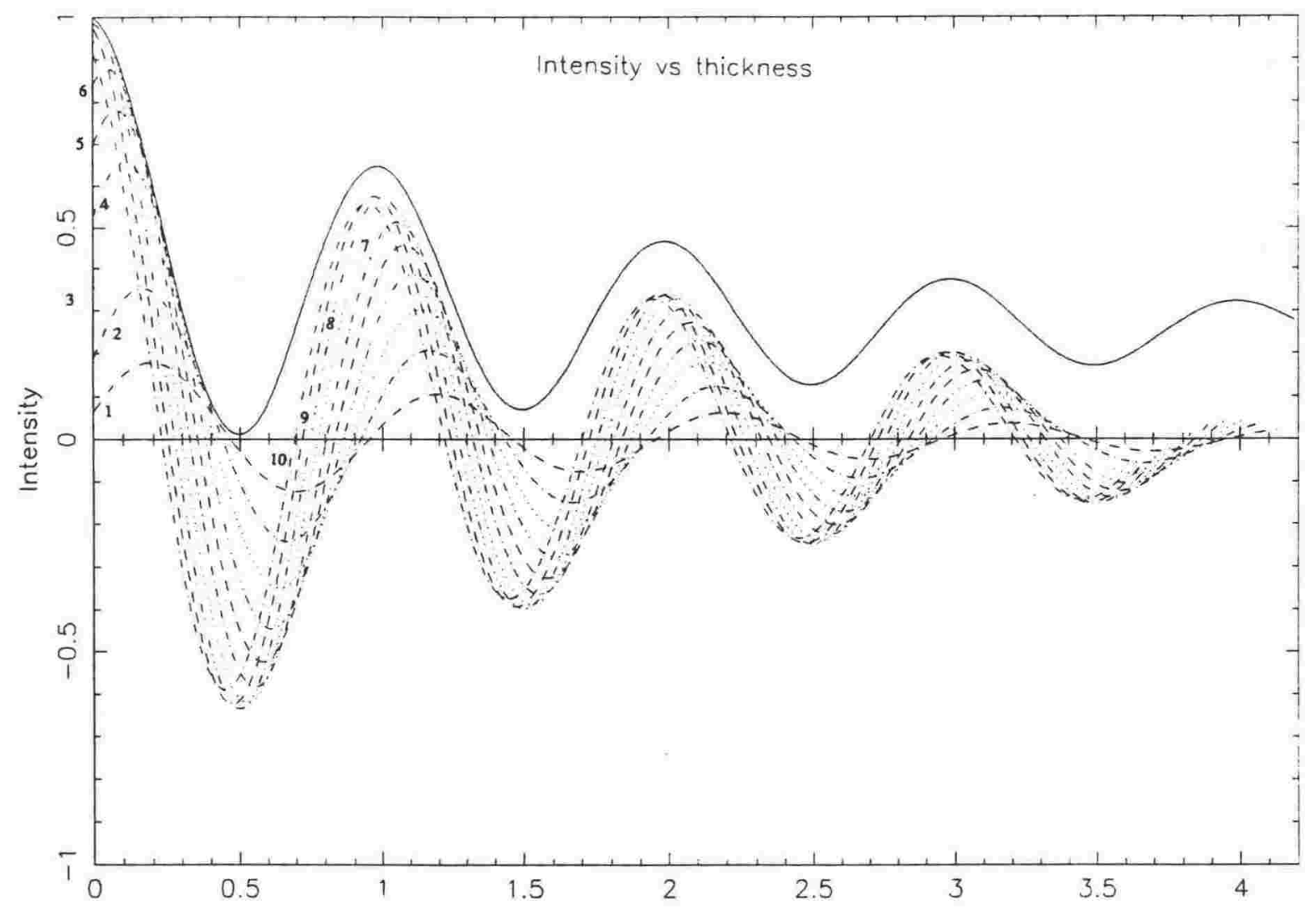

(a)

thickness

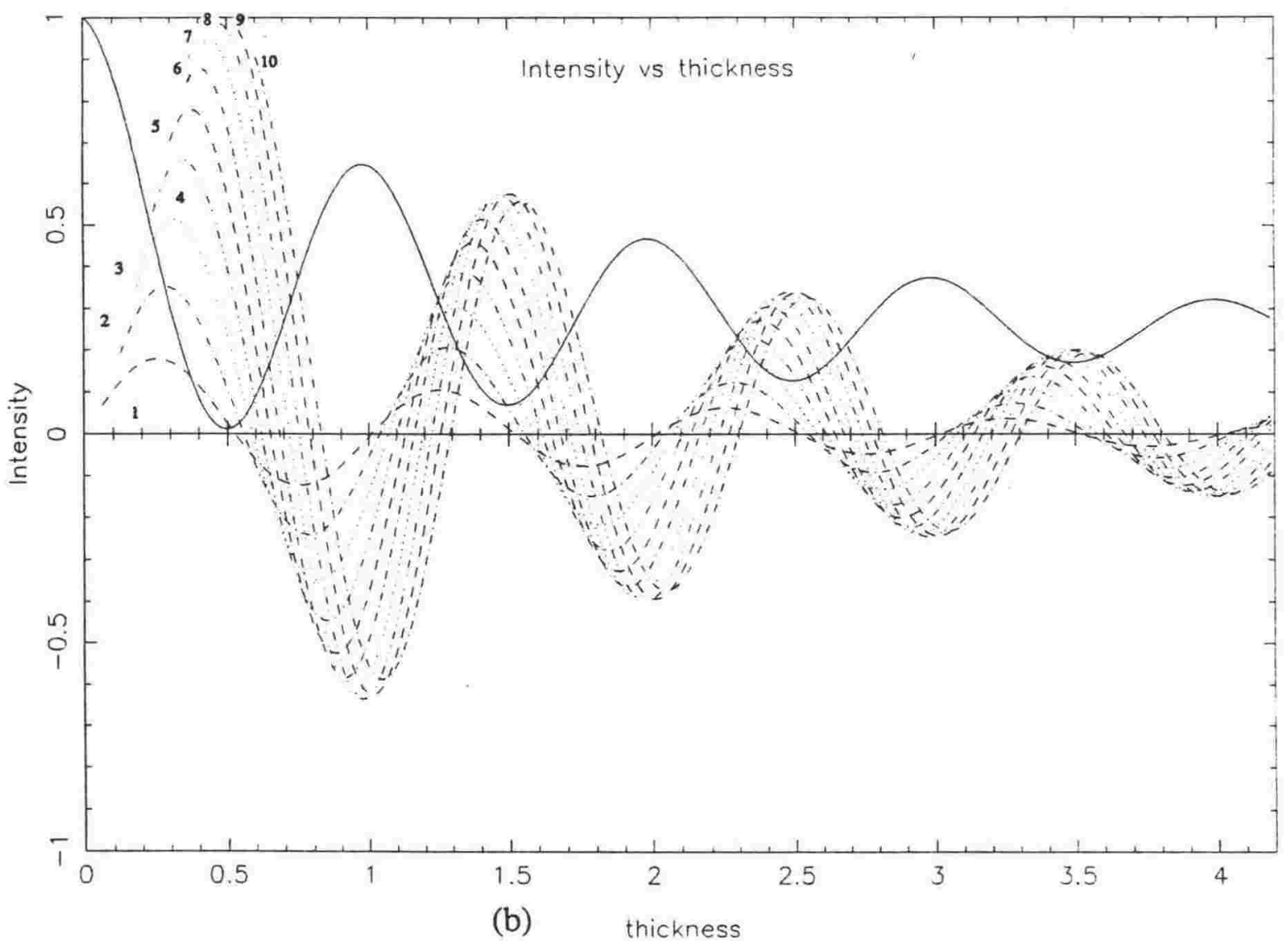

Figure 5.12: The in-focus intensity at the centre of the image of a stack of strain-free bubbles for strong two-beam conditions with $\mathbf{g}=[200]$. In plots (a) and (b) the intensity for stacks of from one to ten bubbles is shown. The information displayed in plots (a) and (b) is the same, but is presented with different choice of horizontal axis. The thickness in plot (a) refers to the amount of pefect crystal material that an electron will go through in passing down the bubble column whereas the thickness in plot (b) includes the bubbles. 
through two columns of metals were considered. The first column passed down through the bubble and the second through perfect crystal at the side of the bubble. The results obtained for a stack of strain-free bubbles in copper are shown in figure 5.12. The bubble radius and spacing are $10 \AA$ and $50 \AA$, respectively.

In figure 5.12 the maximum contrast (i.e., at the intensity peaks) is found to increase initially and then decrease as the number of bubbles in the stack is increased. The maximum contrast stops increasing when the bubble column includes bubbles for more than $0.5 \xi_{\mathrm{g}}$ of its length. This occurs if more than eight bubbles are included in the stack for [111] or [200] reflections or twelve bubbles for $\mathbf{g}=[0,2,-2]$.

Two ways of choosing the horizontal axis have been used. In plot (a) the peaks all lie approximately one above the other. In this plot the "thickness" is the length of perfect crystal that the bubble column includes. (The comparison column passing outside the bubbles has the same length of perfect crystal). The axis of plot (b) and later plots have "thickness" as the length of crystal plus the total "length" of bubble included in the bubble column.

There is little point in simulating a stack of more than six bubbles as observation has shown that stacks containing more than six bubbles are not likely to be found in practice. Appreciable ordering of helium gas bubbles in copper seldom extends over a domain of length greater than about five to six interbubble spacings. The three-dimensional ordering is thought to be similarly limited in extent with each individual domain containing one of several different possible variant bubble structures [45].

In figure 5.13 rocking curves (curves of the variation in intensity with specimen tilt) are presented to show how the intensity at the centre of the image of a stack of bubbles varies for different numbers of bubbles in the stack. Again the bubbles are strain-free and are regularly spaced so that the foil thickness increases in proportion to the number of bubbles included. The rocking curve for a single bubble was given in figure 5.3. Large dips in the intensity of a magnitude roughly proportional to the number of bubbles are found in the curves. E Johnson explained the origin of these dips as due to transmitted electrons which are multiply diffracted, being out of phase and so cancelling with the electrons that are directly transmitted [84]. This occurs when the superlattice constant is approximately equal to an integral number of effective extinction distances (the effective 


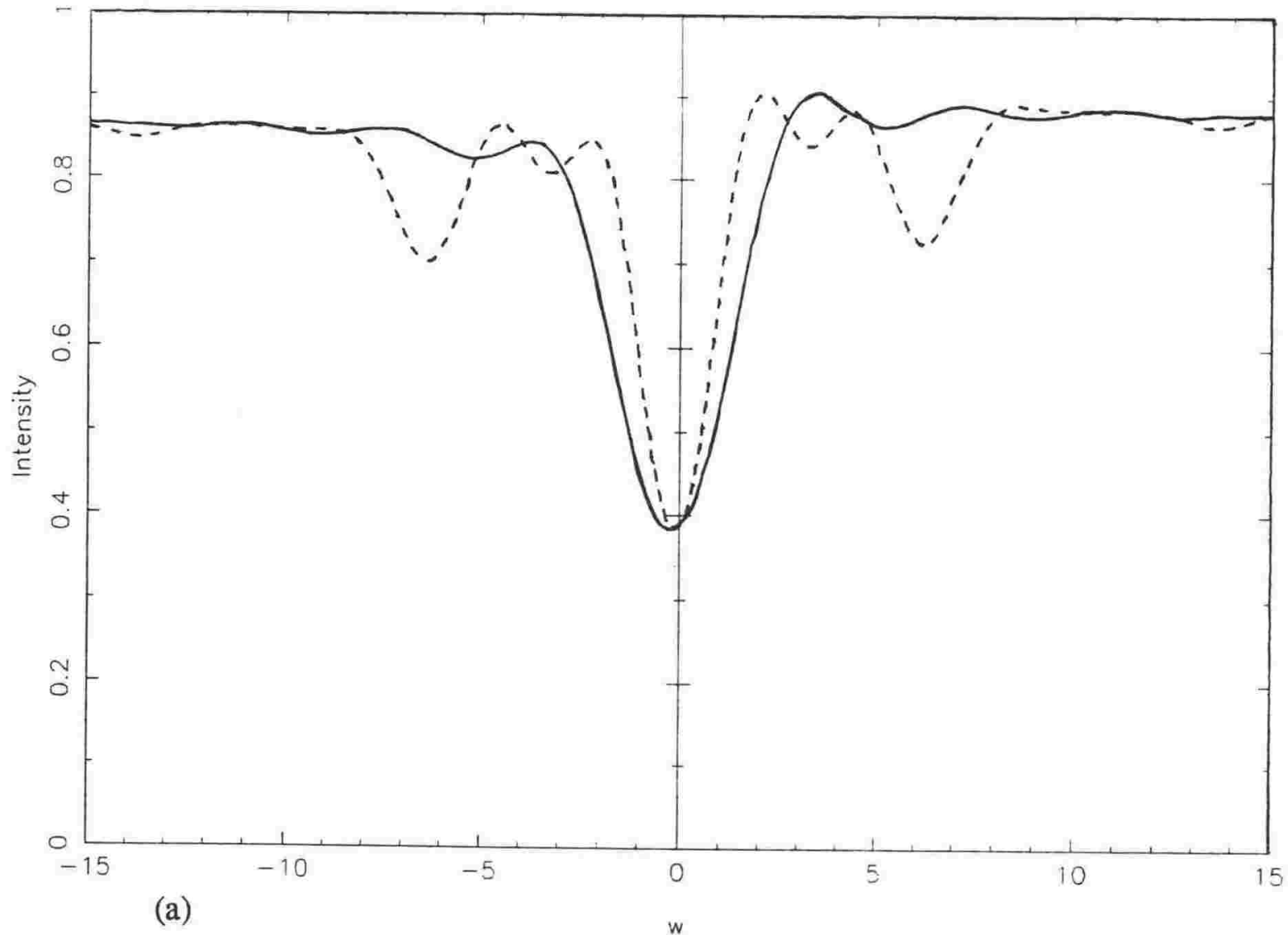

Transmitted Intensity vs w

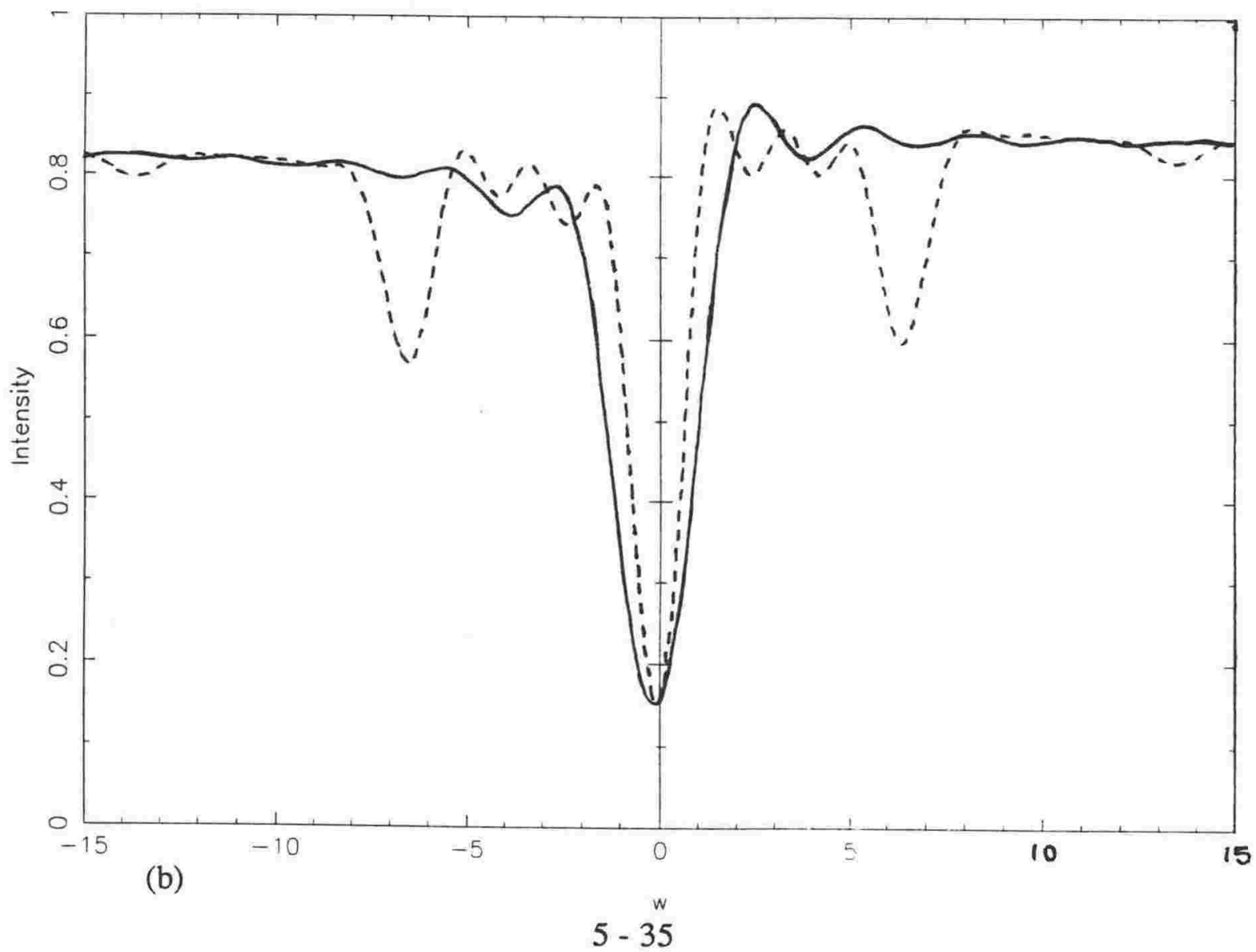

Figure 5.13 (caption on page 5-37) 


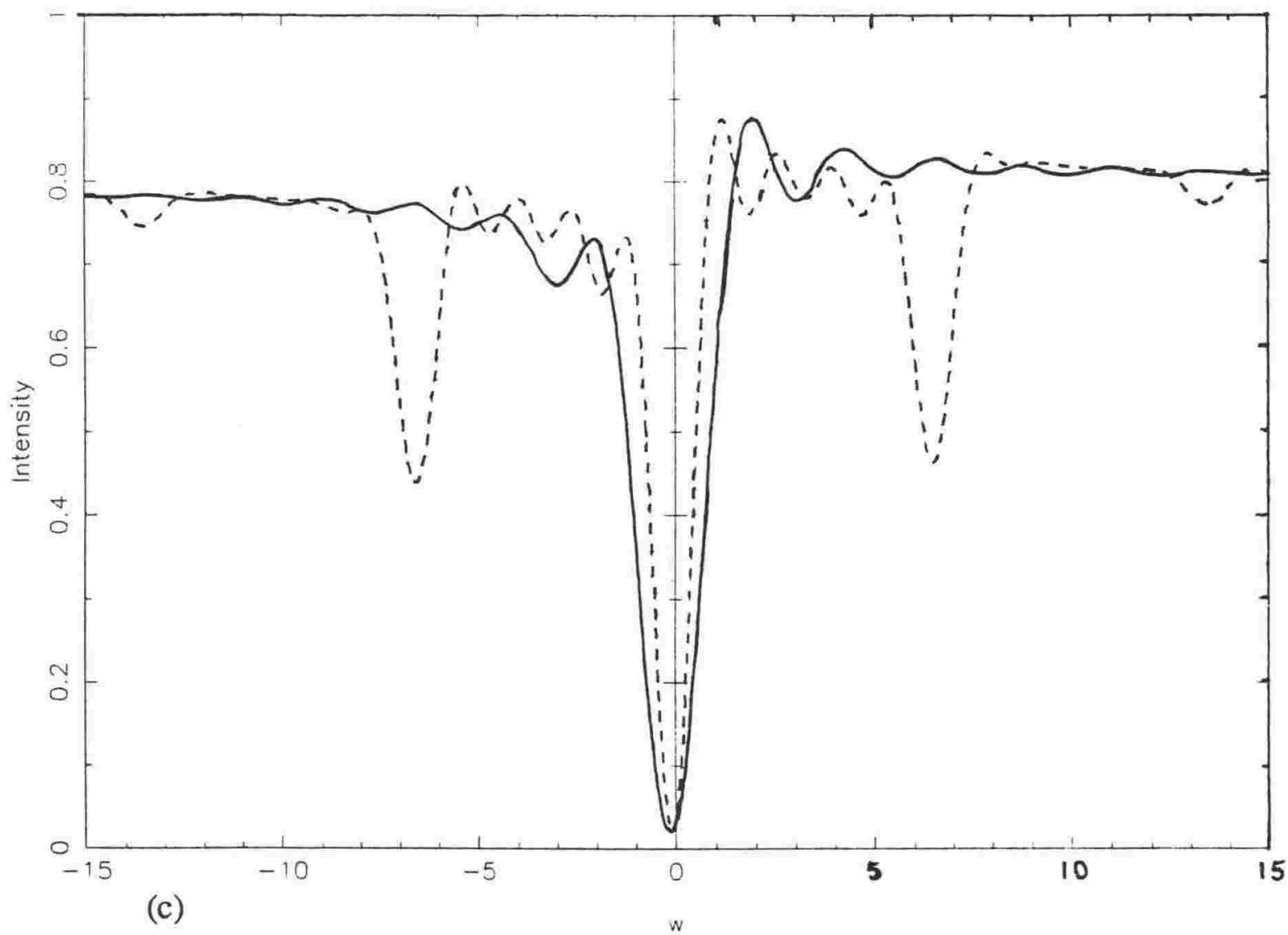

Tronsmitted Intensity vs w

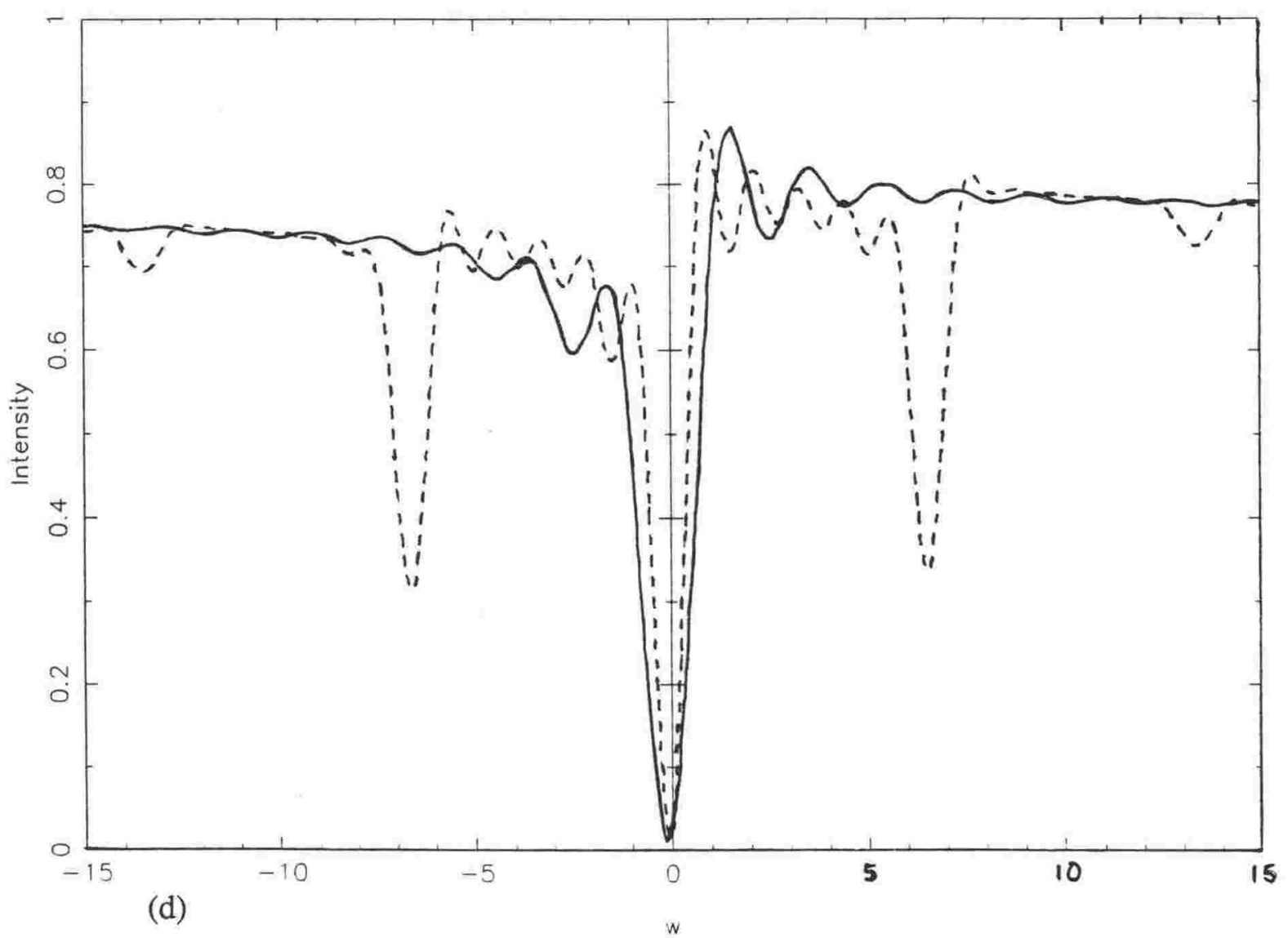

$5-36$

Figure 5.13 (caption on following page) 


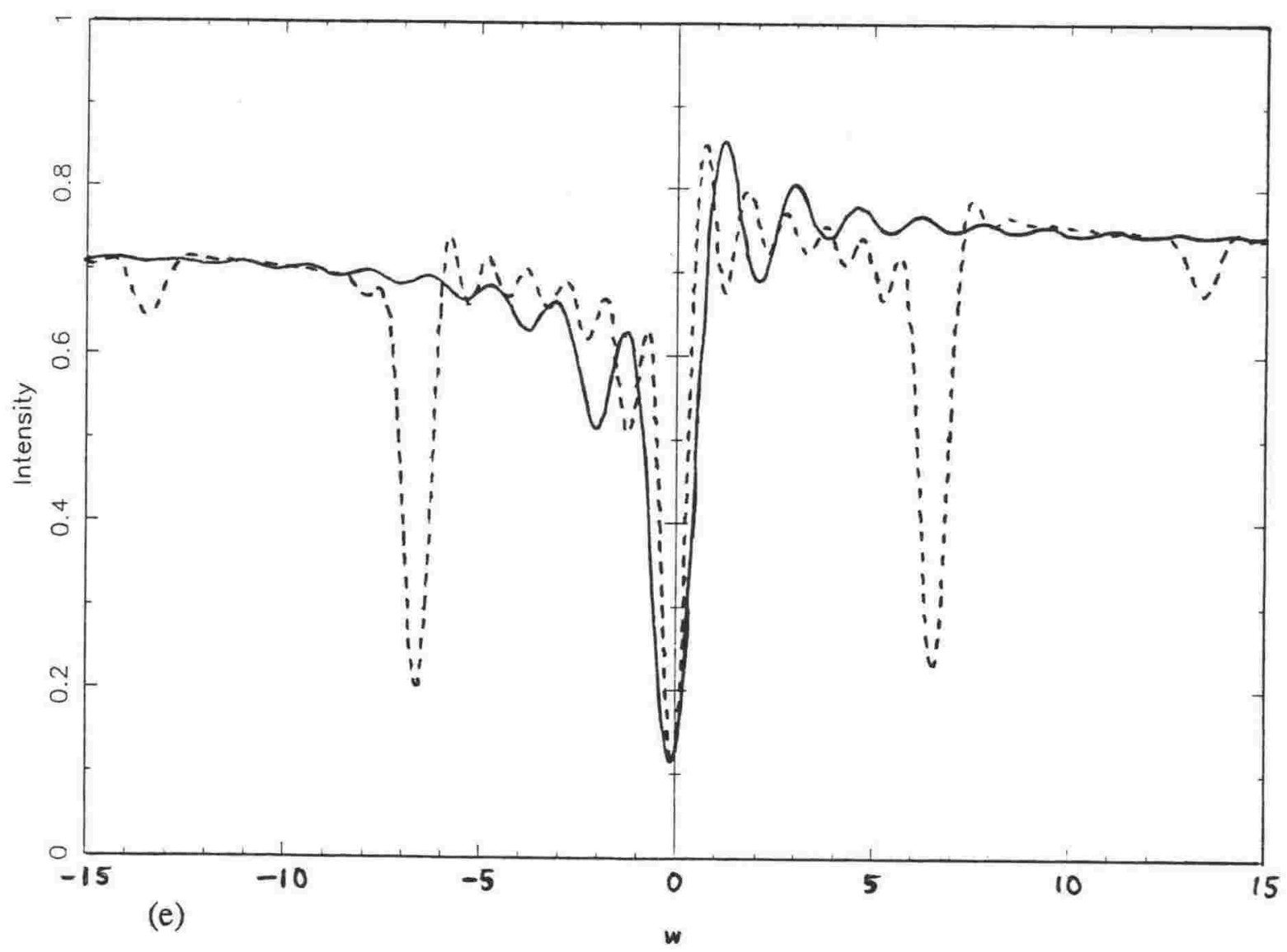

Figure 5.13: Bright-field rocking curves for stacks of (a) two, (b) three, (c) four, (d) five and (e) six equilibrium bubbles. The dashed lines are the rocking curves for a column passing through the bubble stack. The solid lines are the rocking curves for a column passing through a thickness of crystal equal to that in the bubble stack. For example if the stack contained three bubbles with a diameter of $20 \AA$, the centres of which are separated by $50 \AA$ and the centres of the top and bottom bubbles in the stack are $40 \AA$ from the nearest surface, then the foil is taken to have a thickness of $120 \AA$ (equal to four times $30 \AA$ ). Compared with the intensity in the column that passes through the perfect crystal, large deviations can occur in the bubble column. 
extinction distance is reduced from the usual extinction distance by a factor of $\sqrt{ }\left(1+w^{2}\right)$ as proved by the analytical solution to the differential equations of Howie and Whelan in section 2.1).

Gas bubbles are usually identified by their characteristic white appearance when in underfocus and black appearance when in overfocus. Plots of the contrast at the centre of the image of a stack of bubbles for varying degrees of defocus show that this simple interpretation breaks down (and, in fact for one bubble, holds over a restricted range of defocus only). In figure 5.14 the break-down occurs as the number of bubbles in the stack is increased. The curves all converge to the background intensity for large values of the defocus as expected, although some converge to background from above and some from below which means that the appearance of the stack of bubbles can be either white or black depending on the number of bubbles. Stacks of more than five or six bubbles in them appear as black when in underfocus and white when in overfocus except when a small defocus is used (the reverse of the expected behaviour). In these plots the bubbles are constantly spaced so again the foil thickness increases as the number of bubbles is increased.

The same sort of plots are repeated in figure 5.15 with the crystal tilted away from strong two-beam conditions. The conditions are a better model for micrographs of superlattices because the crystal is always tilted away from two-beam conditions to remove or minimise the contrast from any dislocations or strains that are present and thereby enhance both the bubble images and the superlattice reflections in diffraction (section 5.4). A choice of tilt giving $w=15.0$ moves the plots away from the region where dips occur in the rocking curves of figure 5.13. With this larger excitation error stacks of four, five and six bubbles obey the simple criterion over a significant range of the defocus values (for example compare the position of a minimum (at an overfocus around $2200 \AA$ ) with that of a maximum (at an underfocus of $-2000 \AA$ )). Consider an operator that restricts observations of the superlattice to defocus values from $-3000 \AA$ to $3000 \AA$ in a TEM in which the rapid oscillations in intensity for small values of defocus are damped out by the effects listed in section 5.2.1.1. This operator will observe a change in the appearance of the bubble stack from white to black as defocus is increased. The maximum transmitted intensity will be 
Transmitted Intensity / Background Intensity vs Defocus

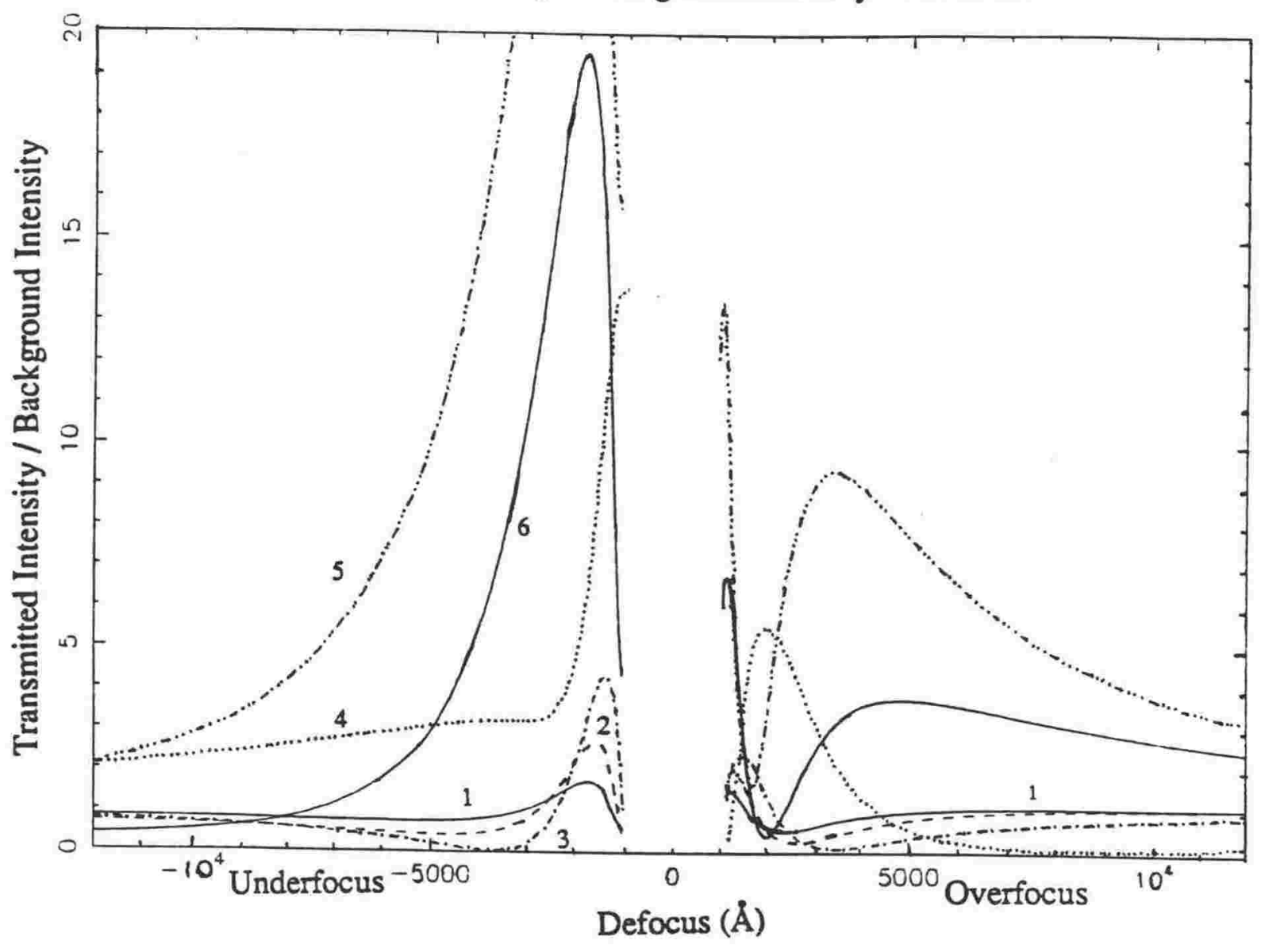

Figure 5.14: Defocussed intensity at the centre of the image of a stack of equilibrium bubbles for $\mathrm{w}=0.0$ and $\mathrm{g}=[200]$. The number of bubbles in the stack is varied from one to six bubbles. The often quoted characteristic of a bubble is that it appears as white in underfocus and dark in overfocus. For one bubble this simple rule is seen to be true over a limited range of defocus. 


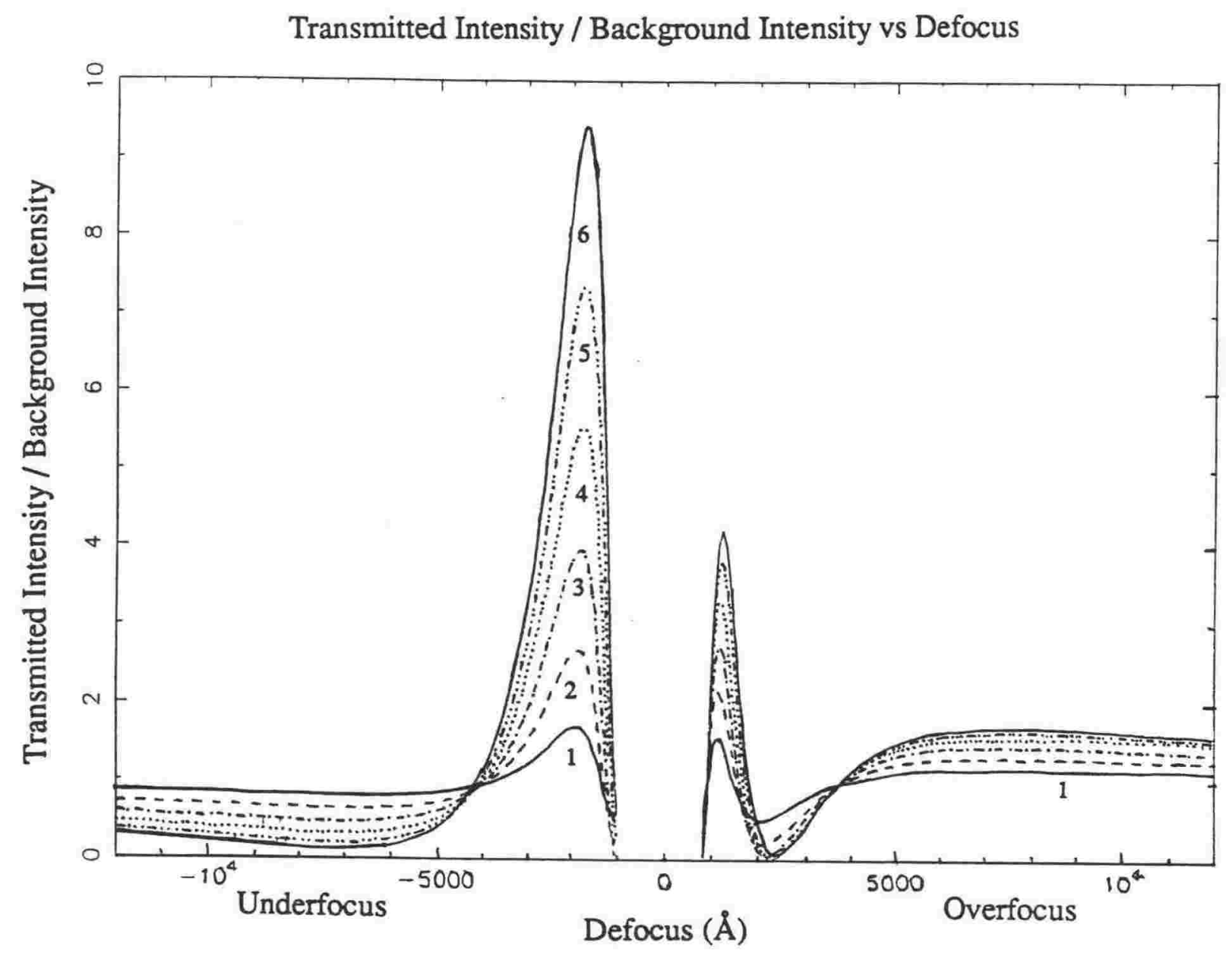

Figure 5.15: Defocussed intensity at the centre of the image of a stack of equilibrium bubbles for $\mathrm{w}=15.0$ and $\mathbf{g}=[200]$. The number of bubbles in the stack is varied from one to six bubbles. The contrast predicted can be compared to the often quoted characteristic of a bubble that it appears as white in underfocus and dark in overfocus. For clarity the central portion of the curves, over which the intensity varies rapidly, is not shown. 
greater than the background intensity by a factor of approximately 1.5 the number of bubbles in the stack.

All the curves in figures 5.14 and 5.15 converge to the background intensity for defocus values of large magnitude. Significant contrast occurs at moderate defocus values and a TEM operator probably will take a photo in this region where the intensity vs. defocus curves have a minimum in overfocus or maximum in underfocus. The peaks in intensity at underfocus are much stronger than the peaks in intensity at overfocus. More detail can be then seen in the overfocus pictures and the choice of exposure times is less critical than in underfocus pictures. Most experimental micrographs are then taken in overfocus. Yet if an operator is trying to determine the bubble density it is better to use underfocus conditions since the contrast will be stronger. Intensity profiles for $\mathrm{w}=0.0$ and 15.0 are shown in figures 5.16 and 5.17 respectively. In figure 5.17 , for one bubble, as the defocus increases the small black part in the centre of the image disappears to be replaced by a white part (a maximum) that extends out to the bubble radius. Still considering one bubble, large intensity variations (greater than about 10\%) from the background do not occur outside about $p=1.5$. This behaviour is very plausible when compared with experiment. The profile through a six bubble stack has many oscillations. The oscillations are larger and extend over more of the profile than those from stacks containing fewer bubbles. Of course beam divergence and coherence effects may damp out these oscillations to some degree. The profiles for more than about three bubbles in a stack begin to approach the appearance of the profiles for six bubbles in a stack (these profiles do not have the correct experimental behaviour in that they display extreme variations in intensity along the profile and have a strong dependence on defocus). Therefore the profiles suggest that the superlattice structure does not extend into the foil for more than about three bubbles.

In figure 5.18 where the foil thickness has been set to $1.675 \xi_{\mathrm{g}}$, the image intensities and contrast are then larger than the curves for other foil thicknesses. This behaviour is expected from figures 5.1 and 5.12. The shape of the curve for one bubble is unaffected by the thickness change but all the other profiles are changed significantly. For example a stack of two bubbles has a large white peak in the centre of the projected position of the bubbles in figure 5.18 at a defocus of $4000 \AA$. However, it has a central dark 
Profile of the Intensity / Background Intensity

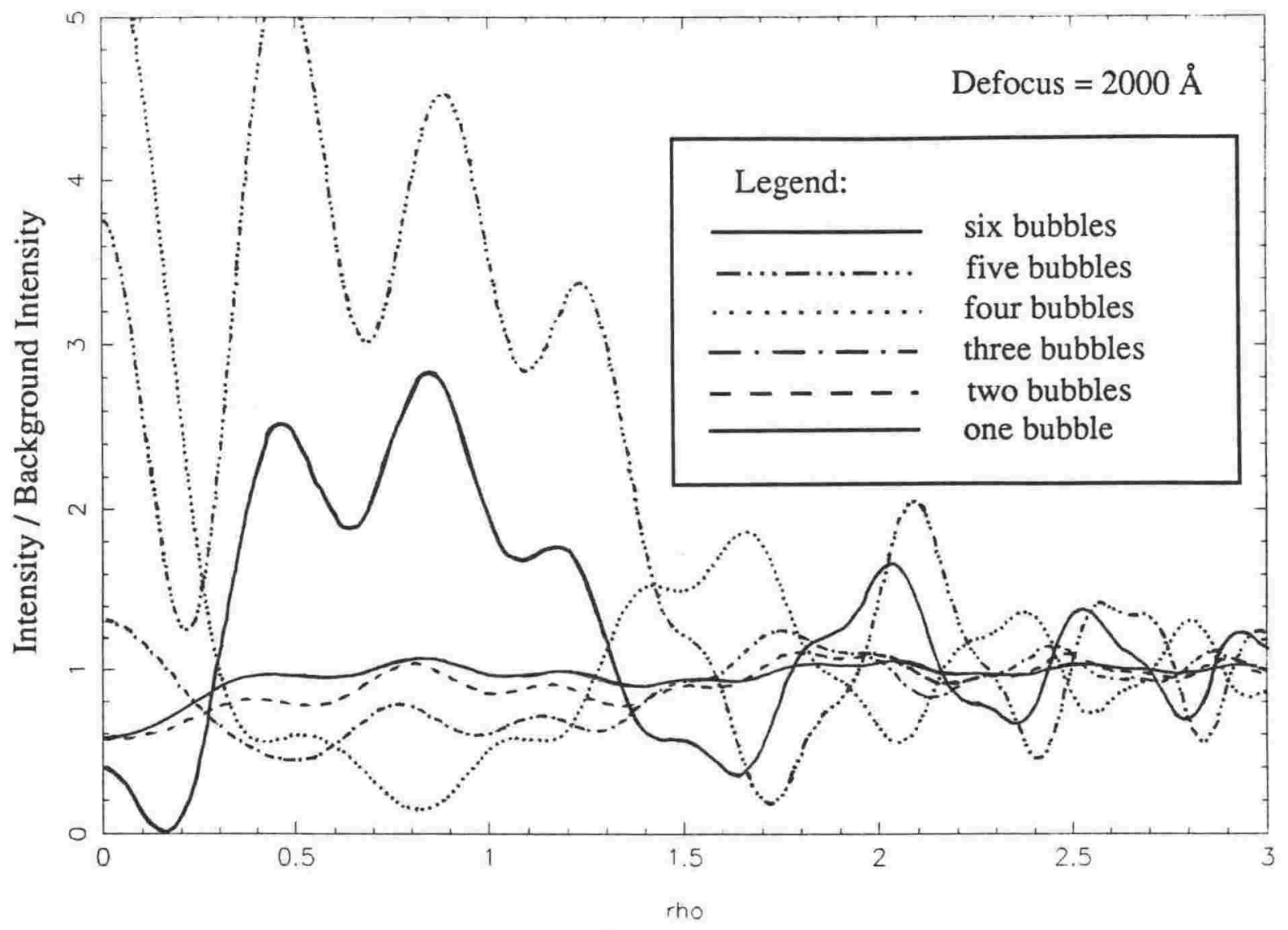

Profile of the Intensity / Background Intensity

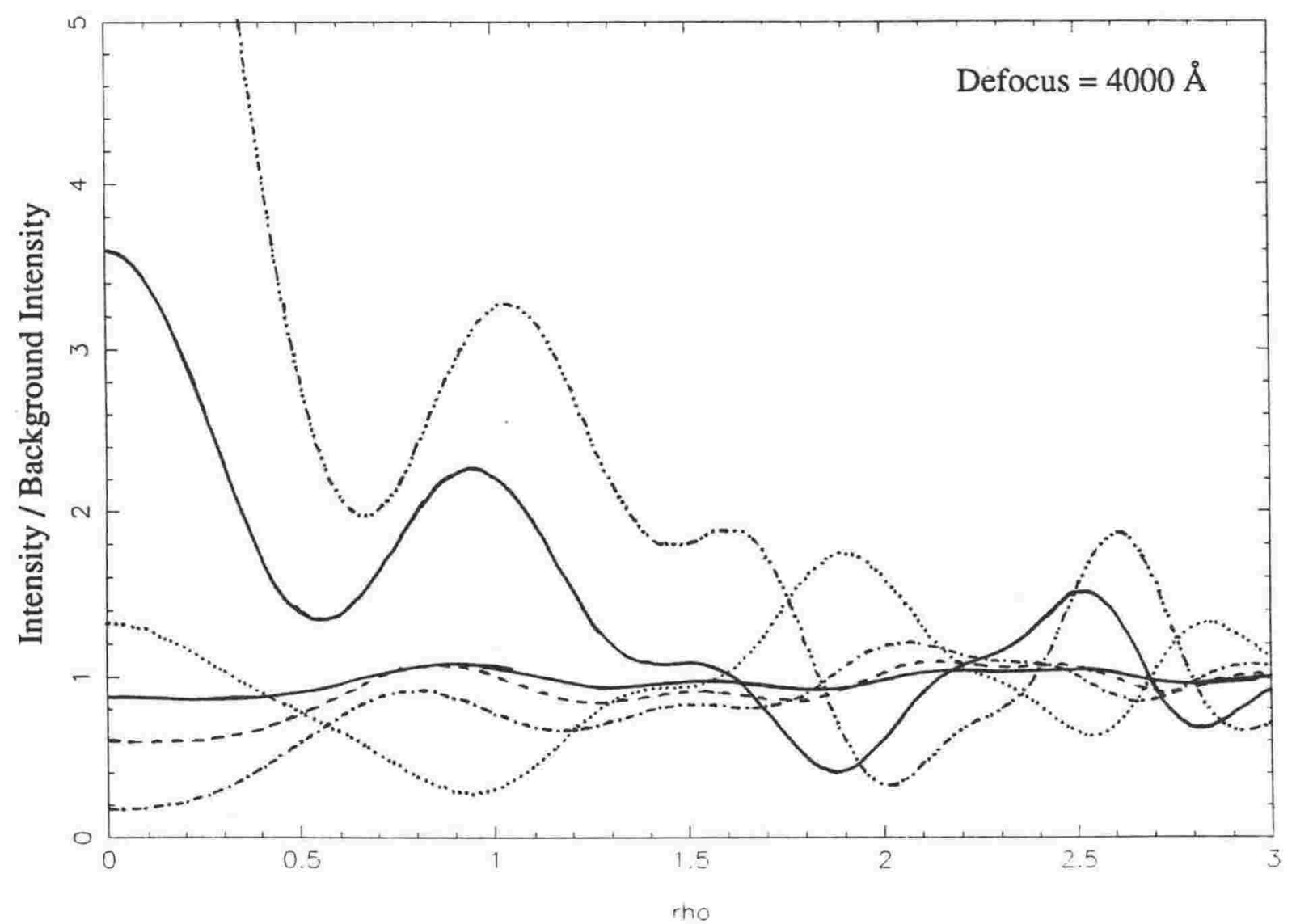

5-42 Figure 5.16 (caption on following page) 

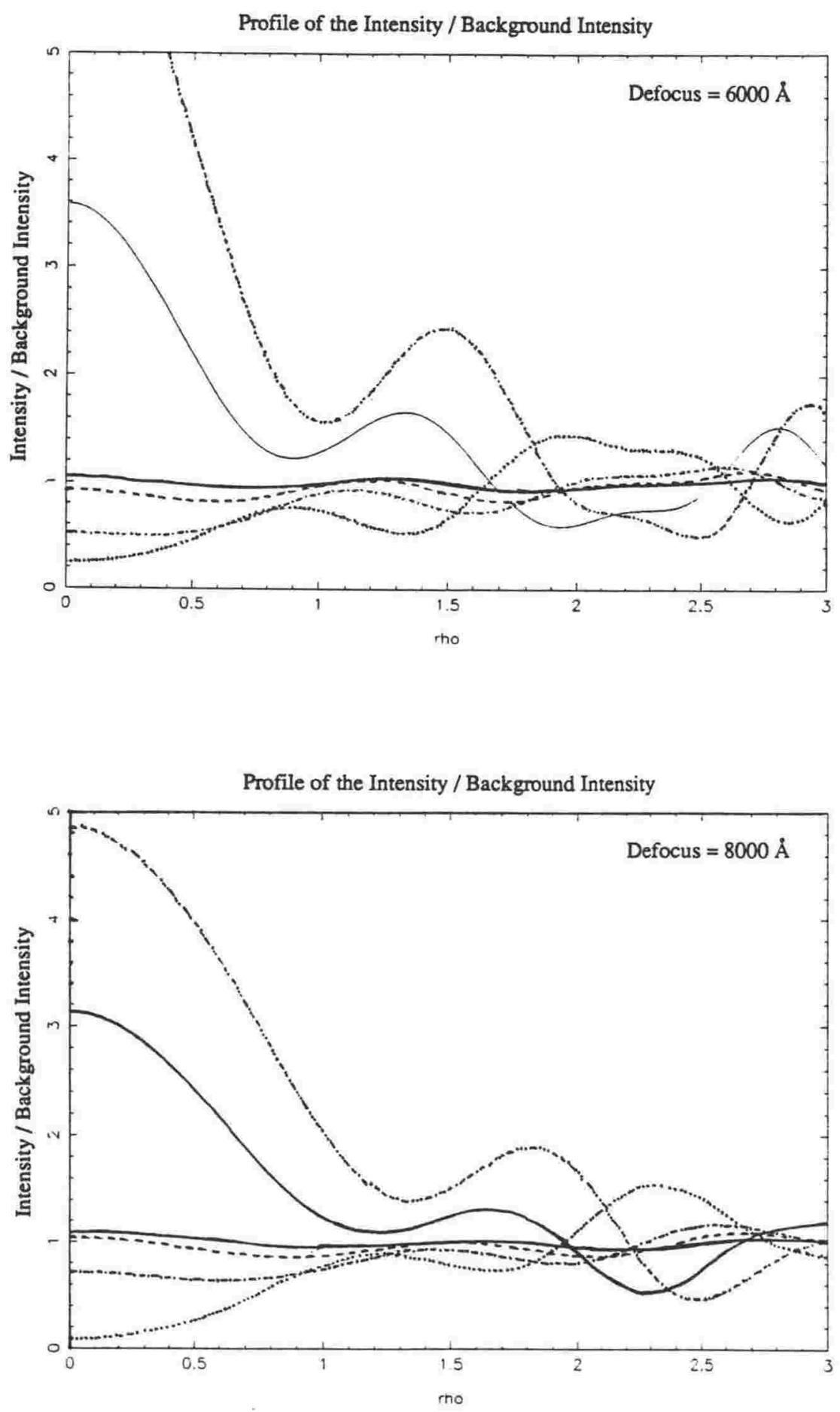

Figure 5.16: Intensity profiles (normalised to the background intensity) for stacks of bubbles containing from one to six equilibrium bubbles, when imaged with $\mathrm{w}=0.0$ and $\mathbf{g}$ $=[200]$. (The curve for one bubble has smaller deviations from the background intensity than the curve for six bubbles). The range of defocus is restricted to $2000 \AA, 4000 \AA, 6000$ $\AA$ and $8000 \AA$. The foil thickness is varied according to the number of bubbles in the stack. The contrast predicted can be compared to the often quoted characteristic of a bubble that it appears as white in underfocus and dark in overfocus. 
Profile of the Intensity / Background Intensity

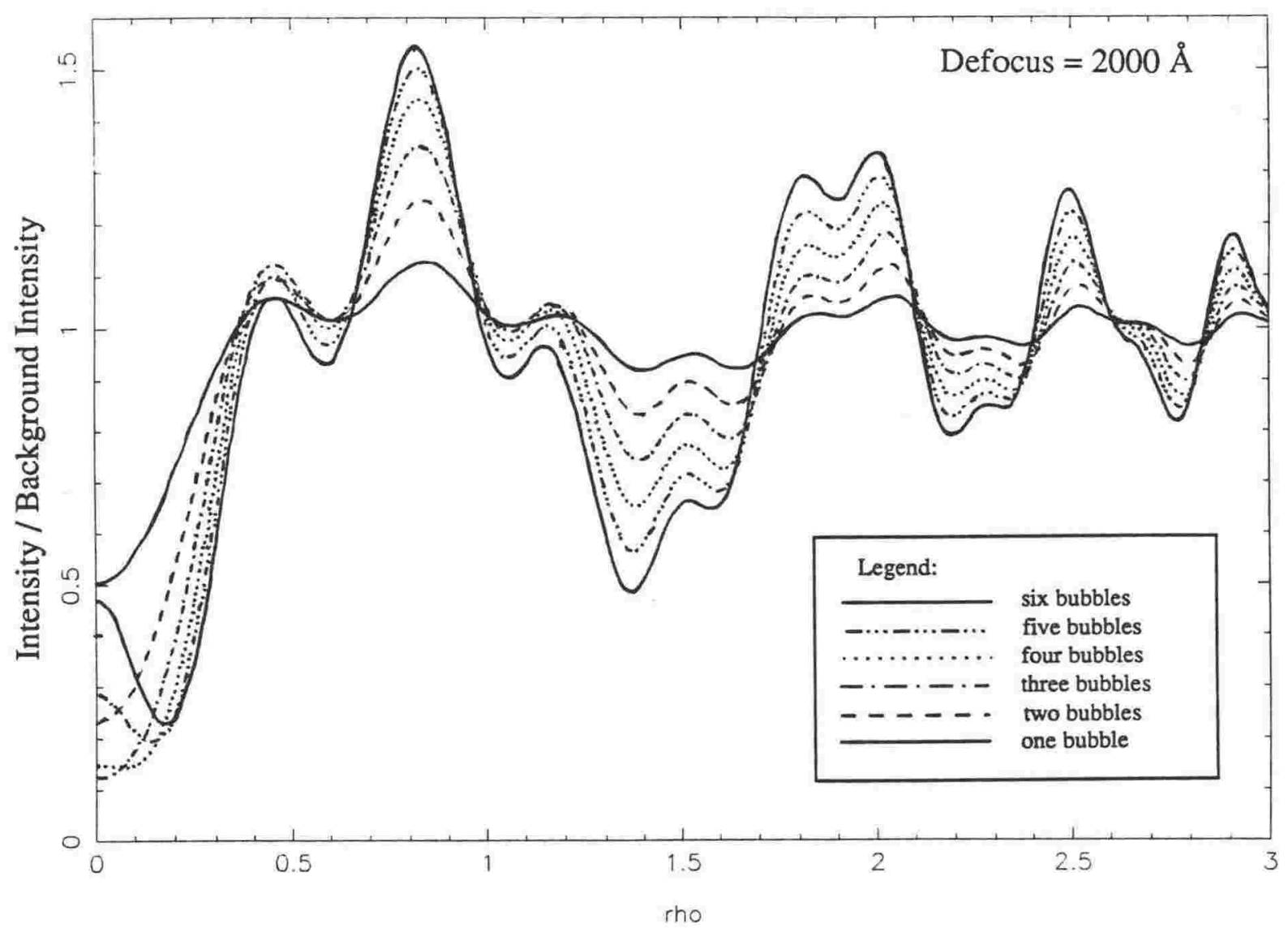

Profile of the Intensity / Background Intensity

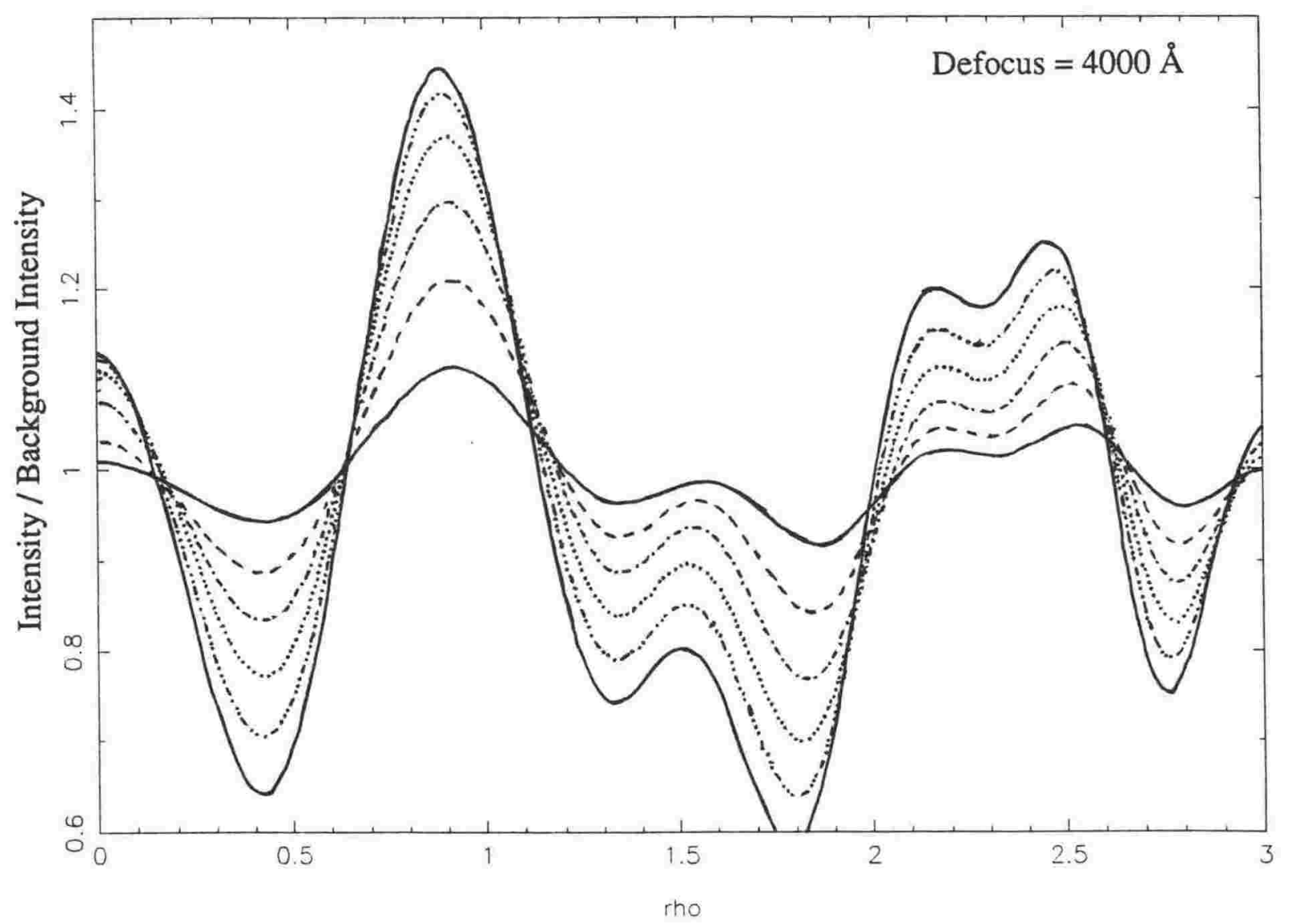

5 - 44 Figure 5.17 (caption on following page) 

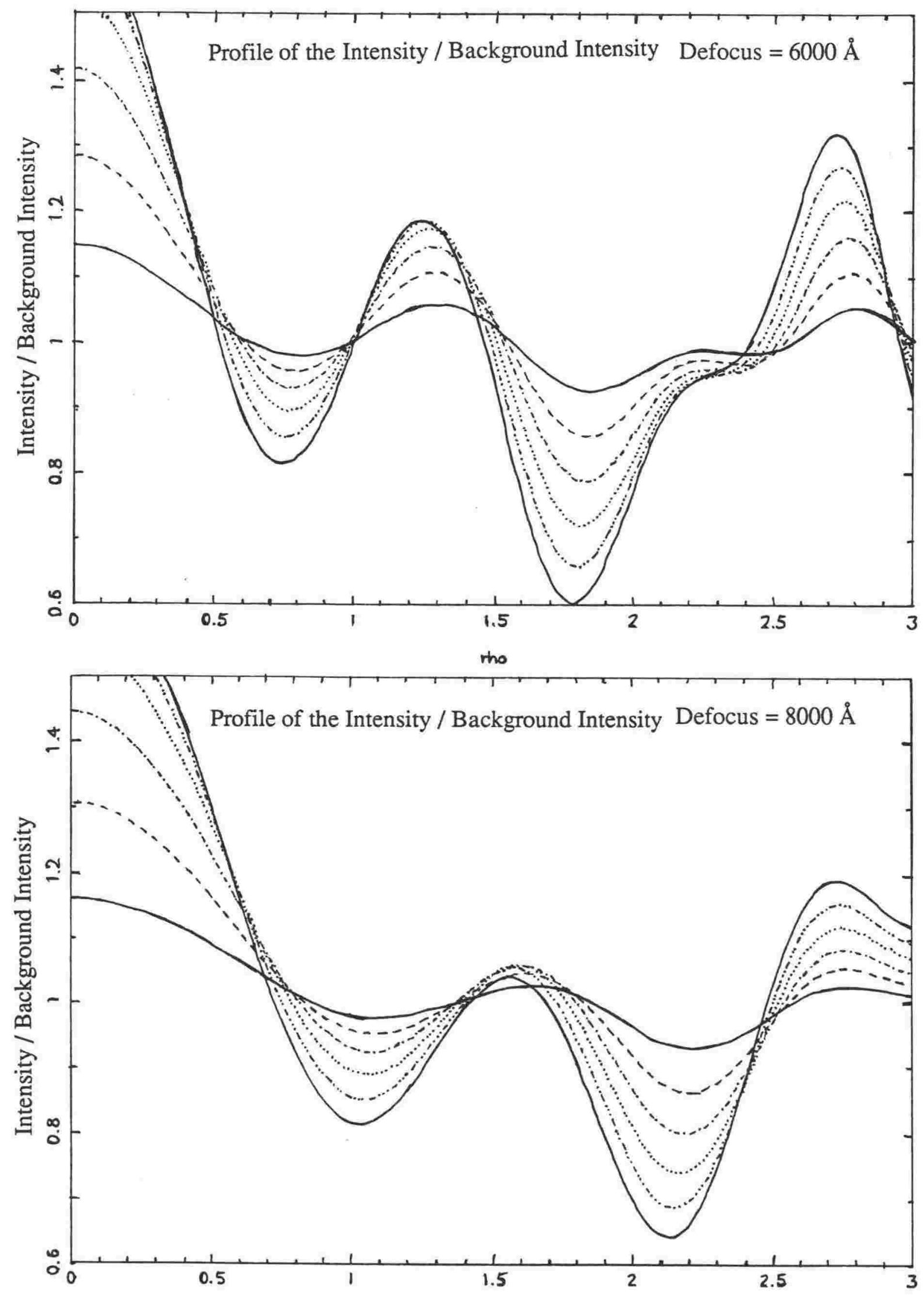

Higure 5.17: Intensity profiles (normalised to the background intensity) for bubbles stacks containing from one to six equilibrium bubbles when imaged with $w=15.0$. The other parameters are as for figure 5.16. Columns that have more than about three bubbles in them do not follow a simple behaviour over even a restricted range of experimentally plausible values for the defocus. 
Profile of the Intensity / Background Intensity

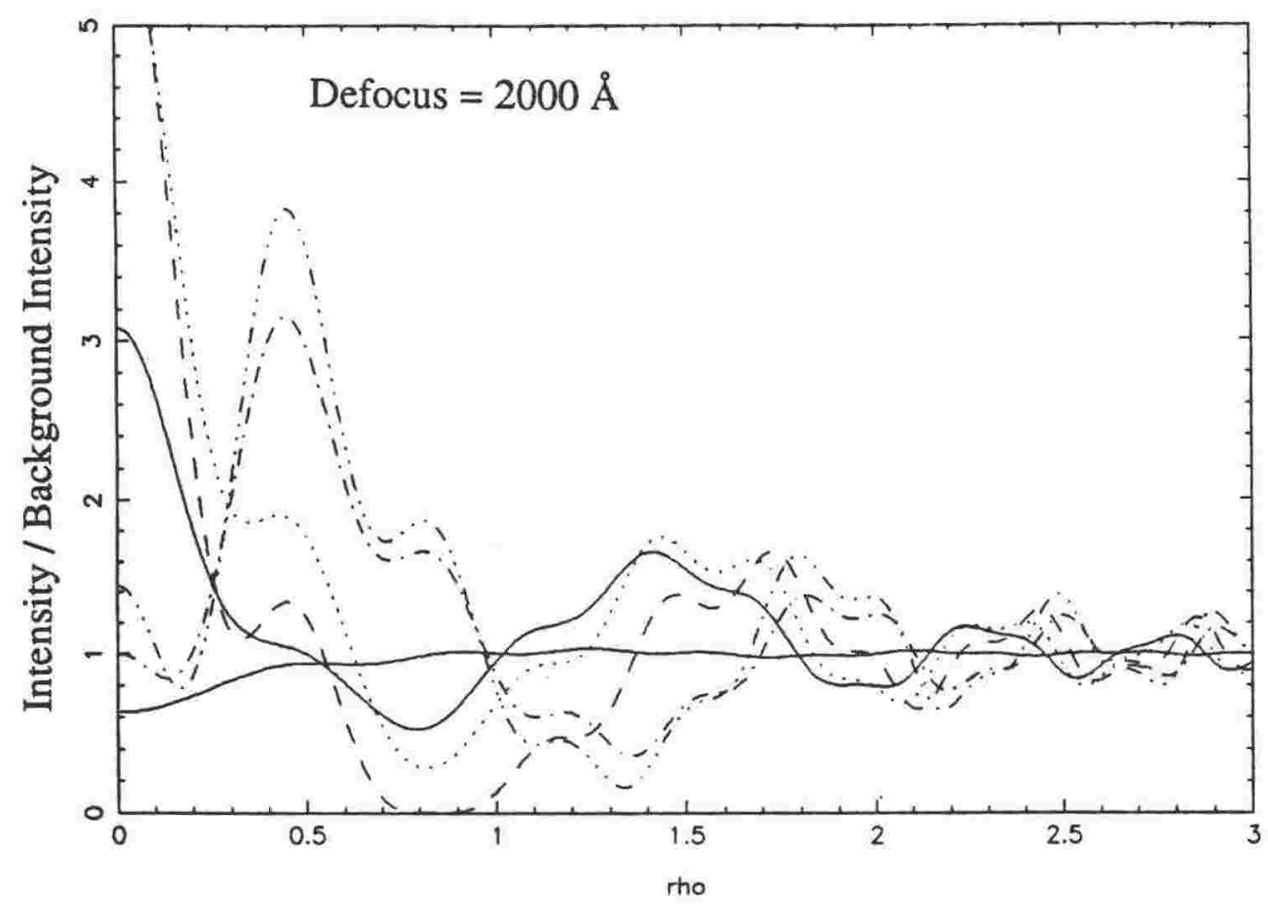

Profile of the Intensity / Background Intensity

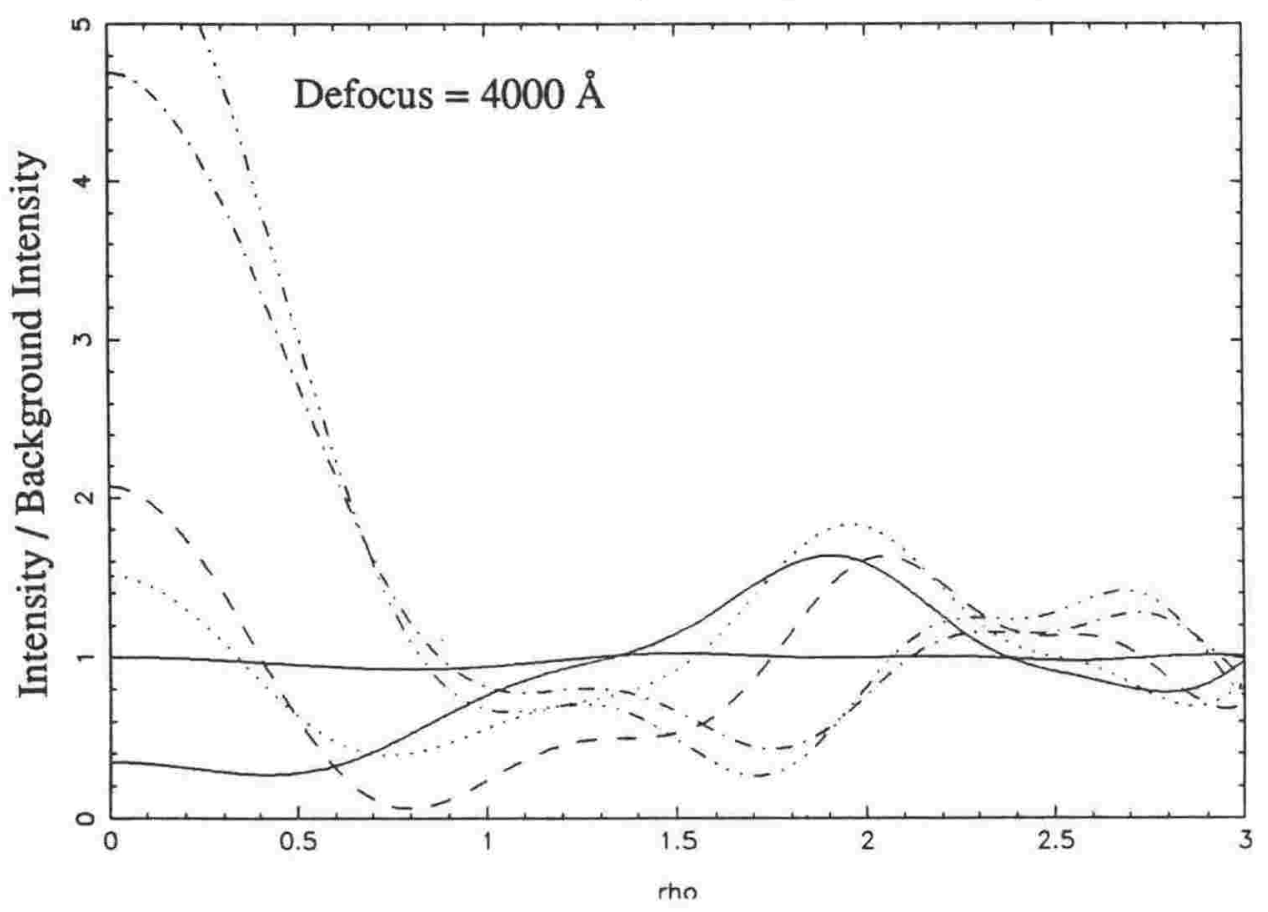

Figure 5.18 (caption on following page) 
Profile of the Intensity / Background Intensity

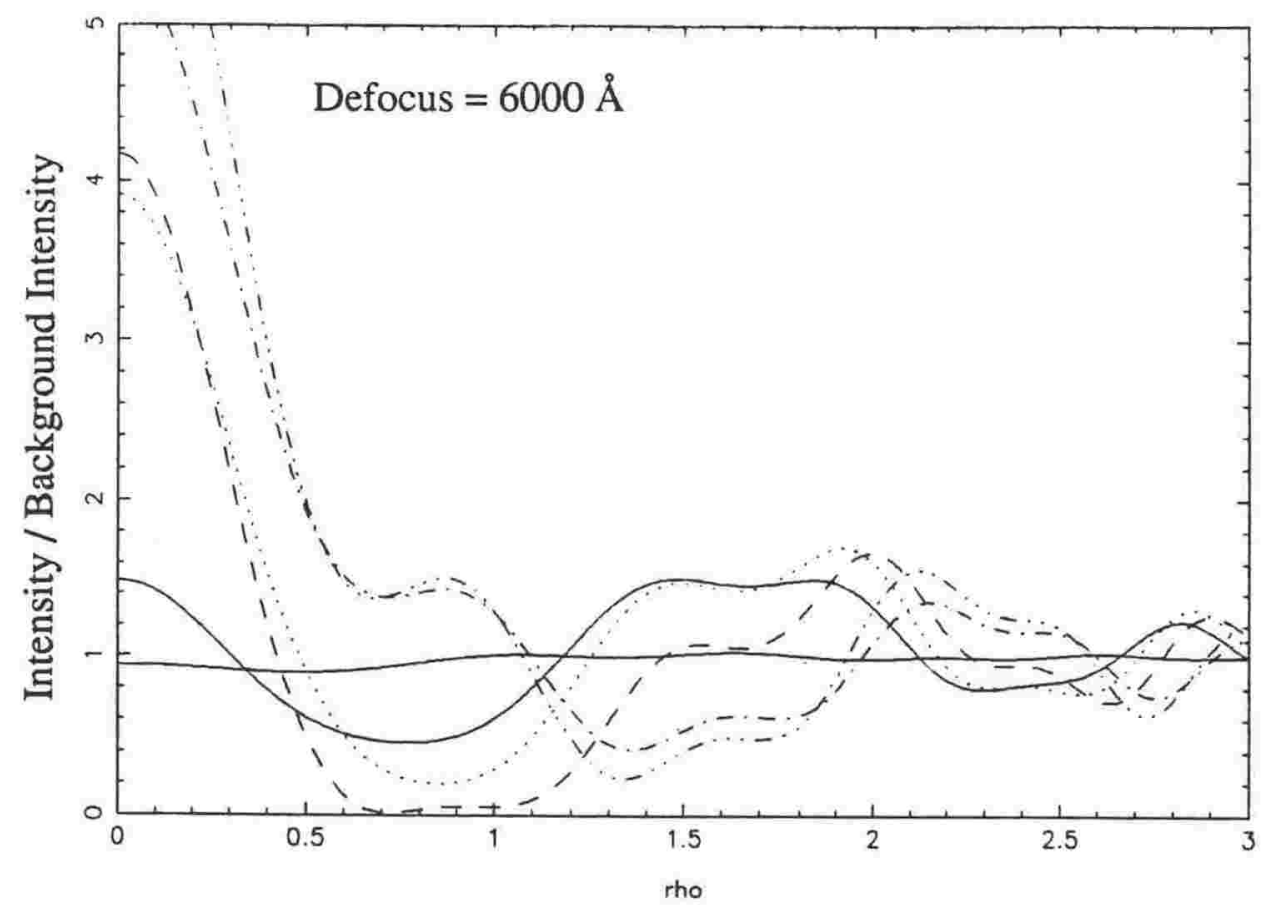

Profile of the Intensity / Background Intensity

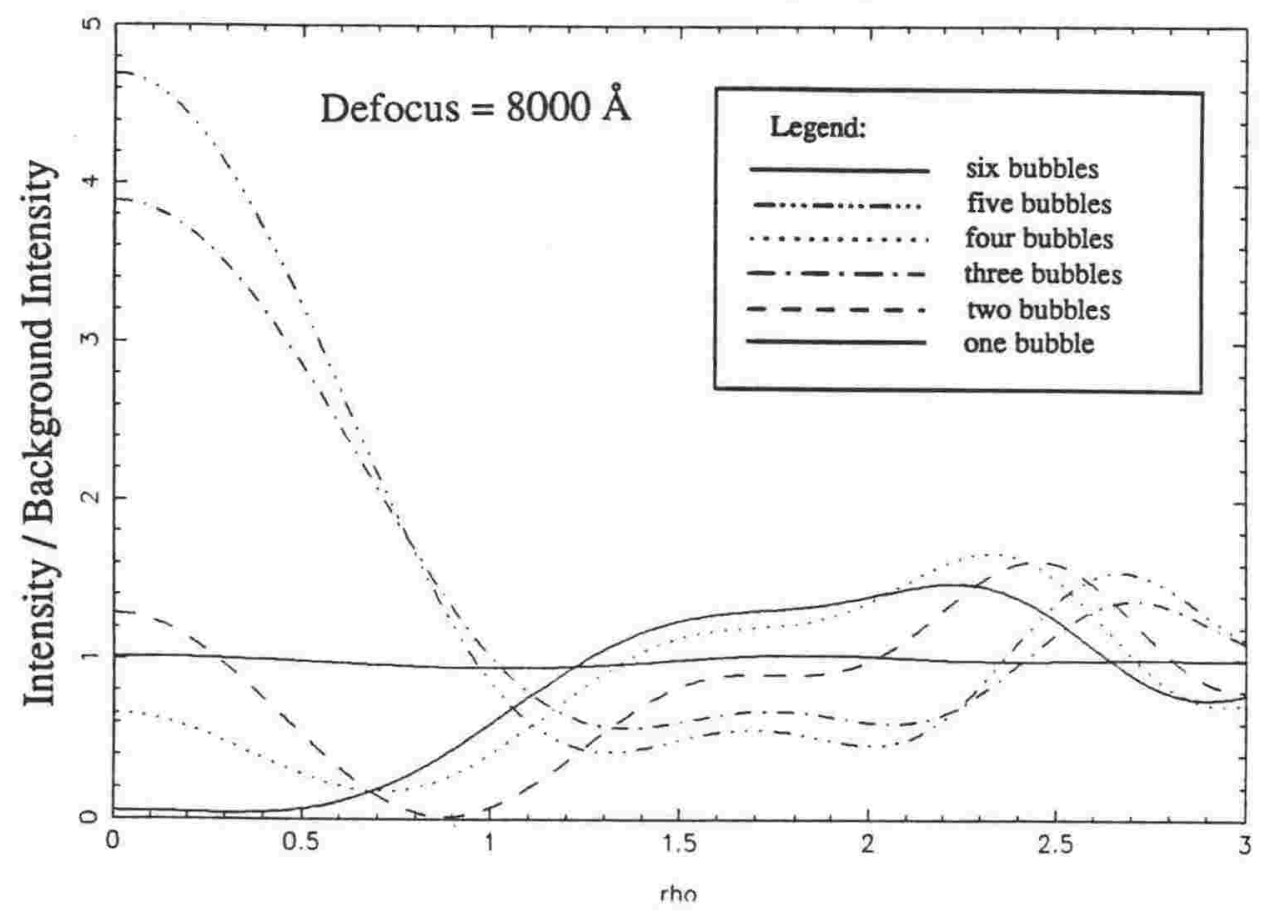

Figure 5.18: BF intensity profiles (normalised to the background intensity) for stacks of bubbles containing from one to six equilibrium bubbles when imaged with a $\mathbf{g}=[200]$ and $w=0.0$. The range of defocus is restricted to $2000 \AA, 4000 \AA, 6000 \AA$ and $8000 \AA$. The foil is of a constant thickness equal to $1.675 \xi_{\mathrm{g}}$. The intensity profiles can be compared against those of figure 5.16 which were for the situation when the foil thickness is varied as the number of bubbles in a stack is changed. The intensity profile through a certain number of bubbles is changed significantly by embedding these bubbles in a different foil thickness. 
minimum in in figure 5.16 at a defocus of $4000 \AA$. It is only for one bubble that the rule "black when in overfocus and white when in underfocus" is obeyed.

\subsection{Helium in a Range of Metals, and Other Gas-metal Combinations.}

The defocussed profiles from a helium gas bubble at equilibrium in a range of metals were simulated to test the validity for different metals of the conclusions reached in sections 5.1 to 5.3. Table 5.3 shows some of the data required for the simulations. The electron scattering factors are from Doyle and Turner [134].

\begin{tabular}{|l|l|l|l|l|l|l|}
\hline Metal & $\mathrm{a}_{1}(\AA)$ & $\mathrm{f}_{\mathrm{e}}(\theta=0)(\AA)$ & $\mathrm{U}_{0}$ (volts) & $\mathrm{V}_{0}$ (volts $)$ & $\xi_{200}(\AA)$ & $\mathrm{ano}_{200}$ \\
\hline $\mathrm{Al}$ & 4.05 & 5.889 & 0.0136 & 16.98 & 693.0 & 0.07 \\
\hline $\mathrm{Cu}$ & 3.61 & 5.600 & 0.1920 & 22.64 & 334.3 & 0.08 \\
\hline $\mathrm{Ni}$ & 3.52 & 6.569 & 0.2136 & 28.85 & 303.9 & 0.08 \\
\hline $\mathrm{Au}$ & 4.08 & 10.573 & 0.2372 & 29.82 & 203.1 & 0.11 \\
\hline $\mathrm{Fe}$ & 2.87 & 7.165 & 0.2308 & 29.04 & 410.4 & 0.03 \\
\hline $\mathrm{He}(\mathrm{gas})$ & 3.57 & 0.418 & 0.0140 & 1.76 & - & - \\
\hline $\mathrm{V}$ & 3.02 & 8.305 & 0.2296 & 28.89 & 478.0 & 0.10 \\
\hline $\mathrm{Cr}$ & 2.88 & 6.969 & 0.2220 & 27.93 & 421.8 & 0.10 \\
\hline $\mathrm{Mo}$ & 3.15 & 10.260 & 0.2500 & 31.45 & 333.2 & 0.10 \\
\hline
\end{tabular}

Table 5.3: Table of lattice constants, $0 \mathrm{keV}$ electron scattering factors, $0 \mathrm{keV}$ zeroth Fourier coefficients of the potential, $0 \mathrm{keV}$ mean potentials, $100 \mathrm{keV}$ extinction distances at room temperature and anomalous absorption ratios $\left(\mathrm{ano}_{200}\right)$ for $\mathbf{g}=[200]$ in a range of metals.

The $\mathrm{Al}, \mathrm{Cu}$ and $\mathrm{Ni}$ values for the anomalous absorption coefficient are the experimental values of Head et al. [82]. The others are from the theoretical values of Humphreys and Hirsch [140]. Any other metals in table 5.3 not mentioned in the above sources are assumed to have an anomalous absorption ratio of 0.10 except helium for which the extinction distances and anomalous absorption ratio are irrelevant. The combination of $\mathrm{He}$ 
and $\mathrm{Cu}$ results in a relative phase change of the beams passing through the bubble to those outside the bubble of about $12^{\circ}$. Other helium and metal combinations give similar phase changes.

Figure 5.19 shows intensity profiles for the same set of imaging parameters in a range of metals. The profiles are insensitive to the metal type. Therefore the conclusions reached above for helium in copper are probably valid for other metals. Hydrogen does not have a mean potential sufficiently different from helium to warrant further simulations either.

\subsection{Comparison to Experimental Through-focal Series}

In this section the position of the maxima and minima in the Frsenel fringes in an experimental through-focal series of a small bubble that is assumed to be at equilibrium is compared to theoretical simulations to deduce the radius of the bubble. Experimental through-focal series can be recorded in two ways in the electron microscope facility at Victoria University. One is to take a sequence of photos by allowing the TEM to vary the defocus automatically between photos. This has the disadvantage when comparing the results to theoretical profiles that the response of the photographic emulsion to the number of incident electrons is non-linear. To match profiles it might be necessary to adjust the theoretical profiles to account for the contrast and exposure of the micrographs. A second way to record a through-focal series is to store the images on a computer disk. The images can be digitised by a Gatan image intensifier or wide angle camera. This second method has the advantage of having a more linear response to the image intensity. Image profiles can be matched without having to adjust the theoretical profiles to account for extraneous imaging parameters. This leaves defocus as the major experimental variable. On-line acquisition of images has the disadvantage that they can be noisier than an electron micrograph taken in the same time and have a limited resolution. This is more of a problem in high resolution microscopy than in the lower magnification techniques used in this thesis. At high magnifications the length of time required to acquire an image with a high signal to noise ratio may be too long. The image probably will drift significantly during this time. 
Profile of the Transmitted Intensity / Background Intensity

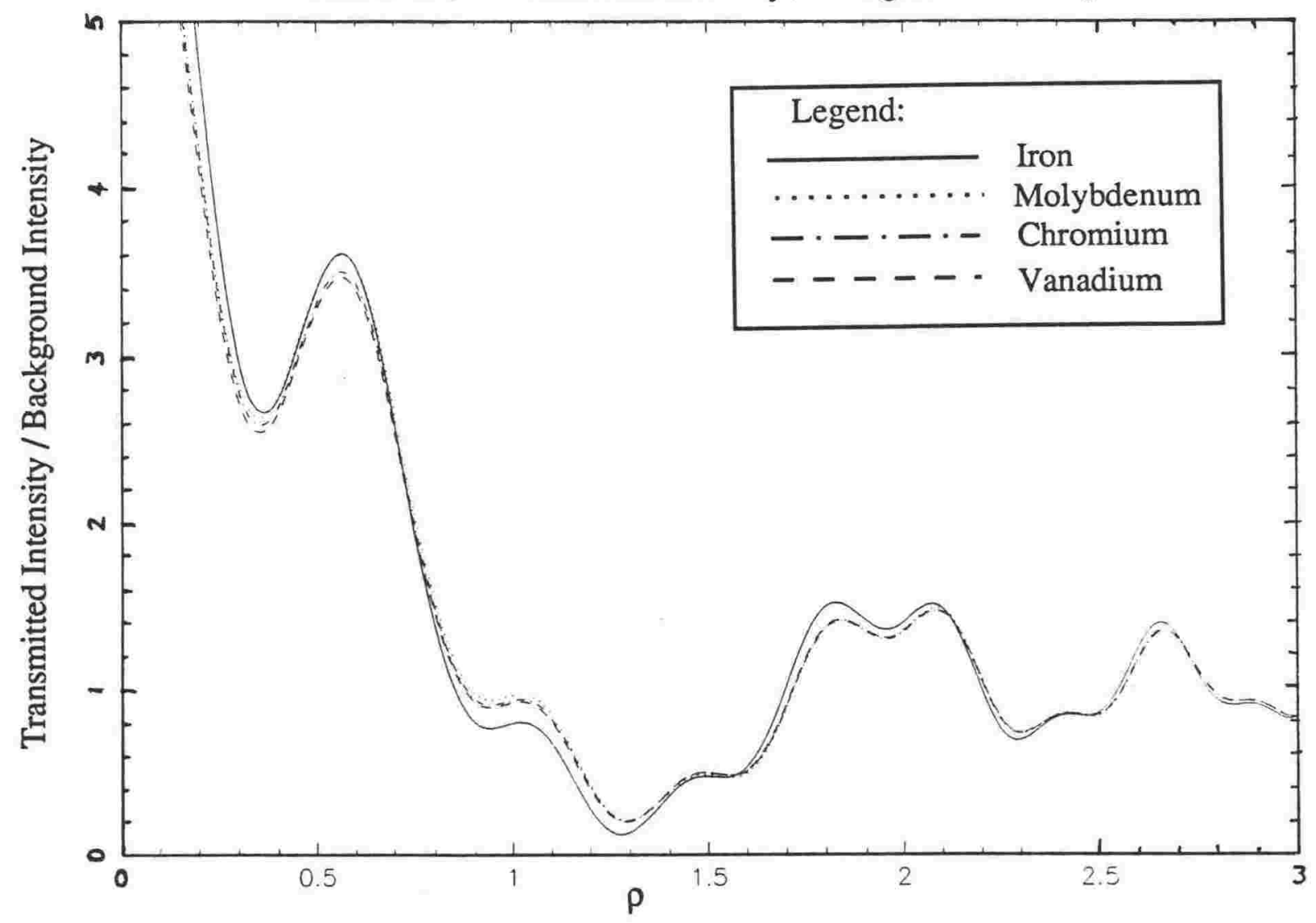

Profile of the Transmitted Intensity / Background Intensity

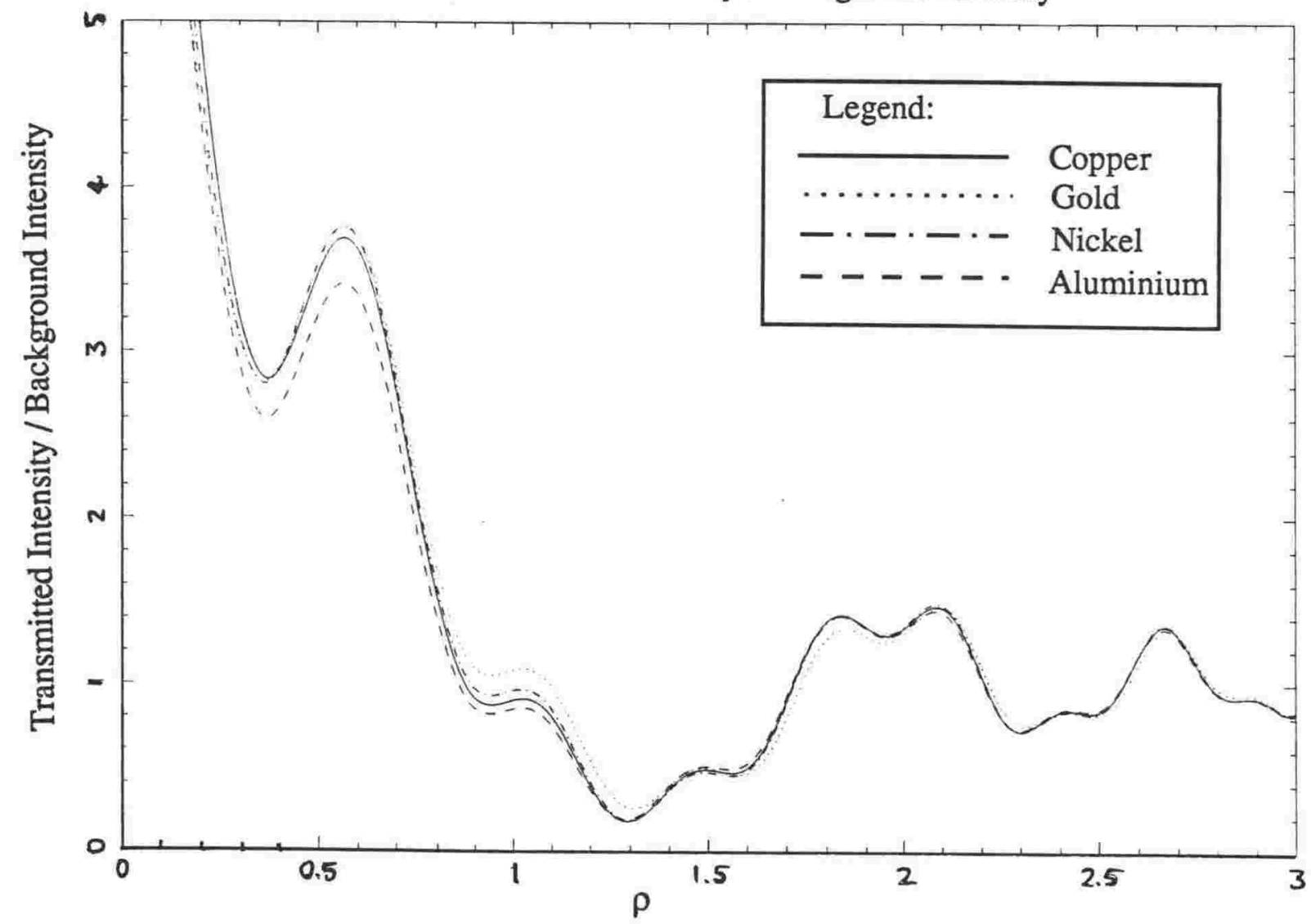

Figure 5.19: Intensity profiles in a range of metals for $\mathbf{g}=[200], \mathrm{w}=0.3$, a foil thickness of $1.75 \xi_{\mathrm{g}}$ and a bubble diameter of $20 \AA$. The profiles are insensitive to the metal type. 
The experimental motivation for producing theoretical plots is to attempt to find accurately the actual bubble radius from an experimental through-focal series. Experimental profiles at any angle across a selected bubble can be obtained from a digitised image using the "extract" command of semper. By matching these experimental profiles or images against theoretical ones covering a range of radii, the actual bubble radius may be determined. In figure 5.20 the behaviour of the effective bubble radius across the range of defocus values is matched to determine that the actual radius of the bubble is approximately $12.5 \pm 0.5 \AA$. The comparison or matching has been done by eye but could have been done more quantitatively by cross correlating theoretical and experimental profiles or images using the semper " $x c f "$ (cross-correlation) command [190].

\subsection{Facetted Cavities.}

Images of facetted cavities have different characteristics from images of spherical cavities [88]. For example, Foreman et al. [88] found that the positions of the maximum and minimum in the intensity profiles are less sensitive to the defocus. Instead the positions are more likely to become centred on the edge of a facet.

Large facetted cavities occur in thin copper and aluminium foils under certain irradiation conditions. Figure 5.21 shows large, facetted cavities that have been found in helium-irradiated aluminium. Notice how the Fresnel fringes change with defocus from having a white boundary inside a black boundary to black inside white. The cavities in copper can be created during irradiations at about $300^{\circ} \mathrm{C}$ and those in aluminium at room temperature. At these temperatures both vacancies and interstitials are mobile. The cavities have sufficient perfect crystal material between them that the experimental images can be matched to simulations without having to consider how the cavities might overlap or interact.

A set of plane normals $\left(\mathbf{n}_{\mathrm{i}}\right)$ and distances $\left(\mathrm{h}_{\mathrm{i}}\right)$ to these planes from the cavity centre can be used to define the facets of a cavity. A point on the foil surface has a position vector $\mathbf{r}_{\mathrm{A}}=(\mathrm{x}, \mathrm{y},-$ the depth of the cavity) in a coordinate system where the origin of the coordinate system is at the centre of the cavity. A point on the exit surface of the foil has a 
Fresnel fringe positions around a bubble

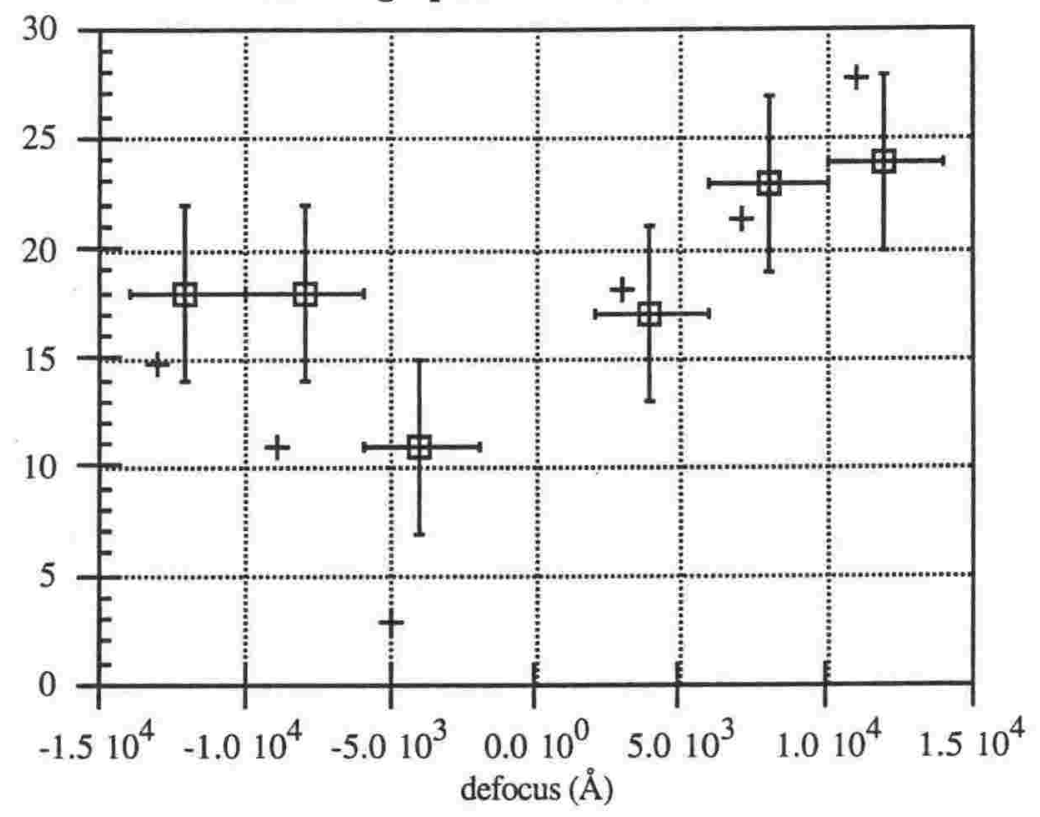

Figure 5.20: Positions of the Fresnel fringes (in Angstroms) versus defocus for experimental micrographs (squares) and simulations (crosses). The specimen is a result of a low-dose helium implantion (see chapter seven for more details). Experimental profiles have been taken through a bubble in a through-focal series. The positions of the Fresnel fringe peaks have been matched to theoretical positions obtained from simulations on a range of bubble sizes at various values of the defocus. To minimise the noise, each experimental profile from which the data were taken is the result of an average over twenty parallel line scans through the bubble. However the profiles are still noisy enough to create discontinuities in the profiles and foil any attempts to measure the bubble radius using the criterion involving the slope of the intensity profile. The nominal magnification printed on the micrographs by the TEM was used to convert the positions of the maximum and minimum in the profiles into distances. The images were taken with $\mathbf{g}=[200]$ and $\mathbf{B}=$ [011]. The other parameters needed for the theoretical profiles are difficult to determine experimentally. The theoretical fit to the experimental data was done for a foil thickness of $1.675 \xi_{\mathrm{g}}$, a bubble depth of $1.675 / 2 \xi_{\mathrm{g}}$, a $25 \AA$ A diameter equilibrium bubble, an electron energy of $120 \mathrm{keV}$ and $\mathrm{w}=0.0$. The experimental position of zero defocus was assumed to have an uncertainity of $\pm 2000 \AA$. 

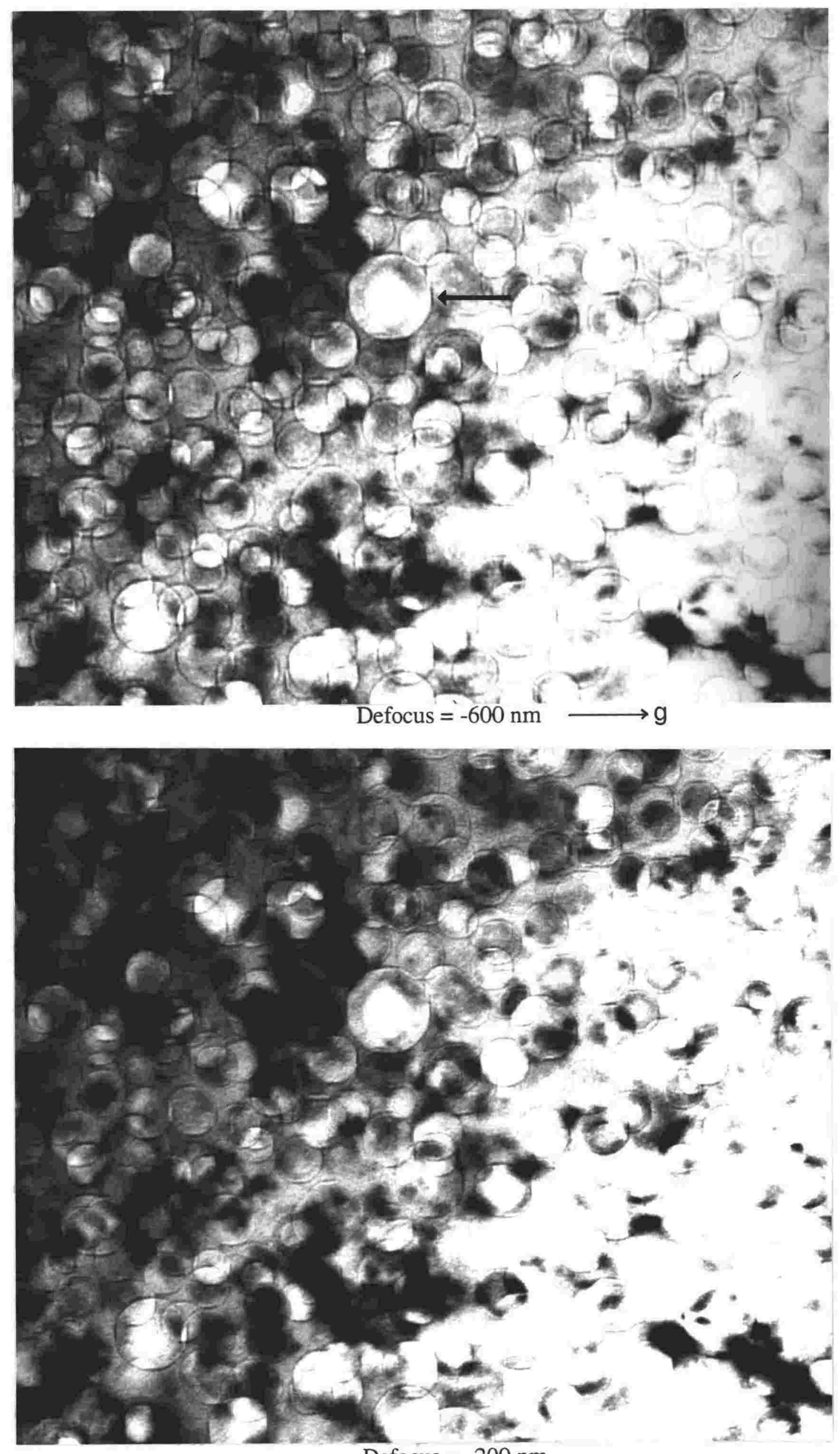

Defocus $=-200 \mathrm{~nm}$

Figure 5.21 (a) (Caption on page 5 - 56) 


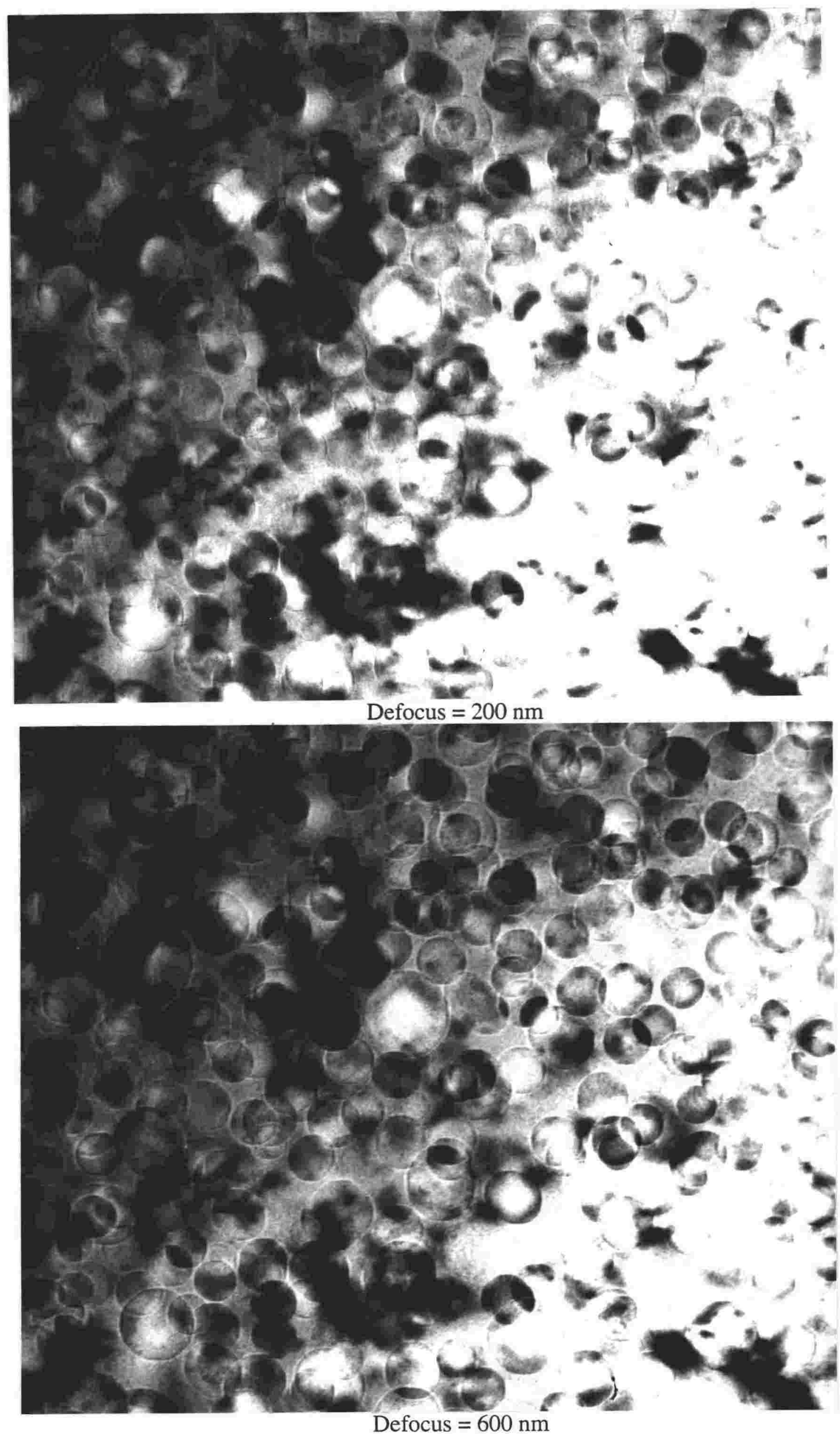

Figure 5.21 (a) (Caption on page 5 - 56) 


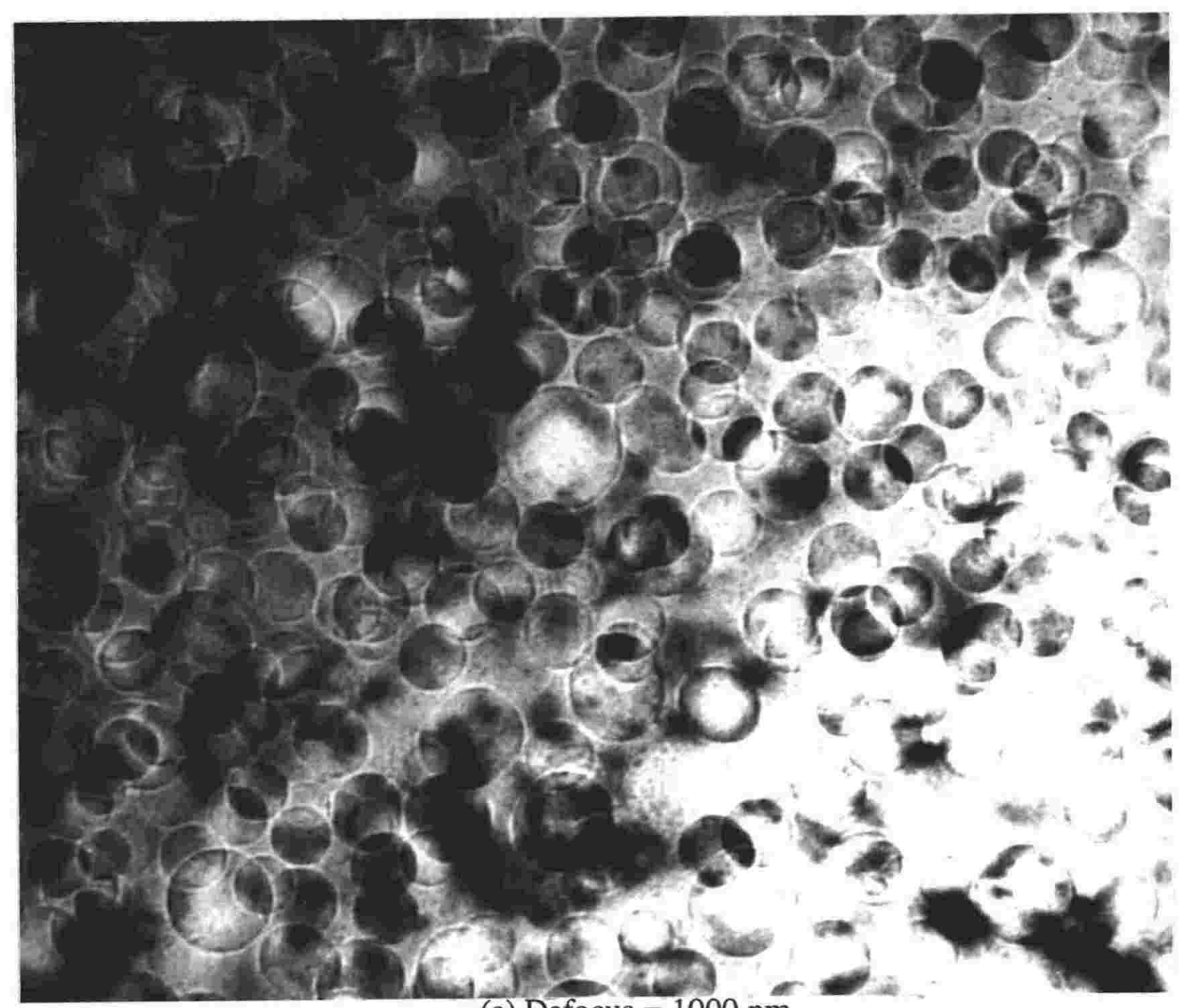

(a) Defocus $=1000 \mathrm{~nm}$

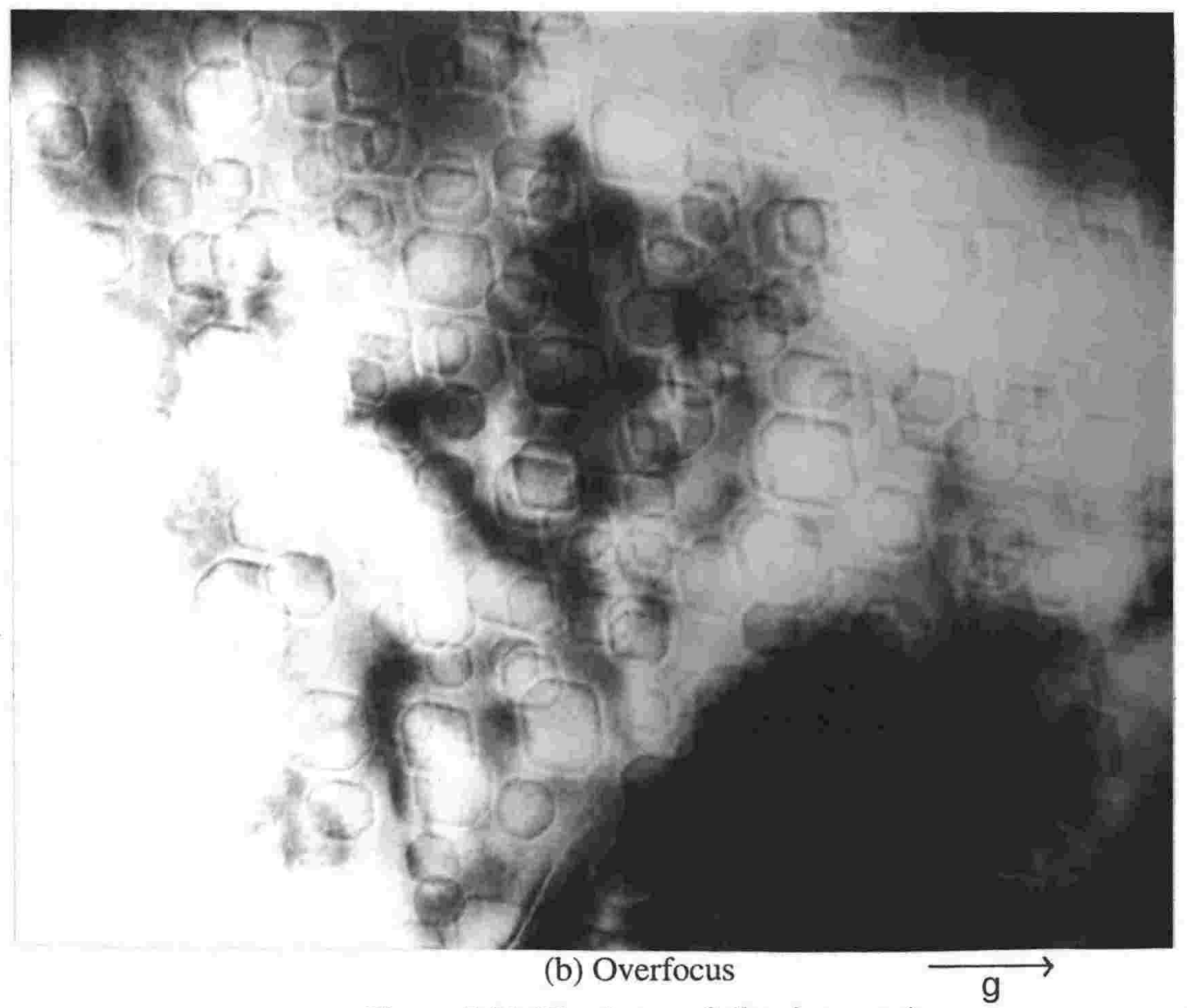

Figure 5.21 (Caption on following page) 

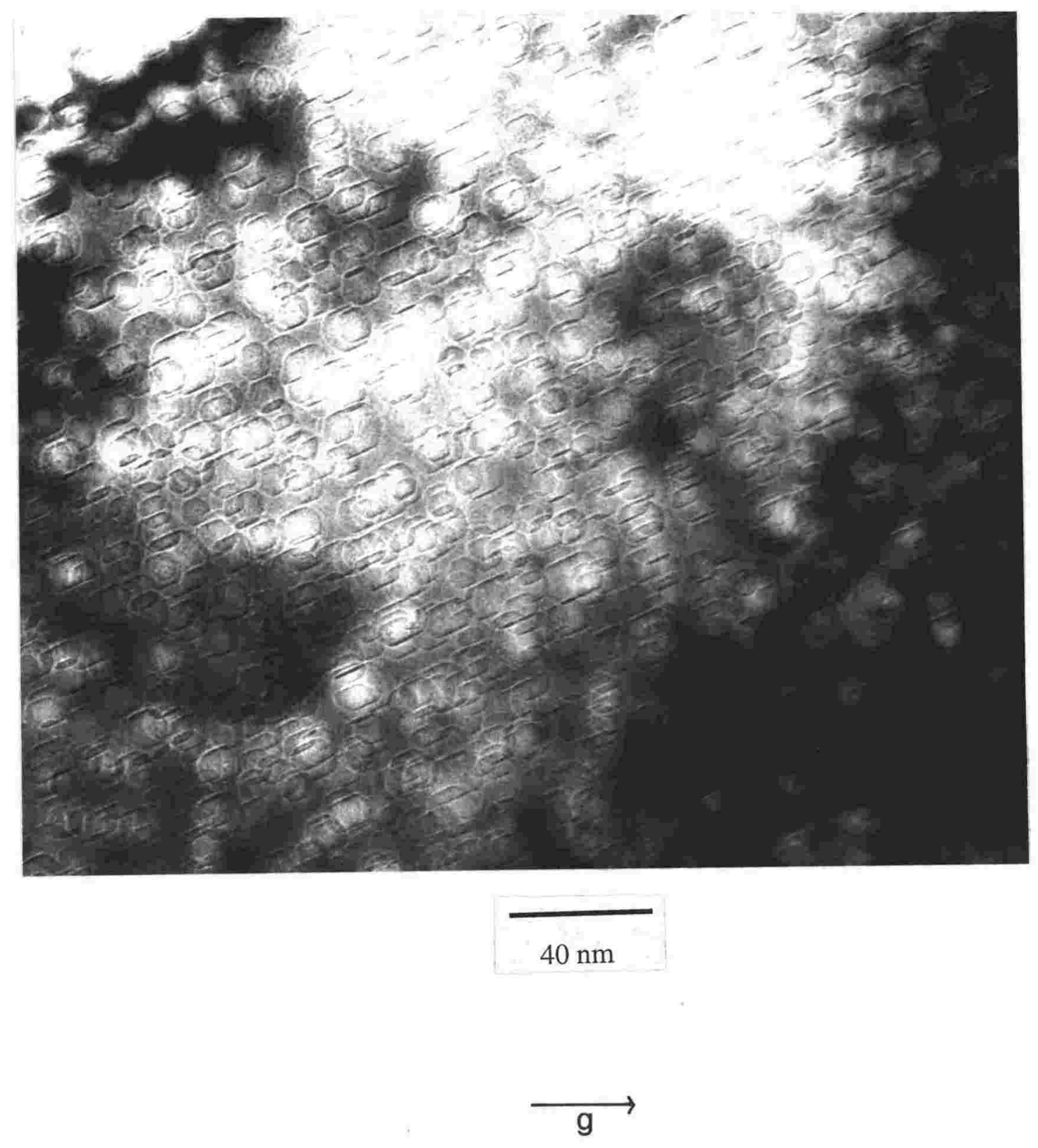

\section{(c) Overfocus}

Figure 5.21: Part (a) shows an experimental five member through-focal series of cubooctahedral cavities in aluminium showing $\{111\}$ and $\{100\}$ facets. Parts (b) and (c) show one member (at overfocus conditions) of similar through-focal series taken at other beam directions. The beam directions and diffracting vectors are: (a) $\mathbf{B}=[0,-1,7]$ and $\mathbf{g}=$ [200], (b) $\mathbf{B}=[15,1,12]$ and $\mathbf{g}=[1,-3,-1]$, (c) $\mathbf{B}=[-6,-5,13]$ and $\mathbf{g}=[3,-1,1]$. The photos are taken at two-beam conditions near simple poles. The g's of the simple poles have been marked on the photos. The defocus increments by $400 \mathrm{~nm}$ between members of the same through-focal series. 
position vector of $\mathbf{r}_{\mathrm{B}}=(\mathrm{x}, \mathrm{y}$, the foil thickness minus the depth of the cavity). Any facet normal, $\mathbf{n}_{\mathrm{i}}=\left(\mathrm{n}_{\mathrm{x}}, \mathrm{n}_{\mathrm{y}}, \mathrm{n}_{\mathrm{z}}\right)$, defines a plane a minimum distance $\mathrm{h}_{\mathrm{i}}$ from the cavity centre, which allows any $z$ coordinates on the plane to be calculated from the standard expression for the equation of a plane:

$$
\mathrm{z}=\frac{\left[\mathrm{h}_{\mathrm{i}}-\left(\mathrm{n}_{\mathrm{x}} \mathrm{x}+\mathrm{n}_{\mathrm{y}} \mathrm{y}\right)\right]}{\mathrm{n}_{\mathrm{z}}} .
$$

The dot products of the $\mathbf{n}_{\mathrm{i}}$ with $\mathbf{r}_{\mathrm{A}}$ and $\mathbf{r}_{\mathrm{B}}$ are calculated separately. The maximum dot products correspond to the planes with $\mathbf{n}$ most closely aligned with $\mathbf{r}_{\mathrm{A}}$ and $\mathbf{r}_{\mathrm{B}}$ respectively. In calculating the wave function the $\mathrm{z}$ coordinates where a column can intersect with some of the top and bottom planes of the cavity is required. Initially the program assumes that the relevant planes (given $\mathbf{r}_{\mathrm{A}}$ and $\mathbf{r}_{\mathrm{B}}$ ) for a column (extending from $\mathbf{r}_{\mathrm{A}}$ to $\mathbf{r}_{\mathrm{B}}$ ) are the ones for which the dot products are maximum. This can be incorrect, so more dot products, $\mathbf{n}_{\mathrm{i}} \cdot \mathbf{r}_{\mathrm{i}}, \mathbf{r}$ $=(\mathrm{x}, \mathrm{y}, \mathrm{z})$, are calculated where $\mathrm{z}$ is found from equation 5.4 above. If any dot product has

$$
\mathbf{n}_{\mathrm{i}} \cdot \mathbf{r}>\mathrm{h}_{\mathrm{i}}
$$

$\mathbf{r}$ then lies outside one of the other facets of the cavity. This plane with its corresponding $\mathrm{z}$ replaces the initial plane and the loop over $\mathbf{n}_{\mathrm{i}}$ is continued until the correct $\mathrm{z}$ coordinates are found.

The facetting of the cavities was deduced by producing a supercell for a cubooctahedral particle using the program of Flueli [186]. In figure 5.22 the cell is projected in the required beam directions using EMS (a software package described in chapter four). A cubo-octahedron is the same shape as the Brillouin zone of a bcc crystal.

Simulations of facetted cavities with no surrounding displacement are shown in figure 5.23. The simulations use the analytical solutions to the Howie-Whelan differential equations of chapter two. Strong two-beam conditions are used to image and simulate the cavities, in contrast to the kinematical conditions on which Foreman et al. [88] based their conclusions. A comparison of figure 5.23 (a) and figure 5.21 shows that a TEM image of a facetted cavity does not faithfully reproduce the facetting or size of the actual cavity structure. In particular the facets on the top of the cavity stand out strongly in the image but are positioned away from the real position of these edges (the real positions are nearer to the 
centre of the cavity image). Some of the parameters used in the simulation of figure 5.23 were chosen by trial and error, with simulations that were an unconvincing fit to the experimental micrographs being discarded. The thickness of the foil is difficult to determine experimentally because of the absence of a feature such as a long straight dislocation that threads through the foil in all the experimental micrographs.

Because of the high density of cavities it was found to be impossible to find the same cavity in all three through-focal series taken at different beam directions. To deduce the $h_{i}$ of a cavity only the one through-focal series can be matched against simulations at a time. In figures 5.24 and 5.25 an experimental through-focal series is matched against simulated intensity profiles. The simulated intensity profiles have large minima and maxima that result from the thickness contrast (they are therefore independent of the defocus), and superimposed upon these minima and maxima are the Fresnel fringes. The separation of the minima and maxima which result from thickness contrast and those that result from the effects of defocus is not so easy on the experimental intensity profiles. The final size of the cavities deduced from the simulations is less accurate than the parameters (obtained by trial and error) used to obtain the theoretical image profiles.

\subsection{Superlattice Spots}

In this section a simulation is made of the superlattice spots obtained in diffraction patterns taken when the diffraction aperture selects out a structure containing ordered equilibrium bubbles.

A selected area diffraction pattern (SADP) is the power spectrum of the wavefunction, and includes all the beams diffracted by the specimen. This Fraunhofer diffraction case is treated in many texts on physical optics [191]. The power spectrum of a micrograph is different from the SADP as the image loses the phase information contained in the wavefunction. Some attempts have been made by others to retrieve this phase information by image processing a through-focal series using the weak phase object approximation WPOA [161]. However, the WPOA is not applicable to images of gas 


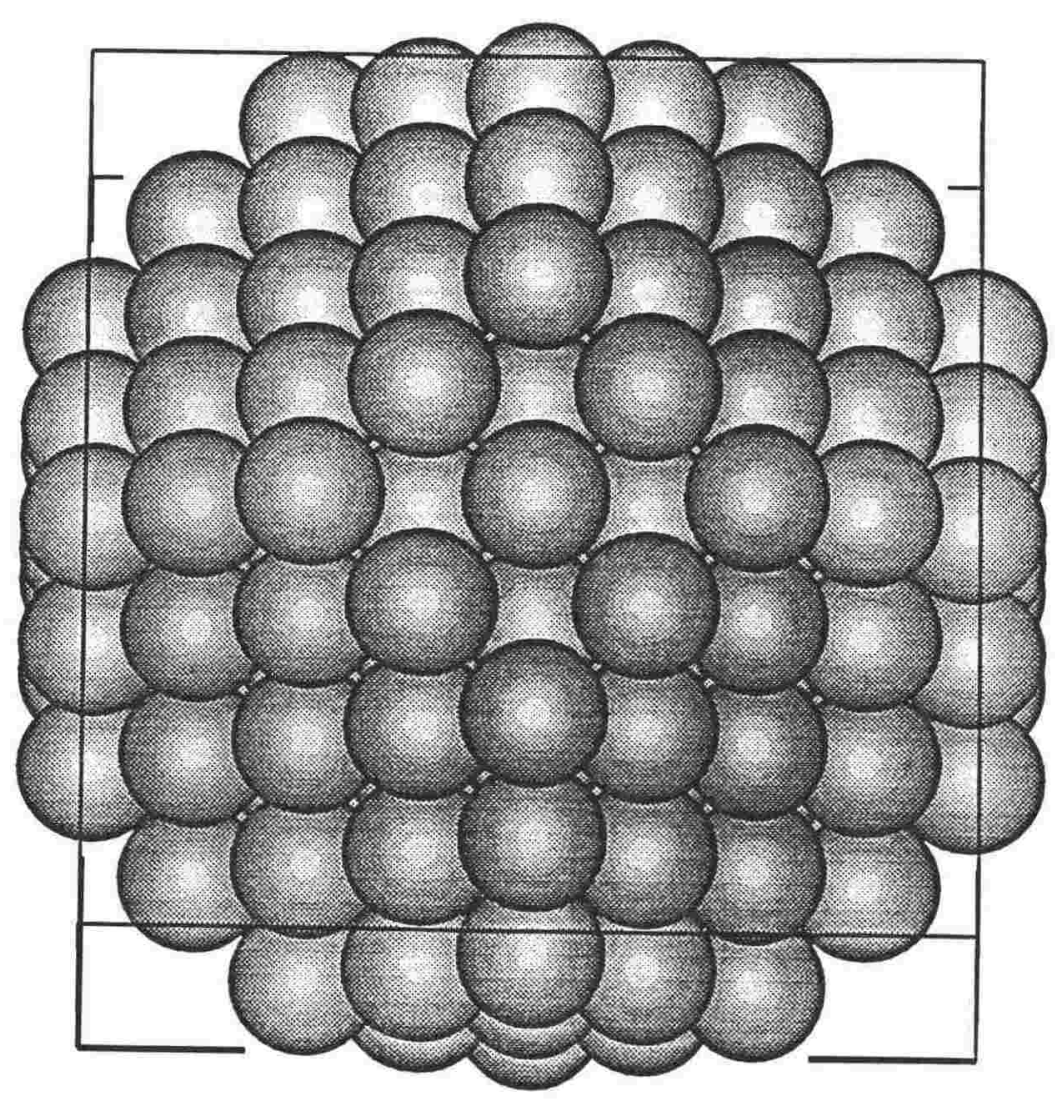

(a)

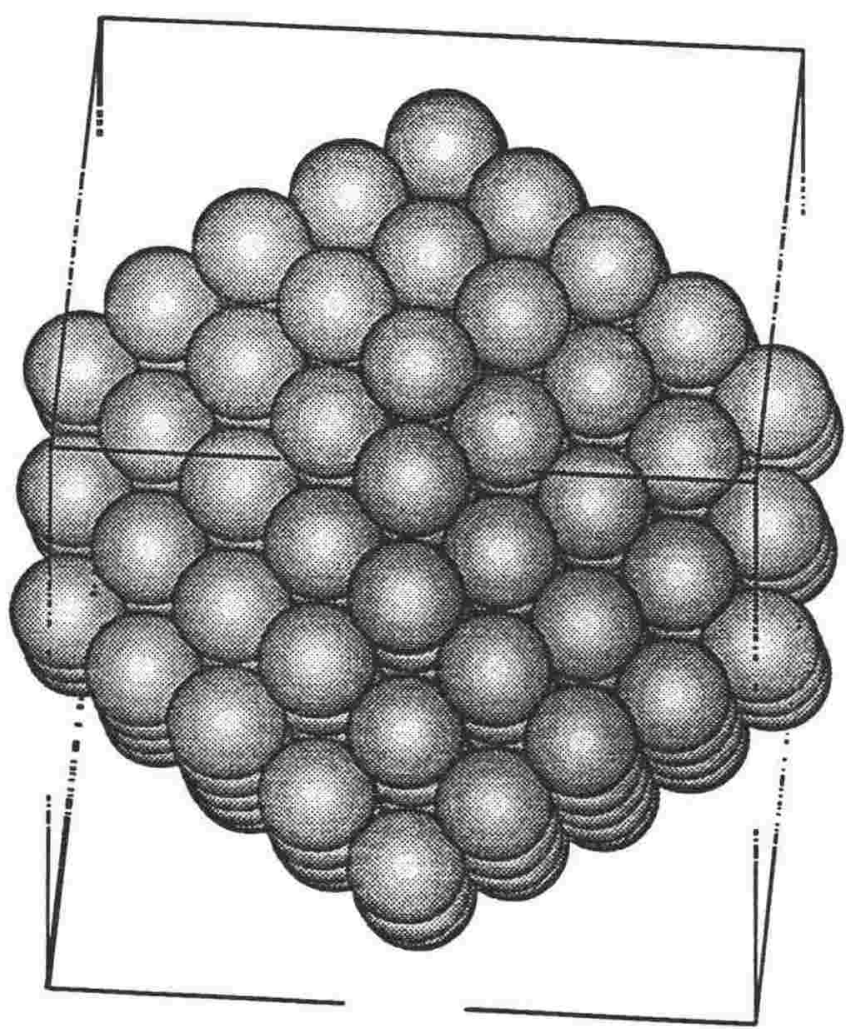

Figure 5.22 (b) (caption on following page) 


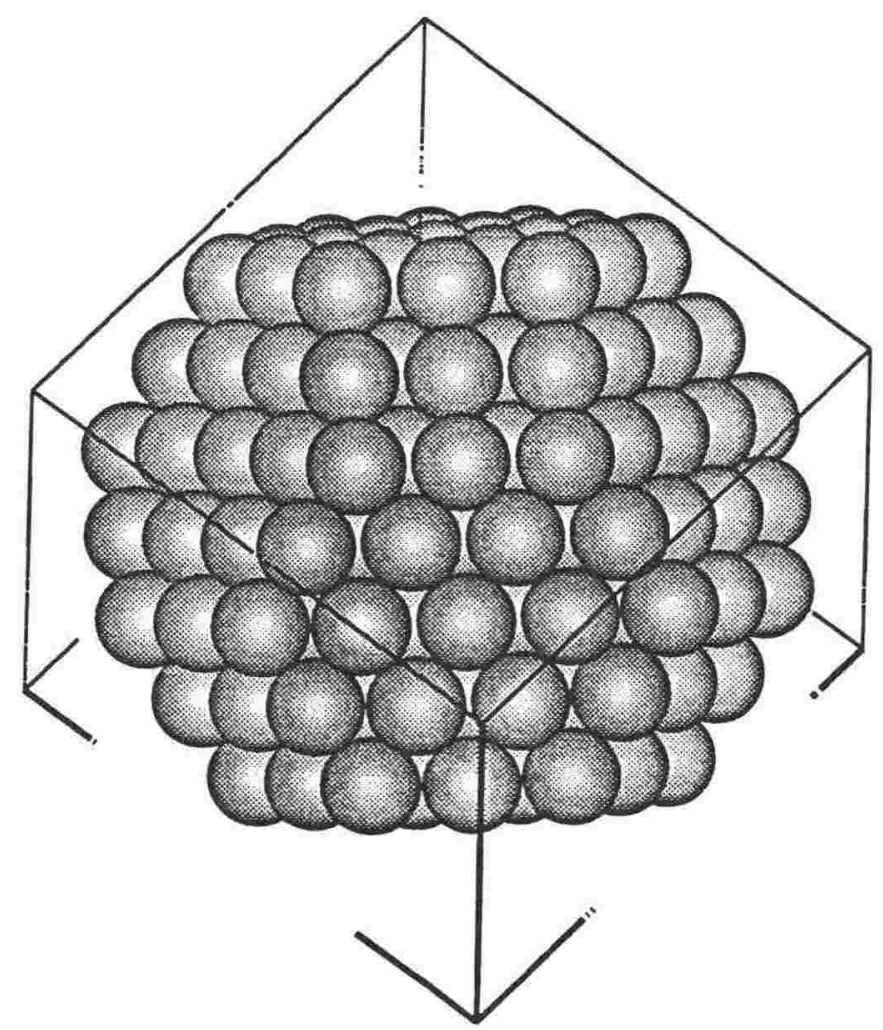

(c)

Figure 5.22: Projections of a cubo-octahedral particle: (a) $\mathbf{B}=[0,-1,7]$, (b) $\mathbf{B}=[15,1$, $12]$ and (c) $\mathbf{B}=[-6,-5,13]$. The appearances of the particle at different beam directions show reasonable similarity to the facetted appearance of some of the particles in the experimental micrographs of figure 5.21 . 


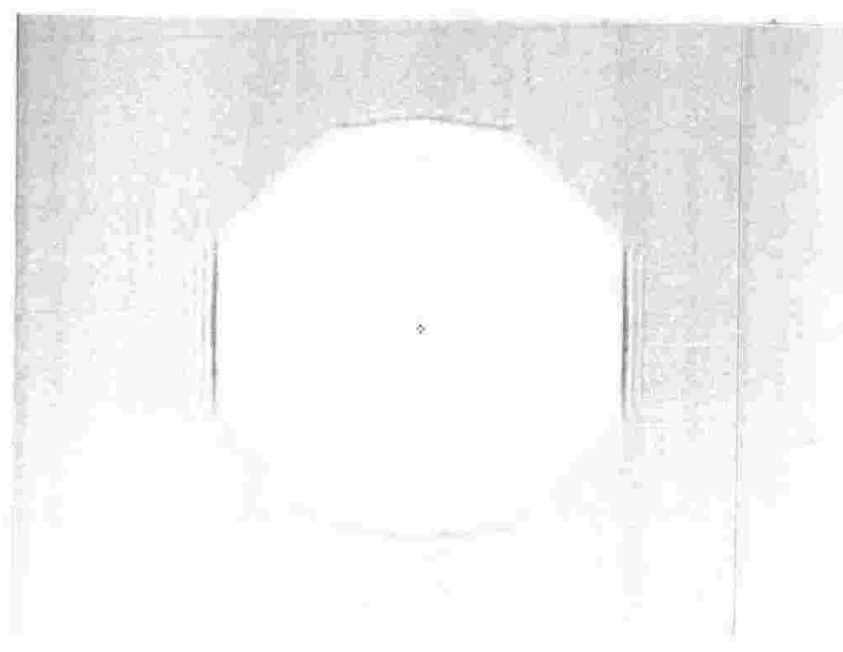

(i)

(ii)

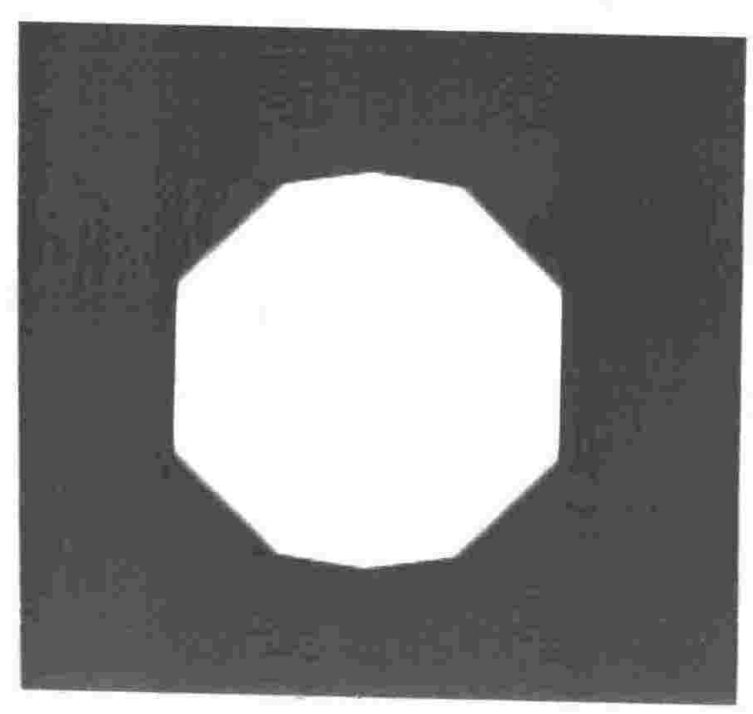

(iii)

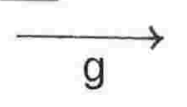

(iv)

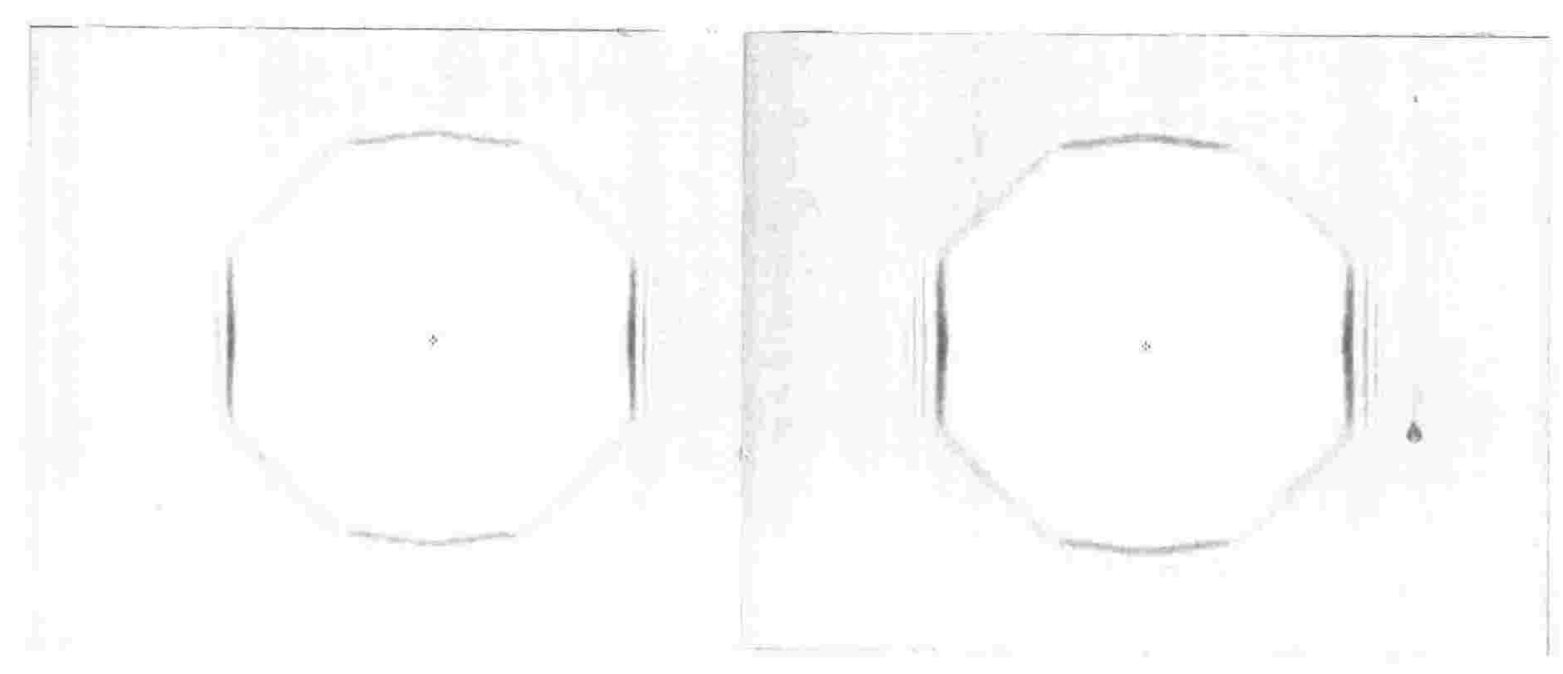

(v)

(vi)

Figure 5.23 (a) (caption on following page)

$$
5-61
$$




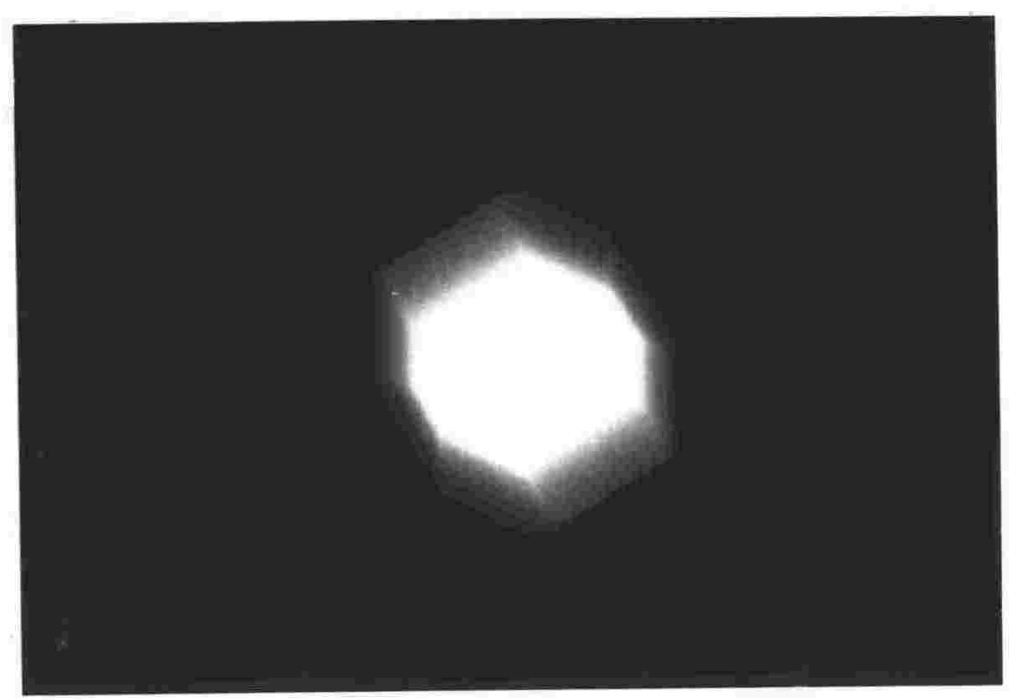

(b) $\longrightarrow \mathrm{g}$

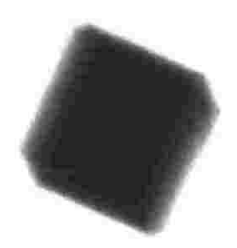

(c) $\longrightarrow \mathrm{g}$

Figure 5.23: Simulations of cubo-octahedral particles in aluminium: (a) $\mathbf{B}=[0,-1,7], \mathbf{w}=$ 2.1 and $\mathbf{g}=[200]$, (b) $\mathbf{B}=[15,1,12], \mathrm{w}=0.0$ and $\mathbf{g}=[1,-3,-1],(\mathrm{c}) \mathbf{B}=[-6,-5,13]$, $\mathrm{w}$ $=0.0$ and $\mathbf{g}=[3,-1,1]$. The foil normal is approximately [001] as the tilts on the specimen holder were approximately zero when at $\mathbf{B}=$ [001]. In part (a) the foil thickness is equal to $2.7 \xi_{\mathrm{g}}$ and the cavity depth is equal to $1000 \AA$ (for consistency amongst the simulations the lengths used in part (a) have been transformed using the beam directions and foil normals for parts (b) and (c) before these latter simulations are calculated). The common simulation parameters are an electron energy of $120 \mathrm{keV}$, and an $h_{i}$ of $164.5 \AA$ for the $\{111\}$ planes and an $h_{i}$ of $190 \AA$ for the $\{100\}$ planes. In part (a) the defocus is (i) $-600 \mathrm{~nm}$, (ii) -200 $\mathrm{nm}$, (iii) $0 \mathrm{~nm}$ (iv) $200 \mathrm{~nm}$, (v) $600 \mathrm{~nm}$ and (vi) $1000 \mathrm{~nm}$; in parts (b) and (c) the simulations are in focus. Because the [200] reflection extinction distance in aluminium is much larger than it is in copper, the images correspond to two-beam conditions even though w has a large value. The appearance of the simulations at different beam directions show reasonable similarity to some of the images of particles in the experimental micrographs of figure 5.24 . 


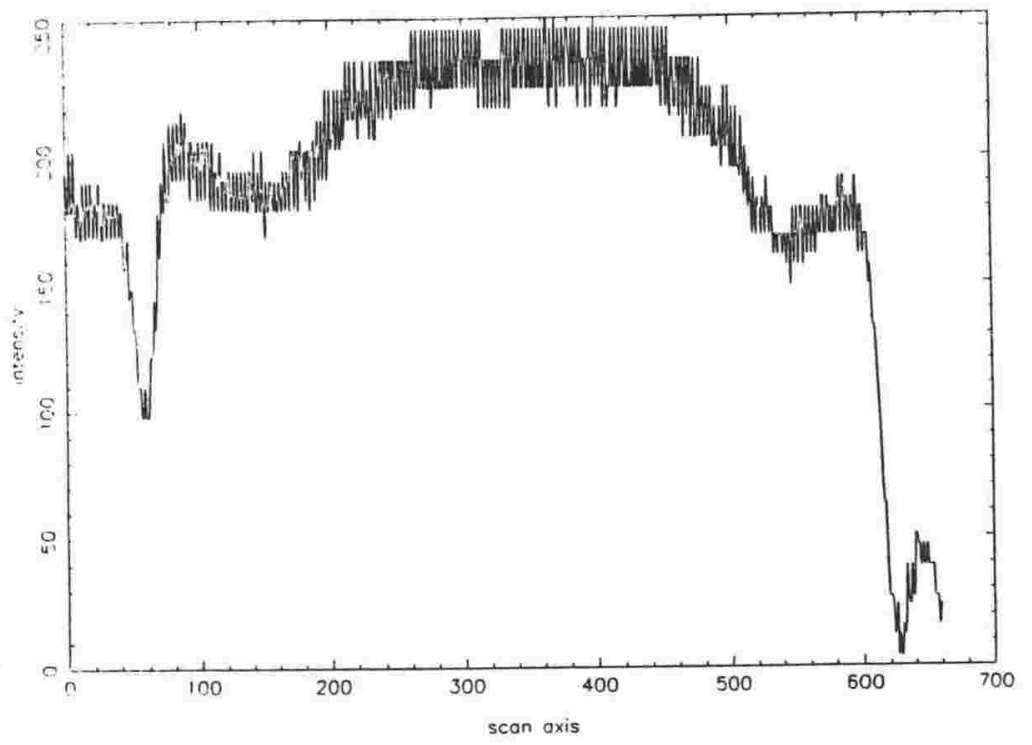

(i) Defocus $=-600 \mathrm{~nm}$

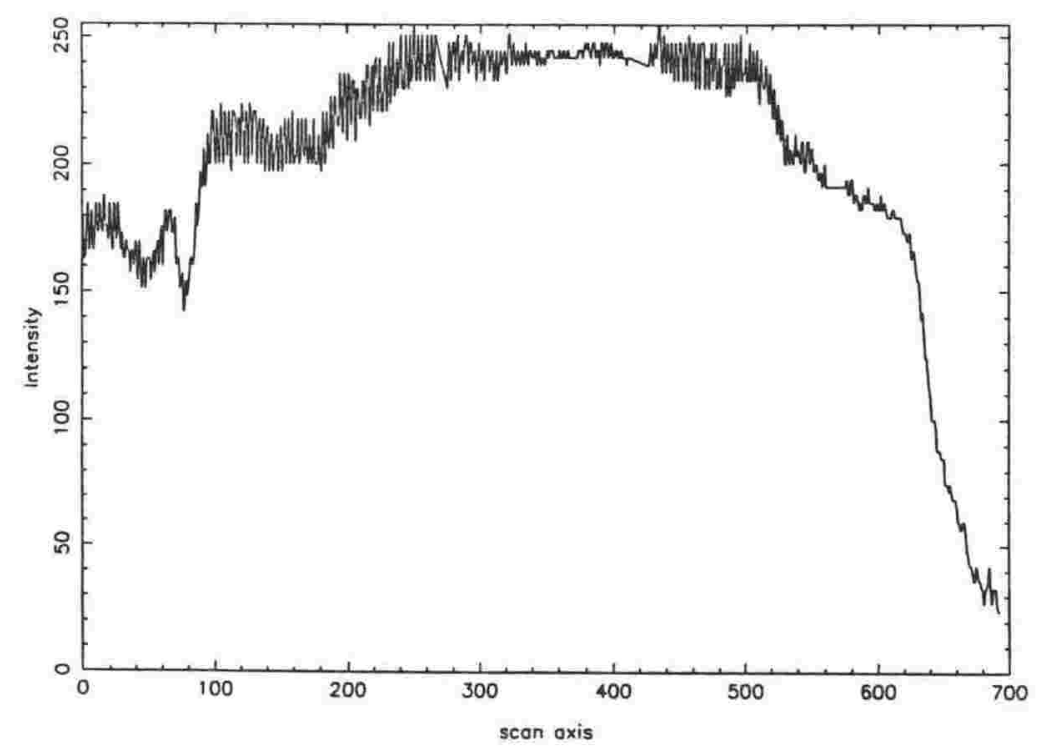

(ii) Defocus $=-200 \mathrm{~nm}$

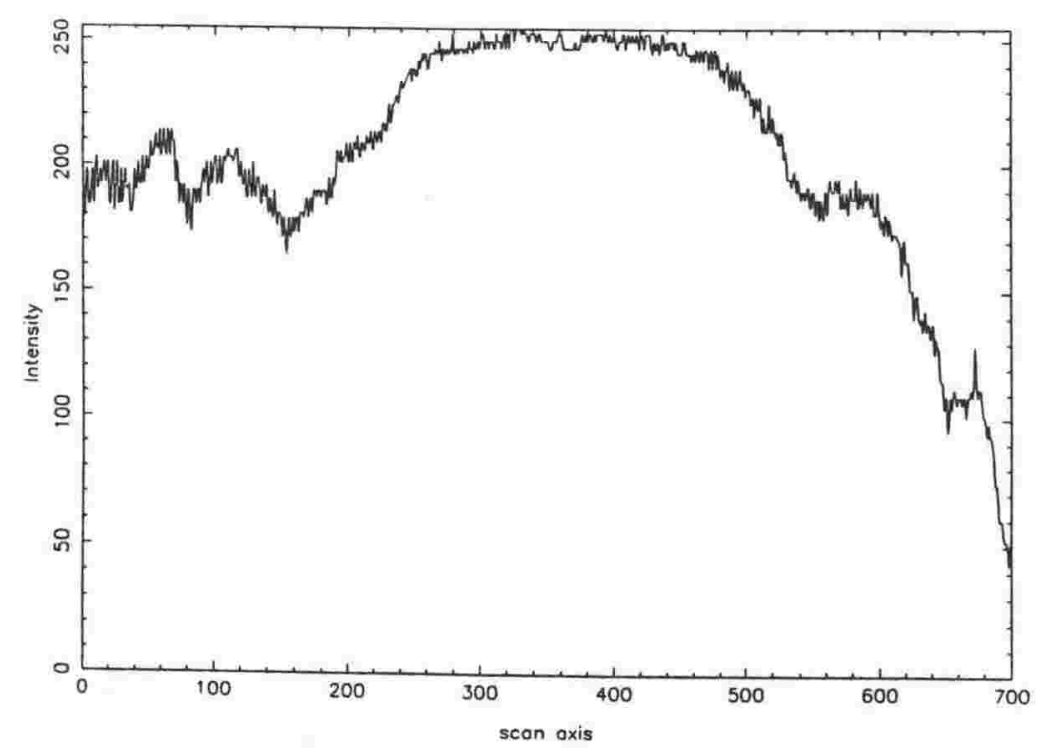

(iii) Defocus $=200 \mathrm{~nm}$

Figure 5.24 (a) (caption on page 5 - 66) 
Intensity scan

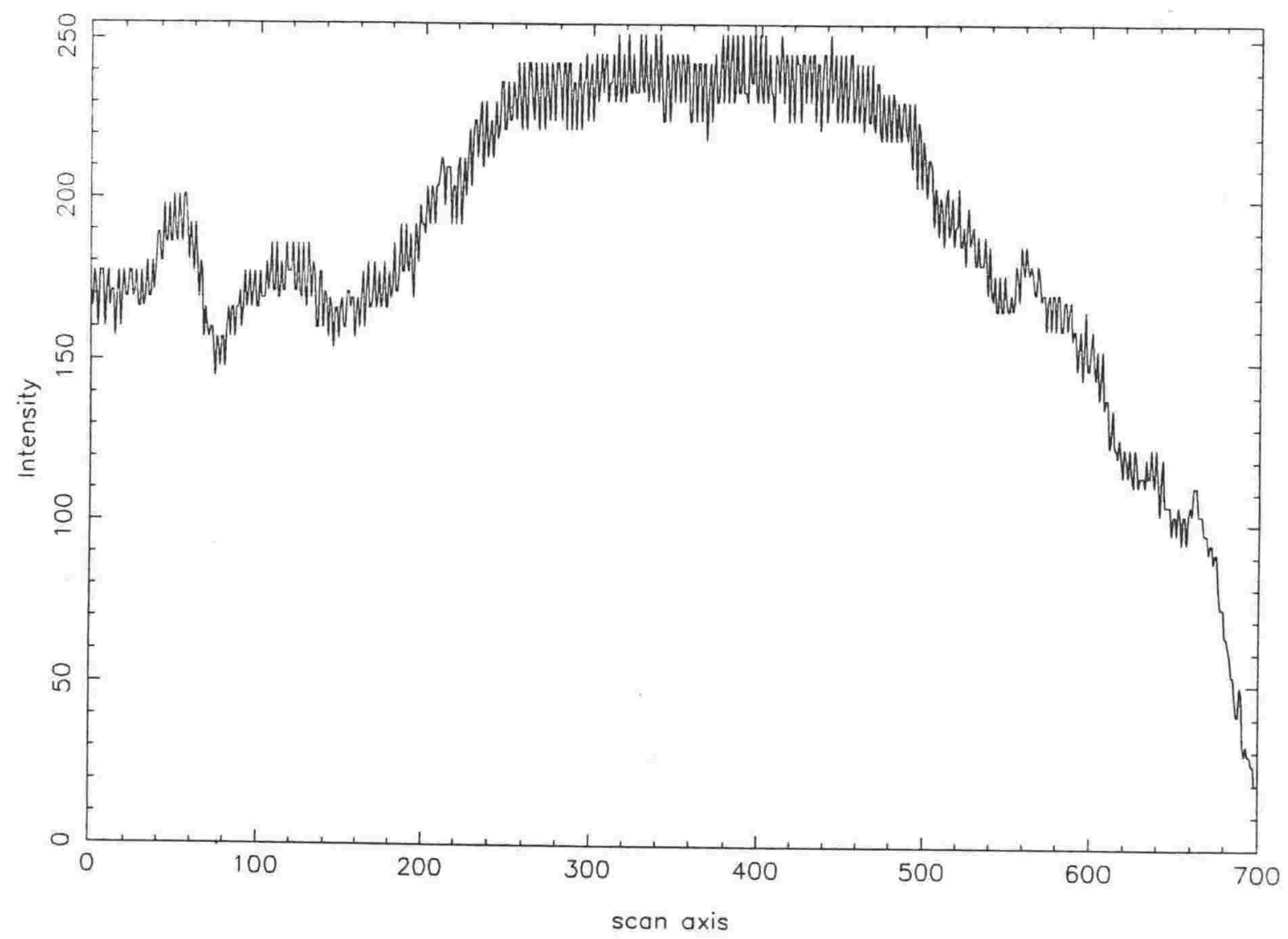

(iv) Defocus $=600 \mathrm{~nm}$

Intensity scan

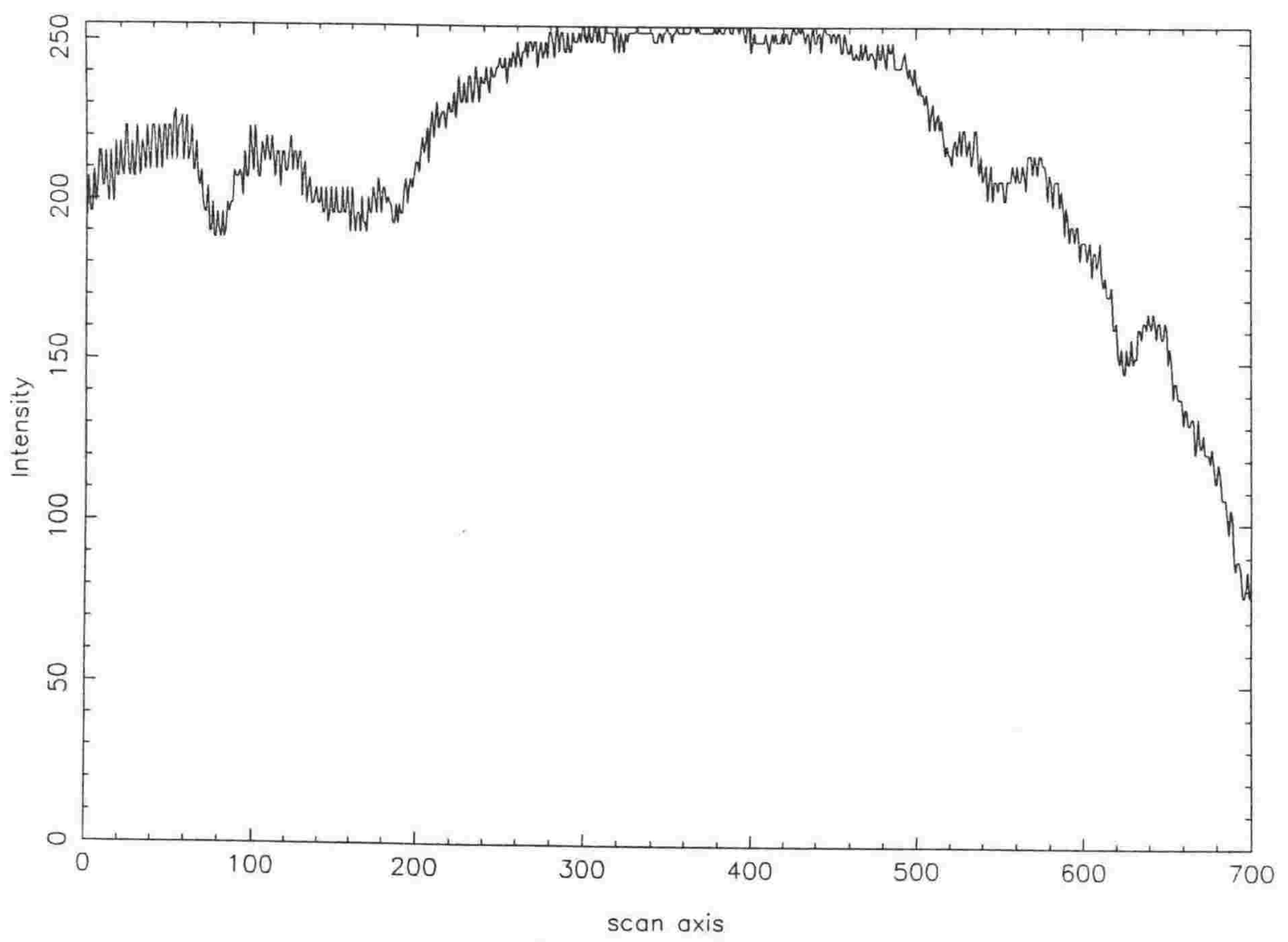

(v) Defocus $=1000 \mathrm{~nm}$

Figure 5.24 (a) (caption on page 5 - 66) 

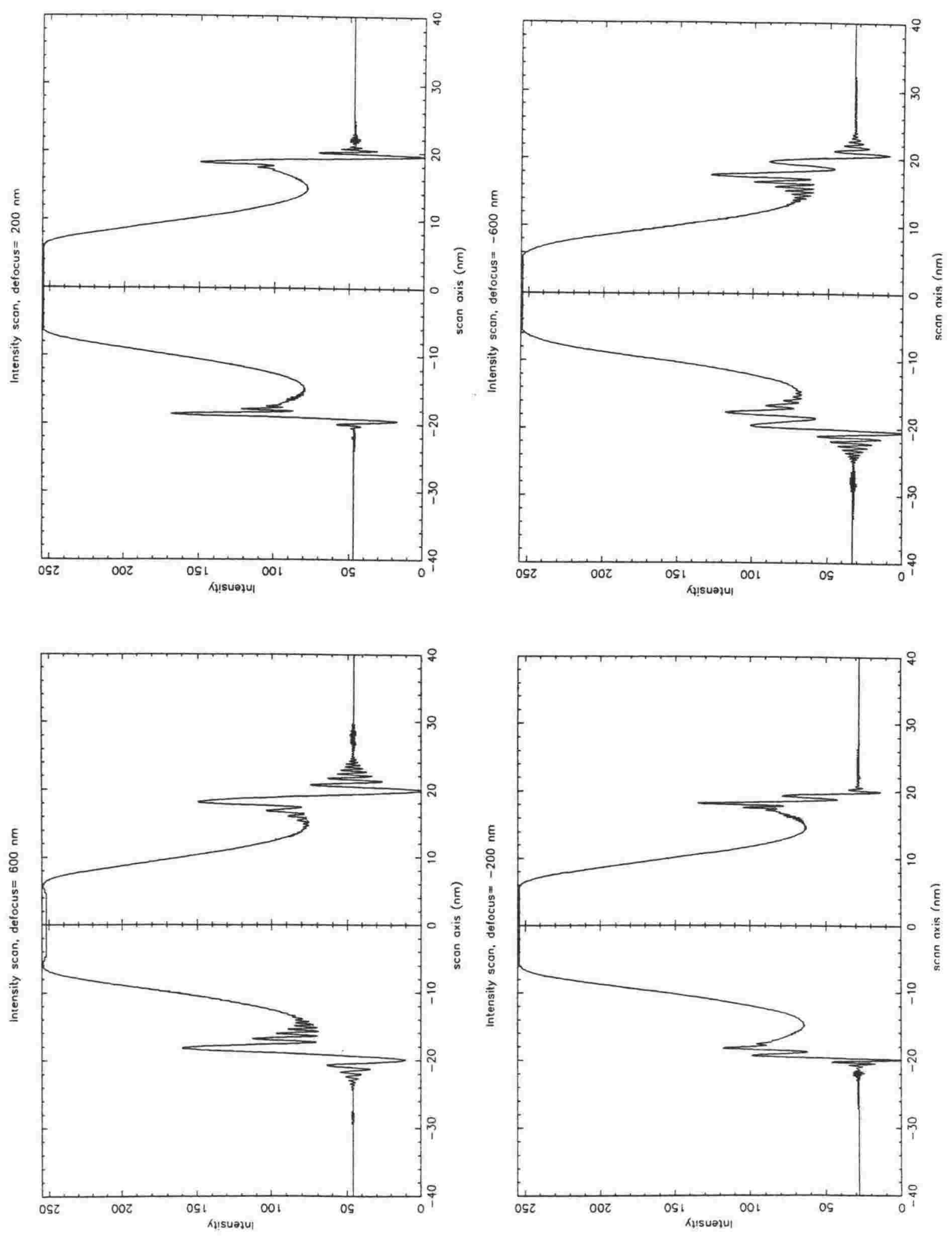

Figure 5.24 (b) (caption on following page) 
Intensity scan, defocus $=1000 \mathrm{~nm}$

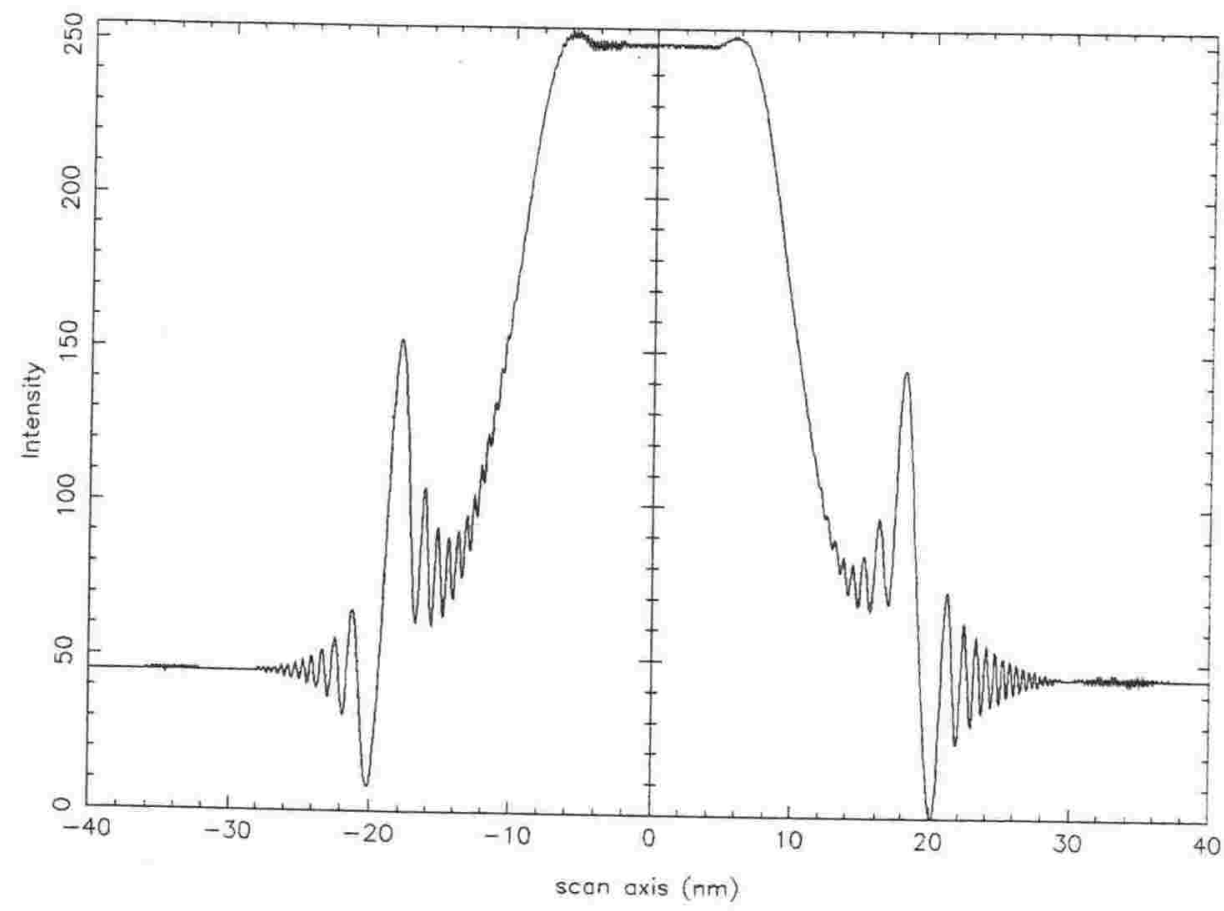

(b)

Figure 5.24: (a) Experimental and (b) simulated intensity profiles, parallel to g, through the centre of the cavity arrowed in figure 5.23(a). The simulations have the same parameters as those used in figure 5.23. In the experimental intensity profiles, from the nominal TEM magnification, 100 units on the horizontal axis is equal to $5.6 \mathrm{~nm}$. The experimental intensity has been scaled to lie in the range 0 to 255 . 


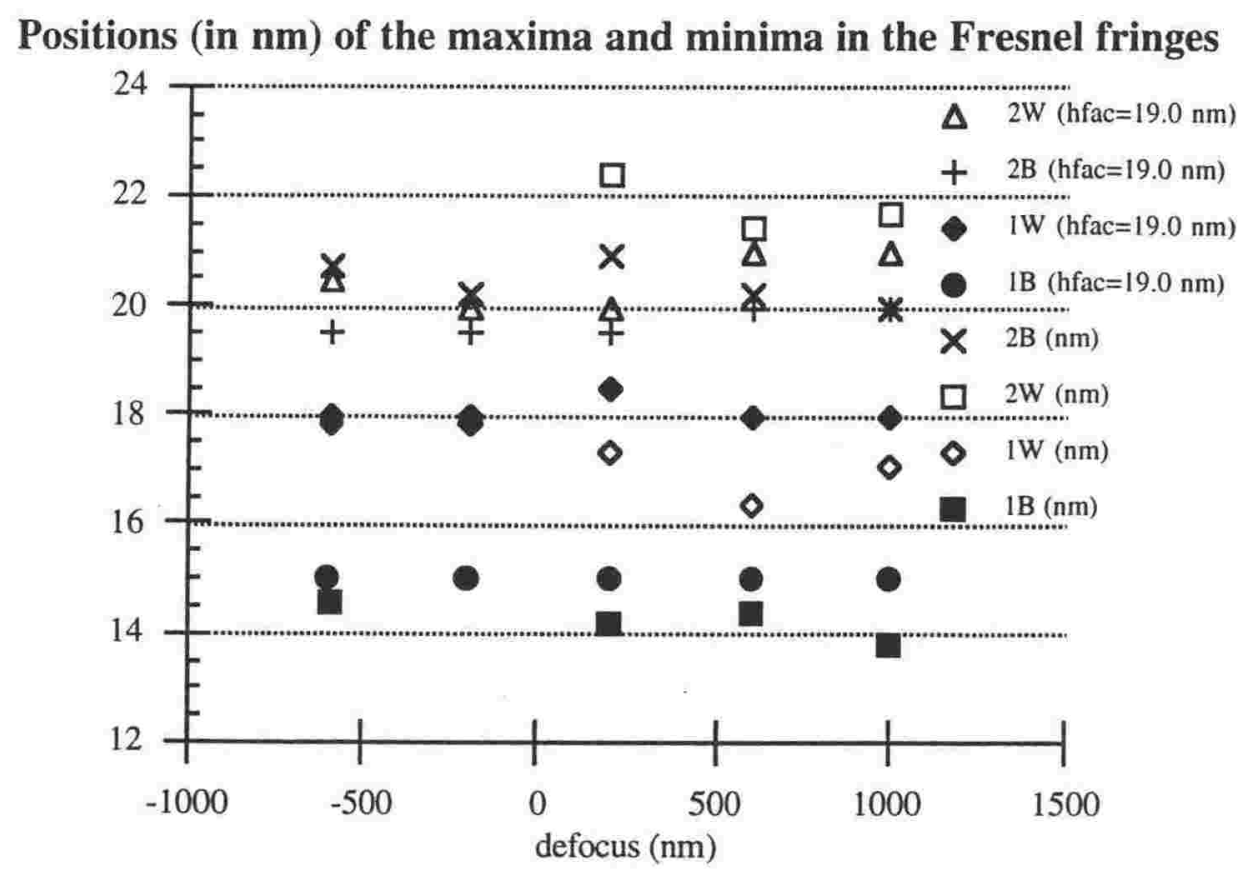

Figure 5.25: Comparison of the positions of the experimental maximum and minimum in the profiles of figure 5.24 to theoretical profiles (labelled for a specified value of hfac where hfac is the minimum distance from the centre of the cavity to a facet.). The "W" indicates that the data is for a maximum in the intensity profile (i.e. a point that is above the background intensity (white)). Similarly the "B" indicates that the data is for a minimum in the intensity profile (i.e. a point that is below the surrounding intensity (black)). The " 1 " or " 2 " in front of the "W" or " $\mathrm{B}$ " indicates that the data is for the first or second maximum or minimum in the intensity profile respectively. The small oscillations in the simulated profiles in figure 5.24 (b) have been ignored because the experimental profiles are too noisy for these oscillations to be detected. The large variation in the peak positions with defocus shows the utility of the simulated profiles as an aid to deducing the cavity radius.

bubbles so deconvoluting the real structure from a through-focal series is impracticable. Instead an image simulation and matching approach is necessary.

The use of the column approximation was discussed in section 2.1, and is valid for images where the beam coherence and resolution are limited. It is assumed that the wavefunction at the exit surface of the crystal after it has undergone scattering from bubbles is accurately calculated by using the column approximation and two beams (as discussed in sections 5.2 and 5.3). The superlattice spots can be then modelled by calculating the power spectrum of this wavefunction. The central spot and the superlattice spots surrounding it 
are determined under bright field conditions whereas any spots around $\mathbf{g}$ can be calculated in the same way using a dark field wavefunction. The region between the 0 th and gth beam will not be accurately modelled unless the Fourier transforms of the BF and DF wavefunctions are added with the appropriate phase factors. Any spots modelled in this way do not contain information about electron waves multiply scattered by bubbles in different columns lying close together laterally. However, because the Bragg angle is equal to about $1^{\circ}$, the diffracted wave does not pass into another column within the small foil thickness, provided the column width is greater than about $0.01 \xi_{\mathrm{g}}$ [75] (about $4 \AA$ in copper). Therefore an electron wave cannot be scattered off two bubbles separated laterally by a superlattice constant which is typically, about $65 \AA$.

The superlattice spots occur because the diffraction aperture is placed around many bubbles with some ordering and the wavefunction at the foil exit surface has some low frequency periodicities. Ignoring the dependence of the diffraction from a bubble upon the depth of the bubble below the top surface of the foil, the wavefunction at the exit surface of the foil is crudely proportional to a projection of the three-dimensional positions of the bubbles upon the foil surface. The superlattice spots are evidence for ordering in three dimensions only if they change as expected for a crystal when the specimen is tilted.

Previous attempts to model the superlattice spots (Stoneham [192] and Cook et al. [193]) calculated the structure factor of postulated bubble structures. This is equivalent to taking a two-dimensional Fourier transform because the change in electron wavevector caused by scattering is approximately perpendicular to the incident electron wavevector. The calculation of the structure factor is approximately independent of the vertical position, parallel to the beam direction, of the bubbles. The z-component of the wavevector does not affect the power spectrum at strong two-beam conditions. This is because every part of the wavefunction at the exit surface of the foil has the same $\mathrm{z}$-component of the wavevector and the same distance to travel to the screen. The structure factor approach is a kinematical theory so it is not as accurate as taking the power spectrum of the wavefunction calculated from a dynamical theory.

Stoneham [192] used the fact that the structure factor of an arrangement of identical bubbles is equal to the structure factor of a single bubble times the structure factor of the positions of the bubbles. Similarly, in the dynamical approach used here it is not necessary 
to calculate the wavefunction scattered by every bubble in the structure. Instead a sum is taken over the different types of bubble wavefunctions in the image. Each sum adds terms containing the Fourier transform of the wavefunction due to a bubble of a certain size and depth. The wavefunctions can be multiplied by the Fourier transform of the positions of bubbles that give this same wavefunction to give the contribution of all the bubbles of a similar type to the superlattice spots.

A Fourier transform of a wavefunction that is rotationally symmetric is

$$
\psi(\mathrm{k})=\int_{0}^{2 \pi} \int_{0}^{R n_{0}} \psi(\rho) \mathrm{e}^{2 \pi \mathrm{ik} \rho \cos (\theta)} \rho \mathrm{d} \rho \mathrm{d} \theta .
$$

The Fourier transform will have rotational symmetry too so that only one angle needs to be included in the integration. Equation 5.2 simplifies to

$$
\psi(k)=\frac{\pi R_{0}^{2}}{2} \int_{0}^{1} \psi\left(\rho^{\prime}\right) \rho^{\prime} J_{0}\left(2 \pi k R_{0} \rho^{\prime}\right) d \rho^{\prime} .
$$

Equation 5.3 can be integrated numerically as were the earlier, similar expressions of Rühle and Wilkens [83] (reproduced in chapter 2).

Stoneham [192] found that the structure factor of a bubble decreases quickly in reciprocal space as $\mathrm{k}$ moves away from the origin. This explains why only first order superlattice spots are found even in well ordered specimens. Figure 5.26 shows the power spectrum of a dynamical wavefunction, calculated using two beams and for various values of the crystal tilt. It is similar in shape to that of Stoneham [192]. Tilting can be used to maximise the height of any superlattice spots relative to the central maximum. Stoneham [192] found that the power spectrum converges to zero at a wavevector roughly inversely proportional to the bubble radius. This roughly corresponds to the position of the zero in the Bessel function in equation 5.3 as a function of the bubble radius. To get second order superlattice spots small, widely spaced, bubbles are required. For example, in figure 5.26, second order spots would only be observed for values of k significantly less than $0.04 \AA^{-1}$, corresponding to the superlattice constant being much greater than $50 \AA$.

A SAD aperture with a radius of $30 \mu \mathrm{m}$ will select a region of $1 \mu \mathrm{m}$ radius on the specimen, containing about 100,000 bubbles if the bubbles are spaced $50 \AA$ apart. This 


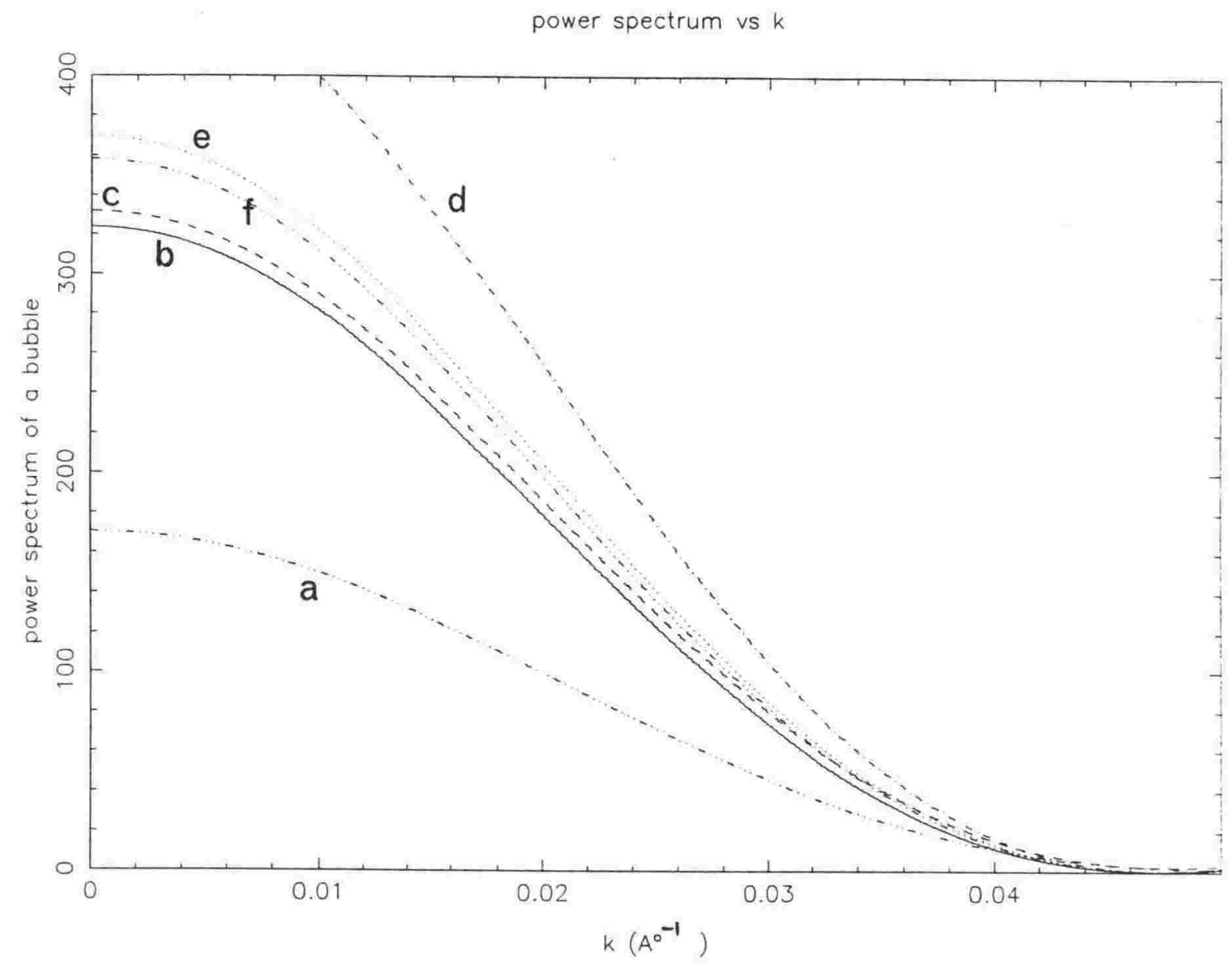

Figure 5.26: Power spectrum of a helium bubble with a $10 \AA$ radius in copper with zero strain field. The foil thickness is $1.75 \xi_{\mathrm{g}}, \mathbf{g}=[200]$, (a) $\mathrm{w}=-1$, (b) $\mathrm{w}=0.3$, (c) $\mathrm{w}=1$, (d) $w=2$, (e) $w=3$ and (f) $w=15$. The power spectrum when $w=-1$ has the lowest intensity which agrees with the experimental observation that it is necessary to tilt the electron beam away from a simple axis in the crystal to increase the intensity of the superlattice spots. 
corresponds to a square of about 300 by 300 bubbles. Since there is no long range order in the bubble positions, it is reasonable to assume that the bubbles lie in locally ordered domains with the positions of the domain centres being random. A Fourier transform or structure factor of the random domain positions is a delta function at the centre of reciprocal space. The intensity of the superlattice spots is determined by the structure factor of just one domain if all of the domains have the same underlying symmetry. Any variations in the bubble positions from the ideal lattice sites within a domain are probably random and could affect the intensity and width of a superlattice spot. One-dimensional profiles through the SADP of an fcc crystal containing bubbles can be generated without knowing the position of the bubbles perpendicular to the direction of the profile. Assuming that the bubbles are spaced about $50 \AA$ apart on a square lattice and that the domain is $\mathrm{n}$ by $\mathrm{n}$ bubbles in lateral extent, the profiles in figure 5.27 result. Damping of the power spectrum causes the peaks to occur slightly before $1 /(50 \AA)$ so that estimates of bubble lattice constants from superlattice spots will be overestimates by about $5 \%$ for the worst case $(n=2)$ in figure 5.27 .

As $\mathrm{n}$ increases the superlattice peak becomes sharper and small subsidiary oscillations appear. This can be understood by analogy with the well known situation of diffraction by $n$-slits. Based on the width of the superlattice spots, the simulated curve for $\mathrm{n}=2$ is the most similar to experimental micrographs. Two effects other than domain size that could contribute to the width of superlattice spots in a practical case are: (i) random displacements of bubbles from ideal lattice sites within individual domains, (ii) limited coherence of the electron beam. The beam coherence is such that the phase of the electrons scattering from different bubbles is probably not the same. A filament in a TEM is of finite size so that regions on the top surface of the specimen separated by a distance of more than the coherence width are scattering electron waves that are not in phase. The coherence width is of the order of tens of Angstroms and varies with the beam current density, type of filament used etc. [162]. In calculating the power spectrum of the wavefunction scattered from a set of bubbles the diffraction intensity from separate patches of bubbles ought to be added. In practice the effects of incoherence have to be neglected and, instead, the Fourier transforms are added in phase, as was done in modelling defocussed intensity profiles in sections 5.1 to 5.3. Figure 5.28 shows some simulated two-dimensional SAD patterns. The occurrence of subsidiary oscillations as $n$ increases cannot be seen but the increased 
SAD intensity vs $k$

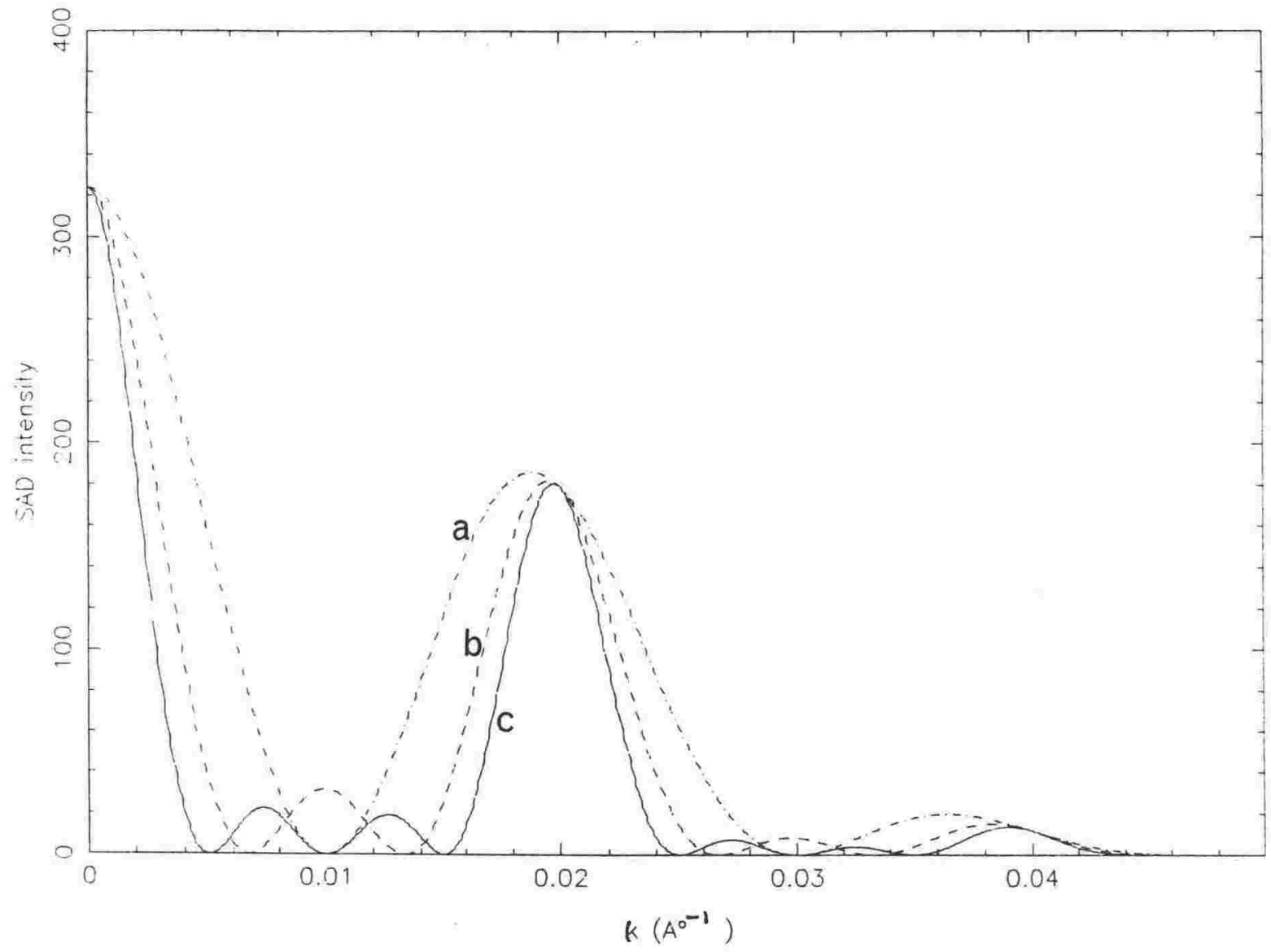

Figure 5.27: $\mathrm{SAD}$ profiles for an $\mathrm{n} \times \mathrm{n}$ square array of helium bubbles in copper with zero strain field for (a) $n=2$, (b) $n=3$ and (c) $n=4$. The foil thickness is equal to $1.75 \xi_{\mathrm{g}}, \mathrm{g}=$ [200] and $w=0.3$. The profiles for a domain that is larger than $2 \times 2$ show a sharp superlattice peak and subsidiary oscillations, features that are not seen in experimental SAD patterns. 


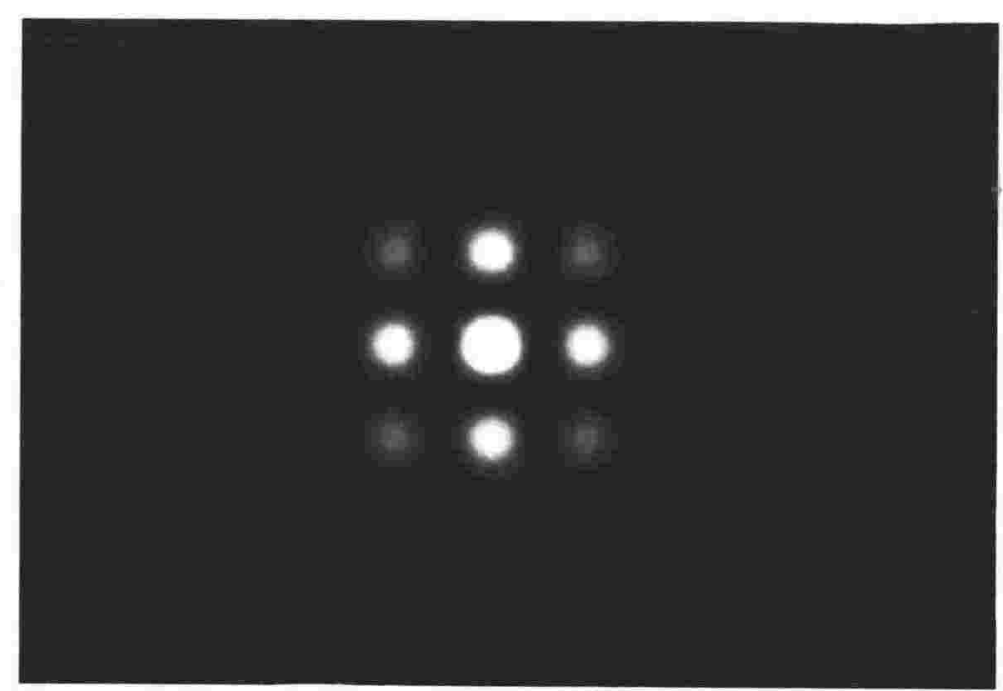

(a)

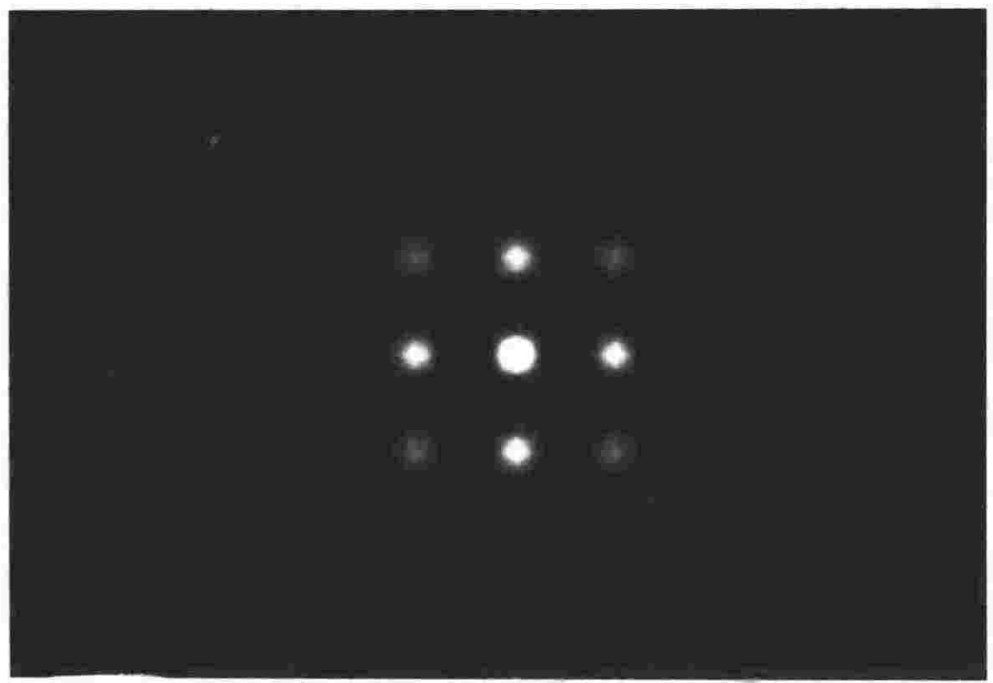

(b)

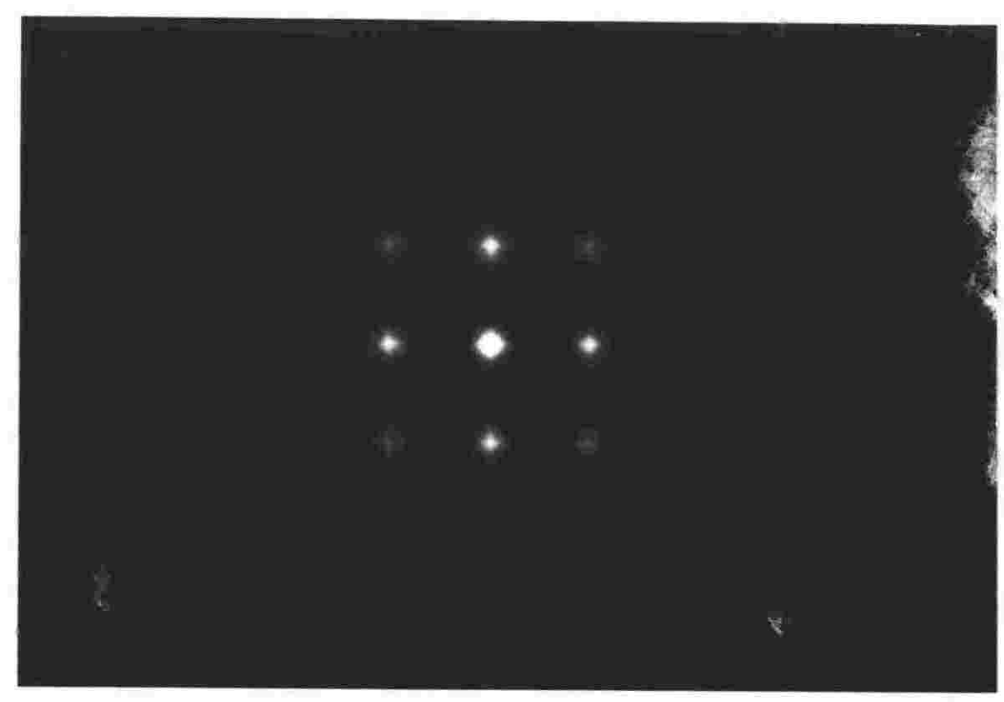

(c)

Figure 5.28: Theoretical SAD patterns for (a) $n=2$, (b) $n=3$ and (c) $n=4$ and a zero strain field helium bubble in copper. The foil thickness is equal to $1.75 \xi_{g}, g=[200]$ and $w=$ 0.3. These images are two-dimensional versions of figure 5.27. 
two-dimensional SAD patterns. The occurrence of subsidiary oscillations as n increases cannot be seen but the increased peak sharpness is noticeable. A comparison of the simulated and experimental SAD patterns to experimental photos again shows that the case of $n=2$ gives the best match.

\subsection{Summary/Conclusion}

An image of a bubble is strongly affected by many parameters - foil thickness, depth of the bubble, bubble radius, diffracting vector, excitation error, bubble pressure and defocus level. Furthermore, the image changes according to whether it is a single bubble which is being considered, or a stack of bubbles. Previous work has looked at some of these factors only, whereas all of them have been considered in this work, as indicated in the table.

\begin{tabular}{|c|c|c|c|c|c|c|c|c|}
\hline Author & Thickness & Depth & Radius & $\begin{array}{l}\text { diffracting } \\
\text { vector }\end{array}$ & $\begin{array}{l}\text { excitation } \\
\text { error }\end{array}$ & Pressure & Defocus & Stacks \\
\hline $\begin{array}{l}\text { Ruhle and } \\
\text { Wilkens } \\
{[83]}\end{array}$ & o & & o & 0 & & & 0 & \\
\hline $\begin{array}{l}\text { Foreman et } \\
\text { al [88] }\end{array}$ & & & o & 0 & & & 0 & \\
\hline $\begin{array}{l}\text { Ashby and } \\
\text { Brown } \\
{[187,188]}\end{array}$ & o & 0 & 0 & 0 & o & 0 & & \\
\hline $\begin{array}{l}\text { E. Johnson } \\
\text { et al [84] }\end{array}$ & & & & o & o & & o & o \\
\hline Stevens & 0 & 0 & o & o & o & o & o & 0 \\
\hline
\end{tabular}


A library of simulated images of an isolated bubble is presented to show the effects of systematic changes in these parameters. It is difficult to summarise the changes when the parameters are varied. It is clear, however, that the bubble images are not always dark in overfocus and white in underfocus, as would have been expected from the "conventional wisdom". The image of a stack of bubbles is even more complicated but has crudely been used to probe the three-dimensional nature of the ordering between bubbles. The results of comparing the simulations with experimental micrographs indicate that vertical stacks of more than three bubbles probably do not occur in practice.

Four criteria were used to determine bubble size. Using these criteria, the ratio of the apparent bubble size to actual size is often significantly different from 1 , sometimes by more than 100 per cent. When plotted as a function of reduced radius, the ratio shows dependence on bubble radius and defocus, and is discontinuous. Comparisons between a through-focal series of bright-field micrographs and simulated intensity profiles provide the best means of estimating the radius of a single bubble. This is a laborious process and even then the radius obtained may be subject to large uncertainties. The bubble could be embedded in any one of a range of metals but the same techniques are still applicable. This image matching approach has also been applied to a facetted cavity.

The presence of superlattice diffraction spots is an indication of the ordering of the gas bubbles in a specimen into a superlattice. These diffraction patterns have been simulated using a dynamical theory rather than the kinematical approach favoured by past authors [193]. The simulations give a more quantitative measure of the degree of local ordering into domains and suggest that the bubble ordering in the gas bubble superlattice is of limited extent. However, it has been found that the effects of beam incoherence may be a limiting factor on extracting more information on the ordering of bubbles from SADP's when the bubbles are of the spacing typically encountered. 


\section{CHAPTER 6. SIMULATIONS: COMBINATIONS OF BUBBLES AND LOOPS}

\subsection{The Simulation Program}

\subsubsection{Introduction}

This section (6.1) describes a program used to simulate the diffraction of electrons from structures containing loops near bubbles while section 6.2 presents the results obtained by running the program. Figure 6.1 shows the main features of the program in a flowchart; these features are briefly introduced in this paragraph and more detail is given in the rest of the section. Inputs are used to specify the geometry of the structure and the diffraction conditions, and are used initially to calculate the Fourier coefficients of the crystal potential and the other parameters shown in the second block of figure 6.1. In brackets next to the parameters are the equations used to calculate these parameters. Several coordinate systems are required in the calculations - for the picture, the foil, the individual bubbles and loops, and the angular dislocations making up the loops - and any particular vector will need to be referred to more than one coordinate system. Transformation matrices are calculated from direction cosines and translation vectors (third block of figure 6.1). After this the numerical integration proper is done. Alternatively the geometry of the structure that is used in the program can be checked by calculating the displacement field due to any combination of loops and bubbles on a column in the sample, on a plane, or within a certain volume. A simulated image is typically calculated as a $41 \times 41$ array and is then read into a Semper picture. The picture is then displayed and magnified four times by a Semper interpolation routine to a picture 164 columns by 164 rows in size before a hardcopy is produced. Details on the simulation system are presented in section 4.4. 
Calculations from inputs: relativistic wavelength (2.3)

g.b and the scattering matrix modulus of the electron

wavevector in the crystal (2.10) relativistic correction factor incident beam direction to give the required excitation error electron scattering factors using the Mott-Bethe formula (2.1) structure factors (2.4)

Fourier coefficients of the potential $(2.5,2.7)$ extinction distances (2.14)

Data:

number of atoms in the unit cell atom type

atom positions in the unit cell accelerating voltage

approximate beam direction foil normal

diffracting vectors

foil thickness accuracy parameter mean potential in the bubble beam on which the aperture is centred $\mathrm{X}$-ray atomic scattering factor fitting parameters

Debye-Waller factors

imaginary part of $\mathrm{U}_{0}$

Burgers vector, loop normal and vector through a vertex of each of the loops

depth of the bubble

Poisson's ratio of the matrix excitation error for a beam length of the picture radius of the bubble strength of the strain around a bubble number of sides to the dislocation loops distance from the centroid of the loop to any of its vertices vector parallel to one side of the picture whether or not to calculate another picture - for batch processing
Set up the coordinate systems for each loop, the bubbles, the foil and the picture:

Construct the picture system using $\mathbf{B}$ and norm - i.e. calculate the direction cosines so that the program can rotate the crystal axes into the picture system

Calculate the direction cosine arrays to convert from the picture coordinates into the OXYZ system of each loop and from OXYZ into the coordinates of each angular dislocation used to construct a loop and to convert the displacements found back into the OXYZ system (3.263.32)

Rotate vectors such as $\mathbf{b}$ into the various coordinate systems

Figure 6.1: A flowchart of the program used for simulating images. The inputs are read in from a file when the program is executed, then some calculations are made which include the setting up of the coordinate systems. A loop is then made over the rows and columns of an array in which the picture is to be stored. During this looping procedure a numerical integration is made down each column of the picture. 
Either simulate a picture using numerical integration or find the displacement field (R)

Calculate $\mathbf{R}$, on a column or a slice through the foil or in a three-dimensional image, due to any one combination of the loops and bubbles
Loop over the picture, integrating down the columns using a Runge-Kutta process (2.18)

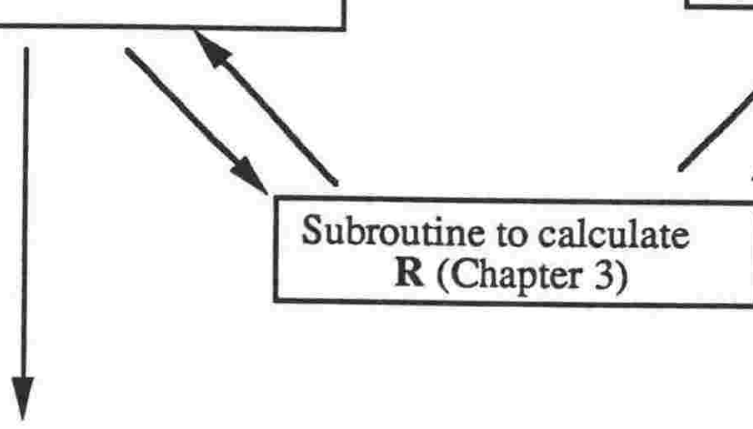

Output the components of $\mathbf{R}$ in a column, a slice or a

three-dimensional array
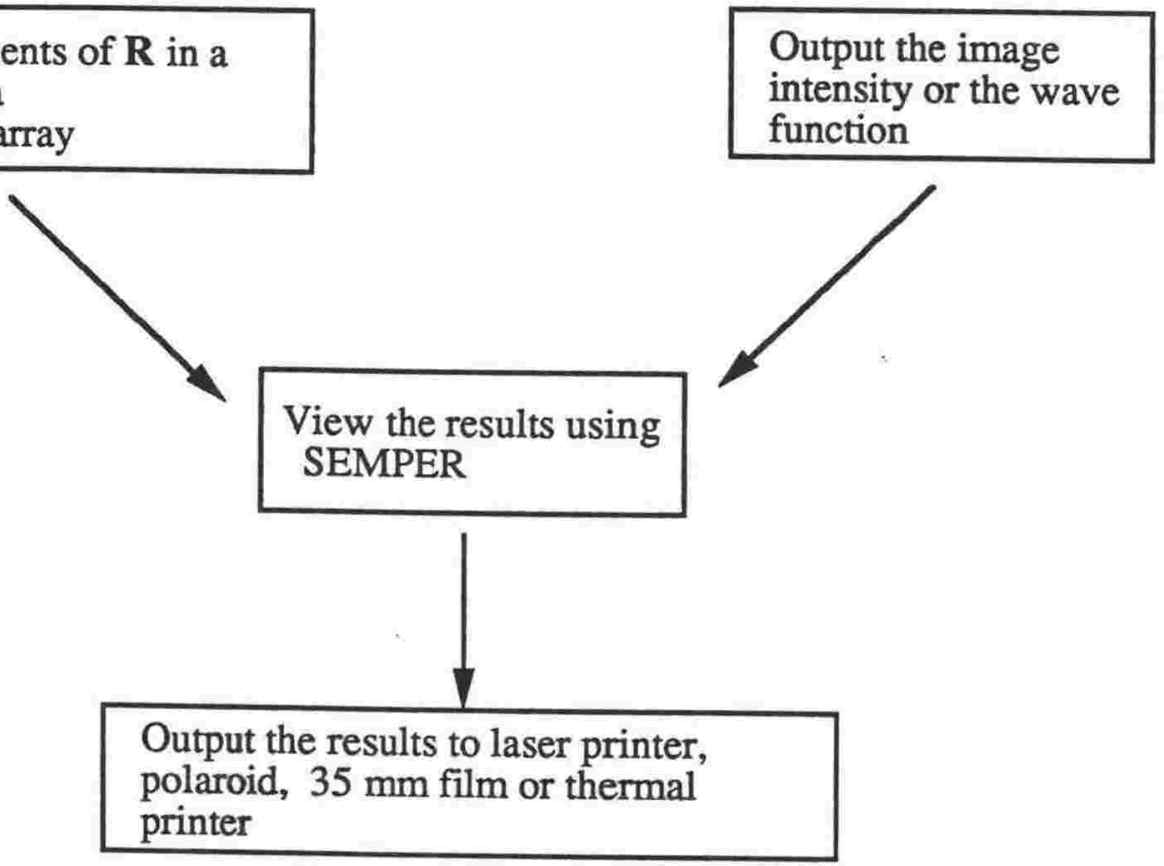


\subsubsection{Calculation of the Scattering Matrices}

It was easiest to convert the flowchart into code that could be compiled and executed by extracting blocks of code from existing programs written by other authors to simulate images of structures different from that considered in this thesis. The "TCBED " program, written in Fortran by Zou and Spence of the Physics Department at Arizona University and obtained by electronic mail from Nestor Zaluzec at Argonne National Labs, was heavily modified to do the simulations. Calculations of the matrix $\mathbf{A}$ are made using the equations of Self et al. [159] (these are the same as those outlined in chapter two) and were tailored to bubbles in copper by using the scattering factors for X-rays in fcc copper. This technique was used instead of the renormalisation technique [194] of the original "TCBED " program. Some of the code in the original program was deleted because there was no need to compute the eigenvalues and eigenvectors of $\mathbf{A}$ or to output Convergent Beam Electron Diffraction (CBED) images as is done in the original program.

\subsubsection{The Numerical Integration}

After the scattering matrices have been calculated the differential equations are numerically integrated using a fourth order Runge-Kutta process with adaptive step width control to satisfy a specified accuracy criterion. Code for the procedure that performs the numerical integration was adapted from the "rkm " and "deriv" subroutines in the "onedis " and "twodis " programs of Head et al. [82]. The "deriv" subroutine first requires the evaluation of the right hand side of equation 2.38 with a subroutine being called to evaluate the resultant displacement field due to all of the defects. This new program is much slower in producing images than their "onedis " and "twodis " programs, partly because of the complicated nature of the displacement field and the need for repeated multiplication of matrices. The main reason for the slowing of the program is because the generalised crosssection construction of Head et al. is inapplicable and so it is necessary to integrate the differential equations down every column of the picture. Simulations of isolated loops produced by the program were checked against published results [62-64] with good agreement always being obtained. 


\subsubsection{The Displacement Field}

In the model of Seitz [119] for dislocation loop punching the loops are prismatic but in the model of Greenwood, Foreman and Rimmer [1] the loops are assumed to be circular. The displacement field due to an hexagonal loop is described in Saldin et al. [62-64], Yoffe [165] and in chapter three. Angular dislocations are connected up to form an hexagonal loop and the resultant displacement field of the loop is found by addition of the displacement fields due to the individual angular dislocations. Saldin et al. [62-64] also constructed approximately circular loops by including a large number of angular dislocations in a polygonal loop but showed that the differences between simulations using the displacement field of an hexagonal loop and the displacement field of a circular loop are small. On the basis of this work, hexagonal loops are used in this thesis.

To calculate the displacement field it is necessary to know how many loops are likely to be in the structure. A typical superlattice structure in copper has bubbles with a radius of about $10 \AA$ and with a spacing between nearest neighbour bubbles of about $50 \AA$. Considering the volume of the bubble and therefore the number of copper atoms displaced by the bubble as it grows, it is estimated that approximately twelve dislocation loops with a radius of $10 / \sqrt{ } 2 \AA$ and a Burgers vector of the $0.5<110>$ type will be punched out along the nearest neighbour $<110\rangle$ directions. However, in practice there probably are more loops of a smaller radius. Section 7.1 should be consulted for other complications on this simple estimate of the number of loops that will be punched out by a bubble of this size.

To give the displacement fields for structures that could occur in specimens containing a superlattice of gas bubbles, in this thesis the displacement fields due to the individual loops and bubbles have been added. All the loops are assumed to be pinned in position around a bubble by the Peierls barrier and by the strain due to the other bubbles and loops. The displacement field that pins the loops in position is assumed not to affect the contrast significantly. 


\subsubsection{The Program Geometry}

Each loop has associated with it a coordinate system described by Saldin [62-64]. To perform a simulation it is necessary to calculate twelve matrices that convert coordinates from the coordinate system of the foil into coordinate systems with an $\mathrm{X}$ axis antiparallel or parallel to each of the twelve loop normals (n). It is also necessary to translate the picture coordinates (i.e. the coordinate system in which the axes are parallel to the edges of the picture) into a system with a local origin at the centroid of each loop before the contribution of this loop to the displacement field is calculated.

The program geometry was checked by generating two-dimensional projections of the structure (as shown for example in figure 6.2). Every element in a three-dimensional array representing the coordinates near the structure was tested to see whether it was within the radius of the bubble or if it was close to any of the segments of the dislocation loops. If an element was close to a segment then its value was set to zero (corresponding to a black point in the pictue) - otherwise it remained at 255. The array was then displayed on the screen of the computer using the "solid" command of Semper. A frame around the edges of the figure (and the cube in figure 6.2c) represents the top and bottom surfaces of the foil and the limits of what is simulated in the picture. A foil that is artificially thin has been used so that the structure is easier to see.

The orientation and magnification of a simulation can be specified by the inputs to the program. The length of side of a picture is always $160 \AA$ unless otherwise stated. To specify the orientation of the images the program uses a vector called norm that is aligned with the horizontal edges of the picture, and points from left to right. This vector is chosen so that a simulation is correctly aligned with the stereogram for whatever beam direction (B) is being used. Stereograms for some simple B's are shown in figure 6.3. Thompson tetrahedra are also useful for understanding the geometry of the structure (Hirth and Lothe [97]). For an fcc material, Thompson tetrahedra represent the, $<110>$ directions (i.e. the directions of the glide planes) by their edges and the $<211>$ directions by lines from the centre of their faces (the (111) planes) to their vertices. The $<211>$ directions are useful because a dislocation with a Burgers vector of [110] can dissociate into two partial 

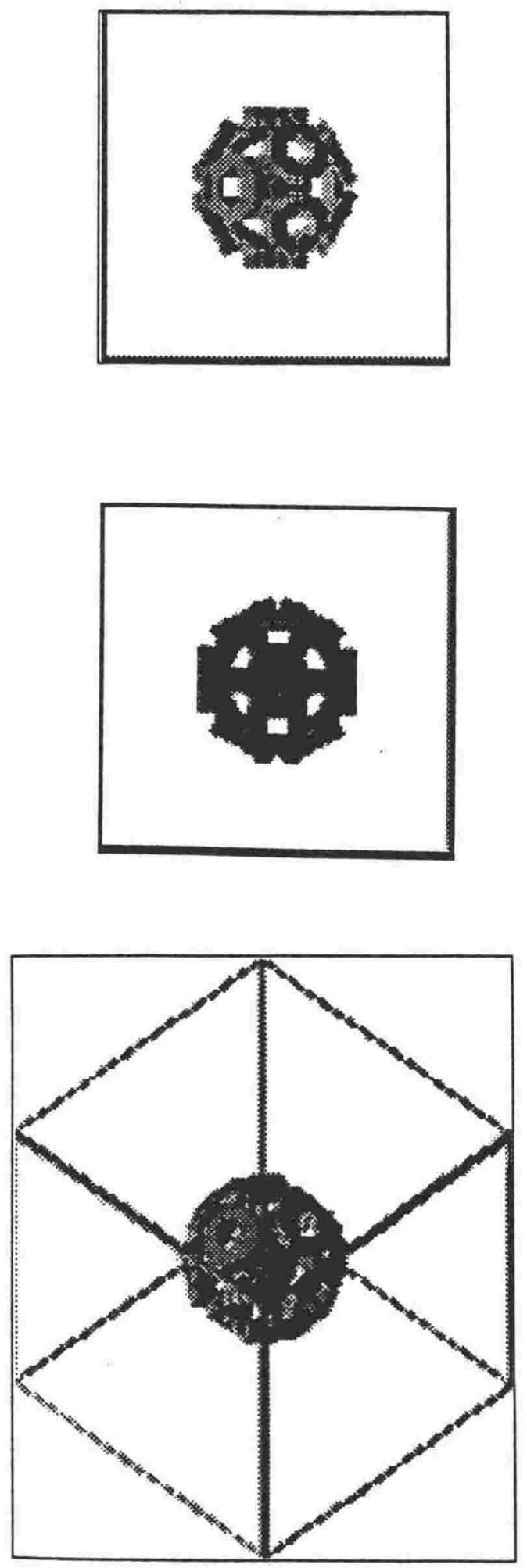

Figure 6.2: Two-dimensional projections of a structure containing a bubble and twelve dislocation loops which are directed along $<110>$ directions. Projections have been made in several directions of the three-dimensional structure. That is, a three-dimensional array has been mapped into several two-dimensional arrays. 

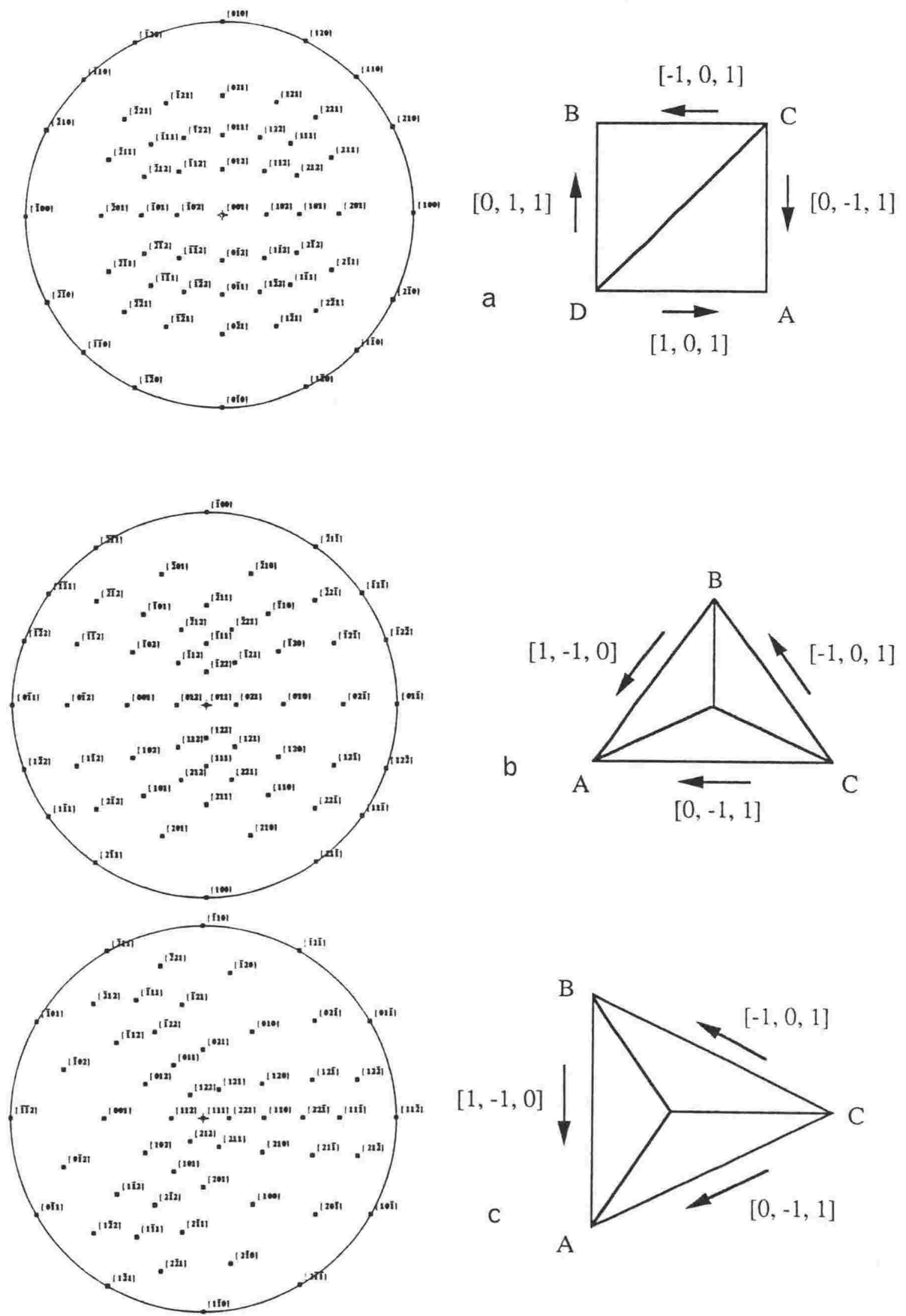

A

Figure 6.3: Stereograms for (a) $\mathbf{B}=$ [001], (b) [011] and (c) [111]. The orientation of the Thompson tetrahedra for these beam directions is also shown. Tetrahedra and stereograms show the orientations of the $<110>$ glide planes and the most commonly used g's. Some of these directions will be used in the simulations in the remainder of this chapter. 
dislocations with Burgers vectors of the $<211>$ type. Some tetrahedra are drawn on figure 6.3 in the correct alignment with the stereograms.

\subsection{Simulations}

\subsubsection{Introduction}

No work has been done previously to simulate images of dislocation loops next to bubbles with the parameters relevant to superlattices of gas bubbles containing helium. In this section this novel structure is simulated, the applicability of the rules for interpreting an isolated defect are tested and whether the contrast of the simulated structure (i.e. a collection of defects) can be separated into its component defects is also tested. In addition the structure is simulated under weak beam conditions [89 and 90] as the higher resolution given by weak beam imaging may be useful when studying defects at the densities thought to be present in materials that have been ion-implanted. A complication is the increased difficulty of interpreting these higher resolution pictures.

Simulations are done over a range of important parameters for imaging superlattices of gas bubbles near dislocation loops. The simulations are compared to simulations when no loops are present. In this way the imaging conditions that give the maximum difference between the two different structures (loops present or not present) is found. This difference is significant and a method for experimentally checking the presence or absence of dislocation loops is proposed in section 6.3.

Authors of the papers cited in the introduction of section 1.1 obtained several general rules for interpreting the appearance of large isolated defects which imply that it is not always necessary to resort to time-consuming computations of images. Experimental micrographs can be sometimes used to determine the vacancy or interstitial nature of a loop (section 4.5). Also the loop's normal (n) and Burgers vector (b) can be found. These parameters have been obtained for dislocation loops that are large and resolvable in section 4.6. 
However, these techniques are not applicable to small dislocation loops, especially when the small loops are near other defects. The most obvious contrast effect characteristic of a small loop that does not have opposite sides that can be distinguished (as for example the sides of the loops in figure 4.12 could be distinguished) is the pair of lobes surrounding the defect. One lobe is black and the other is white. A vector I from the centre of the black lobe to the centre of the white lobe is usually defined. The simulations of other authors [5866] show how this vector varies with respect to the projection of $\mathbf{b}$ upon the image plane, with the diffracting vector $\mathbf{g}$, with $\mathbf{n}$ and with the foil normal $\mathbf{F}$ etc. For edge dislocation loops I is parallel to $\mathbf{b}$.

For edge loops the small effects that result from using isotropic instead of anisotropic elasticity theory are thought to make the neglect of anisotropy in this work a reasonable approximation. Eyre et al. [58-61] matched experimental pictures of dislocations in anisotropic copper with theoretical simulations using isotropic elasticity theory. Ohr [195] has computed images of dislocation loops in anisotropic copper using both anisotropic and isotropic elasticity theory. That paper showed that the effects of anisotropy are a skewing of the black/white lobes and a rotation of $\mathbf{I}$.

\subsubsection{Simulations of Individual Defects}

It is useful to see what part of the contrast in the simulation of a bubble and its twelve dislocation loops is due to the individual defects (i.e. due to single loops or to the bubble alone). To see this, it is possible to limit the program to calculating the diffraction from some of the defects only. In figure 6.4 each dislocation loop has been individually simulated in isolation (figure 6.4a) and alongside an overpressured bubble (figure 6.4b), for $\mathbf{g}=[200]$ and $\mathbf{B}=[001]$. Tables 6.1 to 6.3 summarise the dependence of the image of a defect on the geometry of the structure. By using the $\mathbf{g}=[200]$ column in table 6.1 and the $\mathbf{B}=[001]$ column in table 6.2 the image of a dislocation loop in figure 6.4 can be compared in table 6.3 to the rules for the image type of Eyre et al. [58], as discussed above. It can be seen that the contrast due to the loops in figure 6.4 varies with g.b. Considering now a bubble at equilibrium pressure, it has no surrounding displacement field so that a TEM image of the bubble is determined by thickness contrast. The author has found that if a loop 


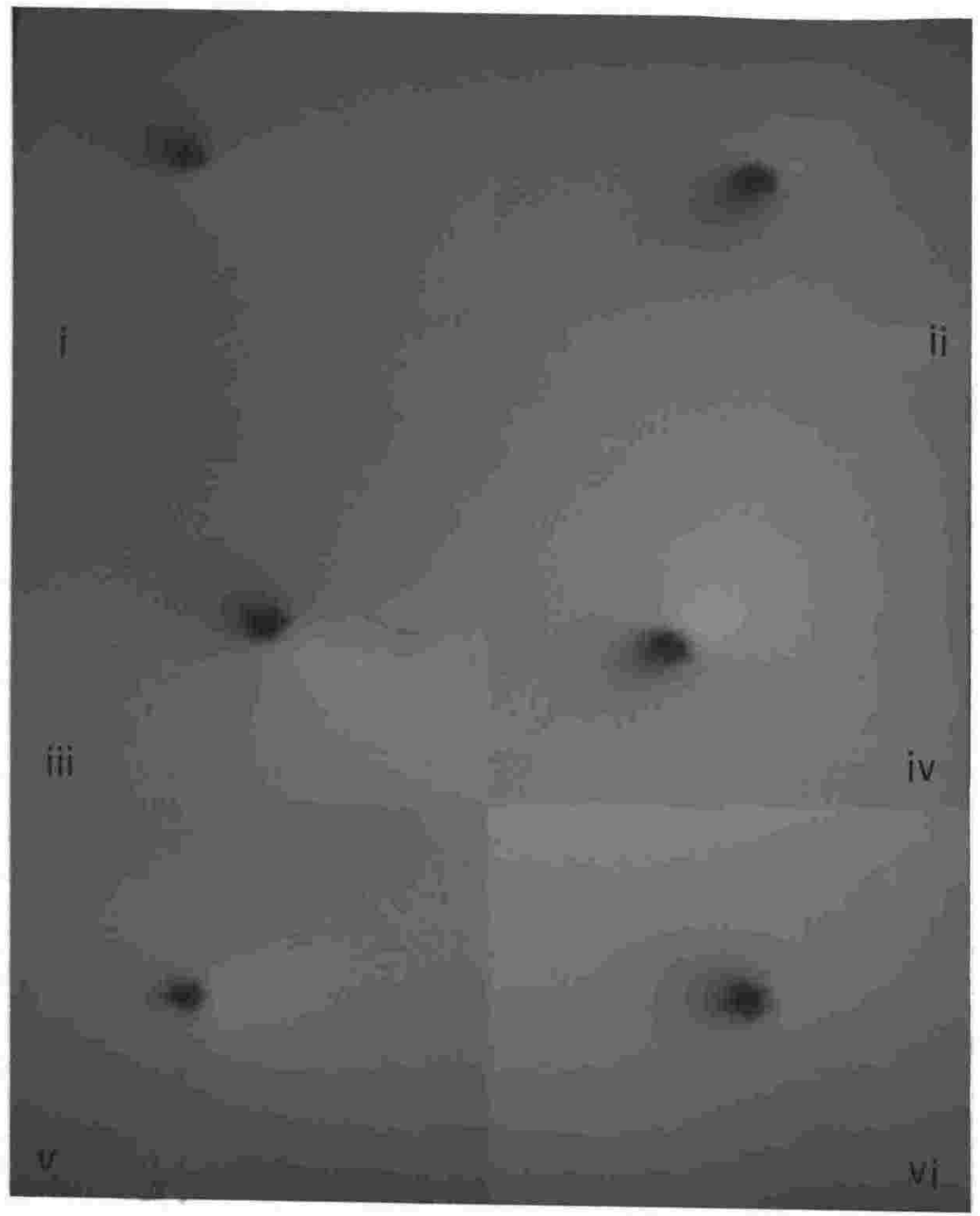

Figure 6.4 (a)

$6-11$ 


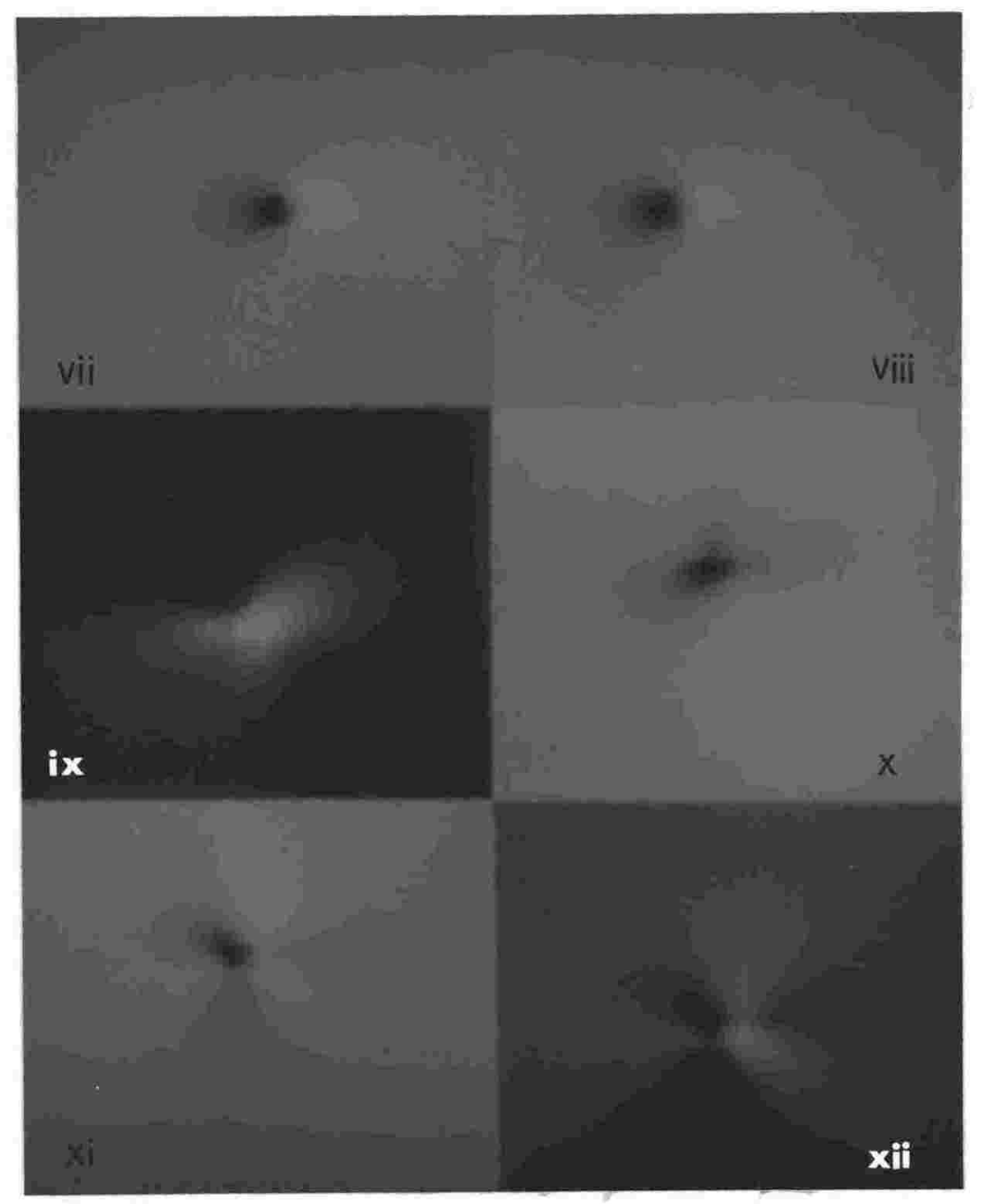

Figure 6.4 (a)

$6-12$ 


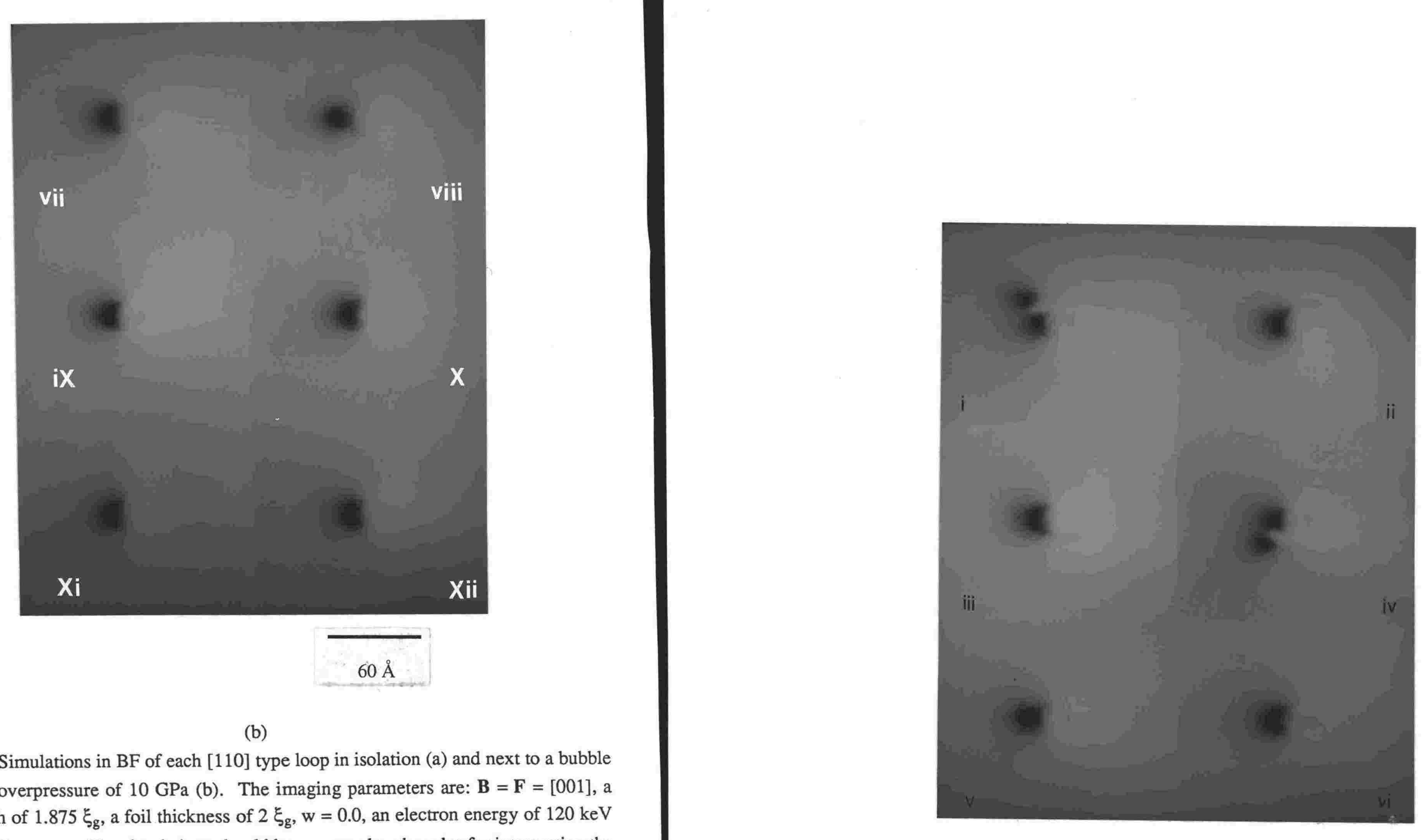

Figure 6.4: Simulations in BF of each [110] type loop in isolation (a) and next to a bubble that has an overpressure of $10 \mathrm{GPa}$ (b). The imaging parameters are: $\mathbf{B}=\mathbf{F}=[001]$, a bubble depth of $1.875 \xi_{\mathrm{g}}$, a foil thickness of $2 \xi_{\mathrm{g}}, \mathrm{w}=0.0$, an electron energy of $120 \mathrm{keV}$ and $\mathbf{g}=[200]=$ norm. The simulations should be compared to the rules for interpreting the images of loops reproduced in table 6.3; loops (i) to (viii) exhibit type 3 contrast and the remaining loops exhibit type 1 contrast. The parameters of the loops are: (i) $\mathbf{b}=0.5[1,-1$, $0]$ and $\mathbf{g} . \mathbf{b}=1$; (ii) $\mathbf{b}=0.5[-1,-1,0]$ and $\mathbf{g} . \mathbf{b}=-1$; (iii) $\mathbf{b}=0.5[-1,1,0]$ and $\mathbf{g} . \mathbf{b}=-1$; (iv) $\mathbf{b}$ $=0.5[1,1,0]$ and $\mathbf{g} \cdot \mathbf{b}=1 ;$ (v) $\mathbf{b}=0.5[1,0,-1]$ and $\mathbf{g} \cdot \mathbf{b}=1 ;$ (vi) $\mathbf{b}=0.5[-1,0,-1]$ and $\mathbf{g} . \mathbf{b}$ $=-1$; (vii) $\mathbf{b}=0.5[-1,0,1]$ and $\mathbf{g} \cdot \mathbf{b}=-1$; (viii) $\mathbf{b}=0.5[1,0,1]$ and $\mathbf{g} \cdot \mathbf{b}=1$; (ix) $\mathbf{b}=0.5[0$, $1,-1], \mathbf{g} . \mathbf{b}=0$ and the angle between $\mathbf{n}$ and $\mathbf{B}$ is $45^{\circ} ;(x) \mathbf{b}=0.5[0,-1,-1], \mathbf{g . b}=0$ and the angle between $\mathbf{n}$ and $\mathbf{B}$ is $45^{\circ}$; (xi) $\mathbf{b}=0.5[0,-1,1], \mathbf{g} \cdot \mathbf{b}=0$ and the angle between $\mathbf{n}$ and $\mathbf{B}$ is $45^{\circ}$; (xii) $\mathbf{b}=0.5[0,1,1], \mathbf{g} . \mathbf{b}=0$ and the angle between $\mathbf{n}$ and $\mathbf{B}$ is $45^{\circ}$. 
with a non-zero g.b is simulated alongside a bubble which is at equilibrium pressure then the contrast in the resultant image is dominated by the contrast due to the loop. This does not agree with the experimental observations, in which large populations of bubbles have been found but the presence of dislocation loops is difficult to confirm, so in this chapter most of the simulations are of more realistic structures in which the bubbles are overpressured.

\begin{tabular}{|c|r|r|r|r|r|r|r|r|}
\hline \multicolumn{10}{|c|}{$+\mathbf{g}$} \\
\hline$+2 \mathbf{b}$ & {$[200]$} & {$[\overline{02} 0]$} & {$[\overline{22} 0]$} & {$[0 \overline{22}]$} & $\overline{1} \overline{11}]$ & {$[1 \overline{11}]$} & {$[220]$} & {$[20 \overline{2}]$} \\
\hline$[\overline{1} 10]$ & -1 & -1 & -2 & 1 & 1 & 0 & 0 & -1 \\
\hline$[110]$ & 1 & -1 & 0 & 1 & 0 & 1 & 2 & 1 \\
\hline$\overline{1} 01]$ & -1 & 0 & -1 & -1 & 0 & -1 & -1 & -2 \\
\hline$[101]$ & 1 & 0 & 1 & -1 & 0 & 0 & 1 & 0 \\
\hline$[\overline{01} 1]$ & 0 & 1 & 1 & -2 & 1 & -1 & -1 & -1 \\
\hline$[011]$ & 0 & -1 & -1 & 0 & 0 & 0 & 1 & -1 \\
\hline
\end{tabular}

Table 6.1. g.b values for the six edge on dislocation loops punched on the [110] glide planes.

\begin{tabular}{|c|c|c|c|}
\hline \multicolumn{4}{|c|}{$+\mathbf{B}$} \\
\hline$+\mathbf{n}$ & {$[111]$} & {$[001$} & {$[011]$} \\
\hline$\overline{1} 10]$ & $90^{\circ}$ & $90^{\circ}$ & $60^{\circ}$ \\
\hline$[110]$ & $35.3^{\circ}$ & $90^{\circ}$ & $60^{\circ}$ \\
\hline$\overline{1} 01]$ & $90^{\circ}$ & $45^{\circ}$ & $60^{\circ}$ \\
\hline$[101]$ & $35.3^{\circ}$ & $45^{\circ}$ & $60^{\circ}$ \\
\hline$[\overline{01} 1]$ & $90^{\circ}$ & $45^{\circ}$ & $90^{\circ}$ \\
\hline$[011]$ & $35.3^{\circ}$ & $45^{\circ}$ & $0^{\circ}$ \\
\hline
\end{tabular}

Table 6.2. Angles between $\mathbf{n}$ and $\mathbf{B}$ for the six edge dislocation loops punched on the [110] glide planes.

\begin{tabular}{|c|l|l|}
\hline Image type & \multicolumn{1}{|c|}{ Diffracting conditions } & Predicted image \\
\hline 1 & $\begin{array}{l}\text { g.b }=0 \text { and angle between } \mathbf{n} \text { and } \\
\mathbf{B}=0 \text { to } 45^{\circ}\end{array}$ & $\begin{array}{l}\text { Invisible or weak } \\
\text { black/white lobes }\end{array}$ \\
\hline 2 & g.b $=0$ and angle between $\mathbf{n}$ and $\mathbf{B}=90^{\circ}$ & 'Butterfly' contrast \\
\hline 3 & $|\mathbf{g} \cdot \mathbf{b}| \neq 0$ but $\leq 1$ & Simple black/white lobes \\
\hline 4 & $|\mathbf{g} \cdot \mathbf{b}|>1$ & $\begin{array}{l}\text { Black/white lobes with } \\
\text { interface structure }\end{array}$ \\
\hline
\end{tabular}

Table 6.3. Image classifications for edge dislocation loops in an fcc or bcc crystal. 


\subsubsection{Simulations of Punched-out Dislocation Loops}

Figure 6.5 shows how the twelve loops punched by an overpressured bubble will appear when imaged with the parameters listed in the figure caption. The loops have different $\mathbf{g . b}$, as shown in table 6.1, and are imaged with different $\mathbf{B}=\mathbf{F}$ values. Table 6.2 shows how the angle between the beam direction and loop normal varies. The changes in $\mathbf{g}$ and the other parameters just mentioned will alter the image characteristics according to the rules in table 6.3. The simulations are aligned with the stereographic projections in figure 6.3 , i.e. the vector pointing from the left-hand bottom corner to the right-hand bottom corner of any simulation is parallel to the vector on the extreme right of the stereogram (for the appropriate value of $\mathbf{B})$. The direction of the Burgers vectors of the dislocation loops can be located on figure 6.3 and matched up with the black/white lobes in figure 6.5. The defects in figure 6.5 have been placed in the middle of the last so-called "layer" in the crystal, as discussed in the next section.

\subsubsection{The Layer Structure}

The image simulations of other authors revealed the dependence of $\mathbf{l}$ on the depth of the defect [187, 188 and 196]. The vector 1 reverses its direction (i.e. the lobes reverse contrast) as the depth of the defect increases, leading to the concept of a layer structure. Saldin [63] among others explained the origin of this using a kinematical theory. The layer structure or Ashby-Brown rules [187 and 188] are shown in figure 6.6 for an isolated defect for the cases of bright field and dark field imaging. The analysis of other authors [58-61] also revealed the transition zones between these layers and the range of validity of these rules, for both edge and non-edge dislocations. If a defect lies more than about 1.25 extinction distances from either foil surface in a foil with a thickness of more than about three extinction distances, then the lobe structure will not appear, and instead a dislocation will be imaged up in BF as a black dot with a fairly uniform intensity. The variation of g.I with distance of the defect from either foil surface is the same for bubbles and for interstitial dislocation loops. This is because the different types of defects both have an antisymmetric (with respect to opposite sides of the defect) derivative of $\mathbf{g} . \mathbf{R}$ with respect to 


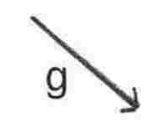

$\longrightarrow$

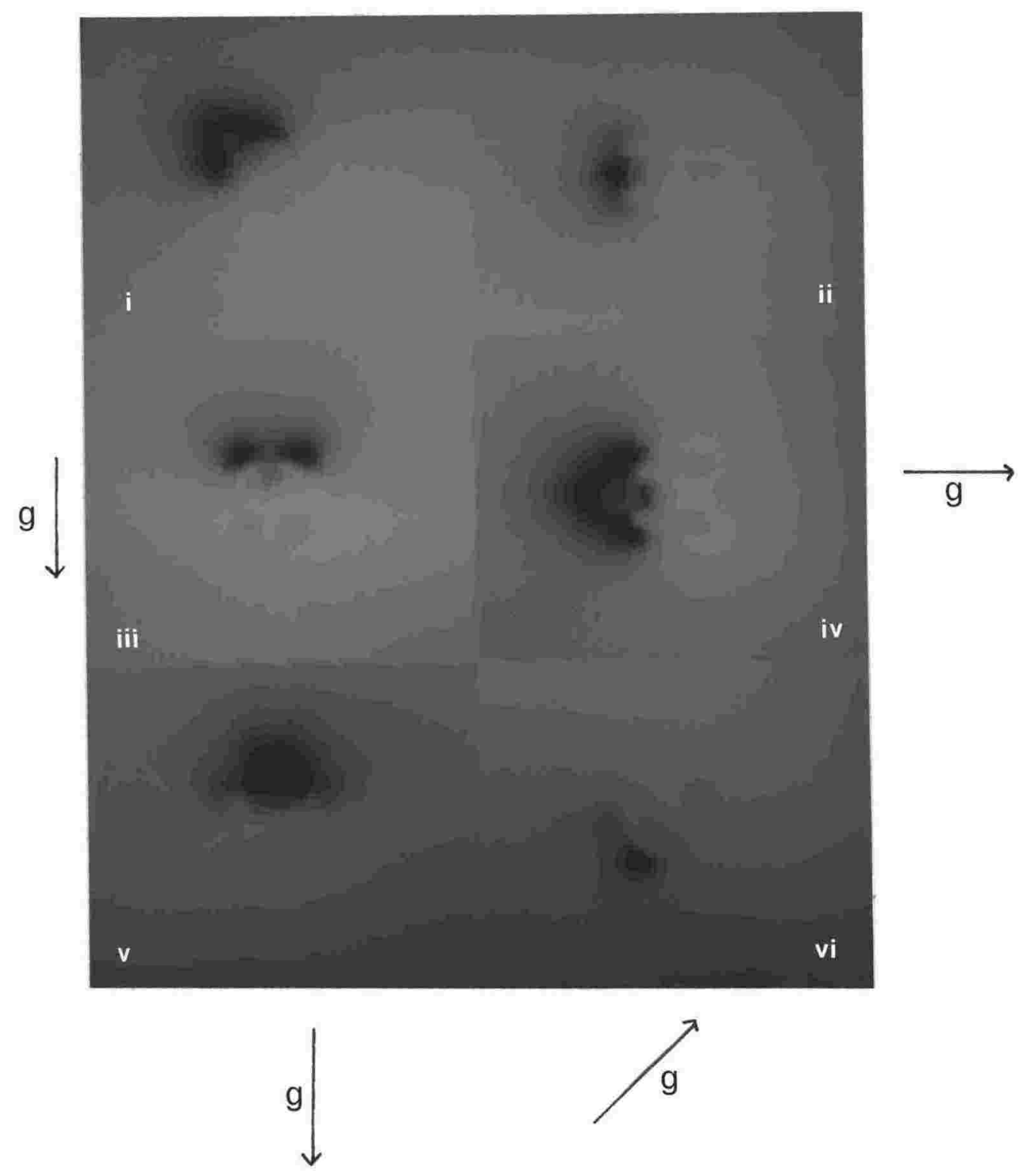

Figure 6.5 (a)

6- 17 
$60 \AA$

(b)

Figure 6.5: Simulations of a bubble and all twelve loops for various combinations of B, F and $\mathrm{g}$ in (a) BF and (b) DF. The common imaging parameters are: a foil thickness of $2 \xi_{\mathrm{g}}$, $\mathbf{B}=\mathbf{F}$, an electron energy of $120 \mathrm{keV}$ and a bubble depth of $1.875 \xi_{\mathrm{g}}$. The $\mathbf{B}$ and $\mathbf{g}$ pairs are: (i) $\mathbf{B}=[001]$ and $\mathbf{g}=[2,-2,0]$; (ii) $\mathbf{B}=[001]$ and $\mathbf{g}=[200]$; (iii) $\mathbf{B}=[011]$ and $\mathbf{g}=$ [200]; (iv) $\mathbf{B}=$ [011] and $\mathbf{g}=[0,2,-2]$; (v) $\mathbf{B}=[111]$ and $\mathbf{g}=[2,-2,0]$; (vi) $\mathbf{B}=[011]$ and $\mathbf{g}=[-1,1,-1]$. The geometry can be understood by comparing the images to the stereograms and tetrahedra in figure 6.3. 


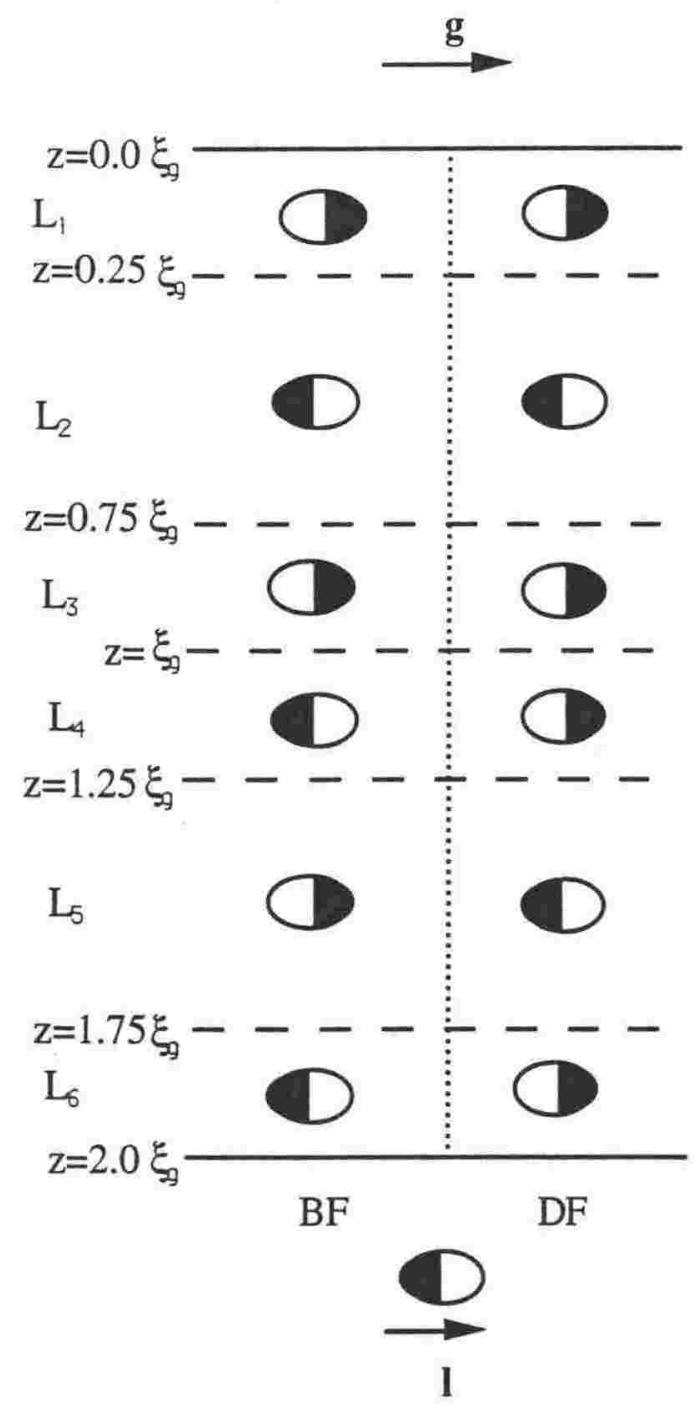

Figure 6.6: The layer structure for interstitial dislocation loops and bubbles. The defect must lie in a layer that is within about 1.25 extinction distances of the surface for the lobe structure to appear. The direction of 1 varies as the depth of the defect is moved between the layers. The detail in this figure is a function of the foil thickness. Transition layers occur in the interface areas near the edges of the layers indicated in the figure; in the transition layers an $\mathbf{l}$ cannot be defined. In a thick foil, e.g. a foil that is $5 \xi_{\mathrm{g}}$ thick, only the first three or four layers near either surface will occur, i.e., a defect near the centre of the foil will not be imaged as a pair of lobes.

the depth $\mathrm{z}$ through the crystal. Vacancy loops have a symmetric $\mathbf{g} . \mathrm{dR} / \mathrm{dz}$ and therefore an $\mathbf{l}$ at any one depth that is opposite to that in figure 6.6. However, the image of a bubble is affected significantly by the thickness contrast and not just by the strain or displacement field contrast. A problem associated with assigning an $\mathbf{I}$ to a bubble is the effect of having 
an excitation error that is non-zero. Yet another factor is the change in mean potential between the matrix and the gas bubble. A value of w that is non-zero will affect the infocus contrast due to the bubble, but the change in mean potential will change the out-offocus contrast only and not the layer structure of the in-focus images. As a consequence in some cases an I cannot be assigned to the bubble.

Figure 6.7 shows that the dependence of the image on the depth of the defects for a structure comprising twelve loops and a bubble is more complicated than the layer structure of an isolated defect. The individual defects have displacement fields of similar magnitude and these fields overlap considerably, so it is sometimes difficult to define an $\mathbf{l}$ for each. Nor is it possible to always define an $\mathbf{I}$ for the structure as a whole. Consider the contrast in a region of the simulation in which a defect is projected onto the plane of the image. The contrast in this region can be significantly different than if the defect were imaged in isolation. If an $\mathbf{l}$ for the entire structure is assigned, the layer structure is similar to that expected for a bubble. This is because the combined $\mathbf{g} \cdot \mathrm{d} \mathbf{R} / \mathrm{dz}$ is approximately antisymmetric, the result of the displacement fields of a loop and the bubble being in opposite directions in the region between them but in the same direction elsewhere. The size of the resultant black and white lobes does vary between layers but the appearance of the images when the structure is at the same distance from the top and bottom surfaces respectively is very similar. For example, figures 6.7 (a) and (f) are similar (although reversed) as are figures 6.7 (b) and (e). However, when the entire structure is within the third and fourth layers (figures 6.7 (c) and (d)) the idea of a layer structure for the entire structure is not really valid. Although the images show the expected reversal, they cannot be said to show distinct black and white lobes.

Figure 6.8 shows the more complicated appearance of the structure in the interface or transition zone between layers. It should be noted, too, that increasing the thickness of the foil whilst keeping the structure in the last layer does not affect the resultant image much. The layer structure and the geometrical symmetry of the defect structure significantly reduces the required amount of computation. The defect structure is symmetrical so often two g's with the same $|g|$, excitation error, $\mathbf{B}$, and $\mathbf{F}$, but different orientation will give the same picture, except the pictures are rotated relative to one another. Individual loops will have their appearance changed significantly but the structure as a whole will look the same. 


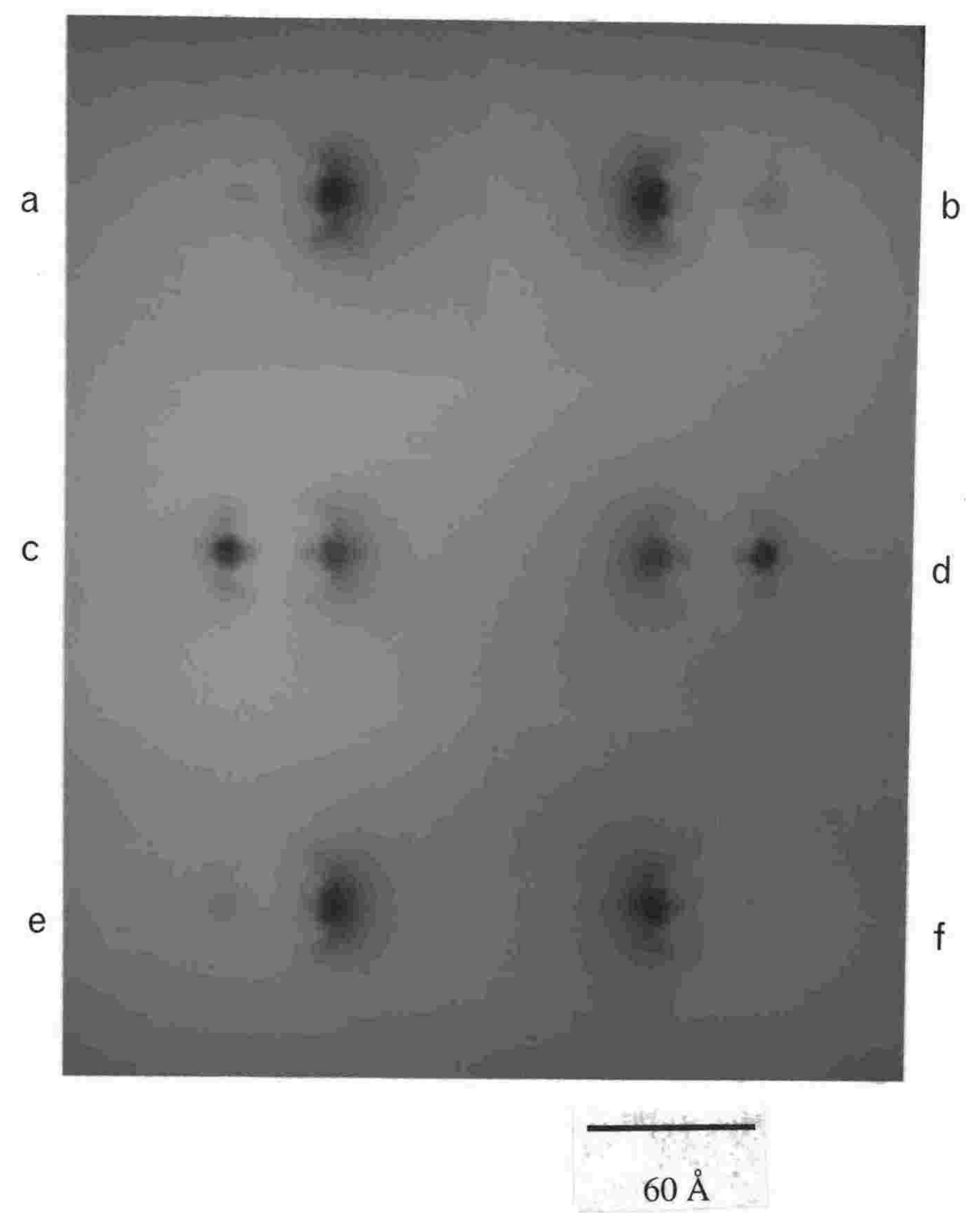

Figure 6.7: The layer structure in the images of a system of one bubble and twelve loops. The imaging parameters in BF are: $\mathbf{B}=\mathbf{F}=[001], \mathbf{g}=[200]=$ norm, an electron energy of $120 \mathrm{keV}$ and a foil thickness of $2 \xi_{\mathrm{g}}$. The depth at which the bubbles lies is (a) $0.125 \xi_{\mathrm{g}}$, (b) $0.5 \xi_{\mathrm{g}}$, (c) $0.875 \xi_{\mathrm{g}}$, (d) $1.125 \xi_{\mathrm{g}}$, (e) $1.5 \xi_{\mathrm{g}}$ and (f) $1.875 \xi_{\mathrm{g}}$. The existence of a layer structure for images of the complicated nature considered here reduces the amount of computation necessary to simulate an image when the structure occurs at many depths in the foil. 


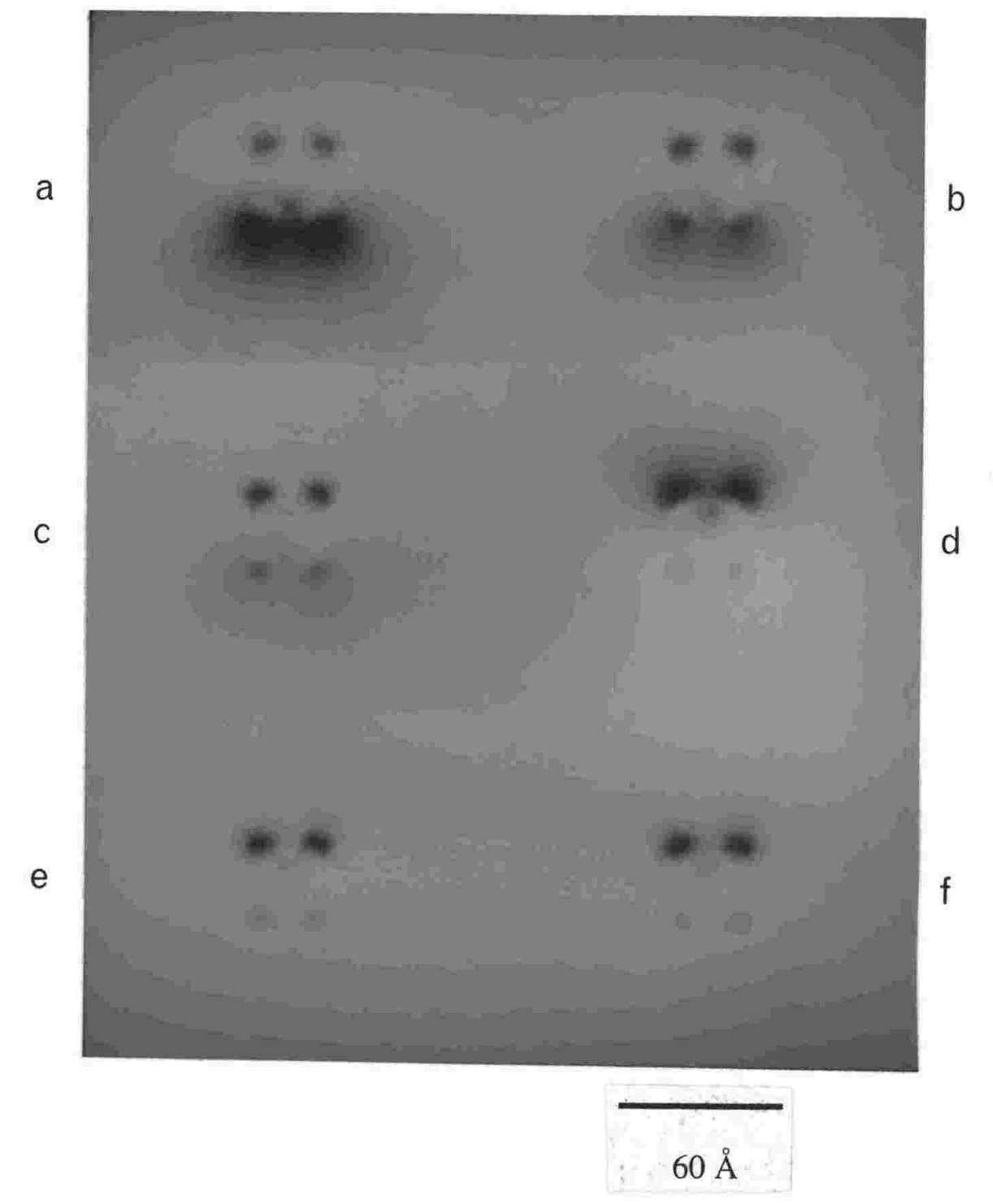

Figure 6.8: Simulations of twelve loops and a bubble as the origin at the bubble in the structure passes through the transition layer region. The imaging parameters in $\mathrm{BF}$ are: $\mathbf{B}=$ [011] $=\mathbf{F}$ and $\mathbf{g}=[200], w=0.0$, an electron energy of $120 \mathrm{keV}$, a foil thickness of $2 \xi_{\mathrm{g}}$ and a bubble depth of (a) $1.65 \xi_{\mathrm{g}}$, (b) $1.7 \xi_{\mathrm{g}}$, (c) $1.725 \xi_{\mathrm{g}}$, (d) 1.75 (e) $1.775 \xi_{\mathrm{g}}$ and (f) $1.8 \xi_{\mathrm{g}}$. As the loops enter the transition region between layers they lose their characteristic black/white lobe appearance, instead appearing as black dots. This behaviour is similar to that which occurs as w increases. 
The picture symmetry also does not vary much between layers as 1 will be rotated by $180^{\circ}$ between some layers and the picture simply rotates with $\mathbf{l}$.

Figure 6.9 shows the effects on the image as the bubble overpressure is increased from zero through to $30 \mathrm{GPa}$. The actual pressure required for dislocation loop punching has been estimated by Jones [54] using the model of Greenwood, Foreman and Rimmer (GFR) [1]. In this model, a bubble of radius $10 \AA$ will be at equilibrium if it has a pressure of $3 \mathrm{GPa}$ and requires an internal pressure of approximately $10 \mathrm{GPa}$ if it to punch out dislocation loops. The more sophisticated model of Wolfer [102] requires that the bubble has a higher overpressure than that predicted by the GFR mechanism [1]. Therefore, in the simulations presented in this chapter the overpressure has been set to $10 \mathrm{Gpa}$ instead of the $7 \mathrm{GPa}$ predicted by Jones.

\subsubsection{The appearance of a Superlattice}

Simulations of two bubbles, with twelve dislocation loops around each bubble, are shown in figure 6.10. Some loops punched by different bubbles are as close to each other as either loop is to the surface of it's bubble. This proximity of the loops causes the formation of a larger region of black/white lobes than usual. Consider the region where the displacement fields of two dislocation loops on the same glide cylinder overlap significantly. The Burgers vectors of the loops are equal in magnitude and opposite in direction. Hence the displacement fields tend to cancel each other out and the intensity between these loops is close to that of the background. In regions close to the core of either dislocation loop the displacement field of this close loop dominates that due to the other loop and the intensity is significantly different from background. This explains why in the interface region between two bubbles the intensity is close to the background intensity. However, then the intensity rapidly increases to give lobes of the usual contrast for a dislocation loop. 


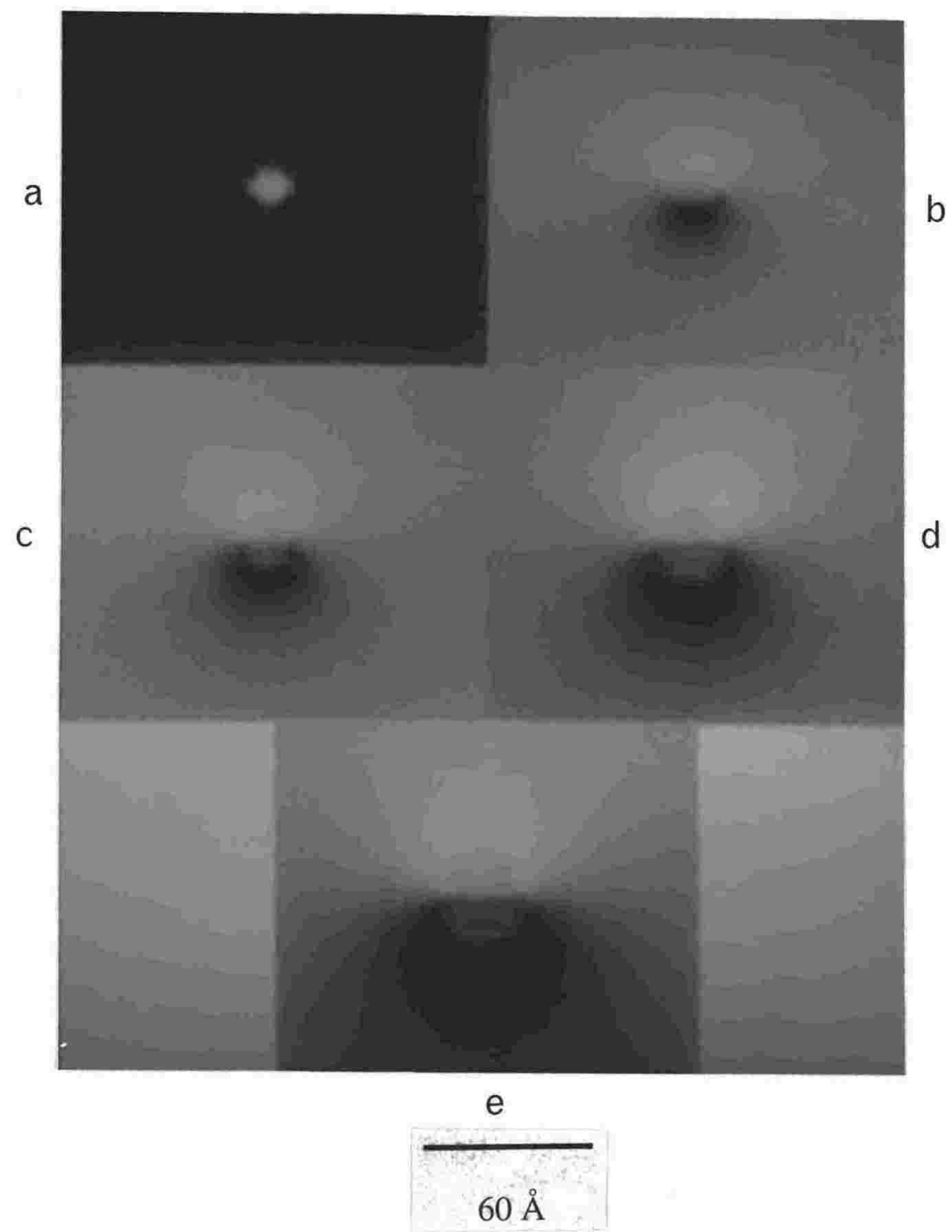

Figure 6.9: The effect of pressure on the image of a $10 \AA$ radius bubble. The overpressure is: (a) $\mathrm{O} \mathrm{GPa}$, (b) $5 \mathrm{GPa}$, (c) $10 \mathrm{GPa}$, (d) $20 \mathrm{GPa}$ and (e) $30 \mathrm{GPa}$. As the bubble pressure is reduced the black and white lobes surrounding the bubble are reduced in size and contrast until the bubble at equilibrium pressure does not have any strain field surrounding it and is imaged up only by thickness contrast. The imaging parameters in BF are: $w=0.0$ and an electron energy of $120 \mathrm{keV}$ with the bubble being positioned at the centre of the last layer in the crystal. 


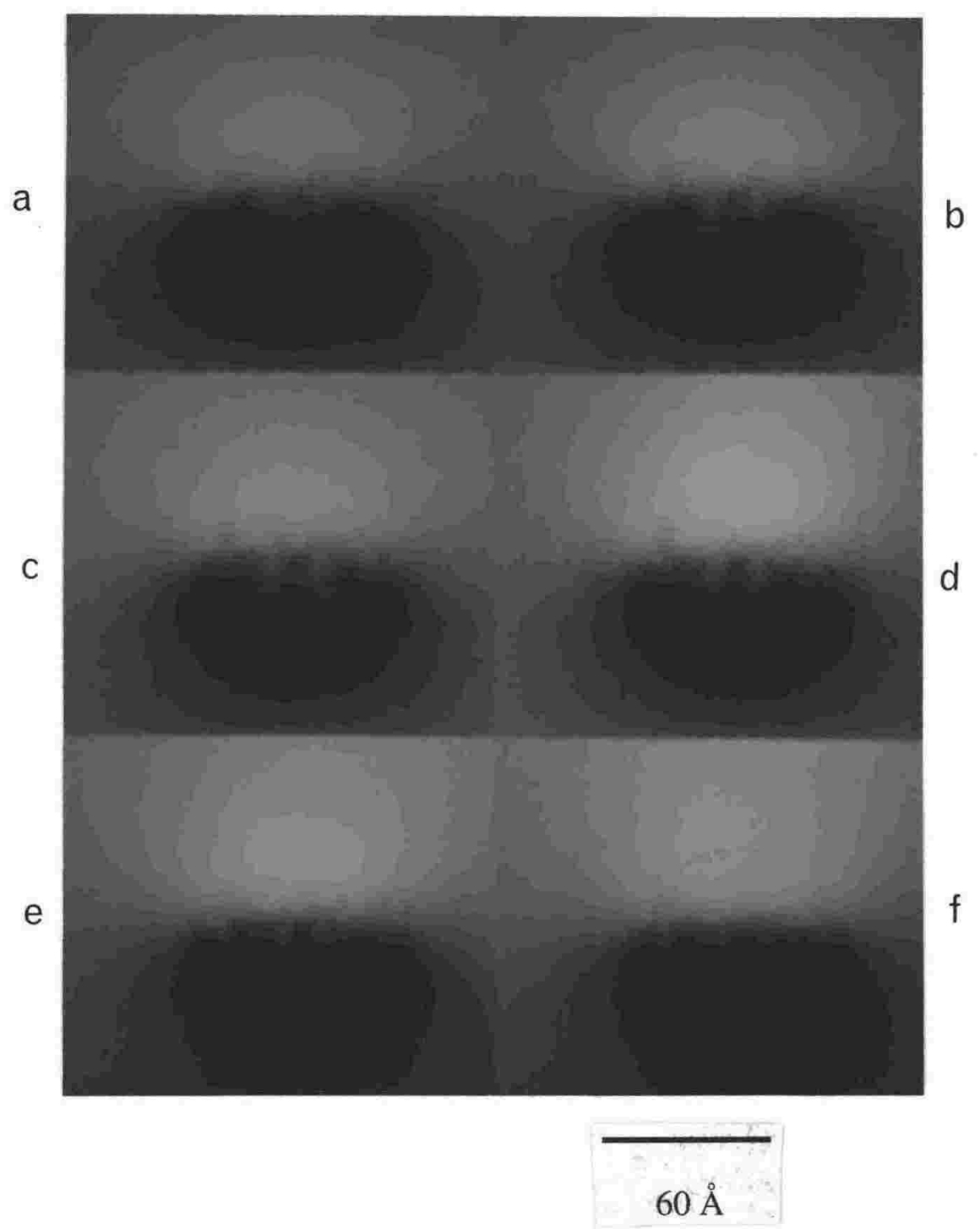

Figure 6.10: Simulations of two bubbles, with twelve dislocation loops around each bubble, as the spacing between the bubbles is varied. The imaging parameters in DF are: $\mathbf{B}$ $=[111]=\mathbf{F}, \mathbf{g}=[2,-2,0]$, a foil thickness of $2 \xi_{\mathrm{g}}$, a bubble depth of $1.875 \xi_{\mathrm{g}}$ and an electron energy of $120 \mathrm{keV}$. The lattice constant takes the values of (a) $40 \AA$, (b) $45 \AA$, (c) $50 \AA$, (d) $55 \AA$, (e) $60 \AA$ and (f) $65 \AA$. When the lattice constant is equal to $65 \AA$ the two bubbles will be spaced about $45 \AA$ apart $(=65 / \sqrt{2} \AA$ ) on a $<110>$ glide cylinder. 
The entire superlattice of bubbles and dislocation loops can be generated by translating the structure simulated in figure 6.10 through the basis vectors of the bubble superlattice. Hence the appearance of many bubbles and loops is shown by the superposition of the simulations above. The appearance of the resultant in-focus image is affected significantly if the projected positions of bubbles overlap with one another. When the projected positions of loops overlap the contrast will be increased or decreased if $\mathbf{g} . \sum \mathbf{b}$ is increased or decreased respectively. When the loops are close, the contrast will be increased or decreased if $\mathbf{g} \cdot \sum \mathbf{R}$ is increased or decreased. How many loops overlap is shown by ball models of the structure of bubbles and loops. This is discussed in more detail in section 6.3.

Figure 6.11 shows the effects of increasing the spacing between the loops and the bubble. The contrast due to individual loops is more readily distinguished as the spacing increases. Figure 6.12 shows the effects of tilting the foil normal (F) away from B. As the tilting occurs the contrast of the defects changes as they move into the transition layers and the black/white lobes become assymetrical.

\subsubsection{Simulations as the excitation error changes}

A more important parameter in the simulations is the excitation error (w). This is a measure of how close the crystal is to strong two-beam $(w=0.0)$ conditions. Figures 6.13 and 6.14 show the effects of changing w. The contrast of the loops in DF (figure 6.13) changes to white dots from the black/white lobe appearance of $w=0.0$. This shows that it is important to be at, or very near to, strong two-beam conditions if the loops are to be imaged via the black/white lobes that they cause. In the images taken under weak beam conditions (figure 6.14), six-beam simulations are used, requiring a different extinction distance to that used in the two-beam calculations, as explained in section 2.4. The loops are resolvable but the images are difficult to interpret.

\subsubsection{Out-of-focus Simulations}

Figure 6.15 shows simulated micrographs of one loop next to a bubble that are defocussed by the approximate Fourier series method of section 5.2. These simulations are 


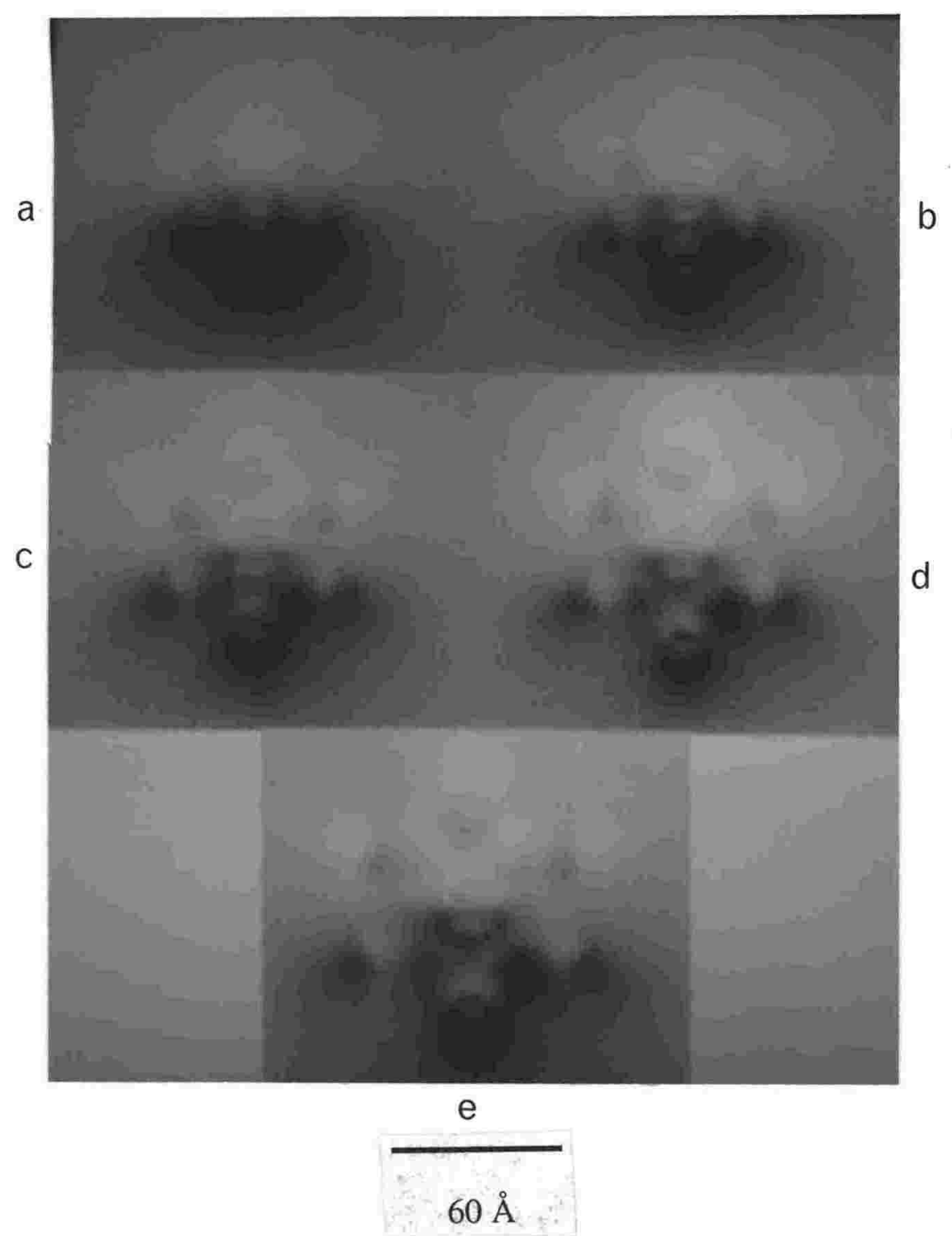

Figure 6.11: Simulations of twelve loops and a bubble as the spacing between the loops and the bubble is increased. The imaging parameters in DF are: $\mathbf{B}=\mathbf{F}=[111], \mathbf{g}=[2,-2$, 0 ], a foil thickness of $2 \xi_{\mathrm{g}}$, a bubble depth of $1.875 \xi_{\mathrm{g}}$ and an electron energy of $120 \mathrm{keV}$. The spacing between the loops and the bubble takes the values of (a) $25 \AA$, (b) $30 \AA$, (c) 35 $\AA$, (d) $40 \AA$ and (e) $45 \AA$. The contrast due to the individual defects becomes more readily distinguishable as the spacing between the loops and the bubble increases.

$$
6-27
$$




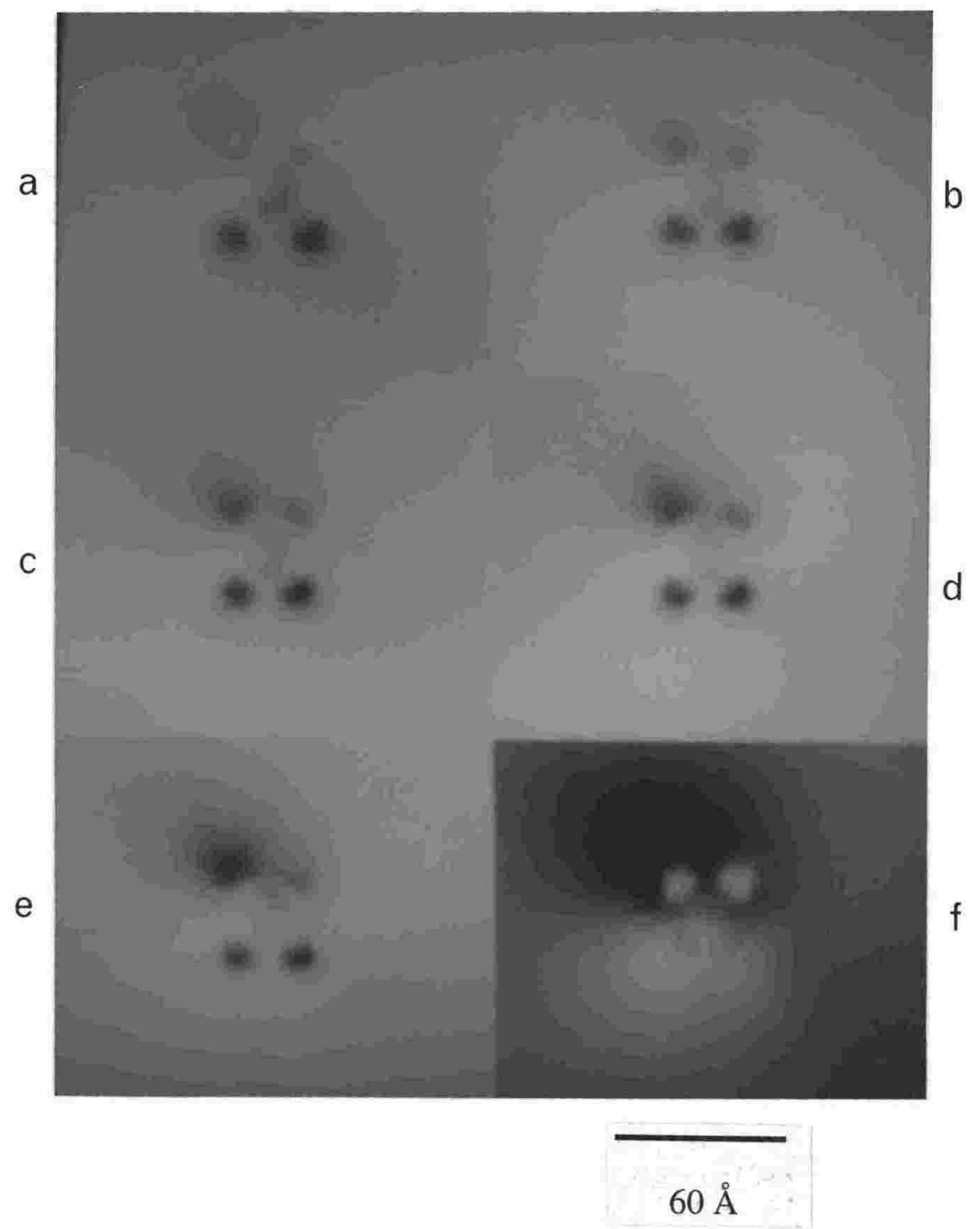

Figure 6.12: Simulations of twelve loops and a bubble as $\mathbf{F}$ is tilted away from $\mathbf{B}$. The imaging parameters in DF are: $\mathbf{B}=[011], \mathbf{g}=$ [200], a foil thickness of $2 \xi_{\mathrm{g}}$, a bubble depth of $1.5 \xi_{\mathrm{g}}$, an electron energy of $120 \mathrm{keV}$ and norm $=[0,2,-2]$. The other parameters are (a) F = [012], (b) [013], (c) [014], (d) [015], (e) [016] and (f) [001]. As the tilt becomes progressively greater (i.e. in going from (a) to $(f)$ ) the black and white lobes become assymetrical. 


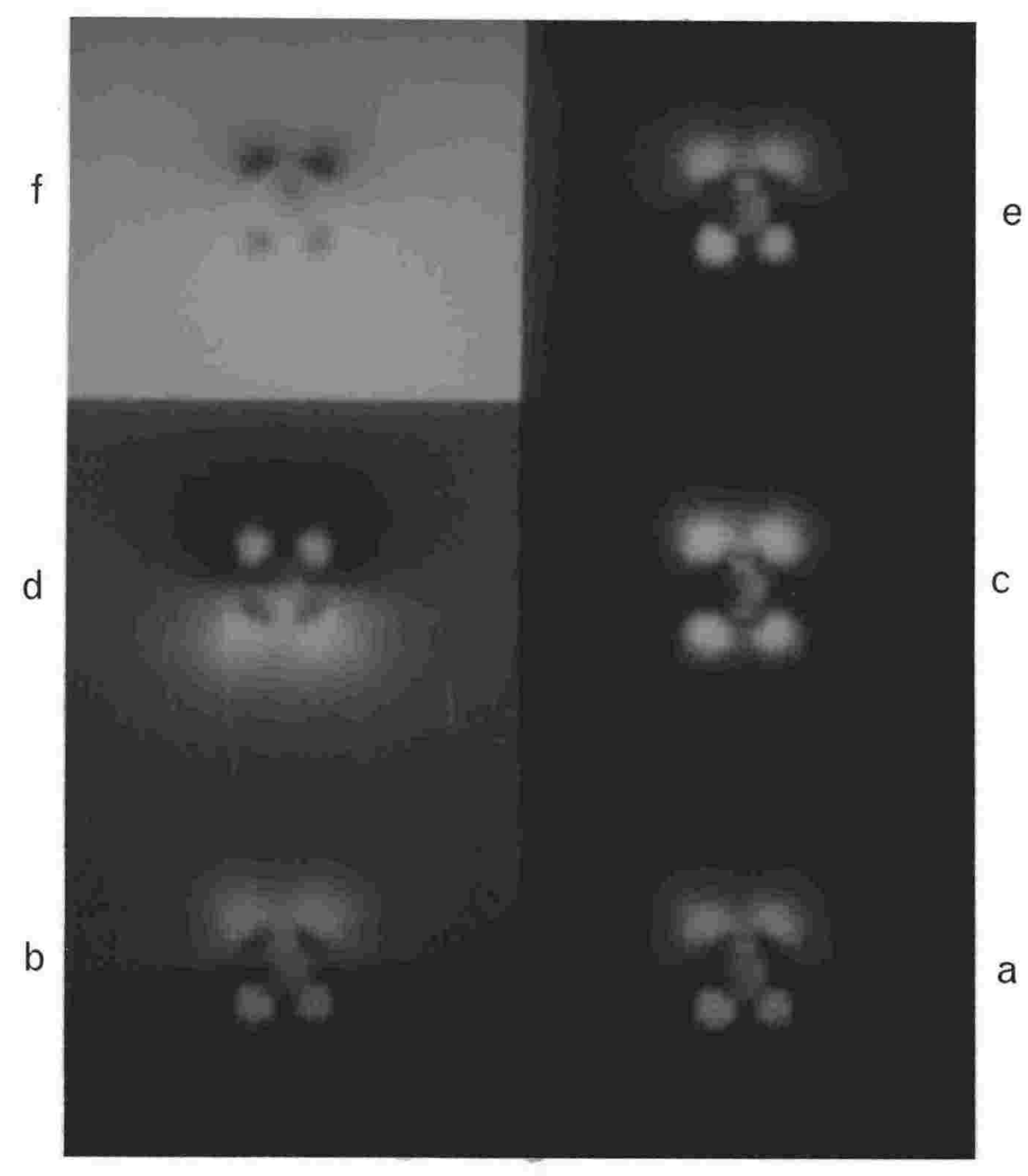

Figure 6.13

$$
\text { 6- } 29
$$




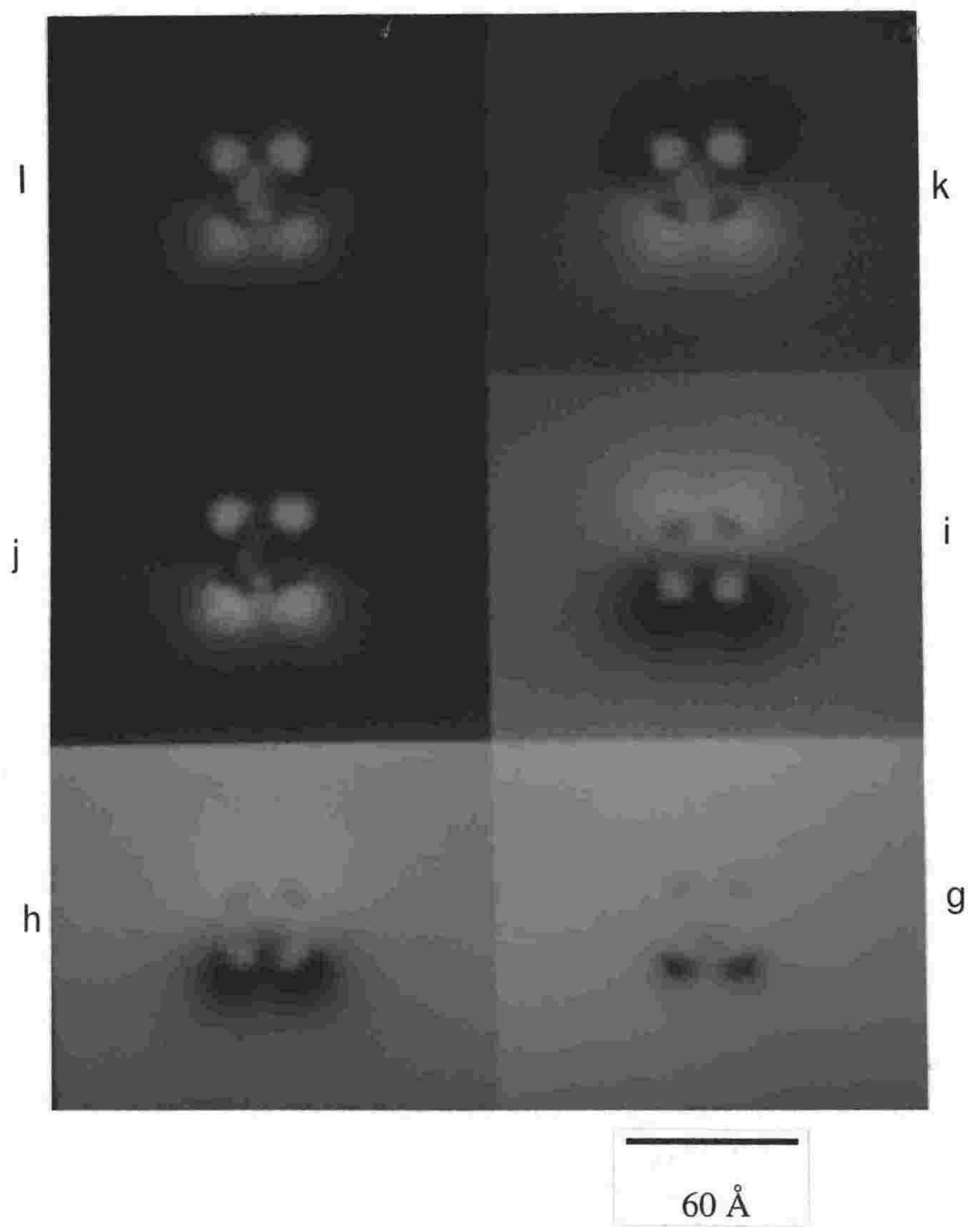

Figure 6.13: Simulations of twelve loops and a bubble as $\mathrm{w}$ is varied. The imaging parameters in DF are: $\mathbf{B}=\mathbf{F}=[011], \mathbf{g}=[200]=$ norm, a foil thickness of $2 \xi_{\mathrm{g}}$, a bubble depth of $1.875 \xi_{\mathrm{g}}$ and an electron energy of $120 \mathrm{keV}$. s $\mathrm{g}$ takes the values of (a) $-0.012 \AA^{-1}$, (b) $-0.010 \AA^{-1}$, (c) $-0.008 \AA^{-1}$, (d) $-0.006 \AA^{-1}$, (e) $-0.004 \AA^{-1}$, (f) $-0.002 \AA^{-1}$, (g) 0.002 $\AA^{-1}$, (h) $0.004 \AA^{-1}$, (i) $0.006 \AA^{-1}$, (j) $0.008 \AA^{-1}$, (k) $0.010 \AA^{-1}$ and (l) $0.012 \AA^{-1}$. As w increases the black and white lobes characteristic of images of the defects taken under strong two-beam conditions begin to disappear until they are imaged only as uniformly dark or bright spots. 


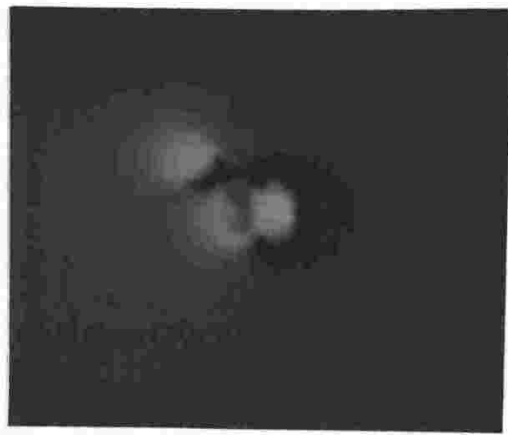

a

$60 \AA$

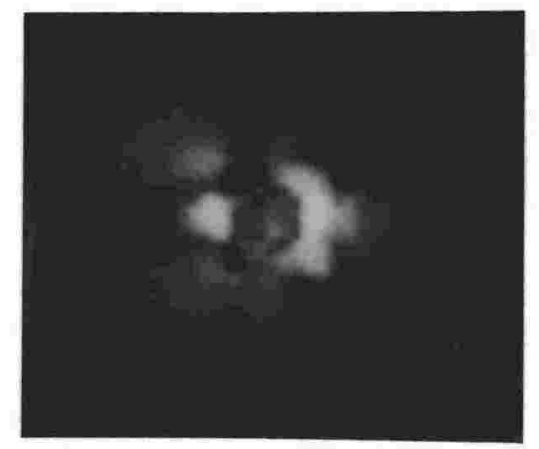

b

Figure 6.14: The figure shows simulations made under weak beam conditions. The imaging parameters in DF are: $\mathbf{B}=\mathbf{F}=[011], \mathbf{g}=[200]=$ norm, a foil thickness of $2 \xi_{\mathrm{g}}$, a bubble depth of $1.875 \xi_{\mathrm{g}}$, an electron energy of $120 \mathrm{keV}$ and $\mathrm{s}_{\mathrm{g}}=-0.0247 \AA^{-1}$. Six beams in a systematic row are included, with the imaging done with $\mathbf{g}=[200]$. Part (a) shows one loop next to a bubble, and part (b) shows six loops next to a bubble. The images simulated at weak beam conditions have a higher resolution than images taken at strong two-beam conditions but are difficult to interpret because of their complicated nature and the similarity of the images with the defocussed simulations taken at strong two-beam conditions that are shown in figure 6.15 . 

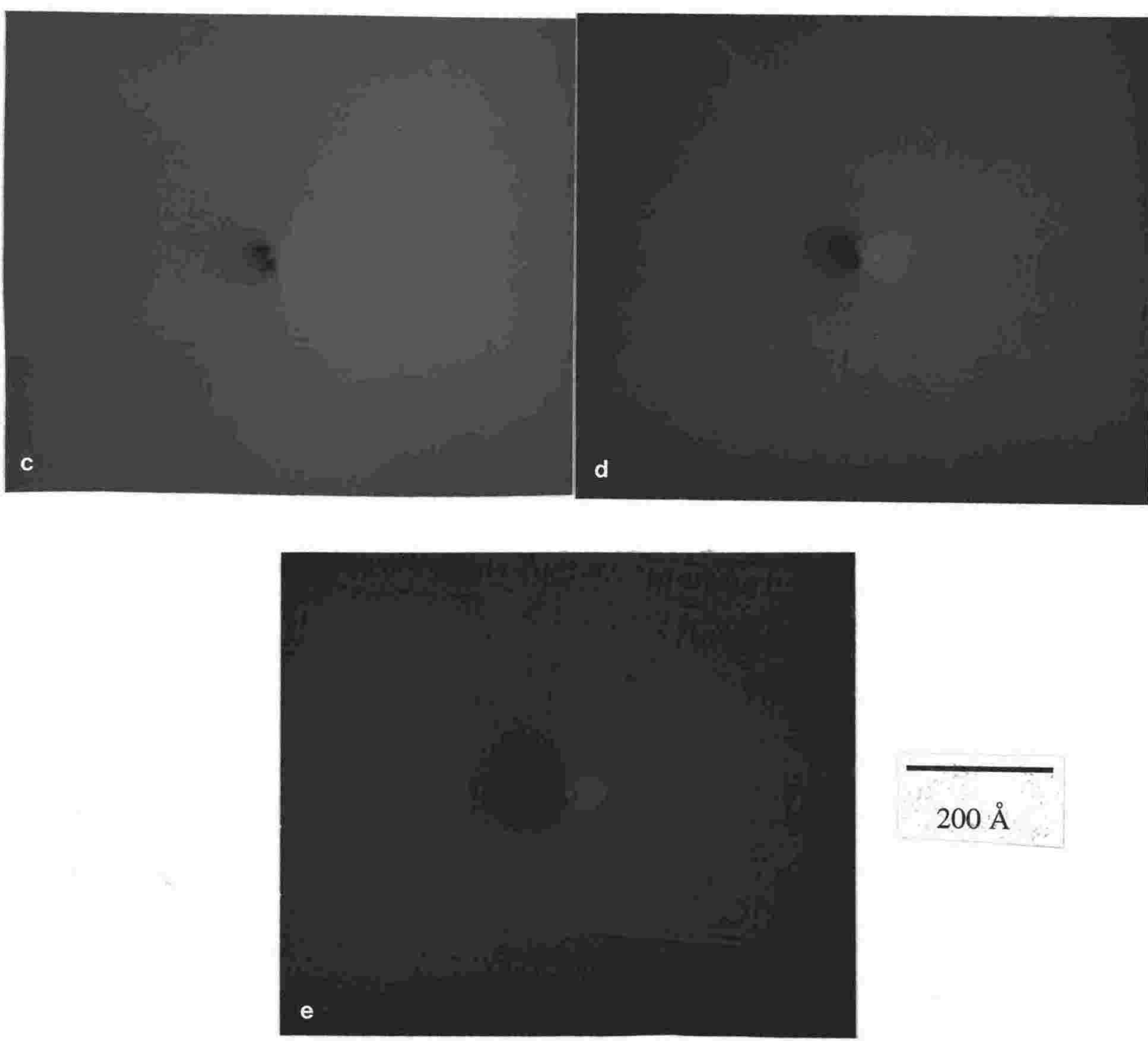

Figure 6.15: The figure shows defocussed simulations of a loop next to a bubble. The imaging parameters in BF are: $\mathbf{B}=\mathbf{F}=[011], \mathbf{g}=[200]=$ norm, a foil thickness of $2 \xi_{g}$, a bubble depth of $1.875 \xi_{\mathrm{g}}$, an electron energy of $120 \mathrm{keV}$ and $\mathrm{w}=0.0$. The defocus values are: (a) $1500 \AA$, (b) $750 \AA$, (c) $0 \AA$, (d) $-750 \AA$, (e) $-1500 \AA$. The pictures are $800 \AA$ square (to avoid errors in the approximate Fourier series method which has been used to defocus the images) instead of the $160 \AA$ square used in the other pictures.

$$
6-32
$$




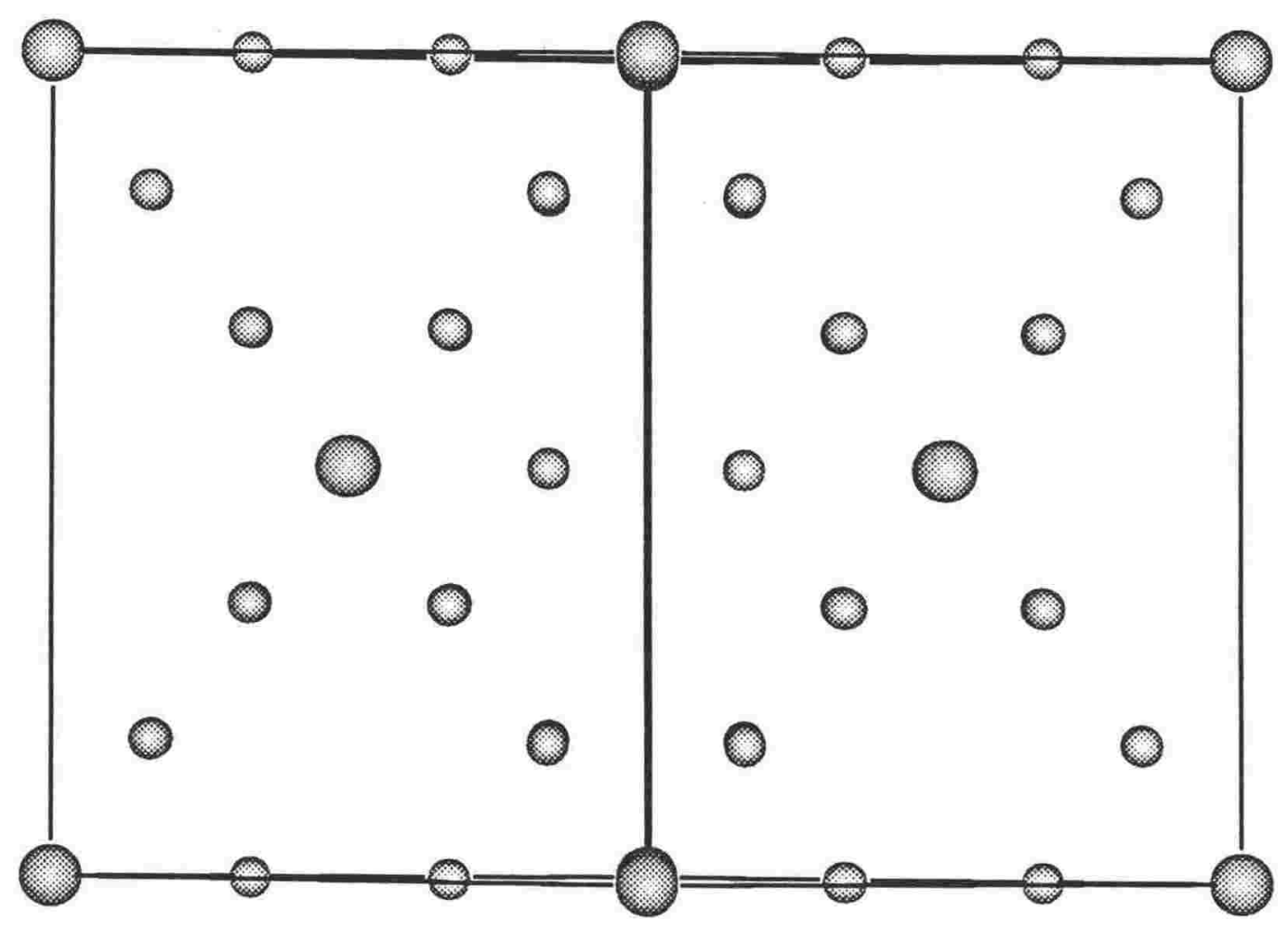

Figure 6.16: View of a system of loops (represented by small spheres) on $\langle 110\rangle$ glide cylinders and bubbles (large spheres), looking down $\mathbf{B}=[110]$. This perspective can be compared to the stereograms and tetrahedra of figure 6.3 . 
of the structure imaged with a higher magnification in figure 6.4 (b) (i). The black/white lobe of the loop extends up towards the top left hand corner of the images as shown in figure 6.4 (a) (i). The simulations in figure 6.15 show that the contrast due to the bubble is still comparable to that due to the dislocation loop. The loop also loses its characteristic black/white lobe appearance when it is out of focus and the black/white lobes are blurred by the Fresnel fringes. However the Fresnel fringes around the bubble are stronger and more symmetrical than the fringes around the loop. The simulation in figure 6.15 probably explains why experimental evidence for the presence or absence of dislocation loops has been slow to appear. In a typical electron micrograph of a superlattice of gas bubbles that is taken under out-of-focus conditions it is hard to separate the contrast due to the loops from that due to the bubbles.

\subsection{Summary: Best Conditions for Imaging Loops and Prospects for Finding Loops.}

In this chapter simulations were performed of the images that would be obtained in a TEM for a set of dislocations near a bubble. Section 6.1 describes the program used to perform the simulations. The results obtained by running the program are presented in section 6.2. The simulations show that the images from a structure containing a set of loops and bubbles is complicated. However, in some circumstances (as will be shown in chapter 7) comparison of the simulations with experimental micrographs can still be used to obtain an estimate of the density of dislocation loops.

To observe dislocation loops it is desirable that as much intensity as possible is associated with them and, furthermore, that their projection on the image does not overlap with that of the bubble. The maximum spacing between loop and bubble is achieved when the beam direction $\mathbf{B}$ is perpendicular to the loop normal $\mathbf{n}$. The diffracting vector is then chosen to give a large value for g.b. Tables 6.1 to 6.3 are useful for this purpose. Consider, for example, a loop with $\mathbf{n}$ parallel to $[0,-1,1]$. A beam direction at right angles to this is $\mathbf{B}=[011]$. Choosing a diffracting vector $\mathbf{g}=[0,2,-2]$ gives an image showing a distinct loop of strong contrast. 
Another consideration is how many loops are projected onto one another for a certain B. Choosing B, such that loops with middle of the range values for $\mathbf{g} \cdot \mathbf{b}$ all project approximately into one image, may give stronger contrast than the image of (mainly) a single loop having a large value of g.b. Ball models and computer-drawn projections of the unit cell of the bubble superlattice and dislocation loops can be used to see how many loops project into one image. Projections were done with the EMS programs of Stadelman [183]. Assumed positions of the dislocation loops and bubbles were used to generate an EMS supercell file. Typical results are shown in figure 6.16. The bubbles and the loops are both represented by spheres, the ratio of the radii being that given by the theory of dislocation loop punching which was outlined in chapter one. A complication is that the position of the image of a dislocation loop depends on the local curvature of the lattice planes and the excitation error w. The ball models are a crude guide only in deciding how many images of dislocation loops will project with a significant degree of overlap.

The simulations of section 6.2.3.5 show that strong black/white lobes characteristic of dislocation loops can occur in in-focus experimental micrographs. Strong two-beam conditions are required as shown in section 6.2.3.4. Thus, provided well-focussed images and strong two-beam conditions are obtained, experimental micrographs can be used to establish the presence of dislocation loops in a superlattice of gas bubbles. However, it should be noted that either poor ordering or the presence of many loops could easily result in the black/white lobes being smeared out in the image to form a continuous dark band that can not be interpreted. 


\section{CHAPTER 7: EXPERIMENTAL EVIDENCE FOR DISLOCATION LOOPS NEAR BUBBLES}

\subsection{Variations in the Structure with Changes in the Dose, the Depth and the Irradiation Conditions}

\subsubsection{The Density of Dislocation Loops}

\subsubsection{Introduction}

This section contains the results of experimental efforts to image dislocation loops in ion-implanted metals. The section starts with a discussion of the experimental problems that occur when imaging defects at high density. After this discussion an alternative approach is introduced, that of preparing ion-implanted metals containing a lower density of defects in which the dislocations can be easily seen. In section 7.1.2 the experimental evidence for the punching of dislocation loops is reviewed in the light of the results presented in this thesis. The theoretical framework is also reviewed and a suggestion made as to what causes the formation of a lower density of defects in specimens implanted to a lower dose. Building on these observations of specimens implanted to a lower dose, a discussion is presented in section 7.1.3 on the implications for specimens containing a superlattice of gas bubbles. The experimental observations in low dose irradiations suggest that diffusion related processes are more important in the growth and ordering of bubbles than the punching of dislocation loops.

\subsubsection{Experimental Problems in imaging a High Density of Defects}

Attempts to find dislocation loops in copper specimens containing superlattices of gas bubbles by tilting to strong two-beam conditions were complicated by the diffuseness of the Kikuchi lines. A related problem is that the diffracted beams are strong for a larger range of specimen tilt than is typical for a pristine crystalline specimen. Figure 7.1 contrasts the diffraction patterns in a copper specimen which is unirradiated and a copper specimen 


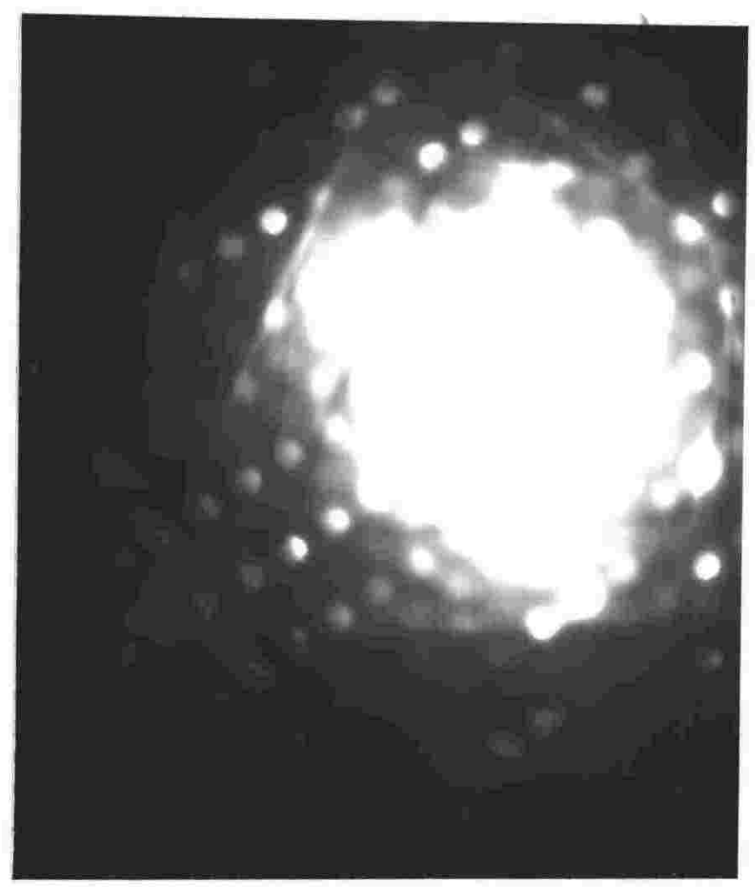

(i)

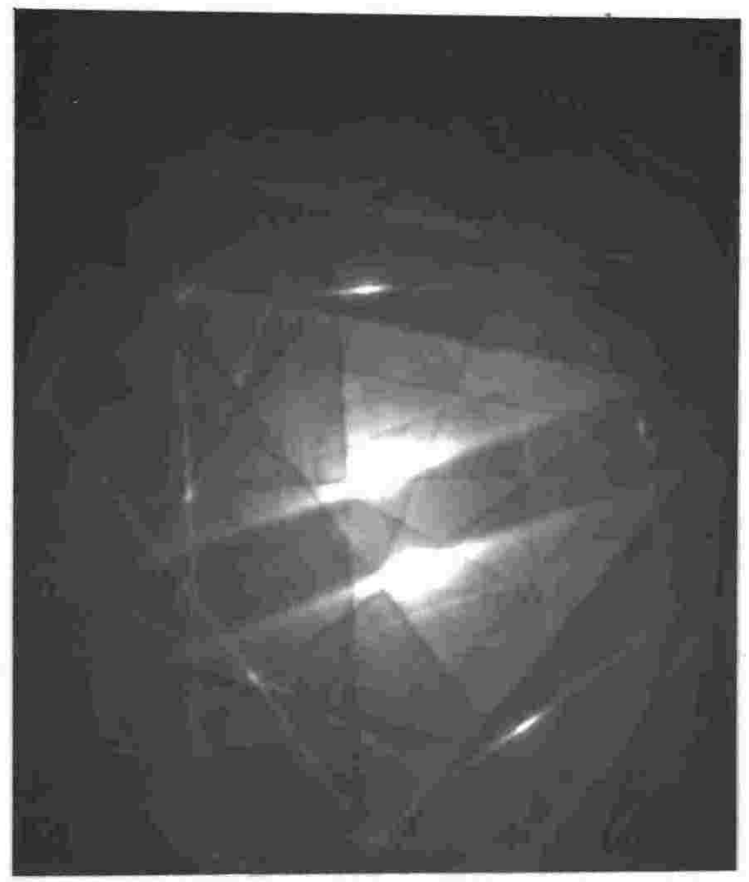

(ii)

Figure 7.1: CBED patterns in (i) irradiated and (ii) pristine copper. The Kikuchi lines in the irradiated specimen are much more diffuse than those in the unirradiated specimen and there are a larger number of reflections excited. This characteristic of irradiated specimens makes controlled tilting of the specimen difficult. 
that contains a superlattice. In the irradiated specimen the Kikuchi lines are much fainter and broader than those in the unirradiated specimen. The irradiated material contains such a high density of bubbles and other defects that the crystallinity of the material has deteriorated significantly.

A superlattice of gas bubbles could be inserted into a crystal by cutting out spheres of material and filling them with gas. In this model the material between the bubbles is still crystalline and undistorted. If the bubbles were overpressured the matrix would be strained. Consider the experimental situation when the TEM is in diffraction mode and the electron beam is converged down to make the Kikuchi lines stand out more strongly than occurs in SAD. The pattern of the Kikuchi lines varies markedly as the specimen is tilted. It becomes difficult to tilt the specimen in a controlled fashion to follow the Kikuchi lines from pole to pole in the crystal and to two-beam conditions. Figure 7.1 has been used in this thesis to support a claim that the crystallinity of the material has deteriorated significantly due to the ion-implantation process. The material between bubbles may locally have the structure of a distorted crystal, but on a larger scale the patches of crystal are rotated relative to one another.

A similar transfer of intensity from the low order diffraction spots into the higher order spots and the loss of intensity in the low order Kikuchi lines has been observed by Wilkens and Rapps [197] in a crystal that had been heavily deformed by electron irradiation. This transfer of intensity into what were weak reflections as a result of the electrons being scattered by the deformation in the metal can be understood using the weak beam theory developed by Cockayne [89 and 90]. Wilkens and Rapps [197] and Humble et al. [198] proposed this observation as a method of measuring high dislocation densities. Wilkens and Rapps used a statistical theory of electron diffraction to consider the scattering off a high density of the same type of defect. This approach has not been followed in this thesis because it was anticipated that different mixtures of bubbles and loops would give the same transfer of intensity into the higher order spots. That is, it would be impossible to uniquely identify the density of bubbles and loops. The Wilkens' theory cannot treat the broadening of the diffraction spots so the existence of superlattice spots is not likely to be useful in determining the population of the bubbles and loops. Even if the diffraction patterns could be used to determine the density of loops and bubbles it would give no 
information as to whether the loops were punched out by neighbouring bubbles along glide cylinders. The diffraction patterns could also not give information about whether the loops were formed by radiation damage independent of any bubble ordering or growth process. Experimental images and simulations of the structure are required to check on the theory of Dubinko et al. [7] of how bubbles order by punching out dislocation loops.

Figure 7.2 illustrates the difficulties in interpreting experimental micrographs of bulk specimens irradiated to the dose at which superlattices form. In the BF and DF pictures black and white lobes characteristic of loops and bubbles are visible with a range of directions for $\mathbf{l}$, not just $\mathbf{l}$ parallel to $\mathbf{g}$. Some loops are present but not at the densities to be expected if every bubble were to punch out approximately twelve loops. The weak beam simulations of figure 6.14 showed that a bubble has a ring around it similar to Fresnel fringes when imaged under these conditions, whereas the loop retains its black/white lobe structure more. However the differences in image characteristics are not strong enough to readily distinguish loops from bubbles in the photos taken at weak beam conditions.

\subsubsection{Experimental Results from Low Dose Irradiations}

The size of the bubbles and their degree of ordering varies with depth. Dislocation tangles occur near the surface, then small bubbles, larger bubbles and then smaller bubbles again as the depth below the surface increases. The scale of the depth dependence is determined by the angle of the irradiation and the mean projected range of the helium ions. The variation in the structure with depth can be used to obtain larger bubbles without a dislocation tangle by ion beam thinning the front and the back of a specimen after jet electropolishing has been used to perforate it.

By using lower doses, in irradiations of copper discs that have been prethinned, gas bubbles that have more material between them are attainable. The bubbles in the specimen are not ordered. To attain these specimens, doses are used that are equal to about a tenth of the dose used to form blisters on a specimen. The specimens have Kikuchi lines that are more diffuse than the unirradiated specimens but the lines are still sharp enough to readily

tilt the specimen between axes and to two-beam conditions. If the dose is lowered below 


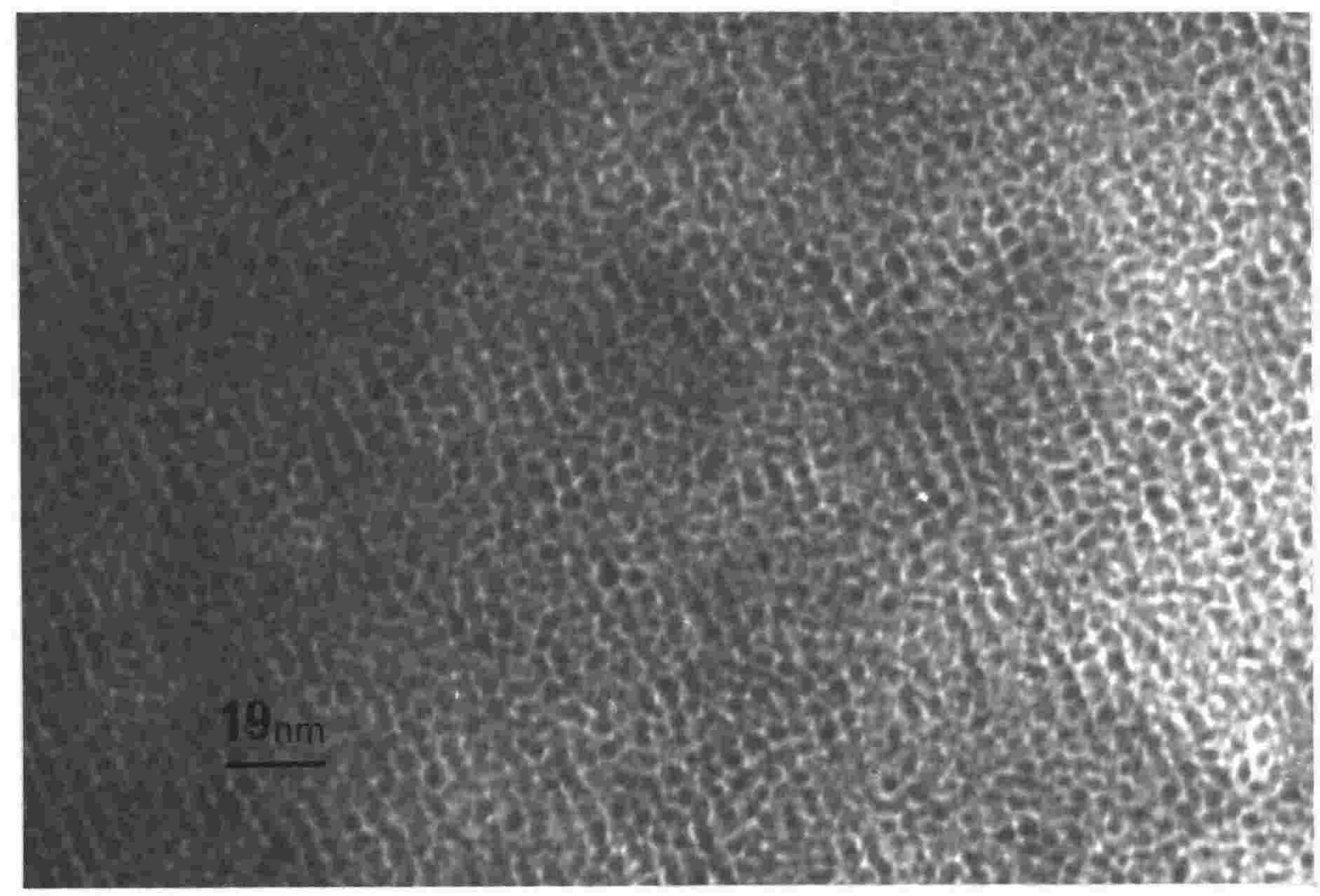

(i)

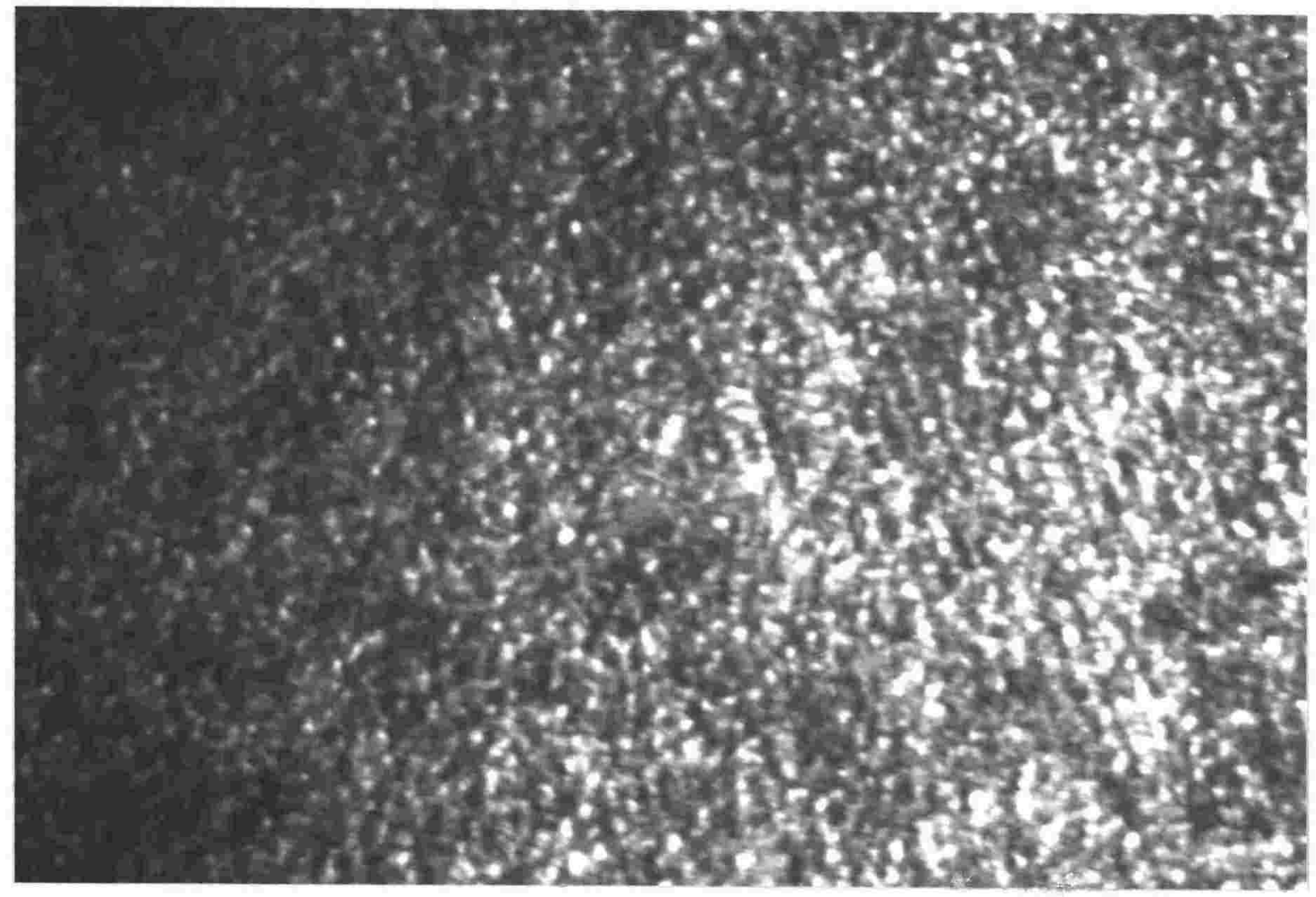

(ii)

Figure 7.2: (i) BF (overfocussed) and (ii) weak beam images of the same region of superlattice, imaged under the same diffraction conditions. The high density of bubbles makes it difficult to distinguish any dislocation loops that might be present. 
about a sixteenth of that required for blistering, dislocation tangles but no bubbles are obtained.

The technique required to obtain a low density of bubbles in a material with reasonable crystallinity is to first irradiate a $3 \mathrm{~mm}$ diameter copper disc that has been prethinned to perforation. During irradiation the effect of the hole in the specimen is that in the thin regions which are electron transparent most of the helium ions do not come to rest either so no bubbles will occur there. Also the hole allows the relief of the lateral stress found in specimens irradiated in the orthodox manner. The hole also provides a sink for any mobile interstitials or helium ions close to the peak in the damage profile. Normally in the irradiation of bulk specimens the peak in the damage profile occurs about a micron below the foil surface. (The position of this peak can be worked out by simulations which use Monto-Carlo methods [45]).

Ion beam thinning of the specimen from the back is the second step. Figure 7.3 shows the satisfactory structures that may be attained after two hours of thinning with a 30 $\mu \mathrm{A}$ current of $4 \mathrm{keV}$ Ar ions. This amount of ion beam thinning is roughly equivalent to the removal of a thickness of material equal to $11 \mu \mathrm{m}$ from the back of the specimen. There are several assumptions in calculating the amount of material removed by the ion beam thinning process. The main assumption are (i) the ion current (which is integrated to give the number of ions) strikes only the $3 \mathrm{~mm}$ disc and not the specimen holder, (ii) secondary emission from the disc can be neglected and (iii) the ion current is uniformly distributed over the specimen. The $11 \mu \mathrm{m}$ figure could easily be out by a factor of five. Assuming a wedge angle of $30^{\circ}$ for the prethinned foil then the hole radius is increased by about $9 \mu \mathrm{m}$ by the ion beam thinning.

In figure 7.3 the black/white lobes that have $\mathbf{l}$ parallel to $\mathbf{g}$ (e.g. the defects in the square boxes in figure 7.3 (b) (ii)) as expected have a central region of near background intensity. The existence of these lobes with a surrounding strain field means the defects contain gas that is overpressured and therefore the defects are bubbles and are not voids. Some of the lobes have $\mathbf{l}$ rotated away from $\mathbf{g}$ (e.g. the defects in the circles in figure 7.3 (b) (ii)), and nor are they separated by a region which has an intensity near that of the background. These lobes are dislocation loops of similar size to the gas bubbles. There are both black and white bubbles in the in-focus micrograph. This confirms the plots of 


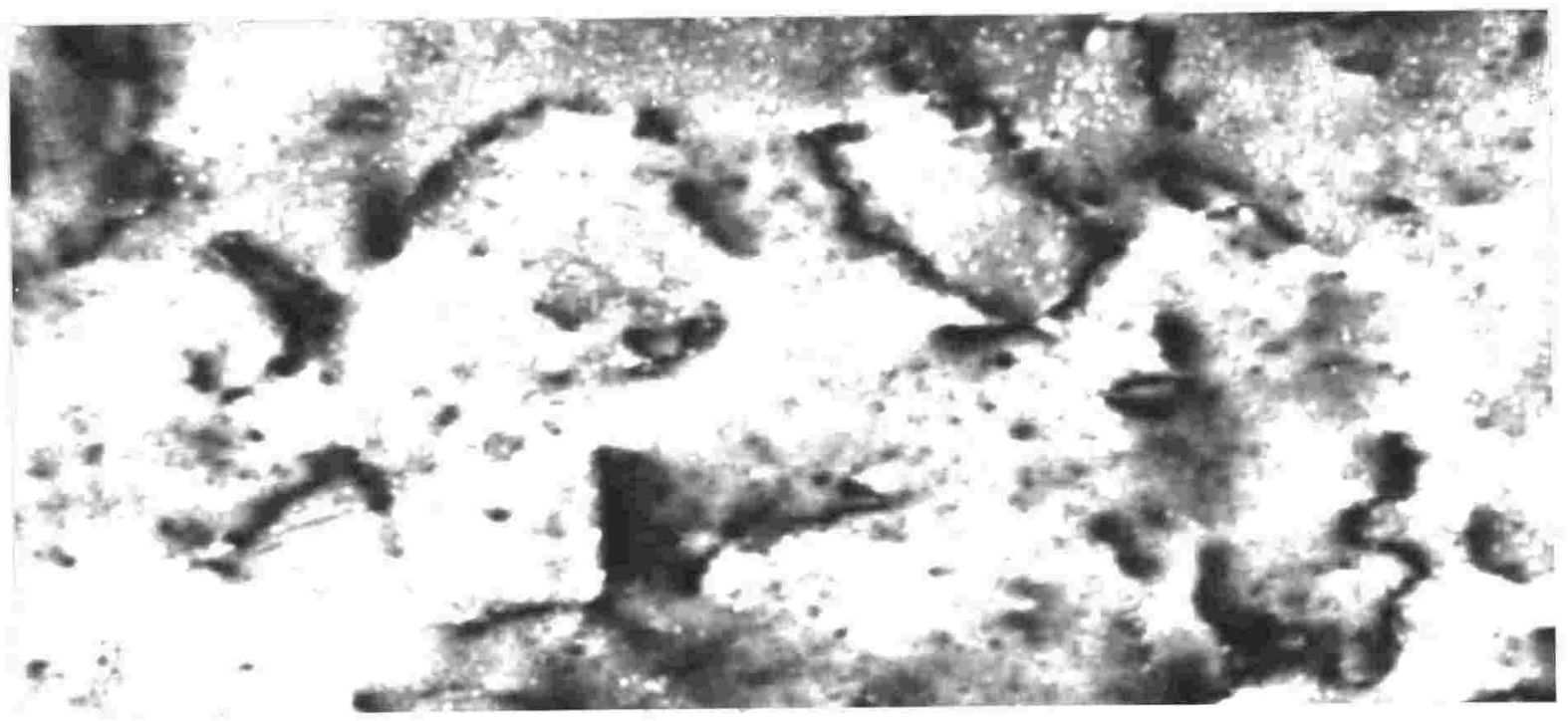

(i)
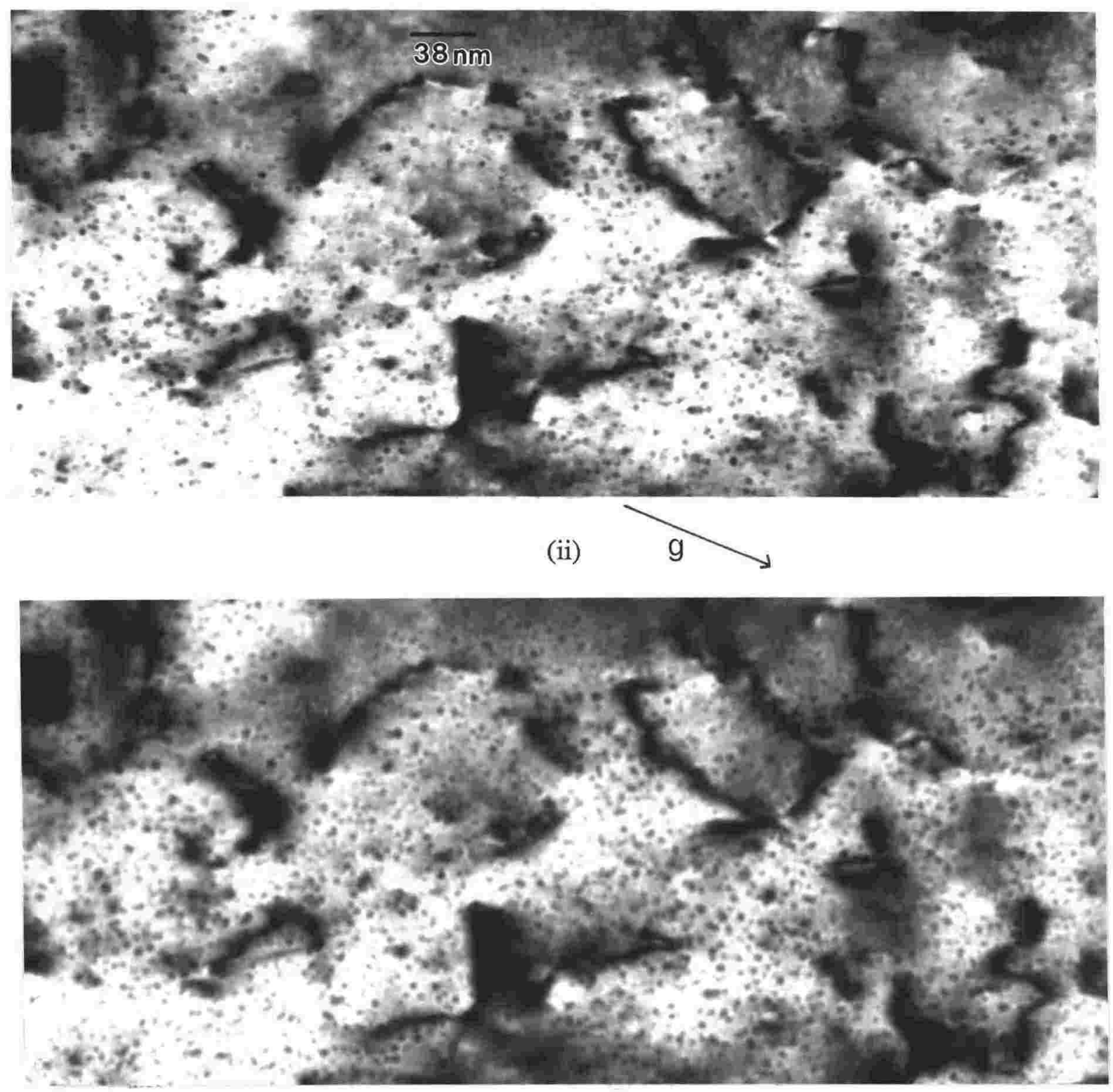

Figure 7.3 (a), (iii) 


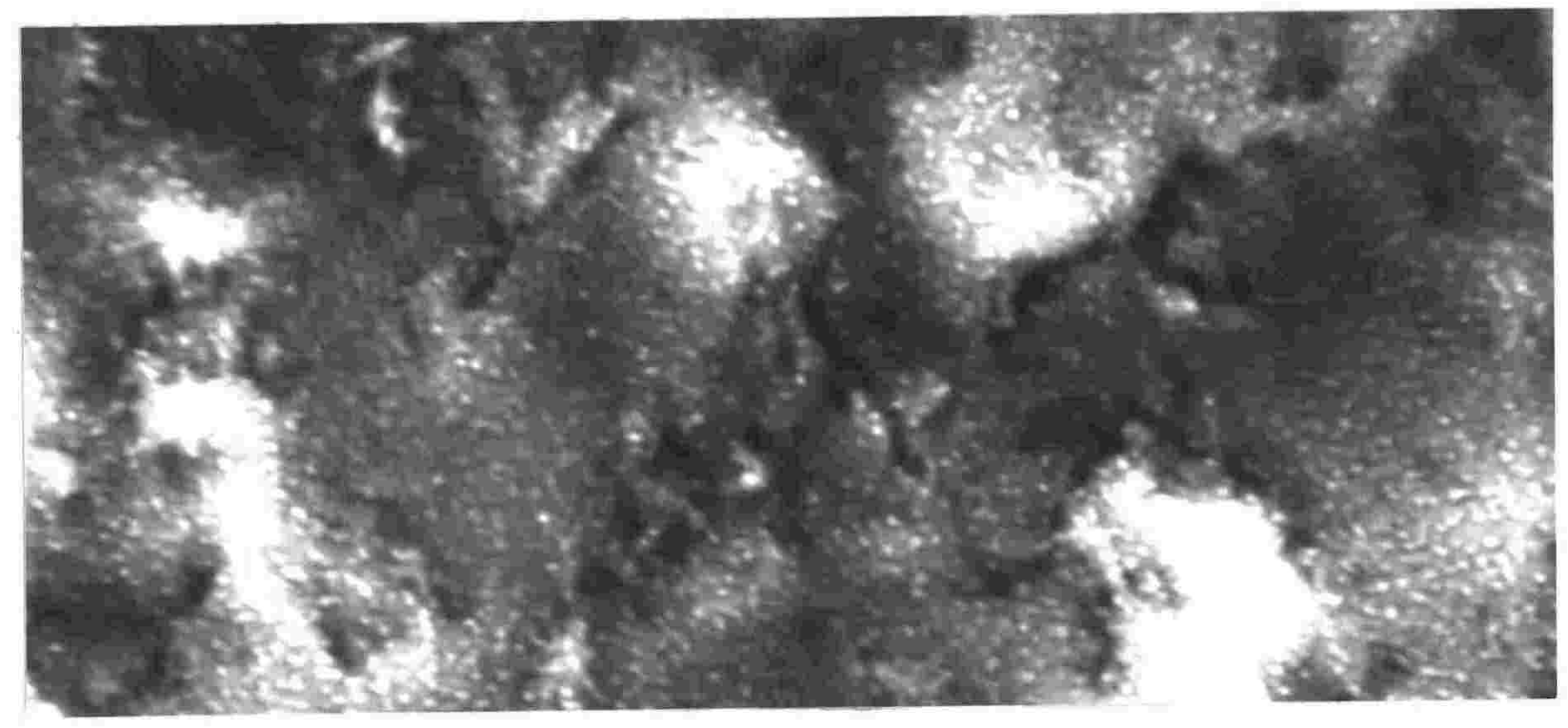

(i)

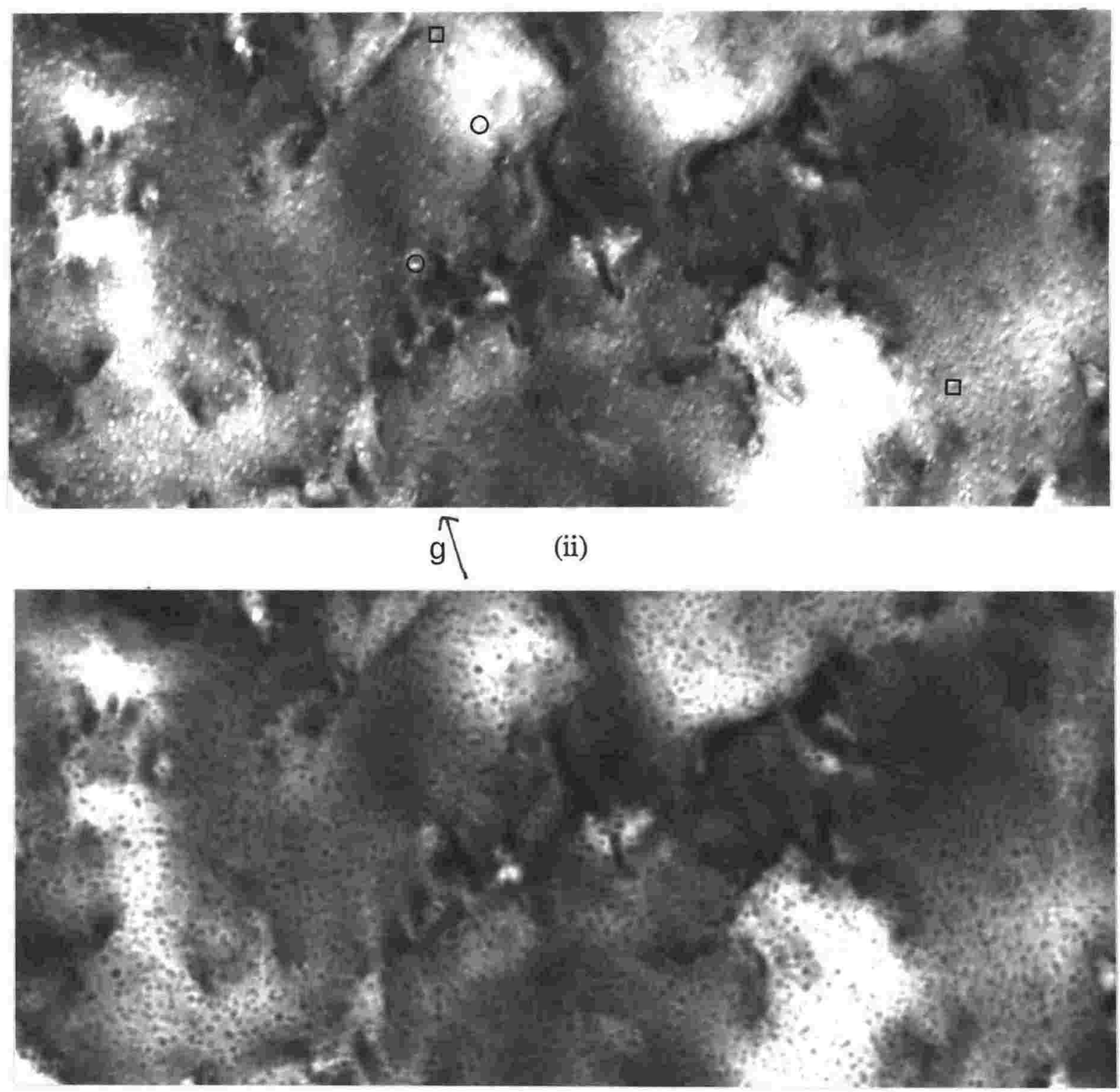

Figure 7.3 (b), (iii) 


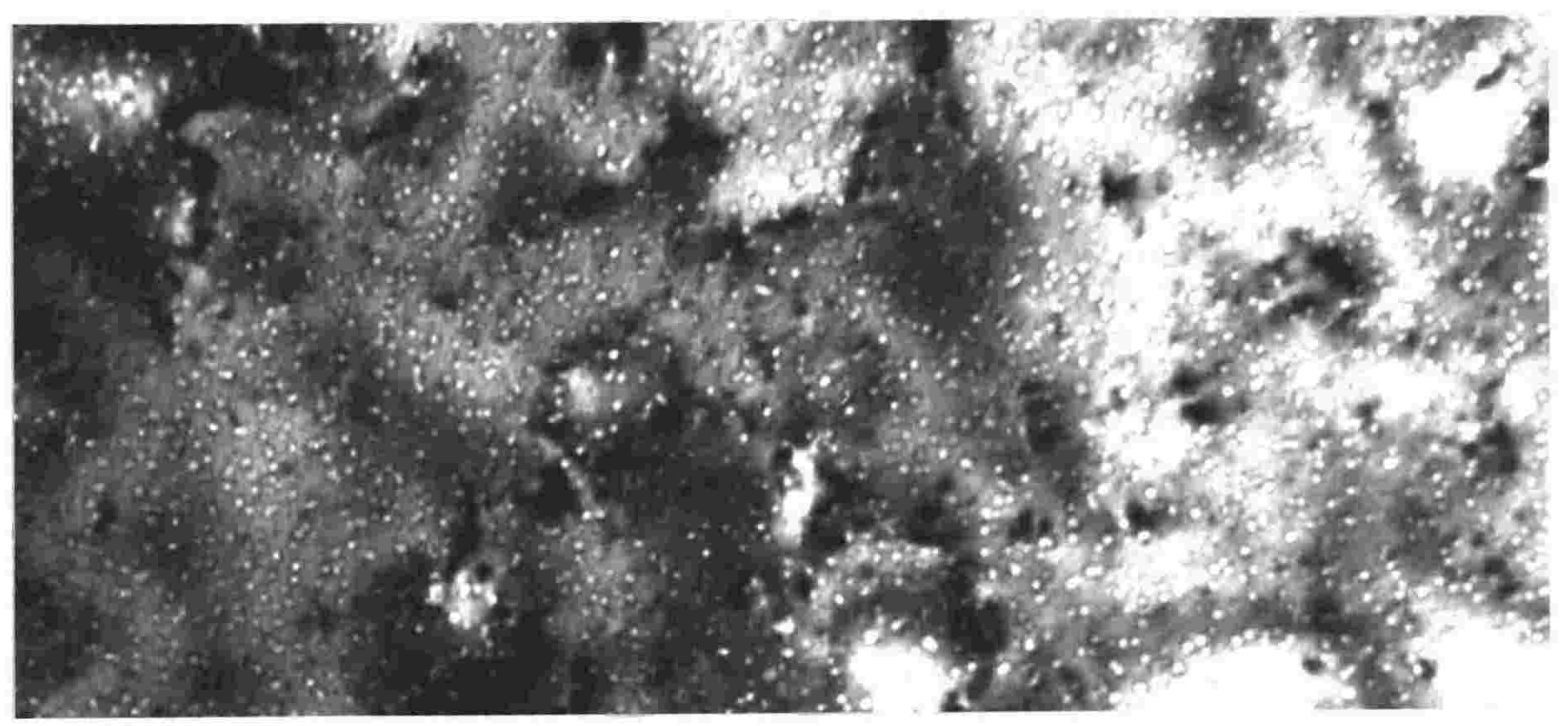

(i)
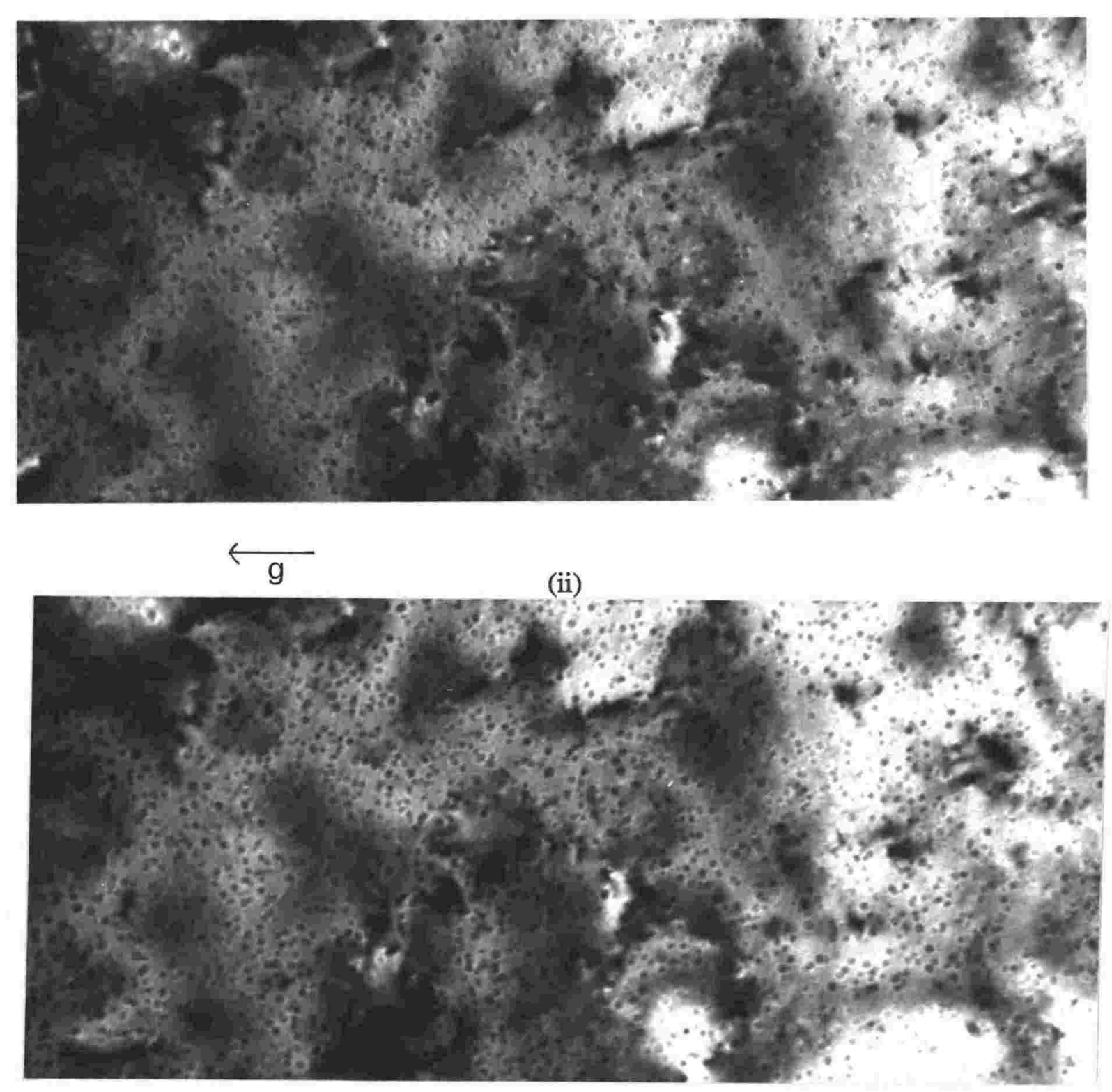

Figure 7.3 (c), (iii) 


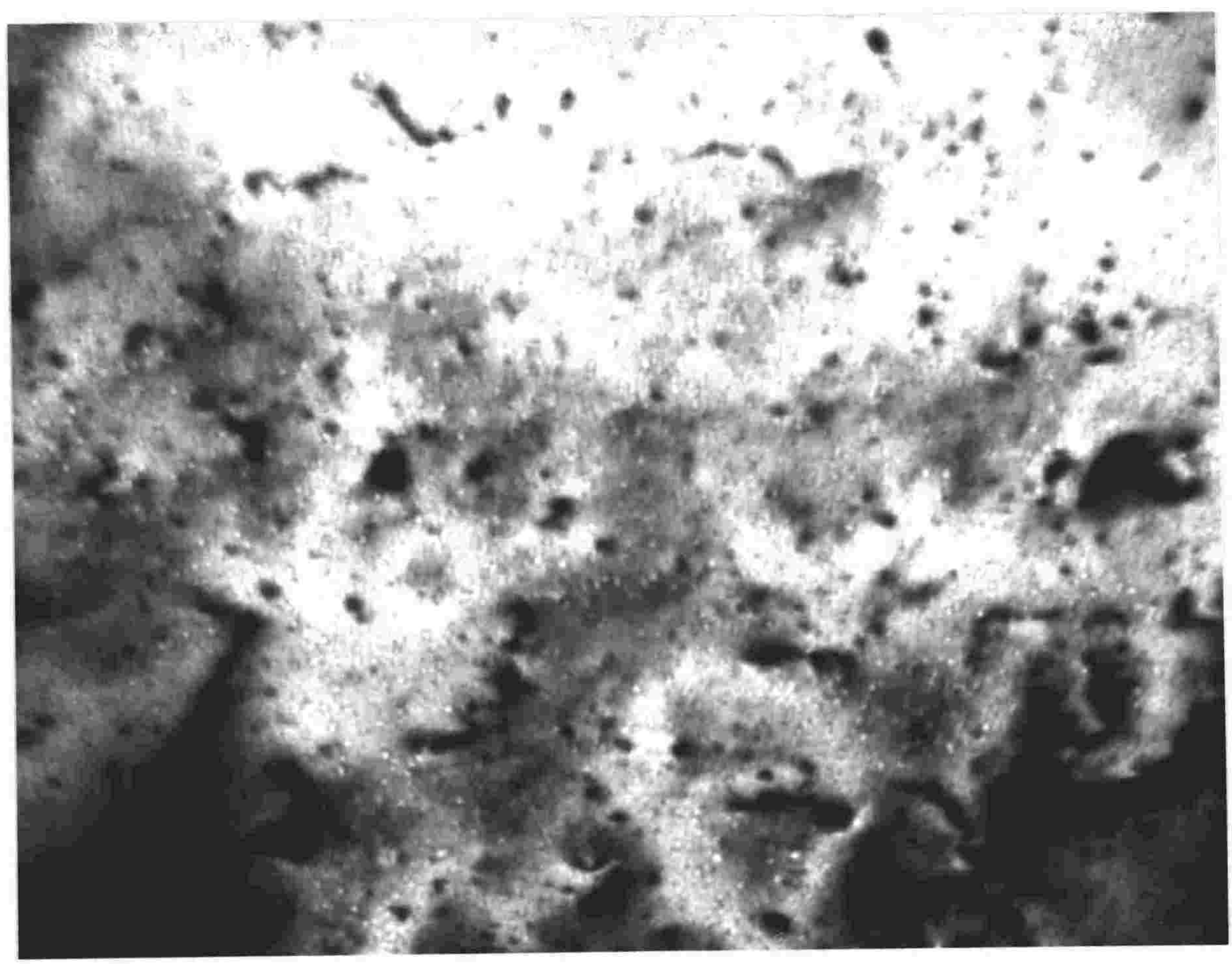

(i)

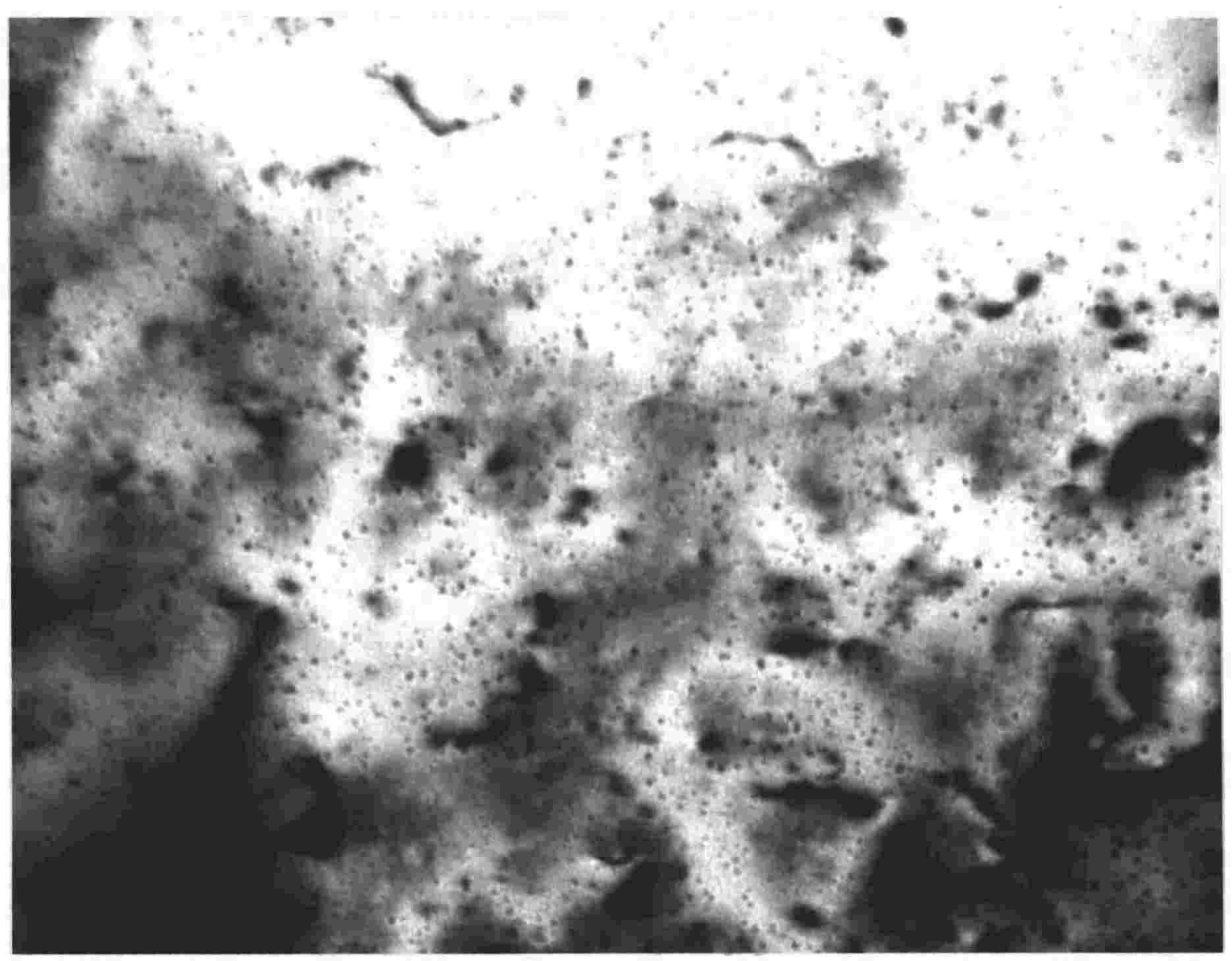

Figure $7.3 \mathrm{~d}$ (ii) 


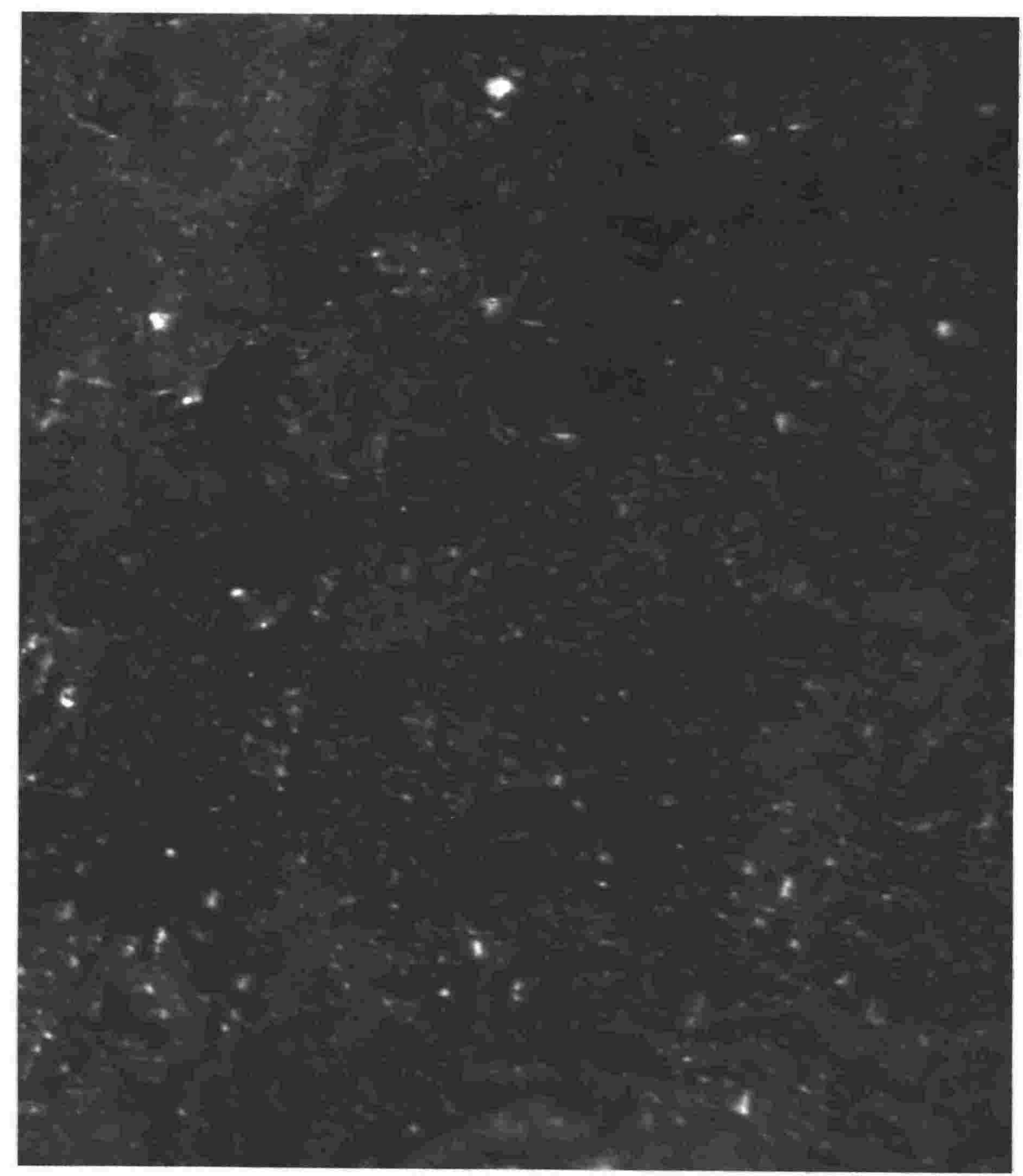

(d), (iii)

Figure 7.3: Three sets of through-focal series of a low density of bubbles obtained with two-beam conditions in a low dose irradiation of a prethinned copper disc. The beam directions and diffracting vectors used are: (a) $\mathbf{B}=$ [101], two-beam conditions, $\mathbf{g}=$ [111], (i) underfocus, (ii) in-focus and (iii) overfocus; (b) $\mathbf{B}=$ [112], two-beam conditions, $\mathbf{g}=$ [220], (i) underfocus, (ii) in-focus and (iii) overfocus; (c) B = [001], two-beam conditions, $\mathbf{g}=$ [200], (i) underfocus, (ii) in-focus and (iii) overfocus and (d) $\mathbf{B}=[101], \mathbf{g}=$ [111], (i) $\mathrm{BF}$, underfocus, (ii) BF, overfocus and (iii) weak-beam condition. Defects with I parallel to $\mathbf{g}$ may be either loops or bubbles, whereas defects with $\mathbf{I}$ rotated away from $\mathbf{g}$ are dislocation loops. The low observed density of dislocation loops supports a model for bubble growth based on diffusion related processes. 
contrast at the centre of the bubble versus depth in chapter five. Also there are more bubbles visible with strong contrast in underfocus conditions than there are in the overfocus or in the in-focus micrographs. This confirms other plots of chapter five. It also means estimates of bubble volume fractions and bubble densities made from overfocus micrographs are likely to be significant underestimates of the true bubble volume fraction. From chapter six, any loops that lie approximately outside or between the first three layers of either surface of the foil will appear as black dots. This contrast occurs instead of the usual black/white lobes. Thus just counting black/white lobes that are not separated by a region of near background intensity can cause an underestimation to be made of the dislocation loop density.

\subsubsection{The Theoretical Density of Dislocation Loops in the Low Dose Irradiations}

An estimate of the number of loops punched by a bubble is obtained by equating the final volume of the bubble to the volume occupied by the interstitial planes that form the loops (that is equal to the area of the loop times the magnitude of the Burgers vector). In a crude estimate the radius of all the loops is set equal to the final radius of the bubble divided by the square root of two (as required by the Greenwood, Foreman and Rimmer mechanism discussed in chapter 1). Using this crude estimate a helium bubble in copper that has a final radius of $10 \AA$ will have punched out sixteen dislocation loops with a [110] type Burgers vector. Similarly a helium bubble in copper that has a final radius of $20 \AA$ will have punched out thirty two dislocation loops. In practice a larger number of loops will be punched out by the bubble, but many of them will have smaller radii than the last loop punched out. An added complication in estimating the density of dislocation loops is that the nucleation centre must grow by diffusion related processes to be larger than just a few atom sites before it becomes valid to describe the further growth as being due to the punching of dislocation loops. Hence not all of the final volume of the bubble should be equated to the volume of the dislocation loops. In the simulations in chapter six it was estimated that twelve dislocation loops with a radius equal to $7.07 \AA$ are punched out by a gas bubble with a radius of $10 \AA$. This number of loops was used instead of sixteen 
because of the considerations above and as it corresponds to the symmetrical situation of one on every glide cylinder. This symmetry allows the simulations to be applied to a large number of geometries with the minimum amount of time-consuming computation.

The bubbles in figure 7.3 , having a diameter of approximately $40 \AA$, are bigger than those typically observed in superlattices of helium gas bubbles in copper. Therefore the bubbles in figure 7.3 are slightly more likely to punch out loops if they have the same pressure as the bubbles in the superlattice case (i.e. the bubbles have a higher overpressure because the equilibrium pressure is lower). However, the density of loops in the figure is lower than the approximately fifty or more loops expected to be punched out by a bubble with a diameter of $40 \AA$. The figure of fifty loops to be punched by a bubble with a radius of $20 \AA$ corresponds to about four loops on every glide cylinder. Any loops that are visible cannot be readily associated with the glide cylinders of nearby bubbles.

It is possible that punched out loops have glided to the foil surface where the glide cylinders are not blocked by another bubble. On a fine scale, the effects on the material close to a segment of a dislocation loop are identical to those of the same length of a long straight dislocation. Therefore a differentiation of equation 1.20 followed by a division by the circumference of the loop can be used to calculate the force per unit length on the loop which tries to expel it from the foil. It can be shown, using the result of the differentiation, that the force per unit length on a small loop is larger than that on a large loop. In addition the larger dislocation loops are more likely than smaller loops to have their paths to the surface obstructed by another defect. For these reasons it is less likely that a high density of larger dislocation loops could be expected to disappear. The gas bubbles shown in the figure probably grew by diffusion related processes during the irradiation. However the possibility that the dislocation loops may have been lost from the prethinned discs can not be ignored.

While dislocation punching won't occur until the nucleation centre has grown by diffusion related processes, there is also a point at which loop punching is taken over by displacement events. Predictions have been made about this by Evans [125]. Evans described a theory for the mechanism by which blisters form in metals that have been irradiated with helium. This mechanism models the growth of a bubble as being initially due to the punching of dislocation loops then being by displacement events and includes the 
effects of varying the dose. A Gaussian profile for the range at which the helium comes to rest is assumed. This appears reasonable from Monto-Carlo simulations. A more significant assumption is that the density of sites on which bubbles nucleate is uniform throughout the foil. The number of loops punched out by a bubble with a diameter of $40 \AA$ therefore may be a lot less than fifty if the bubble stops growing by punching out dislocation loops after it grows to a diameter of $10 \AA$ for example. Practical computation of this bubble radius requires the mean range and standard deviation of the distribution of the implanted helium to be estimated from Monto-Carlo simulations. An estimate of the bubble density to compare the theory of Evans [125] against experimental micrographs would require a knowledge of the thickness of the foil. Any small inaccuracies in this thickness result in a large uncertainty in the calculation of the radius of the bubble at which the punching of loops stops. This occurs because the equations of Evans for the bubble pressure depend exponentially on the density of the bubbles. Accurate estimates of the number of loops to be expected to be punched in specimens of the dose in figure 7.3 are then impractical.

\subsubsection{Experimental Evidence for the Punching of Dislocation Loops by Small Bubbles}

In the following sections the experimental evidence, for the punching of dislocation loops by bubbles of the size of those found in superlattices is reviewed in the light of the results presented in this thesis.

\subsubsection{Quenched Metals}

Small loops of radius 10 to $20 \AA$ are not found in quenched metals [2-6]. However, these papers are invoked by many authors as providing experimental evidence for the relevance of the punching of dislocation loops to the growth of small bubbles. It is interesting that in the quenched copper in chapter four, rows of loops from about $300 \AA$ in diameter and upwards were observed. Smaller loops were not found which suggests, that, in that case, a bubble grows by diffusion related processes until its diameter is about $300 \AA$. 
The bubble pressure and radius are then enough to satisfy the GFR inequality of chapter one and loops begin to be punched.

Many displacements per atom are predicted to be caused by the incoming helium ions in the Evan's theory for blistering [125]. In quenched metals the gas is diffused in thermally and so the gas does not possess anything like the energy of helium ions in an irradiation. In quenched metals, therefore, the growth mechanism by displacement damage cannot be used to stop the growth by loop punching. Continued punching of loops in quenched metals will then cause long rows of loops to be formed like those in chapter four that will not be found in irradiated metals. In quenched copper diffusion processes seem responsible for early bubble growth with dislocation punching becoming important for bubble sizes near $300 \AA$.

For quenched metals the comparison of loop spacings found from experiment with the theory of Bullough and Newman [112] gives a value of the critical shear stress that is much lower than that for irradiated metals [99]. This suggests that for bubble growth in irradiated metals diffusion related processes will be even more favoured than the punching of dislocations.

\subsubsection{Tritium Charging}

Metal foils charged with tritium by thermal diffusion and then aged contain bubbles of helium -3 . The ${ }^{3} \mathrm{He}$ produced by the $\beta^{-}$decay of the tritium, has a recoil energy in the decay which is insufficient to cause displacement damage. Bubble growth at least in part is by dislocation punching; the loops are about $30 \AA$ or bigger, much closer in size to the $10 \AA$ to $20 \AA$ expected in specimens containing superlattices. However because there is no displacement damage these observations provide little insight into the behaviour expected in irradiated metals.

\subsubsection{Low Energy Implantations}

Other papers most commonly cited as providing experimental evidence of the relevance of loop punching are those of Evans et al. [200 and 201]. The Evans paper 
describes how $3 \mathrm{keV}$ helium ions were used to create helium-vacancy complexes in the near surface region of a molybdenum foil. (This sort of treatment could be done in an ion beam thinner that accelerates helium ions instead of argon ions. It is impossible with the Van de Graaff accelerator at Victoria University which has an accelerating voltage that is unstable below about $100 \mathrm{kV}$.) Thermal helium desorption spectroscopy has shown that the implanted helium ions have an energetic preference to come to rest in a vacancy or hole in the host matrix. These helium-vacancy complexes $(\mathrm{HeV})$ can act as nucleation sites for bubbles.

A $150 \mathrm{eV}$ helium irradiation of the surface of the specimen after it has been "seeded" with $\mathrm{HeV}$ sites by the $3 \mathrm{keV}$ irradiation will result in most of this low energy helium being lost to the surface. Yet some helium will nucleate bubbles or platelets at the $\mathrm{HeV}$ complexes by diffusing into the bulk of the material. The bubbles were observed by Evans to grow by trapping the helium and punching out dislocation loops.

However this situation in which the dislocation loops are formed is different from the irradiation conditions that cause the formation of superlattices of bubbles. The major difference is that because of the low energy of the helium irradiation there is a much lower displacement damage in the region where the bubbles form. In the Evans et al. experiment the displacement damage does not terminate the process of punching loops, nor does it control the bubble growth.

\subsubsection{Low Dose Irradiations of a Prethinned Copper Disc}

The discussion in section 7.1.2.3 is however relevant to the mechanism by which the bubbles in figure 7.3 grew in the irradiation of the prethinned copper disc. In this specimen the nearby foil surfaces and hole are a sink for the interstitials created in the early stages of the irradiation and for the helium atoms. This is because the concentration of interstitials and helium atoms is zero at the foil surfaces, so that these defects will diffuse to the surfaces down the concentration gradient. In contrast the vacancies are relatively immobile at room temperature. The density of $\mathrm{HeV}$ complexes, which act as nucleation sites for bubbles, will then be lower than in an irradiation of a bulk specimen because of the 
loss of helium to the surfaces. Vacancy clusters can collapse to form vacancy loops but the nucleation sites cannot do this because they contain helium.

As the irradiation continues the nucleation sites begin to compete with the surfaces for many of the incoming helium atoms because these sites are closer to where the damage is created and the helium comes to rest than the surfaces. The same number of vacancies will be created by the irradiation of the prethinned specimen as are created in the same thickness of a bulk specimen in an implantation at the same energy and dose etc. However, there are fewer nucleation sites available, so that per bubble, there will be more vacancies and helium atoms to grow with than normal. The result is the formation of a lower density of larger bubbles.

The diffusion coefficients of the defects obey

$$
\mathrm{D}=\mathrm{D}_{0} \exp \left(-\frac{\mathrm{E}_{\mathrm{m}}}{\mathrm{kT}}\right)
$$

where $D_{0}$ is a constant that is different for interstitials, vacancies, helium vacancy complexes and helium atoms. $\mathrm{E}_{\mathrm{m}}$ is the activation energy for a defect to jump from site to site in the lattice. Self interstitial atoms (copper atoms that are usually referred to as SIAs in the literature) have jump frequencies that are of the order of hundreds of $\mathrm{GHz}$. The jump frequencies of vacancies are of the order of $\mathrm{Hz}$ at room temperature in copper. $\mathrm{HeV}$ complexes are more mobile than the vacancies but less mobile than the interstitials. Interstitial helium atoms, like interstitial copper atoms, are also more mobile than the vacancies but less mobile than the SIAs (Glyde and Mayne [203]).

For comparison the ideal gas laws can be used to calculate the average kinetic energy and hence the rms velocity of a helium gas atom at room temperature. This turns out to be $1300 \mathrm{~m} / \mathrm{s}$, so a helium atom will be hitting the walls of a bubble with a frequency of the order of hundreds of $\mathrm{GHz}$ to maintain the bubble pressure and keep the SIAs out. If the bubble pressure is high enough the bubbles can emit SIAs instead of just keeping out the SIAs. However, the pressure required to do this is even larger than the pressure required for the punching of dislocation loops.

An SIA will collide with a bubble an order of magnitude less frequently than the jump frequency between lattice sites because the superlattice constant is approximately 
twenty times larger than the lattice constant. In figure 7.3 the density of bubbles is probably too low for the mechanism by which the SIA flux can order the bubbles to operate because the bubbles do not lie in the shadows of the other bubbles (section 1.4). The vacancies are not very mobile at room temperature but, because of the exponential dependence of the diffusion coefficient, are very mobile at $700{ }^{\circ} \mathrm{C}$, for example. The vacancies could however be stimulated by the radiation to migrate to a bubble. In the early stages of bubble development, bubble growth probably occurs by the coalescence of the $\mathrm{HeV}$ complexes not lost to the surfaces.

The parameters of a bubble superlattice are insensitive to the irradiation temperature over a large range of temperature despite the significant effect of temperature on vacancy mobility. This has often been invoked as a reason for a pressure driven model for bubble formation [45]. However, in the prethinned case the vacancy mobility is significant because the proximity of the foil surfaces to the nucleation sites for the bubbles influences the density of interstitials and helium available for the bubbles. That is, the density of the interstitials and helium is reduced significantly, but the density of the vacancies is not.

Consider the bubble pressure in the structure in figure 7.3 in comparison to the bubbles in an orthodox irradiation. Because there is a lower density of bubbles and the surface is competing for helium, the ratio of helium to vacancies in a bubble will be lower than normal if the vacancies have enough radiation-stimulated mobility to reach the bubbles. However for many of the vacancies the bubbles are more distant on average than in an orthodox irradiation. If all the vacancies created in the irradiation can reach bubbles then the bubbles in the prethinned specimen will have a lower pressure than those created in a bulk irradiation. The bubbles will then be less likely to punch out loops when they are at the same radius as the bubbles in an orthodox irradiation. In the prethinned irradiation vacancies that are within a certain volume of a bubble, as in an orthodox irradiation, will still reach the bubble. The final bubble is larger so therefore more vacancies are required to form the bubble and on average these vacancies will have to travel further. If the vacancy mobility is not sufficiently radiation-stimulated to allow the vacancies to travel the extra distance to a bubble then the bubbles will have a higher pressure than those created in a bulk irradiation and are more likely to punch out loops. Of the two alternatives considered in which the vacancies can or cannot reach the growing bubbles it is more likely that the 
vacancy mobility is not radiation-enhanced by the factor of $10^{9}$ required to bring it up to the same level as that of the interstitials. Instead the vacancies probably recombine with the very mobile SIAs before getting near a bubble. The bubbles are therefore more likely to have a higher pressure than normal.

It is possible that the bubbles found in low dose irradiations of a prethinned copper disc after ion beam thinning are not helium bubbles but are argon bubbles. To check this out it would be possible to do experiments based on EELS or thermal desorption spectroscopy. Even if the bubbles contain helium or argon or a mixture of both gases the low density of dislocation loops that has been observed around bubbles of the radii found in figure 7.3 still tends to support diffusion related growth of gas bubbles.

\subsubsection{Electron Irradiations}

Shiraishi et al. [202] prepared specimens of an aluminium-lithium alloy that contained helium gas bubbles by using the $(n, \alpha)$ reaction of ${ }^{6} \mathrm{Li}$ with a thermal neutron. The specimens were then irradiated in separate TEMs with either $200 \mathrm{keV}$ or $1 \mathrm{MeV}$ electrons. During the electron irradiation the gas bubbles punched out loops. The mechanism proposed was that the electrons knock interstitials into the gas bubbles which decrease the bubble volume and increase the pressure so that the GFR inequality is satisfied. This mechanism of forming dislocation loops by using electrons is not relevant to helium irradiations for which the superlattice has been formed prior to examination in the TEM.

Evans et al. [200 and 201] also observed electron beam induced punching of loops by helium bubbles formed in molybdenum by the low energy technique outlined in section 7.1.2.3. Evans proposed different possible explanations to that of Shiraishi et al. [202]. He claimed that the loops were punched by the bubbles after the excitation of the helium atoms in the bubbles caused the pressure of the helium in the cavity to rise. Alternatively the electrons could be transferring enough energy to the molybdenum atoms to overcome an activation barrier for loop nucleation. 


\subsubsection{Implications for Implantations to a Higher Dose}

In this section a discussion is presented of what the implications of the observation of specimens implanted to a lower dose are for specimens containing a superlattice of gas bubbles. In the theory of Dubinko for bubble ordering [7] the bubbles first grow by diffusion related processes. The bubbles attract each other and coalesce until the remaining bubbles are spaced along the glide cylinders of nearest neighbour bubbles only. Thereafter the bubbles repel each other by punching out loops. Figure 7.3 shows bubbles that are not ordered and so are probably not lying on the glide cylinders of other bubbles. Dubinko requires a set of initial conditions to explain the ordering of gas bubbles via the punching of dislocation loops. The stability of the bubble structure in figure 7.3 suggests that the attraction between the bubbles is not strong enough for the structure to reach these initial conditions and the net force on a bubble due to the surrounding bubbles is smaller than that required to overcome the Peierls barrier.

The bubbles in the low dose irradiation have a radius that is approximately twice that of the specimens containing superlattices. Hence the structure found in the low dose irradiation is not a precursor of a superlattice in the same region of the specimen. For the reasons outlined in section 7.1.2.4 the bubbles in figure 7.3 are thought to have a higher pressure than those in an orthodox irradiation at the same stage in their growth. That the bigger bubbles have grown without punching loops and have displaced a larger amount of matrix material than the smaller bubbles in a superlattice supports diffusion related ordering and growth of the gas bubbles.

It is worth considering if the occurrence of a higher bubble density in a specimen would make the specimen more likely to reach the initial conditions that Dubinko requires in his model in which bubbles order by punching out dislocation loops. Consider bubble structures with bubble radii half those found in figure 7.3 but which contain the same total amount of helium and vacancies in the bubbles. The equilibrium bubble pressure will be doubled if this new structure can be created. The structure will have four times the number of bubbles at the new equilibrium pressure at approximately $0.63\left(=0.25^{1 / 3}\right)$ times the interbubble spacing. If the number of helium atoms and vacancies could be increased by a factor of ten with this extra helium going to more $\mathrm{HeV}$ complexes to form ten times the 
number of bubbles of the same volume and equilibrium pressure, then the interbubble spacing will be reduced by a factor of 0.467 . The postulated structure will have the same order of bubble radii and bubble spacings as found in superlattices -that is forty times the bubble density of figure 7.3.

The Willis-Bullough expression [104] for the attractive force between two bubbles is

$$
\mathrm{F}_{\mathrm{e}} \alpha(\mathrm{PR}-2 \gamma)^{2}\left(\frac{\mathrm{R}}{1}\right)^{7}
$$

where 1 is the interbubble spacing, $\mathrm{R}$ the bubble radius and $\mathrm{P}$ the bubble pressure. The PR factor is approximately unaltered as $\mathrm{R}$ varies in the more sophisticated theory of Wolfer [101-103]. Therefore, ignoring the prefactor, the attractive force between two bubbles would be about forty one times greater if the structure in figure 7.3 is changed as above. This would make the initial conditions of Dubinko more likely.

Consider the effects if two bubbles of the same radius were to coalesce as a result of the attractive force in equation 7.2 as required by the initial conditions of Dubinko. Assume the new bubble is constrained by the matrix to the same total volume as the two original bubbles. The resultant bubble will have a radius that is equal to 1.26 times the original radius of either initial bubble but the density of helium atoms in the resultant bubble and the pressure will be unchanged. (Even in the high pressure regime where the ideal gas law breaks down, pressure is still a function of gas density.) However, the equilibrium pressure has decreased and the new bubble is overpressured. If enough bubbles can coalesce the resultant bubble could punch out dislocation loops. If eight bubbles with a radius of $5 \AA$ coalesce as described above one bubble with a radius of $10 \AA$ will result. If the initial bubbles are slightly above the equilibrium pressure of about $6 \mathrm{GPa}$ at this $5 \AA$ radius [54] (so that they can attract each other) the resultant bubble requires a pressure of only $2.6 \mathrm{GPa}$ to be in equilibrium. The final bubble would require a pressure for loop punching of about $0.22 \mu=16.6 \mathrm{GPa}$ (figure 1.9). If the final bubble maintains the bubble pressure of the original bubbles it will then be overpressured by $3.4 \mathrm{GPa}$ but is still short of the pressure required to punch out loops by about $10.6 \mathrm{GPa}$. Instead it is more likely that the bubbles order according to the SIA diffusion model. 


\subsection{Summary}

In this chapter the results of attempts to image any dislocation loops that are present in ion-implanted metals are presented. Specimens containing superlattices of gas bubbles are not as crystalline as unirradiated specimens. This deterioration in crystallinity makes it more difficult to orientate the specimens to diffracting conditions where the loop contrast would be strong. To overcome this problem lower dose implantations on a copper disc that was prethinned by jet electropolishing were performed to create specimens that contained a lower density of small gas bubbles. In this case there is sufficient crystallinity retained to allow the specimens to be tilted to obtain strong two-beam conditions. Some dislocation loops are observable in these specimens but they are at a low density. This may suggest that the bubbles are formed primarily by diffusion related processes rather than by the punching of dislocation loops. Alternatively, the dislocations may have been lost from the prethinned discs.

The implications of this observation for the density of loops in specimens irradiated to a higher dose are interesting. Bubbles in specimens irradiated to different doses have a different size and probably a different pressure. Experimental micrographs of the specimens containing superlattices taken at approximate strong two-beam and weak beam conditions do exhibit the presence of some dislocation loops. However, it is difficult to find a bubble that has punched out the full complement of twelve loops along the glide cylinders towards the nearest-neighbour bubbles. (This sort of structure would form images like those calculated in the simulations of chapter six.)

A review is given in this chapter of the experimental evidence that has lead many authors to suggest that the punching of dislocation loops is the most important mechanism for bubble growth and bubble ordering. It is pointed out that most experimental observations of loops around bubbles have been for bubbles (usually formed by quenching) that are much larger than those found in superlattices. Larger bubbles have a lower equilibrium pressure and thus are more likely to have reached the degree of overpressure required to punch out loops. Consequently, the results for large bubbles may not be a good guide to the superlattice case. A further difference is the high level of displacement damage 
that occurs during implantation. This could have the effect of annealing the metal so the dislocation density in the implanted layer is lower than might otherwise be expected.

The suggestion is also made that in high dose irradiations diffusion related processes may be more important for bubble growth and ordering than is the punching of dislocation loops. It is important to note, however, that these diffusion processes could be strongly influenced by the stress fields around individual bubbles - stress fields that reflect the tendency of overpressured bubbles to punch out dislocation loops. The stress fields resulting from this tendency (rather than the presence of actual dislocation loops on common glide cylinders linking nearest-neighbour bubbles) have also been invoked by Johnson et al (see for example ref. 8) to explain the origin of the spatial variants [42] they found in fcc bubble superlattices. 


\section{CHAPTER 8: CONCLUSIONS}

This chapter is a review of the main conclusions reached in the preceding seven chapters. More details on the conclusions can be found in the summaries at the end of each chapter. The aim of the thesis is to develop and evaluate methods based on transmission electron microscopy (TEM) for measuring the density of dislocations in some selected metals that have been ion-implanted. Towards this end the initial chapters introduced some necessary background information and theory. Chapter one contains an introduction to the mechanism by which dislocations are punched out by overpressured gas bubbles. The discussion of this mechanism required a digression into isotropic elasticity theory. In the first chapter a brief review is made of the previous work done on structures containing gas bubbles, especially how they order and grow.

The microstructure of an ion-implanted metal is investigated by TEM. An understanding of the mechanism by which electrons are scattered by possible structures is required if images obtained using TEM are to be correctly interpreted. Chapter two summarises the theory of image formation in TEM. In particular the Howie-Whelan differential equations are derived. These equations are the most commonly used set of equations for modelling the images of dislocations or gas bubbles. More complicated theories of how weak beam and high resolution images are modelled are summarised in the remainder of the chapter.

The modelling of the electron diffraction from defects requires that the displacement fields can be calculated. Chapter three gives equations for the displacement field around a gas bubble, a long straight dislocation and an angular dislocation. A polygonal dislocation loop can be constructed from a set of angular dislocations with the same Burgers vector, arranged so that the dislocation lines of adjacent dislocations lie in opposite directions outside the dislocation loop. Each angular dislocation has a coordinate system associated with it. To obtain the displacement field at a point near a dislocation loop, the coordinates of this point need to be transferred into the axes of each angular dislocation. The displacement field due to each dislocation is then calculated. 
The results are then transferred back into the original coordinate system and are summed to give the resultant displacement field.

Chapter four departs from the previous chapters in emphasising the experimental techniques required to prepare the specimens before micrographs can be obtained in the TEM. The specimens are cleaned by mechanical polishing, bath electropolishing and annealing in vacuum. They are then ion-implanted in a particle accelerator or are quenched to attain a lower density of defects. Jet electropolishing is used to perforate the specimens, then any further thinning is done by an ion beam. The computer system used to implement the theory discussed in chapters one to three is discussed in detail. The micrographs and simulations presented in chapter four enable the Burgers vectors of some dislocation loops and straight dislocations to be obtained. The results gave the author experience in the image matching of structures simpler than those typically encountered in ion-implanted metals.

Gas bubbles are the most obvious defects in ion-implanted metals. They are important enough for chapter five to be devoted entirely to the imaging of gas bubbles without worrying about the presence of any dislocations. Initially the image of a single isolated bubble was considered before extending the work to imaging stacks of bubbles. A comparison of the experimental micrographs with theoretical profiles of the intensity diffracted from a stack of bubbles indicates that in the vertical direction the ordering of gas bubbles seldom extends over more than three bubbles. Simulations of superlattice spots suggested that the lateral ordering of the gas bubbles is limited, although the effects of beam coherence make this conclusion somewhat tentative. Intensity profiles through defocussed images of an isolated single bubble showed that measurements of the bubble radius could easily be about $200 \%$ out from the real value. A more accurate measurement of the radius is obtained by simulating the positions of the Fresnel fringes in an experimental through-focal series. The dependence on defocus of the image of a large array of ordered bubbles of similar radius has not been studied in this thesis.

Dislocation loops are present in several models of how gas bubble superlattices evolve. In chapter six, the simulations give an indication of the contrast to 
be expected from a system of dislocation loops punched by a growing gas bubble. The calculations have assumed that it is permissible to use isotropic elasticity theory. Limitations put upon any conclusions drawn from this work are outlined in Eyre et al. [58-61].

\section{The main conclusions resulting from the simulations are:}

(i) Several parameters that have successfully been used to characterise the diffraction from isolated dislocation loops can be carried over to the more complicated structures considered in this thesis. For example, the parameters are the 1 vector and the black/white lobe structure and its dependence upon the loop normal, the diffracting vector, the Burgers vector, the beam direction and the foil normal.

(ii) Any interpretation of experimental micrographs needs to consider how many loops are projected onto one another, the depth of the loops and what $\mathbf{g} . \Sigma \mathbf{b}$ is. Ball model diagrams are essential for interpretation.

(iii) When loops are close together the resultant contrast is determined by $\mathbf{g} \cdot \Sigma \mathbf{R}$. This results in rapidly varying black or white lobes appearing in the images, separated by a region of near background intensity.

(iv) The images with a significantly non-zero excitation error give black or white dots in BF and DF respectively instead of black/white lobes. The two beam approximation can lead to significant errors as the excitation error increases to the weak beam condition, but six beam simulations show that the dislocation loops become resolvable. However, the image becomes difficult to interpret with the density of defects included here. This limits the applicability of weak beam imaging techniques to this structure.

(v) The contrast due to any dislocation loops present is comparable to that due to the thickness and strain contrast of the gas bubbles at strong two-beam and in-focus conditions. This is also true with micrographs obtained with out-of-focus conditions. To detect the presence or absence of dislocation loops in gas bubble superlattices, the crystal should be tilted to strong two-beam conditions for a range of g's around simple B's, and the micrographs should be well focussed to attempt to obtain the black and white lobes characteristic of dislocation loops. In practice it may be necessary to take experimental 
through focal series to find the in-focus condition. Black/white lobe images with I rotated away from $\mathrm{g}$ will be loops whereas those with I parallel to $\mathrm{g}$ could be loops or bubbles.

The dislocation loops have been assumed to be edge in character with a Burgers vector of the [110] type in an fcc crystal. Because edge dislocation loops have a higher energy per unit length than screw dislocations the loop normal may try to rotate away from the Burgers vector. However, as $\mathbf{n}$ rotates the total length of dislocation line in the loop will increase so that the strain energy of the loop may actually increase. Any screw character that results from external stresses will alter the detailed appearance of the micrographs, and may need to be considered to obtain good matches between experimental and theoretical micrographs. If the present work was extended to bcc materials, perfect loops with $\mathbf{b}=a / 3<111>$ should be considered. Defects with this Burgers vector can occur in fcc materials but, because the so-called Frank dislocation loop that results incorporates a stacking fault, the defect cannot glide and consequently is not responsible for the growth and ordering of gas bubbles. Other Burgers vectors would need to be considered in hep materials.

In practice, experimental micrographs taken at strong two-beam conditions are difficult to obtain in ion-implanted metals because the irradiation process has reduced the crystallinity of the material. In chapter seven the best micrographs obtained in ionimplanted metals are presented. Implantations to a lower dose give specimens that are crystalline enough to discover that the density of dislocation loops is significantly below that to be expected if the bubbles found had grown by the punching of dislocation loops alone. The suggestion is made that the bubbles probably grew by diffusion related processes. The implications of this observation in specimens irradiated to a lower dose are that specimens containing superlattices of gas bubbles also grow by diffusion related processes. A review of the experimental evidence that has lead other authors to suggest that the punching of dislocation loops is the most important mechanism by which small gas bubbles grow was reviewed in chapter seven and found to be unconvincing. The 
simplest conclusion is that, on average, small gas bubbles do not acquire a high enough pressure to punch out dislocation loops.

A possible direction for future work is to use an electron energy loss spectroscopy (EELS) apparatus to measure the gas pressure inside bubbles. Pressure measurements on the prethinned specimens of chapter seven would be especially interesting to do, and also comparison measurements on the gas pressure in material irradiated in the bulk. This has obvious implications for the mechanism by which dislocation loops are punched.

Higher resolution micrographs of the prethinned specimen may also be useful. Bubbles occur at a low density out toward the hole in the specimen, so the material should be thin enough to obtain structure images. The multislice simulations of chapter four are more appropriate to the structure images of copper attainable with a higher resolution TEM than that available at Victoria University. The TEM at Victoria University can only really produce lattice fringe pictures, and not structure images of copper. Of course, in very thin material any dislocations are more likely to have escaped from the foil, so any results that do not show dislocations may be inconclusive. However, if HREM images of bubbles were obtained, they could be used to measure the displacement field around a bubble and hence the bubble overpressure by measuring the distortion of the lattice fringes. If no distortion is present then it may be possible to make a measurement of the equilibrium pressure of the bubble.

Throughout this thesis the displacement field, around a structure containing twelve loops next to a bubble, that is required to perform image simulations has been calculated by adding together the analytical displacement fields due to the individual defects. The neglect of the interactions between the defects and the effects that this has upon the resultant displacement field have been assumed to be negligible.

It would be interesting to use a technique such as finite element analysis to calculate the displacement field around the structures. Techniques based on finite element analysis require the minimisation of the energy of the displacement field, as calculated on a triangular mesh of points about the structure, using an iterative steepest descent method. 
The displacement field is considered to have been calculated by this technique when the energy of the field calculated in succesive iterations is the same to within a specified level. In addition to applications in elasticity theory, finite element analysis is commonly used to solve problems involving heat transfer and electromagnetism in situations with a complex geometry.

If the displacement field could be calculated using finite element analysis in a reasonable time on the more powerful computers now becoming available, then it might be possible to perform realistic image simulations on more complicated structures than those considered in this thesis. As a superlattice of gas bubbles grows and evolves there is expected to be some coarsening of the dislocation structure. This coarse structure might be more readily modelled using finite element analysis instead of superimposing the analytical solutions for the displacement fields about defects. (The analytical solutions in elasticity theory only really apply when specific boundary conditions are valid). 


\section{Appendix A:}

\section{List of Symbols}

a: the dislocation loop radius or bubble radius

A: a matrix describing the electron scattering or a rotation matrix

A: the anomalous absorption coefficient, the axial astigmatism or an area

$A_{k}$ : the components of a vector

$a_{1}$ : lattice constant

ano: anomalous absorption ratio

b: $\quad$ Burgers vector

b: magnitude of the Burgers vector

B : electron beam direction

$\mathrm{B}_{\mathrm{j}}$ : Debye-Waller coefficient (depends on temperature)

$b_{e}$ : edge component of the Burgers vector

$b_{s}$ : screw component of the Burgers vector

$b_{\mathrm{x}}$ : the $\mathrm{x}$ component of the Burgers vector

$\mathrm{b}^{\mathrm{i}}\left(\mathbf{k}_{0}, \mathbf{r}\right): \quad$ ith Bloch wave

c: $\quad$ velocity of light

C: matrix of coefficients for the Bloch waves

$\mathrm{c}(\mathbf{k})$ : the micrograph contrast

$\mathrm{c}_{\mathrm{ijkl}}$ (or $\mathrm{c}_{\mathrm{mn}}$ ): the elastic constants of the material

$\mathrm{C}_{\mathrm{s}}$ : the spherical aberration coefficient

$\mathrm{d}$ : the rms effective focus spread or the distance from the centre of a bubble to the foil surface

$\mathrm{d}_{\mathrm{g}}$ : $\quad$ spacing of the planes corresponding to the gth reflection

$\mathrm{D}_{\alpha}$ : a scaling constant

$\mathrm{D}_{0}$ : a diffusion constant that is different for interstitials, vacancies and helium atoms

e: $\quad$ electron charge

E: energy to form a dislocation loop or the voltage the electrons are accelerated through

$\mathrm{E}(\mathrm{k})$ : complete elliptic integrals of the second type 
$\mathrm{E}\left(\mathbf{k}_{1}, \mathbf{k}_{2}\right)$ : envelope function used to include the effects of beam divergence and defocus spread in the contrast transfer function of a lens

$\mathrm{E}_{\mathrm{m}}: \quad$ the activation energy for a defect jump to occur

F: free energy

F: foil normal or point force vector

$\mathrm{F}(\mathrm{k})$ : complete elliptic integrals of the first type

f: $\quad$ an arbitrary function

$\mathrm{f}_{\mathrm{e}}$ : atomic electron scattering factor

$\mathrm{F}_{\mathrm{e}}$ : the attractive force between two bubbles

$\mathrm{f}_{\mathrm{X}}$ : atomic $\mathrm{X}$-ray scattering factor

$\mathrm{F}_{\mathrm{n}}: \quad$ coefficients in a polynomial

$F_{g}: \quad$ structure factor for the gth reciprocal lattice vector

g: diffracting vector

h: Planck constant

$\mathrm{h}_{\mathrm{i}}$ : distances to a set of planes

I: the intensity

$\mathrm{I}_{0}$ : the background intensity

i: $\quad$ index that can take the value 1,2 or 3

j: $\quad$ index that can take the value 1,2 or 3

$\mathrm{J}_{0}$ : $\quad$ zero order Bessel function

$\mathrm{J}_{1}$ : first order Bessel function

$\mathrm{k}$ : index that can take the value 1,2 or 3 ; or the magnitude of the wavevector or Boltzmann's constant

k: wavevector

K: wave vector in the crystal

l: $\quad$ index that can take the value 1,2 or 3 ; or the spacing between bubbles

I: black/white lobe vector

$\mathrm{m}$ : index that can take the value 1,2 or 3

$\mathrm{m}_{\mathrm{e}}: \quad$ the mass of the electron

n: Loop normal

$\mathrm{n}$ : index that can take the value 1,2 or 3 ; or the refractive index

$$
\text { A - } 2
$$


$\mathrm{n}_{\mathrm{i}}: \quad$ plane normals

norm: a vector that points from the bottom left corner of a simulation to the top right corner

p: $\quad$ propagation function or a constant

$\mathrm{P}$ : the gas pressure in the bubble

$\mathrm{P}_{\alpha}: \quad$ constants

$\mathrm{P}_{\mathrm{n}}: \quad$ a Legendre polynomial of degree $\mathrm{n}$

$\mathrm{p}_{\mathrm{rz}}: \quad$ shear stress

Q: variable used to find the displacement field around a bubble

Q $: \quad$ constants

$\mathrm{q}_{\mathrm{n}}: \quad$ the transmission function for the nth slice

r: position vector

r: the bubble radius or magnitude of the position vector

$\mathbf{R}(\mathbf{r})$ : displacement vector describing deformation of the perfect crystal

$\mathrm{R}_{\alpha}$ : constants

$r_{d}: \quad$ the dislocation core cut-off parameter

$\mathbf{r}_{\mathrm{j}}$ : $\quad$ position of the $\mathrm{jth}$ atom in the unit cell

$\mathrm{r}_{l}: \quad$ the radius of the dislocation loop

$\mathrm{r}_{0}$ : the radius of the bubble

S: $\quad$ Amplitude of the diffracted beam

$\mathrm{S}_{\alpha}: \quad$ constants

$\mathrm{S}_{\mathrm{g}}$ : Reciprocal space distance or direct lattice spatial frequency

$\mathbf{s}_{\mathrm{g}}$ : excitation error for the gth reflection

t: foil thickness

T: Amplitude of the central beam, a temperature or the foil thickness

$\mathrm{T}_{\mathrm{m}}$ : the melting temperature of the metal

u: Dislocation line direction or displacement vector

$\mathrm{u}$ a displacement field component

$\mathrm{u}_{\mathrm{j}}$ : the displacement components of the material

$\mathrm{U}_{\mathrm{g}}$ : gth Fourier coefficient of the potential (proportional to $\mathrm{V}_{\mathrm{g}}$ )

$\mathrm{u}_{\mathrm{ij}}$ : the Green's function for the elastic displacements 
v: a displacement field component

V: volume or visibility

$\mathrm{V}(\mathbf{r})$ : potential that the electron is moving through

$\mathrm{V}_{\mathrm{c}}$ : volume of the unit cell

$\mathrm{V}_{\mathrm{g}}$ : gth Fourier coefficient of the potential

w: excitation error or a displacement field component

W: work done

$\mathrm{w}(\mathbf{k})$ : the contrast transfer function of the lens

$\mathrm{x}_{\mathrm{j}}$ : $\quad$ spatial coordinates

$\mathrm{x}$ : spatial coordinate

y: spatial coordinate

z: $\quad$ spatial coordinate

Z: $\quad$ atomic number

Greek symbols:

$\alpha: \quad$ parameters

$\beta$ : defocus parameter or the rms width in section of the angular brightness distribution of the illumination

$\beta_{\mathrm{g}}^{\prime}$ : parameter related to the derivative of the displacement field

$\gamma$. the surface tension of the host metal or the relativistic correction factor

$\Gamma: \quad$ gamma function

$\gamma^{(i)}$ the distance parallel to the Brillouin zone boundary between the ith dispersion surface and the Ewald sphere for the 0th reflection

$\delta: \quad$ a measure of the strength of dilatation or overpressure in the bubble

$\delta_{\mathrm{ij}}: \quad$ the Kronecka delta function

$\Delta: \quad$ the defocus (overfocus positive)

$\Delta_{\mathrm{n}}$ : the difference between the wave function that has passed through the bubble and the perfect crystal wave function, normalised by the perfect crystal wave function

$\varepsilon_{\mathrm{kl}}: \quad$ the strain tensor

$\varepsilon_{\mathrm{ijk}}: \quad$ the Einstein permutation operator

$\xi$ : the defocus or a coordinate 
$\xi_{\mathrm{g}}: \quad$ real part of the extinction distance of the gth beam

$\xi_{\mathrm{g}}^{\prime} \quad$ imaginary part of the extinction distance of the gth beam

$\theta_{\mathrm{g}}: \quad$ Bragg angle for the gth reflection

$\kappa_{0}: \quad$ the real part of the wave vector inside the crystal that is determined by the mean potential of the crystal

$\lambda$ : electron wavelength or the Lame constant

$\mu: \quad$ shear modulus

v: Poisson's ratio

$\eta$ : the normal absorption coefficient or a coordinate

$\rho: \quad$ loop spacing or bubble radius parameter

$\sigma:$ the interaction constant

$\sigma_{\mathrm{ij}}: \quad$ the stress tensor

$\phi_{\mathrm{g}}: \quad$ Darwin amplitude of the gth beam

$\chi$ : $\quad$ wave vector in vacuum

$\psi: \quad$ the Airy stress function, the wavefunction or some other function

$\psi^{(\mathrm{i})}: \quad$ Bloch wave amplitudes

$\Omega: \quad$ the solid angle subtended by the dislocation 


\section{References}

[1] G.W. Greenwood, A.J.E. Foreman and D.E. Rimmer, J. Nucl. Mat. 4 (1959) 305.

[2] R.S. Barnes and D.J. Mazcy, Acta Mctallurgica 11 (1963) 281.

[3] G. Thomas and M.J. Whclan, Phil. Mag. 4 (1959) 511.

[4] D.A. Jones and J.W. Mitchell, Phil. Mag. 3 (1958) 1.

[5] P.G. Partridge, Central Elcctricity Gencrating Board Research and Development Department, Berkeley Nuclear Laboratories, Job no. 71306.

[6] R.E. Smallman and A. Eikum, Lattice Defects in Quenched Metals, Academic Press Inc., New York (1965) 591.

[7] V.I. Dubinko, V.V. Slezov, A.V. Tur and V.V. Yanovsky, Rad. Eff. 100 (1986) 85.

[8] P.B. Johnson, Proc, of NATO Advanced Research Workshop, Bonas, France: Fundamental Aspects of Inert Gases in Solids, eds. Donnelly S.E. and Evans J.H., Plenum, New York (1991) 167.

[9] R.M.J. Coterill, Phys. Letters Vol. 60A no. 1 (Jan. 1977) 61.

[10] C. Kittel, Introduction to Solid State Physics, 6th ed. (1986) 570.

[11] G. Dearnaley, Surf. Engng. 2 (1986) 213.

[12] P.B. Johnson, T.G.L Shirtcliffe and G. Dearnaley, Victoria UNiversity of Wellington Phys. Dept report $1 / 81$.

[13] G.L. Kulcinski, J.L. Brimall and H.E. Kissinger in Radiation Induced Voids in Metals, ed. W.

Corbett and L.C. Iannicllo, Albany, USAEC-CONF-71061, (1972) 449; J. Nucl. Mater 40 (1971) 166.

[14] D.J. Mazey, S. Francis and J.A. Hudson, J. Nucl. Mat. 47 (1973) 137.

[15] A. Risbet and V. Levy, J. Nucl. Mat. 50 (1974) 116.

[16] L.J. Chen and A.J. Ardell, J. Nucl. Mat. 75 (1978) 177.

[17] S.B. Fisher and K.R. Williams, Rad. Eff. 32 (1977) 123.

[18] J.H. Evans, Nature 229 (1971) 403.

[19] A.M. Stoneham, Proc. of the International Conf. on Fundamental Aspects of Radiation Damage in Metals Vol. 2, cd. M.T. Robinson and F.W. Young Jnr. (1975) 1221.

[20] A.M. Stoneham, Harwell Unclassificd Report no. AERE - R7934 (1975) 319.

[21] K. Krishan, Rad. Eff. 66 (1982) 121. 
[23] R.S. Barnes and D.J. Mazcy, UKAEA Unclassificd Rcport, AERE R-3348 (1960) and Phil. Mag. $5(1960) 290$.

[24] S.L. Sass and B.L. Eyrc, Phil. Mag. 27 (1973) 1447.

[25] P.B. Johnson and D.J. Mazey, Nature 276 (1978) 595.

[26] P.B. Johnson and D.J. Mazcy, Harwell Report AERE R-9290 (1978).

[27] P.B. Johnson and D.J. Mazey, Nature 281 (1979) 359.

[28] P.B. Johnson and D.J. Mazcy, Rad. Eff. 53 (1980) 195.

[29] P.B. Johnson and D.J. Mazey, J. Nucl. Mat. 93/94 (1980) 721.

[30] P.B. Johnson and D.J. Mazey, Harwell Report AERE R-9685 (1980).

[31] P.B. Johnson and D.J. Mazcy, J. Nucl. Mat. 91 no. 1 (1980) 41.

[32] D.J. Mazey and J.H. Evans, J. Nucl. Mat. 138 (1986) 16.

[33] A. vom Felde, J. Fink, Th. Müller-Heinzerling, J. Pflüger, B. Scheere, G. Linker and D. Kaletta, Phys. Rev. Letters 53 (1984) 922.

[34] S.E. Donnelly and C.J. Rossouw, Science 230 (1985) 1272.

[35] J.H. Evans and D.J. Mazcy, J. Phys. F.: Met. Phys. 15 (1985) L1-L6.

[36] J.H. Evans and D.J. Mazcy, J. Nucl. Mat. 138 (1986) 176.

[37] C. Templier, H. Garem and J.P. Rivicre, Phil. Mag. A 53 (1986) 667.

[38] P.B. Johnson, R.W. Thomson and D.J. Mazcy, Nature Vol. 347 no. 6290 (1990) 265.

[39] P.B. Johnson and D.J. Mazey, J. Nucl. Mat. 170 (1990) 290.

[40] P.B. Johnson, D.J. Mazey and J.H. Evans, Harwell Report AERE R-10874 (1983).

[41] P.B. Johnson, D.J. Mazey and J.H. Evans, Rad. Eff. 78 (1983) 147.

[42] P.B. Johnson, A.L. Malcolm and D.J. Mazcy, Nature 329 (1987) 316.

[43] P.B. Johnson, A.L. Malcolm and D.J. Mazey, J. Nucl. Mat. 152 (1988) 69.

[44] P.B. Johnson, A.L. Diprose and D.J. Mazcy, J. Nucl. Mat. 158 (1988) 108.

[45] A.L. Malcolm, PhD. Thesis, Victoria University of Wellington (1988).

[46] T.R. Armstrong, P.B. Johnson and W.R. Jones, J. Nucl. Mat. 114 no. 1 (1983) 1.

[47] T.R. Armstrong, R.C. Corliss and P.B. Johnson, J. Nucl. Mat. 98 no. 3 (1981) 338.

[48] T.R. Armstrong, J. Nucl. Mat. 84 (1979) 118.

[49] P.B. Johnson and T.R. Armstrong, Nucl. Instr. and Meth. 148 (1978) 85.

[50] P.B. Johnson and T.R. Armstrong, Appl. Phys. Letters Vol. 31 no. 5 (1977) 325.

$$
R-2
$$


[52] V.A. McGlone, PhD. Thesis, Victoria University of Wellington (1989).

[53] T.R. Armstrong, PhD. Thesis, Victoria University of Wellington (1980).

[54] W.R. Jones, PhD. Thesis, Victoria University of Wellington (1985).

[55] P.B. Johnson, Rad. Eff. Vol. 32 (1977) 159.

[56] P.B. Johnson and W.R. Joncs, J. Nucl. Mat. 120 (1984) 125.

[57] D.M. Maher and B.L. Eyre, Phil. Mag. 23 (1971) 409.

[58] B.L. Eyre, D.M. Maher and R.C. Perrin, J. Phys. F.: Metal Phys. Vol. 7 no. 8 (1977) 1359.

[59] B.L. Eyre, D.M. Maher and R.C. Perrin, J. Phys. F.: Metal Phys. Vol. 7 no. 8 (1977) 1371.

[60] S.M. Holmes, B.L. Eyre, C.A. English and R.C. Perrin, J. Phys. F.: Metal Phys. Vol. 12 no. 9 (1979) 2307.

[61] C.A. English, B.L. Eyre and S.M. Holmes, J. Phys. F.: Metal Phys. Vol 10 (1980) 1065.

[62] D.K. Saldin, A.Y. Stathopoulos and M.J. Whelan, Phil. Trans. Roy. Soc. Lond. A 292 (1978) 523.

[63] D.K. Saldin, D. Phil. Thesis, University of Oxford Oct. 1975.

[64] D.K. Saldin and Whelan M.J., Phil. Trans. R. Soc. Lond. A 292 (1978) 513.

[65] S.M. Holmes, D. Phil. Thesis, University of Oxford (1975).

[66] M. Wilkens, Diffraction and Imaging Techniques in Material Science, eds. S. Amelinckx R. Gevers and J. van Landuyt, North Holland: (1978) 185.

[67] P.B. Hirsch, A. Howie, R.B. Nicholson, D.W. Pashley and M.J. Whelan, Electron Microscopy of Thin Crystals, Krieger, Huntington, New York (1977).

[68] M.J. Whelan and P.B. Hirsch, Phil. Mag. A Ser. 8 Vol. 2 no. 21 (Sept. 1957) 1121.

[69] M.J. Whelan and P.B. Hirsch, Phil. Mag. A Ser. 8 Vol. 2 no. 21 (Sept. 1957) 1303.

[70] H. Hashimoto, A. Howic and M.J. Whclan, Phil. Mag. A Ser. 8 Vol. 5 (1960) 967.

[71] H. Hashimoto, A. Howie and M.J. Whelan, Proc. Roy. Soc. A 269 (1962) 80.

[72] A. Howie and M.J. Whclan, Proc. Roy. Soc. A 263 (1961) 217.

[73] A. Howie and M.J. Whelan, Proc. Roy. Soc. A 267 (1962) 206.

[74] A. Howie, Proc. Roy. Soc. of London A 271 (1963) 268.

[75] A. Howie and Z.S. Basinski, Phil. Mag. 17 (1968) 1039.

[76] A. Howie and U. Valdre, Phil. Mag. 15 (1967) 777.

[77] A. Howie and C.H. Sworn, Phil. Mag. 22 (1970) 861.

$$
\text { R - } 3
$$


[79] A.K. Head, Aust. J. Phys. 20 (1967) 557.

[80] P. Humble, Aust. J. Phys. 21 (1968) 325.

[81] P. Humble, Phil. Mag. A Vol. 51 no. 3 (1985) 355.

[82] A.K. Head, L.M. Clarcbrough, P. Humble, C. Forwood and A.J. Morton, Computed Electron Micrographs and Defect Identification, Amsterdam, North Holland, (1973).

[83] M. Rühle and M. Wilkens, Crystal Latticc Defects 6 (1975) 129.

[84] E. Johnson, E. Kynde and L.T. Chadderton, J. of Microscopy 116 part 1 (1979) 15.

[85] W. Gruschel, A. Laupheimer and M. Wilkens, Proceedings 10th Intemational Congress on Electron Microscopy, Hamburg Vol. 1 (1982) 97.

[86] W.J. Gruschel, Computer Physics Communications 16 (1979) 175.

[87] W.J. Gruschel and M. Wilkens, Phys. Stat. Sol. (a) 89 (1985) 467.

[88] A.J.E. Foreman, H.S. von Harrach and D.K. Saldin, Phil. Mag. A 45 no. 4 (1982) 625.

[89] D.J.H. Cockayne, D. Phil. Thesis, University of Oxford (1970).

[90] D.J.H. Cockayne, Diffraction and Imaging Techniques in Material Science, eds. S. Amelickx, R.

Gevers and J. van Landuyt, North Holland: (1978) 153.

[91] A.R. Wilson, Acta Cryst. A39 (1983) 282.

[92] A.R. Wilson and D.J.H. Cockayne, Phil. Mag. A Vol. 51 no. 3 (1985) 341.

[93] G.R. Anstis and D.J.H. Cockayne, Acta Cryst. A35 (1979) 511.

[94] D.J.H. Cockayne, J.R. Parsons and C.W. Hoclke, Phil. Mag. 24 (1971) 139.

[95] D.J.H. Cockayne, J. Appl. Cryst. 8 (1975) 222.

[96] I.L.F. Ray and D.J.H. Cockayne, Phil. Mag. 22 (1970) 853.

[97] J.P. Hirth and Lothe, The Theory of Dislocations, Wiley Interscience, (1968).

[98] B.A. Bilby, R. Bullough and E. Smith, Proc. Roy. Soc. A 231 (1955) 263.

[99] R.E. Smallman, Modern Physical Mctallurgy, Butterworths, 4 th edition (1990).

[100] F. Kroupa, Czech. J. Phys.10 (1960) 284.

[101] W.G. Wolfer and W.J. Drugan, Phil. Mag. A Vol. 57 no. 6 (1988) 923.

[102] W.G. Wolfer, Phil. Mag. A Vol. 58 no. 2 (1988) 285.

[103] W.G. Wolfer, Phil. Mag. A Vol. 59 no. 1 (1989) 87.

[104] J.R. Willis and R. Bullough, J. Nucl. Mat. 32 (1969) 76.

[105] E.P. Eernisse and S.T. Picraux, J. Appl. Phys. 48 (1977) 9.

$$
\text { R - } 4
$$


E. Gerritsen, Doctoral Thesis, Rijksuniversitcit Groningen (1990).

[107] L. Graaback, J. Bohr, E. Johnson, H.H. Andersen, A. Johansen, L. Sarholt-Kristensen, Mat. Sci. Eng. A115 (1989) 97.

[108] R.F. Egerton, Electron encrgy-loss spectroscopy in the electron microscope, Plenum Press, New York (1986).

[109] W. Jager, R. Manzke, H. Trinkaus, G. Crecclius, R. Zeller, J. Fink and H.L. Bay, J. Nucl. Mat. $111 \& 112$ (1982) 674.

[110] S.E. Donnelly, D.R.G. Mitchell and A. van Veen, Proc. of NATO Advanced Research Workshop, Bonas, France: Fundamental Aspects of Inert Gases in Solids, eds. Donnelly S.E. and Evans J.H., Plenum, Ncw York, (1991) 357.

[111] J.B. Adams, W.G. Wolfer, S.M. Foiles, C.M. Rohling and C.D. Van Siclen, Proc. of NATO Advanced Research Workshop, Bonas, France: Fundamental Aspects of Inert Gases in Solids, eds. Donnelly S.E. and Evans J.H., Plenum, New York (1991) 3.

[112] R. Bullough and R.C. Newman, Phil. Mag. 5 (1960) 921.

[113] G.N. Watson, The Theory of Bessel Functions, Cambridge University Press (1952).

[114] I.S. Gradshteyn and I.M. Ryzhik, Table of Integral Series and Products, 4th Edition Academic Press (1965).

[115] CRC Handbook mathematical companion.

[116] C.S.Barrett, Structure of Metals, McGraw-Hill New York (1952) 297.

[117] P.B. Johnson, 4th Annual Solid State Physics Meeting, AIP/NZIP Pakatoa Island, NZ (Jan 1980). Summary in Victoria University of Wellington Phys. Dept. Report 1/82.

[118] J.H. Evans, NATO Meeting Corsica (1989).

[119] F. Seitz, Phys. Rev. 79 (1950) 723.

[120] W. Weertman, Phys. Rev. 107 (1957) 1259.

[121] J.H. Evans, R. Bullough and A.M. Stoneham, Proc. Albany Conf., ed. J.W. Corbett and L.C. Ianiello (1972) 522.

[122] A.M. Stoneham, Harwell Voids Symposium AERE-R7934, ed. R.S. Nelson (1974) 319.

[123] V.K. Tewary and R. Bullough, Harwell Unclassified Report no. T.P. 479 (1972) 1.

[124] J.D.Eshelby, Proc. Roy. Soc. London Scr. A 248 (1957) 376.

[125] J.H. Evans, J. of Nucl. Mat. 68 (1977) 129.

[126] V.I. Dubinko, P.N. Ostapchuk and V.V. Slczov, J. Nucl. Mat. 161 (1989) 239.

$$
\mathrm{R}-5
$$


J.M Cowley, Diffraction Physics, Amsterdam: North-Holland (1975),

[128] C.G. Darwin, Phil. Mag. 27 (1914) 315.

[129] H.A. Bethe, Ann. Phys. 87 (1928) 55.

[130] N. Kato, J. Phys. Soc. Japan Vol. 7 no. 4 (July-Aug. 1952) 397.

[131] N. Kato, J. Phys. Soc. Japan Vol. 7 no. 4 (July-Aug. 1952) 406.

[132] N. Kato, J. Phys. Soc. Japan Vol. 8 no. 3 (May-June 1953) 350.

[133] N.F. Mott, Proc. Roy. Soc. (London) A127 (1930) 658.

[134] P.A. Doyle and P.S. Turner, Acta Crystallography, A24 (1968) 390.

[135] K. Fujiwara, J. Phys. Soc. Japan Vol. 14 no. 11 (Nov. 1959) 1513.

[136] K. Fujiwara, J. Phys. Soc. Japan Vol. 16 no. 11 (Nov. 1961) 2226.

[137] K. Molière, Ann. d. Phys. Lpz. 34 (1939) 461.

[138] H. Yoshioka, J. Phys. Soc. Japan Vol. 12 no. 6 (June 1957) 618.

[139] Y. Kainuma, Acta Cryst. 8 (1955) 247.

[140] C.J. Humphreys and P.B. Hirsch, Phil. Mag. 15 (1967) 507.

[141] L. Sturkey, Proc. Phys. Soc. 80 (1962) 321.

[142] H. Niehrs and F.N. Wagner, Z. Phys. 143 (1955) 285.

[143] F. Fujimoto, J. Phys. Soc. Japan Vol. 14 no. 11 (Nov. 1959) 1558.

[144] J.M. Cowley and A.F. Moodic, Proc. Phys. Soc. B 70 (1957) 486.

[145] J.M. Cowley and A.F. Moodie, Proc. Phys. Soc. B 70 (1957) 497.

[146] J.M. Cowley and A.F. Moodie, Proc. Phys. Soc. B 70 (1957) 505.

[147] J.M. Cowley and A.F. Moodie, Acta Cryst. 10 (1957) 609.

[148] J.M. Cowley and A.F. Moodie, Acta Cryst. 12 (1959) 353.

[149] J.M. Cowley and A.F. Moodie, Proc. Phys. Soc. (1958) 533.

[150] J.M. Cowley and A.F. Moodie, Acta Cryst. 12 (1959) 360.

[151] J.M. Cowley and A.F. Moodie, Acta Cryst. 12 (1959) 367.

[152] J.M. Cowley and A.F. Moodic, Proc. Phys. Soc. 76 (1960) 378.

[153] P. Goodman and A.F. Moodic, Acta Cryst. A30 (1974) 280.

[154] D.F. Lynch and M.A. O'Keefe, Acta Cryst. A28 (1972) 536.

[155] G.R. Anstis, D.F. Lynch and M.A. O'Kecfe, Acta Cryst. A29 (1973) 138.

[156] M.A. O'Keefe, Acta Cryst. A29 (1973) 389.

[157] D.F. Lynch, A.F. Moodie and M.A. O'Kecfe, Acta Cryst. A31 (1972) 536.

$$
R-6
$$


[159] P.G. Self, M.A. O'Keefe, P.R. Buseck and A.E.C. Spargo, Ultramicroscopy 11 (1983) 35.

[160] P.M. Fields and J.M. Cowley, Acta Cryst. A34 (1978) 103.

[161] W. O. Saxton, Computer Techniques for Image Procesing in Electron Microscopy, Academic Press, New York (1978).

[162] J.C.H Spence, Experimental High Resolution Microscopy, Oxford University Press (1981).

[163] eds. P. Buseck, J.M. Cowley and L. Eyring, High-Resolution Transmission Electron Microscopy and Associated Techniques, Oxford University Press, New York (1988).

[164] M. Abramowitz and I.A. Stegun, Handbook of Mathematical Functions, Dover New York (1970).

[165] E.H. Yoffe, Phil. Mag. 5 (1960) 161.

[166] S. Timoshenko and J.N. Goodier, Theory of Elasticity, McGraw-Hill Book Company Inc, New York (1934).

[167] R. Mindlin and D. Cheng, J. of Applied Physics no. 21 (1950) 926.

[168] A.E.H. Love, Mathematical Theory of Elasticity, Cambridge University Press, London, (1927), fourth edition.

[169] J. Ingram, J. of Applied Physics Vol. 40 no. 13 (1970) 5030.

[170] T. Corfiatis, MSc. Thesis, Victoria University of Wellington (1987).

[171] P. Callaghan, BSc(Hons) project.

[172] P.B. Johnson, PhD. Thesis, Victoria University of Wellington (1966).

[173] W.R. Wampler, private communication.

[174] W.R. Wampler, T. Schober and B. Lengeler, Phil. Mag. Vol. 34 no. 1 (1976) 129.

[175] W.R. Wampler, Doctoral Thesis, Institut fur Festkorperforschung, Kernforschungsanlage Julich (1976).

[176] D.H. Housden, private communication.

[177] P.V. Shigolev, Electrolytic and Chemical Polishing of Metals, 2nd Edition, Freund Publishing House (1974).

[178] J.W.Edington, Practical Electron Microscopy in Materials Science, Philips Technical Library Macmillan, Vols. 1-5 (1975).

[179] S. Kikuchi, Jap. J. Phys. 5 (1928) 83.

[180] M.v. Laue, Ann. d. Phys. Lpz. 23 (1935) 705.

[181] J. Gjonnes, Acta Cryst. 20 (1966) 240.

$$
\mathrm{R}-7
$$


[182] M.H. Loretto, Electron Beam Analysis of Materials, Chapman and Hall (1984).

[183] P.A. Stadelman, Ultramicroscopy 21 (1987) 131.

[184] J.R. Moon, Worked Examples in Stereographic Projection, The Institution of Metallurgist, Monograph no. 2 (1978).

[185] P.B. Johnson, K.J. Stevens and R.W. Thomson, Nucl. Instr. and Meth. B 62 (1991) 218.

[186] M. Flueli, Docteur es Sciences Degree, Ecole Polytechnique Federale de Lausanne (1989)

[187] M.F. Ashby and L.M. Brown, Phil. Mag. 8 (1963) 1649.

[188] M.F. Ashby and L.M. Brown, Phil. Mag. 8 (1963) 1083.

[189] P.B. Johnson, private communication.

[190] Semper manual, Synoptics Ltd, U.K. (1990).

[191] M. Born and E. Wolf, Principles of Optics, New York, Pergamon Press (1959).

[192] A.M. Stoneham, J. Phys. F: Metal Phys. Vol. 4 (1974) 1335.

[193] C.L. Cook, P.B. Johnson, O. Jedlinska and Z. Kolodziejski, unpublished work, Victoria University of Wellington.

[194] J.M. Zuo, J.C.H. Spence and M. O'Keefe, Phys. Rev. Letters, A34 (1978) 138.

[195] S.M. Ohr, Ninth International Congress on Electron Microscopy, Toronto Vol. 1 (1978) 326.

[196] K.G. McIntyre and L.M. Brown, Journal of Physique (1966) 178.

[197] M. Wilkens and P. Rapps, Phys. Stat. Sol. (a) 44 (1977) 173.

[198] P. Humble and A.H. Head, private communication.

[199] W. Jager, R. Lasser, T. Shober and G.J. Thomas, Rad. Eff. 78 (1983) 165.

[200] J.H. Evans, A. Van Veen and L.M. Caspers, Scripta Metallurgica (1983) 549.

[201] J.H. Evans, A. Van Veen and L.M. Caspers, Scripta Metallurgica (1981) 323.

[202] K. Shiraishi, A. Hishinuma and Y. Katano, Rad. Eff. Vol. 21 (1974) 161.

[203] H.R. Glyde and K.I. Mayne, Phil. Mag. 12 (1965) 919. 